


1 


1. 1905. Shull, George Harrison. Galtonian regression in the "pure line."

2. 1905. Shull, George Harrison. Species and varieties: their origin by mutation (a review).

3. 1907. Shull, George Harrison. Importance of the mutation theory in practical breeding.

4. 1907. Shull, George H. Hementary species and hybrids of Bursa.

5. 1907. Shwll, George Harrison. The significance of latent characters. Some latent characters of a white bean.

6. 1908. Shull, Geo. H. The composition of a field of maize.

7. 1908. Shul7, George Harrison. Some new cases of Mendelian inheritance.

8. 1908. Shull, George Harrison. The pedigreeculture: its aims and methods.

9. 1908. Shull, George Harrison. A new Mendelian ratio and several types of latency.

10. 1909. Shull, George Harrison. A pure-line method in corn breeding. 


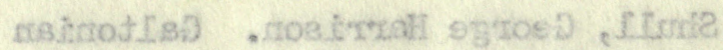
, does of

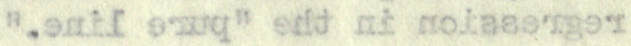

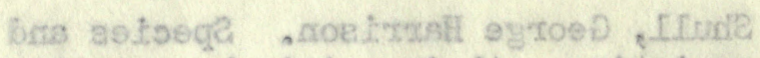

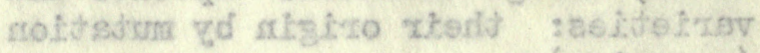

. vops as - (wo tvox B)

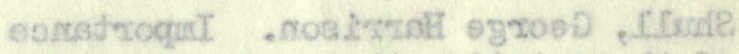

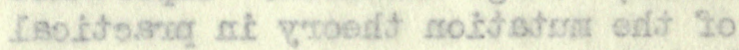
- entibeerd

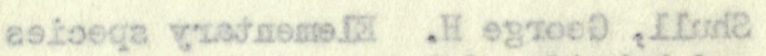
. Toer ad

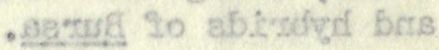

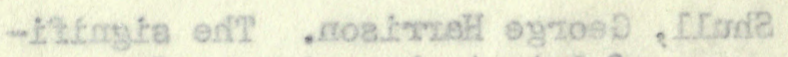

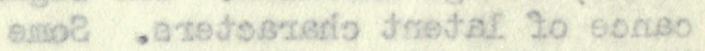

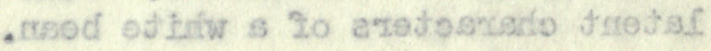

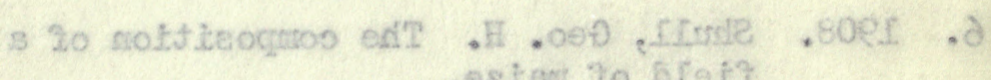

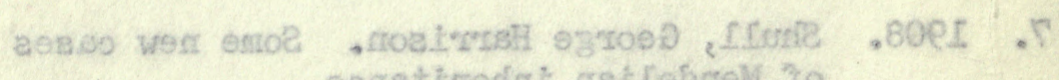

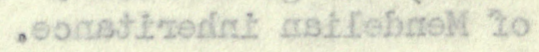

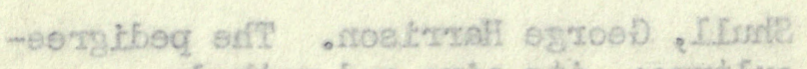

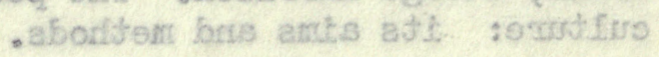

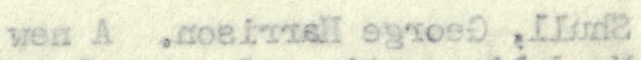
.8005 .8 $.80 p r$ ie

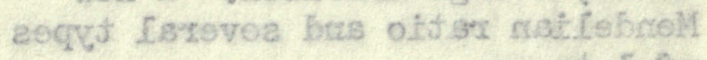
. Toes . - tonejat jo

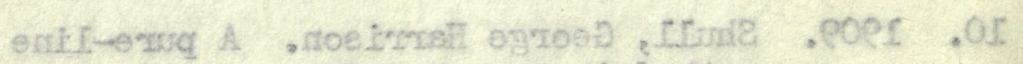
- gritbeord arios mí bonfítom 
11. 1909. Shull, George Harrison. A simple chemical device to illustrate Mendelian inheritance.

12. 1910. Shull, George Harrison. Germinal analysis through hybridization.

13. 1910. Shull, George Harrison. Hybridization methods in corn breeding.

14. 1910. Shull, George Harrison. Results of crossing Bursa bursa-pastoris and Bursa heegeri.

15. 1910. Shull, George Harrison. Color inheritance in Iychnis dioica $\mathrm{L}$.

16. 1910. Shull, George Harrison. Inheritance of sex in Lychnis.

17. 1911. Shull, George Harrison. Defective inheritance-ratios in Bursa hybrids.

18. 1911. Shull, George Harrison. The genotypes of maize.

19. 1911. Shull, George Harrison. Reversible sexmutants in Lychnis dioica.

20. 1911. Shull, Geo. H. Experiments with maize (notes for students). 

21. 1912. Shull, George Harrison. Inheritance of the heptandra-form of Digitalis purpurea L.

22. 1912. Shull, Geo. H. "Genotypes," "biotypes," "pure lines" and "clones."

23. 1912. Shull, Geo. H. "Phenotype" and "clone." ,

24. 1912. Shull, George H. Heredity (a review).

25. 1912. Shull, G. H. "Genes" or "gens"?

26. 1912. Shyll, George H. A pilgrimage to Brunn.

27. 1912. Shull, George Harrison. The primary color-factors of Lychnis and colorinhibitors of Papaver Rhoeas.

28. 1912. Shull, Geo. H. Hermaphrodite females in Lychnis dioica (a review).

29. 1913. Shull, George Harrison. Über die Vererbung der Blattfarbe bei Melandrium.

30. 1914. Shull, G. H. Heredity and sex (a review).

31. 1914. Shull, George Harrison. Sex-limited inheritance in Lychnis dioica $\mathrm{L}$. 

32. 1914. Shull, George Harrison. A peculiar negative correlation in Oenothera hybrids.

33. 1915. Shull, G. H. Genetic definitions in the New Standard Dictionary. 

[Reprinted from Torreya, Vol. 5, No. 2, February, 1905.]

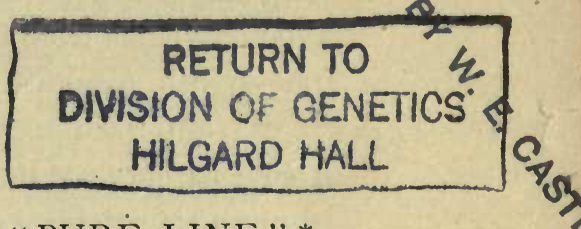

GALTONIAN REGRESSION IN THE "PURE LINE" *

By George Harrison SHUlL

Among the experiments undertaken this year at the Station for Experimental Evolution for the purpose of investigating the inheritancè of characters in plants, was one intended to be essentially a repetition of Johannsen's studies $\dagger$ in the inheritance of seed-weights in beans. The variety of Phaseolus viulgaris chosen for this study proved to be unsatisfactory from a technical standpoint and it is not proposed to pursue the experiment further with this material, though several subsidiary questions may be taken up in other plants. The relation between the results of Johannsen on beans and those of Galton on sweet-peas + have appeared on further analysis to be in need of reinterpretation rather than reinvestigation, and the writer feels justified, therefore, in taking this abandoned experiment as a text for such reinterpretation.

From a number of statistical studies upon various characters in man and animals and a single series of experiments in sweet-peas, Galton derived his law of natural inheritance and its corollary - the law of regression from mediocrity. $\|$ The law of natural inheritance is, briefly, that the offspring of any

* Presented before Section G, A. A. A. S., at Philadelphia, December 30, Ig04, under title of "Inheritance in Pure Lines."

†Ueber Erblichkeit in Populationen und in reinen Linien. Jena : Fischer, 1903. + Natural inheritance. New York: Macmillan \& Co., 1889.

|| This has frequently been called " regression toward mediocrity," but as the coefficient of regression is measured from the mean condition of the population confusion has arisen through expressing it in this way. Galton's own inconsistency in discussions of regression is doubtless responsible for this confusion. He first presents it clearly as a deviation from mediocrity, but later says there is "no regression at all" when this deviation is equal in the two kinships under comparison, and the coefficient of regression is unity. $C f$. Natural inheritance $95-98$ with $132-133$.) 
parentage, when considered in its entirety, inherits one-half its characteristics from its parents, one-fourth from its grandparents, one-eighth from its great-grandparents and so on. The law of regression from mediocrity points out that the children of extreme parents are not on the average so extreme as their parents, though they deviate in the same direction from the mediocre condition of the race. As an example of regression, take Galton's results on sweet-peas: The diameter of parent seeds which produced plants having on the average seeds of the same diameter was $3.94 \mathrm{~mm}$. Assuming this to be the mediocre condition of the strain he was using he found that whatever the parental deviation from this diameter the mean filial deviation was in the same direction, but only one-third as great. Thus the offspring from seeds $5.34 \mathrm{~mm}$. in diameter produced seeds having an average diameter of $3.94+\frac{5.34-3.94}{3}=4.41 \mathrm{~mm}$. (observed diameter, $4.44 \mathrm{~mm}$.).

Johannsen obtained similar results in beans when he compared the average weight of seeds in the offspring with the weight of the parent seeds, if the latter were selected solely with reference to the weight of the individual seeds and without regard to the pre-parental ancestry; but when he separated the individual "pure lines" he found that the mean weight of seeds in the offspring is the same on the average as that of the preceding generations in the same "line," in other words, plants produced from small seeds bear seeds of the same average weight as do plants which are produced from large seeds having the same ancestry.

By the "pure line" Johannsen means a series of individuals related only through the process of self-fertilization. On $a$ priori grounds it seems proper to apply the term to every series of individuals that do not combine the elements of two or more ancestral lines through the equivalent of a sexual process. Thus, so far as hereditary qualities are concerned, there should be no reason to expect in a self-fertilizing population, conditions different from those in a population related through budding or other method of vegetative reproduction, provided of course, that the self-fertilizing population has not been so re- 
cently modified by a cross as to allow the analysis and recombination of characters derived from different ancestral lines.

The complete return of the offspring of an extreme parent, to the mean condition of the "pure line" to which it belongs, or in technical language the entire want of "regression" in the "pure line," is presented by Johannsen as a fundamental exception to the conclusions of Galton.

Weldon and Pearson have criticized * the work of Johannsen in considerable detail and although the tone of their criticism is adverse throughout, they grant that his main contention may well be true, that small seeds and large seeds of the same plant do not give rise to plants bearing small seeds and large seeds respectively. If read aright, their criticism must be held to be confirmatory in so far as Johannsen's data are capable of biometric analysis. Certainly their conclusion that his results are closely identical with those found for other plants and for animals when we compare mean parental and mean filial characters, agrees precisely with that reached by Johannsen, for these means represent the condition in the population or mixture of several "pure lines," and not in the individual "pure line."

The relation between this work of Johannsen and that of Galton on sweet-peas may now be considered. In the first place, the actual results were the same when the treatment of the material was the same, and in so far the work of Galton was confirmed; but when the "pure lines" were followed separately they were found to offer an apparent exception in the complete return of the offspring of extreme parents to the mean condition of the "pure line." Instead of this being fundamentally opposed to Galton's results, however, it is the condition which should have been derived a priori from Galton's " Law of natural inheritance."

Regression is lucidly explained by Galton † as due to the fact that the child inherits partly from his parents, partly from his more remote ancestry, and that if " traced far backwards his ancestry will be found to consist of such vafied elements that they are indistinguishable from a sample taken at haphazard from the

* Inheritance in Pluseolus vulgaris. Biometrika, 2 : 499-503. N 1903.

† Natur.l in'heritance, I05. 
general population, ... in other words it will be mediocre." Now, if the mean condition of the parental generation and of each preceding generation in the same line deviates to the same degree from the mean condition of the population, it becomes an inevitable inference that in so far as hereditary influences are concerned, the offspring must have the same mean character regardless of the largeness or smallness of the individual seeds from which those offspring have developed.

This "fixity of type" which Johannsen finds in the "pure line " was recognized by Galton in his treatment of pure breeds* and it seems strange that he did not perceive that his sweet-peas which he recognized and described as a self-fertilizing population were at variance with this fixity of type in the pure breed. Johannsen has brought harmony in Galton's results. where there was a previously unnoted discord, and has confirmed the laws of " natural inheritance" and of "regression from mediocrity" as applied to the characters of self-fertilizing populations.

An important point which is brought out by these results of Johannsen both from a scientific and an economic standpoint is that the weight or size of an individual seed is not the hereditary unit, but the character of all the seeds of each plant considered as a whole. A plant which produces small seeds in general, may produce some seeds which are larger than the smallest seeds of another plant which produces large seeds in general, so that when the student of heredity wishes to use seed-characters or presumably any other repeated character, he must seek the general condition of the character in question in each plant and not depend upon the character of single seeds or single other repeated organs.

The economic application of this important principle is obvious. It has been very generally maintained by horticulturists that varieties deteriorate as the result of the selection of small seeds, tubers, etc., for propagation, but this proposition, while satisfying a certain sense of logic, has rested on no scientific research. The fixity of type in the "pure line" which now appears to be established, shows that no such deteriorating effect

* Natural inheritance, 189. 
will be produced so long as the seeds are large enough to produce vigorous plants.

The farmer and the plant-breeder may plant the small potato tubers or the small seeds without any danger of deterioration in the yield and quality of the crop provided they select these tubers or seeds from plants which yield the largest quantity and the finest quality of tubers or of seeds.

Station for Experimental Evolution,

Cold Spring Harbor, Long Island. 


The unusual simplicity, directness and beauty of the language used, the purity of its Anglo-Saxon English, in connection with the largeness of its theme, renders the new book at once a classic, and although "Die Mutationstheorie" must always stand as the epoch-making work, it is "Species and Varieties" that will be found most frequently back to back with Darwin's "Origin of Species" on the shelves of the general libraries, and that will make the name of de Vries known as Darwin's is to every man and woman of intelligence regardless of vocation.

As compared with "Die Mutationstheorie," the new book shows many evidences that the author has profited by the discussions which have been aroused by that work, and he has very carefully defined his position in regard to points in which he has been misconstrued. Ardent Darwinians immediately attacked the new theory because it appeared to be offered as a substitute for the theory of "Natural Selection." In evident response to these attacks, the author has joined his views in a masterful way to those of Darwin, showing that there is no conflict, and making the reader feel that the theory of mutation was the next step. logically, as it certainly has been the next important step historically in the development of a satisfactory conception of the origin of specific and varietal differences.

The basis of the author's views is the conception of characterunits as the ultimate bearers of heredity, a conception that, though seemingly too simple and inelastic to be entirely satisfying to the physiologist, has been brought into the greatest prominence and furnished support amounting at least to partial demonstration in the work of Mendel and of those who have since confirmed and extended Mendel's results, in the renaissance and extension of which Professor de Vries himself had such prominent part.

Recognizing as did Darwin that by far the greater part of our knowledge of evolutionary processes is necessarily based upon the results of economic practice, Professor de Vries has made a careful experimental analysis of horticultural and agricultural processes, and it is this part of his work which commends itself especially to the thinking scientist. 
By showing that the years devoted by the horticulturist to "fixing" new garden varieties have for their purpose the elimination of the effects of "vicinism," $i$. $\epsilon_{\text {. }}$, the chance crosses with neighboring species or varieties, and by distinguishing between ever-sporting varieties and those which possess only an ordinary degree of fluctuating variability, the way has been cleared for a proper appreciation of the true relations between the garden and nature. It is doubtful however whether physiologists will agree that the cases of "double adaptations" in nature, and the relation of juvenile to adult leaf-characters, are to be classed with the ever-sporting varieties of the garden, for in the former cases definite laws of occurrence of the alternative characters are discernible, while in the ever-sporting varieties no such laws have yet been detected and they seem in many instances to be closely related to fluctuating variations.

The book is divided into six sections... After an introductory lecture on the theories of evolution. and methods of investigation, the conception of elementary species as distinct from systematic species is developed, and a definite and distinctive significance is attached to the term, "variety," which is quite different from its usually loose usage for any assemblage of forms. less extensive than the systematic species. A variety as conceived by de Vries is not qualitatively like a species, being distinguished from the species to which it belongs and from which it has been derived in the possession or lack of some single definite character, or two or three single characters at most while species differ from one another in almost every character. The several different kinds of varieties, progressive, retrogressive, degressive, and ever-sporting, are thoroughly considered, along with the included subjects of latency and atavism.

The fifth section deals with mutations, the evening-primroses naturally having an important place, but the number of other fully authenticated cases described will doubtless give surprise to some readers who may have thought that the mutation theory rests only on the behavior of Onagra Lamarckiana.

The last section is devoted to individual and partial variability or "fluctuation" as it is called. This process, which has. been 
held by Wallace and the "Neo-Darwinians" as practically the only source of evolutionary changes, is held by Professor de Vries to have no effect whatever in giving rise to new specific and varietal distinctions, though it is of great importance both in nature and in culture, in that it allows a certain amount of adaptive change or amelioration within the species.

The editor professes to have changed as little as possible the original diction of the author, and for this the reader will be grateful both because it leaves unmodified the simple, genial flavor of the author's personality and because no material change is conceivable which would not have resulted in a more involved style. Some changes might have been introduced, however, which would have been distinct improvements, and it is to be hoped that in succeeding éditions these changes will be made. Thus the description of the zygomorphic or bilateral flowers of Digitalis as "symmetrical" is using in an unusual though literally correct sense a word that has long been in use in descriptive botany with a totally different meaning. Another even less desirable practice of quite similar character is the interchangeable use of "retrogression" and "regression" for the mutative loss of a character. "Retrogression" was the term first applied by the author to this process and there is no reason why it should not be used exclusively in biological terminology in this very definite sense. "Regression" already has a distinctive significance in connection with "fluctuation" and is used in its proper sense in Section F. which is devoted to that subject. Much confusion will be avoided if in future editions "retrogression" be substituted for "regression" wherever the mutative loss of a character is intended. An added complication in this connection is found on page 22I, where, presumably by a typographical error, " degressive evolution" is rendered " regressive evolution." A number of other typographical errors occur, but in most cases the context prevents misinterpretation. Aside from these the press-work leaves little to be desired.

The year 1904 will always be memorable in the annals of American science because of the number of distinguished foreign scientists who visited this country during that summer. Of these 
none was received with more genuine appreciation and honor than Professor de Vries. No more fitting memorial of his summer in America could have been left to his delighted hosts than this series of charming lectures on the most fundamental problems of biology, and one may safely predict that the work will further stimulate the interest that has awakened everywhere in experimental research in variation and heredity, the two fundamental processes of organic evolution.

George Harrison Shull.

Station For EXPERIMENTAL Evolution,

Cold Spring Harbor, New York, April, 1905. 




\section{IMPORTANCE OF THE MUTATION THEORY IN PRACTICAL BREEDING}

BY

GEORGE HARRISON SHULL

Cold Spring Harbor, N. Y.

2 atify. ens. 


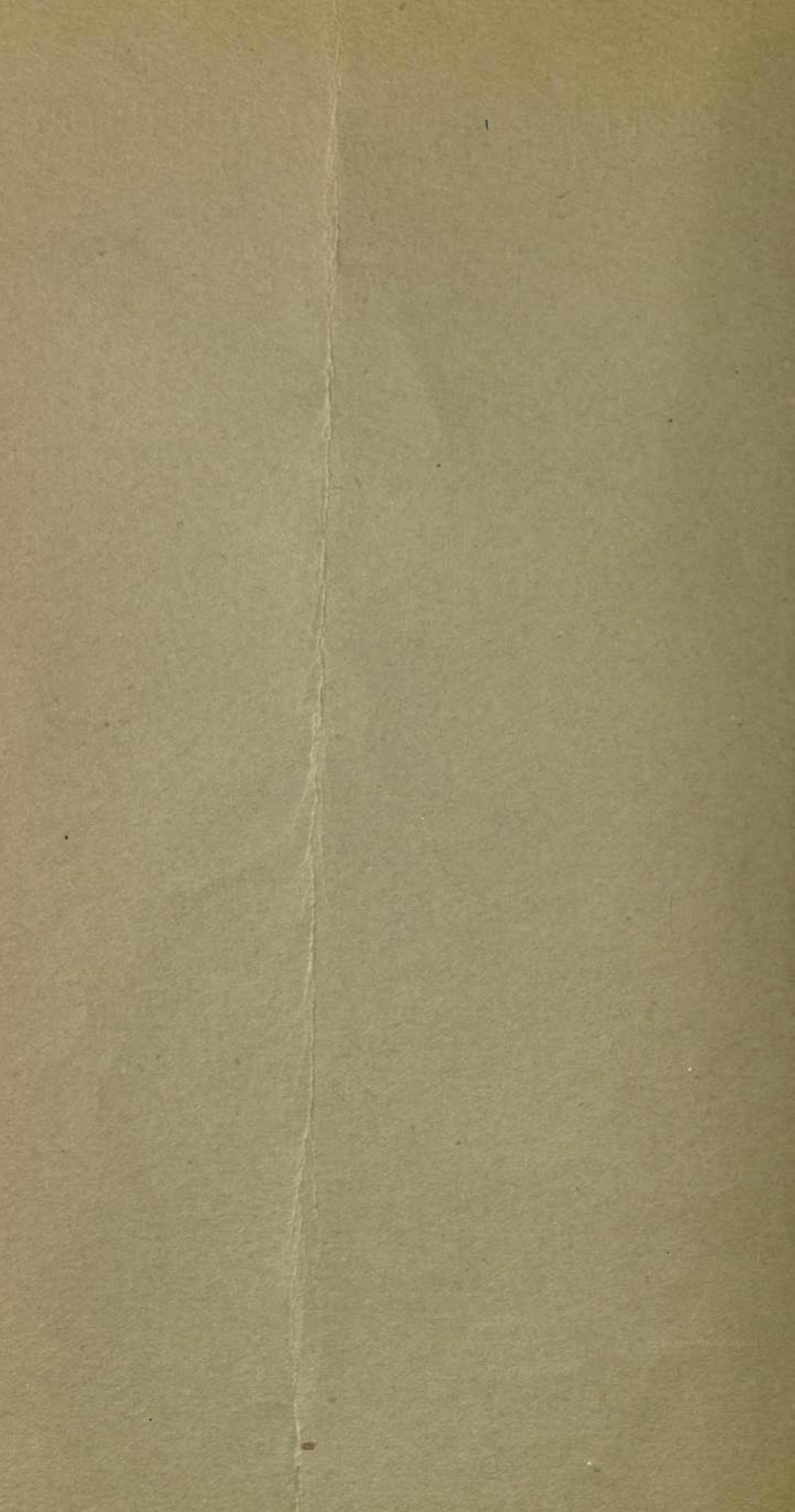




\section{IMPORTANCE OF THE MUTATION THEORY IN PRACTICAL BREEDING.}

By George Harrison Shuld, Cold Spring Harbor, N. Y.

The time is long past when the practical man who is looking for immediate economic values is inclined to ignore the work done by the devotee of pure science. Conversely, the scientist appreciates as never before, the reciprocal relation existing between his work and that of the man who would turn every available resource to the production of something useful to man.

Nowhere is this improved relation between the theoretical and the. practical better exemplified to-day than among breeders, and the establishment of the American Breeders' Association is a noteworthy indication of the existence of this improved condition. At the same time this organization fosters and promotes the reciprocity that shall increase the volume and the value of both scientific and economic breeding.

It is now a truism that all economic breeding must be in as great degree as possible scientific, and that all scientific breeding is likely to prove in the long run economic. Much of the scientific work being done, seems rather remotely if at all economic, and it is not always easy to indicate wherein any specific result is of practical value. Experience has shown, however, that all science is so closely and so complexly interrelated, that few scientific researches are conducted, whose economic bearings do not sooner or later become manifest, though their true economic worth may not be recognized for years.

While it is not fair to the scientist to insist that he shall be able to point out the economic value of all of his results-in the search for truth he must not be so hampered-it is fair to ask of science that, when any of its results have a large and important bearing upon economic problems, these results shall be made as available as possible for the use of those who can turn them to immediate practical account.

The mutation theory, formulated by de Vries and supported by a great mass of data which he had gathered during twenty years of careful and energetic work, has this large bearing. It struck a fundamental key in biological science, and is now one of the principal foci about which biological research and controversy are centering. Almost daily some new scientific support for the theory is given from 
experimental sources, while with similar frequency controversial papers unsupported by experimental evidence, are being directed against it. Under these conditions it is not difficult to foresee the outcome. In science, truth will prevail, and the accumulation of experimental data can never be overthrown by volumes of speculative character with whatever of warmth or asperity they may be written. The fact that the mutation theory is a subject of controversy need not concern us here, however, since even its most strenuous opponents grant that it is supported by garden experience. As the practical breeder is little concerned with what goes on outside of field and garden cultures, there can be no controversy regarding the truth of the mutation theory in its applicability to economic breeding.

The central doctrine of the mutation theory, is the origin of new forms-whether we call them species, elementary species, varieties, or strains-by the sudden appearance of an aberrant specimen that gives rise at once to the new strain by producing offspring like itself. The alternative conception is that usually attributed to Darwin, in which new hereditary lines are established by the gradual cumulative action of the environment in selecting those slight variants which are best qualified to meet the conditions under which they must live.

Every breeder is familiar with the large number of unexpected variations or sports that have been the starting points for new strains both in plants and in animals; and the value of these sports to the breeder is too well understood to need comment. It should be said, however, that there is grave danger of ruining the reputation of the words "mutant" and "mutation," and I earnestly ask your assistance to prevent this. Certainly not every aberrant form we see is a mutant, and when plants are capable of propagation by some vegetative means, as by bulbs, tubers, buds, cuttings, grafts, etc., many striking individual variations may be preserved and extensively multiplied, which are not mutations at all. No combination of characters resulting from the resolution and recombination of the parental qualities in hybrids ought ever to be called a mutant, however striking or unexpected it may be, because in these cases no really new quality has been produced, but only new combinations of qualities already in existence. Mutations can only be recognized as such with certainty when they arise in pure-bred strains. They are not to be distinguished from fluctuations by being large and striking variations but by the fact that they represent a fundamental change in the internal composition or structure of the vital substance, which renders the characteristic qualities of the new form transmissible through sexually produced seeds. Variations that are thus transmitted are often very insignificant, quantitatively, compared with others that are not so transmitted. We must be careful, therefore, not to handle the word "mutation" too lightly, since we can know that a plant is a mutant only after a sufficient number of seed generations have been produced to demonstrate the permanent character of the variation.

Many economic varieties are the result of hybridizing natural species, and this is especially true of perennials, which possess means 
of vegetative reproduction, but the number which are the result of mutations and of the crossing of the products of mutations which have arisen in gardens, is enormous. If the mutation theory holds for plants and animals in a state of nature, and this appears daily the more probable, then mutation is the basis for the origin of every permanent variety or strain. In nature, mutations have been preserved because they were adapted to the life conditions in which they originated; in the garden they have been preserved because they pleased the eye of man or promised to minister to his wants or needs.

As a typical instance of the utilization of mutations in the production of valuable modifications in an otherwise comparatively worthless plant, take the little red poppy of the English cornfields, known to the botanist as Papaver rhoas. Until 1882 this plant had received little attention from the horticulturist. Few and slight variations had doubtless been observed, but the form, size, and color of the flowers were fairly constant as still seen to-day in the uncultivated specimens growing abundantly as weeds in the English fields. A quarter of a century ago a mutational change in the color of the flowers of one of these little poppies attracted the notice of Rev. W. Wilks, the present secretary of the Royal Horticultural Society. He removed the plant to his garden and raised numerous seedlings from it. Without crossing with any other species he developed in a very few years the beautiful Shirley poppy with all its numerous color variations, ranging from the intense red with a black cross in the center as seen in the original wild form, through shades of red and pink to salmon and white and nearly sky-blue with a white or black cross, or a black cross margined with white, or no cross at all, with the petals margined with red, white, or blue, and often with the most delicate shadings, veinings and stripings. Owing to the methods used in the development of the Shirley poppy, many of the steps are not as well known as would be desirable, but the fact that none of the new qualities were brought in by hybridization leaves all to be explained by mutation.

Other equally impressive examples of the myriad forms that may arise in this way are seen in stocks, sweetpeas, and garden beans, and out of your own knowledge you may add many more quite as typical. These instances are typical of all the familiar cases not only in that they represent the production of numerous new constant forms without hybridization, but also because they agree with the general experience that the sports that have been used as the starting points of new strains have been those that had to do with some large and striking character, such as color of the flowers, cut or color of the leaves, habit of branching, etc. In other words, these striking variations have been observed and utilized in the formation of new strains not so much because they were sought for and found, but because they could not be overlooked.

In all such cases the individual plants stand out with such prominence that seed of the mutant is likely to be saved apart from the seeds of the parental form, thus leading to its rapid segregation. Some recent results of breeding show that mutation is just as preva- 
lent in grasses and grains as in flowers, but they have not been noticed generally because the external characters which distinguish each newly arisen form from its parent, will be observed in most cases only upon careful examination by a trained eye. Yet the value of these mutations in the field crops is economically greater than that of the striking changes that have added to the attractiveness of our flower-gardens, because, however small or insignificant may be the external characters by which the several mutants of grass or grain may be recognized, each elementary form has its own characteristic size, shape, weight, number, and nutritive value of the seeds, adaptability to particular climatic conditions, resistance to disease, or the attacks of insects, etc.; and some of these new forms are sure to prove distinctly better in some or all of these respects, than those already in cultivation.

This emphasizes one of the most important gains that come to plant breeding through the recognition of the mutation theory. When sports or mutants are recognized as normal products of a natural process, the eye of the breeder learns to seek for mutational variations, and this search must result in the discovery of many instances of mutation of lesser magnitude-though possibly of even greater economic value-than was formerly required to force themselves upon his attention. He will also look for mutations in plants whose individual characters had before passed almost unnoticed. It is plain, therefore, that the greatest advance to result from the mutation theory will be made in the breeding of those economic plants in which the individual loses itself to a great extent in the population, as is the case in the field crops, for in these it indicates the desirability of handling the individual in much the same manner as it would be handled in the development of a new variety of flowers.

In the various grass, grain, clover, cotton, and other field crops that are raised from seed, most of the work of improvement has been carried forward thus far on the basis of mass-selection instead of individual selection, the chief incentive to this method being the general belief in the injurious effects of in-breeding. Many excellent gains have been made by this method, but it is now obvious that it is not calculated to produce either the best attainable result or the largest return for a given expenditure of energy, and these are the prime economic considerations. Rimpau required twenty years for the isolation of the famous Schlaustedt barley which has added so largely to the value of the German barley crop, and this length of time was consumed because he did not realize that his problem was one of isolation instead of fixation. It is probable that the use of some good isolation method would have accomplished an equal result in onefourth the time.

Every method which carefully tests the transmissible characteristics of the individual, and bases its selections upon performance records is in essential harmony with the conception that each economic variety is a composite group of distinct elementary forms that have origi- 
nated and do originate one from another by mutation. Several such methods have already been devised and their value is becoming daily more appreciated. One of the earliest of these isolation methods was that used by Vilmorin in the improvement of the sugar beet. It is known as "Vilmorin's Isolation Principle," and consisted as applied to beet culture, in the growing of the offspring of individual seed plants in parallel rows-one row from each parent-and the use of a portion of each row in determining the percentage of sugar of the particular hereditary line to which that row belonged. Seed plants for the next year's crop were then selected from the rows showing the best performance record, $i$. e., the highest sugar content associated with other desirable qualities. Johannsen has demonstrated that this method is fundamental in the improvement of beans by selection, since what appeared to be a homogeneous variety possessing only fluctuating variations was found capable of being at once separated into a number of distinct hereditary lines each with its own characteristic size, form, and weight of seed, width of hull, etc. The earrow test is essentially the same method applied to the breeding of corn.

"The centgener method," designed by Mr. Hays for use in grainbreeding experiments at the Minnesota State Experiment Station, is one of the best and most complete isolation methods yet devised, and the splendid results in the immediate discovery of several wheats of surpassing merit have brought the method into deserved prominence.

To still another agricultural genius is due the credit of having worked out an isolation method in the breeding of economic seed crops, and of having achieved noteworthy results quite independently of the mutation theory, but his method like those already mentioned owes its preeminent success to its accord with that theory. This man is Dr. Hjalmar Nilsson, Director of the Swedish Agricultural Station at Svalöf. Dr. Nilsson has doubtless had from the first a clearer conception than had any one else, of the true constitution of the economic crops, for within a year after he was made Director of the Svalöf Station in 1890, he had recognized the composite character of the several named horticultural and agricultural varieties, and had decided that the elementary species is the unit with which the economic breeder must deal. Up to that time the work of the Swedish Station had consisted largely in the testing of named varieties, each variety being accepted as a horticultural unit; but, upon the adoption of the new point of view, the director and his corps of assistants set to work to separate the elementary forms making up the several varieties, and to study and tabulate their characteristic differences. They found that every elementary species has its own normal mean condition of its several morphological and physiological features including all those characters which determine the success or the failure of an economic crop. In the fifteen years during which this discovery has formed the basis of operations at Svalöf, that station has become famous for its achievements, despite the fact that all of its publications have been 
rendered nearly inaccessible to the breeders of other countries by being printed exclusively in the Swedish language.

In the last five or six years, careful pedigreed cultures of a large number of these isolated elementary forms have been made at Svalöf, and a number of mutants have been seen to originate in purebred, carefully guarded lines, so that there can be no doubt that the elementary species of the various grains are the products of mutuation either directly or through the combination and resolution of the characters of two or more other elementary species by means of hybridization. Indeed, every logical consideration as well as all the experimental data now available lead conclusively to the view that the very existence of distinct elementary species differing from each other by unit characters is indubitable evidence that they originated by mutation.

In discussing the importance of the mutation theory, it is not to be forgotten that mutation and Mendelism are counterparts of each other and both depend for their validity, upon the existence of unit characters. These three-mutation, Mendelism, and unit charactersare all part and parcel of one consistent view of the world of living matter. Together they constitute the new biological knowledge which has revolutionized the discussion of breeding problems, and which has already entered in large measure into the methods employed by the breeder. Of the supreme importance of this new discipline as a whole nothing need be said, and it would be futile to attempt to dissociate the theory of mutation from the conception of unit characters and Mendelian inheritance in order to weigh the importance each would have for practical breeding when standing alone. Neither Mendelian inheritance nor the mutation theory could have assumed by itself the sudden importance they have now attained; but the appearance of both at the same time, both telling the same story of the existence of character-determining units, have wrought so great a change in biological thought as to amount practically to a revolution.

Always in revolutionary times the sane, conservative man guards himself against being carried too far afield, and it may not be out of place to say here, that although we must recognize the great value of the new theories, they are not everything that the breeder needs to keep in mind. There are many characters that are not unit characters, variations that are not mutations, and inheritance that is non-Mendelian, and such characters, such variations, and such inheritance are also important to the breeder. Indeed, another great advantage the breeder gains through recognition of the mutation theory comes from the strong emphasis that theory places upon the fact that variations are not all alike. A sharp distinction is drawn between mutations and fluctuations and the breeder who would derive the greatest possible benefit from the mutation theory will carefully keep this distinction in mind to serve as a guide to the technique that may be expected to best meet the requirements of each particular case.

In the selection of a permanent seed-strain, mutation must be the 
basis either directly or indirectly, but many elementary forms have a wide range of fluctuating variability and there is in such cases the capacity for great improvement within the elementary species. In the improvement of strains by the selection of the best fluctuating variants, however, unceasing attention is necessary: for the possibility of fixing an extreme of the fluctuating kind lacks experimental support. No matter how long the selection of the same extreme is carried on, the instant the selection ceases to counteract the regression, a deterioration begins, and in the course of time there is a complete return to the norm of the particular species or strain in question. When a new elementary species originates by mutation, a new norm is produced and if this is a higher norm with respect to the aggregate of economic features than that possessed by the parental strain, a distinct and permanent advance has been achieved. A new range of fluctuating variability has also been produced and if this range is greater than that of the parent, new possibilities of improvement within the elementary species is provided for.

It has been found that mutants arise in a species with something like a constant frequency and that the economical way to secure mutants is to raise enormous numbers of seedlings among which to search for them. It is confidently expected that at some not distant time, ways will be found to increase the relative number of mutants, or even to influence the direction of mutation. A first important step has been made in this direction by Dr. D. T. MacDougal, the Director of the Department of Botanical Research of the Carnegie Institution at Washington, who has apparently induced mutations in several species by injecting into the developing ovaries various solutions of mineral and organic substances, but aside from the suggestion offered by these interesting experiments, we seem to be at present entirely at the mercy of nature. However, nature treats us well if we stand equipped with as complete understanding of her ways as science provides, ready to take advantage of and preserve every advance she makes.

In summing up, it may be said that the value of the mutation theory in its bearing upon practical breeding lies in the better appreciation it gives of the significance of the phenomena with which the breeder must deal. New methods and the extension of old methods to new material are indicated, the characteristic features of the method being the production of enormous numbers from which to select, the complete isolation of each individual whose characters suggest the possibility that it may be the starting point of a new strain, the complete control of the fertilization processes, and the rearing of the offspring of the guarded plants under conditions that will allow all distinguishing characteristics to reach a normal development.

But even if no changes in method were suggested-if old methods adopted empirically should be found to harmonize perfectly with the theory so that no improvement could be made-the value of the mutation theory is great in the feeling it gives the breeder that he 
is handling the normal and eternal forces of nature. An added interest attaches to each new variation when it is realized that every instance of mutation is a part of the order of nature, and not a monstrous departure from natural conditions; and the sense of exaltation that comes from dealing with the processes and products of universal evolution cannot fail to increase enthusiasm and efficiency in the breeder's work. 




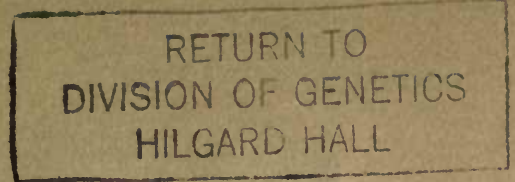

[Reprinted from Scrence, N. S., Vol. $X X V .$, No. 641, Pages 590-591, April 12, 190\%.]

\section{ELEMENTARY SPECIES AND HYBRIDS OF BURSA ${ }^{2}$}

THE rearing of over 20,000 pedigreed specimens of Bursa Bursa-pastoris (L.) Britton, has demonstrated the presence of at least four elementary species, all of which breed true when self-fertilized or crossed within the limits of the same elementary form. From over thirty hybrid families the fact is derived that these several elementary forms hybridize in strictly Mendelian fashion, each form which went into the cross coming out again in the perfectly pure extracted dominant or recessive form of the parents.

The existence of elementary forms in nature within the recognized limits of the species, differing from one another as do the elementary species of Bursa, in the possession of definite characters that behave as hereditary units, presents a condition that is not unique, but one which has an important bearing upon some of the questions that have been recently discussed. Several atypic plants have appeared in the cultures, which have bred true to their atypic characters, when the assumption that they were due to chance crosses would have required that they split into the atypic and typic forms in the ratio $3: 1$. These occurred in families of which the pollination was not guarded and their status as

${ }^{2}$ Extract from paper read before the joint meeting of Section F and Section G, A. A. A. S., New York City, December -, 1906. 
mutants is in consequence not considered sufficiently secure to be presented in detail at present as proofs of mutation. The fact that throughout these cultures the differentiating characters behaved as units in the Mendelian sense appears to me indubitable evidence that the several elementary species have arisen through mutation and hybridization.

On the basis of allelomorphic differences between different species these elementary species of Bursa represent the closest possible relationship between forms belonging to distinct types, since they are seen in most cases to differ from each other by a single distinguishing unit or by two units at most. The fact that Bursa Bursa-pastoris is everywhere recognized as a variable species, and the presence of several distinct forms in each of the localities from which material was derived for these studies, show that these elementary species generally grow in actual contact with each other. There is neither geographical isolation nor complete physiological isolation, yet these nearly related elementary forms maintain themselves absolutely distinct. This should convince any one who may still entertain any doubt regarding it that in the presence of Mendelian hybridization, no form of physical isolation is necessary for the maintenance of closely related forms. Moreover, these observations on Bursa show that Mendelian behavior is a strictly normal natural process and in no manner dependent upon the artificial conditions supplied by garden practise.

When a new form arises, differing from the parent in one or several unit characters, these new characters may be either dominant or recessive to the corresponding character of the parent. Less rarely they are neither dominant nor recessive. The chances of survival under these several possible conditions seem 
to need discussion, since, in several recent conversations, I have found the notion to prevail that recessiveness is a handicap, and allusions based upon the same idea have found their way into print. This view is quite erroneous; not only has the dominant form no advantage in the competition which the newly arisen elementary species must encounter, but it can be shown that under certain conditions the reverse is true.

If the dominant and recessive forms are equally adapted to the particular environment in which they live, there is absolutely no advantage in favor of either. The second generation of a Mendelian monohybrid contains the same number of pure recessives as of pure dominants, and the heterozygotes continue to produce in each succeeding generation just as many recessives as extracted dominants. The chances that extracted dominants will selffertilize or that they will cross with other extracted dominants are exactly the same as the chances that recessives will self-fertilize or cross with other recessives. In like manner extracted dominants and recessives will cross with heterozygotes with equal frequency, and the quantitative results in these two cases will be exactly parallel, in one instance giving fifty per cent. of pure dominants, in the other case fifty per cent. of pure recessives. In this equal fashion the struggle will continue indefinitely so long as the premise holds, that the two forms are equally well suited to the conditions under which they must grow.

The situation is different when natural selection favors one or the other of the competing forms. A single extreme case will suffice to demonstrate: Let us suppose that the new form is dominant over its parent, but so poorly adapted to the particular habitat in which it originated that it can not successfully compete with the parent form. All the 
hybrid offspring resulting from crosses between the mutant and its parent will have the unadapted new form, and when the selection becomes extreme, not only will all the purebred specimens of the new form be destroyed, but all the hybrids as well, and in this way every vestige of the new form will be entirely lost. Assuming, on the other hand, that the mutant is recessive to its parent but that in other respects the conditions are the same as before, the extreme selection that is assumed to destroy all the recessive individuals, leaves the heterozygotes living because they have the successful form possessed by the parent species. These successful heterozygotes give rise to a progeny in the next generation including the recessive form, and also a considerable percentage of heterozygotes that may carry the form on to still another generation, and in this way the recessive mutant may be preserved indefinitely under the protection of the dominant characteristics of its more successful parent. Such prolongation of the life of a recessive may serve to tide it over times of special stress, or may continue its existence until the various distributing agents have carried it beyond the limits of the habitat in which it is a failure into others in which it may become a success.

George H. Shull

Station for Experimental Evolution,

Cond Sprivg Harror, L. I. 


\section{RETURN TO \\ DIVISION OF GENETICS \\ HILGAK HALL}

2 THE SIGNIFICANCE OF LATENT CHARACTERS

\section{SOME LATENT CHARACTERS OF A WHITE BEAN}

BY

DE. GEORGE HARRISON SHULL

[Reprinted from Science, N. S., Vol. XXV., No. 646, Pages 792-794, May 17, 1907; No. 647, Pages 828-832, May 24, 1907.] 

[Reprinted from Sciencre, N. S., Vol. $X X V$., No. 646, Pages 792-794, May 17, 1907; and No. 647, Pages 828-832, May 24, 1907]

\section{THE SIGNIFICANCE OF LATENT OHARACTERS ${ }^{3}$}

THose of you who were present at the last annual meeting of the Botanical Society, at New Orleans, will remember that I presented a paper upon the latent characters of a white bean, showing that the appearance of two new characters in the $F_{1}$ hybrid offspring of a white bean when crossed with a plain brown or yellow bean, demonstrated the presence of a color-pattern, and of a pigment-changer as 'latent' characters in the white bean, latency meaning simply invisibility and not dormancy. On this basis it was predicted that in the second generation five forms would appear according to the well-known tripolyhybrid ratio, $27: 9: 9: 3: 16$. These forms in the order of the ratio are purple mottled, black (dark purple), brown mottled, brown, and white. I show you to-day samples of these five predicted types taken from the second generation.

The ratios of these several groups have not yet been determined because not all of the material has been worked over, but the presence of the predicted types-especially the presence of the two forms, plain black and brown mottled, which were not known to have ever occurred in the ancestry on either sidesufficiently demonstrates the correctness of my interpretation of the allelomorphic composition of the parents. Some additional unexpected types were found which must await further breeding experiments before their significance can be profitably discussed.

${ }^{1}$ Read before the Botanical Society of America, at New York, December 29, 1906. 
It will be remembered that the condition I assumed for these hybrid beans was used to bring into harmony with simple Mendelian hybrids the apparently anomalous results of Tschermak, Emerson, Lock, Bateson, Correns, Cuénot and Castle. The prediction that the same conception of latent characters in the sense of invisible, not inactive ones would without doubt give a solution to the intricate and otherwise apparently inexplicable behavior of stocks and sweet-peas, as studied by Bateson, was fulfilled with unexpected promptness, as the third report ${ }^{2}$ to the Evolution Committee presented in March, 1906, and published later in the same year, adopts the same theory and shows that in this way practically all of the apparent anomalies of stocks and sweetpeas may be explained upon the simple basis of typical Mendelian behavior without recourse to the hypallelomorphs or compound units earlier assumed by Bateson.

This complete demonstration that latent characters, at least in many cases, are not inactive units that may be rendered active by some unknown influence, but are, instead, units that produce a visible character only when acting in conjunction with one or more other units, justifies me in calling attention again to the significance of such characters.

In order to see the bearing of these results upon the process of evolution it is necessary to realize that what we call a unit character is not necessarily produced by the activity of a single allelomorph, and I consider it probable that few visible characters are so produced. It makes no difference how many internal units are involved in the production of any so-called unit-character, so long as there

${ }^{2}$ Bateson, W., Saunders, Miss E. R., Punnett, R. C., 'Experimental Studies in the Physiology of Heredity,' Reports to the Evolution Committee of the Royal Society, Report III., 53 pp., London, 1906. 
is a difference of only one unit involved in the cross. Thus, allelomorphs $A B C D E F G H$ may determine a single characteristic and $A B C D E F G$ an alternative characteristic. If plants having characters so determined are crossed together, they will behave as if these were unit characters, though according to our assumption one is determined by the presence of eight dominant units, the other by seven.

The best actual examples we now have of the compound nature of certain apparently simple external characters are seen in the splendid results of Professor Bateson's studies on stocks and sweet-peas. In stocks, for instance, canescence is found to depend upon the simultaneous presence of three dominant allelomorphs wholly uncorrelated and each acting in the normal Mendelian manner. In one strain of sweet-peas two such dominant units are necessary to produce any color whatever and another unit determines whether that color shall be blue or red. This condition produces the remarkable result that the first generation hybrid between two white-flowered parents have blue or red flowers.

Similar conditions were presented in two of the papers given Jesterday (December 28, 1906) on the joint program of Sections $F$ and $G$ of the American Association for the Advacement of Science, viz., the appearance of a ' latent' agouti factor in certain guinea-pigs, and an invisible red factor underlying black in certain fowls as reported by Dr. Castle ${ }^{3}$ and Dr. Davenport." The characters of both these apparently anomalous hybrid products were recognized as atavistic or reversionary. The same is true of the purple-flowered hoary stocks produced from glabrous white and glabrous cream-colored strains. The same was true of

Castle, W. E., 'On a Case of Reversion Induced by Cross-breeding and its Fixation.'

'Davenport, C. B., 'Reversion.' 
flower and seed-coat color of beans and peas as found by Tschermak and Lock, and is no doubt the correct explanation of the purple mottling in my hybrid beans. Indeed, so many instances are now on record in which a cross results in reversion, that generalizations can be made with some degree of security.

These reversions indicate that the original character was compound, being determined by the simultaneous action of two or more, possibly many, dominant units, and that the later specific or varietal derivatives were produced by the disappearance of one or more of these original units as a dominant characteristic. Thus in the example assumed above in which the original character was determined by the dominant units $A B C D E F G H$, the later derivatives may be $A B C D E F G h, A B C D E F g H$, $A B C D E f g H$, etc., through all the possible permutations. May we not perhaps get in this way a comprehensive view of at least the later stages of evolution as a process of analysis due to the disappearance of one unit after another?

All the visible variations of the present plant and animal world were once involved in some generalized form or forms, and the process of differentiation pictures itself to us as a true process of evolution brought about by the change of individual character-determining units from a dominant to a recessive state. This conception results in an interesting paradox, namely, the production of a new character by the loss of an old unit.

When I first became interested in the Mendelian discipline one of the most difficult things for me to understand was the fact that, somehow, every dominant character in a plant or animal finds its recessive counterpart in all of its near relatives not possessing the character in question. For a time credulity balked and I was compelled to look upon character-units as figures of speech. The origin 
of forms from a common parent by the loss of dominancy in its several character-determinants accounts for the general presence of a recessive unit, corresponding with each dominant unit, in all the nearly related forms.

No suggestion has been made as to the nature of the change by which a dominant allelomorph becomes recessive, but if this change be looked upon as a degenerative one which may be followed later by complete disappearance of the unit it would account for the fact that hybrids between nearly related forms are usually Mendelian, while those between more distant ones are not.

I may summarize briefly as follows:

(a) What appear to be unit characters may be, and probably usually are, compound characters.

(b) New characters appear by the change of one or more character determinants from the dominant to the recessive condition.

(c) Some of the partial products resulting from this process of analysis have no externally apparent distinguishing characteristic, and these supply instances of so-called 'latent' characters.

(d) Mendelian hybridization results in an $F_{1}$ which is a partial or complete synthesis of an ancestral condition.

(e) This conception gives an explanation of the general presence of recessive units corresponding to the dominant units in each closely related form.

( $f$ ) If the change from the dominant to the recessive condition is a degenerative process which may be followed by complete disappearance of a unit, an explanation is found for the fact that Mendelian behavior is a function of nearly related forms but not of more distantly related ones. 
SOME LATENT CHARACTERS OF A WHITE BEAN ${ }^{1}$

IN order to secure material to display as simple illustrations of Mendel's laws of dominance and gametic purity, I made reciprocal crosses last year (1904) among four different varieties of the common bush bean. These varieties were the 'Prolific black wax', with purple-black seeds, the 'Ne plus ultra' with yellow-brown seeds, the 'Long yellow sixweeks' with seeds of a light greenish-yellow color, and the 'White flageolet,' whose seedcoats are wholly without pigment, being transparent when saturated with liquids, but nearly white because of the inclusion of air when dry.

I have this year (1905) the first generation of the hybrids, and all the crosses behaved in the expected way except those in which the 'White flageolet' entered as one of the parents. The crosses between black and brown, black and yellow, and between brown and yellow showed in every case the complete dominance of the darker pigment over the lighter. Thus, the first-generation hybrids between black and brown beans and those between black and yellow were in every case indistinguishable in color from the black parent, whether the black bean supplied the egg or the sperm. Similarly the hybrids between the brown and yellow were in each case not to be distinguished from the brown parent. But the crosses between each of these three pigmented varieties and the 'White flageolet' gave $F_{1}$ hybrids so different from either parent that, if unknown, their origin would never be guessed. These seemingly anomalous hybrids were quite indistinguishable from one another, whether the pigmented parent was black, brown, or jellow. They were characterized by a dark purple pigment, and by an aggregation of the pigment-bearing

${ }^{1}$ Read before the Botanical Society of America, at New Orleans, December, 1905. 
cells to form a mosaic or mottled surface. Although these hybrids were quite unexpected, it was recalled that Tschermak ${ }^{2}$ had secured similar results in a number of cases, and Emerson $^{3}$ describes them also in crosses between 'Ultra' and 'Navy,' this being presumably the very same cross that I present here as 'Ne plus ultra' $X$ 'White flageolet.'

Very similar phenomena have been seen by Tschermak," Bateson, and Lock" in peas, by Tschermak ${ }^{4}$ and Bateson ${ }^{8}$ in stocks, by Batoson $^{5}$ in sweet peas, by Correns ${ }^{7}$ in Mirabilis, by Cuénot ${ }^{8}$ in mice, and by Castle in guineapigs. It is not because of the newness of the phenomenon, therefore, that I draw attention to the subject, but for the purpose of dis-

${ }^{2}$ Tschermak, E., 'Weitere Kreuzungsstudien an Erbsen, Leukojen und Bohnen,' Zeitschr. Landro. Versuchsw., 7: 533-638, 1904.

'Emerson, R. A., 'Heredity in Bean Hybrids,' Ann. Rept. Agr. Exp. Sta. Nebraska, 17: 33-68, 1904.

- Tschermak, loc. cit.

'Bateson, W., Saunders, Miss E. R., Punnett, R. C., 'Experimental Studies in the Physiology of Heredity,' Second Report to the Evolution Committee of the Royal Society, London, 1905.

'Lock, R. H., 'Studies in Plant Breeding in the Tropies,' Ann. Roy. Bot. Gard. Perideniya, 2: 299356, 1904.

${ }^{7}$ Correns, C., 'Ueber Bastardirungsversuche mit Mirabilis Sippen,' Ber. d.d. Bot. Ges., 20: 594-609, 1903; 'Zur Kenntniss der scheinbar neuen Merkmale der Bastarde. Zweite Mittheilung über Bastardirungsversuche mit Mirabilis Sippen,' Ber. $d$. d. Bot. Ges., 23 : 70-85, 1905.

- Cuenot, 'Le loi de Mendel et l'herédité de la pigmentation chez les souris,' Arch. Zool. expér. et gén., -: 27, 1902; 'L'herédité de la pigmentation chez les souris, (2me note), Arch. Zool. expér. et gén., 4 Se., 1: 33-41, 1903.

'Castle, W. E., 'Heredity of Coat-characters in Guinea-pigs and Rabbits,' Publ. Carnegie Institution of Washington, No. 23, 78 pp., Feb., 1905. 
cussing the phenomenon of latency in the light of the accumulated data.

The appearance of hereditary characters which are not traceable to the immediate ancestry offer the most difficult problems with which the student of heredity must deal. These characters may be recognizable as having belonged to the more remote ancestry of the form in question, i. e., they are atavistic, or they may be wholly new. Sometimes they. occur under known conditions, at other times there is no clue to the causes upon which they may depend.

These bean hybrids which possess characters not seen in either parent furnish good examples of latency and if we can determine whence the new characters came we shall be far advanced toward a correct conception of latency. Taking, for instance, the hybrids between the yellow and white and between the brown and white, two characters may be recognized as new, viz., the dark pigment and the mottled color-pattern. The fact that the very same characters appear in the hybrid offspring, no matter what the character of the pigmented parent, leaves little doubt that both of these new characters are traceable to the white parent, and we should seem to be warranted in saying that this white bean contains latent purple and latent mottling. Tschermak ${ }^{10}$ takes just the opposite view, however, and would say that the purple mottling is latent in the pigmented bean, and that the white bean acts simply as a releasing agent which allows the latent character to become manifest. Bateson ${ }^{11}$ also subscribes to the same view. On Tschermak's hypothesis it would appear to me a remarkable coincidence

${ }^{10}$ Tschermak, E., loc. cit.

${ }^{11}$ Bateson, W., Saunders, Miss E. R., Punnett, R. C., 'Experimental Studies in the Physiology of Heredity,' Second Report to the Evolution Committee of the Royal Society, London, 1905. 
that the black, brown and yellow varieties should all contain the same latent characters, and I am forced to the conclusion just stated, that the novelties which appear in these hybrids are directly derived from the white parent. The new characters thus lose the mystery that otherwise invests them. They appear not as released prisoners or awakened sleepers, which had for some inconceivable reason remained inactive, but are combination phenomena.

The color characters of these beans are not dependent upon a single pair of units, but upon three pairs, viz.:

1. Pigment vs. no pigment.

2. Modifier which changes pigment to purple vs. no modifier.

3. Mottled color pattern vs. self color.

Of these three characters, the brown and yellow beans contain only the dominant pigment character and might be represented by the formula, $\mathrm{Pbm}$; the black bean contains the pigment and the modification to purple, $P B m$; and the white bean contains both the modifier and the mottled color-pattern but no pigment, $p B M$. It is only because of the lack of pigment that these two characters possessed by the white bean are not apparent. They are latent only in the sense that they are invisible. Whenever the 'White flageolet' is crossed with any variety of self-colored bean, the three dominant allelomorphs, $P B M$, are brought together with the result seen in these hybrids, namely, a first generation characterized by dark purple mottled seeds.

The great advantage of this explanation over that of Tschermak is that it brings these apparently aberrant results into harmony with typical Mendelian cases, and allows with a reasonable degree of accuracy, a prediction as to the composition of subsequent generations.

On the assumption that the $F_{1}$ hybrids between the 'White flageolet' and either of the 
non-purple self-colored beans contain the three dominant allelomorphs, $P B M$, associated with the corresponding recessive allelomorphs, $p b m$, the following forms and proportions may be expected in the second generation. In each 64 plants belonging to the second generation there should be

$27 P B M=$ purple mottled like $F_{1}$.

$9 \mathrm{PbM}=$ brown mottled or yellow mottled.

$9 P B m=$ purple self-colored or black.

$9 p B M=$ white, exactly like the white parent.

$3 \mathrm{Pbm}=$ brown or yellow self-colored.

$3 \mathrm{pBm}=$ white (with the modifier but no mottling).

$3 p b M=$ white (without the modifier but with mottling).

$1 p b m=$ white (with neither modifier nor mottling).

Owing to the fact that the internal composition of the white beans has no external manifestation, the four white classes having different allelomorphic composition are indistinguishable from one another, thus resulting in a frequently found ratio for tripolyhybrids, $27: 9: 9: 3: 16$. $=64$

In this interpretation of latent characters are to be found explanations of several observed phenomena. Tschermak, Bateson and Emerson have noted that the behavior of a given character in one strain can not be used safely as a criterion for predicting the behavior of an apparently like character in another strain. A good illustration of this is seen on comparing with these hybrids of the 'White flageolet' bean, Emerson's cross between the ' Ultra ' and 'Marrowfat,' the latter being likewise a white bean. The $F_{1}$ hybrids were brown mottled, thus showing that the "Marrowfat' differs from the 'Navy' or 'White flageolet' in having no dominant pigmentchanging allelomorph. The gametic formula of the 'Marrowfat' is, no doubt, $p b M$, and in the table above, representing the $F_{2}$ of the 
cross between ' Ne plus ultra' and 'White flageolet' it is seen that one of the white derivatives from this cross has the samo gametic composition as the 'Marrowfat.'

Emerson attempted no explanation of the different behavior of these beans, simply presenting them as exceptions to Mendel's laws or as evidences of the limitation of the usefulness of those laws in predicting the results of hybridization. Practically all of the exceptional results obtained by him cease to be exceptional when we cease to look upon the products of his crosses as monohybrids with respect to seed color. His second generation hybrids were classifiable into four categories instead of the three he expected, but his expectation was based upon the assumption that the black, brown, white, etc., are unit characters, and that the mottled hybrids were simply mosaics or blends between the white and the self-colored parents. The simple assumption, demonstrated in my hybrids, that the pigmentation and the mottling are distinct unit characters, harmonizes his results perfectly, though the numbers with which he dealt were too small for the satisfactory determination of the agreement or disagreement with the theoretical ratios of a dihybrid.

In discussing the appearance of purple spotting as a novelty in peas, Lock ${ }^{12}$ follows Tschermak in referring the latent character to the pigmented parent, saying that "On crossing $A(B)$ ( $B$ being latent) with $a b$ we get: $F_{1} A B a b$ ( $B$ latent having become $B$ active)." If instead he had considered that $A b$ is crossed with $a B$, the $A$ producing the pigment and the $B$ aggregating it into spots, he would get the same $F_{1}$, namely $A B a b$, but would have avoided the difficulty of a capricious unit which may be active or inactive under condi-

"Lock, R. H., 'Studies in Plant Breeding in the Tropics,' Ann. Roy. Bot. Gard. Perideniya, 2: 299-356. See p. 341 . 
tions that can not be determined. He would then have had no latent character in any other sense than that it was invisible owing to the absence of pigment.

The explanation here offered is essentially that presented by Cuénot for mouse hybrids, in which one unit is assumed to give pigmentation and another to determine the color which this pigment will exhibit. Cuénot considers the various colors to be latent in the albino and he is supported in this respect by my hybrids, but I prefer not to call this character a latent pigment but an active pigmentchanger.

This reference of various colors to the action of a pigment-changer requires that the pigments upon which the various colors depend shall bear some simple relation to each other. I have made some preliminary studies on the pigments of these beans and have partially demonstrated this simple relation by converting the yellow and brown pigments to black by the use of alkalies but I have not yet been able to reverse the process. It is easily demonstrable that the black (dark purple) bean contains anthocyan, and this gives a simple explanation of the correlation between black seed-coats and red flowers, observed by Mendel and all other students who have chanced to use black-seeded peas or beans.

That the yellow, red, and black pigments of animals are closely related is also well known, and there can be no doubt that the 'latent black' which Castle ${ }^{18}$ reported in certain albino guinea-pigs is to be interpreted exactly as Cuénot's mice, the black being due to the presence of a melanizer which is a unit character wholly independent of the pigment-producing unit. The fact that half the gametes of this individual carried the so-called 'latent black' simply showed the animal to be heterozygous with respect to this allelomorph, and

${ }^{18}$ Castle, W. E., loc. cit. 
the extracted recessives which did not in subsequent generations produce any black offspring could not do so for the simple reason that the pigment-changing unit had acted in a perfectly normal way and had been absolutely separated out into the black offspring while its recessive counterpart was segregated with equal purity into the non-black.

A very important consideration in this connection is the frequency with which the new character is atavistic. This shows the process by which these various color varieties were originally produced. The original character was compound and the new variety was produced by the loss of one or more of the components. In other words these varieties are retrogressive. Beginning, for instance, with a purple mottled bean, one variety was formed by the loss of the mottling, another by the loss of the pigment, and another by the loss of the pigment-changer. Then by hybridization every possible combination of these three characters became the constant characteristics of distinct strains. When these varieties are crossed together the original variety may be reproduced by bringing together the several component parts of the original compound character.

There are still many mysteries regarding latent characters or qualities, but I believe the considerations here presented bring a large number of otherwise anomalous phenomena into perfect harmony with typical Mendelian cases of alternative inheritance. It appears to me certain that this conception of latent characters as invisible ones, which has already been used by Correns ${ }^{14}$ to interpret in part the behavior of Mirabilis hybrids, can be extended to clear up his remaining difficulties, and that Bateson will find in the same conception an explanation of the complex behavior of his 
sweet peas and stocks without resort to the inexplicable synthesis and resolution of supposed hypallelomorphs.

George Harrison Shull

Station for Experimental Evolution,

Cold Spring Harbor, Long Istand,

December, 1905 





\section{The Composition of a Field of Maize.}

\section{indered}

BY

\section{GEO. H. SHULL.}

Reprinted from

vOLUME IV, 1108

AMERICAN BREEDERS' ASSOCIATION. 



\title{
THE COMPOSITION OF A FIELD OF MAIZE.
}

\author{
By George H. Shull, Cold Spring Harbor, N. Y.
}

While most of the newer scientific results show the theoretical importance of isolation methods, and practical breeders have demonstrated the value of the same in the improvement of many varieties, the attempt to employ them in the breeding of Indian corn has met with peculiar difficulties, owing to the fact that self-fertilization, or even inbreeding between much wider than individual limits, results in deterioration.

The cause of such a result is wholly unknown at present. The old hypothesis which sought an explanation of the deleterious effects of inbreeding in the inharmonious or unbalanced constitution produced by the accumulation of disadvantageous individual variations, can hardly stand in the face of the fact that a very large number of plants normally self-fertilize and a noteworthy few have even given up sexual reproduction entirely, without in the least degree lessening their physiological vigor and evident chances of success in competition with sexually produced plants. The dandelion is propagated parthenogenetically, i. e., its seeds are produced without fertilization, but only the advocate of an unwarrantable theory will maintain that this plant is on that account undergoing a process of deterioration which threatens it with summary extinction. Many species of violets produce most of their seeds from flowers that never open, and one of the most vigorous forms of the small-petaled evening primrose (Oenothera cruciata) does the same. In the breeding of tobacco, it is now well known that cross pollination within the limits of a single strain produces inferior offspring and only self-fertilization gives offspring of the highest degree of vigor, though hybrids between distinct strains of tobacco often display a vigor superior to that of either parental strain. Examples could be continued indefinitely, but even one instance in which long-continued inbreeding results in no injurious effects would be sufficient to diseredit the old hypothesis. ${ }^{a}$

Some results of the pedigree-breeding of maize at the Cold Springs Harbor Station for Experimental Evolution have suggested a different explanation of the deterioration which has been universally observed in self-fertilized maize. For several years a series of investigations on Indian corn has been in progress at the Station, which involved parallel cultures of cross-pollinated and self-fer-

a For a good discussion of inbreeding see A. D. Shamel, on "The effect of inbreeding in plants," Yearbook U. S. D. A., 1905, pp. 377-392. 
tilized lines of as nearly equivalent parentage as possible. Although a study of the injurious effects of self-fertilization was not the aim of the investigation, it was immediately apparent in the smaller, weaker stalks, fewer and smaller ears, and the much greater susceptibility to the attacks of the corn-smut (Ustilago maydis). These results were almost as marked when the chosen parents were above the average quality as when they were below it, which in itself refutes the idea that the injurious effect is due to the accumulation of deficiencies possessed by the chosen parents.

All the cross-bred rows were similar in structure, vigor, variability, etc., but each self-fertilized row could be seen to differ from other self-fertilized rows in ways capable of description in fairly definite terms. Without entering into a description of all the different self-fertilized rows, a comparison between the two rows showing the greatest contrast will suffice to illustrate and serve as a basis for the conclusion to be arrived at. Following the method everywhere known among breeders as the "ear-row test," I planted parallel rows from ears having given numbers of rows of grains, one self-fertilized and one cross-fertilized ear form each row-class, i. e., one row was planted from a self-fertilized ear and beside it a row from a cross-fertilized ear having 10 rows of grains each; one row each was planted from two ears having 12 rows of grains; and so on.

Taking for comparison the row produced from a self-fertilized ear having 12 rows of grains, and the row produced from a selffertilized ear having 14 rows of grains, the following differences were observed. In each row the variability was slight and the different qualities noted were characteristic of the entire row. The characters are given in contrasted pairs (designated as a and b), the qualities of the row originating from a 12-rowed ear (a) being given first in each pair: (a) Average height, 61/4 feet, (b) average height, $81 / 2$ feet; (a) stalks moderately stocky, (b) stalks slender; (a) strong tendency to sucker near the ground, (b) no suckers; (a) leaves broad, dark green, and spreading, (b) leaves rather narrow, light green, not strongly spreading; (a) ears rather strongly diverging on long shanks, the latter usually as long as the internodes or longer, (b) ears erect on short shanks which are usually not over half the length of the internode; (a) husks with well-marked leafy appendages, (b) husks without appendages; (a) grains flinty, (b) grains starchy; (a) most common number of rows to the ear, 10, (b) most common number of rows, 14 .

Most of the features here contrasted differ more or less in both cases from the cross-fertilized rows derived from the same original stock. If self-fertilization is assumed to be the direct cause of any of the above characteristics of the one row, it is obviously illogical to attribute the opposite characteristic possessed by the other row to self-fertilization as a direct result. We come to the conclusion therefore that the observed differences between these rows are not 
directly attributable to self-fertilization, but must be due to an indirect effect. The distinguishing characters of these two rows are permanent inheritable qualities and each therefore represents what is known as an elementary species or biotype, as Johannsen has appropriately named the elementary form-group. Self-fertilization has simply isolated the two described forms by separating them from their hybrid combinations with other elementary species.

By rearing under different conditions or by selecting in different directions, it is possible to get a number of somewhat different strains within the same biotype; but, if we do not distinguish clearly between biotypes and these strains which differ only because of the different treatment they have received, only confusion can result. The difference between biotypes and the different strains of a single biotype lies in the nature of the characters which they possess as regards their heritability. Two biotypes will remain distinct from each other without resort to selection as long as. they are kept pure-bred and are grown under like conditions, provided those conditions are sufficiently favorable generally to allow such characters as each possesses to develop normally. Two strains within the same biotype may be just as distinct from each other as some biotypes are, but when they are grown under the same conditions, constant selection will be required to keep them distinct, and if selection is omitted the distinguishing characteristics quickly disappear, usually within several generations. Such characters as can only be retained in a pure-bred race by constant selection or by culture under a particular set of external conditions are called fluctuating characters. Now, the inheritance of the fluctuations in any character follow a well-known law, usually known as Galton's law, whose essential feature is the lagging of the average value of single characters of the offspring behind the average of the parents with respect to the same characters. In other words, the average condition of children with respect to any fluctuating character, stands between the average condition of the parents and the average condition of the biotype to which they belong.

It fnllows from this law that when a given degree of a fluctuating condition is continuously selected under fairly constant cultural conditions, the ideal which is followed in the selection marks the theoretical limit of progress which will take place in the direction of that ideal, and there will always be some lagging back of the average condition, which lagging becomes less and less, the longer the selection is continued. To be specific, in the strain of maize with which I started the most frequent number of rows of grains per ear was 14. According to theory, if we are dealing here with a fluctuating character of a single biotype, we should never expect to be able to exceed 20 rows on the average, by continuously selerting 20 rowed ears for seed, and in like manner we could never hope to get a strain whose average number of rows is less than 12 , by continuously selecting 12 , provided no change in the conditions of the cul- 
ture tended to increase the number of rows generally in the former instance or to decrease them generally in the latter. Not the least significant contrast therefore between the two self-fertilized strains above described is that which deals with the number of rows of grains on the ears. In the case of the selection to 14 rows, the result shows a considerable predominance of 14 among the ears of this year's (1907) crop, nearly 40 per cent falling into that single class as compared with 38 per cent in the same class among the unselected population with which the experiments were begun four years ago. As 14 rows was the original prevailing class or "modal" class as it is called, it is quite what we would expect, to find that continued selection of this modal number has simply increased the relative value of class 14 . In the case of selection to 12 rows on the other hand we are met with a surprise, for instead of the average number of rows being between 12 and 14 as the supposition that we are dealing with the fluctuations of a single elementary species whose normal mode is 14 rows, would lead us to expect on theoretical grounds, we find the prevailing class to be 10 , with nearly 39 per cent in that class. Knowing that this row was the result of continued selection of 12-rowed ears, one would infer from the data of this year's crop that the original condition of the populationthe normal condition for the race to which this row belongs-is probably 10 rows or possibly even 8 rows as the modal class, instead of the 14 rows possessed by the original stock from which all my cultures came. It is demonstrated therefore that these two rows belong to distinct races or elementary species of corn, though the original stock appeared to be fairly homogeneous. Most of the other self-fertilized rows showed by various marks that they were likewise to be considered members of distinct biotypes, instead of fluctuant parts of a single biotype as I believed they were when I began my investigation.

The obvious conclusion to be reached is that an ordinary cornfield is a series of very complex hybrids produced by the combination of numerous elementary species. Self-fertilization soon eliminates the hybrid elements and reduces the strain to its elementary components. In the comparison between a self-fertilized strain and a cross-fertilized strain of the same origin, we are not dealing, then, with the effects of cross and self-fertilization as such, but with the relative vigor of biotypes and their hybirds. The greater vigor of the cross-fertilized rows is thus immediately brought into harmony with the almost universal observation that hybrids between nearly related forms are more vigorous than either parent.

The components of a hybrid strain may be separated by means of cross-fertilization just as surely as by self-fertilization, if the parents of the cross are rigidly selected, generation after generation, for definite characteristics; but the process of segregation will be in this case much slower, because in each cross some of the elements which were eliminated from the mother will be reintroduced by the 
father, and vice versa. For this reason the deterioration which comes from close inbreeding coupled with cross-fertilization should not be as rapid though just as sure as by self-fertilization. This again is in accord with such observations as are on record.

As most of the important characteristics for which the corn breeder strives are closely related to the question of physiological vigor the fundamental problem in breeding this plant is the development and maintenance of that hybrid combination which possesses the greatest vigor. Up to a certain point the common empirical method of selection will mostly eliminate only those components which do not contribute to the best possible result, and the more rigid the selection during this period the more rapid will be the improvement of the selected strain; but if the selection is continued in the same rigid manner after these inferior components are eliminated, it may lead to the loss of one after another of the component biotypes which had added to the physiological vigor of the strain and there will then be a resultant deterioration, especially if among the characteristics which guide the selection are some which are unrelated to vigorous growth. The fundamental defect in every empirical scheme of corn-breeding which simulates the isolation methods of the breeder of small grains, lies in the fact that there is no intelligent attempt in these methods to determine the relative value of the several biotypes in hybrid combination, but only in the pure state.

In the present state of our knowledge it is impossible to predict from a study of two pure strains what will be the relative vigor of their hybrid offspring. That is an important relation which future investigations must unlock for us. The problem of getting the seed-corn that shall produce the record crop, or which shall have any specific desirable characteristic combined with the greatest vigor, may possibly find a solution, at least in certain cases, similar to that reached by Mr. O. I. Simpson in the breeding of hogs by the combination of two strains which are only at the highest quality in the first generation, thus making it necessary to go back each year to the original combination, instead of selecting from among the hybrid offspring the stock for continued breeding. That is, it may be found that the desirable combination of elementary species of Indian corn will be best attained by separating and recombining in some definite manner the different elementary species, or on the other hand it may be found that selection according to the empirical methods now most approved can be carried to a point at which the most efficient combination has been isolated from the less efficient components and may then be maintained only by a relaxation of the rigid selection.

Such questions as these cannot be settled in the study, but only in the field by means of carefully conducted experimentation. I hope that those experiment stations which are dealing with the problems of the improvement of maize will undertake the solution 
of these fundamental problems and that as a consequence the technique of corn-breeding will find a basis in scientific knowledge quite different from the present more or less blind conflict between empirical selection and the little understood injurious effects of inbreeding.

In conclusion I wish to say that the idea that in breeding maize we are dealing with a large number of distinct elementary species or biotypes is not presented here as a new idea, for De Vries, in his little book on "Plant breeding" presents this view, and Dr. East in a recent bulletin from the Connecticut Station has indicated the great complexity of the corn breeder's problems owing to the concurrence of these elementary species and fluctuating variations. I have aimed simply to point out how my own experience in cornbreeding supports the same view. I think, however, that the suggestion here made, that continuous hybridization instead of the isolation of pure strains is perhaps the proper aim of the corn breeder, is new and it is this view that I wish to submit for your consideration. 



\section{T.

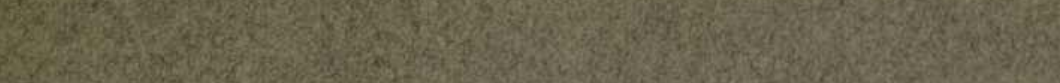

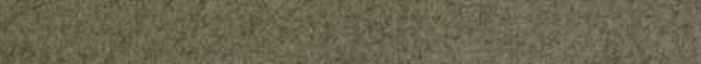

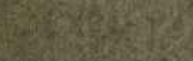

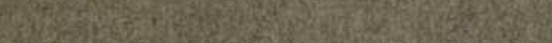

(4) 202

6.

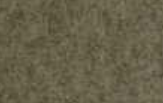

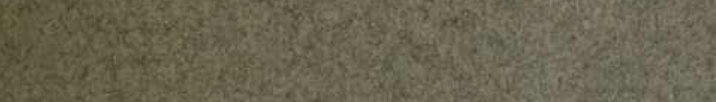

3.5.

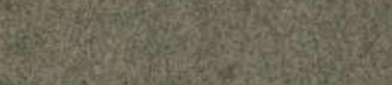

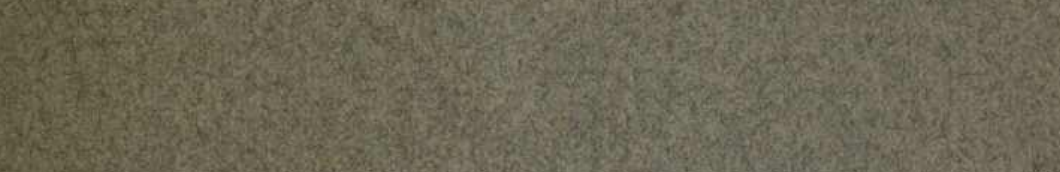

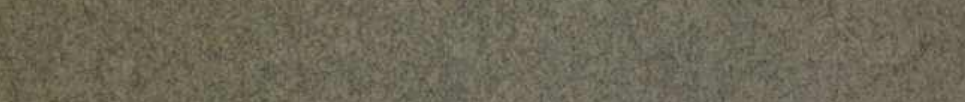
(4)

Fis.

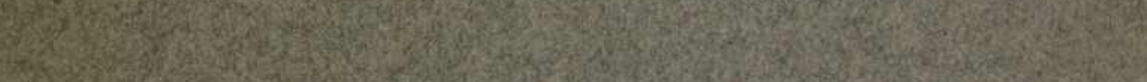

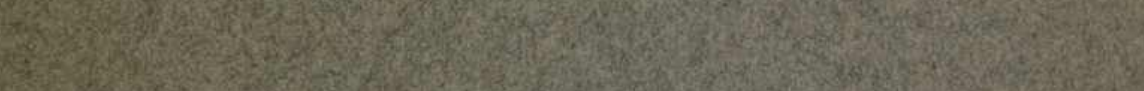

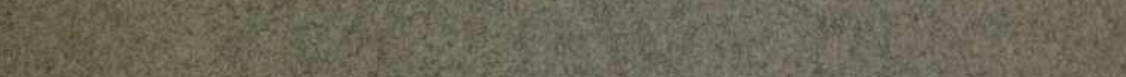

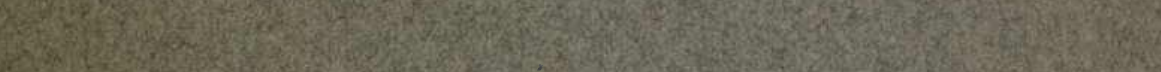

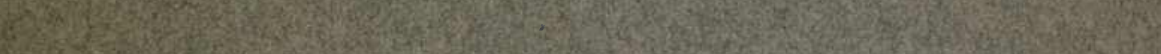

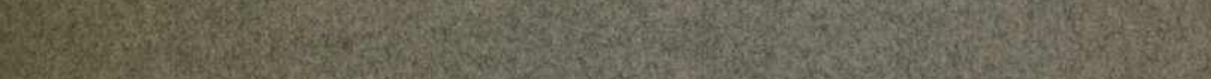
6 3.

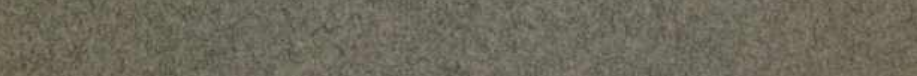

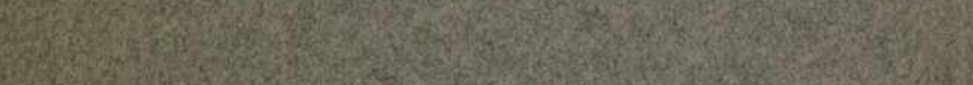

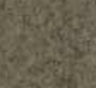

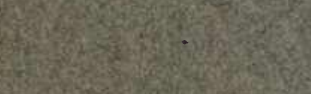



Reprinted from the BotaniCaL GazeT1E 45: 103-116, February 1908

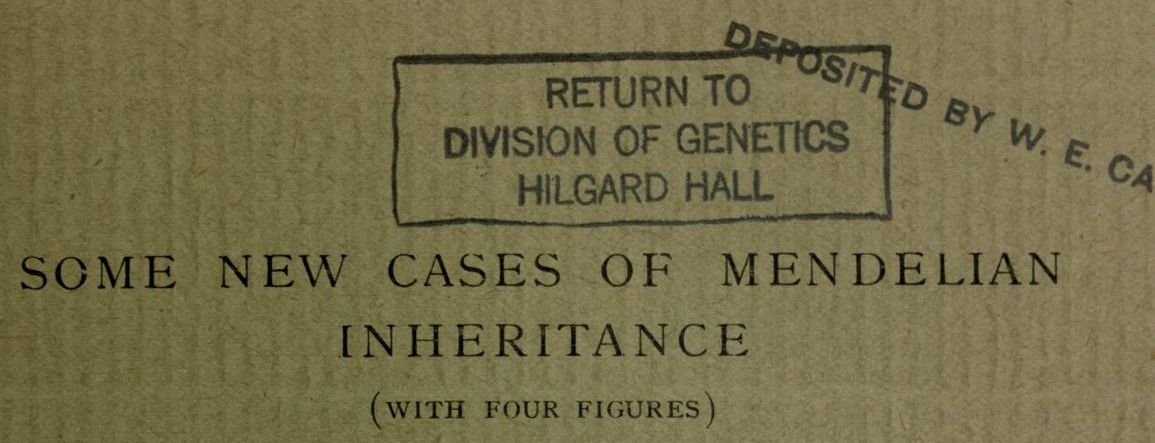

GEORGE HARRISON SHULL 



\title{
SOME NEW CASES OF MENDELIAN INHERITANCE
}

\author{
GEORGE HARRIS ON SHULL
}

(WITH FOUR FIGURES)

In crosses between nearly related elementary forms, numerous investigations have shown that a very wide range of characteristics in a great many species of both plants and animals behave in the Mendelian way; that is, they give a uniform progeny in the first hybrid generation $\left(F_{1}\right)$, and show perfect segregation of the various characteristics in $F_{2}$ and later generations. So consistent have been the results that there can be no question that MENDEL's law has fundamental and widespread applicability, but it has also been demonstrated that it has its limitations. Where these limitations lie and why it is thus limited may not be known until a much larger mass of data is on record than is now available.

Several of the cultures at the Station for Experimental Evolution, upon which no report has yet been published, present new instances of Mendelian heredity under conditions which make their consideration desirable, even though only a preliminary account can be given at this time.

\section{Helianthus annuUs L.}

Among II 2 plants of the so-called "Russian" sunflower (Helianthus annuus var.) grown at the Station during the summer of $\mathbf{1 9 0 4}$, all but one had the usually unbranched stem surmounted by a single large head. Sometimes these had several weak branches in the axils of several of the lower leaves, but there were never more than three or four of these lateral branches, and none ever stood higher on the stem than the fifth node above the cotyledons. The one exceptional individual had strong branches in nearly all of the axils and bore a number of somewhat smaller heads, but was not observed to differ in other respects from its unbranched neighbors (figs. I, 2). The seeds had been purchased at a seed store, and nothing is known of the antecedents of these plants.

ro3]

[Botanical Gazette, vol. 45 
Not until late in the season was it decided to investigate the hereditary qualities of the branching habit, and consequently the pollination was not guarded. Attempts at getting self-fertilized seeds from other plants proved unsuccessful, however, and all my experience in breeding the sunflowers during the past four years indicates that they are entirely self-sterile. From this it may be safely inferred that the

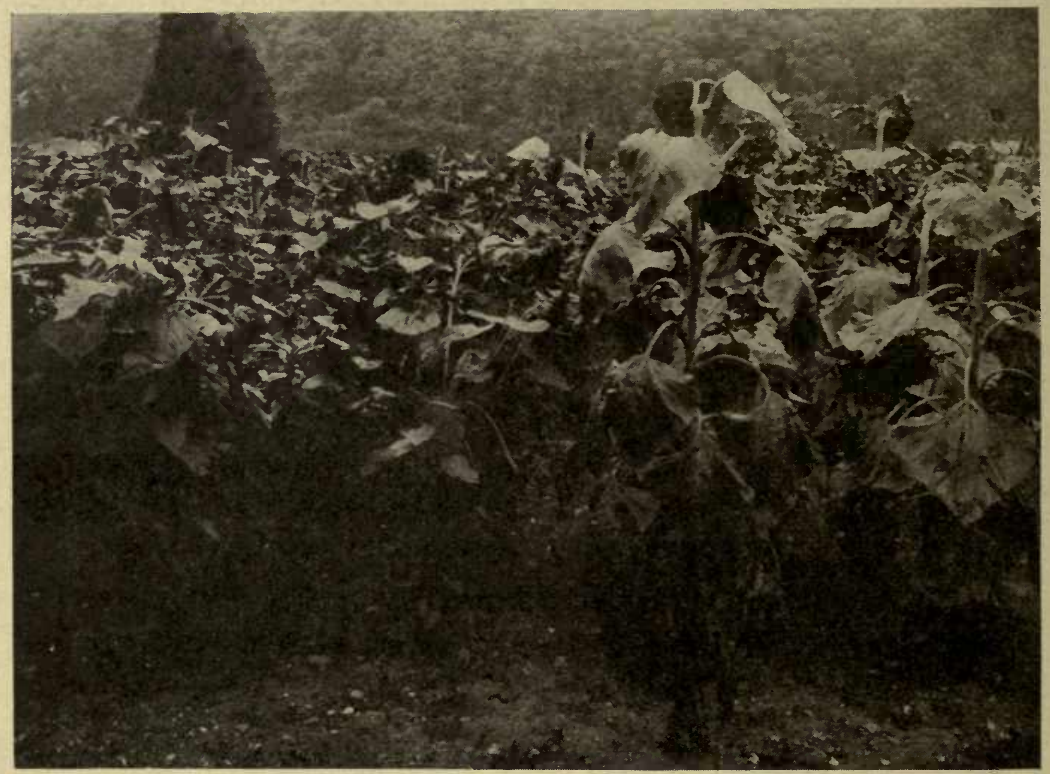

FIG. I.- "Russian" sunflower (Helianthus annuus var. hort.) showing two purebred strains; unbranched on the left, branched on the right.

branched individual (pedigree no. o4Io9) was cross-fertilized; and as it was the only individual of its kind, it must have been fertilized by means of pollen from the unbranched individuals. Assuming that this branching habit is a Mendelian character, several possible assumptions would lead to different expectations regarding the offspring of this cross: (a) If the unbranched habit were dominant over the branched, all the offspring should be unbranched; (b) If the branching habit were dominant, there would be two cases: (I) when the branched parent is a pure dominant, the offspring should be all branched; and (2) when the branched parent is a heterozygote (DR), 
the offspring should consist of both branched and unbranched individuals in the ratio $\mathrm{I}: \mathrm{I}$.

In the summer of 1905,59 offspring were reared from this cross, 28 branched and $3 \mathrm{I}$ unbranched, showing either that branching is

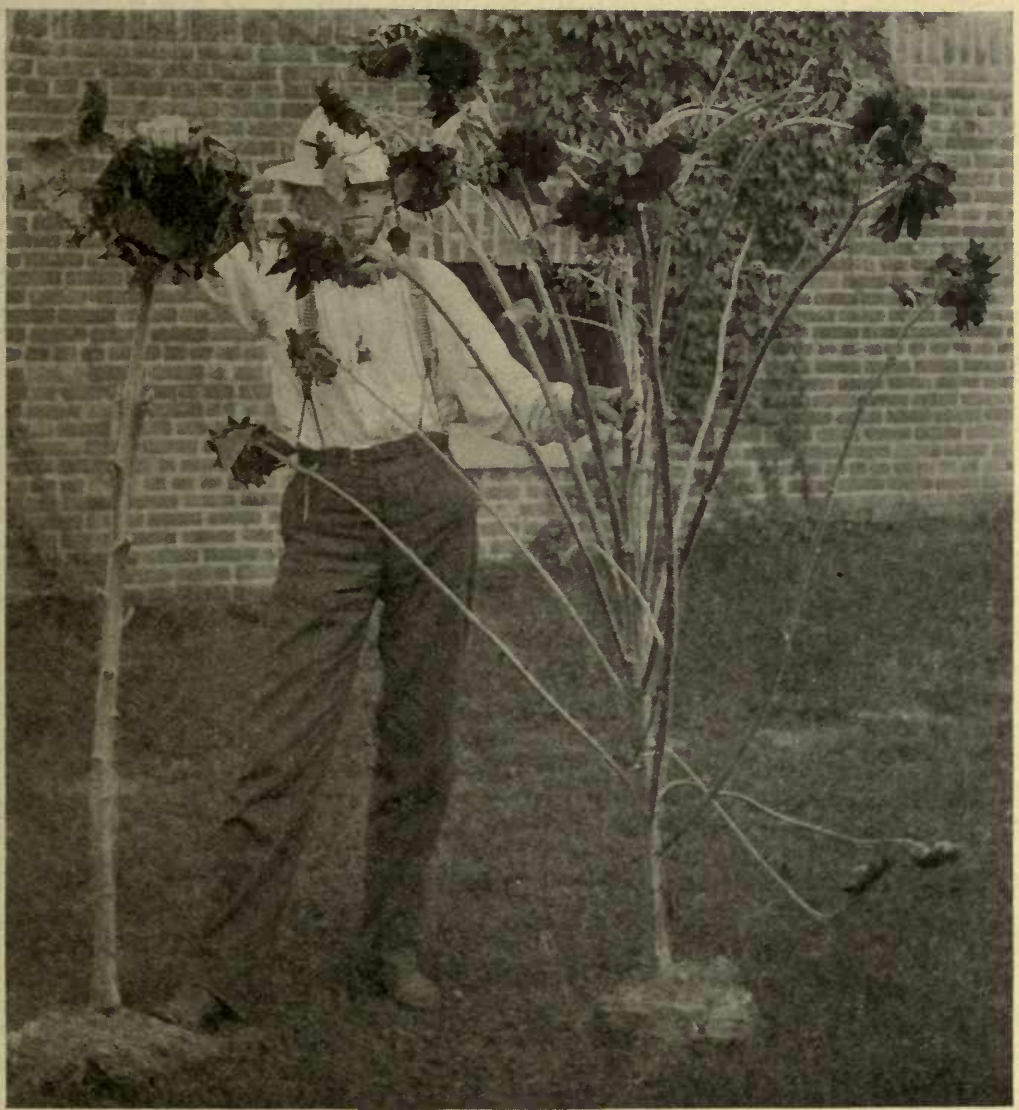

FIG. 2.-Individuals representing two elementary strains of the "Russian" sunflower; leaves removed to display the branching habit.

dominant, and the original branched plant was a heterozygote, or that branching is not a Mendelian character. About half of the unbranched plants showed a few weak branches about the base, but none had more than four branches, while none of the branched individuals had 
less than nine branches, the average number of branches in the latter being about 15 .

During the same season, 8I offspring of an unbranched plant (04108) which had also been left unguarded showed 72 unbranched and 9 branched. This result would find a ready explanation in case branching is a Mendelian unit-character, simply as the usual result of vicinism. On this assumption the proportion of branched offspring in this family indicates that the pistil-parent (04108) received about one-fifth of its fertilizations from the branched specimen (04ro9) which was growing near it, and the other four-fifths from the I Io unbranched plants, several of which were growing equally near.

In 1906 the Helianthus cultures were started several weeks too early (April 2) and became tall and slender from long crowding and insufficient illumination before they could be set into the garden. Some of these began to bloom shortly after they were transplanted, and the branching habit was very much deranged. Two families, (05149 and 05150) raised from branched parents having had the pollination fully controlled, resulted in 54 branched and 2 nearly unbranched in the one case, and 68 branched and 2 unbranched in th ${ }^{\circ}$ other, but as the whole branching system was considerably modified the classification could not be made with security. The expectation in these cases, since both parents were DR, would have been branched and unbranched approaching the ratio $3: 1$. The results secured indicate either that we are not dealing with a Mendelian charecter or that the conditions of the culture caused an excessive development of branches in normally unbranched individuals. The latter suggestion is strengthened by a small family (05145) reared from fully guarded unbranched parents from the same hybrid family to which the branched parents of the last-mentioned two families belonged. Both parents should be in this case extracted recessives (RR), and all of the offspring should belong to the unbranched class. Only six offspring were produced, and all showed some branching, ranging from one strong branch below the middle to twelve branches from the middle to the top of the stem, the latter type of branching being characteristic of the branched form. The results in 1906 seem therefore to oppose distinctly the idea that the branching character is Men- 
delian, but still leave a doubt on account of the obvious derangement produced by ill-treatment.

In the summer of 1907 the seeds were sown at the proper time (April 30) and the Mendelian character of the branching habit was fully established by the following facts: (a) Reciprocal crosses (06356 and 06357 ), fully controlled, between two strongly branch $\approx d$ specimens belonging to the second of the two DR families mentioned in 1906 (namely, no. 05150) gave 65 fully branched individuals in the one family, 13 in the other family, and no unbranched specimens in either, thus showing that at least one of the chosen parents was an extracted dominant (DD). The number of branches in both these reciprocal families ranged from to to 25. (b) Properly controlled pollinations w re made between two specimens of the last family (05 I45) mentioned for 1906, supposedly a recessive family. The pistilparent had two strong branches, one of which showed an abnormal bifission. The pollen-parent had one strong lateral branch which exceeded the main stem. The cross was made between two heads borne by lateral branches. Of the 22 offspring produced (06353), I 9 were wholly unbranched, two had one small branch, each near the base of the stem, and one had two small branches similarly located. This demonstrated that both parents were pure recessives and showed that the branches produced the preceding year had no hereditary significance. (c) Another sowing ( $\left.0635^{2}\right)$ was made of unguarded seed from the same pistil-parent as the last. The $9 \mathrm{I}$ offspring consisted of 67 individuals having less than 7 branches each and 24 having more than 7 branches each, the latter group having an average of about 16 branches and the former group an average of less than two branches ( $f g .3$ ). As the branched type does not usually have more than 4 or 5 vacant axils above the highest branch, one individual having 8 branches and to vacant axils probably belongs with the unbranched class. The result in this family shows that about one-fourth of the pollen received by the unguarded mother came from branched individuals, and as this fact was made obvious in the first generation it gives further proof of the dominance of the branching habit over the unbranched.

Furthermore, it illustrates well how impossible it would be to discover Mendelian ratios in cross-fertilizing species or varieties without careful control of fertilization. This requirement accounts for 
two things, namely, the fact that MENDEL's law was not discovcred by economic plant-breeders and gardeners long ago, and that in certain quarters the notion prevails that Mendelian inheritance is a function only of self-fertilizing or inbred strains.

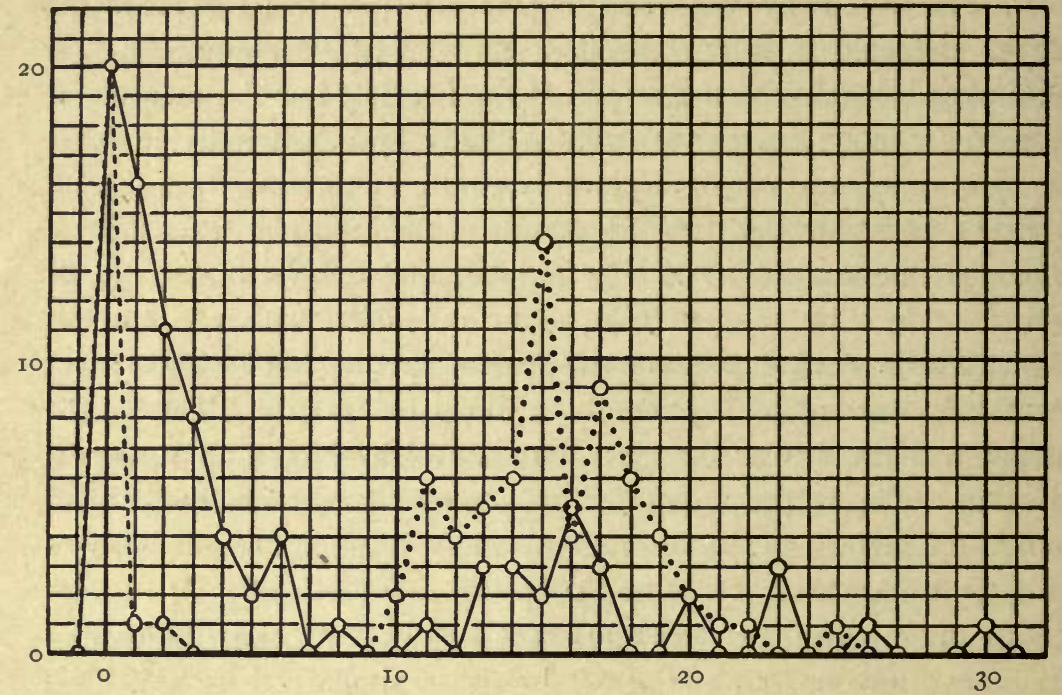

FIG. 3.-Curves showing the extent of branching in three families of "Russian" sunflower; the dotted line at left represents the offspring of two unbranched parents; the dotted line at the center represents the offspring of two branched parents, one of which must have been an extracted dominant; the unbroken line shows the condition of a family from the mother of the pure unbranched family, but with pollination unguarded; the dominance of branching produces a branched offspring in each case that successful fertilization took place by means of pollen from a branched plant.

The foregoing account of experiments on the inheritance of the branching habit in Helianthus annuus may be recapitulated in the form of a family tree thus:

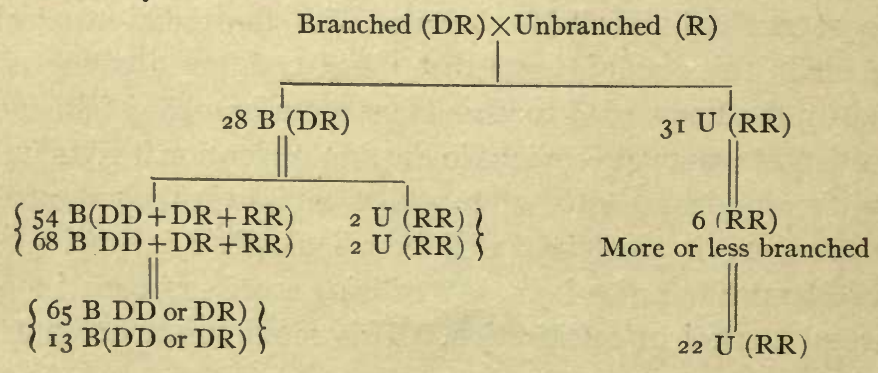


During the summer of 1905 experiments were also begun for the purpose of testing the relationship between the Russian sunflower and the wild Helianthus anmuus of the prairie region. Seed of the latter had been received from Nebraska through the kindness of Dr. C. E. Bessey. Several points of contrast were noted between the plants produced from this seed and the Russian sunflower, the most noteworthy being with regard to branching habit and the color of the disk. The lower branches of the wild sunflower, when given space for full individual expression as they are in my cultures, diverge almost horizontally, having on this account a slightly wider spread than branches arising at higher points on the stem. At the time the first head is ready to spread its rays, the whole plant has a nearly conical form. The development of the primary head checks the growth of the main stem, and some of the upper branches then usually elongate so as to overtop the central axis, thus giving the mature plant a more columnar form. My branched type of the Russian sunflower has all the branches strongly ascending, the lower being enough longer than the upper to reach almost the same level, thus giving the mature plant a corymbose or broadly obconical form.

I have not yet reached a conclusion in regard to the hereditary behavior of these two types of branching, as it is not easy to find decisive criteria for the exact classification of the two forms and their combinations. It has been apparent, however, in my cultures that there is a combination of both types of branching in many if not all of the first-generation hybrids, and at least some segregation in the next generation. The indefiniteness of the character will probably make it difficult to reach satisfactory quantitative results, but the attempt is being made.

A very much simpler character to deal with is the color of the disk. All of the western Helianthus annuus that I have thus far observed have a deep purple disk, the color being found in the tips of the paleae, which are of a deep, metallic purple; the margin of the corolla, which is brownish purple; and the style and stigmas, which are reddish purple. In all my Russian sunflowers, the tips of the paleae are yellowish green, the corolla is a clear lemon yellow, and the styles and stigmas usually have the same color as the corolla; but in a small proportion the stigmas are margined with a narrow line of deep 
crimson, which gives the stigmas the appearance of being orange colored, unless examined closely. No study has been made of the inheritance of this stigmatal margin, but it seems not unlikely that it too may prove to be a Mendelian character. Although the color of the disk affects several organs of very different morphological value, it proves to be a simple character, showing the same kind of correlation that is found in peas and beans which have purple flowers and black seeds, and other plants which have purple flowers associated with more or less purple in the stems.

The cross made during the summer of 1905 between the purpledisked wild sunflower and the yellow-disked, branched, garden sunflower, produced in 1906 a progeny (05153) consisting of 26 with purple disks and 27 with yellow disks; when I would have expected all purple on the assumption that both parents were pure-bred and purple dominant over non-purple, as has been the general experience with the purple color-character in other plants. The conclusion reached was that either the purple disk is not a Mendelian character or the purple parent was a DR instead of a pure-bred purple. As yellow disks have not appeared among my cultures of wild sunflowers during three years, the assumption that the one plant which I used for the cross was itself a hybrid seemed very unlikely, and the probability that this strictly alternative character was not behaving according to Mendelian expectation led me to watch the culture the following season (1907) with great interest. Five $\mathrm{F}_{2}$ families were raised, two reciprocal families of purple by purple, representing the hypothetical cross $\mathrm{DR} \times \mathrm{DR}$; two reciprocal families between purple and yellow, representing the cross $\mathrm{DR} \times \mathrm{R}$; and one family of yellow by yellow, representing the cross $\mathrm{R} \times \mathrm{R}$. The results were as follows:

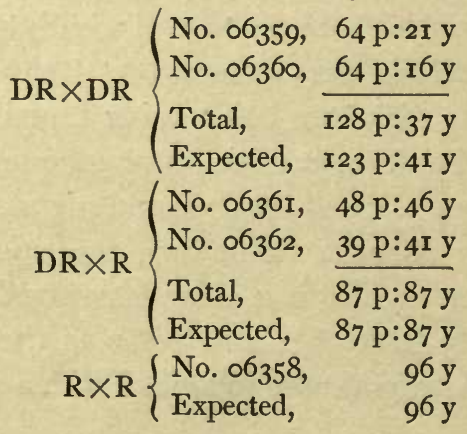


From all of these results it must be concluded that the purple disk is a strict Mendelian character, and that the first purple-disked parent was a heterozygote. The original cross is being repeated to make the latter point more certain.

\section{LyChNIS DIOICA L.}

This plant is a native of Europe and was doubtless very often cultivated in the gardens of a generation or more ago. It has now commonly escaped from the gardens and is maintaining itself quite generally along roadsides in the eastern United States. The flowers show considerable variation in the intensity of their purple color, and white-flowered ones are quite common. Purple and white flowers have been crossed in so many species and found to follow MENDEL's law, that there would have been little incentive to study this particular character in Lychnis dioica, had it not been for the fact that this species is dioecious, and that in consequence self-fertilization could not be held responsible for the Mendelian behavior if it should be found to exist. Statements in the manuals regarding the dioecious habit of this species indicate that it also occurs as a hermaphrodite, but among about five thousand individuals which I have examined only one was noted which had both stamens and pistils, and that one had but a single flower with both kinds of sporangia.

During the summer of 1905 I made all possible combinations with white and purple flowers, but in one case unfortunately used two different purple-flowered individuals for the pollination of the same white-flowered plant and collected the seeds from both crosses together. The offspring from these crosses reared in 1906 were as follows:

$$
\begin{array}{lr}
\text { No. } 05212, \mathrm{w} \times \mathrm{w}, & 90 \mathrm{w} \\
\text { No. } 05213, \mathrm{w} \times 2 \mathrm{p} \text { s, } 66 \mathrm{p}: 4 \mathrm{I} \mathrm{w} \\
\text { No. } 05114, \mathrm{p} \times \mathrm{p}, & 65 \mathrm{p}: 23 \mathrm{w} \\
\text { No. } 05115, \mathrm{p} \times \mathrm{p}, & 96 \mathrm{p}
\end{array}
$$

The fact that two white-flowered parents produced nothing but white-flowered offspring, and that in one case two purple-flowered parents produced only purple-flowered young, shows pretty conclusively that we are dealing here with a Mendelian pair of characters, even though the one family (05II4), which probably represents the cross $\mathrm{DR} \times \mathrm{DR}$, shows a considerable departure from the expected 
ratio of $3 \mathrm{p}: \mathrm{Iw}$. The ratio of purple to white in family no. $05^{2} \mathrm{I} 3$ can have no significance, since one of the two purple plants used as the pollen parents was probably a $\mathrm{D}$ and the other a $\mathrm{DR}$.

To test the assumption that family no. 05II4 represented the cross $\mathrm{DR} \times \mathrm{DR}$, and to get further evidence on the Mendelian character of the purple color in this species, a series of over eighty crosses was made within this single family. All of the pistillate plants received pollen from a single white staminate plant, and pollen from each of the staminate plants was used to fertilize the different flowers of a single white pistillate plant.

This method was employed because it is the simplest way in which the correct classification of every individual of the first generation of hybrids may be attained. Every single cross in the series had the recessive white as one of its members, so that only three possible combinations could occur among the resultant hybrid families, namely, $\mathrm{D} \times \mathrm{R}, \mathrm{DR} \times \mathrm{R}$, and $\mathrm{R} \times \mathrm{R}$, giving respectively, according to expectation, roo per cent., $5 \circ$ per cent., and o per cent., of purple-flowered offspring, in the ratio $\mathrm{r}: 2: \mathrm{I}$. About 50 plants from each of these 83 crosses were reared to maturity in 1907 , and, when classified according to the percentage of purple individuals in each family, showed 13 families with more than 95 per cent. purple, 48 having 30-70 per cent. purple, and 22 with less than 5 per cent. purple, the expectation being 2I with 100 per cent. purple, 42 with 50 per cent. purple, and 21 with o per cent. purple. The result is close enough to expectation to demonstrate the correctness of the Mendelian law in regard to the flower-color of Lychnis dioica L.

The diagram (fig. 4) showing the distribution of percentages of purple individuals in the heterozygote families may be used to call attention to what we ought to mean by "Mendelian expectation." Very often when a rather small series of observations shows a considerable departure from the ratios $I: I, r: 2: 1,3: 1,9: 3: 4$, or whatever other ratio represents the theoretical limit appropriate to the conditions of the particular experiment in hand, the statement is made that the results are not in accord with "Mendelian expectation." When the number of observations is small, such a statement is usually due to the fact that too much is expected. This will become clear when attention is called to the fundamental basis of the Mendelian ratios. Equal 
numbers of both eggs and sperms carry the antagonistic or alternative qualities making up a pair of Mendelian units. These different germ cells unite according to the laws of chance, and we should mean therefore by the expression "Mendelian expectation" that our observations if seriated in the form of a curve will present a normal probability curve within the limits of probable error. Not until the number of observations becomes infinite have we a right to expect absolute agreement with the theoretical ratios.

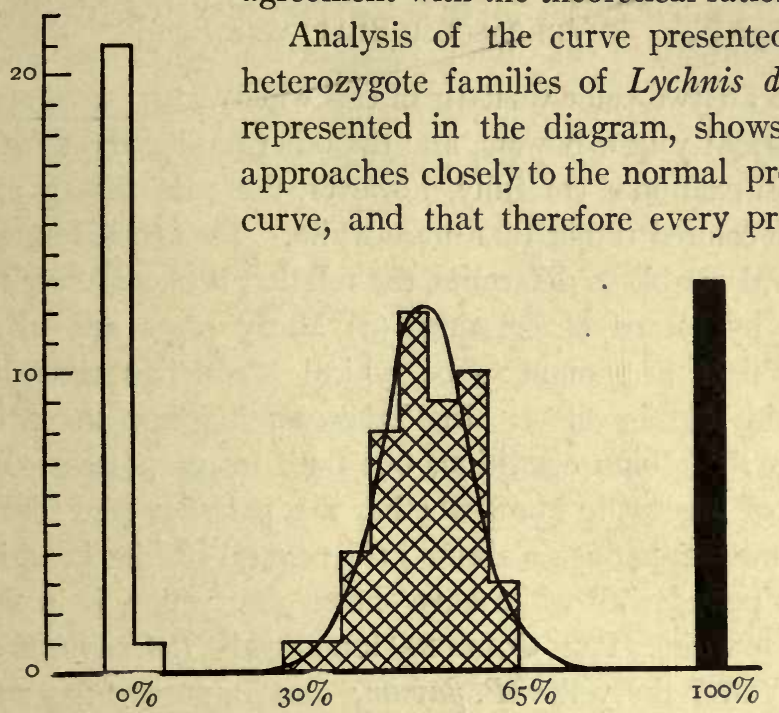

FIG. 4.-Lychnis dioica L.: variation in the percentage of purple-flowered offspring in hybrid families of the third generation having the form $(D+2 D R+R) \times R$; lefthand group represents the extracted recessives $(R \times R)$, the right-hand group the extracted dominants $(D \times R)$, and the middle group the heterozygotes $(D R \times R)$; superposed upon the heterozygote group is the normal curve having the same standard deviation, showing the close agreement with a perfect chance distribution.

pectation is fulfilled. As the departure of variates from the mean is a fundamental part of the law of chance, the fulfilment of expectation is just as complete in the case of the one $\mathrm{DR} \times \mathrm{R}$ family that had only 35 per cent. of purple-flowered individuals, or the three similar families that had about 65 per cent. of purple flowers, as in the twelve families which consisted of about 50 per cent. purple. This is an important fact that needs to be taken into account by both the student and the critic of Mendelian inheritance. 
The results here presented both in regard to Lychnis and Helianthus show that Mendelian phenomena stand in no relation to selffertilization, since neither of these species can self-fertilize. Emphasis upon this point should not be needed, since many of the most typical cases of Mendelian inheritance occur among animals, where the sexes are almost invariably separated.

\section{Verbascum BlattaRia L.}

There are two color-varieties of this widely distributed species, one having bright-yellow flowers, and the other having the flowers almost white. If examined carefully, however, the pale flowers are seen to be cream colored rather than pure white. Two considerations made it appear desirable to determine the relation between these two colorvarieties by means of crossing. (a) Many cases are on record in which albinos are found to be typical Mendelian recessives when paired with certain colors. In Verbascum Blattaria the pale flowers approach the albino condition, but both forms possess some color, and it is of interest to know whether this pale-flowered form behaves in the same manner as an albino. (b) Several studies in which yellow has been paired with white have shown that yellow is in these cases recessive to white. Thus CORRENS ${ }^{\mathrm{I}}$ found white Polemonium coeruleum dominant over the yellow P. flavum; and BATESON ${ }^{2}$ has shown that yellow-flowered stocks (Matthiola) are recessive to the white-flowered. CorRens' results with Polemonium stood alone at the time my experiments with Verbascum were begun, as an instance in which a white-flowered variety dominated one with colored flowers. It seemed important on this account, also, to know what would be the behavior of the yellow-flowered Verbascum Blattaria when crossed with its pale-flowered form.

To secure a solution to these problems, several crosses were made during the summer of 1904. The seeds were sown in the spring of

x Correns, C., Weitere Beiträge zur Kenntnis der dominierenden Merkmale und der Mosaikbildung der Bastarde. Ber. Deutsch. Bot. Gesells. 2 I : I95-201. I903. (See p. I98.)

2 Bateson, W., Saunders, Miss E. R., and Punnett, R. C., Reports to the Evolution Committee of the Royal Society, II. 1905, and III.rgo6. 
1905, but the plants did not bloom until the summer of 1906. The results then stood as follows:

$$
\begin{array}{lcc}
\text { No. } 0446, & \mathrm{y} \times \mathrm{y}, & 75(\text { ?) } \mathrm{y} \\
\text { No. } 0447, & \mathrm{w} \times \mathrm{w}, & 53 \mathrm{w} \\
\text { No. } 0448 \times 47, & \mathrm{w} \times \mathrm{y}, & 8 \mathrm{y} \\
\text { No. } 0448 \times \text { self, } & \mathrm{y} \times \mathrm{y}, & \text { (?) y (number unrecorded) }
\end{array}
$$

Among the members of these families all possible combinations were again made. The offspring of these crosses were mostly induced to bloom the first season by starting early and giving the seedlings the best possible conditions. The results of the second generation were thus secured during the summer of 1907 , and showed the following composition:

\begin{tabular}{cc} 
No. 0624I, D $\times$ R, & $26 \mathrm{y}$ \\
Expected, & $26 \mathrm{y}$ \\
No. 06244, DR $\times$ DR, & $5 \mathrm{y}: 7 \mathrm{w}$ \\
Expected, & $9 \mathrm{y}: 3 \mathrm{w}$ \\
No. $06243, \mathrm{R} \times \mathrm{DR}$, & $\mathrm{I} 3 \mathrm{y}: \mathrm{I} 8 \mathrm{w}$ \\
No. $06245, \mathrm{DR} \times \mathrm{R}$, & $5 \mathrm{y}: 8 \mathrm{w}$ \\
\hline Total, & $\mathrm{I} 8 \mathrm{y}: 26 \mathrm{w}$ \\
Expected, & $22 \mathrm{y}: 22 \mathrm{w}$ \\
No. 06242, & $90 \mathrm{w}$ \\
Expected, & $90 \mathrm{w}$
\end{tabular}

Although some of these ratios depart rather strongly from the theoretical limiting ratio, this is due entirely to the small numbers of observations, as pointed out above in the discussion of Lychnis. It is plain therefore that the color-forms of Verbascum Blattaria constitute a Mendelian pair, and that the bright yellow is dominant over the pale. The dominance of yellow in this cross is just the reverse of the behavior of yellow in Polemonium and Matthiola, and teaches that there are two kinds of yellows in plants, one of which is dominant to white, the other recessive to white. A microscopic examination of the petals of Verbascum shows that BATESoN's ${ }^{3}$ distinction between plastid-colors and sap-colors is probably fundamental, as the yellow color in this species proves to be a sap-color, and its behavior in combinations with white is the same as that of the blue, purple, and red

3 Loc. cit. 
sap-colors; while the yellow color of Matthiola and of Polemonium flavum are plastid-colors and this must account for their different behavior. In Mirabilis CoRRENS 4 has noted a yellow-flowered variety, M. Jalapa gilva, in which the yellow color seems to be dominant. I do not know that any examination has been made to determine the basis of the yellow color in Mirabilis, but I predict that it will be found to be a sap-color. HuRST ${ }^{5}$ reports, on the contrary, that the yellow of Antirrhinum majus, which is recessive to white, is a sap-color. If this statement is correct, this species secms to stand alone at present in the possession of a sap-color recessive to white. Why a plastidcolor should be recessive to the absence of that color calls for an explanation which science is not yet ready to give. This difference between the two kinds of yellow recalls a similar situation in poultry, in which white is usually dominant over colors, but in at least one strain (Silky) the white is recessive.

Station for Experimental Evolution

Cold Spring Harbor, L. I., N. Y.

4 Correns, C., Zur Kenntnis der scheinbar neuen Merkmale der Bastarde. Ber. Deutsch. Bot. Gesells. 23:70-85. 1905.

5 Hurst, C. C., Mendelian characters in plants and animals. Rep. 3 d Internat. Conference on Genetics, 1906. Roy. Hort. Soc. I 907: 114-ז 28. figs. 6. 




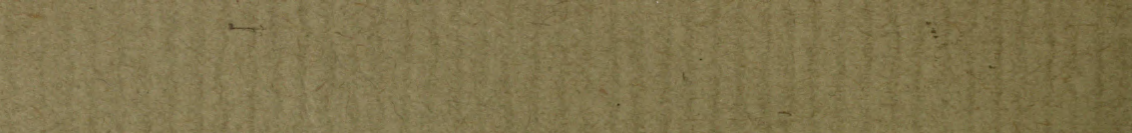

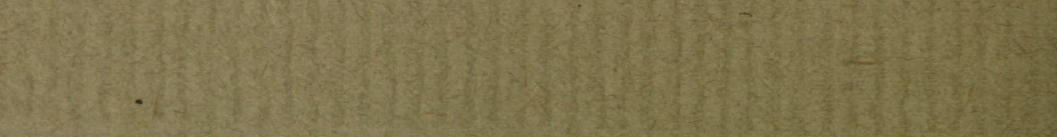
$\cos$

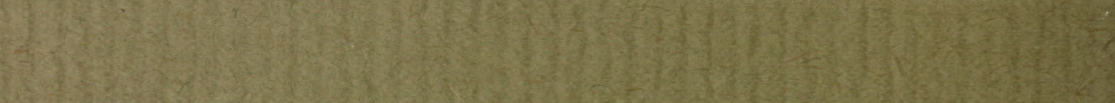

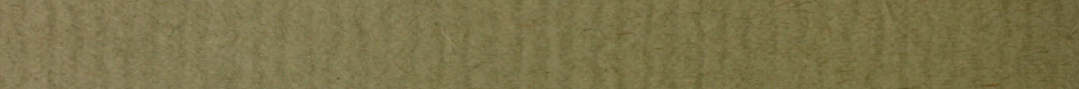

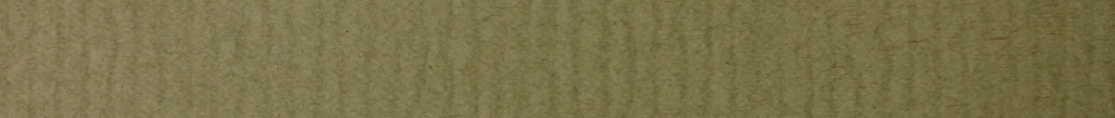

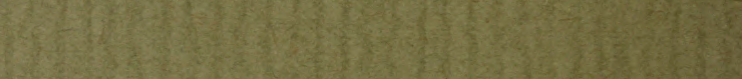

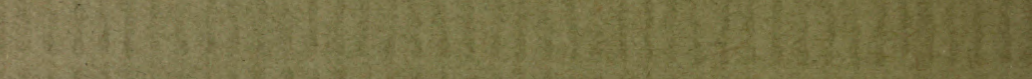

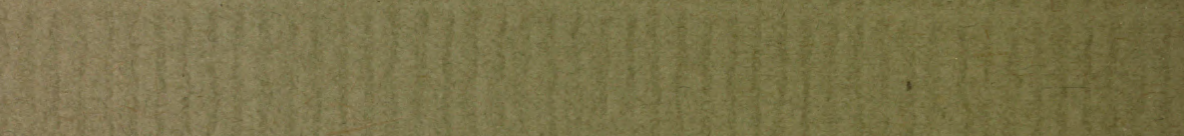

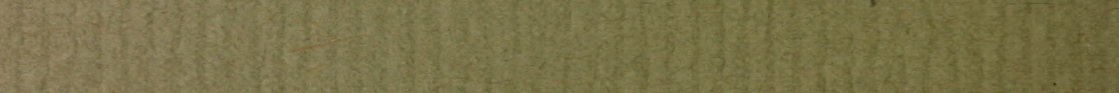

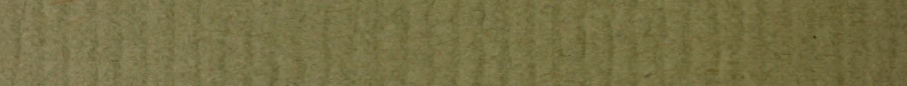

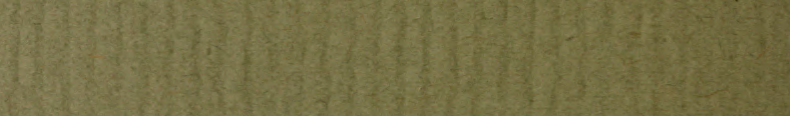
2.

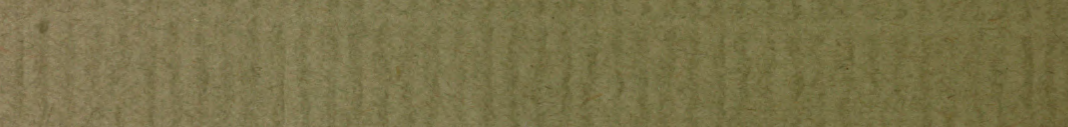

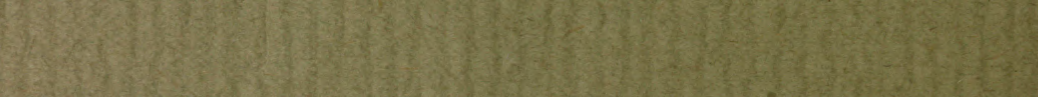

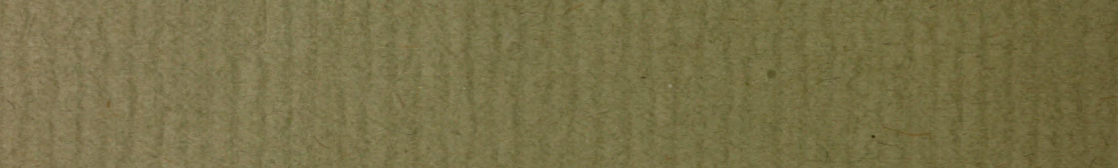

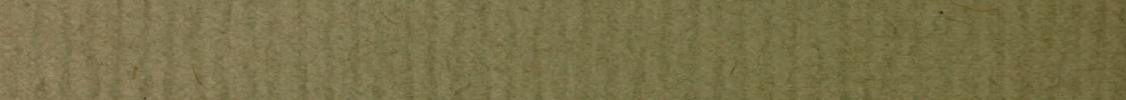

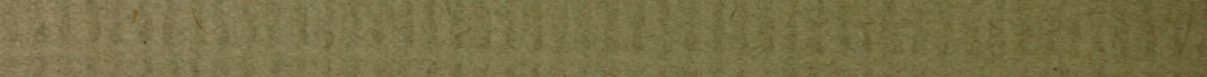

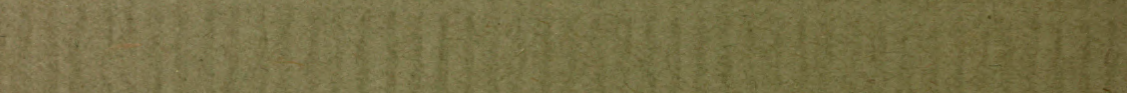

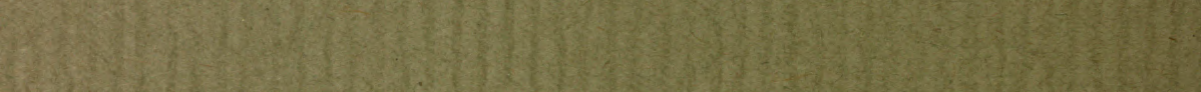

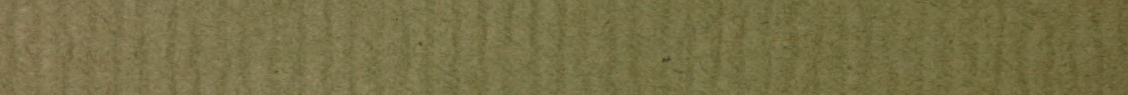

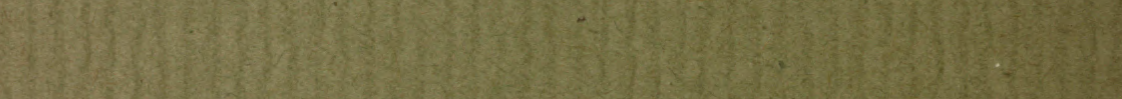

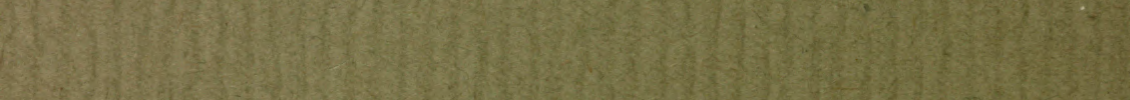
3
3

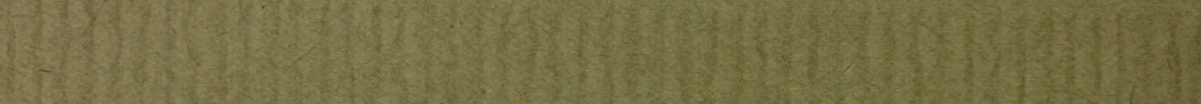

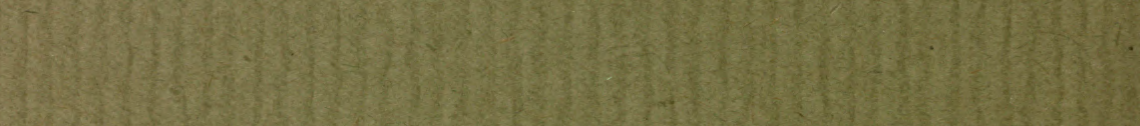

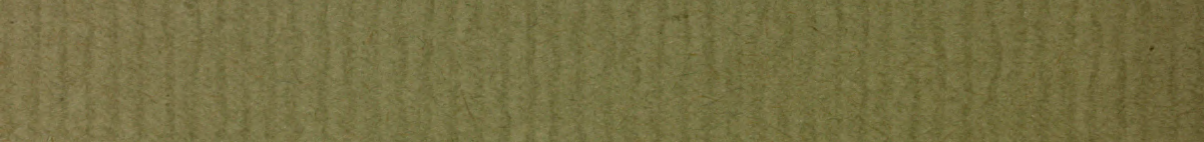

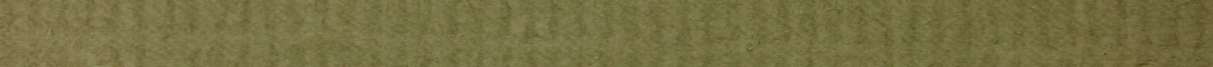

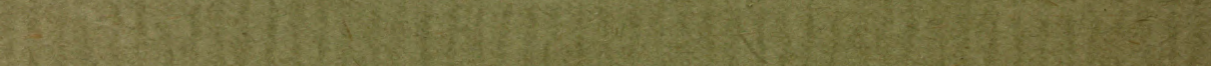
1950 a

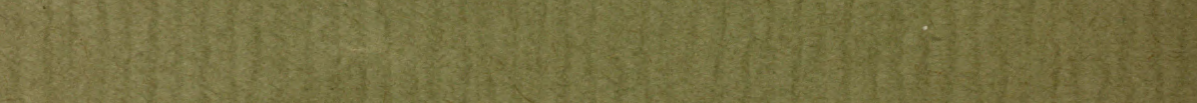

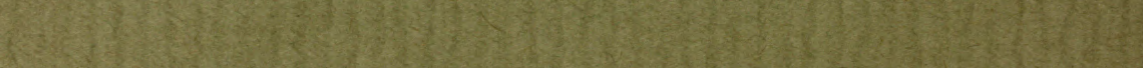

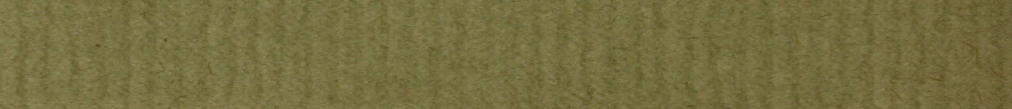




\section{THE PLANT WORLD}

At Flagazine of Gentexal Batann

\section{FEBRUARY, 1908}

\section{THE PEDIGREE-CULTURE: ITS AIMS AND METHODS.}

\section{By Dr. George Harrison Shull.}

There is a considerable body of data now on record of every degree of accuracy and inaccuracy, upon which it is easy to base discussions of variation, heredity, evolution and kindred topics if one has-a good imagination and a sufficient faith in the correctness of data he may wish to cite, and the eternal verity of certain hypotheses which were put forward by some ancient seer, or which hang like a cloud on the horizon of his own inner consciousness. The experimentalist lacks this sublime faith. His motto is "Ignoramus, in hoc signo laboremus." Investigation shows him that the available data are scanty indeed that are not written all over with question-marks, which would need to be exchanged for exclamation-points in order to justify the strenuous attitude taken by some writers who seem to assume that these momentous questions are either already settled or are beyond the legitimate sphere of experimentation.

From some of these contemners of experimentation comes the criticism that the distinctive feature of the pedigreeculture method is isolation unlike anything which commonly occurs in nature, and that the results can have valid application only to those rare situations in which similar isolation is found in nature. This statement of the case is quite erroneous and may prove misleading to those not familiar with the scope of the pedigree-method. The distinctive feature of the pedigree-culture method is not isolation, but perfect knowledge of ancestry to a certain number of terms. No 
student of "Genetics", as Professor Bateson aptly calls the new science which has the pedigree-method as its peculiar instrument, has limited his attention to the study of "pureline" cultures and it is recognized by all such students that the usefulness of the perligree-method has not been exhausted until every possible combination has been made with the material in hand. Thus, in the case of the most gigantic piece of pedigree-culture work that has yet been done, namely, the studies upon the Oenotheras by de Vries, followed on a large scale by MacDougal and many others, there is abundant published data showing the result of crossing each of the several mutants with their parent and with each other. It may be granted that the cause of science will be advanced by the repetition of some of this work for the sake of confirmation or of further analysis, but the points to be made here are that these combinations have been made and that the making of such combinations is a prominent feature of all pedigree-culture studies that have been made or are being made. It is needless to urge that in nature no other than the possible combinations exist, and that therefore when with respect to any species or group the full scope of the pedigreemethod has been utilized we have the fundamental data upon which alone can be based any proper conception of what goes on in nature, and that the data so found when properly confirmed by repetition are applicable to all nature in which the particular species or group in question is involved.

The pedigree-method with the resultant perfect knowledge of ancestry has given to biological science the two most illuminating suggestions which have come to it from any source in many years, namely Mendelian inheritance, and the Mutation theory, with their common postulate, the existence of unit-characters and character-bearing units or determinants. It is not known how universal these great truths are but evidence is accumulating daily to show that they are truiy of wide-spread and fundamental value. The only test of the extent of their applicability is the one by which they were discovered, namely, the pedigree-method. 
To attain to perfect knowledge of ancestry, the ideal toward which the pedigree-culturist strives, many precautions are necessary, and as the fundamental character of the problems of genetics is bound to attract increasing numbers of investigators, it is thought that the writer's experience in the conduct of experiments involving the pedigree-breeding of plants at the Station for Experimental Evolution may be of use to others who have occasion to take up such work, and this is the author's motive in presenting the following account of methods used.

Perfect knowledge is a high ideal and not absolutely attainable, because some sources of error can not be eliminated. This is due principally to the fact that they are of the nature of catastrophes which can not be foreseen; but if reasonable care is taken the results will usually be so decisive as to leave no doubt of their correctness, and in cases of doubt, where it may be assumed that some unexpected and unknown error has crept in, the cultures may be and should be repeated. The same accident is not likely to occur a second time to the same pedigree. A pedigree with a questionmark in it is usually no better and often very much worse than no pedigree, and any material whose pedigree is in doubt should be at once eliminated, or if the material is rare and valuable in itself, it should be treated as any other unknown material, namely, assigned a distinct pedigree-number or other designation which gives no suggestion of relationship to other known material. Cultures grown from such questionable material may indicate its relationship by the characters presented, but knowledge of this relationship rests purely on inference, and that inference is just as trustworthy in itself when no hint of probable derivation has been noted as when the record indicates a probable but doubtful origin.

The most important factor in the elimination of errors is a high ideal in this regard on the part of all those who have anything to do with the cultures. Mind and senses 
must be on the alert for every possible source of contamination of pollen and of seed, or of mistaken labels and records. Briefly the method I am following at the Station for Experimental Evolution is as follows:

Seeds of individual plants, whose pollination has been controlled when that has been practicable, are collected and to the seeds of each such individual a pedigree-number is assigned, giving reference to a record, or description of parent and parentage. These are sown in earthen pans of sterilized soil at such time that the offspring may be expected to reach maturity at the close of the first season. For most biennials and perennials this planting must be done before the rst of March, while the annuals are not sown until about the Ist of May or later. When sufficiently developed, the young seedlings are set in 3 -inch pots, each of which receives a wooden label bearing the pedigree-number of the mother plant. As a rule no selections are made in removing from the seedpan. If all are not to be reset the requisite number is secured by taking all consecutively, beginning at one side of the pan, until the desired number has been removed, after which the contents of the pan are dumped.

At the proper season such as are destined for out-of-door culture are reset to the garden, and those which are to be used for indoor culture may or may not be reset to larger pots, as the needs of each particular case requires. A label once attached to a plant accompanies it throughout its life, and is copied on the seed packet which contains its seeds. The chance of error is slight after the plants have been potted singly and each has received its appropriate label. The most critical points in the technique are the control of fertilization, the sowing of the seeds, and care of the seedpans. These matters, together with the question of labels and records, may be discussed with profit to others engaged in pedigree work, or contemplating taking it up.

In regard to the control of fertilization the general method consists (a) in preventing self-fertilization or cross- 
fertilization as may be required by the particular experiment in hand, (b) the protection of the pollen to be used from mixture with pollen from unknown sources, (c) the conveyance of the pollen to the stigma of the desired mother-plant, and (d) the continued protection of the latter until the possibility of its accepting pollen from any other source is past. In carrying out this program each individual case will require special refinements of technique which can only be de. rived by a study of the peculiarities of the particular species under experimentation. For example, plants which, like Helianthus annuus and all dioecious species, cannot fertilize themselves, will not require the removal of the stamens, and this fact greatly simplifies the technique in some cases which would otherwise present peculiar difficulty.

When self-fertilization is possible, the conduct of "pureline" experiments is peculiarly easy, the only important requirement being the exclusion of foreign pollen. On the other hand, if the experiment with self-fertilizing material requires that a cross be made, the stamens must be removed from the plant chosen as the mother, before the anthers dehisce, the time at which this must be done being different in different plants, according to the relative precocity of stamens and pistils. In Oenothera it is necessary to castrate 24 hours in advance of anthesis, while in strongly proterogynous flowers the anthers may be removed at the time of pollination. Both the stigmas and the pollen must be protected from insect visits and other agencies for the carriage of pollen. For this purpose the best method is to enclose the entire inflorescence (or in some instances the single flowers) in bags of paraffin paper. These are made in various sizes to order by A. G. Stursberg, New York City. I have found the most generally useful sizes to be: $8 \mathrm{in}$. long $x_{3}$ in. wide, 10x4, 10x $73 / 4,15 \times 4,17 \frac{1}{2} \times 6,19 \frac{1}{2} \times 15^{1 / 2}$, and $20 \times 93 / 4$. These may be attached to the plants in various ways. I have used ordinary white cotton pack-thread, and raffia, but have found the nurseryman's $3 \frac{1}{2}$-inch wired tree 
labels by all odds the most convenient, as they can be attached or detached almost instantly, and the label may bear a statement of the particular cross that is being made. In order to secure a satisfactory quantity of seed it is nearly always necessary to repeat the process of pollination several times between the same two parents, and the inscriptions on the tags prevent errors in the way of mixed parentages, even when a large number of sister plants are being operated upon simultaneously.

For removal of the anthers I find most useful a pair of forceps, different sizes and styles being best suited to different cases. A needle-pointed forceps serves for both dissection of the buds and removal of the anthers in the case of Bursa, but in most flowers of larger size a broader pointed forceps is preferable. I prefer to remove the anthers with as little injury to adjacent parts as possible, but when the perianth is thick and elastic so as not to allow easy access to the stamens on merely opening the buds, as is the case in Verbascum, it is desirable to remove it. For this purpose a small curved dissecting scissors is very satisfactory.

For the conveyance of the pollen to the chosen stigma the method must again be varied to meet the requirements of the material. In the majority of cases it is most satisfactory to detach the flower which is to supply the pollen and carry it in such a way that nothing comes in contact with the anthers, which are then touched directly to the stigma. The pollen plant is uncovered only long enough to allow the removal of the flower to be used, and the pistil plant only long enough to receive the pollen, the bag being replaced in both cases as quickly and carefully as possible. In certain cases this ideal technique is impossible. For example, in Helianthus annuus the method of handling individual flowers is not feasible, and here it is my custom to use camel's-hair brushes. One brush is used for each pair of heads to be crossed. When not in use this brush is kept securely enclosed in an ordinary seed-bag which bears the pedigree-number of 
the two plants upon which it has been used. The pollen from the head of one of the plants is brushed into one Syracuse watch-glass and instantly covered with another. In this way it is carried to the other plant of the pair and applied carefully to the circle of receptive stigmas. Then the pollen is brushed from this plant into the same dish and returned to the first member of the pair, to be brushed upon its receptive stigmas. The dishes are then deposited in a jar of 90 or 95 per cent alcohol, where they remain until the following day, when they are washed and used again. The same brush is used day after day on the same pair of plants until the operation is completed, when it, too, is consigned to the alcohol jar. In this case, as in all others, the protecting bags are removed from each plant no longer than is absolutely necessary for the performance of each operation.

In controlling the pollination of Indian corn still another method is used. Both ears and tassels are enclosed in paraffin paper bags before the external appearance of stigmas and anthers. Pollen is produced in great quantity which collects in the bag enclosing the tassel. This bag is removed from any plant desired and carried to the plant to be pollinated. The silks are uncovered and immediately sifted over with the pollen, which trickles from a small hole torn in one corner of the bag. The silks are then quickly re-covered and the pollen bag is returned to the plant from which it was taken, to be used again the next day or the second day after in a repetition of the process. This work is usually done in the afternoon when almost no pollen is falling from other plants, in order that the entrance of foreign pollen during the momentary uncovering of the silks, shall be reduced to a minimum.

Probably the most potent sources of error in these various methods consists: (a) In the possible fertilization by foreign pollen which has been scattered upon the foliage of the plant and so enclosed within the bag; and (b) its introduction by the wind or insects in the brief period during 
which the bag is removed for the purpose of castration and pollination. The audacity of some of the short-tongued bees has necessitated the sacrifice of a number of flowers after the greatest care had been used in controlling their pollination. Experience has taught in all those cases in which the entrance of foreign pollen would make itself plainly apparent in the result, that the precautions taken gives essentially perfect control of pollination.

(To be continued.) 


\section{THE PEDIGREE-CULTURE: ITS AIMS AND METHODS.}

By Dr. George Harrison Shull.

(Conclusion.)

The handling of the seeds is the next crisis in the pedigree method. Each seed-bag is labelled at the time the seed is collected, and to make doubly sure of the correctness of the record the wooden label is taken from the seed-bearing plant and enclosed with the seeds until a later date when the pedigree records are being made. The wooden label is then removed and carefully compared with the data on the bag and in the record.

As all garden soil contains numerous viable seeds it is necessary that the soil to be used in the germination of pedigree seeds, shall be freed from all other seeds. For this purpose the autoclav sterilizer has proved very satisfactory. This consists essentially of a steam-tight drum fitted with a thermometer and steam gauge. An inch of water is poured into this drum and the soil is put into it in suitable bailed receptacles made to fit the interior of the sterilizer. When the lid is clamped on, heat is applied to the bottom of the drum until the steam pressure within is raised to $5-\mathrm{I} 5$ pounds to the sq. in. and this pressure is sustained for about five hours. If it were necessary this process might be repeated the following day, but experience has shown that few if any seeds can withstand the temperature of $110^{\circ} \mathrm{C}$. for 5 hours when in the somewhat moist state in which they are sure to be in the soil. When gas is available the problem of heating 
the autoclav needs no other solution. As the Station for Experimental Evolution has no gas, resort was had to an alcohol blast-lamp. At first the autoclav radiated more heat than the lamp could supply, before it reached an adequate temperature, but this was remedied by jacketing the drum with felt herbarium driers.

If an autoclav is not available the soil might be freed from seeds by boiling or baking but in both cases the mechanical condition of the soil is apt to be greatly injured. My experiments were begun without a sterilizer but instead of germinating the seeds in garden soil I used sand from a glacial deposit taken in such manner as to avoid the possibility of contamination with seeds which might have been carried to the surface of the sand by the wind or any other agency. The desired result was attained in this way, as no foreign seedlings appeared, but at the expense of failure to get satisfactory germination in many species for whose early development the mechanical qualities of sand are apparently unsuited.

The care and handling of the sterilized soil present many problems. No permanent supply can be safely kept and all soils should be sterilized from day to day as they are needed. Those who handle the soil must keep their clothing brushed and their hands and nails clean. The soil-bench is carefully swept and covered with clean newspapers before the soil is turned out of the vessels in which it has been sterilized. The sifters and other implements used in handling the sterilized soil, if of a character which makes their perfect cleansing difficult, must never be used for unsterilized soil. I use only square earthen seed-pans $30 \mathrm{~cm}$. at the top, $25 \mathrm{~cm}$. at bottom and $7 \mathrm{~cm}$. deep. These are thoroughly scrubbed before using. Wooden boxes of similar dimensions were used at first, but the difficulty of freeing them from seeds which might remain in the joints led me to discard them after once using. After the seed-pan has been made ready for the sowing of the seeds, it is removed to a safe distance from 
the pile of sterilized soil and from other seed-pans and after the seeds are sown, a label is inserted, which bears the same number as that on the seed-packet. Then the pan is set in a shallow vessel of water until it is thoroughly subirrigated, after which it is covered loosely with a clean glass plate and removed to its final place in the propagating house. Only one lot of seeds is handled at a time, and the hands are thoroughly cleaned after completing the sowing of one lot of seeds before opening the seed-bag or handling the pan which is to receive the seeds of another pedigree. All watering of seed-pans is preferably done from below, because the slightest carelessness in throwing a jet of water on a seedpan might transfer a seed from one pan to an adjoining one. If surface watering becomes at any time advisable, the individual pans should be removed to a safe distance from all others during the operation. In the case of surface-watering it is also necessary to take thought as to the source of water used. If from a protected well, it is all right, but at the Station for Experimental Evolution the propagating house is supplied from a reservoir which receives water from an open stream, and there is no safety in using this water on the seed-pans unless it is first filtered, because many small seeds find their way into the reservoir and might be easily conveyed into the seed-pans by surface watering.

All of the foregoing matters are controlled with comparative ease, but there are sources of error which are much more difficult to manage. The director of the experiments understands the necessary precautions and takes them, and so do his foreman and others regularly engaged about the cultures, but not so the casual visitor. The plumber comes in to repair a pipe. He does not take kindly to a request that he brush the hayseed from his clothing before he enters the propagating house. He is sure they will not shake off while he is at work. Visitors want to see the cultures, and it is disconcerting to them to be asked to keep their fingers out of the seed-pans. I have often wondered what about a 
seed-pan seems so attractive to the fingers of the thoughtless. The soil looks so smooth and moist that everybody wants to feel just how soft and how moist it is, never dreaming that every time an unguarded finger passes from one seed-pan to another, a fatal question-mark is put into a pedigree that may have cost several years of patient care, and which may have within it the power to solve some problem of far-reaching scientific importance. At one time I discarded six pedigree seed-pans, because earth was thoughtlessly shaken from the roots of lettuce-plants growing in a box of unsterilized soil near the pedigree cultures, by one who did not at the moment see the importance of being careful. To satisfy myself that there was cause for discarding these pans, one of them was saved and in a few days several native weeds were found germinating. As the pedigree-culture was itself that of a native weed, no plant in the pan could be with certainty held to belong to the pedigree indicated by the label. This episode led to the apparently selfish precaution of keeping the pedi gree-cultures under lock and key, admitting no one excepi when a responsible guide was at hand to guard the seed-pans from thoughtlessness and ignorance; and I would advise the same precaution on the part of every pedigree-culturist. who wants to be sure of his pedigrees.

Two other instances of error of similar catastrophic type have occurred, which may prove of interest. My headgardener and I were greatly surprised at one time to find violet seedlings coming up in a number of Bursa seed-pans and we were at first nonplussed as to their origin until it was observed that the majority of these grew along the farther edges of the pans, thus indicating that they had come from the direction of the walk. Following this clew it was found that a few violets were fruiting in pots under an adjoining bench. Though these fruiting specimens were three feet below the level of the seed-pans and four feet or more to the side, the force with which the capsules dehisced was sufficient to lodge a considerable number of the seeds in the 
seed-pans, thus demonstrating the efficiency of the violet's method of dissemination. As none of the cultures then in the seed-pans were violets, no harm was done by this unexpected accession of violet seeds, but it is plain that special precau. tions would be necessary in the study of the violets by the pedigree method, in order to preclude the possibility of contamination with seeds of unknown parentage.

In times of heavy winds it is usually impossible to close ventilators so tightly or the house in general so hermetically that no dust or other debris carried by the wind can find its way through the crevices. Shortly after such a dust storm two specimens of Erechtites and one of Oxalis were seen to germinate in pans with which the usual precautions had been taken. As fragments of corn-leaves and of the inflorescences of Panicum capillare (?) had been found in the propagating house after the storm, it was natural to surmise that the several seeds which appeared where they did not belong had entered in the same way.

When plans are being laid for the prosecution of pedigree cultures on a fairly comprehensive scale the adoption of some system of labeling and note-keeping which shall be sufficiently complete and yet fairly simple is imperative. As the personal equation of the investigator is always an important factor in the adoption of such a system, I do not present the methods used by me as those which are likely to seem ideal to all people, but there may be some suggestions at least which will be of value to others, either as a good way to do it or the way not to do it.

As the number of pedigrees handled is large, the only feasible system of designating them is one whose chief characteristic is the Arabic numeral. I have developed a system which in different phases uses both the Roman numerals and the letters of the alphabet, but which uses the Arabic numerals alone for the permanent designation, or pedigree-number proper. All Arabic numbers used refer to specific individuals. Roman numerals are used only in the garden to designate the 
separate rows of each culture, always beginning with (I) at the label-stake and always counting in the same direction across the garden. Letters of the alphabet always refer to types or classes of plants distinguished by morphological differences sufficiently described in the pedigree-records, and, when practicable, also represented by specimens in the herbarium.

It is so generally necessary to make notes regarding individuals before it can be known whether they will ever be used as progenitors, that it is necessary to assign some temporary designation which may afterwards be replaced by a permanent number when it is decided to use them. For this temporary label I use the Arabic numeral in curves, thus ( $\mathrm{I}$ ), (2), etc. All notes entered in the note book regarding any particular individual refers to it under the number thus assigned. This refers only to pot cultures in which each plant has its own label bearing the pedigree-number of its mother. In the garden, the individual plant is not given a separate label, but is referred to in terms of its location. Thus "III. I 3 " indicates the thirteenth plant in the third row, counting the row bearing the label-stake as the first.

In the construction of the permanent pedigree-numbers, there are several things to be kept in mind: The tracing of pedigrees through successive generations must be facilitated; connection of each member of the pedigree with the notes regarding its characters, and those of its family must be made as perfect and simple as possible; and numbers must be kept from getting too large, as the likelihood of incorrect writing and reading of large numbers is very much greater than small numbers. To attain these ends I begin all pedigree numbers with the last two digits of the year in which the mother of the corresponding culture was grown, and follow this by the number of the page in my notebook for that year, on which the current notes are entered regarding the offspring of this particular plant. Thus 06230 indicates a plant, the complete history of whose offspring during one generation is 
to be found on p. 230 of my 1906 notebook. At the head of that page is given the pedigree-number of the grandmother as well as the mother, thus " 0557.230 Oenothera Lamarckiana Ser." The maternal year-number, 06 , is not necessary here because the entry occurs in the 06 notebook. In tracing out the complete history of the pedigree, I am at once referred by this number to p. 57 of the 05 notebood where I find the data for the parental family with with 04156.57 at the top, indicating that on p. 156 of the 04 note-book will be found the occasional notes on the grand-parental generation. At the top of that page appears the number 030.156 indicating that there was no entry in the 03 notebook and that therefore this was the beginning of the culture. In this particular instance there was no 03 notebook since the Station for Experimental Evolution was opened in 1904.

During the course of each season the pedigree-numbers for that particular year become quite familiar. These familiar numbers are carried along on the seed-bag and on the labels in the seed-pans, as a sort of introduction to the new family. Thus in the seed-pan from which the 06230 plants mentioned above were taken, appeared the label 0557.230 just as it did in the note-book. The characteristics of the 0557 family are still fresh in mind and serve as a key to the new family, without always needing to refer back to the records. On removing the plants from the seed-pans to pots, each must have its individual label. The new family has become established in the mind of the operator, and much labor is saved in the writing of labels by dropping the grand-parental number and prefixing the year-number to that part of the former number following the period, thus resulting in the "06230" first introduced above by way of example.

In keeping of notes it will have been already observed that I enter all the data concerning the members of any family on one page of my note-book. These notes are entered in chronological order and dated. Whenever an individual is observed which varies in any notable way a parenthetical number 
is added to its label, and this becomes its individual name for the rest of the season. Its label may then appear " 06230 ( $\mathrm{I}$ )" and its peculiarity is described in the note-book on p. 230 under the caption (I). Whenever thereafter a note is made concerning this individual it is referred to as ( $\mathrm{I}$ ) in the note-book. When a second aberrant individual is noted its label becomes "0623o (2)" and so on. These parenthetical numbers are always copied onto the seed-bag when the seed is collected, but are displaced by the regular pedigreenumbers when arrangements are made for the use of particular individuals in the continuation of the cultures.

As only a small proportion of the individuals of one season's pedigrees can be used for their continuation in the next season, it will be seen that the notes on these elected specimens will be scattered among notes on others which it is decided to follow no farther. It is a matter of great convenience therefore to bring together all of the entries regarding any individual to be used as the parent of a new culture and put them into shape for the most ready reference. This I do in the form of a card catalog, using the ordinary library card of good heavy grade. As this is the most accessible reference to the characteristics and history of each unit in the direct line of the pedigree, the cards must be substantial enough to withstand continuous use. At the top of the card appears the double pedigree-number exactly like that at the top of the page in the note-book, together with its equivalent parenthetical designation, thus permanently recording the equivalence between the new number and the one by which this individual was known in the note-book, e. g. "06230.I6=(I)". In the upper right hand corner of the card is the characteristic of the year, ( 07 in this case) indicating that the offspring of this plant will bear the label 0716 . This is necessary because in some cultures two or more generations may be raised in one year, or a pedigree may be omitted for one or more years and then resumed. On the next line appears the name of the species. Below this is given 
the history of the particular plant "( $\mathrm{I}$ )" which has now become " 16 ", stating among other things how it was pollinated. Then follows a statement of the characteristics of this plant, and finally, if it has been cross-pollinated, a description of the pollen-parent. These cards form a complete and permanent record of all the factors entering into the direct pedigreed lines. They are arranged alphabetically by species, (Oenothera Lamarckiana in this case), then according to the year number ( 07 in the example cited) and finally according to the value of the permanent individual pedigreenumbers ( 16 in the example given).

Besides the records already mentioned I have an index to the cultures, arranged according to the pedigree-numbers, which saves the necessity of referring to the larger notebooks when it is only desired to know what species is concerned in a culture bearing any given pedigree-number. The head-gardener has his note-book also in which is noted every act of his which in any way affects the cultures, as seedsowing, potting, and repotting, transplanting to the garden, all deaths and other casualties, etc. All these notes are entered in his note-book affixed to the pedigree-number found on the labels and are arranged chronologically, each note being made at the close of the event recorded and before a new task is taken up, thus insuring that the entries shall represent observations and not remembrances. The Station for Experimental Evolution has been very fortunate in the choice of its head-gardener, and I take pleasure in thus publicly acknowledging my indebtedness to the honesty, faithfulness and accuracy of Mr. Leo Macy for a large measure of the all but perfect control which it has been possible to attain in the plant-breeding work at the Station.

There is no way in which pedigree-cultures may be carried on for scientific purposes which does not involve the copying of pedigree-numbers, and it is necessary that this work be done with the greatest care. In such work it is well to acquire the habit of looking a second time to see that the 

[Reprinted from The American Naturalist, Vol. XLIL, July, 1908.

\section{A NEW MENDELIAN RATIO AND SEVERAL . TYPES OF LATENCY}

\section{DR. GEORGE HARRISON SHULL}

Station for Experimentau Evolution of the Carnegie Institution, Cold Spring Harbor, N. Y.

\section{INTRODUCTION}

In two papers presented before the Botanieal Society of Ameriea at its annual meetings in New Orleans (1905) and New York (1906), I discussed the question of latency as exemplified by certain color-characters in common garden beans (Phaseolus vulgaris). These papers were published in reversed order in Scrence, May 7 and 24, 1907.

It was shown that certain eharacters appeared in the hybrids, of which no indication was found in either parent, and the origin of these novelties was traced to unseen Mendelian units possessed by the white bean (White Flageolet) used in the various erosses. The new characteristies were a mottled color-pattern, $M$, and a blackener or enzyme, $B$, which acts upon brown or yellow pigments, $P$, to produce anthocyan, the presence of the latter resukting in black or various shades of violet to reddish purple seed-coats. It was assumed that the brown and yellow beans used in these erosses have the gametie formula, $\mathrm{Pbm}$, the black bean the formula, $P B m$, and the white the gametie formula, $p B M$. In erossing the whito bean with any of the self-colored beans the three dominant units were brought together, resulting 

[Reprinted from The American Naturalist, Vol. XliI., July, 1908.

\section{A NEW MENDELIAN RATIO AND SEVERAL . TYPES OF LATENCY}

DR. GEORGE HARRISON SHULL

Station for Experimental Evolution of the Carnegie Institution, Cold Spring Harbor, N. Y.

\section{INTRODUCTION}

IN two papers presented before the Botanical Society of America at its annual meetings in New Orleans (1905) and New York (1906), I discussed the question of latency as exemplified by certain color-characters in common garden beans (Phaseolus vulgaris). These papers were published in reversed order in Science, May 7 and 24, 1907.

It was shown that certain characters appeared in the hybrids, of which no indication was found in either parent, and the origin of these novelties was traced to unseen Mendelian units possessed by the white bean (White Flageolet) used in the various crosses. The new characteristics were a mottled color-pattern, $M$, and a blackener or enzyme, $B$, which acts upon brown or yellow pigments, $P$, to produce anthocyan, the presence of the latter resulting in black or various shades of violet to reddish purple seed-coats. It was assumed that the brown and yellow beans used in these crosses have the gametic formula, $P b m$, the black bean the formula, $P B m$, and the white the gametic formula, $p B M$. In crossing the white bean with any of the self-colored beans the three dominant units were brought together, resulting 
in a purple mottled $\mathrm{F}_{1}(P B M)$. It was the occurrence of this purple mottled $\mathrm{F}_{1}$, no matter which pigmented bean was used, that led to my conclusions regarding the latency of a mottled color-pattern and a melanizer in the white bean, and also to the prediction that $\mathrm{F}_{2}$ would consist of the five forms-purple mottled, black, brown mottled, brown (more properly, dark orange), and white, -in the well-known tri-polyhybrid ratio, $27: 9: 9: 3: 16$.

\section{An Unexpected Ratio and its Significance}

At the time my last report was made, the count of the $\mathrm{F}_{2}$ hybrids had not been completed, but the five predicted types were clearly presented. On summing up the results of the census of the numerous $\mathrm{F}_{2}$ hybrid families, it was found that the ratio was not as predicted, but the mottled and self-colored beans occurred in all cases in approximately equal numbers, resulting in the ratio $18: 18: 6: 6: 16$, or, reduced to its lowest terms, $9: 9: 3: 3: 8$. To be exact, in the cross between Ne Plus Ultra (dark orange yellow, called "brown" in my notes) and White Flageolet, 10 families gave 133 purple mottled, 114 black, 40 brown mottled, 50 brown, 105 white, and 6 doubtful. Similarly, in the cross between Long Yellow Six Weeks -(light yellow) and White Flageolet, 13 families gave 154 purple mottled, 159 black, 39 yellow mottled, 59 yellow (or brown), 160 white, and 12 unclassified. In the cross between Prolific Black Wax and White Flageolet, 3 families gave 53 purple mottled, 59 black, 44 white and 4 unclassified.

On comparing these results with those published by Tschermak ${ }^{1}$ it is found that they are in perfect accordance with them, as he also found in a number of similar crosses, an equality between the mottled and self-colored beans. But our conclusions were diverse as to the source of the mottled pattern, I assuming that the mottled factor was brought into the combination by the white

\footnotetext{
${ }^{1}$ Tschermak, E. Weitere Kreuzungsstudien an Erbsen, Levkojen und Bohnen. Zeitschr. Landw. Versuchsw., 7, pp. 533-638, 1904.
} 
bean as a simple Mendelian unit, while he assumed that a mottled factor was carried as a "cryptomere" by the pigmented bean and that the white bean acts simply as a releasing agent or activator which allows or compels the latent mottling to become apparent.

The ratio $18: 18: 6: 6: 16$ must have at first a very unfamiliar look to the student of genetics. It was not explained by Tschermak, but was separated by him into two groups of $9: 3: 4$, wherein the interrelations of the several terms need no explanation.

The census of my second generation was completed shortly after the appearance of De Vries's ${ }^{2}$ interesting account of "Twin hybrids" in Enothera, and the suggestion lay at hand that this ratio presented by Phaseolus might be a case of twin di-hybrids, the first and second terms of the ratio, as also the third and fourth terms, being in each case different phases or aspects of a single unit, which might be expressed thus $9 \mathrm{~A}: 9 \mathrm{~V}: 3 \mathrm{~B}: 3 \mathrm{C}: 8 \mathrm{~W}$. While such an hypothesis would fit the conditions presented by the $\mathrm{F}_{2}$, it was seen very soon that it does not harmonize with the occurrence of a uniformly purple mottled $\mathrm{F}_{1}$, nor with the splitting phenomena of $\mathrm{F}_{3}$, a portion of which has been already examined. A consideration of the $\mathrm{F}_{1}$ and $\mathrm{F}_{3}$ shows that there are three distinct units involved, as was stated in my earlier papers, namely - a pigment factor, $P$, a blackener, $B$, and a mottled pattern, $M$.

If all of these characters behaved according to the simple Mendelian method, the ratio would be that previously predicted, and out of every 64 individuals, on an average, 27 would have purple mottled seeds, and 9 black. In order that the number of individuals having purple mottled seeds shall be equal to the number having black seeds, it is necessary that of the 27 that should on theoretical grounds be purple mottled, 9 must show no purple mottling but must be black, though it contains the dominant mottle factor, $M$. This group of 27 purple mottled

${ }^{2}$ De Vries, H. On Twin Hybrids. Bot. Gaz., 44, pp. 401-407, D 1907. 
individuals belonging to the theoretical $\mathrm{F}_{2}$ ratio consists of the following eight types:

$\begin{array}{rl}1 & P B M P B M \\ 2 & P B M P B m \\ 2 & P B M P b M \\ 2 & P B M p B M \\ 4 & P B M P b m \\ 4 & P B M p B m \\ 4 & P B M p b M \\ 8 & P B M p b m \\ 27 & \end{array}$

There is only one basis on which a group of 9 individuals having a common gametic feature may be derived from this group, namely, on the ground of homozygosis with respect to any single allelomorph. Thus, there are 9 homozygotes with respect to $P$ (1 $P B M P B M$ $+2 P B M P B m+2 P B M P b M+4 \cdot P B M P b m), 9$ homozygotes with respect to $B(1 P B M P B M+2 P B M P B m+$ $2 P B M p B M+4 P B M p B m)$, and 9 homozygotes with respect to $M$ (1 $P B M P B M+2 P B M P b M+2 P B M p B M$ $+4 P B M p b M)$, and the assumption that any one of these groups will give self-colored beans will answer the requirements of the empirical $\mathrm{F}_{2}$ ratio, 18:18:6:6:16.

The only way in which it is possible to decide which of these three possible groups of 9 homozygotes is responsible for the equality of the mottled and self-colored types is to test their applicability to the other generations, since they all fit equally well the ratio found in the second generation. If homozygotes with respect to $P$ hide the presence of $M$, it will be impossible to find an individual with mottled seeds which will not give a progeny, one fourth of which will be white-seeded; but of the $\mathrm{F}_{3}$ families already examined, a number have been found which, while continuing to give mottled and selfcolored beans in the ratio $1: 1$, have failed to produce any whites. If the homozygotes with respect to $B$ are responsible for the latency of $M$, some brown or brown 
mottled offspring would be produced by every purplemottled parent, and there would be no equality between the purple-mottled and black in many families of the third and subsequent generations; but those $\mathrm{F}_{3}$ families which have been thus far investigated show a number of instances in which purple mottled parents produce no brown or brown mottled young and there is a continued equality between the mottled and self-colored offspring of such parents. The remaining possibility, namely, that individuals which carry the mottled pattern, $M$, but are homozygous with respect to that character, are not mottled but self-colored, is the only one that fits all of the observed facts. The mottled color-pattern must be heterozygous in order to become apparent in the hybrids.

We may then indicate the composition of the group of individuals of $\mathrm{F}_{2}$ which carry the dominant mottling factor, $M$, and the expectation as to the composition of the offspring which each will produce in $\mathrm{F}_{3}$ as follows:

$1 P B M P B M=\mathrm{Bl}(\mathrm{Bl})$ ( $M$ latent in all).

2 PBMPBm = PM(1PM:1Bl) ( $M$ latent in $\frac{1}{2}$ the $\left.\mathrm{Bl}\right)$.

$2 P B M P b M=\mathrm{Bl}(3 \mathrm{Bl}: 1 \mathrm{Br})$ ( $M$ latent in all).

$2 P B M p B M=\mathrm{Bl}(3 \mathrm{Bl}: 1 \mathrm{~W})(M$ latent in all $)$.

4 PBMPbm = PM $(3 \mathrm{PM}: 3 \mathrm{Bl}: 1 \mathrm{BrM}: 1 \mathrm{Br}) \quad$ ( $M$ latent in $\frac{1}{2}$ the self-colored).

$4 P B M p B m=\mathrm{PM}(3 \mathrm{PM}: 3 \mathrm{Bl}: 2 \mathrm{~W})$ ( $M$ latent in $\frac{1}{2}$ the $\mathrm{Bl}$ and $\frac{3}{4}$ the $\left.\mathrm{W}\right)$.

4 PBMpbM $=\mathrm{Bl}(9 \mathrm{Bl}: 3 \mathrm{Br}: 4 \mathrm{~W})$ ( $M$ latent in all).

8 PBMpbm = PM(9PM:9Bl:3BrM : 3Br: 8W) (M latent in $\frac{1}{2}$ the self-colored and $\frac{3}{4}$ the W).

It will be seen from this scheme that the mottled colorpattern could exist and does exist as a latent ( $i$. e., invisible) character in pigmented beans just as well as in the white bean, which is contrary to the assumption made, when I insisted that the mottled pattern must have come from the white bean. It is also obvious that the mottled 
pattern could not exist in both the pigmented and white beans used in these crosses, as in that case the $\mathrm{F}_{1}$ hybrids would have been homozygous with respect to this character and would have been black self-colored instead of purple mottled. In attempting to settle the question as to the origin of this mottled color-pattern I have carefully examined the results recorded by Tschermak and find evidence that at least one pure-bred pigmented bean must possess the mottled pattern while another does not. Whether the white beans used by him carried latent $M$ can not be settled at present, but it is now plain that he may have been right in referring the mottling factor to the pigmented beans. My White Flageolet as well as all the white beans used by Tschermak may not have the mottled pattern, and the gametic formula of the White Flageolet should then be written $p B m$, instead of $p B M$.

This question can only be settled by further careful crossing. The evidence derived from Tschermak is as follows: In the cross between "Hundert für eine" (light yellowish brown) and "Mettes Schlachtschwert" (white) there was no mottling in the offspring; "Hundert für eine" crossed with "Schwarze Neger" (black), both selfcolored, gave mottled offspring. Now according to my hypothesis, if "Schwarze Neger" carries the mottled pattern, "Hundert für eine" does not have it, and in turn, "Mettes Schlachtschwert" must not have it. If "Schwarze Neger," on the other hand, does not carry the mottled pattern, "Hundert für eine" has it, and in this case "Mettes Schlachtschwert" must also carry it. We can not say certainly, therefore, that the white "Mettes Schlachtschwert" does or does not have the mottled pattern, but on theoretical grounds either condition would be equally possible.

Among the corollaries of the explanation here given for the ratio $18: 18: 6: 6: 16$ is not only the fact already given that the mottled pattern may be carried by the pigmented bean invisibly quite as well as by the white bean, but also, since the mottled beans are heterozygous with re- 
spect to $M$, it would be impossible to have any of them breed true, $i$. e., the mottled bean is in the same category in this respect as the famous Blue Andalusian fowl. This conclusion is supported by 48 families of the third and fourth generations reported by Tschermak and by over sixty families of the $\mathrm{F}_{3}$ from my own mottled hybrids which have been already examined. Not one instance has been found in which the offspring of a mottled hybrid were even approximately all mottled.

The existence of pure-bred mottled races raises the interesting question as to what relation exists between these mottled hybrids which are heterozygous and can not breed true and the true-breeding mottled varieties. Tschermak ${ }^{3}$ shows that in crosses between constant mottled races and self-colored races, the mottled pattern acts as a typical Mendelian dominant, the hybrids splitting in $\mathrm{F}_{2}$ and subsequent generations in the ratio, 3 mottled: 1 self-colored.

\section{Latency due to Separation}

With respect to the question of latency since the purple mottling may not be a latent character of the White Flageolet, the type of latency discussed in my previous papers was only certainly exemplified by the pigment-changer, $B$, carried by the white bean. This type of latency is discovered by the production of a novelty when two allelomorphs are brought together, one or each of which, when acting alone, produces no visible character. Thus the black or purple color of these hybrids is due to the combination of the yellow or brown pigment of the pigmented parent and the colorless pigment-changer borne by the white parent. It may be called latency due to separation since patency is brought about by recombination. In my first paper on latency, ${ }^{4}$ issue

${ }^{3}$ Loc. cit.

${ }^{4}$ Shull, G. H. Some Latent Characters of a White Bean. Science, N. S., 25, pp. 828-832, May 24, 1907. 
was taken with Lock $^{5}$ regarding his assumption that novelties which appeared in crosses between certain peas were due to inactive units which became active on crossing. Lock ${ }^{6}$ has since reconsidered that case and independently come to the same conclusion that I reached, namely, that the spotted seed-coat was introduced by the white-coated pea in which it was invisible owing to its separation from the pigment-producing factor. This is not an uncommon type of latency and seems to be the only type included by writers who have treated the subject of latency from the Mendelian view-point. It gives rise to such modifications of the Mendelian ratios as $9: 3: 4$, $9: 7$, $27: 9: 9: 3: 16,27: 9: 28$, etc., instead of the theoretical $9: 3: 3: 1$ and $27: 9: 9: 9: 3: 3: 3: 1$. Some of these modified ratios are of more common occurrence, and are more familiar, than the unmodified ones, perhaps owing to the fact that albinism has been so frequently involved in the Mendelian investigations. Characteristics which are rendered latent by separation in the course of Mendelian hybridization have been called "masked" characters by Punnett. $\quad$ This is not a particularly apt term for latent characters of this type, and would be much more appropriately applied to cases of latency due to hypostasis discussed below.

\section{Latency due to Combination}

The existence of mottling as a latent characteristic in pigmented beans, due to the fact that it only becomes apparent when in the heterozygous condition, is obviously of an entirely different type. Instead of being a phenomenon of separation, it is due to the union in the same zygote, of two dominant allelomorphs, either of which alone will produce a manifest character, but

${ }^{B}$ Lock, R. H. Studies in Plant Breeding in the Tropics. Ann. Roy. Bot. Gard. Peradeniya, 2, pp. 299-356, 1904. See p. 241.

${ }^{8}$ Lock, R. H. On the Inheritance of Certain Invisible Characters in Peas. Proc. Roy. Soc., B, 79, pp. 28-34, 1907.

${ }^{7}$ Punnett, R. C. Mendelism. 2d ed., pp. viii + 85, 1907, London: Macmillan \& Co. See pp. 47-53. 
which, when acting together, produce none. This may therefore be called latency due to combination, since patency is brought about by separating the two allelomorphs whose union effaces their characteristic manifestation. If the White Flageolet carries the mottling factor, $M$, as was at first supposed, the appearance of mottling as a novelty in the first generation hybrids was due not alone to that fact, but just as much to the fact that the pigmented bean does not carry the mottled factor; or if, on the other hand, it should prove true on further investigation that the white bean does not carry the mottled factor, the mottled $\mathrm{F}_{1}$ is due to this very fact, quite as much as to the fact that the colored bean does possess it.

The conclusion, reached in my previous papers, that the primitive bean was probably purple mottled and that the purple mottled condition is therefore an atavistic one, seems to be left in some doubt, because of the existence of two types of mottling, one of which behaves as a typical Mendelian unit as shown by Tschermak, the other having the peculiar faculty of losing its external manifestation the instant it becomes homozygous. I have no doubt that in some form the mottling unit is a primitive one, but whether the ancestral bean possessing that unit was mottled or self-colored would depend entirely on which type of the mottling unit it carried. In order to breed true it is necessary that both eggs and sperms shall all carry the mottled factor, and if this mottled factor were of the latter type, the beans produced by the union of such sperms and eggs, being homozygous with respect to mottle, would be self-colored, while if the mottle was of the former type, the homozygous beans would be mottled. The conclusion as to the allelomorphic composition of the original bean is probably correct, but as to its external appearance, it may as well have been black as mottled.

The peculiar behavior of the purple mottled allelomorph in my hybrids and in most of Tschermak's, may have a 
very important bearing upon the interpretation of what are known as mid-races, $i$. e., races which regularly produce two forms in about equal proportions, for, as has been seen, the mottled beans of all the hybrid families which did not have a mottled bean as one of its original pure-bred ancestors, constitutes a mid-race. This fact was recognized by Tschermak (loc. cit., p. 47), though he attributed it to an unexplained effect of cross-fertilization, and not to the characteristic behavior of a definite Mendelian allelomorph. Other mid-races may likewise represent instances of latency due to combination. Wherever there is a double series of characters occurring in about equal numbers in the progeny of a self-fertilized individual, this type of latency should be looked for.

Purple punctation and brown flecking, which occur as novelties in the seed-coats of hybrid peas, were found by Tschermak to behave in a manner quite analogous to the mottling in beans, the first generation showing dominance of the novelty and subsequent generations always splitting into the punctate and non-punctate or the flecked and unflecked, respectively, and these no doubt are also cases of latency due to combination. Lock ${ }^{8}$ has shown, on the other hand, that when certain mottled and spotted peas are crossed with self-colored peas, the mottling and spotting act as typical Mendelian dominants capable of extraction as characteristics of pure-breeding races, which ought to be expected, since the homozygous parental strains possessed these characters. The apparent discrepancy between his results and those of Tschermak will be fully explained if we assume that there are two types of these color-pattern characters in peas, as there are in beans.

In all of these cases of latency due to combination, the two units involved are of the same kind, so that the latency occurs only in the homozygous individuals, thus resulting in a striking contrast between homozygotes and

${ }^{8}$ Lock, R. H. On the Inheritance of Certain Invisible Characters in Peas. Proc. Roy. Soc., B, 79, pp. 28-34, 1907. 
heterozygotes. There are many other cases in which the homozygote and heterozygote show marked and often unexpected differences, the case of the Blue Andalusian fowl being one of the best known of these, but the heterozygous type of the Blue Andalusian fowl or other similar forms is not a case of latency at all, since no hidden allelomorph is brought to light as a result of heterozygosis, but only an unexpected external manifestation.

\section{Latency due to Hypostasis}

A third type of latency has also appeared in these bean hybrids, as best exemplified by a cross between the Prolific Black Wax and the Ne Plus Ultra, and between Prolific Black Wax and Long Yellow Six Weeks. In both of these crosses, besides the black and orange or black and yellow which were expected in the ratio $3: 1$, there have appeared a considerable number of beans of a dark seal brown or a dark greenish brown eolor. It is certain that these dark brown beans owe their color to the latency of a dark brown factor in the black bean. It has not been an infrequent occurrence to find black beans, not quite perfectly matured or which have been more or less weathered, that show this brown color apparently underlying the black. In this case the appearance of the novelty is due to the presence of a characteristic which can not be seen (i. e., which is latent), for the simple reason that the black pigment possessed by the same bean is so intense as to cover over and hide the brown pigment. The independence of the brown and black pigments allows them to be separated into different individuals upon crossing the black with some other color.

Letting $D$ represent this dark brown factor, the gametic formula for the black bean is $B D$, and for the orange brown and yellow beans, $b d$. This assumption leads to another rather unfamiliar modification of the Mendelian ratio, since the $\mathrm{F}_{2}$ should consist of black, brown and orange or yellow in the ratio $12: 3: 1$. The actual ratios are in essential accord with this expectation though there 
are rather wide discrepancies due to the fact that the categories were not as carefully distinguished at first as they should have been. Thus in the case of the cross of Prolific Black Wax (black) with Ne Plus Ultra (dark orange or "brown") many of the dark brown beans were recorded at first simply as "brown," and the ratio found, 174 black:47 seal-brown:26 "brown," shows clear evidence of the extent of error thus produced. A deficiency of black is also apparent and is no doubt due to the recording of some weathered blacks, as dark brown. In the cross between Prolific Black Wax and Long Yellow Six Weeks, the deficiency in the blacks and corresponding excess in the dark brown is even more striking, giving the ratio, 155 black:55 dark brown:9 yellow:5 unclassified, theory requiring 168 black:42 dark brown:14 yellow. This factor $D$ is also found to be present in the White Flageolet, where, like the black factor, $B$, it is latent by separation.

The occurrence of dark brown as an invisible character in the black bean may be called a case of latency due to hypostasis, following the terminology suggested by Bateson. ${ }^{9}$ The unexpected character is not inactive, but its characteristic manifestation is invisible because it is hidden or inhibited by some other quality, and can only become visible when the overlying or inhibiting quality is removed by some means.

This type of latency is no doubt very common, as it is involved in many cases of simple dominance, as that term is generally understood. If the "presence and absence" hypothesis has general validity (and there is a very great preponderance of evidence in favor of it), the term "dominance" should be limited to the relation of the presence of any characteristic to the absence of that same characteristic, and should not be used for the relation between two different positive allelomorphs by virtue of which one hides the presence of the other. Bateson

\footnotetext{
${ }^{\circ}$ Bateson, W. Facts Limiting the Theory of Heredity. Science, N. S,
} 26, pp. 649-660, November 15, 1907. 
applies the terms "epistatic" and "hypostatic" to the relative capacity of one unit to hide or to be hidden by another, owing to what I call latency due to hypostasis. As a simple illustration, a cross between a pea with yellow cotyledons, $Y$, and one having green cotyledons, $G$, shows $Y$ dominant over its absence, $y$, and not over $G$. This would become immediately obvious if we could cross the yellow pea with still another type, say with one having colorless cotyledons. The correct gametic formula for the yellow pea is not $Y$ but $Y G$, in which the green is latent owing to the fact that $Y$ is epistatic to $G$. The gametic formula of the green pea is $y G$.

That this is a correct interpretation of the apparent dominancy of one positive allelomorph over another is shown by some of my bean crosses. Thus Ne Plus Ultra (dark orange yellow) crossed with Long Yellow Six Weeks (light yellow) produced in $14 \mathrm{~F}_{2}$ families, 382 orange yellow:130 light yellow, an apparent dominance of orange over light yellow. That the light yellow is latent in Ne Plus Ultra and is not the recessive condition of the orange yellow allelomorph is proved by the fact that in the $\mathrm{F}_{2}$ families of the cross between White Flageolet and Ne Plus Ultra, light yellow beans appear. Letting $O$ represent the orange allelomorph and $Y$ the yellow one, the gametic formula of Ne Plus Ultra with respect to these two factors is $O Y$, that of the yellow bean is $o Y$, and that of the white bean likewise $o Y$.

The ratio, 12:3:1, presented by the crosses of Prolific Black Wax with Ne Plus Ultra and Long Yellow Six Weeks, has been reported for but one other case so far as I know, though it ought not to prove very uncommon. It will appear in the $\mathrm{F}_{2}$ of any cross which produces an $\mathrm{F}_{1}$ of the form $A B C a b$ with $B$ hypostatic to $A, C$ hypostatic to both $A$ and $B$, and neither $A, B$, nor $C$ latent from any other cause. In these beans the crosses are of the type $A B C \times a b C=A B C a b, i . e$, both $B$ and $C$ are latent in the one parent and no latent characters are demonstrated in the other. The same ratio will result from a 
cross of the type $A b C \times a B C=A B C a b$ provided the same relations exist among the several allelomorphs as before. In this case the character $C$ is latent by hypostasis in both parents. This condition has been realized by Toyama ${ }^{10}$ in hybrids between the common Japanese white silk-worm and the Siamese striped silk-worm in both of which a "pale," unmarked type is latent by hypostasis. The $\mathrm{F}_{1}$ is uniformly striped like the Siamese, and the $\mathrm{F}_{2}$ consists of striped, "white," and "pale" in the ratio $12: 3: 1$. Toyama's statement that the "pale" character was in the "dormant" state indicates a misconception of the nature of latency due to hypostasis.

\section{Latency due to Fluctuation}

Another very potent cause of latency is to be found in fluctuation. It is well known that many of the less marked qualities of plants do not appear under unfavorable conditions of growth. By growing the offspring of these poorly developed individuals under favorable conditions they may be shown to possess all the characters of other members of the race to which they belong. Invisibility produced by this cause may be called latency due to fluctuation. Patency is brought about by good feeding, room for full individual expression, etc. As a specific example, I may mention my experience with several biotypes of Bursa bursa-pastoris (L.) Britton. These differ from one another by certain characteristic lobings of the leaves, and these characters have proved, on crossing, to be typical Mendelian unit-characters. However, by growing the plants belonging to any of the several biotypes under sufficiently unfavorable conditions they may be made to produce seeds while bearing only the unlobed juvenile type of leaf. The Mendelian rosette characters are then wholly invisible or latent. If the

${ }^{10}$ Toyama, K. Studies on the Hybridology of Insects. I. On some silkworm erosses with special reference to Mendel's Law of Heredity. Bull. Coll. Agr. Tokyo Imp. Univ., 7, pp. 259-393, pls. VI-XI, July, 1906.- See pp. 348-353 and pl. X, III, $a, b$, and $c$. 
offspring of sush plants are grown under favorable conditions the latent characters are again rendered patent, showing that the loss of external manifestation has had no influence upon the allelomorphs themselves; they were present in the badly developed specimens, but were invisible because a sufficiently late stage of differentiation was not attained to permit them to express themselves.

Another striking case in which the latency of a Mendelian character, perhaps due to fluctuation, has been fully demonstrated, is in the cross between blue and white Indian corn investigated by Lock. ${ }^{11}$ The blue is, in general, dominant over the white, but the white grains are always in excess of expectation, sometimes more, sometimes less; subsequent breeding tests with the whites show that a sufficient proportion of them are heterozygous, instead of extracted recessives, to make up the deficiency found in the number of blues in the preceding generation. It is not impossible, as Lock suggests, that further investigation of this case will discover some other cause than fluctuation for the latency of the blue aleurone layer in these white-grained heterozygotes.

The classic case of so-called "double adaptation" in Polygonum amphibium which is pubescent in its terrestrial form and glabrous when grown as an aquatic, and other cases of the same kind, present illustrations of latency due to fluctuation, instead of being due to the presence of two antagonistic determinants whose activities are mutually exclusive as suggested by De Vries. ${ }^{12}$

The very common occurrence of latency due to fluctuation must have an important bearing upon the significance of cultural conditions for the production of variations. There has been much diversity of opinion on this point, the general impression being that cultivation and the removal of competition are very potent in inducing

${ }^{12}$ De Vries, H. Species and Varieties, their origin by mutation, pp. xviii + 847. 1905. Chicago: Open Court Pub. Co. See p. 430 et seq.

${ }^{11}$ Lock, R. H. Plant Breeding in the Tropies. III. Experiments with maize. Ann. Roy. Bot. Gard. Peradeniya, 3, pt. 2, pp. 95-184, November, 1906. See pp. 144-163. 
variation, and that in consequence of this fact it is improper to apply principles derived under cultivation to plants growing free in nature. There can be no doubt that good cultural conditions render patent many internal characters which are invisible under conditions of poor nutrition and crowding, and this fact together with the fact that many of the common culture-plants are complex hybrids, may fully account for the general impression regarding the effects of culture. There is no satisfactory evidence that good feeding and other conditions usually supplied under tillage have any effect in the production of the mutations upon which the external characters no doubt ultimately depend.

\section{General Considerations}

It is obvious from the foregoing results and discussions that latency is not a simple phenomenon, but may be due to a number of different circumstances. The point which I have strongly emphasized in my two preceding papers on the subject of latency-namely, that cases of latency must be explained, not upon the ground of inactivity or dormancy of characteristics, but simply on their invisibility - is fully borne out by all the facts here presented. The several different types depend upon the different causes for the invisibility of the characteristics.

Of the four types of latency here recognized, the first three types - those in which latency is due to definite interrelations between Mendelian units-will give rise to definite characteristic ratios which are as constant for each case as the typical ratios are for typical Mendelian phenomena. This is not so with latency due to fluctuation, as the variable conditions upon which the fluctuations depend may be such that any proportion of the individuals from none to all may have the character in question latent. This is not only true of the characters of pure-bred types as exemplified by Bursa bursa-pastoris, but is even more apt to be true of heterozygotes, thus resulting in many deviations from the correct ratios, as 
seen in Lock's blue $\times$ white corn cross and doubtless in very many other cases.

It is probable that many discrepancies between actual and theoretical ratios are due to some sort of latency. This will generally be detected readily in subsequent generations, and no one should be hasty in declaring that a character which is of the splitting kind is non-Mendelian until the various types of latency are considered which may have taken part in modifying the ratios. "Variable potency," "contamination", or "impurity" of the gametes, and "alternating dominance" will all need to be reconsidered and in some cases reinvestigated, before they can have any secure standing as exceptions, amendments or additions to the simple law of "purity of the gametes" which is the essence of Mendelism.

There is still another way in which unexpected ratios may be produced, without in any way affecting the fundamental principle of the purity of the gametes, their production in equal numbers, and their union according to the laws of chance, and while the question of latency is not involved in this case, it deserves to be mentioned in this connection. Baur ${ }^{13}$ has shown that in a variegated race of Antirrhinum, the variegation belongs only to the heterozygote. The extracted recessives are green and the extracted dominants fail altogether to appear, owing evidently to the fact that the zygote so formed is incapable of development, the ratio resulting from selffertilization of the heterozygotes being therefore $2: 1$. It is conceivable that every degree of inefficiency of zygotes formed by the union of two particular allelomorphs might occur and thus quite various modifications of the expected ratios be the result, when those ratios are determined by a count of the successful zygotes. This cause for the failure of the expected ratios is certainly of rare occurrence, but like questions of latency it can be demonstrated

${ }^{18}$ Baur, E. Untersuchungen über die Erblichkeitsverhältnisse einer nur in Bastardform lebensfähigen Sippe von Antirrhinum majus. Ber. Deutsch. Bot. Gesell., 25, pp. 442-454, 1907. 
without difficulty by breeding tests, and these should be made before any new principle is invoked, or the old and well-founded principles are declared invalid, in the attempt to account for such discrepancies.

\section{Summary}

The foregoing discussion and conclusions may be summarized thus :

In certain bean hybrids, mottled seed-coats depend upon the presence of a mottling allelomorph in a heterozygous condition, the homozygous condition giving unmottled seeds. This peculiar situation results in a tripolyhybrid ratio, $18: 18: 6: 6: 16$, instead of the usual ratio, $27: 9: 9: 3: 16$.

Latency is held to mean invisibility, and not inactivity or dormancy, and four types are recognized, according to the different causes of invisibility; still other types may be found. The four types discussed in this paper are:

(a) Latency due to separation, in which an allelomorph when acting alone has no external manifestation and is only rendered patent by combining it with another allelomorph. Such latency gives rise to the ratios $9: 3: 4,9: 7$, $27: 9: 9: 3: 16$ and $27: 9: 28$, instead of the theoretical, $9: 3: 3: 1$ and $27: 9: 9: 9: 3: 3: 3: 1$.

(b) Latency due to combination, in which two dominant allelomorphs, each giving rise to a peculiar character when acting alone, lose their external manifestation when co-existing in the same zygote. Upon self-fertilization this type of latency gives rise to such ratios as $1: 1,3: 3: 2$, $18: 18: 6: 6: 16$, etc., and may be found to account for the existence of certain mid-races, and other cases in which a double series of characteristics are presented in nearly equal numbers.

(c) Latency due to hypostasis, in which the presence of one allelomorph can not be detected owing to the presence of another allelomorph, the character produced by the latter being unmodified by the activity of the former. This type of latency is exemplified by the black bean 
which hides the presence of a wholly distinct brown allelomorph, and a dark orange bean which carries invisibly a light yellow allelomorph. This condition gives rise in one series of crosses to the ratio, $12: 3: 1$. Properly the term "dominance" should be limited to the relation between any positive characteristic and its own absence. Whenever one positive character seems to dominate another positive character, the latter is latent by hypostasis in the individual possessing the former.

(d) Latency due to fuctuation, a very frequent phenomenon in which characteristics disappear under conditions of poor nutrition, etc. Cultivation under favorable conditions makes such characteristics patent and this fact may account in part for the general impression that cultivation induces variation. Cases of "double adaptation" are examples of this type of latency.

Many discrepancies between theoretical and empirical inheritance-ratios are due to latency, and care should be taken to investigate the possible latencies which may be present before declaring that a character is non-Mendelian, because of a discrepant ratio. "Variable potency," "contamination" or "impurity" of the gametes, and "alternating dominance" which have been proposed to account for the appearance of various novelties, or of deviations from expected ratios, can have no secure standing until the question of latency in the sense of invisibility has been taken into account.

A modification of expected ratios may rarely result also from the failure of certain allelomorphs to make vigorous zygotes when joined together in certain combinations. 



\section{A PURE-LINE METHOD IN CORN BREEDING}

BX

DR. GEORGE HARRISON SHULL

Cold Spring Harbor, N. Y.

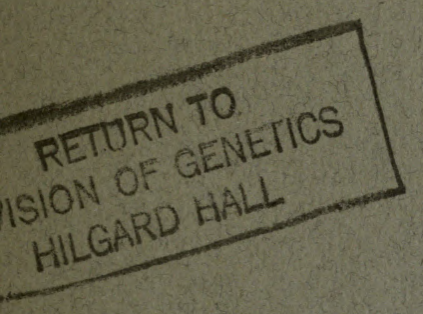

Index ${ }^{-1}$ 
Thy

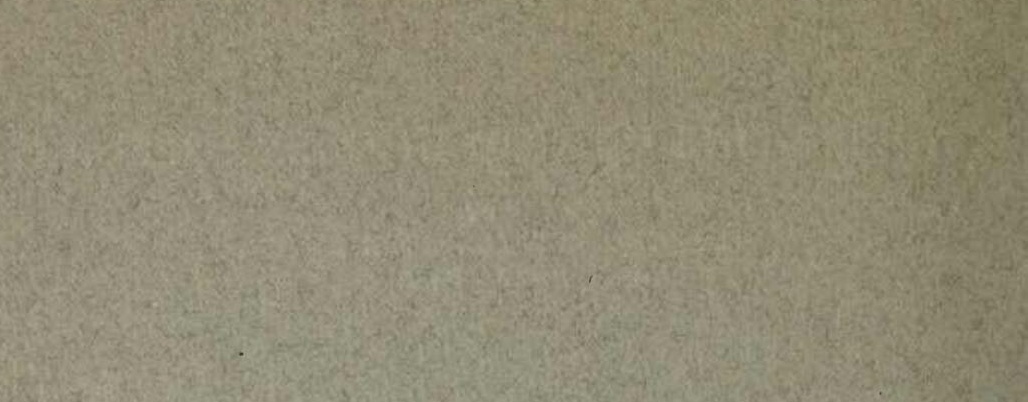
19.8. 1.

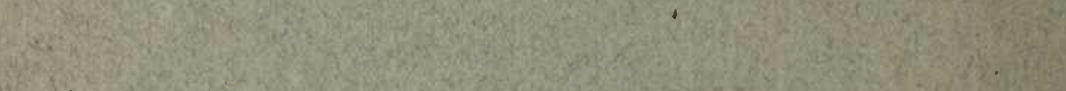

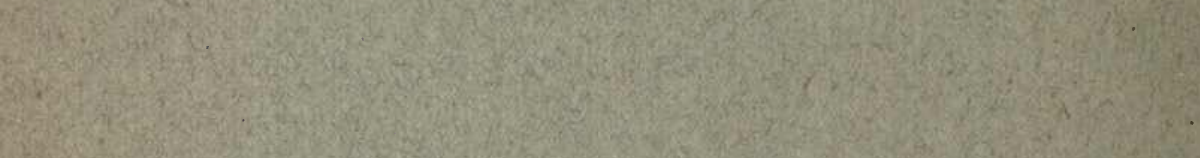
Q

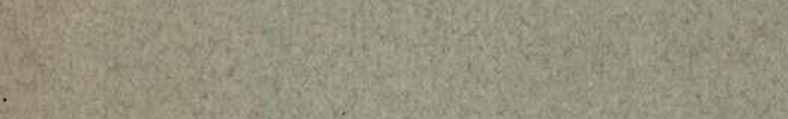
-

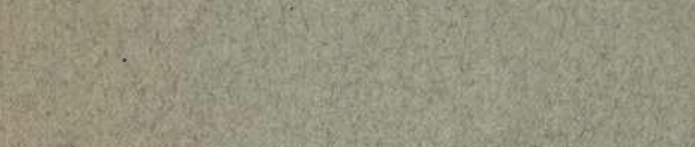

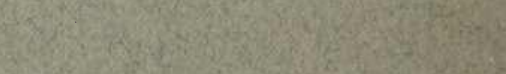

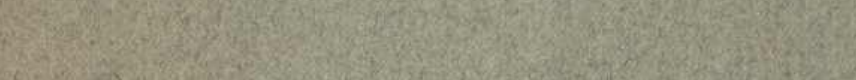

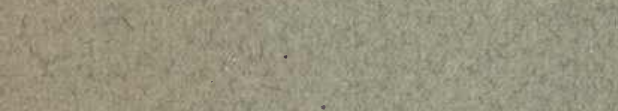
(3) $\sin ^{2} \sin ^{2}$

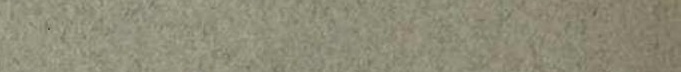




\title{
A PURE-LINE METHOD IN CORN BREEDING.
}

\author{
By Dr. George Harrison Shull \\ Cold Spring Harbor, N. Y.
}

Last year I described ${ }^{1}$ a series of experiments with Indian corn which led me to the conclusions: (I) that in an ordinary field of corn the individuals are generally very complex hybrids; (2) that the deterioration which takes place as a result of self-fertilization is due to the

${ }^{1}$ The Composition of a Field of Maize. Report American Breeders' Association, 4:296-30I, I908. 
gradual reduction of the strain to a homozygous condition; and (3) that the object of the corn-breeder should not be to find the best pureline, but to find and maintain the best hybrid combination.

The continuation of these studies during the past year have given still further proof of the correctness of the first two of these propositions, and besides has given unexpected suggestions for a new method of corn breeding by which the essential feature of the third proposition may be realized. It is my purpose to discuss this new method briefly in the following pages. I will first, however, describe the results of the past year's experiments in so far as they bear upon the points in which we are interested here. For convenience I will refer to the two self-fertilized families contrasted in my paper last year as "Strain A" and "Strain B." It will be remembered that these two families resulted from the self-fertilization of different, apparently equal, individuals; but that notwithstanding this fact, they differed from each other in height and stockiness of stems, width and greenness of the leaves, length of shank of the ears, appendages of the husks, quality of the grains, and the number of rows of grains on the ears. (See fig. I.)

In addition to the parallel cultures of self-fertilized and crossfertilized families which have been continued from the beginning of these experiments in $1904, I$ had during the past season the $F_{1}$ offspring of a cross between two sibs in Strain A, and two families representing reciprocal crosses between Strain A and Strain B. It was observed that every one of the mentioned characteristics which distinguished Strains A and B, remained constant distinguishing features in the purebred families, but in regard to the number of rows on the ears, it is now obvious that Strain A has the normal mean number 8, as compared with I4 in Strain B, for in this year 89 per cent of the ears produced by Strain A had only 8 rows of grains, though the selection of ears for seed in this strain during three years was for 12 rows on the ear, and only in the last year was an 8-rowed ear used because a suitable 12-rowed ear was not available. This result is a striking confirmation of the suggestion made last year that according to the law of regression the occurrence of a mean number of rows less than 12 in Strain A indicated that the normal number of rows for this strain is Io or possibly only 8 .

The cross between two sibs in Strain A was grown beside the selffertilized family belonging to the same strain, and these two families were so similar during the entire period of their development that they were considered identical, but at the end of the season it was found that the cross-bred family was a trifle taller and produced over 30 per 

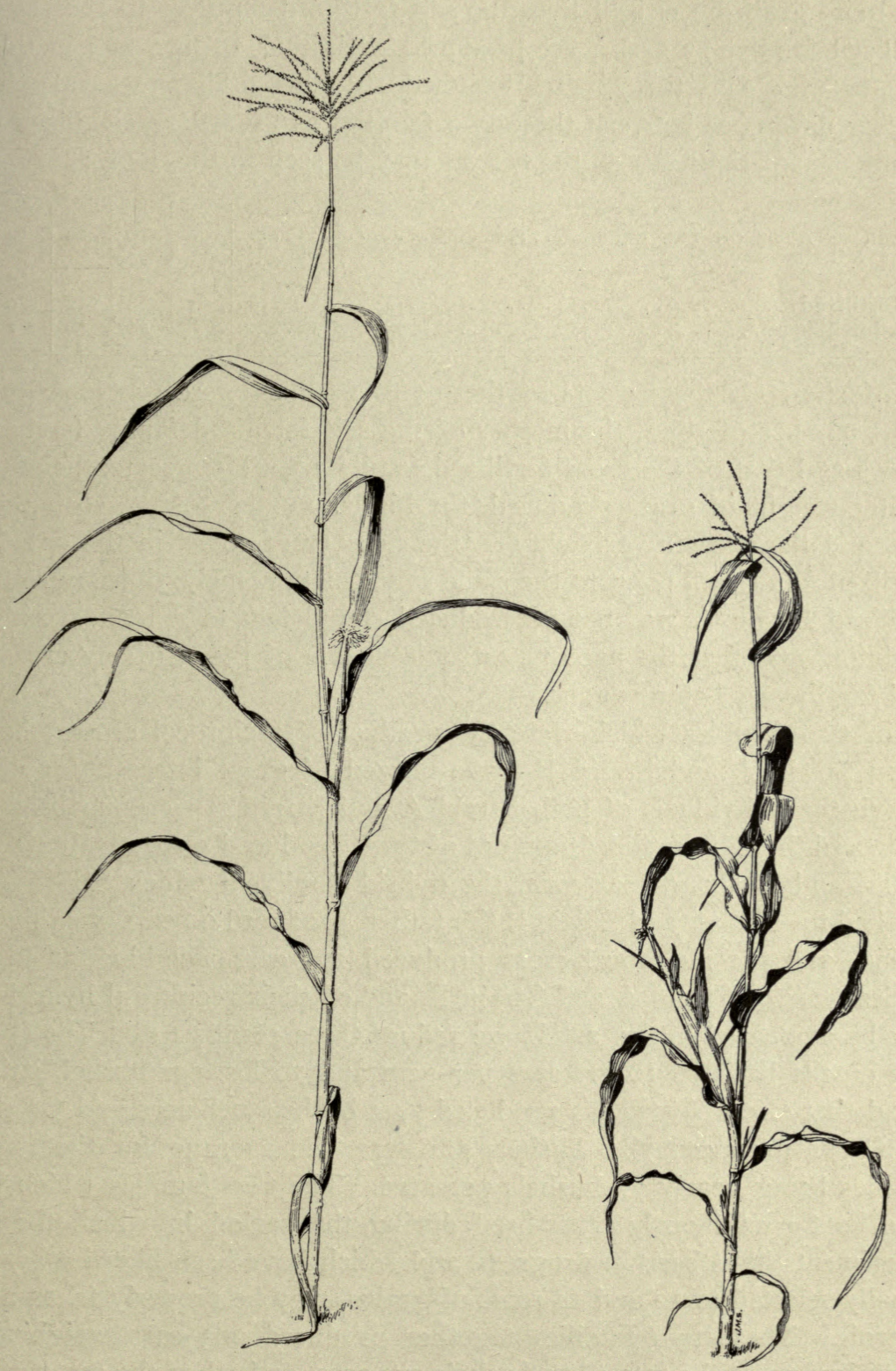

FIG. I. TYPICAL SPECIMENS OF STRAIN A (AT RIGHT) AND STRAIN B (LEFT), SHOWING CONTRAST OF VEGETATIVE CHARACTERS. DRAWN BY J. MARION SHULL, FROM A PHOTOGRAPH. 
cent more grain by weight than the self-fertilized family. In the selffertilized family, 73 ears were produced, weighing $12 \mathrm{lbs}$., and in the cross between sibs the 78 ears weighed $161 / 2 \mathrm{lbs}$. There was also a striking difference between these two families as regards variability in the number of the rows on the ear, as may be seen in this table:

\begin{tabular}{|c|c|c|c|c|}
\hline NUMBER OF ROWS ON THE EARS $\ldots \ldots \ldots \ldots$ & 8 & ro & 12 & $I_{4}$ \\
\hline $\begin{array}{l}\text { Self-fertilized. . . . . . . . } \ldots \ldots \ldots \ldots \\
\text { Cross-fertilized . . } \ldots \ldots \ldots \ldots\end{array}$ & $\begin{array}{r}65 \\
8\end{array}$ & $\begin{array}{r}6 \\
50\end{array}$ & $\begin{array}{r}2 \\
19\end{array}$ & $\begin{array}{l}0 \\
I\end{array}$ \\
\hline
\end{tabular}

Unfortunately the parents of these two families were not identical in the number of rows, the mother of the self-fertilized family having 8 rows and that of the cross-fertilized family Io. The greater height, greater weight of grain produced, the higher number of rows on the ears, and the greater variability in the number of rows, in the crossfertilized family, all point to the same conclusion, namely, that my selffertilized Strain A was not yet reduced completely to a homozygous condition, and that the parents, or at least one of them, of my crossbred family was heterozygous.

The two families which were the product of reciprocal crosses between Strain A and Strain B, have proved of great interest, for although the individuals of both Strain A and Strain B were small and weak, and the self-fertilized families of these produced respectively only I2 lbs. and I3 lbs. of ear-corn, the hybrid family in which Strain A supplied the mother and Strain B the father, produced 92 ears weighing $48 \mathrm{lbs}$., and the reciprocal cross produced Ioo ears weighing $55 \mathrm{lbs}$. Typical ears of Strain A and Strain B, and of their reciprocal hybrids, may be compared in fig. 2. If we reduce these results to bushels per acre on the basis of 10,000 ears per acre and 7o lbs. per bushel, it is found that Cross $\mathrm{A} \times \mathrm{B}$ has produced 74.4 bushels per acre and Cross $\mathrm{B} \times \mathrm{A}$ has produced 78.6 bushels per acre, the average for the two families being nearly 77 bushels per acre. The two families which I have kept continuously cross-bred during the period in which these experiments have been in progress, and which have been likewise continually selected to 12 and I4 rows of grains, may be properly taken as controls. These two families together produced 203 ears weighing I0 $7 / 2 \mathrm{lbs}$., or at the rate of 75 bushels per acre, and when the comparison is extended so as to include my other continuously crossed families- 8 families in all-it is found that these produced collectively at the rate of a little less than 75 bushels per acre. 
My farmer friends, especially here in the heart of the corn country, will not be greatly impressed with these yields of $75-78$ bushels per acre, but I must call attention to the facts that the light gravelly soil of Long Island bears a very unfavorable comparison with Mississippi valley alluvium for the production of Indian corn, and further that the summer of 1908 was notable for one of the longest periods without rain that has ever been experienced there. The important point will

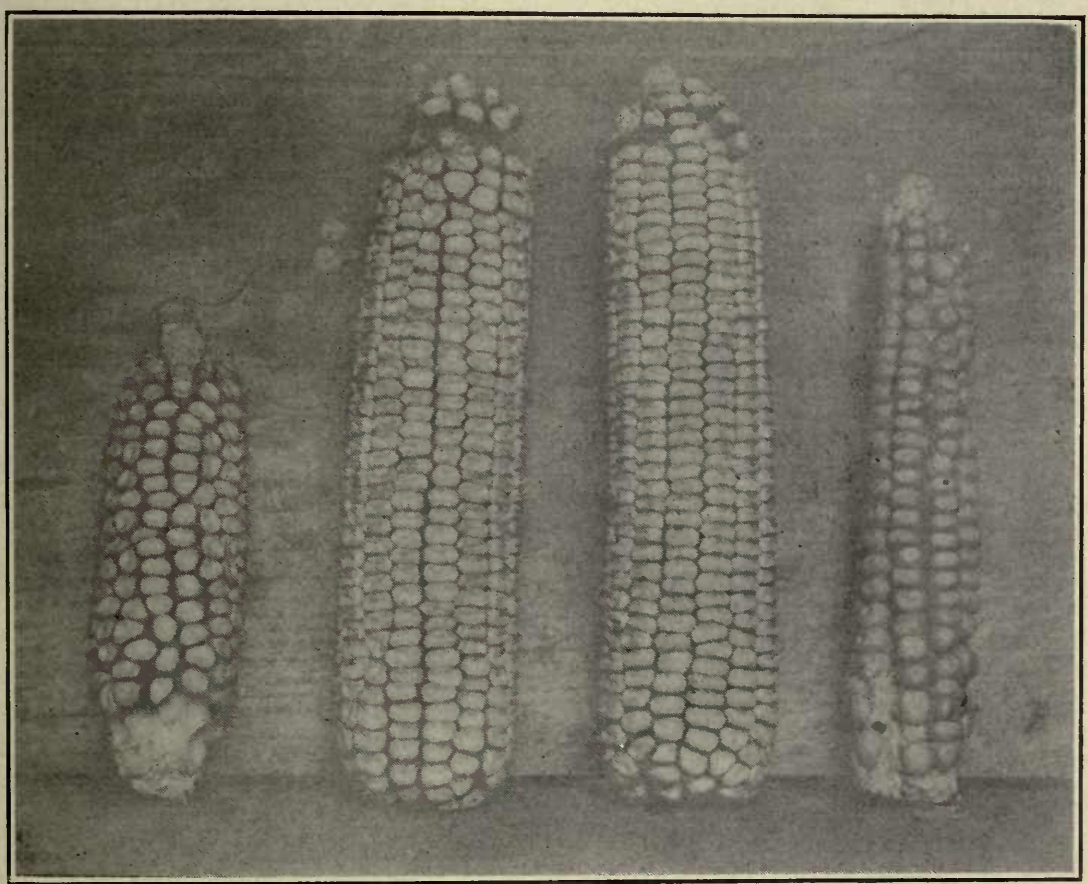

FIG. 2. TYPICAL, EARS OF STRAIN A (AT RIGHT) AND STRAIN B (LEFT) AND OF THEIR RECIPROCAL HYBRIDS. EACH HYBRID STANDS NEAREST ITS MOTHER STRAIN,

not be missed however that the crosses between two self-fertilized strains yielded a little more grain than those strains which had been kept carefully cross-fertilized by hand. To be sure, the difference is not great enough to seem of any particular significance in itself, but it must be remembered that the two self-fertilized strains, $\mathrm{A}$ and $\mathrm{B}$, have been essentially unselected, being simply those two strains which lave first approached the pure homozygous state as a result of selffertilization. It is scarcely conceivable that other pure strains crossed 
together should not give in certain combinations considerably greater yields than those produced by the combination of Strains A and B. At any rate the result is sufficiently striking to suggest that the method of separating and recombining definite pure-lines may perhaps give results quite worth striving for.

This suggestion will be more readily appreciated perhaps if I discuss briefly the theoretical aspect of this method of pure-line breeding as compared with the method now in use among the most careful cornbreeders. In the light of my results, the constant precautions that are taken in the method now in use to prevent in-breeding, have for their real object the retention of the most efficient degree of heterozygosis or hybridity, and it is obvious that the selection of the most vigorous individuals for seed, really picks out those individuals which have this most efficient degree of hybridity.' While I have not investigated the inheritance of the various characteristics of the pure lines of maize and am not in position to say that they all follow Mendel's law, many investigations of particular characteristics in corn have shown that those characteristics are Mendelian. Even if some of the differentiating characteristics of corn should not prove to be Mendelian, it seems not improper to discuss the two methods on the Mendelian basis.

In the method which selects for seed the most heterozygous individuals, the characteristic splitting and recombination of unit-characters must produce an offspring of quite various degrees of heterozygosis. Some individuals will be as complex as the selected parents, others will have many of the same units in the homozygous condition, and thus be less complex and consequently less vigorous. According to the laws of chance a few individuals in the field may be expected to be almost or quite completely homozygous, and as a result will be very inferior in vigor and will produce but little grain. The result of such a process must always be to give a crop of lower average yield than the average of the selected seed. Moreover, these different combinations of unitcharacters and different degrees of hybridity in the offspring of a complex hybrid must introduce a certain amount of heterogeneity into the crop which will have the effect to also lower the average quality with respect to any other desirable points which have been used as guides in the selection of the seed-corn, and efforts at the attainment of homogeneity by the method now in use tend to lessen physiological vigor, and therefore lessen the yield, owing to the fact that such homogeneity in the offspring of hybrids is to be attained only through homozygosis in respect to all those characteristics which affect the 
form and size of the ear, width, depth, shape, and composition of the grains, and any other feature in which homogeneity may be desired. This is doubtless the explanation of the interesting experience related by Mr. Joseph I. Wing at the meeting of the American Breeders' Association in Columbus two years ago. His father had selected a very fine deep-grained variety of corn in which great uniformity had been attained but only at the expense of decreased yield.

- In the pure-line method outlined below all individuals in the field will be $F_{1}$ hybrids between the same two homozygous strains, and there are theoretical grounds for expecting that both in yield and uniformity superior results should be secured. Thus, every individual will be as complex as every other one and should produce an equal yield of grain if given an equal environmental opportunity, so that in so far as hereditary influences are concerned the vigor of the entire crop should be equal to the best plants produced by the methods now in use. This would seem to result necessarily in a larger yield than can be produced by the present method. But not only will all the plants in the field have the same degree of complexity, but they will all be made up of the same combination of hereditary elements, and consequently there must result such uniformity as is at present unknown in corn.

With such a prospect as this, I believe we will be sufficiently interested to make the discussion of the method by which such results are to be attained worth while. The question naturally arises as to whether the technique of the new method will be sufficiently simple to make it practicable. To this question I believe I can safely answer that the pure-line method will be considered simpler than the elaborate one now in use among the most careful breeders, $e . g$., those at the Illinois, Connecticut, and Ohio State Experiment Stations. The process may be considered under two heads: (I) Finding the best pure-lines; and (2) The practical use of the pure-lines in the production of seed-corn.

( I) In finding the best pure-lines it will be necessary to make as many self-fertilizations as practicable, and to continue these year after year until the homozygous state is nearly or quite attained. Then all possible crosses are to be made among these different pure strains and the $F_{1}$ plants coming from each such cross are to be grown in the form of an ear-to-the-row test, each row being the product of a different. cross. These cross-bred rows are then studied as to yield and the possession of other desirable qualities. One combination will be best suited for one purpose, another for another purpose. Thus, if the selffertilized strains be designated by the letters of the alphabet, it may 
be found that Cross $\mathrm{C} \times \mathrm{H}$ will give $\mathrm{s} 2 \mathrm{O}$ bushels per acre of high-protein corn, that $\mathrm{F} \times \mathrm{L}$ produces a similar yield of low-protein corn that $\mathrm{K} \times \mathrm{C}$ gives the highest oil-content accompanied by high yield, and so on. Moreover, it seems not improbable that different combinations may be found to give the best results in different localities and on different types of soils. The exchange of pure-bred strains among the various experiment stations would greatly increase the number of different possible hybrid combinations and facilitate the finding of the best combination for each locality and condition.

(2) After having found the right pair of pure strains for the attainment of any desired result in the way of yield and quality, the method of producing seed-corn for the general crop is a very simple though

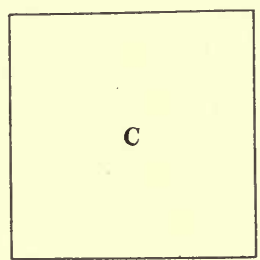

PLOT I

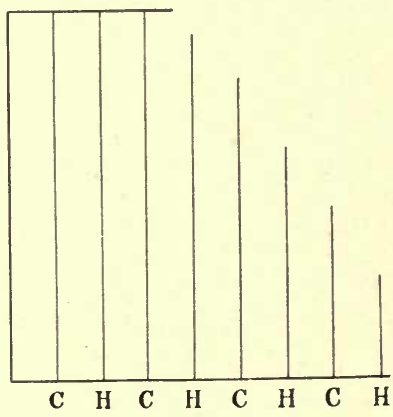

PLOT II

FIG. 3. ARRANGEMEN'T OF THE TWO ISOLATED PLOTS FOR THE, PURE-LINF IMETHOD OF CORN BREEDING.

somewhat costly process. Two isolated plots will be necessary, to which I may conveniently refer as Plot I and Plot II. (See fig. 3.) In Plot I will be grown year after year only that pure strain which investigation has proved to be the best mother-strain for the attainment of the desired end. Thus, if it has been found, as in the example already cited, that Cross $\mathrm{C} \times \mathrm{H}$ gives the desired result, Plot I will be occupied by Strain C. This will require no attention from the breeder's point of view except that any exceptionally vigorous or aberrant individuals should be eliminated, as such plants might be safely assumed to be the result of foreign pollinations. In Plot II Strain C and Strain $\mathrm{H}$ are to be planted in alternate rows, and all of Strain $\mathrm{C}$ is to be detasseled at the appropriate time. All the grain gathered from the detasseled rows will be seed-corn for the general field-crop, and that gathered from the tasseled rows will be pure-bred Strain $\mathrm{H}$ to be used again the 
following year in the same way. Here again in pure Strain $\mathrm{H}$ all exceptionally vigorous or aberrant individuals should be discarded as being probably due to the entrance of foreign pollen.

I am not prepared at present to say what will be the probable cost of seed-corn when produced by this method, but have reason to suppose that it would be more expensive than by the present method; nor can I surmise what relation this increased cost will bear to the increased yield that will be produced. These are practical questions which lie wholly outside my own field of experimentation, but I am hoping that the Agricultural Experiment Stations in the corn-belt will undertake some experiments calculated to test the practical value of the pure-line method here outlined. 




|Reprinted from the Plant World, Vol. 12, No. 7. July, 1909.1

\section{A SIMPLE CHEMICAL, DEVICE TO ILLUSTRATE MENDELIAN INHERITANCE.}

\section{By George Harrison Shuli.}

At the recent meeting of the Botanical Society of America at Baltimore, Dec. 31, 1908, I performed an experiment to illustrate how the absence of a character may be dominant over its presence. The experiment was of such simplicity and appeared to have such pedagogic value that I was asked to describe it in the PLANT WORLD for the benefit of teachers.

In order to make the bearing of the experiment perfectly clear, I will first give a brief statement of just what is involved in Mendelian inheritance, when considered from the standpoint of the "presence and absence" hypothesis, now generally accepted by the foremost experimental students of heredity.

The presence and absence hypothesis assumes that in every case in which two plants or animals which may be crossed together, differ from each other in a simple unit-character, their difference from each other is to be described in the terms of presence and absence of a single character and not as a pair of contrasted characters. For example, when a yellow-seeded pea is mated with a green-seeded one, instead of saying that the contrasted characters are yellow vs. green, we should say "yellow vs. absence of yellow." The pure-bred yellow pea is believed to possess all the elements contained by the green pea, with a single pair of units added which makes it yellow instead of green. If the green pea, which I call in such a case the "negative homozygote," is represented by the letters $G G$, intended to indicate that it has inherited its greenness from both its parents, then the pure-bred yellow pea should be represented by $Y Y G G$, to show that both greenness and the added unit for yellowness was inherited from both parents. This forms what I call the 
"positive homozygote." Every vegetative cell of the greenseeded pea-plant contains the pair of units or "genes" * GG, 1.ut when the germ cells are being formed, these two genes serarate so that each egg-nucleus and each sperm-nucleus has only one $G$, or green-producing gene.

In the same manner, every vegetative cell of the positive homozygote, or pure-bred yellow-seeded pea-plant contains the two pairs of genes, $Y Y G G$, since it received a $Y$ and a $G$ from each of its parents, and all of its male and female germ-cells will contain the two genes $Y G$. Now, when a cross is made between these two kinds of peas, we either apply a pollen-grain of the green-seeded fea-plant to the stigma of the yellow-seeded peaplant and allow a germ containing a single $G$ to find its way to an egg-cell which contains $Y G$, or we reverse the process, and by applying the pollen of the yellow pea to the stigma of the green pea, allow sperms containing $Y G$ to reach eggs containing only $G$. The results of these two processes are the same, for $G \times Y G=$ $Y G \times G$, and every vegetative cell of the plants produced by such a crcss will contain the three genes, $Y G G$, and as the green seeded character, $G$, can not be seen when $Y$ is contained in the same nucleus, all of the hybrid plants of the first generation from such a cross will have yellow seeds, and we say that yellow is dominant over green. **

When these hybrids produce germ-cells the three genes, $Y G G$, separate again so that half the egg-nuclei contain $G$ and half contain $Y G$ and similarly half the sperm-cells contain $G$, and half contain $Y G$. When sperms that are mixed in this way are applied to egg-cells having the same mixture, some $G$ sperms will fuse with $G$ eggs, to form $G G$ plants (negative homozygotes), some $Y G$ sperms will fuse with $Y G$ eggs to form $Y Y G G$ flants (positive homozygotes), some $G$ sperms will fuse with $Y G$ eggs to form $Y G G$ flants (heterozygotes), and some $Y G$

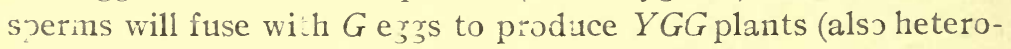
zygotes). Thus, it is seen that three kinds of plants are produced

*This word has bsen projosed by Dr. W. Johannsen for the internal units or determivarts. upon who e presence the proluction of any simple or unit character depends. It has tile advant ige of being short and of miking no apparent assumption as to the 11 inite nature or behavior of such determining factors.

**Professor Bateson suggested that we use "epistatic" and "hypostatic" to represent

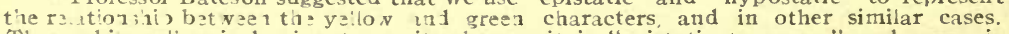
Thu , whice yeliow is dominant over its absence it is "epistatic to green," and green is hypostatic to yelıow." 
which may be represented by $G G, Y G G$, and $Y Y G G$, and the only difference in these plants is in the number of $Y$ genes they contain. Taking the negative homozygote, $G G$, we make the heterozygote by adding one $Y$ gene, and the positive homozygote by adding two $Y$ genes. If $Y$ is dominant over its absence, and this is much the commonest form of Mendelian inheritance, the $Y G G$ individual looks like the $Y Y G G, i . e$. both will have yellow seeds. If the absence of $Y$ were dominant over its presence the $Y G G$ individual would look like the $G G$ individual, both having green seeds, and if dominance were only partial, the $Y G G$ individual or heterozygote would be intermediate between $G G$ and $Y Y G G$, having greenish-yellow seeds. The important point to emphasize is that the heterozygote and

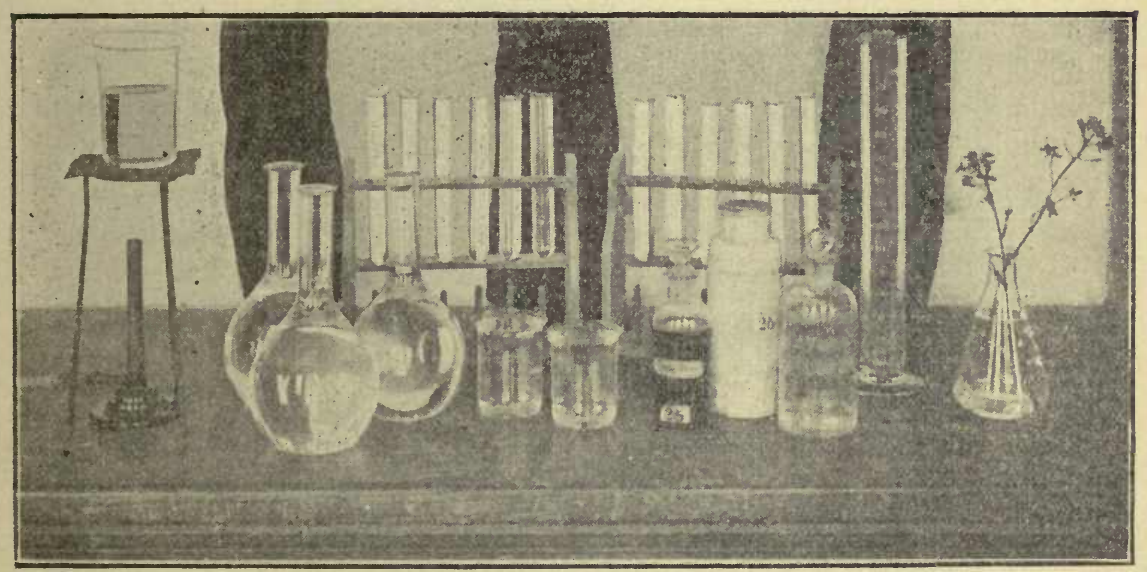

Fig. 1. Material needed for illustration of Mendelian inheritance. positive homozygote are the same as the negative homozygote, with one or two portions of something (a $Y$ gene in this case) added.

Material needed for the demonstration: Solution of litmus, sodium hydroxide or aqua ammonia, hydrochloric acid; 3 halfliter flasks, glass graduate, 1 dozen $15 \mathrm{~cm}$. test-tubes, and two test-tube stands. Also, if convenient, a blue and a red flower of any species, and if these flowers are at hand, an alcohol lamp, or a bunsen burner if gas is available, ring-stand, wire gauze, and several small beakers. See Fig. 1. 
To a moderately dilute solution of litmus add a small quantity of sodium hydroxide or aqua ammonia. In the presence of the class, divide this solution into two of the flasks, calling attention to the fact that the same solution is put into both. Then add to the one flask in the presence of the class a certain quantity $*$ of hydrochloric acid, which changes the solution from blue to red. See that it is perfectly clear to every member of the class that there is now just one difference between the solutions in the two flasks, namely, the one contains a certain portion of acid, the other has none. As it is desired that the solutions in these two flasks should represent respectively the negative and positive homozygotes which are to be crossed together, and as they are homozygotes because they inherited the same character from both parents, the acid should be added in two equal portions with the explanation that one acid-producing unit or gene came from the mother and one from the father. The experiment will be rendered more inpressive if a red flower and a blue flower of any variety, such as the sweet-pea, is standing in a vase on the teacher's desk, for then it may be explained that while it would take two years to demonstrate Mendelian inheritance by an actual cross between these two flowers, the whole process may be shown in a few minutes by letting the blue solution represent the blue flower and the red solution the red flower. See that the class appreciates the fact now that just as in the case of the solutions, the red and blue flowers differ from each other in only one quality, one-half of which came from one of its parents, the other from the other farent.

Several different experiments may be performed as follows:

(A) To demonstrate the more common type of Mendelian inheritance, that in which presence is dominant over absence: Add sufficient acid to the solution in the one flask to change the color from blue to red, and then add a second equal quantity. These two solutions now represent the two plants to be crossed. Fill four test-tubes one-quarter full from each flask and let these represent the germ cells of the two plants to be crossed. See that is it still kept in mind that the red germ-cells are exactly

*The amount of acid to be added will differ according to the requirements of the particular experiment to be performed, and this must be determined by the teacher in a
preliminary experiment. 
the same as the blue ones with a single added unit of acid. Have one test-tube rack for the pollen-parent which produces the sperms or male germ-cells (all red for instance), and the other for the pistil-parent which produces the eggs or female germcells (all blue). Then take the test tubes with the red solution one by one and empty them into those having the blue solution, explaining that similarly in fertilization a sperm meets and fuses with an egg in the formation of a new plant. The result of this process is the production of four red solutions representing the first generation hybrid between the red and the blue solutions; $i$. e. the dominance of the red or acid over its absence.*

Next explain the production of the second generation hybrids, thus: Each vegetative cell of the first generation hybrid contains side by side the elements received from the two parents, one of these having a unit of acid the other lacking it. When the germ-cells of these hybrids are being formed these elements received from the parents are separated again so that half the eggs have the acid unit and half lack it, and in the same way half the sperms have the acid and half lack it. Illustrate these germ-cells of the hybrids by placing in the "female" test-tube rack two test-tubes one-quarter filled with the original blue solution, and two one-quarter filled with the original red solution. In like manner illustrate the fact that the male germ cells are of two kinds, half having the acid unit and half lacking it, by a similar set of test-tubes in the "male" test-tube rack, two with the blue solution and two with the red. As the testtubes are taken one by one from the "male" rack and emptied into the test-tubes on the "female" rack, see that the combinations are fully understood. One sperm having the acid unit fertilizes an egg which also has the acid unit, illustrated by pouring the red solution from a test-tube of the "male" rack into one with a red solution on the "female" rack, resulting in a purebred red, (positive homozygote). Another red or acid-bearing sperm meets an egg which lacks the acid, as the test-tube con-

*It may seem better, pedagogically to have blue "dominate" over red, as it actually does when blue and red flowers are crossed. This can be easily managed by making the original solution which represents the negative homozygote acid for this particular experiment, adding two equal portions of alkali to produce a blue positive homozygote. As the present experiment was devised to show the dominance of absence over presence it was thought best to make (B) coincide with actual experince in blue $X$ red crossds, giving 3 blue to 1 ted. The experiment is equally apt ether way and no misapprehension need result. 


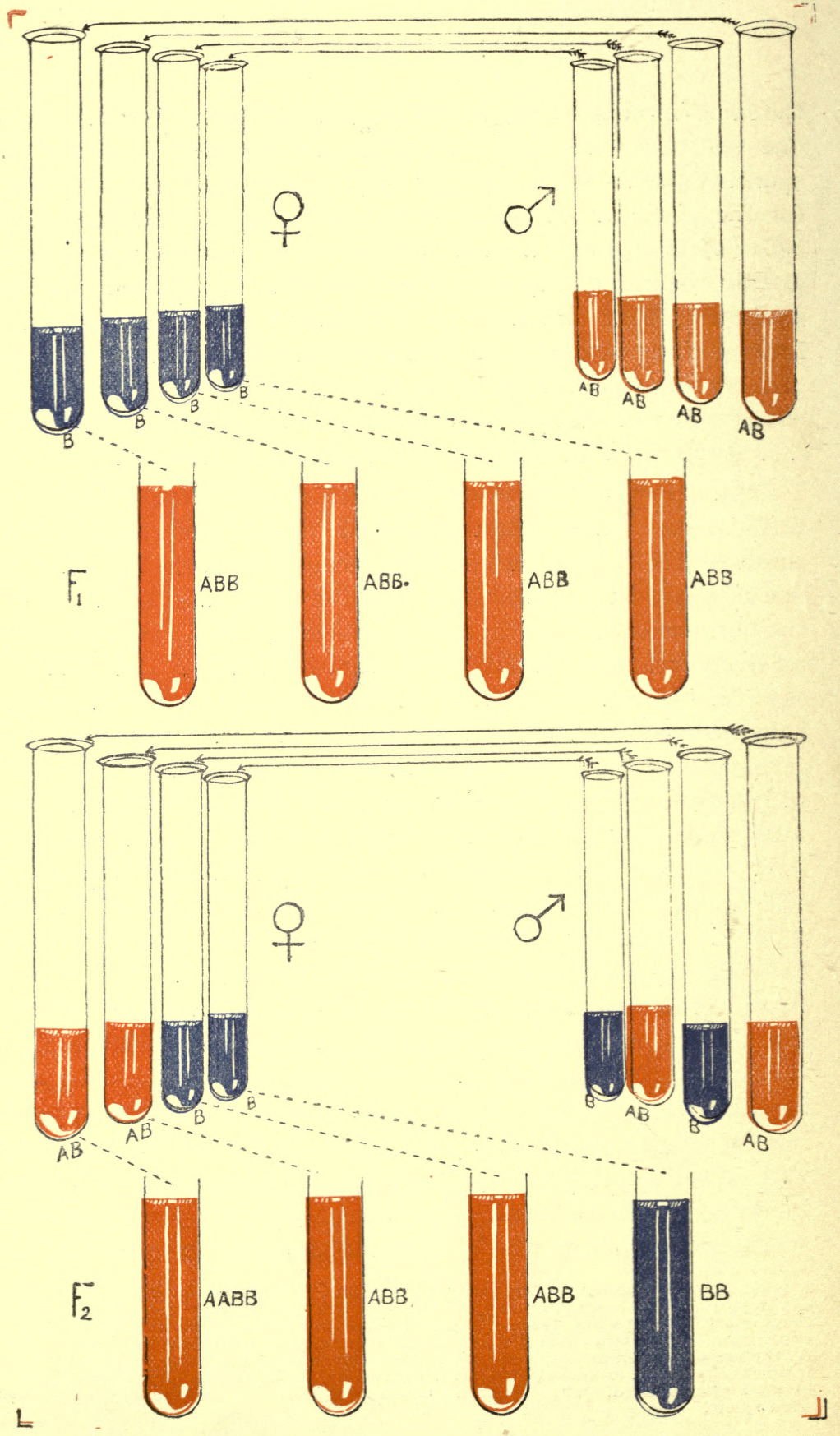


taining the other red solution is taken from the "male" rack and its contents poured into one of the blue solutions on the "female" rack. When all the combinations have been made the four test-tubes on the" female" rack represent the second generation hybrids and show 3 red to 1 blue. That one of the three red individuals of this second generation was a positive homozygote and the other two were heterozygotes, should also have been carefully noted by the class.

(B) To demonstrate the dominance of absence over presence: Starting again with the two flasks containing an alkaline solution of litmus, determine how much acid must be added just to make the solutions safely acid; then take half of this quantity to represent the acid-producing unit coming from each of the parents. When this half portion of acid has been added the solution should remain blue, but on adding the second half portion the color will change to red, so that now you are again prepared to make a cross between a blue and a red individual. The rest of the experiment is conducted exactly as in (A), but the first generation will show four blues, and the second generation will show 3 blue to 1 red, that is, the absence of the added acid unit is dominant over its presence.

(C) To show the equivalence of reciprocal crosses, regardless of the fact that egg and sperm differ much in size: Take the situation described under (A) after preparation has been made for the demonstration of the second generation. Fill up the testtubes on the female rack with water until they are half or two-thirds full, leaving those on the male rack one-fourth filled with the more concentrated fluids. As this is done it should be explained that just as the water is neither acid nor alkaline and does not modify the reaction which takes place when the two fluids are brought together, so the food-materials whose accum:1lation makes the egg larger than the sperm are neutral substances which do not affect the behavior of the hereditary qualities. If desired, this difference between the egg and sperm may be represented throughout all of these experiments, by adding water to the test-tubes on the female rack in all cases, but as the aim should be to keep the demonstration as simple as possible, it would seem best usually to follow the plan 
suggested here, of making the equivalence of reciprocal crosses a separate experiment.

Finally, to show how appropriately this experiment represents the actual cross between the red and blue flower, dip the red flower into boiling water a moment and then hold it in the fumes of ammonia or dip it into a dilute solution of ammonia or of sodium hydroxide. Similarly take the blue flower and dip it into the boiling water, and then into a dilute solution of hydrochloric acid, in this way demonstrating that the difference between the blue and red flower is due simply to the fact that the cell-sap of the blue flower is alkaline, and the cell-sap of the red flower is acid. 
GERMINAL ANALYSIS THROUGH HYBRIDIZATION.

BY GEORGE HARRISON SHULL.

Reprinted from

Proceedings of the American Philosophical Society

Vol. XLIX, No. 196, 1910 



\section{GERMINAL ANALYSIS THROUGH HYBRIDIZATION.}

By GEORGE HARRISON SHULL.

(Read April 23, 1910.)

The study of the various characteristics of plants and animals as independent units has made hybridization a valuable instrument in experimental morphology, and has given to the name of Mendel an enduring place as a true prophet in the history of biological progress.

The importance of the Mendelian contribution can scarcely be over-estimated. Before the "re-discovery" a decade ago, no one but Mendel had given an approximately correct interpretation of the composition and behavior of hybrid progenies, and the process of hybridization was therefore of no particular consequence for general biology. The hybrid individual was taken as the unit and comparisons between the hybrids and their parents were made in generalized terms involving the general aspect or tout ensemble. As only rarely were all the characteristics of either parent recombined in one of the offspring, the phenomena of segregation and recombination were considered of relatively rare occurrence, and described in terms of atavism or "throwing back" to the ancestral condition.

An important cause for the long delay in the discovery of the Mendelian phenomena was the distinction made between the offspring of species-crosses, which alone were distinguished as "hybrids," and the cross-bred offspring of more closely related forms, which were stigmatized as "mongrels." The difficulty of making species-crosses, the consequent rarity of such hybrids, and the usually uniform type of the offspring produced, all gave the impression of their greater scientific importance at a time when rarity and uniformity of phenomena instead of their general occurrence and variability seem to have made the stronger appeal.

Retrinted from Proceedings American Philosophical Society, Vol. xlix., roro. 
Koelreuter, the first hybridologist, started the current in this direction by devoting his attention so strongly as he did to the phenomenon of sterility in hybrids, which he considered an important test of the specific distinctness of the parents. The very fact of fertility in the progeny of a cross seemed in later years to terminate its interest for him and only in rare instances in his writings do we find any data as to the characteristics of individuals belonging to second and later generations.

Gaertner dealt with the subject of hybridization in a much broader way and arrived at many interesting generalizations. However, he also worked almost wholly with species-crosses, purposely choosing his material with as wide differences as possible in order to facilitate definiteness of descriptions, but in this very effort to gain definiteness, the opportunity for studying the second and later generations was usually lost through the sterility of the first generation hybrids. He did, however, make some studies on such well-known Mendelian material as peas, sweet peas and Indian corn, but only in the last did he study a second generation, and in this the complexities introduced by "xenia" were doubtless the chief cause of his failure to find the simple law of segregation.

Practically all other hybridizers from Gaertner's time on to the beginning of the present century, considered the mere securing of hybrid individuals and their systematic description as the matters of prime value. Thus it was that the combination phenomena of hybridization alone occupied the stage, and the separation of the parental characters and their recombination in different individuals was only imperfectly recognized as variability and returns to one or other of the two parental types.

Two French investigators, Godron and Naudin, who were working synchronously with Mendel, seem to have come very near sharing Mendel's great discovery, but each of these two investigators by a strange chance observed a different phase of the Mendelian phenomena, Godron reaching the conclusion that in mongrels ("métis") all progenies return in several generations to the parental types and then breed true, while Naudin thought he had demonstrated that the progenies continue to vary after the $F_{2}$ and 
never become fixed. However, in the work of Godron and Naudin, their near discovery of Mendelian segregation was not due to a deliberate consideration of the various characteristics as units, but rather to the fact that several of the forms which they used in their cultures, differed from each other by single unit-characters, as exemplified for instance by the purple color of stems in Datura Tatula, contrasted with the green stem of D. Stramonium, or the usual prickly fruit of the Daturas contrasted with the smooth fruit of a var. "capsulis inermibus."

The Mendelian method of following single characteristics possessed by the parents, not only into their $F_{1}$ progeny, but also through the second, third, and later generations, brought to light a regularity of behavior which has served to shift the stress from the simple combination phenomena involved in hybridization, to the phenomena of separation and recombination of such elementary differences as existed between the two parents.

The result of this important innovation of method has been to demonstrate beyond a peradventure, that many of the distinguishing characteristics of adult plants and animals are predetermined by corresponding differences in the constitution of the germ-cells; that these differences may be of an elementary character, capable of separation into different germ-cells; that when two parents used in any cross, differ by such elementary characters, half the resultant germ-cells have the capacity to produce any given elementary character of the one parent, the other half possess the capacity for the production of the corresponding or alternative characteristic of the other parent; that as a rule such unit-characters are wholly independent from one another and capable of rearrangement in every possible combination with one another; and, finally, that it is purely a matter of chance, which available type of sperm shall fertilize any given egg.

The separation of the unit-characteristics into different germcells in every possible combination with other characteristics gives the power in many cases to recognize all the unit-differences which served to distinguish the two parents. By the study of the hybrid progenies we are thus given an insight into certain phases of the 
protoplasmic constitution and the "mechanism" of heredity, which has been totally unattainable by other means.

In making analyses of such hybrid progenies and in working out the nature and delimitations of the unit-differences involved in Mendelian crosses, no assumption need be made as to the ultimate nature of the "genes" ${ }_{1}$ or determiners. The attitude of nearly all experimenters in the field of genetics is one of more or less consciously suspended judgment on this point, and I believe that no other attitude is justified at the present time. So far as I am aware no investigator of the Mendelian phenomena "sees only particles" as Dr. Riddle ${ }^{2}$ has erroneously assumed, although it must be confessed that his speech does sometimes seem to symbolize them. The Mendelian interpretations do not "stand as a formidable block in the path of progress," nor as any block at all, since all terminology is more or less symbolic, and comes to mean new things as rapidly as new truths are brought to light. All investigators in this field will be appreciative of the service Dr. Riddle has performed in bringing to their notice the recognized facts in the process of melanin formation, though they can scarcely fail to regret his unfamiliarity with the present state of genetic science, and with the attitude of those engaged in the investigations. If he had been thus familiar with work in genetics, he might very easily have shown that the facts of melanin chemistry are in harmony with the mass of other data for which the "Mendelian interpretation" has proved so illuminating.

Although the question of epigenesis versus preformation is emphasized as a fundamental difference between Riddle's views and those of the Mendelians, this supposed difference is mainly imaginary. Riddle's assumption of different "strengths" in the germcells as a possible method of accounting for the production of different colors or other characters in adult animals, involves a preformation of the same order as that assumed by the investigators of

${ }^{1}$ The genes are the differences, of whatever nature, whose existence in the germ-cells determines the capacity of the unit-characters to be present or absent in the individuals developed from those germ-cells.

${ }^{2}$ Riddle, O., "Our Knowledge of Melanin Color Formation and its Bearing on the Mendelian Description of Heredity," Biol. Bull., 16: 316-35I, May, I909. 
Mendelian phenomena. Every thremmatologist is too familiar with the facts of ontogeny to give the slightest credence to anything approaching the old emboitement hypothesis, but he must accept as a philosophical necessity the fact that there can be no action without an agent. There can be no "strength" without something to be strong. Preformation and epigenesis are simply inseparable phases of a single philosophical unity and any attempt to separate them is fallacious.

The statement that the "nature of present Mendelian interpretation and description inextricably commits to the "doctrine of particles,'” presents Mendelism and its investigators in a false light, as no such commitment is involved. Despite the enormous activity and splendid progress that has been made in these ten years in tracing the Mendelian behavior until it has become evident that it is a well-nigh universal phenomenon,-no doubt practically co-extensive with sexual reproduction,- the changes in descriptive terminology to which Dr. Riddle deprecatingly refers, have been remarkably slight, and one reads Mendel's original account with wonder that it should still be so modern. Mendel's genius grasped the essentials of this type of inheritance so completely and presented it with such fulness and clarity, that it may doubtless always serve as a good elementary presentation of the subject. But while Mendel's paper is in such essential accord with "present interpretation" as to seem strictly modern, there occurs throughout his whole admirable discussion, not one word af suggestion that he attributes the occurrence of any external character to the presence of an internal particle.

Modern Mendelians as a rule have specifically declined to postulate the presence of material "particles" as the physical bases of unit-characters. Bateson, who has done more than any other to demonstrate the wide applicability of the Mendelian method, clearly placed himself from the first in opposition to any purely morphological interpretation of Mendelian phenomena by giving to his reports to the evolution committee the title: "Experimental Studies in the Physiology of Heredity," and he has from first to last carefully guarded all statements with reference to the nature of the genes, in such manner as to be entirely non-committal. Other investigators have either wholly ignored the question, or have usually 
couched their suggestions in such terms as to show that they were open to any new light upon the subject.

Although I have never looked upon the Weismannian conception of character-determiners as at all plausible, I do not agree with Dr. Spillman ${ }^{3}$ that the facts presented by Riddle "disprove" the "particle hypothesis." The only manner in which Riddle despatched(?) the "particle hypothesis" was by ruling the observed facts of Mendelian heredity out of court. If the Mendelian phenomena are real, and no one can do careful investigation in this field without becoming convinced that they are, the postulation of "particles" or "bullets" having certain chemical and physiological properties, and behaving during the reproductive process in some such manner as the cytologists are fairly agreed that the chromosomes behave, would offer a complete explanation, and the correctness of such an explanation can not be disproved except by proving that some other method of determination is the true one. However, while the particle hypothesis is not disproved, I have no doubt that the Weismannian, and perhaps also the De Vriesian conception of the genes will seem less and less plausible as new facts accumulate.

In Dr. Spillman's brilliant development of what he calls the "teleone hypothesis," a suggestion is offered which virtually makes the chromosomes the "bullets" whose differential properties determine the unit-characters. This interpretation of the Mendelian phenomena has much to commend it, especially as it calls for no structure and no type of behavior which are not already generally recognized as being universally present in the formation of the germcells, and it has the added advantage that it seems to be capable of experimental tests.

I can not see, however, that Dr. Spillman has presented "an explanation of Mendelian phenomena without resorting to the idea of unit-characters." If he appears to do so, it is only because he gives to them a new name. The unit-characters are the empirical phenomena for whose explanation the "bullets," "teleones" or genes of any other sort, are devised. It is no new idea that these

'In conversation.

"Spillman, W. J., "Mendelian Phenomena Without De Vriesian Theory," Amer. Nat., 44: 214-228, April, 1910. 
unit-characters are "differentials," as this was recognized by Mendel himself and has been common knowledge to all investigators of Mendelian heredity since.

The length of hair in guinea-pigs and rabbits, the stature of peas, sweet peas, beans, etc., the length of styles in Primula and Enothera, density of the heads in wheat and barley, and in fact practically all other characters with which Mendelian investigators have worked, have been so obviously differentials that it is impossible to assume that any Mendelian has ever meant anything else by the expression, "unit-character."

This being true, the contention of Riddle that even in the absence of a given unit-character there is not a complete absence of the particular manifestation in which the essence of that unit-character consisted, or in other words, that the unit-character is simply a phase or "strength" of some " rather general protoplasmic power," is not likely to seriously disturb the Mendelians, since that is a fact with which they have long been familiar.

It appears to me that the unnecessary shifting of the terminology of clearly distinguishable empirical phenomena is undesirable. The unit-characters are real things capable of repeated demonstration. They are still differential characters, and possess the capacity to behave as units, entering into various combinations with other unitcharacters and capable of reëxtraction from them, or of being absent altogether, regardless of the manner in which their behavior is explained. The genes, on the other hand,- the ultimate organs of the protoplasm or conditions of the protoplasmic substance upon whose existence depends the capacity to give certain series of reactions, or to pass through certain cycles of ontogenetic development,-are purely inferential. Their nature is not yet capable of demonstration. They are "unknown gods" to whom each new prophet may appropriately apply a new name whenever he ascribes to them new attributes.

While the ultimate nature of the genes lies wholly beyond the powers of present-day analysis, and there is nothing therefore to warrant a departure from the prevailing attitude of suspended judgment, the more intimately the unit-characters themselves are studied, the better will be the basis provided for an understanding of their 
common properties, and thus finally for an approximation to the nature of the genes which determine them.

The most hopeful directions of approach in the effort to learn more of the true inwardness of the unit-characters, are those of chemical analysis and experimental cytology. As applied to unitcharacters, these are almost untouched fields at present, though several investigators have made a beginning. Miss Wheldale, especially, has made a hopeful beginning in the investigation of the chemistry of anthocyanin colors which have continually exhibited typical Mendelian behavior. Several unit-characters which have been recognized and described heretofore only in terms of colorfactors, now seem to be capable of description in terms of a chromogen (present in all sweet peas and stocks investigated), and of activators, peroxidases, peroxides, and reducers, thus making the various colors " the result of definite oxidation stages of the chromogen." Riddle has come to much the same conclusion in regard to the nature of the melanin colors, from a consideration of the work of Bertrand, Gessard, Spiegler and others.

In experimental cytology there seems to have been nothing done as yet, which can throw light on the nature of those unit-characters involving the structure and size of parts. How are the number and direction of cell-divisions that shall take place in any cell-lineage determined? Are these also referrable to the presence of definite chemical substances or to definite configurations of protoplasmic molecules? To these questions I believe no satisfactory answer is now possible, but that these processes are controlled in many instances by characteristics possessed by the germ-cells, rests upon aboundant experimental evidence.

While waiting for further information from the chemist and the cytologist, there is still abundant room for the work of the experimental breeder. Owing to the characteristic distribution of the genes at the time of germ-cell formation already described, Mendelian hybridization provides a partial analysis of the germ-plasm, and thus gives some insight into the constitution of living protoplasm. It is of great importance that such analysis be continued until all the unit-differences of plants and animals have been studied, 
for only when this is done can the full scope and significance of the Mendelian phenomena be understood.

It need scarcely be pointed out that the complete tracing of the germinal analysis which takes place in Mendelian hybrids, is attended with many difficulties. The unit-characters represent capacities for reaction in a certain, very specific way to given conditions of environment. Individuals having the same unit-composition may react in a totally different way to a different environmental complex. Some unit-characters are so sensitive to slight differences of environment that they offer a wide range of fluctuation, or they may represent such a slight differential as to be readily distinguishable only in their plus-fluctuations. Two or more unitcharacters may even be indistinguishable from one another as Nilsson-Ehle ${ }^{5}$ has shown to be the case in certain unit-characters of wheat and oats, and East ${ }^{6}$ in endosperm colors of corn. Many unit-characters are quite invisible except when occurring in combination with some one or more other characters, and this fact has led to what is called the "factor hypothesis." That the factors are real unit-characters, differing in no essential way from ordinarily visible unit-characters, is now in a fair way to be demonstrated by such work as that of Miss Wheldale, and others who are working along similar lines. The implication by some writers that the factor hypothesis is a late development of Mendelism is not correct, as Mendel himself suggested it tentatively. The difficulty of tracing invisible characters necessarily made the development of knowledge regarding them slower than that regarding the easily visible characters, but the essential correctness of Mendel's suggestion has been abundantly substantiated.

All of the foregoing difficulties can be overcome, and are continually being overcome by careful analysis and patient, long-continued breeding tests.

Finally, since we are examining the Mendelian process as one of germinal analysis it is appropriate to discuss for a moment the

"Nilsson-Ehle, H., "Kreuzungsuntersuchungen an Hafer und Weizen," 4to, pp. I22, 1909, Lund: Hakan Ohlsson.

"East, E. M., "A Mendelian Interpretation of Variation that is Apparently Continuous," Amer. Nat., 44, 65-82, Feb., I9ı. 
"insoluble residue." Although Mendelian behavior has proved to be nearly universal in those sexually produced plants and animals which are capable of breeding together normally, there are certain clear limitations to the process of analysis. Several instances are known in which differential characters are not segregated, and no analysis takes place with respect to these characters, even when most of the differential characters of the same plants or animals Mendelize in a perfectly typical manner. The relative frequency of this type of behavior may be greater than is now supposed but so far as clear evidence is available permanently blended inheritance of this type is relatively rare except in species-crosses, and in these latter the data is usually too scanty for safe generalization.

Aside from these cases which show a distinctly non-Mendelian mode of inheritance, it must be remembered that Mendelian analysis can be made only in the presence of differential unit-characters possessed by individuals capable of life and of sexual reproduction, and that therefore, there can be no test, except under rare circumstances, of the Mendelian nature of the more fundamental vital characters. This leaves it an open question whether the whole of the germ-plasm is a complex of such genes as those which give rise to the phenomena of unit-characters, or whether, with its wonderful general powers of assimilation, growth and reproduction, it consists of a great nucleus of which the genes are relatively superficial structural characteristics.

However, although nothing inconsistent with life and reproduction are ordinarily amenable to Mendelian analysis, this need not detract from the fundamental importance of unit-characters in the study of heredity and evolution, for the phenomena appearing in these fields are subject to exactly the same limitations. All that we know about heredity and evolution must start with a plasma capable of life and reproduction.

While thus leaving the absolutely fundamental characteristics of living matter untouched, the Mendelian method and its results have brought into harmonious relations many of the most diverse phenomena of phylogenetic differentiation and it is only fair to assume that they hold still greater promise for the future. 




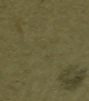

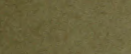

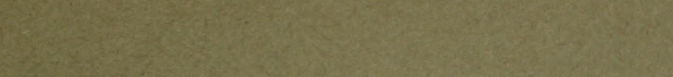

(4)

Q3.

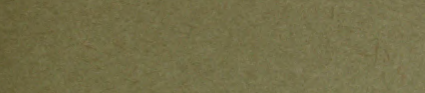

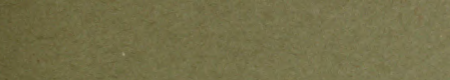

(3)

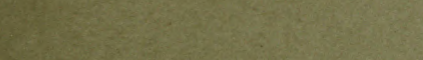

and

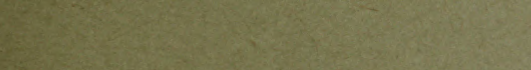

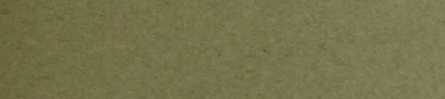

A.

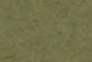

$\frac{2}{4}=2$

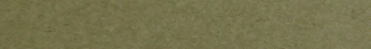

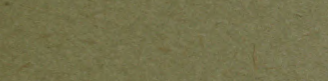

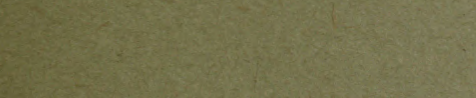

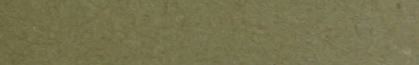

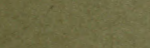

$\log ^{2}$

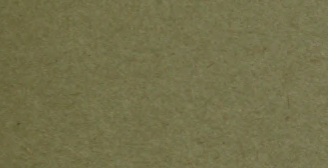

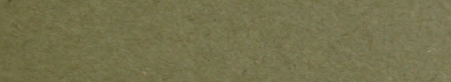

(2)

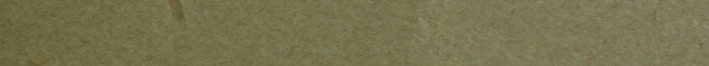

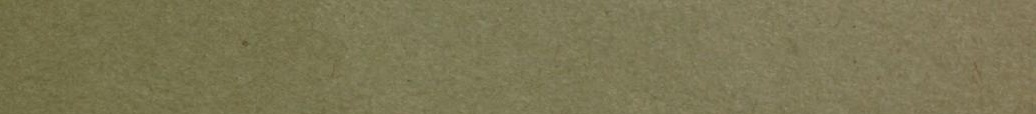

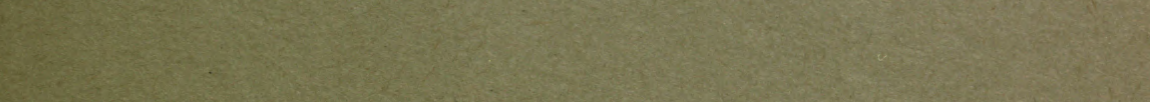

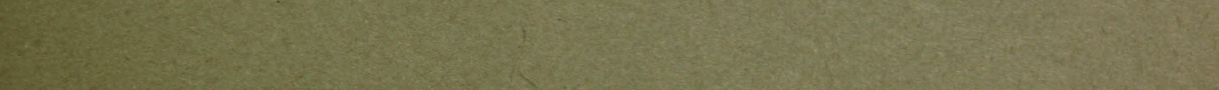
6.

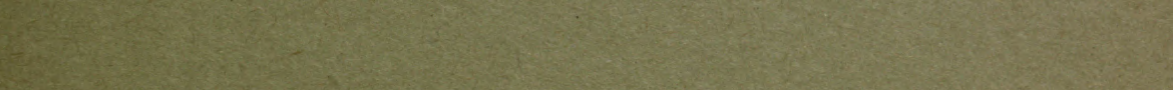
Al

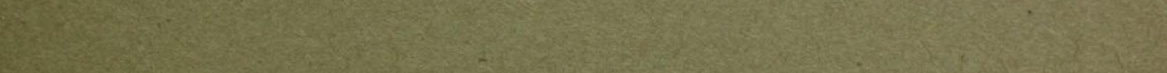

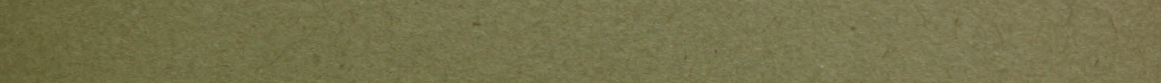

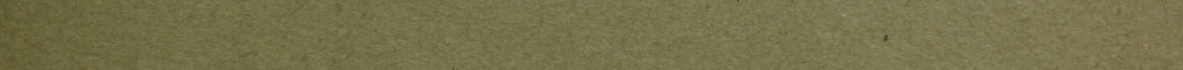

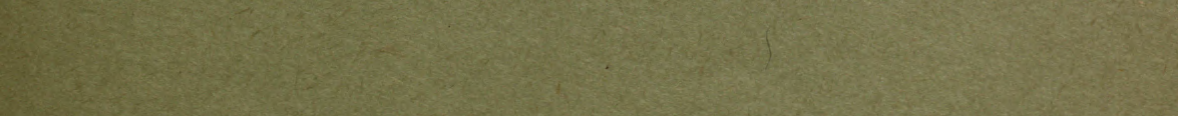
2. 


Reprinted from Vol. I, Number 2. American Breeders Magazine.

\section{HYBRIDIZATION METHODS IN CORN BREEDING.}

George Harrison Shuli, Cold Spring Harbor, Long Isiand, N. Y.

The simultaneous preparation of papers by three different authors about a year ago, advocating the use of more or less definite hybridization in the breeding of Indian corn in lieu of the methods of selection and partial isolation now in general use, probably marks an important step in the improvement of this exceedingly valuable American crop; for the appearance of these papers indicates a growing appreciation of the real biological nature of Indian corn and the requirements necessary to the attainment of the highest and most permanent success in corn breeding.

The three papers to which I refer are, "The distinction between development and heredity in inbreeding," by Dr. E. M. East, in the American Naturalist for March, 1909; a circular of the U. S. Department of Agriculture on "The importance of broad breeding in corn," by Mr. G. N. Collins, issued in June, 1909; and my own paper on "A pure-line method in corn breeding," read before this Association at its last meeting at Columbia, Mo., in January, 1909, and published in its last Annual Report. These three papers are in some 
particulars fundamentally alike, but as they approach the subject from somewhat different points of view it will be interesting to compare them briefly.

The suggestion for a hybridization method in corn breeding is not entirely new. A very clear outline of such a method, with experimental results sufficient to warrant the suggestion, was presented by Morrow and Gardner in Bulletins 25 and 31 of the Illinois Agricultural Experiment Station in 1893 and 1894. These bulletins were evidently unknown to two of the three writers above mentioned, ${ }^{a}$ and the third, Mr. Collins, while referring to Morrow and Gardner's bulletins, makes no statement of the fact that they had devised an adequate hybridization method for the practical utilization of the advantage shown by them to be sometimes attainable by crossing two distinct varieties.

In my paper on "The composition of a field of maize," read before the American Breeders Association in Washington two years ago, I pointed out the fundamental defect of the method now in general use, which simulates to a degree the isolation methods so successfully used in the improvement of small grains, and I suggested there that "continuous hybridization is perhaps the proper aim of the corn breeder." The conceptions which formed the basis of that paper were the complex hybridity of corn as ordinarily grown and the stimulating effect of heterozygosis or hybridity. It was shown that this stimulating effect comes into play in corn breeding because selffertilizations result in the partial or complete isolation of many quite distinct strains, and that cross-fertilization must therefore result in the production of hybrid combinations of these pure strains.

My suggestion for a pure-line method in corn breeding was a direct logical sequel of this original paper on "The composition of a field of maize." Dr. East's article on inbreeding above mentioned is also a sequel to the same paper, as shown by his references to it and also by his excellent discussion of the stimulation which results from hybridity, in regard to which he has arrived at views identical with those entertained by me at the time my original corn paper was written. It may be said, therefore, that Dr. East's paper and my

${ }^{a}$ I am indebted to Prof. W. J. Spillman for calling my attention to these bulletins. Dr. East has informed me since this was written and read at Omaha, that he, too, knew of the existence of Morrow and Gardner's hulletins or. corn, but was not aware that these authors had described a method for continuous hybridization in the culture of this crop.-G. H. S. 
own have grown out of the conception, first presented by myself two years ago, that what may appear to be a uniform variety of Indian corn is really a series of very complex hybrids involving numerous distinct biotypes, ${ }^{b}$ which may be isolated from their hybrid combinations by self-fertilization and which owe their smaller size and inferior yielding qualities, not to any injurious effect of inbreeding as such, but to the fact that self-fertilization gradually results in their reduction to a pure homozygous state. They are thus deprived of the stimulus which had been derived from crossing with other biotypes.

It appears that my paper of two years ago was unknown to $\mathrm{Mr}$. Collins, although it was read in Washington and published in the Annual Report of the American Breeders Association in 1908. As Dr. East and I have both performed many experiments which have led us to place great confidence in the practical importance of the discoveries of Mendel, De Vries, and Johannsen, it is interesting to read in Mr. Collins's paper that these new results from the scientific side are "particularly dangerous" when applied to corn breeding. As Dr. East and I by the application of the newer biological conceptions have arrived at practically the same method which Mr. Collins recognizes as necessary for the best results in corn breeding, it should become evident to him that there is no such danger as he fears in the application of the latest scientific results to practical work.

The crossing of relatively homozygous strains or of distinct biotypes in corn in order to secure the stimulus of hybridity, as advocated by Dr. East and myself, involves a much more definite conception, however, than that suggested by the term "broad breeding." The idea of hybridization between distinct strains or between biotypes calls for the use each year of those two parental strains or biotypes which careful tests have shown'to result in the greatest capacity for yielding an excellent $F_{1}$ (first generation hybrid) progeny. Although Mr. Collins advocates a method which is essentially identical with that proposed by Morrow and Gardner fifteen years ago and which has been invented anew by Dr. East, the idea of broad breeding would logically require the working in of a new variety or strain each year, instead of going back to the same relatively inbred strains for each successive crop. In other words, while Mr. Collins has suggested a splendid method of corn breeding, it is not one which corresponds

A biotype is a group of individuals which do not differ from one another in any hereditary quality, and which therefore constitute a pure race. 
well with the theory upon which he bases his suggestion. If the "broad breeding" idea is taken as a basis and a new variety is brought into the combination every year, it is plain that it must be done blindly, since the influence which this new variety will have upon either the quality or quantity of the crop cannot be known. However, the method advocated by Mr. Williams, $c$ of the Ohio Agricultural Experiment Station, would overcome to a certain extent the blindness of this "introduction of new blood" by first testing its influence.

In the method of Morrow and Gardner, which, as we have seen, has been endorsed by East and Collins, involving the use of strains that, according to my experiments, are already complexly hybrid, there must be more or less resolution of characters in the resultant cross-bred plants, since the crop would not consist of $F_{1}$ plants with respect to all the numerous characters in which the two chosen parental strains differed, but would be, with respect to many of them, the $\mathrm{F}_{2}$ (second generation hybrid) in which splitting up of characters occurs. The method which I described last year under the name of a "pureline method" is the only one yet suggested in which all the plants in the resultant progeny would be first generation hybrids in regard to all the qualities which served to distinguish the parents. This does not prove, however, that my pure-line method is better than the method of Morrow and Gardner. It may be true, as Dr. East says, that the pure-line method is "more correct theoretically but less practical" than the method of Morrow and Gardner which he describes. It is conceivable that the method of using highly developed strains which have been produced by line-breeding and continual selection of the best and most vigorous ears may produce such high yields when crossed together that the expense and trouble of isolating pure biotypes will not be justified; but such a conclusion can be properly reached only as the result of extensive experimentation; and this experimentation, if undertaken in earnest by our State experiment stations, must result in the discovery of the best possible method for the breeding of Indian corn. My anxiety is not for the success of the pure-line method outlined by myself, but that serious experimentation shall be undertaken by every station within the corn-growing region for the purpose of discovering what is the best method. I feel quite

- Williams, C. G., Corn Breeding and Registration. Report American Breeders Association, $3: 110-122,1907$. 
sure that the results of such investigations will lead to the adoption of some hybridization method in the breeding of this crop.

My experiments with corn during the last year have again given the fullest confirmation of my theories regarding the complex hybridity of the plants which compose an ordinary field of corn as grown at the present time; and data have also been secured having a direct bearing upon the applicability and importance of hybridization methods in corn breeding. Last year I presented the results of a single pair of reciprocal crosses between two self-fertilized strains which I called strain $A$ and strain $B$. Those strains in hybrid combination produced a yield a little larger than the average of those families which had not been self-fertilized, but the difference was very slight. As strains $A$ and $B$ had been essentially unselected, being the first two selffertilized strains which had become nearly pure-bred, it was anticipated that a larger number of crosses would discover some strains much superior to $A$ and $B$ as parents of a high-yielding $\mathrm{F}_{1}$ hybrid progeny, as well, perhaps, as some which are inferior. This belief has been fully supported by the results secured in 1.909, for in eight different hybrid combinations which were tested during the past season three have proved better than the combination between strains $A$ and $B$; one other combination, also having strain $A$ as one of the parents, gave a result about equal to that of $A$ and $B$, and three combinations produced somewhat less than those of strains $A$ and $B$. Not all of these hybrid families produced higher yields than the corresponding cultures which had never been self-fertilized, but the three highest yields produced in all my cultures were the result of hybridizing selffertilized strains which had been, no doubt, reduced nearly to a homozygous (pure-bred) state. The average of all the hybrids when compared with the average of all the corresponding cross-bred ${ }^{d}$ families shows the yield of the former to be only insignificantly lower than that of the latter, these averages being respectively 78.9 bushels per acre from the hybrids, and 79.4 bushels per acre from the cross-breds. This shows how effectively the cumulative "injurious effects" of five years of selffertilization may disappear in a single year as the result of crossing.

The large yield of two of my hybrid strains as compared with the product of the best cross-bred families is not a mere chance relation, but is a specific function of the particular hybrid combination

"I use the term "cross-bred" here to denote those families in which all selffertilization has been prevented during the five years these investigations bave been in progress. 
which produced them, as may be shown by two series of facts. In the first place, the hybrids between strains $A$ and $B$ which were reported last year as producing from 74.4 to 78.6 bushels per acre have this year yielded 79.8 bushels per acre. A slight increase in the other cultures for 1909 as compared with those for 1908 makes this slight increase in yield of the hybrid between strains $A$ and $B$ simply a measure of the better climatic conditions of 1909 as compared with

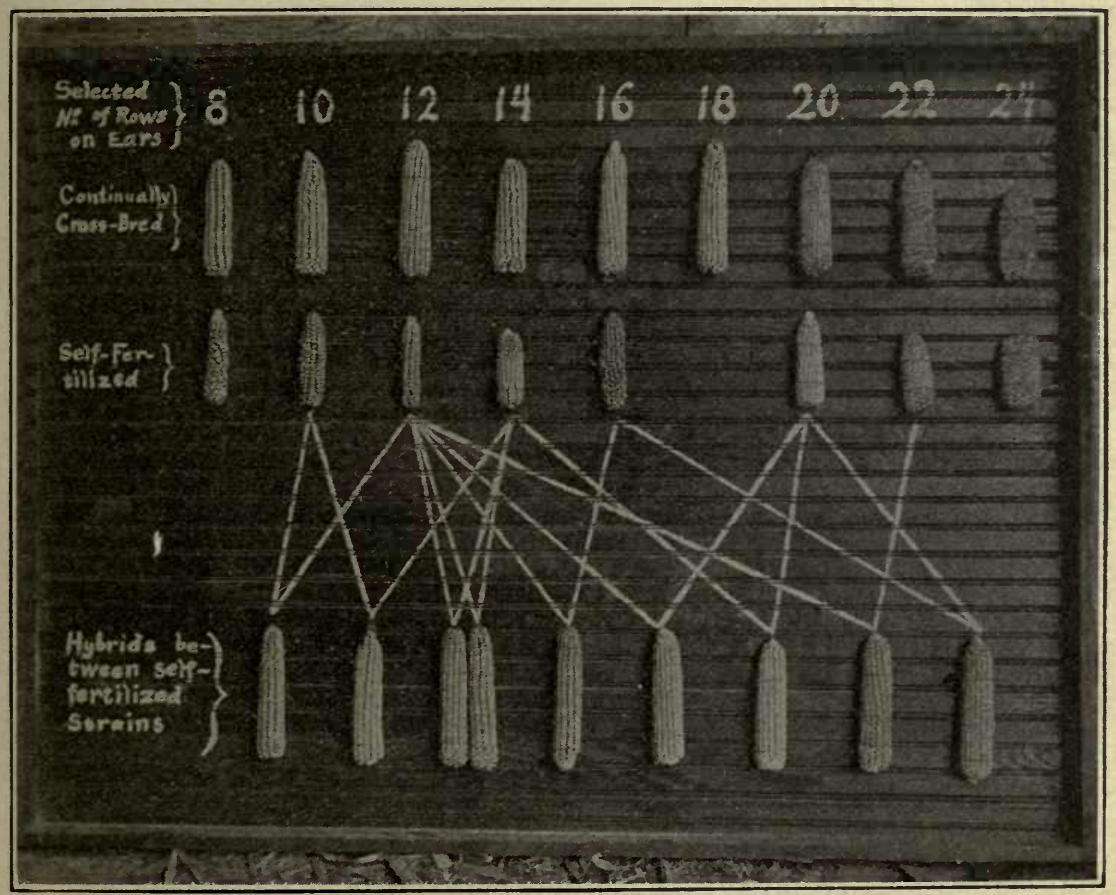

Cross-Fertilized, Self-Fertilized, and Hybridized Corn.

The lines between self-fertilized and hybridized series indicate the parentages of the latter.

1908. The important feature of this result is that the $F_{1}$ hybrids between strains $A$ and $B$ maintain essentially the same yielding capacity in successive years, while other hybrid strains produce quite different yields, varying from 61.5 bushels per acre to 98.4 bushels per acre. This fact, therefore, speaks for the view that the yield is a result of the particular hybrid combination. The second fact which 
supports this view is that reciprocal hybrids $e$ give essentially equal results. Thus, strains $A$ and $B$, between which reciprocal hybrids were reported last year as yielding 74.4 and 78.6 bushels per acre, respectively, produced this year, in reciprocal hybrid families, exactly equal yields, namely, 79.8 bushels per acre. More conclusive still is the result from my best hybrid combination of the past season which has also been tested in reciprocal crosses; see figure. These crosses were made between a self-fertilized strain which had been selected continually to 16 rows and another which had been continually selected to 20 rows of grains on the ear. When the 16 -rowed type was used as the mother, a yield of 98.4 bushels per acre was produced; when the 20-rowed type was used as the mother, a yield of 96.1 bushels per acre was produced. If the production of 98.4 bushels per acre had been purely a chance result which might by equal chance have appeared in any other strain, it is scarcely conceivable that the reciprocal should have so nearly approached the same extreme yield. The lower of these two yields, namely, 96.1 bushels, is 8 bushels per acre above the best yield produced during the same season by any continually cross-bred family of corn in my cultures. From all the results reported in this paragraph, it may be safely concluded that the production of the highest yield requires simply the finding of the best combination of parents and then repeating this combination year after year.

Several new evidences of the correctness of my view regarding the hybrid character of any ordinary vigorous corn plant have resulted from the past season's work. The assumption that self-fertilization results in the isolation of pure homozygous strains or biotypes and that the real purpose of cross-breeding is to secure the stimulus which comes from the heterozygous $f$ association of alternative qualities from the two parents, requires that the first generation of the cross between two pure self-fertilized strains be relatively uniform, and that the second generation, in which these various hybrid qualities are rearranged in every possible combination, shall show greater diversity. I have now reared two families representing the second generation of such a cross between strains $A$ and $B$. The variation in the number of Fows in self-fertilized strains, in $F_{1}$ hybrids, and in $F_{2}$ hybrids, are shown in the following table:

\footnotetext{
e Formed by using one variety as the male parent in one cross and in another cross between the same varieties using the other variety as the male parent; thus $A \times B$ and $B \times A$ are reciprocal crosses, and their progenies are reciprocal hybrids. $t$ Having each separate characteristic derived from only one of the two parents.
} 
TABLE 1.-Variations in number of rows of grains in self-fertilized strains, and in $F_{1}$ and $F_{2}$ hybrids.

\begin{tabular}{|c|c|c|c|c|c|c|c|}
\hline \multirow{2}{*}{ Strain } & \multicolumn{6}{|c|}{ Number of plants having ears with- } & \multirow{2}{*}{$\begin{array}{l}\text { Yield-bushels } \\
\text { per acre }\end{array}$} \\
\hline & $\stackrel{8}{\text { rows }}$ & $\begin{array}{c}10 \\
\text { rows }\end{array}$ & $\begin{array}{c}12 \\
\text { rows }\end{array}$ & $\begin{array}{l}14 \\
\text { rows }\end{array}$ & $\begin{array}{c}16 \\
\text { rows }\end{array}$ & $\begin{array}{l}18 \\
\text { rows }\end{array}$ & \\
\hline Pure strain $A$. & 66 & 5 & 3 & & & & 14.2 . \\
\hline Pure strain $B$. & & & 10 & 18 & 12 & & 12.1 \\
\hline$A \times B\left(\mathrm{~F}_{1}\right) \ldots$ & • & 2 & 18 & 9 & 2 & & 79.8 \\
\hline$B \times A\left(\mathrm{~F}_{1}\right) \ldots$ & & 19 & 58 & 9 & & & 79.8 \\
\hline$A \times B\left(\mathrm{~F}_{2}\right) \ldots$ & 3 & 32 & 57 & 16 & 2 & 3 & 61.0 \\
\hline$B \times A\left(\mathrm{~F}_{2}\right) \ldots$ & & 1 & 26 & 10 & 15 & 2 & 78.0 \\
\hline
\end{tabular}

It is clearly seen that the $F_{2}$ ears show a greater range of variation in number of rows on the ear than those of the $F_{1}$. Since the empirical range of variation is capricious, and therefore is not a satisfactory measure of variability, I have calculated the mean, standard deviation, and coefficient of variation, for the numbers of rows on the ears of each of these six families. The constants thus derived are arranged for comparison in the following table:

TABLE 2.-Mean, standard deviation, and coefficient of variation for number of rows on ear.

\begin{tabular}{|c|r|r|r|}
\hline \multicolumn{1}{|c|}{ Strain } & Mean & \multicolumn{1}{|c|}{$\begin{array}{c}\text { Standard } \\
\text { deviation }\end{array}$} & \multicolumn{1}{|c|}{$\begin{array}{c}\text { Coefficient of } \\
\text { variation }\end{array}$} \\
\hline Pure strain $A \ldots$ & $8.297 \pm 0.055$ & $0.705 \pm 0.039$ & $8.498 \pm 0.474$ \\
Pure strain $B \ldots$ & $14.100 \pm .145$ & $1.363 \pm .103$ & $9.661 \pm .736$ \\
$A \times B\left(\mathrm{~F}_{1}\right) \ldots$. & $12.710 \pm .154$ & $1.271 \pm .109$ & $9.998 \pm .865$ \\
$B \times A\left(\mathrm{~F}_{1}\right) \ldots \ldots$ & $11.767 \pm .070$ & $.956 \pm .049$ & $8.128 \pm .421$ \\
$A \times B\left(\mathrm{~F}_{2}\right) \ldots \ldots$ & $11.841 \pm .110$ & $1.733 \pm .078$ & $14.638 \pm .671$ \\
$B \times A\left(\mathrm{~F}_{2}\right) \ldots \ldots$ & $13.786 \pm .108$ & $1.464 \pm .076$ & $10.623 \pm .559$ \\
\hline
\end{tabular}

It will be seen by noting the numbers in the last column that the inferences as to the relative variability of $F_{1}$ and $F_{2}$, drawn from the range of variation in the several families, were correct. This fact will be even more obvious if the coefficients of variation are averaged in pairs. In this way it may be found that these pure strains had an average variability of 9.081 per cent; their $F_{1}$ hybrids had an average variability of 9.063 per cent; and the $\mathrm{F}_{2}$ hybrids an average variability of 12.63 per cent. My hypothesis requires that in the fertilization of the pure homozygous strains and in the production of the $F_{1}$ hybrids 
between them equal sperms meet equal eggs, so that in each case the resultant offspring should be exactly equivalent in all their hereditary qualities and the coefficients 9.081 per cent and 9.063 per cent must be measures of the non-hereditary variations or "fluctuations," while the coefficient 12.63 per cent in the $F_{2}$ is the result of the concurrence of hereditary and non-hereditary variations.

The number of rows on the ear, which is used here as a measure of the variability, is not in itself of great practical importance, of course, but the general question of variability, which is illustrated by this character, is of very great practical value: The possibility of attaining a fair degree of uniformity in the several desirable qualities will favor a more definite specialization of the crop to meet particular desired ends. Besides, any diversity in the qualities of the crop necessarily means a lower value in regard to each desirable quality than would be attainable if all individuals were brought up to a uniformly high standard.

Another very practical point in regard to this second generation and which emphasizes the importance of utilizing the $F_{1}$ plants for the crop each year, is seen by a comparison of the yields per acre (see Table 1) produced by the $F_{2}$ as compared with $F_{1}$. In both of the $F_{2}$ families the yield is less than the corresponding yield of the $F_{1}$ families, and when taken together this difference amounts to 8 bushels per acre. When considered in connection with the increased variability, this serves to further illustrate the point made in the last paragraph, namely, that the increased range of variation means a decreased yield.

The results of all my investigations to the present time, which seem to demonstrate that there are many distinct biotypes of corn continually mingled together in complex hybrid combinations, and that there is a stimulating effect of heterozygosis, may be summarized in the following statements. The first four of these propositions were demonstrated by data presented in my paper on "The composition of a field of maize"; the next four in "A pure-line method of corn breeding"; and the present paper gives further proof of the correctness of (6), (7) and (8), and adds the last four.

(1) The progeny of every self-fertilized corn plant is of inferior size, vigor and productiveness, as compared with the progeny of a normally cross-bred plant derived from the same source. This is true when the chosen parent is above the average conditions as well as when below it. 
(2) The decrease in size and vigor which accompanies selffertilization is greatest in the first generation, and becomes less and less in each succeeding generation until a condition is reached in which there is (presumably) no more loss of vigor.

(3) Self-fertilized families from a common origin differ from one another in definite hereditary morphological characters.

(4) Regression of fluctuating characters has been observed to take place away from the common mean or average of the several families instead of toward it.

(5) A cross between sibs $g$ within a self-fertilized family shows little or no improvement over self-fertilization in the same family.

(6) A cross between plants belonging to two self-fertilized families results in a progeny of as great vigor, size, and productiveness, as are possessed by families which had never been self-fertilized.

(7) The reciprocal crosses between two distinct self-fertilized families are equal, and possess the characters of the original corn with which the experiments were started.

(8) The $F_{1}$ from a combination of plants belonging to certain self-fertilized families produces a yield superior to that of the original cross-bred stock.

(9) The yield and the quality of the crop produced are functions of the particular combination of self-fertilized parental types, and these qualities remain the same whenever the cross is repeated.

(10) The $F_{1}$ hybrids are no more variable than the pure strains which enter into them.

(11) The $F_{2}$ shows much greater variation than the $F_{1}$.

(12) The yield per acre of the $F_{2}$ is less than that of the $F_{1}$.

[Presented by Committee on Breeding Corn.] 

ADVANCE PRINT FROM THE PROCEEDINGS OF

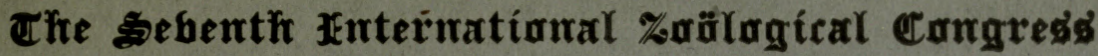

Boston Meeting, August 19-24, 1907

RESULTS OF CROSSING BURSA BURSA-

PASTORIS AND BURSA HEEGERI

GEORGE HARRISON SHULL

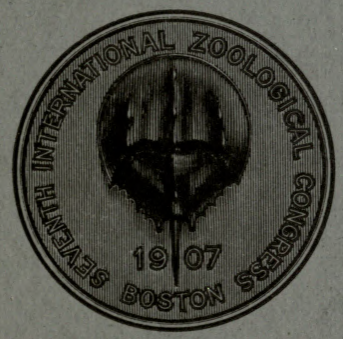

CAMBRIDGE, MASSACHUSETTS

1910 


\section{RESULTS OF CROSSING BURSA BURSA-PASTORIS AND BURSA HEEGERI}

\section{GEORGE HARRISON SHULL}

Ten years ago Solms-Laubach received from Heeger some specimens of a Cruciferous plant which was found growing in nature at Lindau, Germany, which the latter could not identify. In general habit these plants resembled the almost cosmopolitan species, Bursa bursa-pastoris (L) Britton or Capsella bursa-pastoris as it is commonly called by European botanists. They differed from the latter species, however, in having seed capsules oval in outline and circular in cross-section instead of the flat and triangular or obcordate capsules which are characteristic of Bursa bursa-pastoris.

Solms-Laubach and Ascherson to whom he showed the specimens, were both inclined at first to refer them to the genus Camelina, which is characterized by spherical capsules. Cultures made from seed secured from Professor Heeger soon indicated, however, the near relationship of the new form to Bursa bursa-pastoris when in 1898 several apparent reversions to the capsule-form of $B$. bursa-pastoris were noted. In 1900 Solms-Laubach ${ }^{1}$ published an account of the new form, assigning to it the name Capsella heegeri which becomes, according to the American code, Bursa heegeri (Solms-Laubach).

This very distinct species of Bursa has attracted considerable attention for the reason that its occurrence as a component of the flora of a region so well known taxonomically could leave scarcely a doubt of its very recent origin from B. bursa-pastoris by mutation, and it was mentioned by De Vries ${ }^{2}$ as an instance of mutation in nature. Shortly after the publication of the original account, Bursa heegeri disappeared from the type locality at Lindau, and has been reported from nature only once since that time, though it has been widely grown in botanical gardens.

The second report of the discovery of Bursa heegeri in nature was made by Laubert ${ }^{3}$ who discovered it along the Dahlem turnpike in 1905 , but here the likelihood of a new origin is certainly exceeded by the likelihood that a seed was carried to this spot by some agency from a nearby culture, for it had been cultivated for several years at Dahlem in an unprotected bed several hundred meters from the place in which Laubert discovered it. Laubert pointed out that there

1 Solms-Laubach, H., Cruciferienstudien I. Capsella Heegeri Solms eine neuentstandene Form der deutschen Flora. Bot. Zeit., 58, pp. 167-190, Pl. VII. 1900 .

${ }^{2}$ De Vries, H., Die Mutationstheorie. I. Die Entstehung der Arten durch Mutation. pp. $477-478.1901$.

${ }_{3}^{3}$ Laubert, R., Notizen über Capsella Heegeri Solms. Verh. des Bot. Vereins d. Provinz Brandenburg. 47, pp. 197-199, 1905. 
are other characters of the stem and inflorescence in addition to the capsule character noted by Solms-Laubach, which serve to distinguish $B$. heegeri from its supposed parent, but on the other hand he found that in both species there occur frequent instances of abnormal pistillate but sterile flowers in the lower portion of the flower-stem, and he took this fact together with the occurrence of capsules occasionally simulating those of $B$. bursa-pastoris, formed when $B$. heegeri is attacked by Cystopus and Peronospora, as additional proofs of the correctness of the view that $B$. heeger $i$ is a derivative from $B$. bursapastoris. Of the manner of its derivation from $B$. bursa-pastoris nothing is known, of course, but Potonie ${ }^{1}$ suggests that its origination was a pathological phenomenon with atavistic significance; in other words, that it is a reversion induced by some pathological condition.

The prominent part which mutation may have taken in the production of new species makes it of great interest to know in each case just what will be the result both of self-fertilization and of crossing the mutant with the parental form, for its behavior in these two cases is the important factor in determining its power of maintenance at the time of its origin. The result of self-fertilization was investigated by Solms-Laubach before the publication of his original account, and it was shown that the characters that differentiate $B$. heegeri from $B$. bursa-pastoris are fully hcritable. As the Bursas normally self-fertilize to a predominant extent, the possession of a vigorous constitution would seem to be all that is necessary for successful maintenance.

I have now tested the question as to what will happen when Bursa heegeri and $B$. bursa-pastoris intercross. In order to appreciate the result fully it will be necessary to recount briefly some recently discovered facts regarding the composition of the species $B$. bursapastoris. This species is not of unit value, but is a composite group of elementary species as has been shown by Lotsy, ${ }^{2}$ Shull, ${ }^{3}$ and Almquist. ${ }^{4}$ How many of these elementary species there may be cannot be stated at this time, but Almquist has described and named over 70 . In my own cultures I have observed that some of the differentiating characters used by Almquist disappear upon continued breeding, and I think it very probable that not all of the 70 elementary species described by him will stand the test of longer culture under more carefully controlled conditions, but it seems evident that the number of elementary forms may be considerable. I have studied in their hybrid combinations four of these elementary species, and have

1 Potonie, H., Capsella Heegeri eine pathologische Erscheinung mit atavistischen Momenten? Naturwis. Wochenschr. 21 (N.S. 5), pp. 788-791. 1906.

${ }_{2}$ Lotsy, J. P., Vorlesungen über Deszendenztheorien, mil besonderer Berücksichtigung, der botanischen Seite der Frage. Erster Teil. pp. 179-181. 1906.

3 Shull, G. H., Elementary species and hybrids of Bursa. Science, N. S. 25, pp. 590-591. 1907.

4 Almquist, E. Studien über die Capsella bursa-pastoris L. Acta Horti Bergiana. 4, No. 6, 1907. 
demonstrated their distinctness and permanence beyond a possible question. These four types are distinguished from each other by characteristic lobation of the leaves. For convenience I have assigned to these four forms the names Bursa bursa-pastoris heteris, $B$. $b p$. rhomboidea, B.bp.tenuis, and B. bp. simplex. It has been impossible to determine which of Almquist's elementary forms agree with these, but it is almost certain that each of these four forms is more inclusive than his described forms, and that under each of these headings should be grouped several of his named elementary species. These four types may be briefly characterized as follows:

Type (a). Bursa bp. heteris has the leaves divided to the midrib, the terminal lobe being usually separated from the nearest lateral lobes by clean, deep incisions. The lateral lobes consist essentially of two features, an elongated proximal portion, the "primary lobe," and a more or less rounded or angular portion, the "secondary lobe, in the distal axil of the primary lobe." Several of Almquist's recently described elementary species obviously fall under this head, his B. bp. angustiloba representing the most pronounced type, and Wittrockii, rubella, and others doubtless belong here. From the descriptions and photographs I have been unable to determine which is the first of his elementary species which should be classed here. This is an important matter because it would determine the priority of names.

Type (b). Bursa bp. rhomboidea has the leaves divided to the midrib as in type (a). The lateral lobes have an incision on the distal margin setting off the rounded secondary lobe from an unelongated primary lobe, the secondary lobe corresponding with the rounded distal component of the heteris lobe. There is usually a corresponding incision on the proximal margin of the primary lobe. In the best developed specimens these incisions set off a small terminal portion which is rather blunt or angular at the apex, being generally of rhomboidal form. Less perfectly developed specimens have the incisions very shallow, or nearly wanting. Here may belong Almquist's $B . b p$. polyedra, densa, and alpina.

Type (c). Bursa bp. tenuis.' This differs from both (a) and (b) in that the sinuses do not usually reach the midrib and the terminal lobe is not set off from the nearest lateral lobe by a clean-cut incision reaching almost or quite to the midrib. The lobes are more or less elongated and sharp, entirely lacking the rounded secondary lobe.

Type (d). Bursa bp. simplex. Like the last, this form is scarcely ever divided nearly to the midrib, and the lobes are mostly obtuse, sometimes more or less acutish, but never long and attenuated.

I have studied over twenty families of hybrids, among these four elementary species, and find that they represent the four terms of a typical Mendelian dihybrid, the alternate characteristic being, Aa, an elongated primary lobe vs. no elongated primary; and, Bb, a 
rounded secondary lobe $v s$. no rounded secondary. In the usual manner of designating gametic formulae, B. bp. heteris will be represented as $\mathrm{AB}$, possessing both dominant characteristics; rhomboidea will be $\mathrm{aB}$; tenuis, $\mathrm{Ab}$, and simplex, ab. From this it will be inferred that heteris is dominant over all of the other forms; that rhomboidea and tenuis are both dominant over simplex, and recessive to heteris; and that when tenuis and rhomboidea are crossed together, and when heteris and obtusa are crossed together, heteris appears as the $\mathrm{F}_{1}$, and all four forms will occur in $\mathrm{F}_{2}$ in the ratio $9: 3: 3: 1$. All of these inferences have received complete experimental confirmation.

Now, in the cross between $B$. bursa-pastoris and B. heegeri it is important to note that $B$. heegeri has a rosette possessing the two dominant characteristics of $B . b p$. heteris, though the primary lobe, $A$, is usually wider and more blunt than the best developed examples of the $B . b p$. heteris type, and the rounded secondary, $\mathrm{B}$, is rendered less prominent because of the lesser depth of the sinus which sets it off from the primary lobe. Reciprocal crosses were made between $B$. heegeri and $B . b p$. simplex. The $\mathrm{F}_{1}$ hybrids were essentially uniform, and were of the heteris type, though somewhat stunted because of unfavorable conditions in the propagating-house during the period of their development. All had the flat, triangular capsules of Bursa bursa-pastoris. In $\mathrm{F}_{2}$ there appeared the four forms of rosette already described, in combination with each type of capsule. The rosettes presented many instances of the best developed examples of the four described forms, particularly interesting being the fact that much better developed heteris rosettes occur in the $\mathrm{F}_{2}$ than are seen in purebred $B$. heegeri, though these heteris characteristics must have come directly from the heegeri side of the cross. The numerical results of these crosses may be tabulated thus:

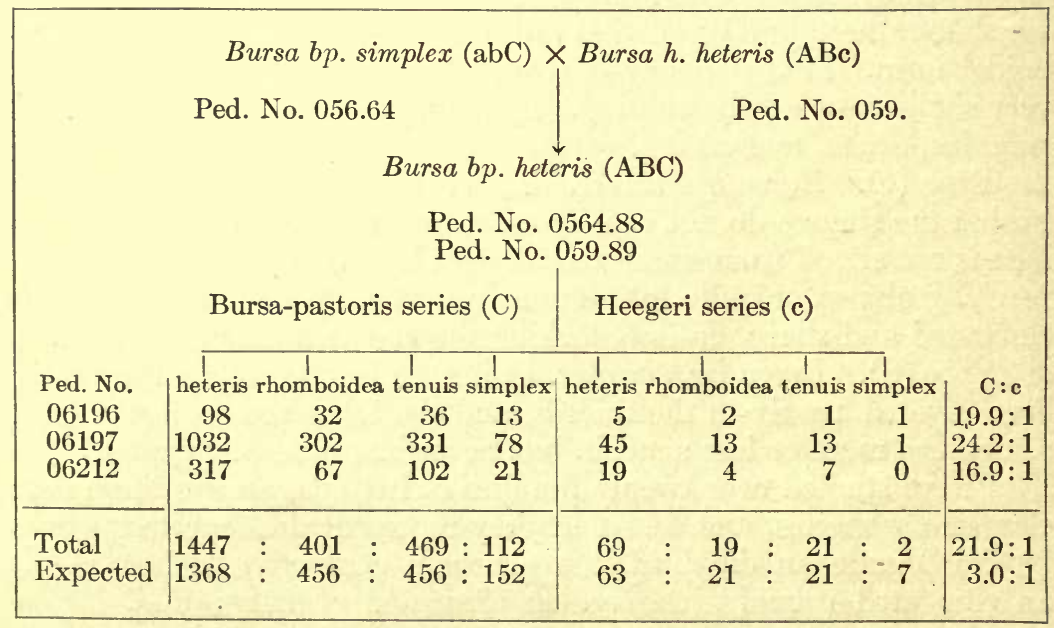


In this table the pedigree numbers 06196 and 06197 represent families in which the bursa-pastoris is the mother, while No. 06212 is the reciprocal cross. It will be seen that there is no essential difference between these two crosses. In the rosette characters the numbers of offspring belonging to the several types are very close to the Mendelian ratios, among both those having bursa-pastoris capsules and those having heegeri capsules, showing by these facts that the heegeri rosette has the same allelomorphic composition as that of bursa-pastoris, and that these rosette characters are not coupled in any but a chance way with the form of the capsule. This independence of characters is thus seen to be a matter of great importance in the production of new elementary forms; for, before this cross was made. there existed, so far as is known, but one elementary species of $B$. heegeri, while out of the cross came four elementary species of B. heegeri.

While there is thus seen to be perfect agreement with Mendelian ratios in rosette characters, the capsules gave a surprising departure. The capsule-form is perfectly alternative; there never appeared in my cultures any intermediate condition. As the bursa-pastoris form is dominant, simple Mendelian expectation would require the appearance of one $B$. heegeri in every four $F_{2}$ individuals, providing this capsule difference be assumed to be due to the presence or absence of a single unit. Out of 2540 plants of the $\mathrm{F}_{2}$ generation observed, only 111 were $B$. heegeri, or approximately one in twentythree. That this result should be consistently given in three different pedigrees representing reciprocal crosses adds greatly to the weight that is to be attached to it. The greatest frequency in any pedigree was one in nearly 18 , and the least frequency was one in 25. I take the fact that the reciprocal crosses gave similar ratios to indicate that the $B$. heegeri capsule is dependent upon something carried by the germ-cells and cannot be a pathological condition transmissible from mother to offspring somatically, a possibility that might account for apparent heritability of characters in a self-fertilized line, but could not well account for equal results in reciprocal crosses. Normal Mendelian phenomena are believed to rest pretty securely on the method of formation of the chromosomes during the reduction division, but no scheme of behavior occurs to me which would result in the distribution of the heegeri determiner to but one cell in 20 .

It is clear that in this case the capacity of $B$. heegeri to maintain itself in competition with its parent would be very slight were it not for the fact that cross-fertilization in Bursa is relatively infrequent.

Briefly summarized my conclusions are:

(1) Bursa heegeri has the same Mendelian units in its leaves as occur in B. bursa-pastoris, which serves further to confirm its derivation from that form.

(2) The crossing of B. heegeri with B. bursa-pastoris gives rise to 
a series of elementary species in the former-corresponding with those in the latter species.

(3) The leaf characters are inherited in strict Mendelian ratios, but the capsule shows a very great departure. The bursa-pastoris capsule dominates the $\mathrm{F}_{1}$ generation, but the heegeri capsule appears in the $\mathrm{F}_{2}$ generation, in only 1 in 18 to 25 individuals.

(4) Under these circumstances the capacity of $B$. heegeri for selfmaintenance in competition with $B$. bursa-pastoris rests upon the comparative infrequency of cross-fertilization.

My sincere thanks are hereby tendered to Dr. E. N. Transeau in whose charge these cultures were left during my absence in California. A large part of the work of grouping the $\mathrm{F}_{2}$ into the proper classes was done by him. 





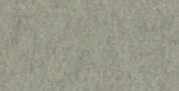

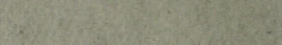

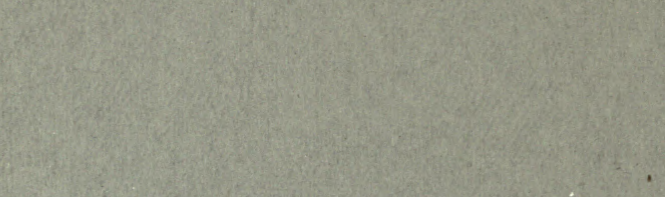

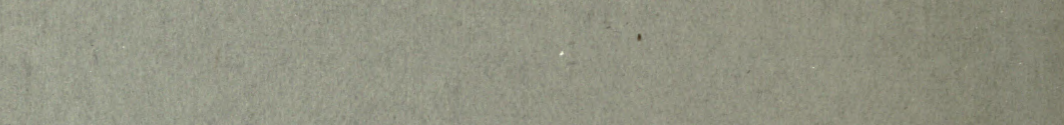

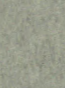


[Reprinted from The American Naturalist, Vol. XI.IV., Feb., 1910.|

\section{COLOR INHERITANCE IN LYCHNIS DIOICA L. ${ }^{1}$}

\section{DR. GEORGE HARRISON SHULL}

Two years ago I showed that in Lychnis dioica $\mathrm{L}$. the purple-flowered form behaves in normal Mendelian manner when crossed with the same type or with the typically white-flowered form of the same species (Shull, 1908). In subsequent work it has been discovered that the purple-flowered plants do not form a single unit-group, but that there are at least two distinct types, one of which has more bluish-purple flowers, the other more reddish-purple. No notice had been taken of such variation in the color characters until last year, although it had been observed that there was some variation in the intensity of color in different plants, and these had been, to a slight extent, recorded in terms of intensity, e. $g$., as "light," "medium" and "dark." Last year several individuals were observed so noticeably distinct because of the bluish character of their flowers, that an effort was made to determine the relationship of this light bluishpurple color to the more common reddish-purple, and several crosses were made representing the combination of "blue" and "red," using a single red-flowered indi-

TABLE I

\begin{tabular}{|c|c|c|c|c|c|c|}
\hline $\begin{array}{l}\text { Ped. } \\
\text { No. }\end{array}$ & Cross. & Red. & Blue. & White. & Theoretical Result. & $\begin{array}{c}\text { Ratio } \\
\text { Involved. }\end{array}$ \\
\hline 0845 & $\times$ Blue & 1 & 83 & 0 & $0: 84:$ & $0: 1: 0$ \\
\hline 0846 & $\times$ Red & 47 & 49 & 0 & $48: 48:$ & $1: 1: 0$ \\
\hline 0844 & White & 52 & 0 & 46 & $49: 0: 49$ & $1: 0: 1$ \\
\hline 0849 & Red & 68 & 22 & 1 & 68: $23:$ & $3: 1: 0$ \\
\hline 0848 & Blue & 27 & 32 & 27 & $32: 32: 22$ & $3: 3: 2$ \\
\hline 0847 & White & 53 & 0 & 46 & $0: 49$ & $1: 0: 1$ \\
\hline 0875 & White $\times$ Blue & 34 & 24 & 41 & $25: 25: 49$ & $1: 1: 2$ \\
\hline \multirow[t]{2}{*}{0876} & White $\times$ Red & 31 & 20 & 0 & $38: 13:$ & $3: 1: 0$ \\
\hline & Total & 313 & 230 & 161 & $310: 225: 169$ & \\
\hline
\end{tabular}

${ }^{1}$ Read before the Botanical Society of America at Boston, December; 1909. 
vidual as the mother in one series of crosses, and a single blue-flowered individual as the mother in another series. The same blue-flowered and red-flowered plants were also erossed at the same time with white-flowered plants. The actual and theoretical results of these eight crosses are given in Table I.

In addition to these families which were bred in such a way as to allow the definite working out of the gametic formulæ of the parents and the theoretical results, bluishflowered plants were also observed in a number of other pedigrees. In some of these families only a small proportion of the individuals had their tints recorded, as they were being especially studied with other objects in view. Such fragmentary records are of no special value in this connection, of course, and they will not be presented, but in Table II. are given all those pedigrees in which approximately all the purple-flowered offspring were recorded either as "blue" or "red."

In this second table it is impossible to vouch for the correctness of the suggested theoretical results, as the gametic formulæ of the parents are in each case very imperfectly known. The column of theoretical results is constructed simply by using that one of the available theoretical ratios which fits most accurately the observed facts. When numbers are so small, mere inspection can not determine with certainty which is the correct theoretical ratio. Thus in No. 08168 the empirical ratio, $19: 11: 31$, is almost equally well referred to either of the available ratios, $1: 1: 2$ and $3: 1: 4$, as it stands about midway between them. Notwithstanding the fact that ignorance of the gametic composition of the parents in this second table makes it impossible to decide in all cases what ratio should have been expected, the results harmonize well throughout with those which comprise Table I., where the theoretical "expectation" is definitely known.

All of the crosses recorded in these two tables seem to be typically Mendelian, with the bluish-purple color hypo- 
TABLE II

\begin{tabular}{|c|c|c|c|c|c|c|c|c|c|}
\hline \multirow{2}{*}{$\begin{array}{c}\begin{array}{c}\text { Ped } \\
\text { No. }\end{array} \\
0850\end{array}$} & Cross. & \multirow{2}{*}{$\frac{\text { Red. }}{67}$} & \multirow{2}{*}{$\begin{array}{r}\text { Blue. } \\
0\end{array}$} & \multirow{2}{*}{$\begin{array}{c}\text { White. } \\
0\end{array}$} & \multicolumn{4}{|c|}{$\begin{array}{l}\text { Probable Theoretical } \\
\text { Result. }\end{array}$} & $\begin{array}{c}\text { Ratio } \\
\text { Invoked. }\end{array}$ \\
\hline & Red & & & & $67:$ & & & 0 & $1: 0:$ \\
\hline 0851 & MITe & 40 & 13 & 0 & 40: & 13 & & 0 & $3: 1:$ \\
\hline 0852 & Vhite & 26 & & 52 & 23: & 8 & $:$ & 51 & $9: 3: 20$ \\
\hline 0853 & ed & 82 & (1) & & $76:$ & & : & 25 & $3: 0:$ \\
\hline 0855 & d & 105 & 0 & 0 & $105:$ & & : & 0 & $1: 0:$ \\
\hline 0856 & ed & 39 & 18 & 39 & $36:$ & 12 & : & 48 & $3: 1:$ \\
\hline 0857 & ed & $t$ & 1 & & 58: & 19 & : & 26 & $9: 3:$ \\
\hline 0858 & Red & 17 & 1. & & $16:$ & 15 & : & 31 & $1: 1:$ \\
\hline 08 & ed & 10 & 0 & 14 & 9: & & : & 15 & $3: 0:$ \\
\hline & & 77 & 0 & 21 & 74: & 0 & : & 24 & $3: 0:$ \\
\hline 08 & & 38 & 0 & 0 & $38:$ & 0 & : & 0 & $1: 0:$ \\
\hline 08 & Red & 101 & 0 & 0 & 101： & & : & 0 & $1: 0:$ \\
\hline 0 & en & 5 & 0 & 6 & $27:$ & 0 & : & 9 & $3: 0:$ \\
\hline & & 3 & ( & 12 & 4: & 0 & : & 12 & $1: 0:$ \\
\hline & ite & 18 & & 6 & $18:$ & 0 & : & 6 & $3: 0:$ \\
\hline 08 & te $)$ & 37 & 0 & 29 & 3 : & & : & 33 & $1: 0:$ \\
\hline & ite & 2 & c & & $20:$ & 0 & : & 21 & $1: 0:$ \\
\hline & & & () & & 65: & 0 & : & $2:$ & $3: 0: 1$ \\
\hline & & 4 & & & $47:$ & 0 & : & 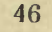 & $1: 0: 1$ \\
\hline 1 & ite & 3 & 0 & & 31: & 0 & : & 53 & $3: 0: 5$ \\
\hline & ite $Y$ & & .8 & & $21:$ & & : & 46 & $9: 3: 20$ \\
\hline & & & & & 8 : & & : & 37 & $3: 1: 4$ \\
\hline 00 & $e x$ & & & & $44:$ & & : & 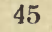 & $1: 0:$ \\
\hline & ite & & & & $29:$ & & : & 29 & $1: 0: 1$ \\
\hline & aite & 23 & 27 & 3 & $21:$ & 21 & : & 43 & $1: 1: 2$ \\
\hline & P & & 0 & 0 & 25: & 0 & : & 0 & $1: 0:$ \\
\hline & ite $x 1$ & 29 & 1 & & $30:$ & 10 & : & 39 & $3: 1:$ \\
\hline & Red & 44 & 13 & & $42:$ & 14 & : & 19 & $9: 3: 4$ \\
\hline & White & 48 & 0 & & 47: & & : & 46 & $1: 0: 1$ \\
\hline & aite $\times \operatorname{Red}$ & 19 & 11 & & $15:$ & 15 & : & 31 & $1: 1:$ \\
\hline & $\mathrm{P}$ & 7 & 0 & & $74:$ & 0 & : & 25 & $3: 0:$ \\
\hline 77 & Red & 72 & 0 & 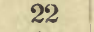 & $71:$ & 0 & : & 23 & $3: 0: 1$ \\
\hline \multirow[t]{2}{*}{08178} & White & 47 & 0 & 47 & $47:$ & 0 & : & 47 & $1: 0: 1$ \\
\hline & Total & 1,426 & 144 & 806 & 1,382: & 143 & & 851 & \\
\hline
\end{tabular}

static or "recessive" to the red. This is one of the first cases of this kind which has appeared, as heretofore the bluish colors have quite generally been found epistatic to the reds. The most important studies which have been made relating to the inheritance of the anthocyan colors are those of Bateson (1902, 1905, 1906, 1909) and his co-workers, on Lathyrus and Matthiola, Miss Wheldale (1907) and Baur (1908) on Antirrhinum, and Tschermak (1901, 1904) on Pisum and Phaseolus. In all of these genera as well as in Clarkia and Salvia (Bateson, etc., 1905), the more bluish anthocyan color is epistatic to the reddish anthocyan. Bateson (1909, p. 41) states in one place, that in Primula Sinensis "blue 
is hypostatic to all the red shades," although the magenta colors are shown to be epistatic to red. This isolated statement regarding the blue color in Primula Sinensis is not supported by any data, and I do not know the chemical relation between it and the magenta colors. Miss Wheldale (1909), who discusses at some length the color series in Primula material secured from Bateson and Gregory, makes no mention of the occurrence of blue, though she ascribes the production of magenta and crimson to the action of a "bluing factor" upon red anthocyan.

Upon comparison of my bluish-purple Lychnis with the colored plates of Primula given by Bateson (1909, p. 294) I think the Lychnis color should be classed as a light magenta rather than a blue, as there is a decided reddish element in this Lychnis color. If Bateson's isolated statement that "blue is hypostatic to all the red shades" in Primula is correct, then that color corresponds in its behavior with this light magenta color in Lychnis. I have not made a thorough investigation of the chemical relations of the two types of purple in Lychnis, but have demonstrated by a few preliminary tests that the reddish-purple color is converted to bluish when treated with alkalies, and that the light bluishpurple is made as bright red as the red-purple type on treating with weak acids, thus indicating a very simple relation between these two colors.

Although the relation between the two types of color in Lychnis is just the reverse of that exhibited by practically all other plants in which similar colors have been studied, I am led to essentially the same conclusions regarding the method of color determination, as those derived from the extensive studies which have been made on Lathyrus, Matthiola, etc. The production of the "lowest stage" of color, i. e., the color which results from the combined action of the least number of genes, is due to the interaction of two independent factors or genes, either of which produces no color when not associated with 
the other. In order to produce a second stage of color it is necessary to assume the occurrence of a third gene which can make its characteristic color-reaction apparent only in the presence of the other two. Thus in Lathyrus, etc., it was assumed that two factors, $R$ and $C$, are necessary to the production of a red anthocyan color, and that a third factor, $B$, modifies this color to bluish. This assumption requires that the presence of each of these three genes be dominant over its absence. An alternative assumption might have been made, viz., that the absence of the third factor is dominant over its presence. Then the lowest grade of color would be a blue color produced by the simultaneous presence of $B$ and $C$, and the red color would appear only when $R$ is present in the homozygous state.

Last year in my discussion of the presence and absence hypothesis (Shull, 1909) it was pointed out that it would be impossible in many cases to determine "whether red flowers are blue flowers with an added factor for acidity or whether blue flowers are red with an added factor for alkalinity," and also that "it is conceivable that both these situations may be presented in different species." The color characters in Lychnis give a very good illustration of these statements.

If we assume the dominance of presence over absence, the lowest grade of color-the bluish-is formed by the combined action of two genes, $B$ and $C$, the one probably representing, according to the studies of Miss Wheldale (1909), the capacity to produce a chromogen of the flavone series, the other representing the production of an oxidase. The red color is in this case produced by an added factor, $R$, which modifies the bluish color produced by $B$ and $C$. The $R$ may be perhaps an acidifier, a reducing agent, or a partial inhibitor of the oxidizing action of $B$. This method of explaining color inheritance in Lychnis presents an interesting reversal of the places occupied by $R$ and $B$ when compared with the situation in other plants. 
If, on the other hand, the assumption be made that, in Lychnis, absence of $B$ is dominant over its presence, the relative positions of $R$ and $B$ may remain the same as in Lathyrus, Matthiola, etc. For in this case $R$ and $C$ will be the two factors necessary to the production of red anthocyan and $B$ the "bluing factor" which is added to it to form bluish anthocyan, the difference between Lychnis and Lathyrus being simply that the $B$ which may be looked upon perhaps as a factor for alkalinity or for an oxidizer, is too weak in its activity in Lychnis to produce its characteristic effect except when present in double quantity or strength, as it is when in the homozygous state.

The data now at hand do not make possible a decision as to whether presence, or absence of the color-modifier, is dominant in Lychnis, as each of these assumptions may be shown to fit all the facts involved in Table I. The gametic formulæ of the six plants involved in these eight crosses are presented in Table III., to enable a comparison of the two methods of explanation.

\section{TABLE III}

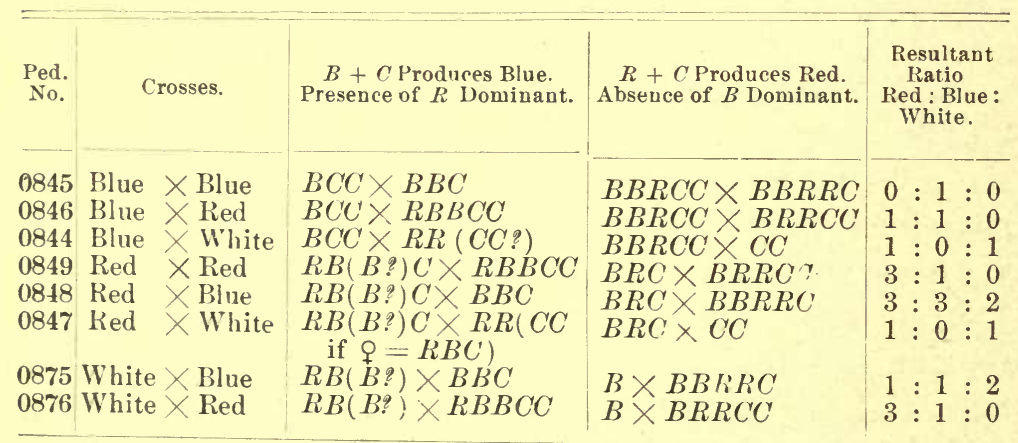

The fact now demonstrated that the purple color in Lychnis is due to the presence of at least three distinct genes instead of only one, as originally assumed by me, has served to elucidate several difficulties which had been encountered. It may be recalled that a rather large range of supposedly fluctuating variability in the 
percentage of purple-flowered individuals in families resulting from crosses of purple with white was shown to form a normal probable error curve, thus conforming very well with the Mendelian hypothesis that gametes of different alternative composition unite according to the laws of chance. While this conclusion is not in any way opposed by my later studies, it is now known that a portion of that apparently fluctuating variation may have been due to the occurrence of a mixture of several different ratios. In accordance with the present demonstration that the purple color is due to three factors, the combined action of two of which are necessary to the production of any color and the addition of a third for the modification of this color, a cross between white and purple must give either all purple, or purple and white in any of the following ratios: $3: 1,1: 1,3: 5$, or $1: 3$, though the ratio $1: 1$ occurs much more frequently than any of the other possibilities. In other words, without any fluctuation at all, purple-flowered individuals, when mated entirely at random with white-flowered individuals, should produce progenies consisting of $25,37.5,50,75$ or 100 per cent. purple-flowered offspring, instead of only 50 or 100 per cent.

I have in several cases found purple-flowered individuals among the offspring of two white-flowered parents. Such occurrence was entirely incomprehensible to me except on the basis of an error in technique. It now becomes obvious that white crossed with white must occasionally give various proportions of purple-flowered offspring. Some such crosses will give all purple while others will give purple and white in ratios $1: 1,3: 5$, or $1: 3$, although a frequent result will be a progeny of all white, which latter alone was expected under the conception that the purple color was due to the presence of a single gene. Although I have reared many families in which both parents were white, I have almost invariably obtained a progeny of all whites, but this is undoubtedly due to the fact that such crosses of white with white were 
nearly always made between sibs in wholly white-flowered families. Such crosses are necessarily homozygous with respect to the absence of one (or both) of the two genes whose joint action is necessary to the production of color. Crossing of white-flowered individuals of different parentage or of white sibs in hybrid families will doubtless quickly demonstrate the production of purpleflowered offspring by white-flowered parents, in the ratios required by theory.

\section{Summary}

The purple color in Lychnis dioica $\mathrm{L}$. is a compound character, produced by the interaction of three distinct and independent genes in a manner exactly analogous to the similar colors in Lathyrus, Matthiola, etc.

The two types of purple color present in different individuals are a reddish and a more bluish-purple, the former being changed to blue by treatment with alkalies, and the latter changed to red by the addition of weak acids.

The bluish or alkaline color is hypostatic to the reddish or acid color, this being the reverse of the condition found in all other plants containing similar series of colors which have thus far been reported, unless possibly an isolated statement should prove correct that in Primula Sinensis "blue is hypostatic to all the red shades."

It is impossible to determine at present whether this reversal of the relation between bluish and reddish anthocyan results from the occurrence of positive characters for both alkalinity and acidity, or whether only one of these exists as a positive character and the alternative color is produced when this positive color-modifier is in the heterozygous state, the latter situation involving the dominance of absence over presence.

The rather wide fluctuation in the percentage of purpleflowered families resulting from the cross of heterozygous purple with white $(i$. e., supposedly $D R \times R)$, reported in a former paper, may have been due in part to 
the mixture of the ratios $3: 1,1: 1,3: 5$ and $1: 3$, all of which are expected Mendelian results, on the basis of present knowledge of the compound character of Lychnis colors.

Crosses between white-flowered plants should, not infrequently, result in progenies of all purple-flowered offspring or of purple and white in the ratios $1: 1,3: 5$, or $1: 3$. These results have not yet been found, owing no doubt to the fact that my crosses between white and white have been almost invariably made between sibs in wholly white-flowered families.

Station For EXPERIMENTAL Evolution,

Cold Spring Harbor, Long Island,

November 19, 1909.

\section{LITERATURE CITED}

1909. Bateson, W. Mendel's Principles of Heredity, pp. xiv + 396, 1909. Cambridge, University Press.

1902. Bateson, W., and Saunders, E. R. Experimental Studies in the Physiology of Heredity, I. Reports to the Evolution Committee of the Royal Society.

1905. Bateson, W., Saunders, E. R., and Punnett, R. C. Reports to the Evolution Committee of the Royal Society, II.

1906. Bateson, W., Saunders, E. R., and Punnett, R. C. Ibid., III.

1908. Baur, E. Einige Ergebnisse der experimentellen Vererbungslehre. Beih. z. Med. Klinik., 4: 265-292, 1909.

1908. Shull, G. H. Some New Cases of Mendelian Inheritance. Bot. Gaz., 45: 103-116, February, 1908.

1909. Shull, G. H. The "Presence and Absence" Hypothesis. Amer. Nat., 43 : 410-419, July, 1909.

1901. Tschermak, E. Weitere Beiträge über Verschiedenwertigkeit der Merkmale bei Kreuzung von Erbsen und Bohnen. Ber. Deutsch. Bot. Gesell., 19: Heft 2, 1901.

1904. Tschermak, E. Weitere Kreuzungsstudien an Erbsen, Levkojen und Bohnen. Zeitschr. f. d. landw. Versuchsw. in Oesterr., 1904.

1907. Wheldale, Miss M. On the Inheritance of Flower-color in Antirrhinum majus. Proc. Roy. Soc., 79: 1907.

1909. Wheldale, Miss M. On the Nature of Anthocyanin. Proc. Cambridge Phil. Soc., 15 (pt. II.): 137-168, 1909. 



Reprinted from the Botanical GazeTte 49: I10-125, No. 2, February 1910

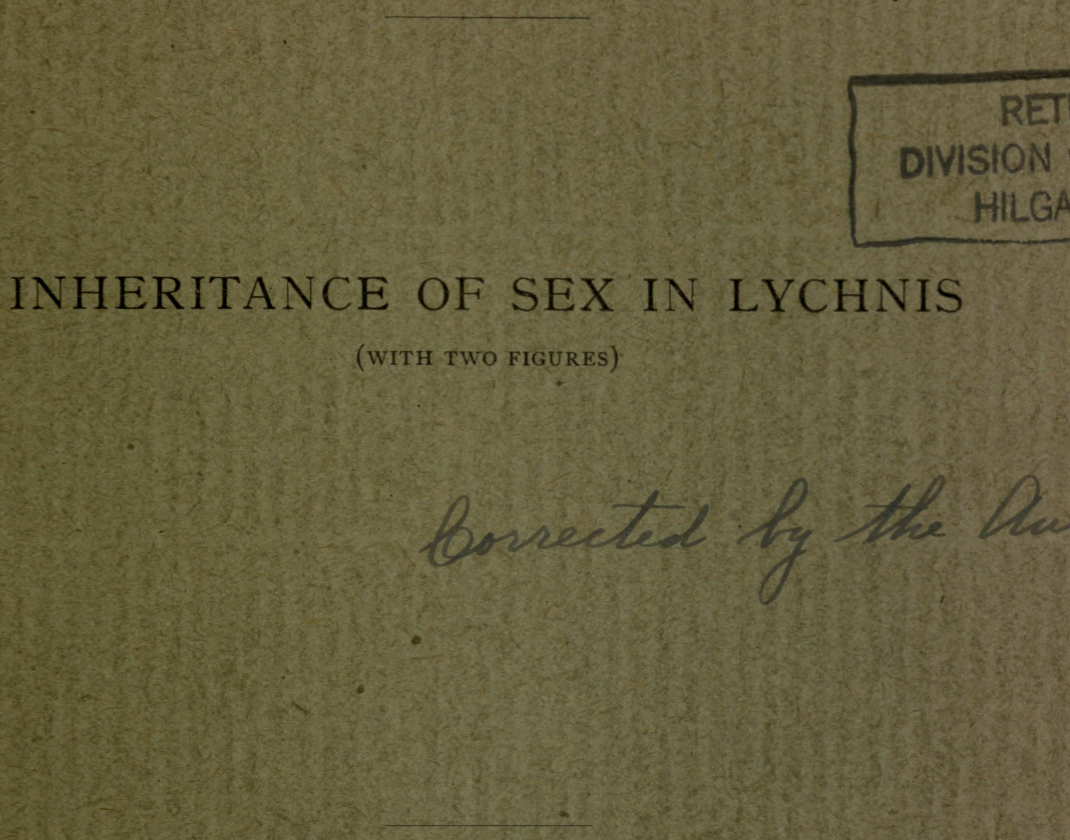

GEORGE HARRISON SHULL 


\section{7.}

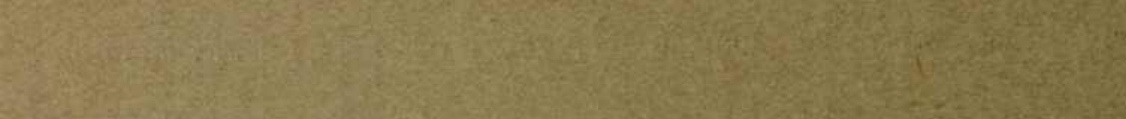

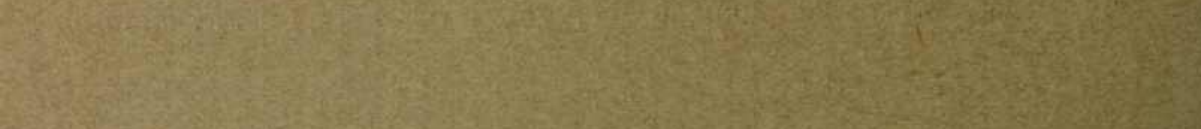

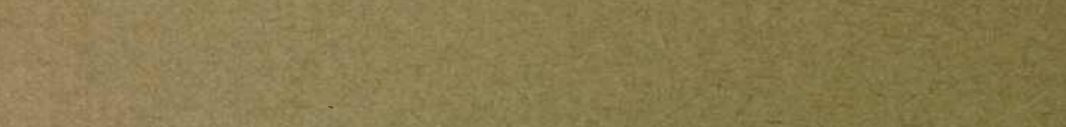

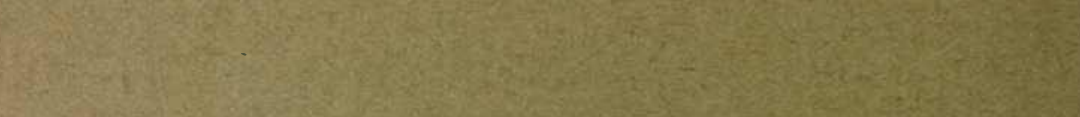

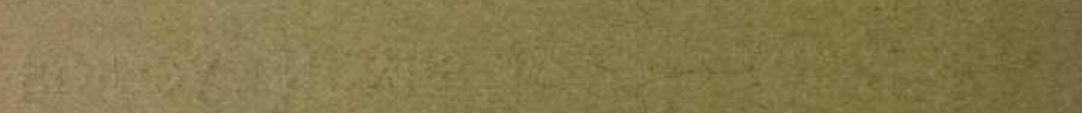

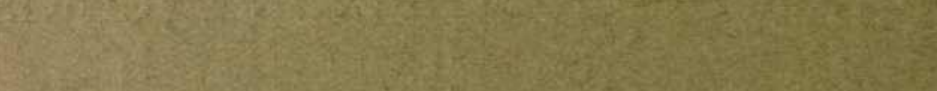

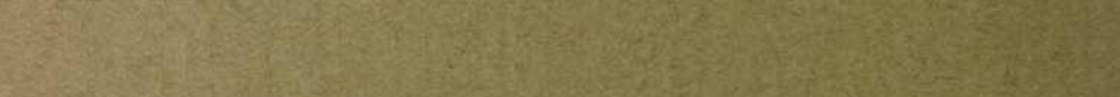
(2)

15.

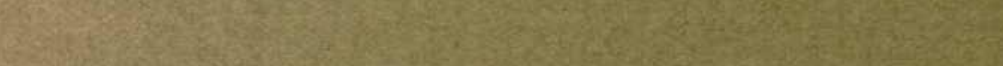

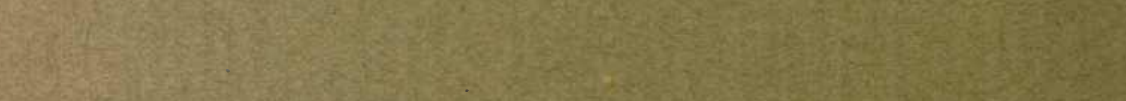

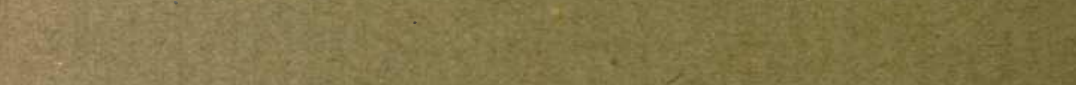

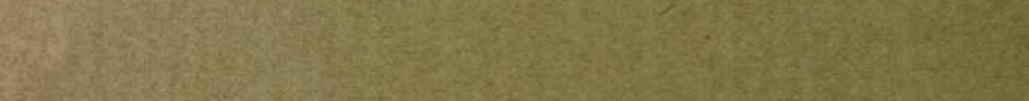

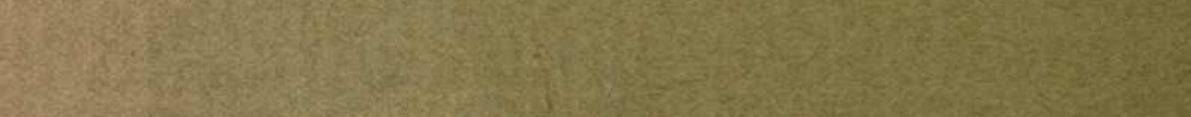

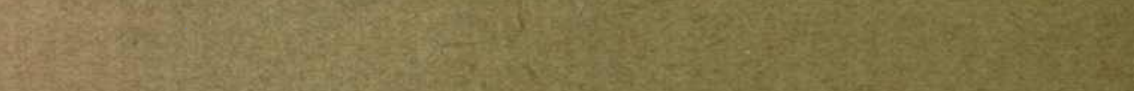

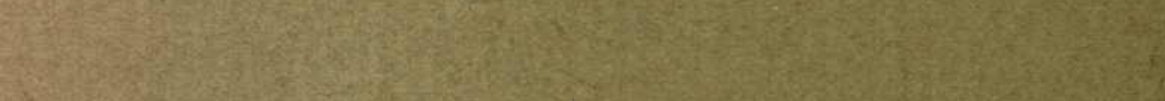

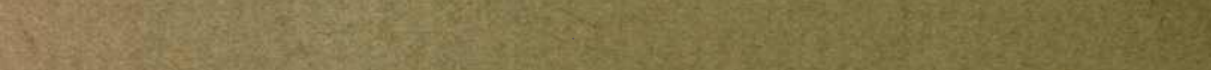
60

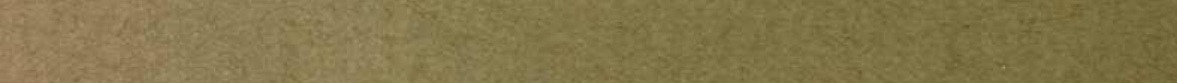

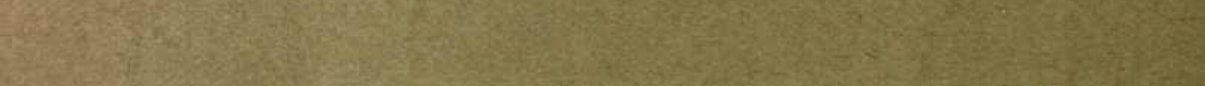
(2)

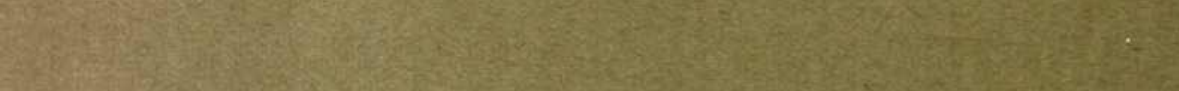

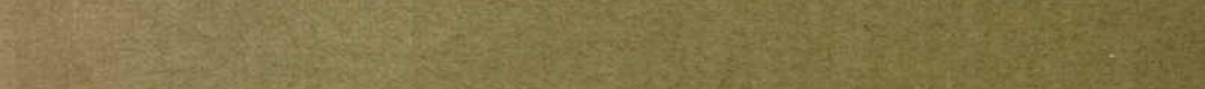

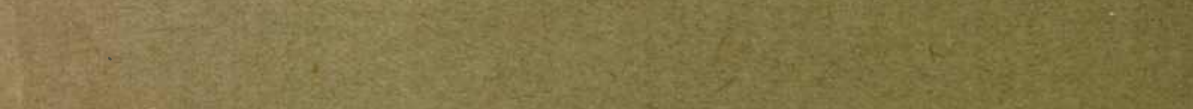

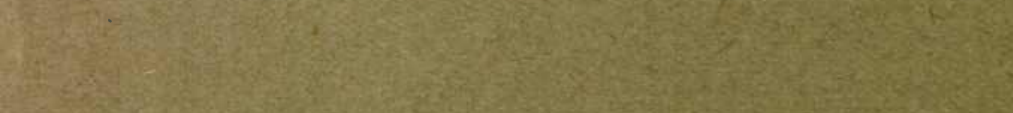

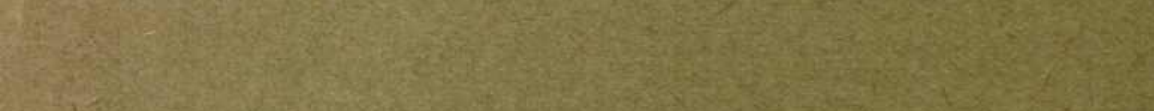

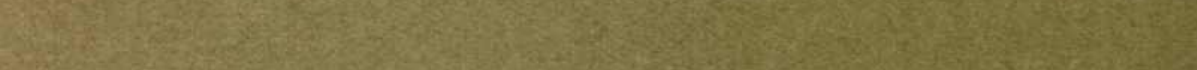
(5) (1) (1)

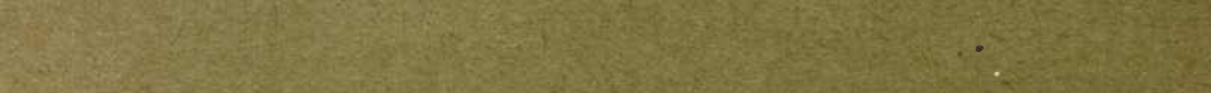

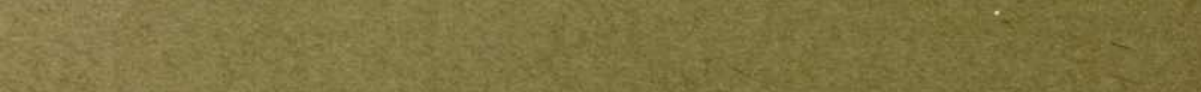

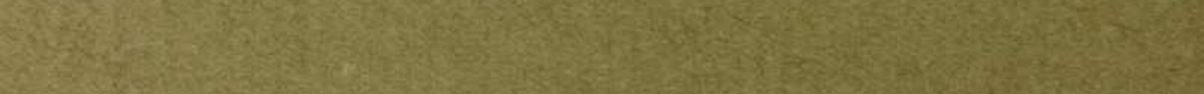
the 


\section{INHERITANCE OF SEX IN LYCHNIS ${ }^{x}$}

GEORGE HARRISON SHULL

(WITH TWO FIGURES)

Since CoRrens (4) made his brilliant investigations with Bryonia, in which he showed that crosses between the monoecious $B$. alba and the two sexes of the dioecious B. dioica do not give equivalent results in regard to the sex of the offspring, and since DonCASTER and RAYNOR (6) published their equally interesting studies with the currant moth, Abraxas grossulariata, and its variety lacticolor, in which it was found that reciprocal crosses were not equal with respect to sex, but that the Mendelian color character of the variety lacticolor is sex-limited, the interest of all students of genetics has been more or less strongly directed toward the problems of sex inheritance, and toward the attempt to describe or explain the heredity of sex on the basis of Mendelian inheritance.

It has been of great interest to find that these two classic cases of Bryonia and Abraxas apparently lead to opposite conclusions as to which sex determines the sex of the offspring, but both seem to favor the conception that one sex is homozygous with respect to sex, and the other heterozygous. BATESON (I) attempts to make the results with Bryonia agree with those in Abraxas, but his interpretation is certainly not as simple as that of CORRENS. BATESON's explanation would require that Bryonia alba be gynodioecious, having larger numbers of pure females than of monoecists, instead of being wholly monoecious as described in the manuals. It does not seem likely that so striking a relation as this would have been overlooked by the taxonomists. Moreover, in the attempt to bring harmony between Bryonia and Abraxas, BATESon introduces fully as fundamental inharmony between the two species of Bryonia, when he assumes that the males of $B$. dioica are pure males with pollen bearing only the male character, while the pollen of $B$. alba is all female. Certainly we are justified in expecting a more complete

I Read by invitation before the American Society of Naturalists, Boston, December 28, I909. 
harmony among the species of a single genus than between a species of plants and a species of animals. Castle (3) has also attempted to relate the Bryonia and Abraxas types of behavior by the assumption that in each case the female is the equivalent of the male plus an $x$ element, the female in Bryonia being a positive homozygote and the male a heterozygote, while in Abraxas the female is a heterozygote and the male a negative homozygote. This explanation recognizes that Bryonia and Abraxas present fundamentally different conditions.

It is not my intention to present a general discussion of the present state of our knowledge regarding the inheritance of sex, as this has been well done recently by Wilson (I2), Castle (3), Bateson (I), Doncaster (5), and Morgan (8). However, it may be said that the number of studies which have been made in this field are entirely too few as yet to warrant far-reaching generalizations on the question of sex inheritance, and further data bearing on the subject will be awaited with great interest. It has appeared to me that a serious criticism can be offered to the results of crosses between Bryonia alba and $B$. dioica as a basis for conclusions regarding the whole problem of sex inheritance. These crosses are interspecific, and, as is very often true of crosses between distinct species, the sterility of the $\mathrm{F}_{\mathrm{I}}$ offspring did not allow any test of the correctness of assumptions regarding the gametic composition of the hybrids. While it is true that in many species-crosses in which the hybrids are fertile, certain characteristics segregate normally, it has not been uncommon to find that many characters do not segregate, or at least that their segregation is very doubtful and obscure. The simple, typical segregation of characters is best exemplified by the most closely related forms, between which also the fertility is most perfect, and this may be expected to hold true of sex characters as well as of other alternative characters.

BAteson (I, p. I66) has repeated the experiments with Bryonia and has fully confirmed the results of CoRrens. BATESon is also convinced of the unsafe character of the Bryonia results as a basis for generalizations, and says that "the relation of dioecious to hermaphrodite and monoecious forms will not in all probability be satisfactorily or rapidly elucidated until some case can be found in which the two types can be crossed together with a fertile result." 
I have some material now in hand which meets this requirement, but have not yet continued the experiments long enough to allow more than a preliminary report upon its behavior. For several years I have been investigating the sex ratios in Lychnis dioica L., and for this purpose have made carefully controlled pollinations yearly in this usually dioecious species. It was my good fortune during the summer of 1908 to find among these pure-bred cultures six hermaphrodite individuals, the first which I had seen in the several years during which I had been working with this species, in which time I had examined some 8000 pedigreed individuals. In the past season I have noted eight hermaphrodites, usually more or less imperfect, in pure-bred normal families including a total of Io,320 individuals. Only two of these eight were well developed and appeared to be fully functional both as males and females.

Although the occasional occurrence of hermaphrodite individuals in this species has been frequently noted, ${ }^{2}$ I have never seen any of them growing wild in the vicinity of Cold Spring Harbor, where the original material for my cultures was collected. STRASBURGER (II) found that hermaphrodite plants in his cultures at Bonn were invariably affected by a smut, Ustilago violacea, which fruits in the anthers, and he ventured the suggestion that all the reported hermaphrodites in this species may have been such diseased individuals; but, fortunately for my experiments, Ustilago violacea has never appeared among my Lychnis cultures, and some, at least, of the hermaphrodite individuals were capable of functioning both as males and females. Five of the hermaphrodites found in I908 were members of a single family (0739). Several of these hermaphrodite mutants did not have the ovaries and pistils fully developed, and consequently my ability to secure offspring from them was somewhat limited. However, during the past summer, I had I3 families in which one of these hermaphrodite plants entered as either the male or female parent. Two of the six original hermaphrodites died before I had opportunity to use them in crossing. Of the remaining four, two were successfully self fertilized, and one of these was also successfully used as a mother in crosses with a normal male.

2 See Penzig, O., Pflanzenteratologie I:300; and KnUth, P., Handbuch der Blütenbiologie $2^{\mathrm{x}}: \mathrm{I} 74, \mathrm{I} 75$. 
For convenience, I will designate the four plants successfully used in breeding by the letters $A, B, C$, and $D$. When $A$ was self fertilized it produced, as a result of two different operations, 33 females and 25 hermaphrodites. When its pollen was used to fertilize four different females, the resultant progenies consisted of 236 females, I6I hermaphrodites, and 2 males. When castrated and fertilized with pollen from a normal male, $A$ gave rise to a progeny of 2 I females, 2 hermaphrodites, and II males. $B$ was also self fertilized and gave a progeny of 1 Io females and 95 hermaphrodites. When pollen of $B$ was used to fertilize three different females, it produced $\mathrm{r} 62$ females and 144 hermaphrodites. It is thus seen that the two plants, $A$ and $B$, showed identical behavior and together produced self fertilized offspring consisting of $\mathrm{I} 43$ females and I20 hermaphrodites, and when crossed with females gave a total of 398 females, 305 hermaphrodites, and 2 males. In any explanation of these results the occurrence of these two males will probably have to be left aside as wholly exceptional. Only further breeding will show whether they were true males, or hermaphrodites with pistils suppressed, perhaps, by some cause external to the germ cells. Plants $C$ and $D$ gave quite a different result. Attempts to self fertilize them and to cross them with normal males all proved futile, though more persistent efforts perhaps might have succeeded. Both were used as pollen parents in crosses with normal females. In such a cross $C$ gave a progeny of 39 females and 55 normal males, and $D$ gave 26 females and 18 normal males. The details of the several crosses are given in table I, p. II4.

Considering first plants $A$ and $B$, and leaving out of consideration for the present the two males occurring in crosses between females and hermaphrodites, and the two hermaphrodites which appeared in the cross between a hermaphrodite and a normal male, it is apparent that the hermaphrodite character belongs only to the males, for in the families in which these hermaphrodites were the pollen parents, the offspring always showed the same ratios of females and hermaphrodites that would have been expected of females and males, had a normal male been used as the pollen parent.

It is clear that the hermaphrodite individuals, $C$ and $D$, belong to an entirely different category from $A$ and $B$, for in the families 
produced by using them as pollen parents, the offspring are exactly the same as if normal males had been used. In $A$ and $B$ the hermaphrodite character is borne by the male germ cells and is fully hereditary. In $C$ and $D$ the hermaphrodite character may have been purely somatic, in no wise affecting the germ cells, and therefore incapable of hereditary transmission; or, in case the factor for hermaphroditism is independent of the sex-producing genes, $A$

TABLE I

\begin{tabular}{|c|c|c|}
\hline Pedigree no. & Cross & Result \\
\hline 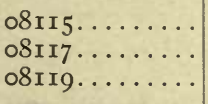 & $\begin{array}{l}\Varangle \times \text { self }(A) \\
\not \times \text { self }(A) \\
\Varangle \times \text { self }(B)\end{array}$ & $\begin{array}{c}24 \%: \text { r } 9 \text { ఫ } \\
99: 6 \nsucceq \\
110 \%: 95 \%\end{array}$ \\
\hline Total ..... & $\Varangle \times$ self & 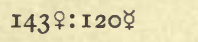 \\
\hline 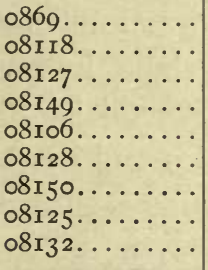 & 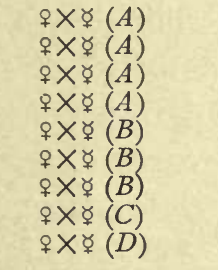 & 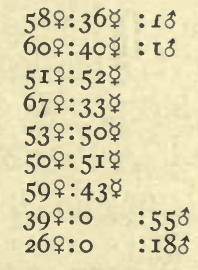 \\
\hline Total ..... & $\wp \times \ngtr\left\{\begin{array}{l}A \text { and } B \\
C \text { and } D\end{array}\right.$ & $\begin{array}{l}398 \%: 305 \%: 2 \hat{\delta} \\
65 \%: 0: 73^{\hat{\delta}}\end{array}$ \\
\hline о8гі6. & $ఫ(A) \times \hat{\jmath}$ & 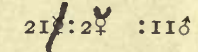 \\
\hline
\end{tabular}

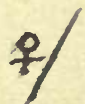

and $B$ may have been homozygous and $C$ and $D$ heterozygous with respect to the hermaphrodite modifier.

That males, which are assumed to be heterozygous and to contain both the male and female tendencies, should occasionally show the development of characters of both the sexes as the result of some accident or environmental influence upon an individual, without in any way changing the character of the germ cells produced by that individual, is quite conceivable. The occurrence of these two different types of hermaphrodite individuals harmonizes well with many experiences met with in other studies in heredity, which have led JoHANnsen (7) to distinguish between genotypes and phenotypes. These four hermaphrodite individuals, $A, B, C$, and $D$, belong to a single phenotype, but to two genotypes. 
If now we compare these results in Lychnis with those of CoRRENS in Bryonia, still leaving out of account the two exceptional males and the two hermaphrodites which occurred in pedigree no. $08 \mathrm{II} 6$, we find that the difference is a consistent one. This comparison may be conveniently made in the following table:

TABLE II

\begin{tabular}{|c|c|c|}
\hline Cross & Bryonia & Lychnis \\
\hline$\$ \times \hat{0}$ & $q$ and $\hat{\jmath}$ & $f$ and $\delta$ \\
\hline$\not \subset \times \hat{~}$ & $q$ and $\delta$ & $q$ and $\delta$ \\
\hline$q \times \not$ & & \& and $\Varangle$ \\
\hline 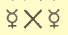 & $\Varangle$ & $q$ and $\varnothing$ \\
\hline
\end{tabular}

In the first place, it is seen that all crosses between normal females and normal males in Lychnis result in females and normal males, just as in Bryonia dioica, and the same thing is true in both cases when the hermaphrodite is fertilized by a normal male. The result of self fertilizing the hermaphrodites of Lychnis, however, is (presumably) quite different from the result of self fertilizing Bryonia alba, for Lychnis gives rise to a progeny of females and hermaphrodites, while Bryonia (presumably) gives rise to only monoecists or hermaphrodite individuals. A similar difference is observable between these two classes of material when normal females are fertilized by pollen of hermaphrodites, as Bryonia produces in this case only females, while Lychnis produces females and hermaphrodites.

These results suggest that the differences between Bryonia and Lychnis are rather simple ones. The fact that the hermaphrodites take the place of males in each family whose male parent was a hermaphrodite, permits no other reasonable conclusion than that the hermaphrodite Lychnis is a modified male; and the capacity of the male to be thus modified so as to allow the development of both male and female organs, strongly favors the view that the male is heterozygous, as has been assumed by Correns (4) and CAstle (3) for Bryonia, and as demonstrated by WILson and his students for many insects.

There are now several possibilities regarding the nature of the females. CAstle (2) long ago suggested that the female, as well as the male, is heterozygous, but in his latest communication on sex (CASTLE 3) he definitely abandons this view and holds that in all 
cases only one sex is heterozygous and the other is homozygous; and this is the conception now generally entertained by those who adopt a Mendelian explanation of sex. According to this view, if the male is heterozygous, as has just been decided to be the case in Lychnis, the female must be homozygous. As there are two kinds of homozygotes, namely, "positive" and "negative" (SHull 9), there remain two possibilities regarding the nature of the female; it may be either a positive homozygote or a negative homozygote. While either of these assumptions will explain about equally well the facts brought to light in Bryonia, neither will fit all those observed in Lychnis without encountering important difficulties.

Making first the supposition that the female is a positive homozygote, as suggested by CASTLE (3), all conditions found in Bryonia will be satisfied if it be also assumed that $B$. alba is a homozygous monoecist (a modified female condition in this case) in which the absence of the monoecious character is dominant over its presence. The sterility of the $\mathrm{F}_{\mathrm{I}}$ hybrids in Bryonia unfortunately makes it impossible to test the correctness of these assumptions. It is less easy to make a positive homozygous condition of the female fit the results found in Lychnis as described in this paper. This can be done, however, by assuming: (a) that all egg cells of both females and hermaphrodites carry the gene for the female sex, and not that for hermaphroditism; and $(b)$ that all the sperms of the hermaphrodites carry a gene for the hermaphrodite modification, regardless whether they possess the gene for the female sex (the " $x$ element") or not. The first of these assumptions seems necessary from the fact that the results are identical in each case, whether a female or a hermaphrodite is used as the female parent; and the second from the fact that females and hermaphrodites result from pollinating a normal female by pollen from a hermaphrodite. The second proposition might be replaced by one involving spurious allelomorphism. It is expected that a second generation will demonstrate the correctness or incorrectness of these several hypotheses.

The alternative assumption, namely, that the female is a negative homozygote, will just as simply represent the conditions of the $F_{I}$ generation. If the monoecious character in Bryonia be considered a modified male condition similar to the hermaphrodite character of 
Lychnis, the difference between Bryonia and Lychnis is reduced to a question of dominance of the hermaphrodite (or modified male) condition. In order that Bryonia alba should breed true, the monoecious (or modified male) character must be homozygous. The fact that in Bryonia a cross between the female B. dioica and the monoecious $B$. alba produces only females, indicates that the monoecious character is capable of coming to full expression only when present in the homozygous state. In other words, while the monoecists are modified positive male homozygotes, absence of this modified male character is almost perfectly dominant over its presence, so that the heterozygous individuals which constitute the $\mathrm{F}_{\mathrm{I}}$ of this cross almost or quite completely lack the male character. In this connection the occurrence of occasional male flowers on a few of these females of the $F_{x}$ may be of interest as showing a slight influence of the unpaired gene for the modified male or monoecious sex.

In Lychnis, on the other hand, the presence of the hermaphrodite character is dominant over its absence, as demonstrated by the fact that the hermaphrodite mutants are heterozygous with respect to sex. In the following table a comparison may be made between the two methods of explaining the character of the $F_{x}$ hybrid generation:

TABLE III

Assumption: The female is a positive homozygote

\begin{tabular}{|c|c|c|c|c|c|c|}
\hline \multirow[b]{2}{*}{ Cross } & \multicolumn{3}{|c|}{ BRYONIA } & \multicolumn{3}{|c|}{ LychNIs } \\
\hline & $\begin{array}{c}\text { Assumed } \\
\text { character of } \\
\text { parental gametes }\end{array}$ & $\begin{array}{c}\text { Resultant } \\
\text { composition of } \\
\text { offspring }\end{array}$ & $\begin{array}{c}\text { Somatic } \\
\text { type of } \\
\text { offspring }\end{array}$ & $\begin{array}{l}\text { Assumed } \\
\text { character of } \\
\text { parental } \\
\text { gametes }\end{array}$ & $\begin{array}{l}\text { Resultant } \\
\text { composition of } \\
\text { offspring }\end{array}$ & $\begin{array}{l}\text { Somatic } \\
\text { type of } \\
\text { offspring }\end{array}$ \\
\hline 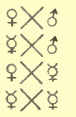 & 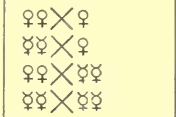 & 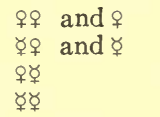 & $\begin{array}{l}q \text { and } \hat{o} \\
\uparrow \text { and } \hat{o} \\
q \\
\Varangle\end{array}$ & 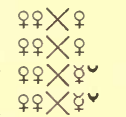 & 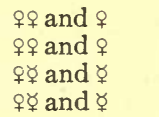 & 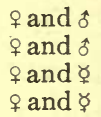 \\
\hline
\end{tabular}

Assumption: The female is a negative homozygote

\begin{tabular}{|c|c|c|c|c|c|c|}
\hline 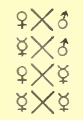 & 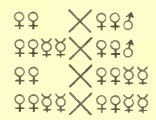 & 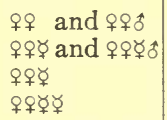 & $\begin{array}{l}q \text { and } \delta \\
q \text { and } \delta \\
q \\
q\end{array}$ & 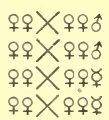 & 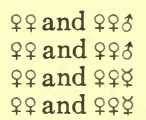 & $\begin{array}{l}q \text { and } \delta \\
\wp \text { and } \delta \\
\uparrow \text { and } \varnothing \\
\wp \text { and } \varnothing\end{array}$ \\
\hline
\end{tabular}

No decision can be made between the two methods of explanation contrasted in this table until the $\mathrm{F}_{2}$ has shown the dependence or 
independence of the gene whose presence results in the hermaphrodite modification of the male. If the females may carry the hermaphrodite modification as a latent character, and if the hermaphrodites of the $F_{x}$ differ in their gametic composition from those of the $\mathrm{P}_{\mathrm{r}}$ generation, the hypothesis that the female is a positive homozygote may be substantiated; but if the hermaphrodites of the $F_{r}$ are identical in their behavior with those of the $P_{I}$, and if the hermaphrodite modification cannot be transmitted through the female, the results will support the hypothesis that the female is a negative homozygote.

It is not at all improbable that the hermaphrodites discussed above as $C$ and $D$ differed from $A$ and $B$ in having the gene for the hermaphrodite modification in the heterozygous state, while $A$ and $B$ were homozygous with respect to this gene. If this should be correct, then $C$ and $D$ were of the type which will appear in the $F_{x}$ of crosses between normal females and such hermaphrodites as $A$ and $B$, in case the hermaphrodite modification is independent of the male germ cells. The occurrence of such hermaphrodites as $C$ and $D$ may be accepted as rather favorable to the view that the female is a positive homozygote.

There is one fundamental difficulty with any hypothesis which assumes that the hermaphrodites are heterozygous. The eggs of these hermaphrodites are of a single kind with respect to sex, all carrying the female character. This difficulty is inherent in the material itself, however, and is not opposed in any way to the assumption that the normal females are either positive or negative homozygotes, for the male germ cells of the hermaphrodites are clearly of two types as required by the hypothesis that these hermaphrodite plants are heterozygous. Cytological investigations of oogenesis in the hermaphrodites may perhaps give a complete solution to the exceptional situation presented by the egg cells. If the females are positive homozygotes there may be simply a failure of those eggs to develop which do not possess the gene for the female sex. If the female is a negative homozygote, there may be a failure of those eggs which possess the male gene, or there may be an exclusion of this gene during oogenesis.

While I have left out of account the two hermaphrodite individuals in the cross (08II6) between a hermaphrodite and a normal male, 
I am not unmindful of their possible importance. The number of offspring of this combination was too small to enable one to be sure that these are not mutants which bear no necessary relation to the fact that their mother was a hermaphrodite. It was found particularly difficult to secure offspring of crosses of this type, as the flowers were very often caused to drop off as a result of the process of castration. Very often also in the flowers of hermaphrodite plants the pistils are immature at the time the anthers are ready to open, and after castration these pistils frequently develop no farther, in which case there is no possibility of effecting fertilization. The occurrence of these two hermaphrodite plants in no. 08II6 offers a further suggestion that the hermaphrodite character may be capable of independent movement, and that consequently it may be carried in some manner or to some degree by the female. This suggestion especially commends itself from the fact that the occurrence of the male and female organs on the same plant, as Correns (4) has pointed out, constitutes in effect a mosaic, and it is well known that mosaic inheritance is often dependent upon the presence of a definite separate unit for the mosaic condition. If further investigation should demonstrate that the hermaphrodite character may be transmitted through the female, as is suggested by this one family, we will be forced to the conclusion that here also the existence of a unit for the mosaic condition is present. In whatever manner the male may be converted into a hermaphrodite, the results seem to demonstrate that in Lychnis, as in Bryonia, it is the male which is heterozygous and which carries both male- and female-producing genes as concluded by CoRRENS, and not the female as assumed by BATESON.

The demonstration that the hermaphrodite of Lychnis dioica is a modified male indicates that STRASBURger (II) was mistaken in his interpretation of the effects produced by Ustilago violacea upon this species. He believed that the infected plants were females in which the development of stamens was stimulated by the attack of the fungus. Instead of this it is probable that they were males in which the disease somewhat lessened or modified the dominance of the male character, thus allowing the female organs to develop; or, if the female is a positive homozygote, the disease may be assumed to have stimulated the single female gene or $x$ element of the male 
to develop the female organs as well as the male organs. STRASBURGER states that the development of the stamens in the diseased plants was correlated with an elongation of the floral axis between the calyx and corolla (a character peculiar to flowers of the male plants), and that not infrequently the infected plants gave the impression that they were males. On the other hand, the fibrovas-

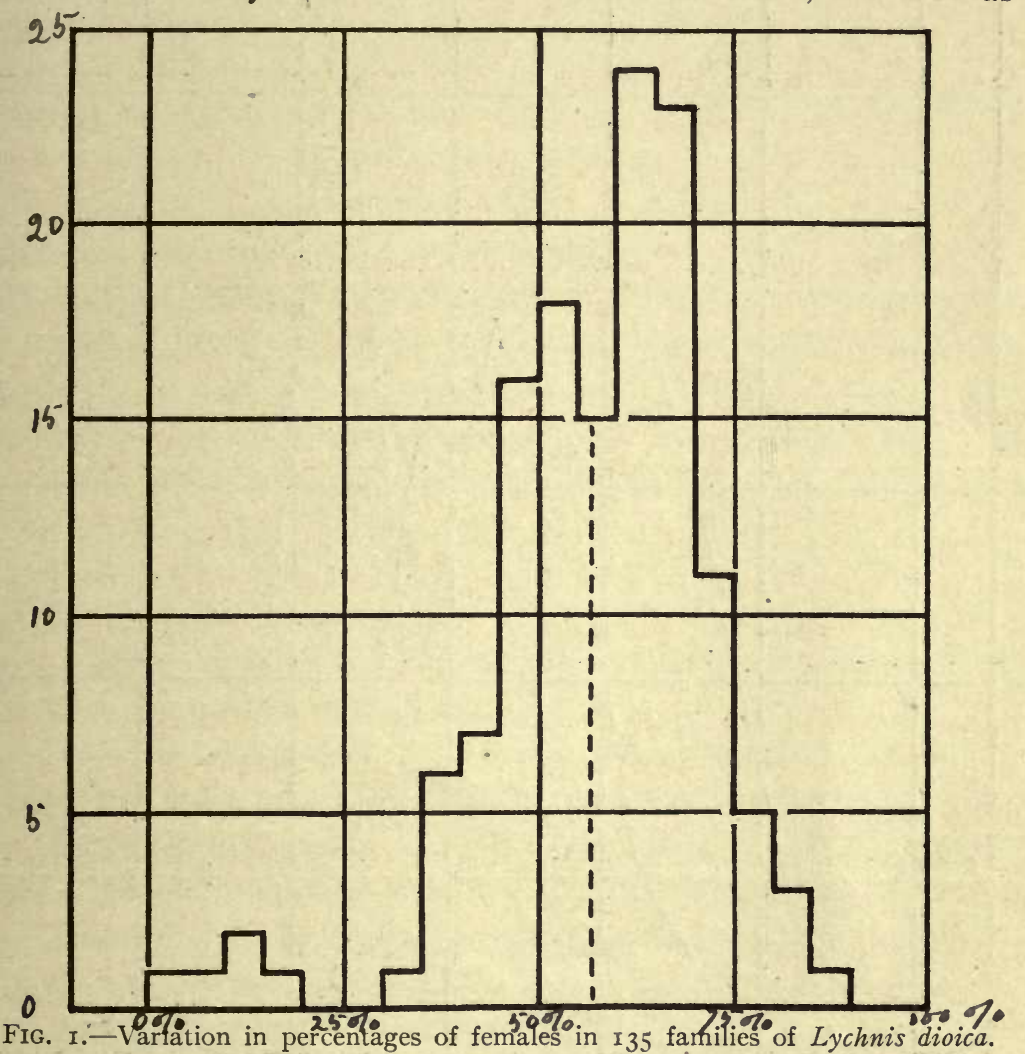

cular system of the calyx in infected plants more nearly assumed the distinctive features of the normal female flowers. The appearance of this secondary female character may be as readily accounted for as the appearance of the female sex organs themselves, on the ground of modified dominance of the male or of the female character in a heterozygous male.

CORRENS has pointed out that the determination of sex ratios is quite a different matter from the determination of sex, and we must 
maintain that this is so, provided sex is inherited as a Mendelian character. But if sex inheritance be not primarily Mendelian, but only associated with and perhaps determined by Mendelian charac-

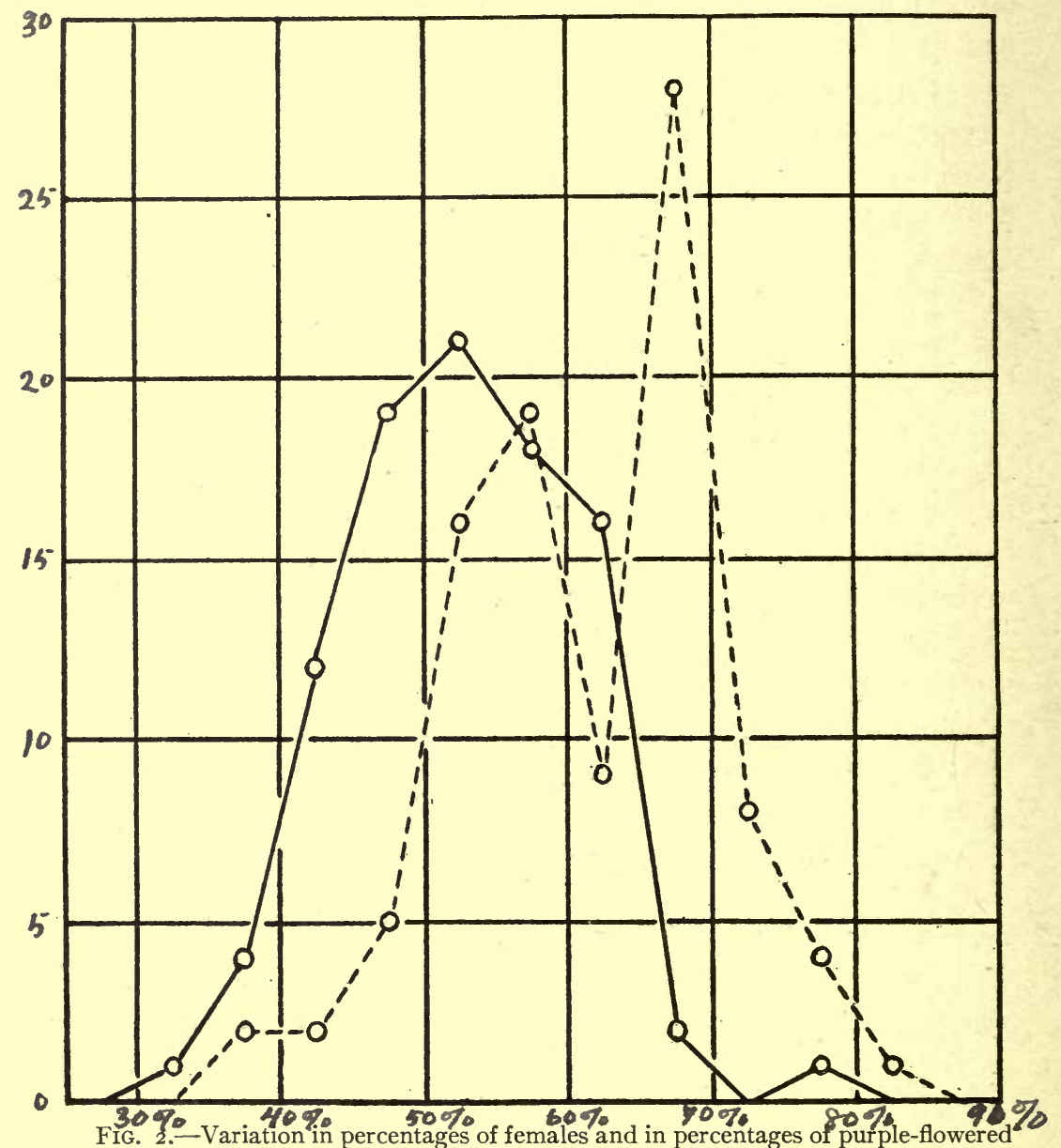
plants in 94 families of Lychnis dioica produced by crossing heterozygous purple with white; broken curve represents females; both curves if monomodal belong to Pearson's Type I. See constants in table IV.

ters in a few cases, the determination of a sex ratio might not be a process distinct from the inheritance of sex itself.

The assumption that the sexes differ from each other by a single unit character, and that one sex is heterozygous and the other homo- 
zygous with respect to this character, would demand equality in the average numbers of the two sexes; but each year Lychnis dioica has produced on the average a considerable excess of females, while the ratios in different families range all the way from less than 5 per cent. female to nearly 90 per cent. female. The percentages of females in $I 35$ families reared during the summer of 1909 are shown in the form of a variation curve in fig. I. It is difficult to believe that a family of 4 females and roo males or of 87 females and ro males is theoretically referable to a ratio of $\mathrm{I}: \mathrm{I}$. In 1909 my cultures of Lychnis dioica, taken collectively, consisted of 6366 females and 483I males (including hermaphrodites), or a ratio of I.32:I, which agrees very well with ratios between I.20:I and I.40: I reported by StRASBURGER (II). After carrying on extensive experiments on the influence of various environmental factors, with negative results, STRASBURGER reached the conclusion that the sex ratio is determined by inherent factors. This is only another way of saying that it is hereditary in some sense. I have been attempting for several years to test the heredity of the sex ratios, but have not yet found the key to the situation. It is hoped that the numerous crosses which have been made will in the near future throw some light upon the significance of these exceedingly variable ratios and allow the causes which determine them to be understood.

In order to compare the variation in these sex ratios with that in a character known to be Mendelian, I have plotted curves representing the percentage of females and the percentage of purple-flowered individuals in all families (94 in number) raised during the years 1907, 1908, and 1909, which were produced by the union of heterozygous purple with white (see fig. 2). The variation constants of the two curves appear in the following table:

TABLE IV

VARIATION CONSTANTS OF CURVES CONTRASTED IN fig. 2

\begin{tabular}{|c|c|c|}
\hline & Percentage females & Percentage purples \\
\hline 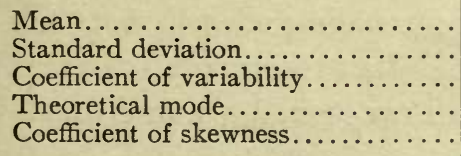 & $\begin{array}{r}61.223 \pm 0.630 \\
9.051 \pm 0.445 \\
14.784 \pm 0.742 \\
62.933 \pm 0.686 \\
-0.0990 \pm 0.0062\end{array}$ & $\begin{array}{r}52.606 \pm 0.551 \\
7.925 \pm 0.490 \\
15.064 \pm 0.758 \\
52.353 \pm 0.600 \\
+0.0282 \pm 0.0062\end{array}$ \\
\hline
\end{tabular}


Aside from the remarkable difference in the value of the means, the two curves are skewed in opposite directions, the strong negative skewness and suggested bimodality of the sex curve, as contrasted with the nearly normal character of the curve for color, being particularly noteworthy. The variability represented by these two curves is properly compared by means of the standard deviations, and not by the coefficient of variability, since we are dealing here with groups of variates having similar theoretical limits of range, and similar expected mean values. On this basis the sex ratios show considerably greater variability than those of the color character. This is especially interesting in view of the fact, pointed out elsewhere (SHull ro), that crosses between heterozygous purple and white Lychnis dioica may frequently result in other ratios than $\mathrm{I}: \mathrm{I}$, owing to the part taken by several distinct genes in the production of the purple colors in this species, and that therefore the data for the color curve may be somewhat heterogeneous, while the sex data are at present assumed to be homogeneous.

\section{Summary}

Hermaphrodite mutants occur in pure-bred families of Lychnis dioica L., the ratio of mutability being somewhat less than $\mathrm{I}: \mathrm{I}$ ooo.

None of these hermaphrodites was diseased and several were fully functional both as females and males, thus negativing the suggestion of StRASBURger that the hermaphrodites in this species may always be due to the attacks of the smut, Ustilago violacea.

In their hereditary relations these hermaphrodites were of two kinds, some behaving like normal males, others having the capacity of transmitting the hermaphrodite character to their male offspring. This second class of hermaphrodites when self fertilized, or crossed with normal females, gives progenies consisting of normal females and hermaphrodites, and when fertilized by normal males gives normal females and normal males.

The hermaphrodite individuals are modified males, for in all families in which these hermaphrodites were the pollen parents the offspring showed the same ratios of females and hermaphrodites as would have been expected of females and males had a normal male been used as the pollen parent. 
These results agree in a general way with those of Bryonia in that they demonstrate the homozygous character of the females and the heterozygous character of the males in Lychnis.

Whether the females are positive or negative homozygotes cannot be determined from the $F_{1}$, as both assumptions can be made to fit the facts by the aid of simple correlative hypotheses whose correctness or incorrectness can be determined only by further breeding.

A fundamental difficulty in either case is found in the fact that the egg cells of the hermaphrodites are apparently of a single type, all possessing the female-producing gene. The assumption that the hermaphrodites are heterozygous leads us to expect equal numbers of two different types of egg cells. Cytological studies may perhaps explain this difficulty. The male germ cells of the same plants are of two types, as required by theory.

The occurrence of two hermaphrodite individuals in a progeny produced by a cross between hermaphrodite and normal male suggests the possibility that the hermaphrodite character may also be transmitted through the female. This fact, together with the occurrence of two genotypes among the hermaphrodites, is held to be slightly favorable to the view that the female is a positive homozygote.

The demonstration that the hermaphrodite individuals of Lychnis are modified males indicates that STRASBURGER was mistaken in assuming that his hermaphrodites were diseased females. They were probably diseased males in which the dominance of the male character was modified by the fungus.

The sex ratios in Lychnis do not accord well with a theory of sex which requires males and females to be present in equal numbers. The ratios found in my cultures are in accord with those found by STRASBURGER, the average for the past year being $\mathrm{I} .32$ females to I male, with a very wide difference in the ratios of different families. The significance of these ratios is not yet understood.

When the variability in the sex ratios is compared with that in ratios produced by crossing heterozygous purple with white-flowered individuals, it is found that not only is there an undue departure in the sex ratios from the expected ratio $\mathrm{I}: \mathrm{I}$, but also that there is greater variability in the sex ratios than in the color ratios, and that the curve is strongly negatively skewed and possibly not monomodal; while the 
color curve derived from the same families is nearly normal; with the mean departing but little from the 50 per cent. required by Mendelian theory.

Station for Experimental EVOlution

COLD SPRing Harbor, LONg IsLAND

\section{LITERATURE CITED}

I. Bateson, W., Mendel's principles of heredity. pp. xiv +396 . Cambridge: University Press. 1909. See pp. I64-195.

2. Castle, W. E., The heredity of sex. Bull. Mus. Comp. Zool. Harvard 40:189. 1903 .

3. - A Mendelian view of sex-heredity. Science N. S. 29:395-400. I909.

4. Correns, C., Bestimmung und Vererbung des Geschlechtes. pp. v+8r. figs. 9. Leipzig: Gebr. Borntraeger. 1907.

5. Doncaster, L., Recent work on the determination of sex. Sci. Progress, no. I3, pp. 90-104. I909.

6. Doncaster, L., ANd Raynor, G. H., Breeding experiments with Lepidoptera. Proc. Zool. Soc. London I : I25. I906.

7. Johannsen, W., Elemente der exacten Erblichkeitslehre. 8vo. pp. vi +516. figs. 3I. Jena: Gustav Fischer. 1909.

8. Morgan, T. H., A biological and cytological study of sex determination in phylloxerans and aphids. Jour. Exper. Zool. 7:239-352. pl. I. figs. 23. Igo9.

9. Shull, G. H., The "presence and absence" hypothesis. Amer. Nat. 43: 4IO-4I9. 1909 .

ro. - Color inheritance in Lychnis dioica. Amer. Nat. 44:83-9r. I910.

i i. Strasburger, E., Versuche mit diöcischen Pflanzen in Rücksicht auf Geschlechtsverteilung. Biol. Centralbl. 20:657-665, 689-698, 72 I-731, 753785. I900.

I2. Wilson, E. B., Recent researches on the determination and heredity of sex. Science N. S. 29:53-70. 1909. 


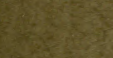

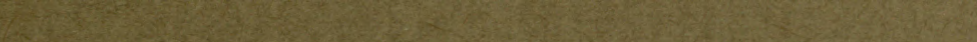

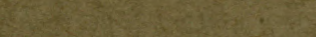

3.

(2)

(3)

4. W.

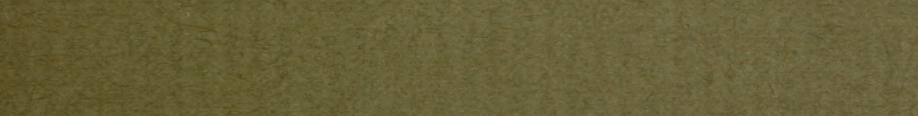

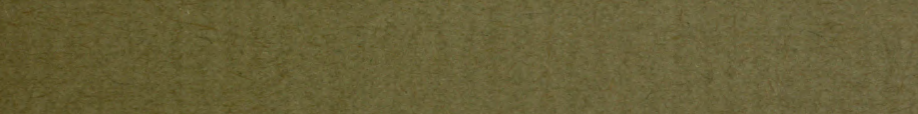
Fis.

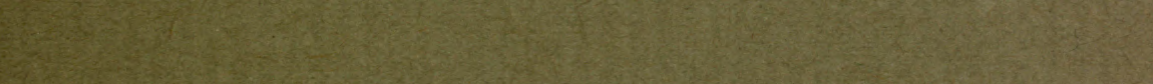

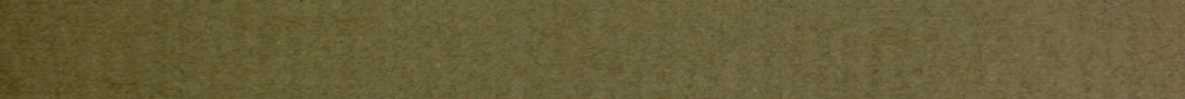
(1) (1)

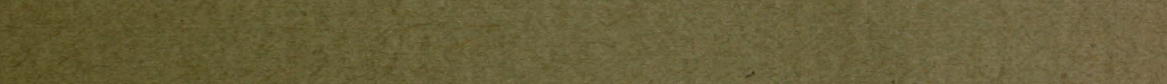

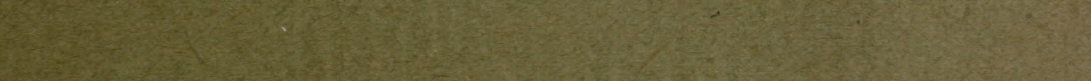

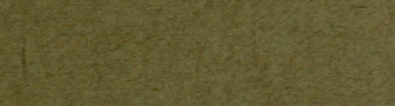
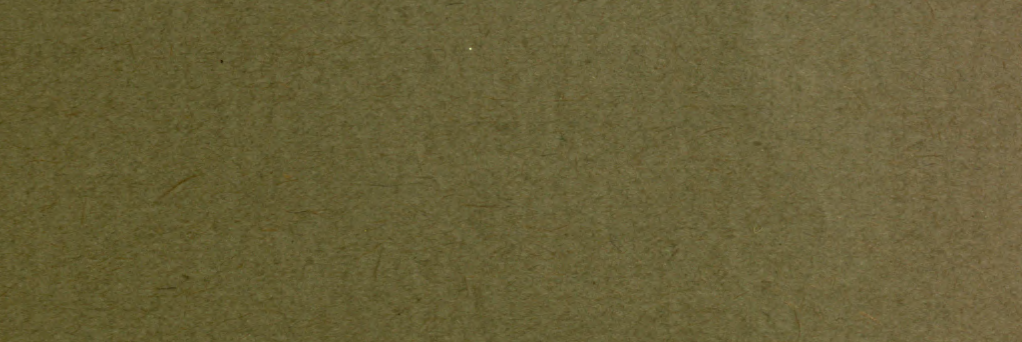

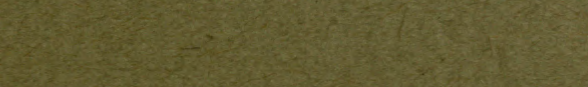

(a)

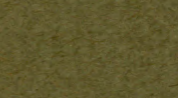

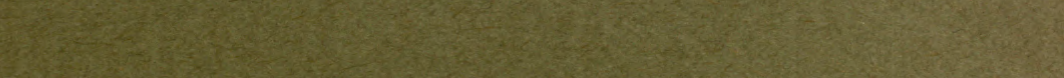

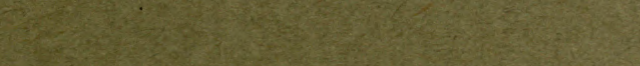

S.

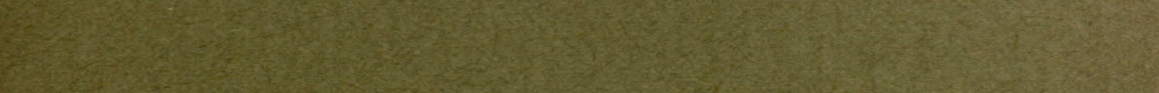

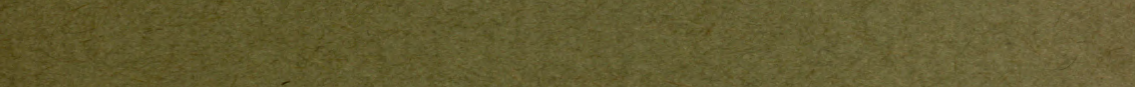

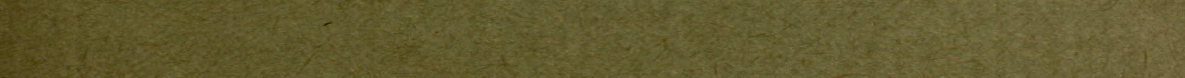
(2) 


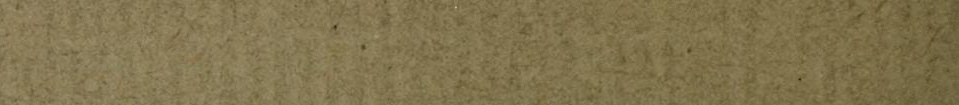

(3)

4.

*

4.)

ond

Whinges

(1) $3 x^{2}=$

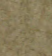

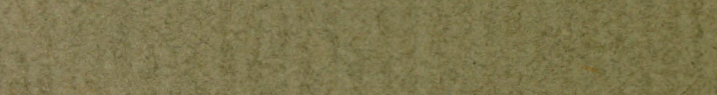

se

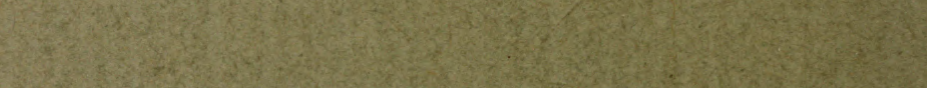

18

- $35 x$

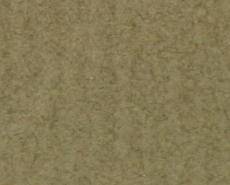

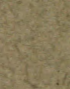

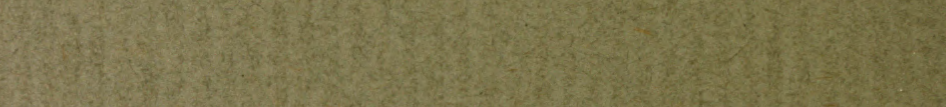

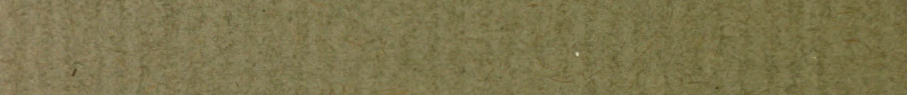
$2 \sin ^{2}$

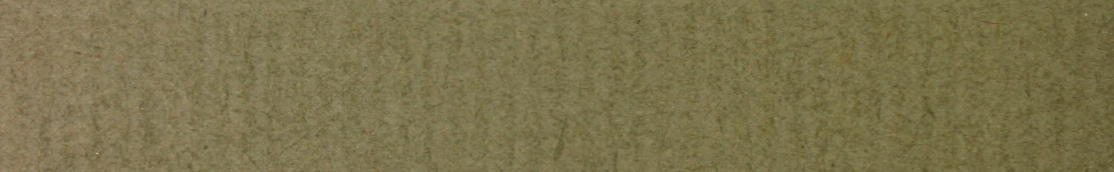

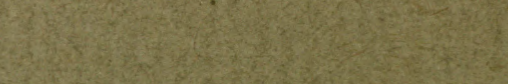

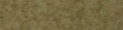
(2)

(

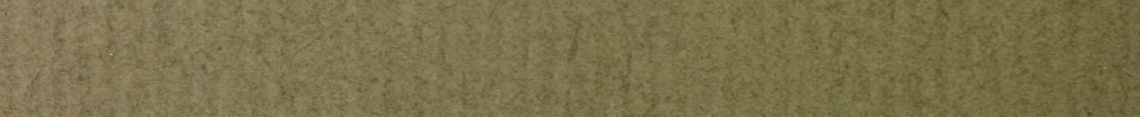

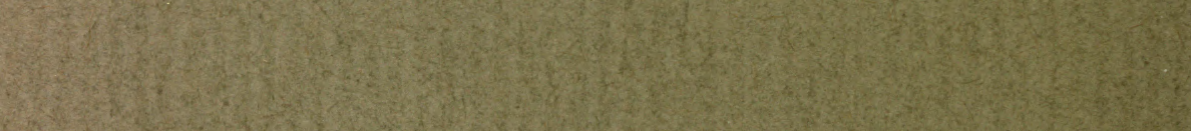
Y.

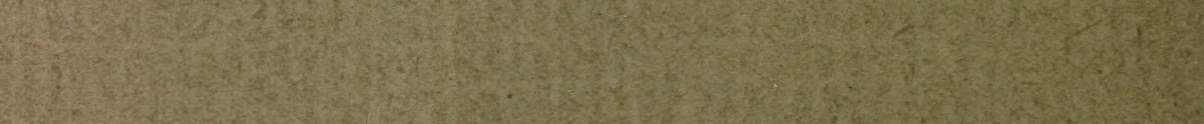

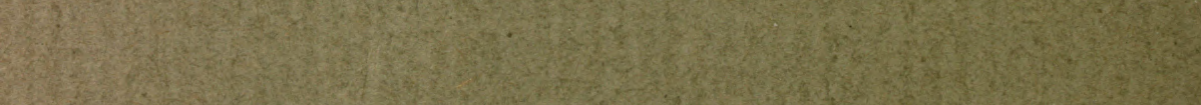

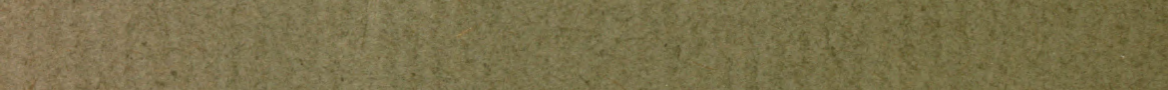

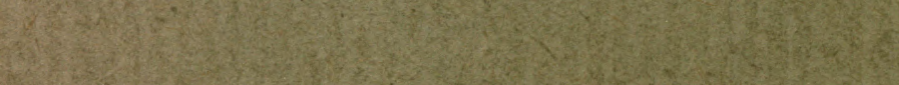

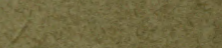

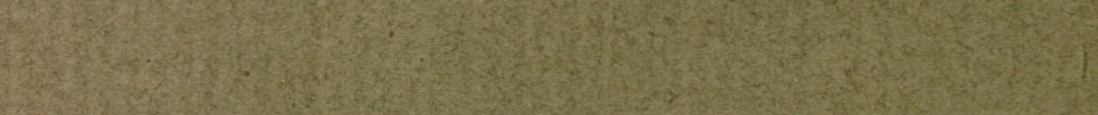

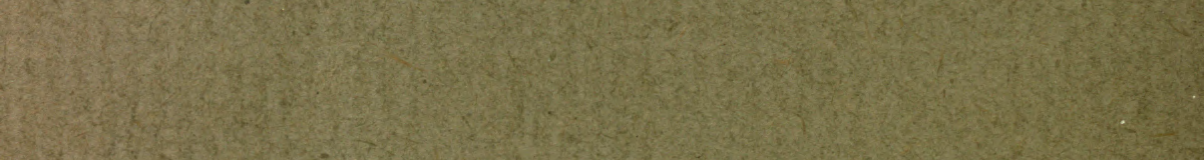

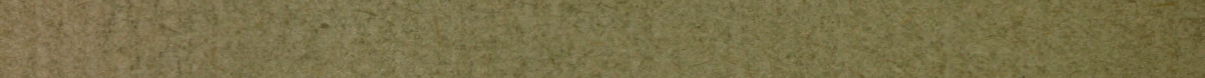

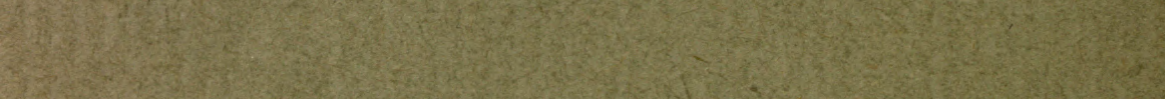

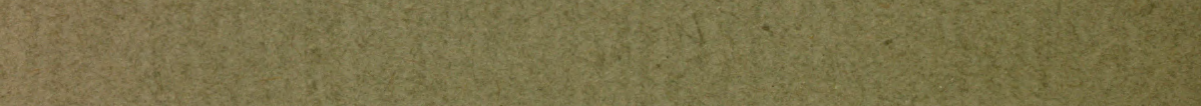

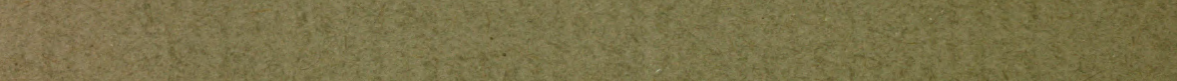

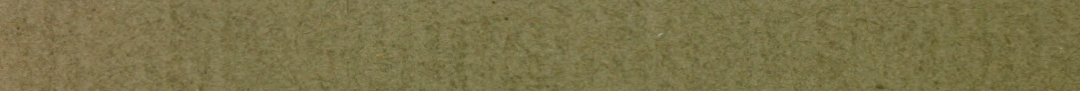

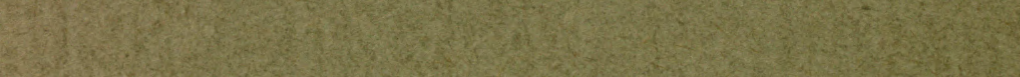

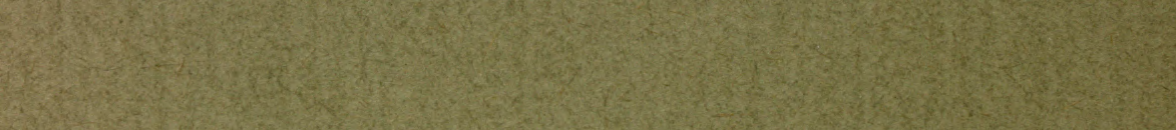

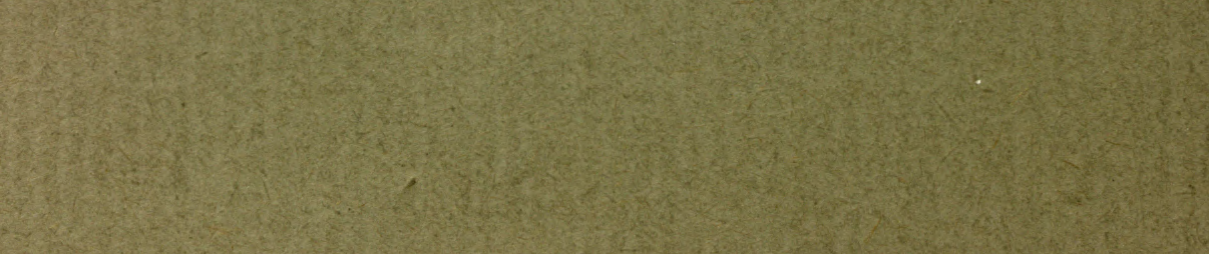




\section{Defective inheritance-ratios in Bursa hybrids.}

\section{By Dr. George Harrison Shull,}

Station for Experimental Evolution, Cold Spring Harbor, Long Island,

New York.

(Sonderabdruck aus dem XLIX. Bande der Verhandlungeu des naturforscherden Vereines in Brünn.)

Several years ago (1905-1907) I was making extensive cultures of Bursa (Capsella) lursa-pastoris, of Bursa Heegeri, and of hybrids between these two species. Bursa Heegeri is generally conceded to have originated from $B$. bursa-pastoris by a recent mutation and has been found in nature only once in a situation which would warrant the belief that it had not been derived from a near-by experimental culture. Although a number of specimens were found by Professor He ger at the original locality on the market-place at Landau, Germany, in 1897, so far as is now known these represented a single elementary form or biotype. Bursa bursa-pastoris, on the other hand, is of almost world-wide distribution, and presents an unknown number, but certainly a large number, of distinct biotypes.

In my cultures of the latter species I found four forms of rosette which were related to one another as the four terms of a Mendelian di-hybrid. These four biotypes were named and described as follows: ${ }^{1}$ )

Type (a). Bursa bursa-pastoris heteris has the leaves divided to the mid-rib, the terminal lobe being usually separated from the nearest lateral lobes by clean, deep incisions. The lateral lobes consist essentially of two features, an elongated proximal portion, the "primary lobe", and a more or less rounded or

1) "Results of crossing Bursa bursa-pastoris and Bursa Heegeri“. $6 \mathrm{pp}$. Proceedings Seventh International Zoological Congress, Boston Meeting, August 19-24 1907. "Advanced reprint" issued in 1910.

"Bursa bursa-pastoris and Bursa Heegeri: Biotypes and hybrids". 57 pp., 23 text-figs., 4 pls., Publ. No. 112, Carnegie Institution of Washington, 1909. 
angular portion, the "secondary lobe", in the distal axil of primary lobe.

Type (b). Bursa bursa-pastoris rhomboidea has the le: divided to the mid-rib as in $B$. bp. heteris. The lateral lobes $h$ an incision on the distal margin setting off the secondary from an unelongated primary lobe. There is usually a co sponding incision on the proximal margin of the primary 1 In the best developed specimens these incisions set off a s] terminal portion of each lateral lobe, which is rather blun angular at the apex, being generally of rhomboidal form. I perfectly developed specimens have the incisions very shallow nearly wanting, but retain the cliaracteristic deep sinuses extenc to the mid-rib.

Type (c). Bursa bursa-pastoris tenuis differs from both and (b) in that the sinuses do not usually reach the mid. The terminal lobe is not separated from the nearest lateral lo by deep, clean-cut sinuses, these more distal sinuses being $\mathrm{r}$ tively shallow, so that one can with but scant propriety sp of the terminal lobe as a definite morphological structure. All lateral lobes tend to be elongated and sharp, and no incis are present to set off a secondary lobe, though in particul vigorous specimens there may be a slight expansion of leaf-ti in the region occupied by the secondary lobe in $B$. bp. he and $B . b p$. rhomboidea.

Type (d). Bursa bursa-pastoris simplex, like B. bp. tenui scarcely ever divided nearly to the mid-rib, and the lateral l are mostly obtuse, sometimes more or less acute, but never and attenuated. No secondary lobing appears except occasion a slight denticulation on the margins.

These descriptions refer to the characters of the clim leaves in well-grown specimens, as all of these types have $j$ nile leaves entirely unlobed and indistinguishable from the $j$ nile leaves of the other forms, and the late rosette-leaves stem-leaves likewise generally lack distinctive features. Un poor cultural conditions plants frequently complete their developm flower, and ripen their seeds, without exhibiting any but th juvenile and senescent characters. The complete procession leaf-forms in typical specimens of the four described biotypes shown in the plates I-IV. 
The production of a considerable number of hybrid families representing the various possible combinations of these four biotypes has demonstrated that their gametic formulae may be aptly represented by the conventional Mendelian symbols, as follows:

$$
\begin{aligned}
& \text { B. bp. heteris . . } A B \\
& \text { B. bp. rhomboidea . } a B \\
& \text { B. bp. tenuis . . } A b \\
& \text { B. bp. simplex . . } a b
\end{aligned}
$$

By comparing these formulae with the descriptions of the different forms it will be seen that $A$ is responsible for the long, sharp character of the primary lobe in $B . b p$. heteris, and the attenuation of the lobes in $B . b p$. tenuis, while $B$ produces the division of the leaf to the mid-rib and the resultant definiteness of the terminal lobe in both $B . b p$. heteris and $B . b p$. rhomboidea, the rounded secondary lobe of $B$. bp. heteris, and the proximal and distal incisions of $B . b p$. rhomboidea.

Bursa Heegeri has the heteris-form of rosette, i. e. with the gametic formula $A B$, but differs so fundamentally from Bursa bursa-pastoris in the characters of the capsules, that if its relationship to the latter species were, not so obvious on other grounds, its capsules would cause it to be placed unquestionably in a separate genus. The flat, triangular or cordate form of the bursa-pastoris capsule is too familiar to need description. The spur-like valves are firm, and fall readily at the slightest touch when the seeds are ripe. The capsules of $B$. Heegeri are of oval form, surmounted by the short, strong, persistent style, and the valves are thin membranous, not the least inflated, and do not fall when the seeds are mature. The seeds are liberated by an irregular break in the central region of each valve.

When this original genotype of $B$. Heegeri was crossed reciprocally with $B$. bursa-pastoris simplex, the rosette-characters segregated in the $F_{2}$, into the four types described above, but the Heegeri-type of capsule appeared in only 111 specimens annong 2540. The distribution of the several characters in the $\mathrm{F}_{2}$ are shown in the following table: 
Table $\mathrm{I}$.

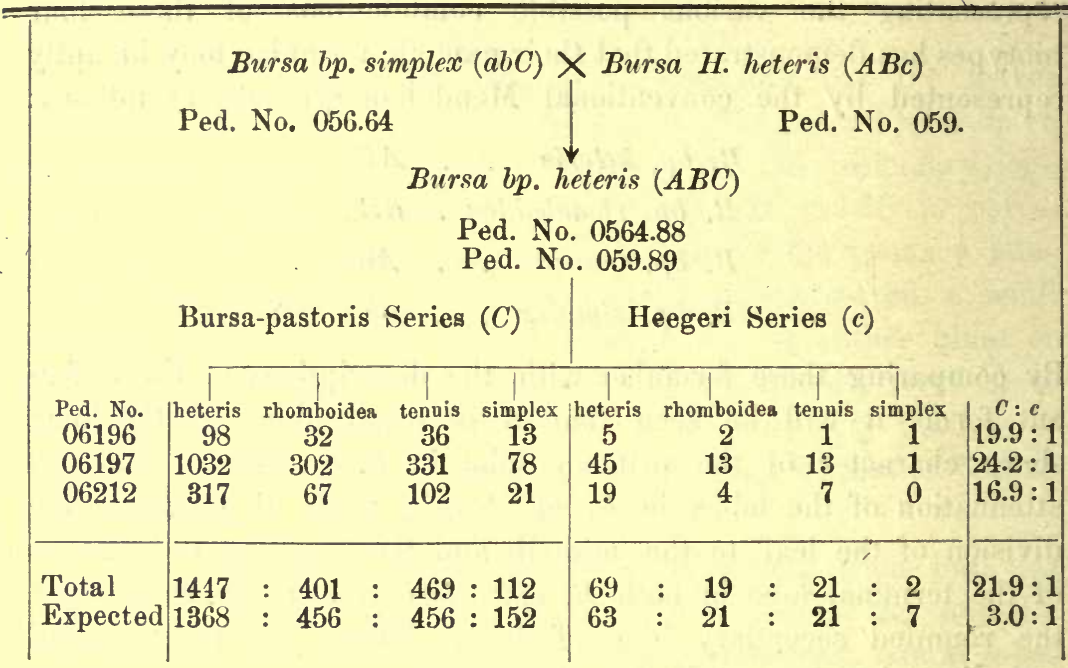

The deficiencies seen to be consistently present in all these pedigrees in the number of rhomboidea and simplex were probably due at least in part to an error of classification, those rhomboideas having the greatest elongation of the terminal segment of the lobes having doubtless permitted their classification as minus-fluctuations of heteris, and the sharpest-lobed simplex as minus-fluctuations of tenuis. The greatest surprise was occasioned by a ratio of about $22: 1$ in the form of the capsules, as it was thought probable that the difference between the two forms would be found to be dependent upon the presence and absence of a single gene, the expected ratio on this assumption being $3: 1$.

At the close of the second generation the cultures were necessarily discontinued, and could not be resumed until in the autumn of 1910 . The appearance of a paper by Nilsson-Ehle ${ }^{1}$ ), showing that certain characters of wheat and oats are independently determined by two or more distinct units or genes, gave the suggestion that the capsule-character of $B$. bursa-pastoris might be determined in like manner by two genes, the absence of both of which produces the Heegeri-type of capsule, although the observed ratio $22: 1$ is a bad approximation to the expected ratio

1) Nilsson-Ehle, H., Kreuzungsuntersuchungen an Hafer und Weizen. pp. 122. 1909. Lund: Hakan Ohlssons Buchdruckerei. 
$15: 1$, considering the number of individuals involved in the $\mathrm{F}_{2}$ cultures and the consistent results yielded by three different pedigrees involving reciprocal crosses. After the pedigrees were all arranged in the spring of 1910 for the testing of this hypothesis, Baur ${ }^{1}$ ) suggested the same possible explanation in a review of one of my papers.

The demonstration of two independent genes for the determination of the same external character is to be found in the composition of the $\mathrm{F}_{3}$ families grown from self-fertilized $\mathrm{F}_{2}$ individuals possessing the dominant character in question, - in this instance the bursa-pastoris type of capsule. Letting the two supposed genes for the triangular capsule be represented by the symbols $C c$ and $D d$, the gametic composition in $\mathbf{F}_{2}$ and the expectation in the $\mathrm{F}_{3}$ is indicated in the following table:

\begin{tabular}{ccl}
$\begin{array}{c}\text { Number of } \\
\mathrm{F}_{2} \text { plants in 16. }\end{array}$ & $\begin{array}{c}\text { Tametic } \\
\text { Composition } \\
\text { in the } \mathbf{F}_{2}\end{array}$ & \multicolumn{1}{c}{$\begin{array}{c}\text { Expected } \\
\text { Results in } \mathrm{F}_{3}\end{array}$} \\
1 & $C D C D$ & All bursa-pastoris. \\
2 & $C D C d$ & All bursa-pastoris. \\
2 & $C D c D$ & All bursa-pastoris. \\
4 & $C D c d$ & Bursa-pastoris and Heegeri, $15: 1$. \\
1 & $C d C d$ & All bursa-pastoris. \\
1 & $c D c D$ & All bursa-pastoris. \\
2 & $C d c d$ & Bursa-pastoris and Heegeri, $3: 1$. \\
2 & $c D c d$ & Bursa-pastoris and Heegeri, $3: 1$. \\
1 & $c d c d$ & All Heegeri.
\end{tabular}

The facts shown in this table may be summarized in the statement that among fifteen families raised from $\mathrm{F}_{2}$ plants having the bursa-pastoris type of capsule, there will be on the average seven $(1 C D C D+2 C D C d+2 C D c D+1 C d C d+1 c D c D)$ which will breed true to that type, four $(C D c d)$ will produce bursa-pastoris and Heegeri in the ratio $15: 1$ as in the $\mathrm{F}_{2}$, and four $(2 C d c d+2 c D c d)$ will give these two types of capsules in the ratio $3: 1$. As in all other recessive types, the offspring of $\mathrm{F}_{2}$ plants having Heegeri capsules should produce no plants with Uursa-pastoris capsules in the $\mathrm{F}_{3}$.

1) Zeitschrift für Induktive Abstamınungs- und Vererbungslehre 3) : $341-342$, Je 1910 , 
Eleven families were grown during the winter of 1910-11 from seeds of $\mathrm{F}_{2}$ plants having bursa-pastoris capsules, and five families from plants having Heegeri capsules. The results are brought together here in the form of a table.

Table III.

\begin{tabular}{|c|c|c|c|c|c|}
\hline $\begin{array}{l}\text { Pedigree } \\
\text { Number }\end{array}$ & $\begin{array}{c}\text { Capsule of } \\
\text { parent }\end{array}$ & $\begin{array}{c}\text { Rosette of } \\
\text { parent }\end{array}$ & Result in $\mathbf{F}_{3}$ & $\begin{array}{l}\text { Ratio } \\
\text { capsules }\end{array}$ & $\begin{array}{c}\text { Ratio } \\
\text { rosettes }\end{array}$ \\
\hline 09258 & bursa-pastoris & heteris & $1 H$. heteris & $0: 1$ & $1: 0$ \\
\hline 09281 & bursa-pasloris & heteris & $307 \mathrm{bp}$. heteris & $1: 0$ & $1: 0$ \\
\hline 09284 & bursa-pastoris & heteris & $\left\{\begin{array}{rrl}31 & \text { bp. } & \text { heteris } \\
11 & \text { bp. } & \text { tenuis } \\
5 & H . & \text { heteris } \\
4 & H & \text { tenuis }\end{array}\right.$ & $4.67: 1$ & $2.47: 1$ \\
\hline 09271 & bursa-pastoris & rhomboidea & 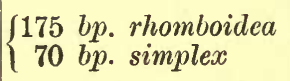 & $1: 0$ & $2.50: 1$ \\
\hline 09272 & bursa-pastoris & rhomboidea & $\left\{\begin{array}{ccc}96 & \text { bp. } & \text { rhomboidea } \\
31 & \text { bp. } & \text { simplex } \\
2 & \text { H. } & \text { rhomboidea }\end{array}\right.$ & $63.5: 1$ & $3.16: 1$ \\
\hline 09273 & bursa-pastoris & tenuis & 375 bp. tenuis & $1: 0$ & $1: 0$ \\
\hline 09274 & bursa-pastoris & tenuis & $\left\{\begin{array}{rl}224 & \text { bp. tenuis } \\
64 & \text { bp. simplex } \\
10 & H . \text { tenuis } \\
2 & H . \\
\text { simplex }\end{array}\right.$ & $24.0: 1$ & $3.55: 1$ \\
\hline 09283 & bursa-pastoris & tenuis & $\left\{\begin{array}{rll}250 & \text { bp } & \text { tenuis } \\
16 & H & \text { Henuis }\end{array}\right.$ & $15.6: 1$ & $1: 0$ \\
\hline 09275 & bursa-pastoris & simplex & $\left\{\begin{array}{l}443 \text { bp. tenuis } \\
213 \text { bp. simplex }\end{array}\right.$ & $1: 0$ & $2.08: 1$ \\
\hline $09275^{1}$ & bursa-pastoris & simplex & $\begin{array}{l}85 \text { bp. tenuis } \\
35 \text { bp. simplex }\end{array}$ & $1: 0$ & $2.43: 1$ \\
\hline 09276 & bursa-pastoris & simplex & 472 bp. simplex & $1: 0$ & $0: 1$ \\
\hline 09282 & bursa-pastoris & simplex & 156 bp. simplex & $1: 0$ & $0: 1$ \\
\hline 09278 & Heegeri & heteris & 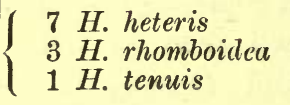 & $0: 1$ & $7: 3: 1: 0$ \\
\hline 09288 & Heegeri & heteris & $1 H$. heteris & $0: 1$ & $1: 0$ \\
\hline 09289 & Heegeri & heteris & $1 H$. heteris & $0: 1$ & $1: 0$ \\
\hline 09277 & Heegeri & tenuis & $\left\{\begin{array}{rl}167 & H . \\
79 & \text { Henuis } \\
\text { simplex }\end{array}\right.$ & $0: 1$ & $2.11: 1$ \\
\hline$|09290|$ & Heegeri & tenuis & $10 \mathrm{H}$. tenuis & $0: 1$ & $1: 0$ \\
\hline
\end{tabular}

1 second sowing. 
Several of these families consisted of but one individual each, and these may be left out of account as having no significance. The most interesting is 09258 in which a bursa-pastoris parent produced a single Hezgeri offspring, thus showing that this parent was heterozygous in respect to capsule-character. Six of the eleven families derived from bursa-pastoris parents bred true to the bursa-pastoris type of capsule, this being slightly in excess of expectation on the assumption that this character is determined independently by two genes, and considerably in excess of the one-third which should have bred true if but one gene differentiated the Heegeri capsules from the bursa-pastoris capsules of the $P_{1}$. However the number of families is wholly inadequate to permit the attachment of any special significance to this closer agreement with the requirements of the two-gene hypothesis. All of the families from parents having Heegeri capsules have bred true to the parental character, as they should do to agree with Mendelian interpretation. The four families which split into the two parental types show the ratios, $4.67: 1,15.6: 1,24.0: 1$, and $63.5: 1$, all of these ratios differing in the same direction but in quite various degrees from the two available ratios $3: 1$ and $15: 1$. Two of the families show a suggestive approximation to the expected ratios, while the other two depart widely from the nearest available ratio $15: 1$. All of these families as well as the three families of the $\mathrm{F}_{2}$ have shown a smaller proportion of Heegeri plants than required by the hypothesis that the bursa-pastoris capsules are determined independently by two genes.

It appears to me that the explanation of these results is to be sought in some modifying influence acting upon the normal Mendelian processes. The ratio 4.67:1 may then represent a modified ratio of $3: 1$, and the other three ratios may be referred to the ratio $15: 1$. These three families taken together give a ratio of $22.2: 1$, essentially identical with the observed ratio 21.9: 1 in the $\mathrm{F}_{2}$, and showing almost exactly the same proportional departure from $15: 1$, that $4.67: 1$ shows from $3: 1$, for $4.67: 3=23.3: 15$.

The nature of the modifying cause or causes which may be operating to produce these defective ratios need not be discussed at length here, as the matter is capable of experimental treatment and is being investigated; but it may be pointed out that either "selective fertilization" favoring the union of unlike gametes, 
or "selective elimination" of the Heegeri homozygotes, would produce the observed results. Of these two sources of modified ratios, "selective elimination" seems to be the more promising, because there appears to be at present no satisfactory evidence that „selective fertilization" occurs in any other organism, while "selective elimination" has been clearly demonstrated in Antirrhinum ${ }^{1}$ ) and in yellow mice. ${ }^{2}$ ) In both of these, the one class of homozygotes is entirely eliminated, so that the normal ratio $3: 1(1+2: 1)$ becomes $2: 1$. If "selective elimination" is the source of the discrepancies between the theoretical and observed ratios in the capsule-characters of Bursa, its operaton must differ from that in Antirrhinum and in yellow mice in two particulars, namely, there must be only a partial elimination of one homozygous class in Bursa, and this elimination must affect the negative, instead of the positive, homozygotes.

The ratios for the rosette-characters in several of the families also deviate considerably from the expected ratio $3: 1$, and it is a pertinent question whether any of these deviations is significant, or whether they may be accounted for by the errors of random sampling, due to the small size of the families. The fact that several of the ratios lie above $3: 1$ and others below, suggests that at least a considerable part of the variation in the ratios is due to purely chance causes of this kind. The ratio $3: 1$ is the ideal, but can be absolutely expected only when the number of offspring is infinite.

Too little attention is paid by students of genetics perhaps to the probable errors of their results. If we follow Johannsen ${ }^{3}$ ) in computing the standard deviation in the case of alternative characters by the formula $\sigma=\sqrt{\%} \mathrm{p}_{\mathrm{v}} \cdot \% \mathrm{p}_{1}$, in which $\% \mathrm{p}_{0}$ represents the percentage of individuals in the one class and $\% \mathrm{p}_{1}$ the percentage in the alternative class, it is easy to determine within what limits a single observed ratio might be referred to $3: 1$ with a sufficient degree of probability. These limits for

1) Baur, E., Untersuchungen über die Erblichkeitsverhältnisse in einer nur in Bastardform lebensfähigen Sippe von Antirrhinum majus. Ber. d. Deutsch. Bot. Gesell. 25 : 442, 1907.

2) Castle, W. E., and Little, C. C., On a modified Mendeliau ratio among yellow mice. Science N. S. 32:868-870, 16 D 1910.

3) Johannsen, W., Elemente der exakten Erblichkeitslehre. pp. VI † 516, 1909. Jena: Gustav Fischer. See p. 57. 
families of various sizes from 100 to 1000 are given in the following table, and are calculated to allow a departure from the observed ratio, equal to three times the probable error. The adoption of three times the probable error as a crtrerion of significant differences is purely arbitrary, and about three families in one-thousand having the given number of individuals could be expected to transgress the limits indicated in the table, and such departures would still be due only to the errors of random sampling. Some biometricians accept 2.5 times the probable error as the limit within which results may not be confidently claimed to be significant.

$$
\text { Table IV. }
$$

$\begin{array}{ccc}\begin{array}{c}\text { Number of } \\ \text { individuals }\end{array} & \begin{array}{c}\text { Observed percentages } \\ \text { theoretically referable to } 75 \%\end{array} & \begin{array}{c}\text { Observed ratios } \\ \text { referable to ratio } 3: 1\end{array} \\ 100 & 60.34 \% \text { to } 85.54 \% & 1.52: 1 \text { to } 5.91: 1 \\ 200 & 64.87 \% \text { to } 82.97 \% & 1.85: 1 \text { to } 4.87: 1 \\ 300 & 66.84 \% \text { to } 81.70 \% & 2.02: 1 \text { to } 4.46: 1 \\ 400 & 68.00 \% \text { to } 80.90 \% & 2.13: 1 \text { to } 4.23: 1 \\ 500 & 68.78 \% \text { to } 80.33 \% & 2.20: 1 \text { to } 4.08: 1 \\ 600 & 69.35 \% \text { to } 79.91 \% & 2.26: 1 \text { to } 3.97: 1 \\ 700 & 69.80 \% \text { to } 79.57 \% & 2.31: 1 \text { to } 3.89: 1 \\ 800 & 70.14 \% \text { to } 79.29 \% & 2.35: 1 \text { to } 3.82: 1 \\ 900 & 70.43 \% \text { to } 79.07 \% & 2.38: 1 \text { to } 3.77: 1 \\ 1000 & 70.68 \% \text { to } 78.87 \% & 2.41: 1 \text { to } 3.73: 1\end{array}$

Only one of the ratios for the leaf-characters in the $\mathrm{F}_{3}$ cul- $^{-}$ tures transgresses the limits indicated in this table. The family 09275 in which 656 individuals gave a ratio of $2.08: 1$, clearly presents a defect not due to random sampling, and the cause of the deficiency was easily discovered. The parent of this family was classified as Bursa bursa-pastoris simplex, and was expected to produce only the parental characters in its offspring; but the progeny consisted of $443 \mathrm{~B} . \mathrm{bp}$. tenuis and $213 \mathrm{~B} . \mathrm{bp}$. simplex, thus demonstrating that the parent was a heterozygote in which the normally. dominant tenuis characters failed to appear. The relative impotency of the tenuis character which allowed it to remain undeveloped in the parent, seems to have affected the offspring in a similar manner, so that without doubt many of the heterozygotes were classified as $B$. $b p$. simplex.

During the early development of this family, it appeared to consist of about three $B . b p$. simplex to one $B . b p$. tenuis, and 
only much later did it become obvious that many of the supposed simplex plants were producing a greater elongation of some of the lobes than is to be found in pure $B$. bp. simplex. The ratio 2.08: 1 here reported for this family, was derived by waiting until the flower-stems were about 5-10 centimeters high, and then calling everything tenuis which produced at least one lobe more elongated than those of pure-bred B. bp. simplex. Plate V shows the most highly developed leaf-characters attained in each of twenty-six individuals taken quite at random from plants in this family, which had been finally classified as B. bp. tenuis. In normal, well-developed specimens of $B$. bp. tenuis there is a long series of leaves in the middle ("climax") region of the rosette, in which there is marked elongation of the lobes (see plate III), but in family 09275 many of the plants which were finally regarded as $B . b p$. tenuis, had but one or two leaves in which recognized tenuis characters appeared. Thus the wide gap which ordinarily separates the dominant and recessive types in these hybrid families of Bursa, was in this particular family not only reduced to zero, but it appears certain that the heterozygous and recessive categories overlapped to such an extent that many individuals which belonged in the former were necessarily classified in the latter: hence the defective ratio is to be explained by the failure of dominance of the tenuis characters in the heterozygotes.

The cause of this failure of dominance is not apparent. The environment has a very considerable influence in determining the various features of Bursa plants, and especially in limiting the development of such distinctive characters as ordinarily appear only in the climax leaves. Crowding in the seed-pans, poor illumination, and other unfavorable conditions, have caused many plants in certain of my cultures, to develop flowers and ripen seeds without having developed their leaves beyond the early juvenile stages. While the rosettes in 09275 were not in any sense juvenile, the simplex characters do represent a less highly specialized type than tenuis, and therefore any influence which tends to abbreviate the cycle of development, might conceivably reduce tenuis plants to a form indistinguishable from simplex. This large family of 656 individuals was germinated in a single square seed-pan, $30 \times 30 \mathrm{~cm}$, and the young plants grew in this seed-pan for seven weeks before they. were potted. Perhaps this long 
crowding might be expected to have some such effect as that observed. So far as known, all other conditions under which these plants grew, were conducive to vigorous development. At any rate this culture was in all other regards on an equal footing with the other cultures which were being grown during the same period, and which gave normal development of the several biotypes.

To test the suggestion that crowding might be responsible for the failure of dominance, a second sowing was made on February 25, 1911, each seed being sown separately and spaced in such a manner that the seed-pan contained only 120 plants. Un April 7 these were potted and their growth has been continuously healthy and vigorous. They have not been subjected at any time to the least injurious crowding, yet they show the same tardy development of the tenuis characters as observed before. On April 22 only three of the 120 plants could be distinguished from $B . b p$. siml lex, though nearly all were sufficiently advanced that if it had been a normal family representing the same hybrid combination, almost a complete separation of the alternative types might have been made. The final census of the plants derived from this second sowing, made on the same basis and with the same care as in the case of the first sowing, showed $85 \mathrm{~B}$. bp. tenuis and $35 \mathrm{~B} . b p$. simplex, - a ratio of $2.43: 1$. The conclusion is reached therefore that the low grade of the tenuis characters in this family is inherent, and not a direct effect of unfavorable conditions of the environment.

This result appears to furnish an illustration of the phenomenon known as "variable potency ${ }^{* 1}$ ), but whether the gene $A$ for the tenuis characters is really different in family 09275 from that in the other families must remain for the present an open question. It is conceivable that the relative inefficiency of $A$ in this family is due to the operation of some other factor which acts as a partial inhibitor, so that although the actual character of $A$ remains unchanged, it must meet a greater resistance, and therefore produces a less effect. To avoid a decision of the question whether the gene has less power or whether it must meet a greater resistance, I use the expression „relative

1) Davenport, C. B., Heredity and Mendel's law. Proc. Washington Acad. Sci. 9 : $179-187,31$ Jy 1907. 
potency". The ninhibiting factor", if such there be, need not even be genotypic in nature, but may be the result of some somatic quality of the parent (such for instance as its state of health) projected to the offspring through influences surrounding the latter during their embryonic development.

\section{Summary.}

Crosses between Bursa (Capsella) bursa-pastoris simplex and $B$. Heegeri heteris have demonstrated the existence of two genes, $A$ and $B$, which determine the differentiating characteristics of the rosettes and which result in the production of four forms in the $\mathrm{F}_{2}$ in the ratio $9: 3: 3: 1$.

They seem to indicate also the presence of two genes, $C$ and $D$, each of which is independently responsible for the bursapastoris-type of capsule. The Heegeri-type appears only in the absence of both $C$ and $D$. On this basis the two forms should appear in the $F_{2}$ in the ratio $15: 1$. The observed ratio was $21.9: 1$. In the $\mathrm{F}_{3}$, some families should give ratios of $3: 1$, and other families should again give ratios of $15: 1$. The observed ratios in $\mathrm{F}_{3}$ were $4.67: 1$, and $22.2: 1$. These observed ratios show a corresponding deviation from the theoretical ratios, and are thought to indicate the action of some modifying influence, such as "selective elimination", distorting the results of an otherwise normal Mendelian segregation.

A defective ratio in the rosettes of one family is shown to be due to failure of dominance, and certain facts are presented, which indicate that this failure of dominance is attributable to a less "relative potency" of the gene $A$ which determines the tenuis-character. This change in the relative potency of $\boldsymbol{A}$ may be due to a decline in the efficiency of the gene itself, or to the operation of some other factor or condition which offers an increased resistance to the development of the tenuis-characters. 
Shull - Defective ratios in Bursa.

Plate I.

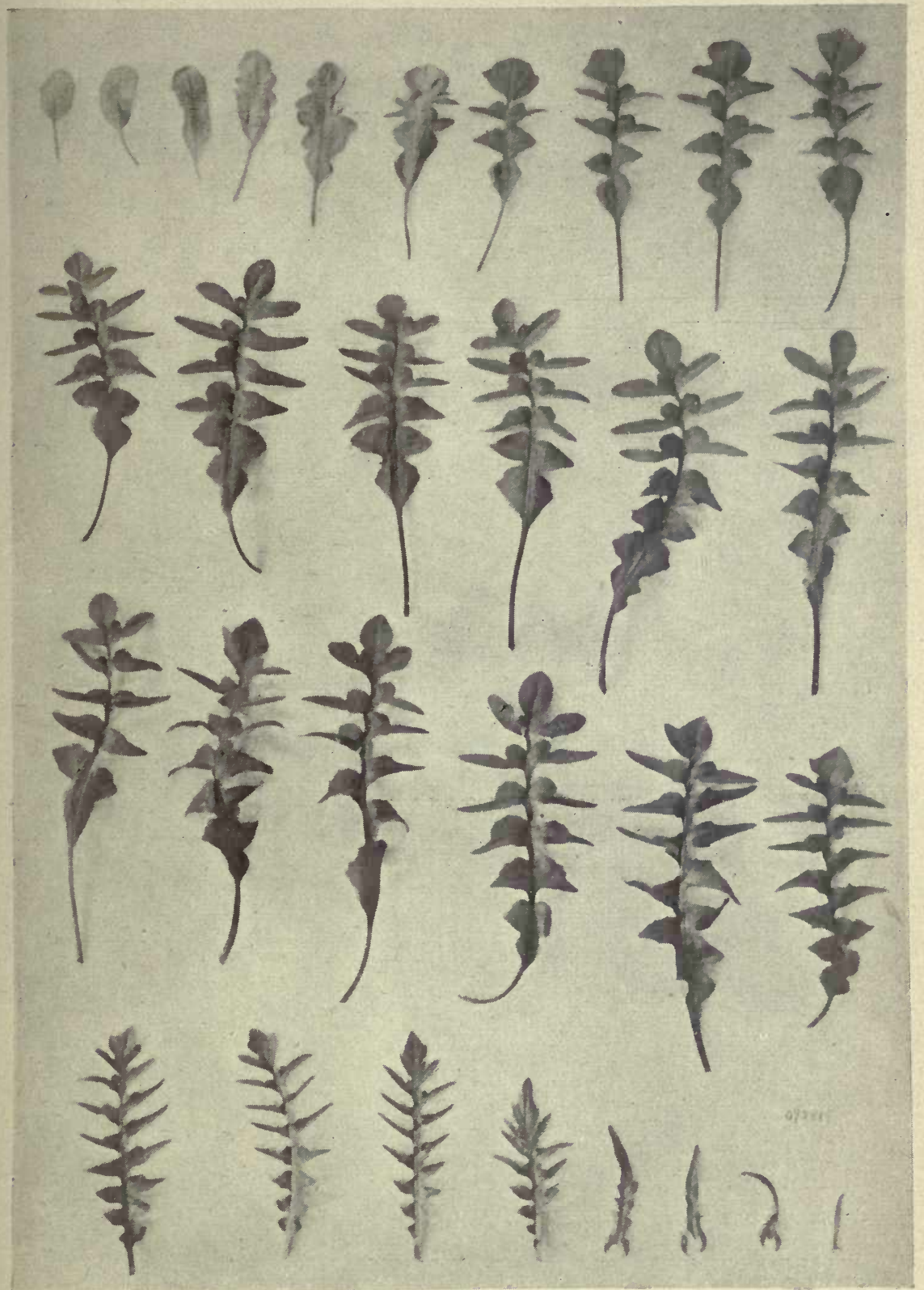

Ontogenetic succession of leaf-forms in Bursa.. . .heteris. 

Shull - Defective ratios in Bursa. Plate II.

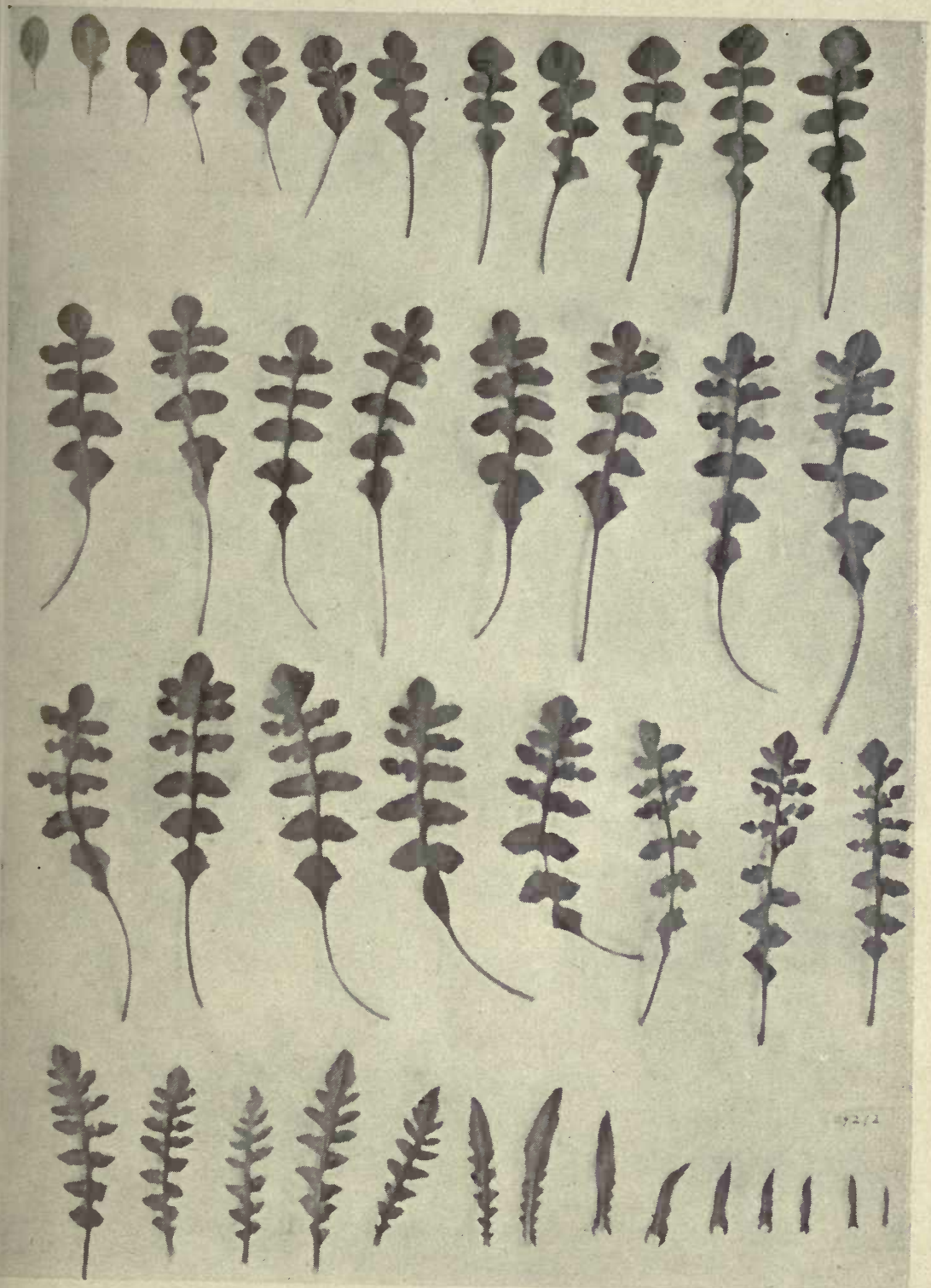

Ontogenetic succession of leaf-forms in Bursa..... rhomboidea. 

Shull - Defective ratios in Bursa. Plate III.

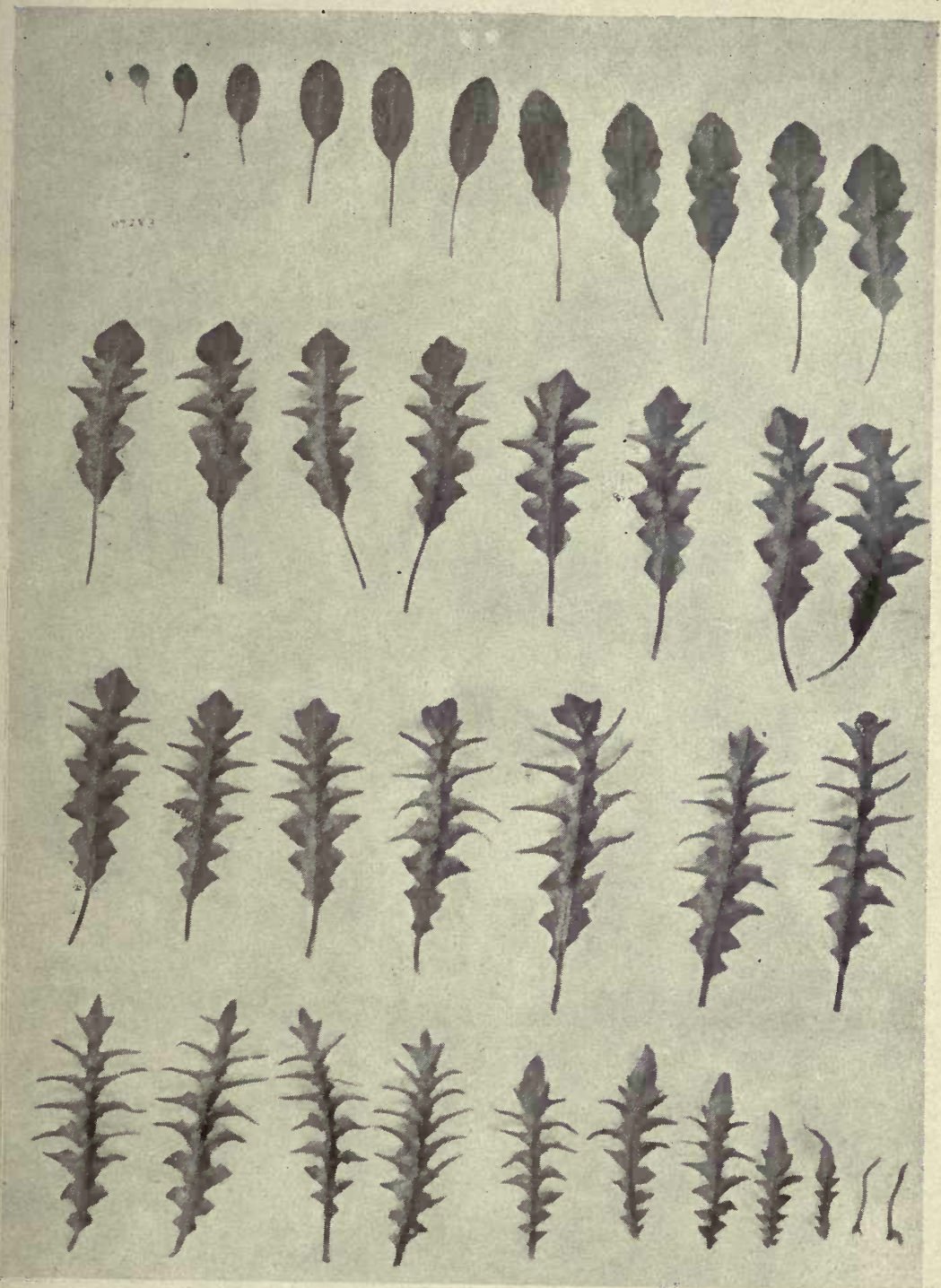

Ontogenetic succession of leaf-forms in Bursa....tenuis. 

Shull - Defective ratios in Bursa. Plate IV.

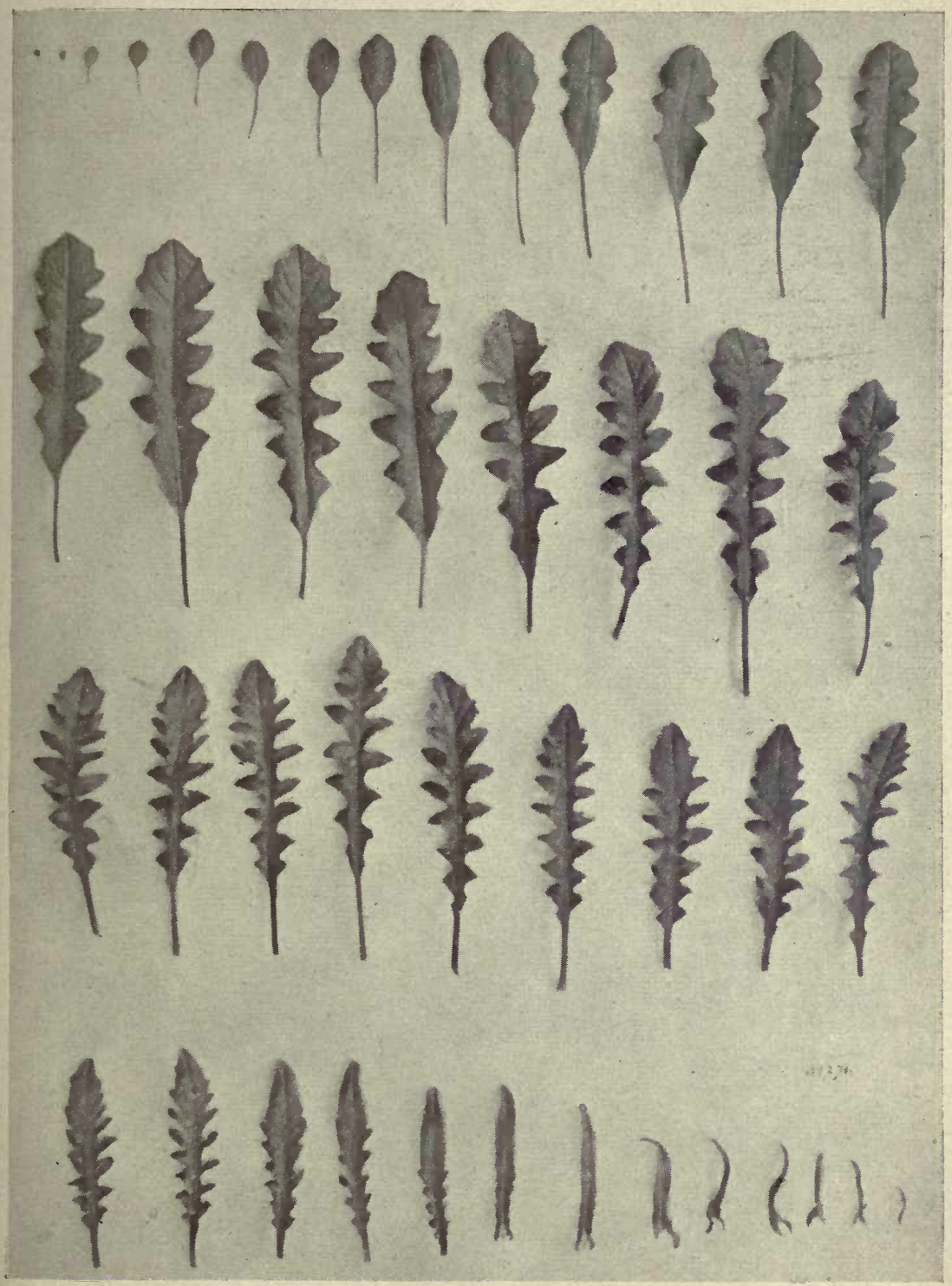

Ontogenetic succession of leaf-forms in Bursa..... simplex. 

Shull - Defective ratios in Bursa. Plate V.

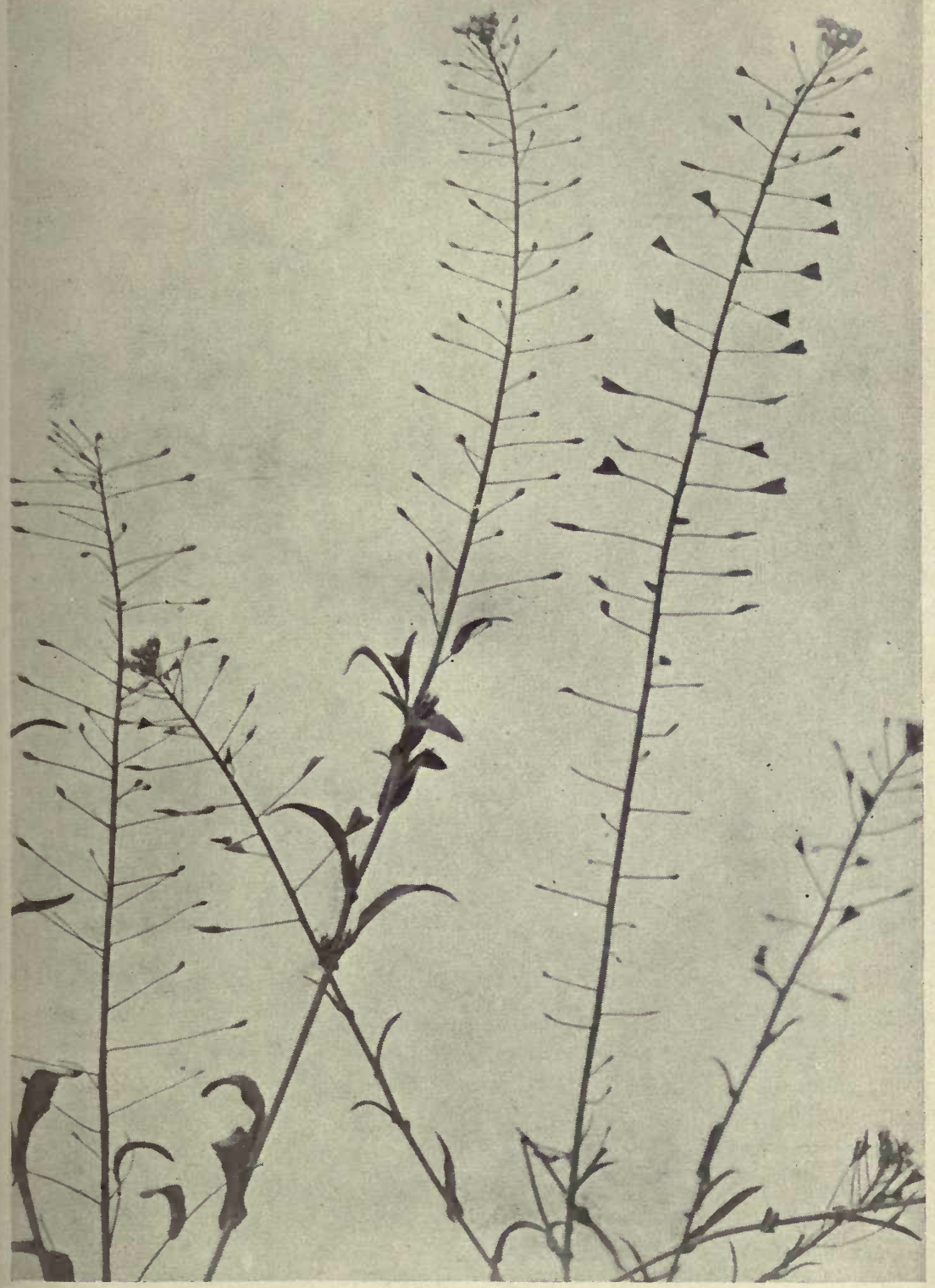

Inflorescences of Bursa bursa-pastoris (at right) and of B. Heegeri (left). 

Shull - Defective ratios in Bursa. Plate VI.

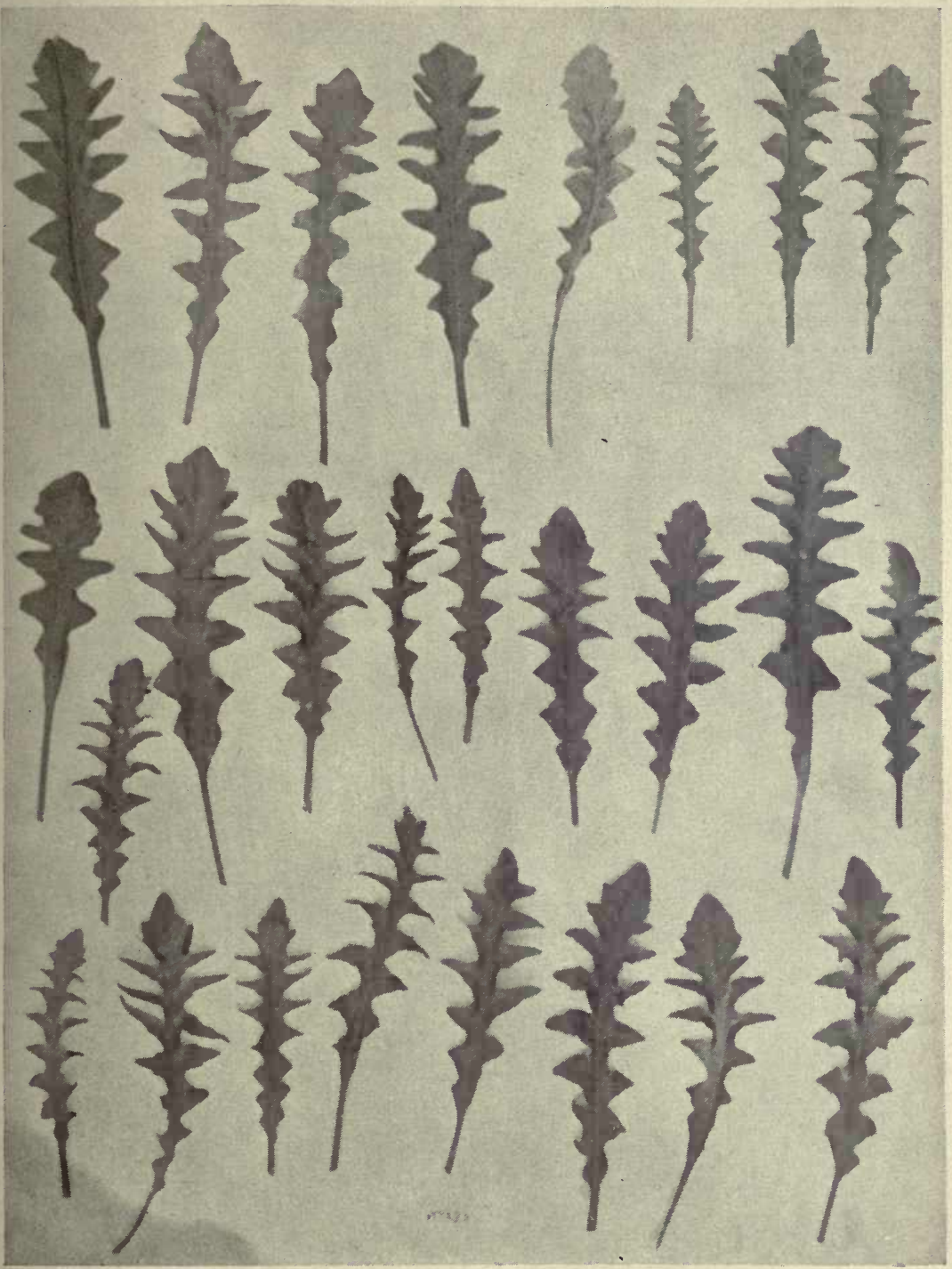

Climax leaves of twenty-six individuals classified as B. bp. tenuis, in a family (09275) which showed an unusually low grade of development of the tenuis characters. 


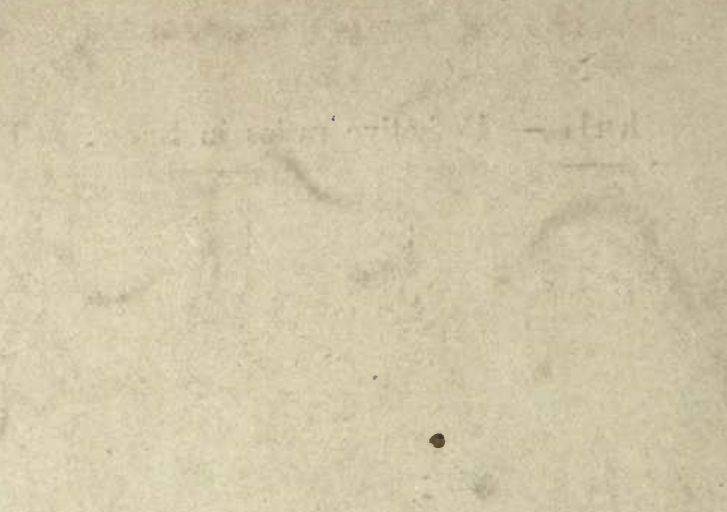


[Reprinted from The American Naturalist, Vol. XLV., April, 1911.]

\section{THE GENOTYPES OF MAIZE ${ }^{1}$}

DR. GEORGE HARRISON SHULL

Station for Experimental Evolution, Cold Spring Harbor, N. Y.

THE doctrine of evolution had to overthrow the conception of permanency of specific types, generally held when Darwin's "Origin of Species" was published, because that conception was then associated with the idea of a separate original supernatural creation of each such type. It was Darwin's great triumph that he succeeded in marshaling such an array of facts pertaining to variability, as to convince the scientific world-and through the scientific world, ultimately the whole world-that everything is in a state of flux, and that there is no such thing as permaneney among living things.

Owing to the work of De Vries and the other early students of modern genetics, permanency of type again demands serious scientific consideration, for such permanency is no longer incompatible with the doctrine of evolution, being now associated with some form of the mutation theory. The old idea of the immutability of specific types was based upon almost total ignorance of genetics, as was likewise the Darwinian conception of fluidity and gradual change, for although many appeals were made by Darwin to the experiences of plant and animal breeders, it is now known that these experiences were the result of no such careful control of conditions or analysis of results as has been found necessary for the discovery of genetic laws. The critical work of the past few years has wrought a great change and the new idea of permanency is gaining ground with the growth of experimental knowledge.

Without granting that we have yet reached a position in which we can say definitely that types are absolutely

\footnotetext{
${ }^{1}$ Read before the American Society of Naturalists, December, 1910.
} 
experimental data now available makes it necessary to recognize a clear distinction between the evolutionary changes in types, on the one hand, and the fluctuations within each type, on the other hand.

Quite naturally the first experimental evidence of the existence of permanent hereditary types involved only such characteristics as are clearly distinguishable upon inspection. Thus Jordan was able to demonstrate that within the systematic species Draba verna there are included as many as two-hundred hereditary forms, whose distinguishing characteristics appear unchanged from generation to generation, in such manner that his pedigrees of these forms were clearly and permanently distinguishable from each other by easily defined morphological features. Such "petites espèces" or "little species" (afterwards called by De Vries "elementary species," and by Johannsen "biotypes" or "genotypes"), have since been observed by Wittrock and his students, and by many others, in a great number of wild species, and they are now quite generally supposed to be of almost universal occurrence.

About 1890 N. H. Nilsson made a similar discovery in connection with his breeding of wheat, oats, barley and other grains at Svalöf, Sweden, but his work remained practically unknown to the scientific world until it was brought to light several years ago by De Vries. Nilsson found in these grains elementary species, each with its own morphological characters and its own specific capacity to vield crops of given size or quality under given external conditions. More recently, sharp-eyed taxonomists have been rapidly raising many of the elementary species of wild plants to the rank of systematic species.

It was natural that the earliest genotypes recognized, such as those of Jordan and Nilsson, should have possessed visibly discrete characteristics, and that they should first have become familiar in normally self-fertilized plants, among which little confusion is occasioned 
by the rare crossing of unlike individuals. Great credit is due to Johannsen ${ }^{2}$ for demonstrating that in such self-fertilized plants, types also exist which are not readily distinguishable by simple inspection, but whose occurrence may be completely demonstrated by the refined methods of the mathematician. Not only has Johannsen's work been so extensive as to justify the conclusions arrived at by him, but various other investigators, working with different classes of research material, have shown that the conditions found by Johannsen in beans and barley are duplicated in many other species and varieties. Perhaps the strongest support in this direction has come from the work of East ${ }^{3}$ with potatoes and that of Jennings ${ }^{4}$ with various microscopic organisms, especially with paramecium.

The fact that Draba verna, and many other wild species in which the existence of numerous elementary species has been demonstrated, as well as wheat, oats, barley and beans, are all predominantly self-fertilizing, and that potatoes and paramecium have an asexual reproduction, has led some to the erroneous notion that the discreteness, uniformity and permanence of the types which have been discovered among these and other similar organisms, are in some way dependent upon the absence of crossing.

It must be admitted that conclusions drawn from selffertilized and asexual material do not necessarily apply to plants and animals whose successful existence is dependent upon repeated crossing. Nevertheless, the conception of pure and permanent genotypes in cross-bred material has become familiar simultaneously, owing to the work done in Mendelian heredity; for homozygous

"Johannsen, W., "Ueber Erblichkeit in Populationen und in reinen Linien,", 68 pp., Jena, 1903.

${ }^{3}$ East, E. M., "The transmission of variations in the potato in asexual reproduction,' Conn. Exp. Sta. Report 1909-1910, pp. 119-160, 5 pls.

"Jennings, H. S., "Heredity, variation and evolution in ProtozoaII. Heredity and variation of size and form in Paramecium, with studies of growth, environmental action and selection," Proc. Amer. Phil. Soc., 47: 393-546, 1908. 
combinations of the various characteristics of plants and animals "breed true" to those characteristics. Just as the first recognition of permanent differences in pure lines involved easily distinguishable characters, so also these first discoveries of permanent pure-breeding genotypes in cross-bred plants and animals involved easily definable morphological characteristics. The demonstration that in normally pure-bred lines there are distinctions more minute than such easily visible features as characterize the elementary forms of Draba and many other species, was an important advance in our analysis of the populations which make up the species of plants and animals. A similar demonstration that populations of cross-breeding plants and animals are composed of fundamentally distinct types, intermingled but not changed by panmixia, and capable of being separated by appropriate means and of being shown to possess the discreteness, uniformity and permanence already demonstrated for the genotypes of self-fertilized and clonal races, will add greatly to the importance of the fundamental conception of permanency of types involved in the work of De Vries and Johannsen.

For the study of this problem there is probably no better plant than Indian corn. It is known to exist in a large number of obviously distinct strains or subspecies which cross together with the greatest ease. Many of its characteristics have been proved by different investigators to be Mendelian unit-characters; such, for instance, as the color of the seed-coat, whether red, dark yellow, light yellow, variegated or colorless, the color of the aleurone layer, whether blue, red or white; the color of the endosperm, whether yellow or white; the chemical composition of the endosperm, whether starchy or sugary, the color of the silks and cobs whether red or white, etc. It has become known also, mainly through the excellent work done at the Illinois State Experiment Station, that oil-content and protein-content of the grains, the position of the ears, the number of ears on the stalk, and 
several other characters, are capable of accentuation by selection, so that different degrees of these qualities are capable of being made characteristics of particular strains of corn, without there being the least evidence as yet that these last-mentioned qualities bear any relation to the unit-characters with which the student of genetics generally deals. A further point in favor of maize as a subject for the study of genotypes among cross-breeding organisms lies in the fact that its flowers are so arranged that, while self-fertilization is possible, it is naturally almost completely excluded, thus ensuring the same relations as are presented by bi-sexual or diœcious plants and animals, while retaining the means of conveniently testing the genotypic nature of each individual by controlled self-fertilizations.

I think I have demonstrated during the last five years that there are many genotypes of Indian corn which, although they can not always be distinguished by defin. able external characteristics, can be proved to be just as certainly and permanently discrete as the types whose distinguishing features can be recognized as Mendelian unit-characters. I shall endeavor to show, in what follows, a portion of the evidence which leads me to this conclusion.

In $1905 \mathrm{I}$ undertook a rather extensive series of com. parisons between cross-bred and self-fertilized strains of Indian corn for the purpose of discovering the effects of these methods of breeding upon variability, and these investigations have been continued each year since that time. Two phenomena immediately attracted my attention: First, the well-known fact that the children of selffertilized parents are inferior to those of cross-fertilized parents in height, yield and other characters dependent in any way upon physiological vigor. In every instance this phenomenon was plainly evident in the very first generation after self-fertilization. This decrease in physiological vigor due to self-fertilization has become an 
extremely important relation in the study of the genotypes, as will be shown later.

The second phenomenon which quickly made itself manifest, was first clearly appreciated in the second generation after the beginning of the experiments; this was the fact that each self-fertilized family possessed morphological features which clearly differentiated it from all other families. In most cases the distinguishing characteristics of these families were of such elusive nature that it was impossible to recognize definite unitcharacters, and indeed, morphological descriptions of the several pedigrees could often be made only in terms of greater or less intensity of the several qualities exhibited. However, the distinctions were real and applied to every member of the particular family. Thus one family might have a very slender, poorly developed male panicle, while another would have more thick and dense branches of the panicle. This difference might be quite small when given in actual measurement but inspection showed that every individual of the one family had the slender, illy developed panicles, while all of the offspring of the other family had the thicker, denser type. Similarly, one family might have a slightly broader and darker green leaf than another, and these characteristic differences were likewise uniformly present in all members of the single families contrasted. No such character as this is capable of being traced through the generations following a cross, in the manner usually pursued by the geneticist, and the matter must be approached by indirect methods. The important point to be kept in mind here is simply that the self-fertilized families, derived originally from a common stock, do differ by morpho. logical characteristics, and that there comes to be great uniformity in regard to the presence of these characteristics in all the individuals of a given self-fertilized family.

This relative uniformity, which is so obvious even to the casual observer, is not sufficient in itself, however, 
to positively demonstrate the existence of distinct genotypes in maize, because the slight variations which must always be present even in the most uniform progeny, can not be certainly distinguished as genotypic or fluctuating simply by inspection. Such demonstration must rest upon a combination of biometric and genetic evidence in order to prove acceptable. Most of the differentiating characters of my several strains of maize are such that they do not lend themselves readily to biometric methods, but the number of rows on the ear is well adapted for such study and several important results have been derived from the consideration of this character. An important proof that the self-fertilized families derived from my common original stock of corn are genotypically distinct, and that they do not owe their different morphological and physiological qualities to fluctuations within a single genotype, was quickly found in the fact that two of these families selected respectively to 12 and 14 rows of grains on the ears, showed a regression of row-number toward different centers instead of toward a common center. The mean of the original population was slightly above 14 rows. The selection to 14 rows was very near this mean and the selection to 12 rows was very near this mean and the selection to 12 rows considerably below it. According to Galton's well-known law of "regression toward mediocrity," the mean of a family whose parents were selected to 12 rows should have lain somewhat above 12 rows, and that selected to 14 rows should have retained the mean approximately at 14 rows. The actual result in the case of selection to 12 rows was the production of a family having a mean row-number considerably below the number of rows selected, and the subsequent generations have since shown a close approach to an 8-rowed condition; while the family whose parents were in each generation selected to 14 rows has always had the mean very near to 14 rows. As these families were grown under as nearly uniform conditions as possible, the fact that the 14-rowed family continues 
to have its mean row-number at 14 shows that the fall in row-number from 12 to 8 in the other family has been due to internal rather than to external causes.

The change in variability in number of rows on the ears has also been studied from year to year. Continued self-fertilization has resulted in a gradual decrease of variability in the number of rows per ear in each of the self-fertilized lines. This is a fluctuating character, and so far as present evidence goes, the number of rows per ear in any strain can not be fixed at a definite number. While it is probable that none of my self-fertilized families has yet reached an absolutely pure-bred condition, several of them have become so nearly pure-bred that their various relations can be used to demonstrate that they are approaching purity as a limit.

In 1909 two of these nearly pure-bred families were compared with their reciprocal hybrids in the first and second generations, with reference to the variability in number of rows. ${ }^{5}$ It was found that the average variability in these two self-fertilized families was 9.08 per cent. The variation in number of rows in their $\mathrm{F}_{1}$ progeny was 9.06 per cent., and in the $\mathrm{F}_{2} 12.63$ per cent. A comparison of these coefficients of variability shows at once that the variation in number of rows in the $F_{1}$ is essentially identical with that in the self-fertilized lines used for the cross. Theoretically this should be so if the strains used were pure genotypes, because in that case all germ-cells in each pure strain were alike, and therefore, when individuals belonging to these two lines were crossed, equal sperms met equal eggs; consequently there should be no variability in their offspring due to germinal differences, but only those due to environment in the widest sense. As the pure-bred families and their $\mathrm{F}_{1}$ and $\mathrm{F}_{2}$ progenies were grown beside each other during the same season, they were subjected to as nearly identical environmental influences as can be attained.

'Shull, G. H., " Hybridization methods in corn breeding," Am. Breeders' Magazine, 1: 98-107, 1910. 
Consequently, when the $F_{1}$ shows the same variability as the pure lines which entered into it we must conclude that there was at least approximate equality among the sperms which came from the one self-fertilized strain, and among the eggs which came from the other. In the $\mathrm{F}_{2}$, on the other hand, genotypic differences appear, owing to the segregation of the different characteristics into the different germ-cells, and to this fact may be ascribed the increased variability in the $\mathrm{F}_{2}$.

While other characters have not been studied by the same methods that have been used in the investigation of the number of rows on the ears, several features associated with the physiological vigor of the various pedigrees have given evidence which appears to me to be strongly corroboratory of the uniformity of the germcells produced by plants which have become pure-bred through continued self-fertilization. The smaller size and less vigor of the offspring of self-fertilized plants as compared with those from a normally cross-bred plant were formerly taken to indicate that self-fertilization is injurious, and Darwin's "Effects of Cross and Self-fertilization in the Vegetable Kingdom" strongly impressed this point of view. I have been able to demonstrate, however, that this supposedly injurious effect of selffertilization is only apparent and not real; or at least that if there is such injurious effect, it is relatively insignificant as compared with the increased vigor due to heterozygosis. The most important evidence of this is found in the fact that the continuation of self-fertilization in any pedigree does not produce a corresponding decrease in vitality and size. The decrease resulting from a second year of self-fertilization is not as great as that from the first year. The third year of self-fertilization produces still less deterioration, and as this process is continued a limit is approached in such manner as to justify the inference that when complete purity is attained no further deterioration is to be expected, thus proving that self-fertilization is not in itself injurious. 
That this is also true of other plants is derivable from Darwin's own work.

This decrease in size and vigor is accompanied by the gradual lessening of variability, and when that state is finally reached in which there is no further decrease in size and vigor, it seems probable that there will be also no further noticeable change in variability. This does not mean, of course, that there will be no variability, for even the most uniform group of plants or animals will of necessity show slight variations produced by different conditions of life, food supply and so forth. But present evidence does not warrant the belief that such fluctuations affect in the least the fundamental qualities of the genotype.

In 1908 I suggested a hypothesis to explain the apparent deterioration attendant upon self-fertilization, by pointing out that in plants, such as maize, which show superiority as a result of cross-fertilization, this superiority is of the same nature as that so generally met with in $F_{1}$ hybrids. I assumed that the vigor in such cases is due to the presence of heterozygous elements in the hybrids, and that the degree of vigor is correlated with the number of characters in respect to which the hybrids are heterozygous. I do not believe that this correlation is perfect, of course, but approximate, as it is readily conceivable that even though the general principle should be correct, heterozygosis in some elements may be without effect upon vigor, or even depressing. The presence of unpaired genes, or the presence of unlike or unequal paired genes, was assumed to produce the greater functional activity upon which larger size and greater efficiency depend. This idea has been elaborated by Dr. East $^{6}$ and shown to agree with his own extensive experiments in self-fertilizing and crossing maize. He suggests that this stimulation due to hybridity may be analogous to that of ionization.

Mr. A. B. Bruce proposes a slightly different hypothe-

${ }^{6}$ East, E. M., "The distinction between development and heredity in in-breeding,", AMER. NAT., 43: 173-181, 1909. 
sis in which the degree of vigor is assumed to depend upon the number of dominant elements present rather than the number of heterozygous elements. While all of my data thus far are in perfect accord with my own hypothesis, and I know of no instance in which self-fertilization of a corn-plant of maximum vigor has not resulted in a less vigorous progeny, it is quite possible that I have still insufficient data from which to distinguish between the results expected under these two hypotheses. However, for the purpose of the present discussion, it is not necessary to decide which of these two hypotheses (if either) is correct. Both of them are based upon the view that the germ-cells produced by any plant whose vigor has been increased by crossing are not uniform, some possessing positive elements or genes not possessed by others.

Several different characters which are more or less dependent upon physiological vigor have been taken into account in my work, each of which gives its own support to the conception upon which both of these hypotheses are based. The number of rows of grains on the ears which has been most extensively used as a measure of variability, and as a guide in selection, is found to be somewhat affected by the vigor of the individual, and it is due to this fact, no doubt, that the row-number is a fluctuating character, even in the pure genotype. Another characteristic which has been used as a measure of vigor has been the yield of corn computed in bushels per acre. $^{7}$ A third characteristic, which was not taken into account at the beginning of the experiments but which has given confirmatory data in the later years, is the height of the stalks, a character which was much used by Darwin as a measure of vigor in his study of the effects of cross- and self-fertilization in plants.

${ }^{7}$ It should be understood that this method of stating yields is seriously defective, in that it implies the existence of a much smaller probable error than is actually present, since each of my pedigrees has usually occupied only about one one-hundredth of an acre. However, I believe that this defect is more than offset by the advantage of using a unit of yield with which all readers are familiar. 
We may now consider the behavior of these several measures of physiological vigor in relation to the theory that distinct genotypes of maize are gradually segregated from their hybrid combinations, by self-fertilization, and that the degree of vigor is correlated with the degree of heterozygosis.

I have kept families selected to given numbers of rows on the ears - one series of families repeatedly self-fertilized and another series repeatedly crossed with mixed pollen in such a manner that self-fertilization is precluded by artificial means. It is not practicable to do this crossing with mixed pollen in such a manner as to duplicate the conditions found in an ordinary corn-field for the simple reason that the number of individuals which contribute the pollen must be more greatly restricted than is true in the open field. While self-fertilization has been entirely prevented, there has been a degree of in-breeding somewhat greater therefore than will occur under non-experimental conditions. This degree of in-breeding is sufficient to slowly eliminate some of the hybrid elements which were originally in my strain of corn and should consequently lead to a gradual deterioration in case my theory of the relation between vigor and hybridity is correct. As a matter of fact, such deterioration has become apparent in the "cross-bred"s families, when measured either by height of stalk or yield per acre, though both of these measures show that the deterioration has been slight. It is so slight, indeed, that it is very much exceeded by the fluctuations from season to season, and may only be demonstrated by the application of a correction which approximately eliminates this seasonal fluctuation. When we compare this continual slight fall in physiological vigor of the cross-

${ }^{8}$ It should be noted that here and in what follows I use the expression "cross-bred", in a special sense, to denote the fact that all self-fertilization has been avoided. The more usual use of the term "cross-bred" to denote a cross between individuals belonging to distinct strains, I replace in this paper by the expression " $F_{1}$,"' as I can see no tangible distinction between such a cross, and hybridization in the older, more restricterl, and more arbitrary sense. 
bred families with the changes produced in the selffertilized families during the same period, there is a striking contrast, for in the latter case there was great decrease in height and yield in the first year, a considerably less decrease in the second year of self-fertilization, still less in the third year, and so on, and while I have evidence that none of my self-fertilized families has yet reached a state of perfect stability, they are at the present time decreasing in regard to both of these measures of vigor somewhat less rapidly under continued self-fertilization than are the families in which self-fertilization has been absolutely precluded.

Necessary corollaries of the view that the degree of vigor is dependent on the degree of hybridity, or, in other words, that it is dependent roughly upon the number of heterozygous elements present and not upon any injurious effect of in-breeding per se, are $(a)$ that when two plants in the same self-fertilized family, or within the same genotype, however distantly the chosen individuals may be related, are bred together, there shall be no increase of vigor over that shown by self-fertilized plants in the same genotype, since no new hereditary element is introduced by such a cross; (b) that first generation hybrids produced by crossing individuals belonging to two self-fertilized lines, or pure genotypes, will show the highest degree of vigor possible in progenies representing combinations of those two genotypes, because in the first generation every individual will be heterozygous with respect to all of the characters which differentiate the two genotypes to which the chosen parents belong, while in subsequent generations, recombination of these characters will decrease the average number of heterozygous genes present in each individual; $(c)$ that crosses between sibs among the first-generation hybrids between two genotypes will yield progenies having the same characteristics, the same vigor, and the same degree of heterogeneity, as will be shown by the progenies of self-fertilized plants belonging to the same first-generation family. 
All of these propositions have now been tested in a limited way. In 1910 nine different self-fertilized families were compared with nine crosses between sibs within the same self-fertilized family; ten crosses between sibs in $\mathrm{F}_{1}$ families were compared with ten self-fertilizations in the same $F_{1}$ families; seven families were raised as first generation hybrids between individuals belonging to different self-fertilized families; and ten families were grown, in which self-fertilization had been entirely precluded during the past five years. The average height of plants in decimeters, the average number of rows per ear, and the average yield in bushels per acre, in these fifty-five families are given in the following table:

\begin{tabular}{|c|c|c|c|c|c|c|c|}
\hline & $\begin{array}{l}\text { Selfed } \\
\times \text { Self } \\
\end{array}$ & $\begin{array}{l}\text { Selfed } \\
\times \text { Sibs }\end{array}$ & $F_{1}$ & $F_{2}$ & $F_{1} \times 2 e^{\prime} !$ & $F_{1} \times \operatorname{Sibs}$ & $\begin{array}{c}\text { Cross- } \\
\text { breds }\end{array}$ \\
\hline Height & 19. & 20 & 0 & 2 & 23 & 23.30 & 22.95 \\
\hline Av & 1 & 13 & 1 & 1 & 13.610 & 13.73 & 15.13 \\
\hline Av. Yiel & 29.04 & 30.17 & 68 & 44.62 & 41.77 & 47.465 & 61.52 \\
\hline
\end{tabular}

An examination of this table indicates to me that on the whole my self-fertilized families are not yet quite pure-bred; for the sib crosses give on the average a slightly greater height, number of rows per ear, and yield per acre than the corresponding self-fertilized families, as shown by a comparison of the first two columns of the table. The same fact is apparent from a comparison of the " $F_{1} \times$ self" and " $F_{1} \times$ Sibs" columns, except that in this case the heights and number of rows per ear are essentially equal while the yield per acre is significantly higher in the sib-crosses than in the selffertilized families. An alternative explanation of these slight differences between the results of self-fertilization and of sib-crosses may attribute them to an injurious effect of self-fertilization, but in any event such injurious effect must be exceedingly slight as compared with the stimulating effect of heterozygosis. My practise of choosing for seed the best available ears tends to delay the attainment of complete genotypic purity, and this fact favors the view that whatever advantages the sib- 
crosses show, are attributable to this lack of purity, rather than to any advantage gained by crossing per se.

The columns of the table representing the $\mathrm{F}_{1}$ and $\mathrm{F}_{2}$ show very plainly the superiority of the former over the latter in regard to both height and yield per acre. The fall in average height from $\mathrm{F}_{1}$ to $\mathrm{F}_{2}$ from 25 decimeters to 23.4 decimeters and the corresponding fall in yield per acre from 68.07 bushels in the $\mathrm{F}_{1}$ to 44.62 bushels per acre in the $\mathrm{F}_{2}$ show in a most striking way the economic advantage of using first-generation hybrids for producing the corn crop. A comparison of the $\mathrm{F}_{1}$ hybrids with the "cross-breds" shows the average yield of the former to be 6.55 bushels per acre greater than that in the families in which self-fertilization had been avoided.

The relation of these results to the experiences of economic breeders of corn may now be considered. Perhaps in no other class of plants has the evidence been so strong for the possibility of gradual improvement through continued selection as in corn, and this method has been generally followed. The selections of particular physical and chemical qualities which have been carried on at various experiment stations have produced noteworthy results. Most important instances of this kind are involved in the breeding experiences of Hopkins, Smith and other breeders at the Illinois State Experiment Station, which have been already mentioned. Here selections for high oil content, low oil content, high protein and low protein, high ears and low ears, and the angle which the ears make with the axis of the plant, as well as selection for increased yields, have all led to the production of strains which possessed the desired qualities to a much higher degree than that in which they existed in the foundation stock when the selection began. All of these results may be readily explained on the ground that some hybrid combinations of genotypes have greater capacity for the production of the desired qualities than other combinations, and that the selection has gradually brought about the segregation of those genotype-combinations 
which had the highest capacity for the production of the desired qualities. At least in regard to yield and not improbably also in regard to the other qualities for which selections were made, the results were dependent, not upon the isolation of pure types possessing the desired quality, but upon the securing and maintaining the proper combination of types. I have shown above that segregation takes place in a manner at least similar to, if not identical with, the well-known behavior of Mendelian characters. As a consequence of this, no strain of corn can be maintained at a high value with respect to any quality whose development is correlated with heterozygosis, except by continued selection for the particular qualities desired. If in any such specialized strain selections should be made for a few years on the basis of some character independent of the one used in establishing the strain, the superior qualities for which it was originally selected would quickly disappear, owing to the breaking up of the efficient combinations which had been segregated and maintained by selection.

The principles here presented have very great potential consequence for the practical grower of corn, and possibly for the breeder of many other cross-breeding plants and of animals. Their importance seems not to have been fully appreciated by any one however, until recently, though several breeders appear to have glimpsed the possibilities at one time or another. Thus G. N. Collins, ${ }^{9}$ of the United States Department of Agriculture, has recently shown that several breeders at different times began experiments to test the value of hybridization in the production of high-yielding strains of corn. The first attempt of this kind which he has found was that of W. J. Beal ${ }^{10}$ at the Michigan Agricultural College in 1876. At Professor Beal's instance several other experiment stations undertook to work in co-operation with the Michigan Station in testing the value of hybrids in

'Collins, G. N., "The value of first generation hybrids in corn,'" Bull. 191, U. S. Bureau of Plant Industry, 45 pp., 1910.

${ }^{10}$ Beal, W. J., Reports, Michigan Board of Agriculture, 1876-1881. 



\section{THE}

\section{Botanical Gazette}

\section{NOVEMBER IOII}

\section{REVERSIBLE SEX-MUTANTS IN LYCHNIS DIOICA ${ }^{\mathrm{I}}$}

GEORGE HARRISON SHULL

\section{(WITH FIFTEEN FIGURES)}

Six hermaphrodite specimens of Lychnis dioica L. were found in cultures of 1908, and eight in I909. With respect to their hereditary behavior in the first generation, when used as pollen parents, these hermaphrodites proved to be of two kinds, the individuals $A$ and $B$ being capable of determining the hermaphrodite character in their male offspring, while individuals $C$ and $D$ behaved exactly like normal males, giving progenies consisting of females and normal males.

The conclusion was reached (SHULL 26) that the hermaphrodites are modified males, because (I) in all families in which the first mentioned type of hermaphrodite was used as the pollen parent the offspring consisted of females and hermaphrodites in the same ratio as would have been expected of females and males if a normal male had been used as the pollen parent, and because (2) the second type of hermaphrodite when used as a pollen parent gave the same result that a normal male would have given.

Accepting tentatively the Mendelian explanation of sex first clearly enunciated by CoRRENS (6), which recognizes the one sex as homozygous and the other sex as heterozygous with respect to a sex-producing gene, it was decided that these hermaphrodites (and therefore also males) must be heterozygous, because ( $\mathrm{I}$ ) the males are capable of being modified in such manner as to display function-

I Read at the meeting of the Botanical Society of America, Minneapolis, December, Igro. 
al organs of both sexes, and because (2) self-fertilized hermaphrodites produce dimorphic progenies, consisting of females and hermaphrodites.

In my first paper on the inheritance of sex in Lychnis (SHULL 26), I represented the sex genes by the conventional signs for the sexes ( $q$, $\hat{b}$, and $\Varangle)$. As these signs were used in my tables with two different meanings - to represent sometimes the character of the genes and at other times the character of the soma-I suspect that readers may have experienced some difficulty in comprehending the tables. I shall therefore adopt here the plan usually followed by students of genetics, of representing the genes by letters, letting $\mathrm{Ff}$ be respectively the presence and absence of a female determiner, $M m$ a male determiner, and $H h$ a hermaphrodite determiner. The conventional signs for the sexes will be used in this paper only in their more usual signification, referring to the nature of the soma, that is, the sporophyte.

If CORRENS' view of sex determination is correct, and the males are heterozygous, the females must be homozygous. Castle (5) suggests that in such a case the females will always be positive homozygotes, having a pair of sex genes $(F F)$ corresponding with a single equivalent gene $(F f)$ in the male. I do not believe that this view can be substantiated, as there seems no good reason why females should not be negative homozygotes in some plants and animals, "neutral" homozygotes in others, and positive homozygotes in a third class. If the females are positive homozygotes, the somatic formula of the two sexes may be represented thus: $F F=q$, and $F f=\hat{\delta}$; if the females are negative homozygotes, the corresponding symbols will be $F F m m=q$, and $F F M m=\hat{\delta}$; and if the female is a "neutral" homozygote, the formulae of the two sexes will be $F F=q$, and $F M=\hat{\jmath}$. Only the first two of these assumptions concerning the nature of the females were considered in my earlier paper, and either was found capable of explaining the results secured in the first generation, provided the presence of a partially independent hermaphrodite factor $(H)$ might also be assumed.

Whether there was any genetic relationship between the hermaphrodites $A$ and $B$ which produced hermaphrodite offspring, and $C$ and $D$ which produced males, could not be determined in the first 
generation, and two explanations seemed possible: (I) these two types of hermaphrodites might be respectively homozygous and heterozygous in regard to a modifying factor $H$, whose presence was assumed, on the suggestion of CORRENS, as possibly necessary for the change of a normal male into a hermaphrodite; (2) the hermaphrodites of the second type $(C$ and $D)$, which gave first gen $€$ ration progenies equivalent to those produced by normal males, might owe their hermaphrodite character to some accident of development which affected the soma alone, leaving the germ cells unchanged. In this case they might be appropriately called "somatic hermaphrodites," to distinguish them from those of the first type $(A$ and $B$ ) which transmitted the hermaphrodite character to their male offspring and which are therefore to be recognized as "genetic hermaphrodites" or true hermaphrodite mutants.

Neither the character of the females nor the relationship of the two types of hermaphrodites could be determined from the results of the first generation, but it was obvious that at least a partial solution could be expected from the second generation. To attain this end a large number of crosses were made in 1909, by using hermaphrodite individuals and their derivatives in various combinations with each other, with unrelated females, and with normal males. The offspring of these crosses were grown during the summer of r9ro, and the 104 families produced from them included 6132 individuals which came to bloom and of which the sex was recorded. These records were made in the writer's absence by Mr. R. Catlin Rose, to whose energy, faithfulness, and care it gives me pleasure to testify.

In order to comprehend fully the problems involved, it will be advantageous to consider some assumptions which were permitted by the results of the $F_{x}$ crosses, and whose availability is partially tested in the $F_{2}$ families reported in the present paper. In this connection it is also important to consider briefly the "presence and absence" hypothesis, a full discussion of which, however, would require too great a digression. Although this so-called hypothesis is frequently referred to by students of genetics, I am not aware that it has ever had a very definite formulation, and it would undoubtedly be defined differently by different students. 
"Presence and absence" came into use in the first place, simply as a convenient method of expression to avoid the confusion which arises when the same dominant character is described as an alternative of several different characters which are hypostatic to it, and which may themselves be present or absent in any particular instance. The very general applicability of this mode of expression naturally suggested to various writers (Hurst I8, SHull 27, etc.) that it might have a more fundamental significance than merely as a convenient form of description. These authors considered it simpler and more practical to suppose that the heterozygous genes are unpaired, and that the "absence" of a character ${ }^{2}$ is unrepresented by any internal unit corresponding with the gene which determines the "presence" of that character. The "presence and absence" hypothesis need not be associated, however, with the conception of unpaired determiners in the heterozygote, for in any pair of organs there may be present a function or feature in one member of the pair which is absent in the other member, or both members may be alike in kind but different in quantity or activity, the differential between the two being in this case the determiner of the alternative characters involved. This excess in one member of the pair would be present, of course, in that member only, and must be absent in its mate. Whether the hypothesis of unpaired genes or that of paired genes represents the true condition in any particular instance, and whether the absence of a character is absolute or only relative, will not interfere in the least with the use of "presence and absence" as the most convenient method of stating a great majority of the alternative characters with which the student of heredity has to deal. For the application of these different phases of the "presence and absence" hypothesis to the sex problem in Lychnis, attention is directed to the following table:

${ }^{2}$ It is to be regretted that some writers have misconstrued the meaning attached by most geneticists to the expression "absence of a character." The absence of the Angora character in cats, rabbits, etc., does not result in a hairless animal, but one with short hair. In Oenothera the absence of rubricalyx pigmentation does not prevent the production of anthocyan in the amount and localization characteristic of $O$. rubrinervis (see Gates, R. R., Studies on the variability and heritability of pigmentation in Oenothera. Zeitsch. Ind. Abst. Vererb. 4:337-372. I9II). 


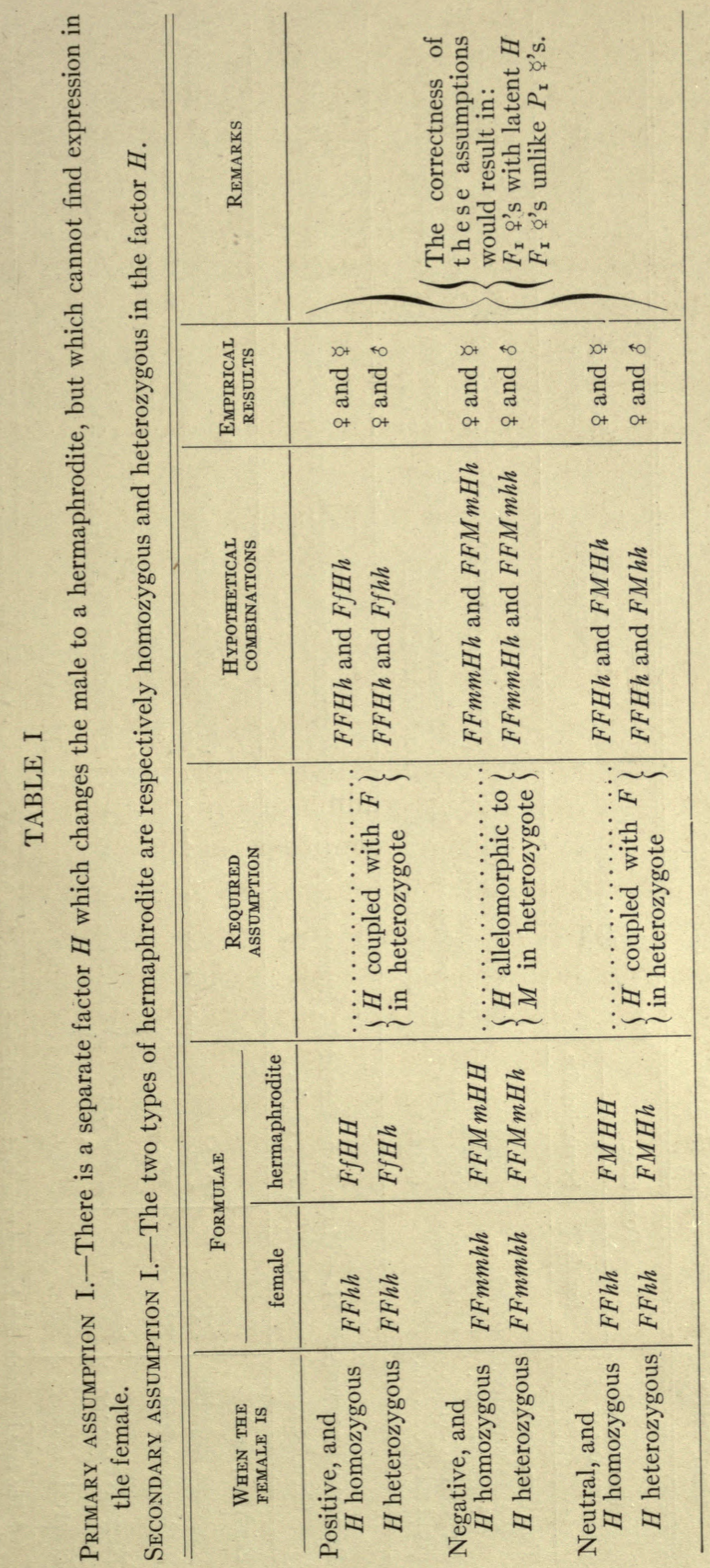




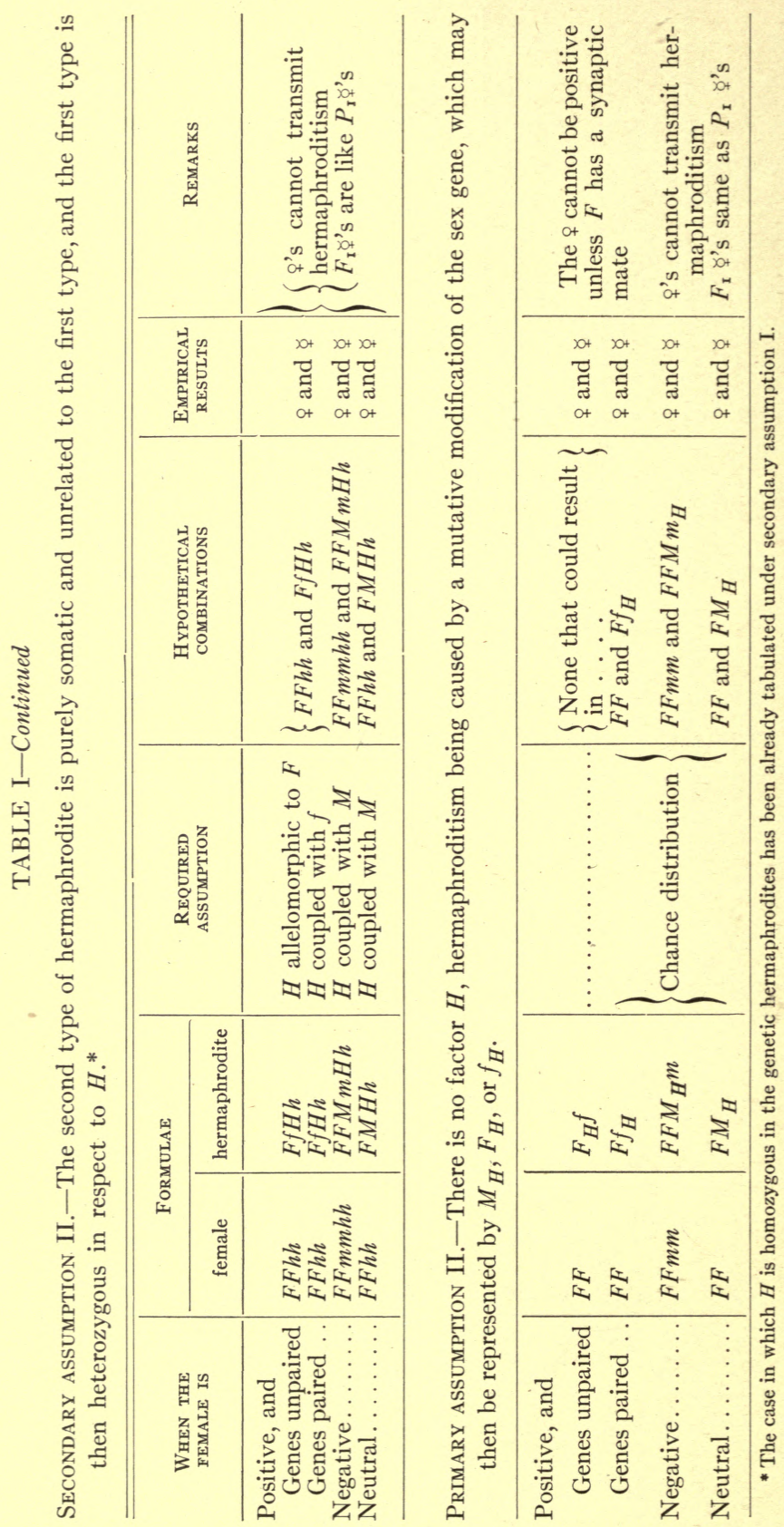


Particular attention should be given to only two points in this table until after the results secured in the second generation have been considered. The assumptions which form the basis of the first section of the table lead to the expectation (a) that females derived from hermaphrodite families, whether they be fertilized by normal males or by their hermaphrodite sibs, will yield families in which the male offspring are hermaphrodite and normal male in equal numbers; and $(b)$ that the hermaphrodites of the second generation when used to fertilize females from normal male families will produce no hermaphrodites, but only females and males. The alternative assumptions involved in the second and third sections of the table, on the other hand, lead to the expectation that, regardless of the origin of the female, no hermaphrodites will be produced normally, except when fertilization is brought about by sperms from a genetic hermaphrodite, and then the result will always be the same whether this hermaphrodite was a mutant or whether it was derived from an antecedent hermaphrodite.

We may now proceed to examine the results of the crosses. This will be most easily accomplished by considering each type of cross separately in the following fourteen cases. In the model pedigrees, illustrated under each case, the oldest ancestors entered in the diagrams are females and males both of which came from normal families, whose matings had been controlled during at least three still earlier generations, and which are known to have been in each such previous generation the result of crosses between females and normal males, and to have belonged to families in which no hermaphrodite mutants appeared. In the diagrams all male and hermaphrodite individuals which appeared as mutants are indicated as such, and it should be understood that any male or hermaphrodite not so marked was a member of a family which consisted of a normal proportion of its own type, that is, either male or hermaphrodite.

\section{CASE I}

Crosses of GENETIC HERMAPHROdite MUTANTS WITH FEMALES

Only 2 of the 8 plants recorded as hermaphrodites in 1909, in otherwise normal male families, were successfully used for breeding. One of these, bred to 2 different unrelated females, produced 72 
females and 88 hermaphrodites (nos. ogr23 and o9r7I). The other, bred to the same 2 females, produced Ir 6 females and 53 hermaphrodites (nos. 09124 and 09172). The result of these four crosses, involving 2 hermaphrodite mutants, was therefore I88 females and I4I hermaphrodites, thus showing that these 2 hermaphrodite mutants were of the same character as the two denomi-

\begin{tabular}{|c|c|c|c|}
\hline Pedigree no. & Result & Pedigree no. & Result \\
\hline \multirow[t]{2}{*}{ 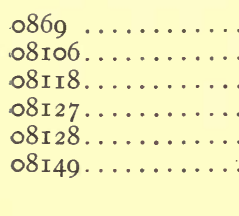 } & \multirow[t]{2}{*}{ 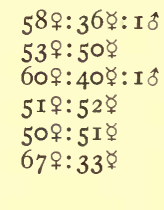 } & \multirow[t]{2}{*}{ 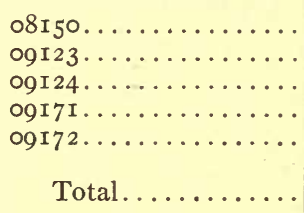 } & 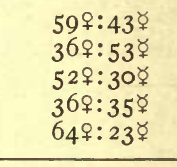 \\
\hline & & & 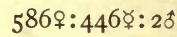 \\
\hline
\end{tabular}

nated $A$ and $B$ in my earlier report. For the sake of completeness, the crosses of $A$ and $B$ already reported are included in the tabulation of these crosses, the total progeny from this type of cross being 586 females, 446 hermaphrodites, and 2 males.

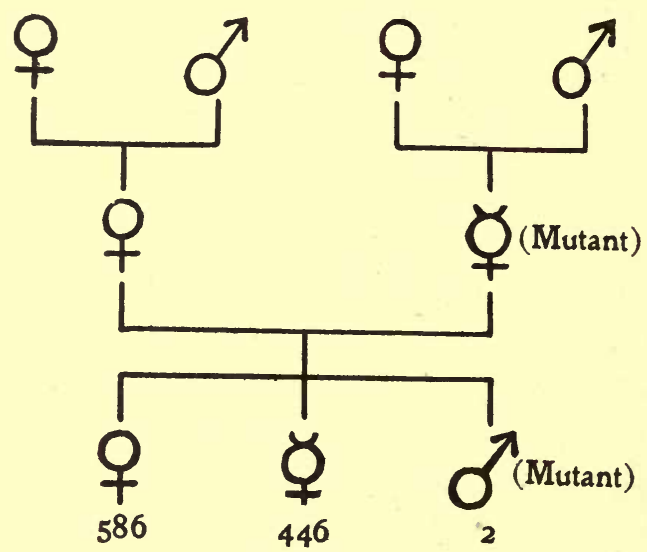

FIG. I.-Model pedigree for case I

Two other individuals, which had a derangement of the sexual characters of such a nature that the lobes of the calyx were transformed into stigmas, and in one instance a small ovary with apparently functional stigmas was present in the center of the flower and 
associated with functional stamens, were of such anomalous character that they have not been included among the 8 recognized hermaphrodites found in normal families in I909, but they will be mentioned later under case XIII in connection with the somatic hermaphrodites $C$ and $D$ of my preliminary report.

CASE II

WHEN GENETIC, HERMAPHRODITE MUTANTS ARE SELF-FERTILIZED

\begin{tabular}{|c|c|}
\hline Pedigree no. & Result \\
\hline 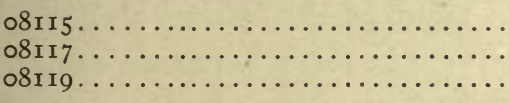 & 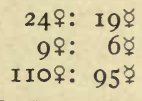 \\
\hline 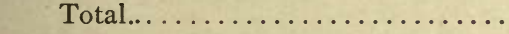 & I43ㅇ: I 20 భ \\
\hline
\end{tabular}

None of the new hermaphrodite mutants discovered in 1909 were successfully selffertilized, and the pedigrees here reported are repeated from my former paper for the sake of completeness. All the self-fertilized hermaphrodites which yielded progenies in my I9Io cultures belonged to a later generation, being offspring of a self-fertilized hermaphrodite and not the progeny of new mutants. They consequently belong to a separate case and will be considered next. The agreement of these results with those under case I leads to the conclusion that the eggs of the hermaphrodite are all of one type, that is, female-bearing, like those of the females. The significance of this result will be considered later.

CASE III

WHEN F HERMAPHRODITES ARE SELF-FERTILIZED

\begin{tabular}{|c|c|c|c|}
\hline Pedigree no. & Result & Pedigree no. & Result \\
\hline 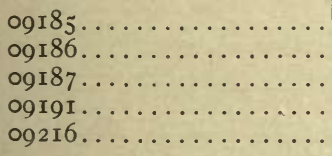 & 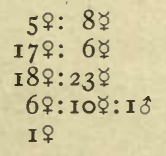 & $\begin{array}{l}09217 \ldots \ldots \ldots \ldots \ldots \\
09218 \ldots \ldots \ldots \ldots \\
09219 \ldots \ldots \ldots \ldots \ldots \\
09220 \ldots \ldots \ldots \ldots \\
09222 \ldots \ldots \ldots \ldots \ldots\end{array}$ & 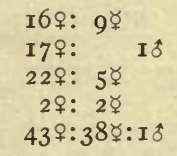 \\
\hline & & Total & I $47+:$ IOI $\varnothing: 30$ \\
\hline
\end{tabular}


The first four of these families were produced by self-fertilizing 4 individuals of pedigree number $08 \mathrm{II}_{5}$, and the rest by selffertilizing 6 individuals of number o8rig. Most of these families were too small to show obvious differences in the genetic composition of the different parent plants, or between them and the

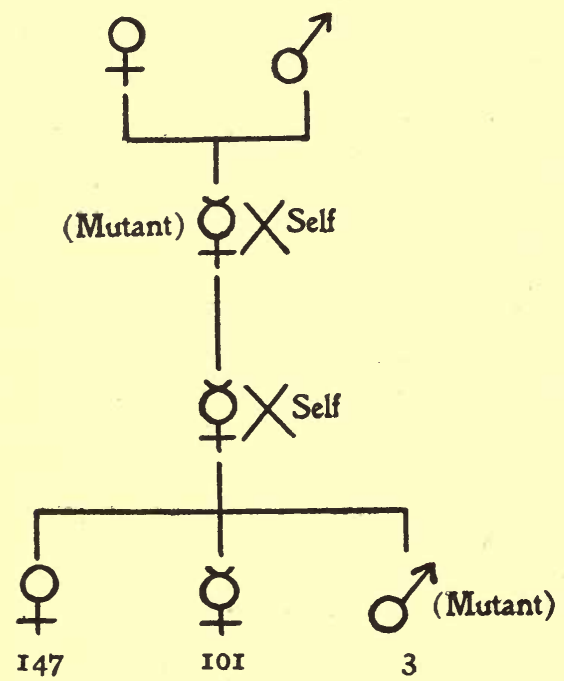

Fig. 3.-Model pedigree for case III hermaphrodite mutants tested under case II. The small size of the families is due to the comparatively poor development of the ovaries and stigmas in most hermaphrodites, and the consequent difficulty of securing large quantities of seeds by self-fertilization. Most of the attempts to selffertilize the hermaphrodites resulted in failure, and only in a small proportion were any seeds produced. The total result agrees with results secured from the observation of larger families, and it is fair to assume that the relatively large differences shown by some of these families are not significant because of the smallness of the progenies. This conclusion will be fully justified I believe, when it is observed under case IV that the very same plants, which produced the somewhat variable progenies shown above, gave uniform results when they were crossed with an unrelated female.

\section{CASE IV}

WHEN HERMAPHRODITES FROM THE PROGENY OF A SELF-FERTILIZED HERMAPHRODITE MUTANT ARE CROSSED WITH AN UNRELATED FEMALE

The families $09 \mathrm{r} 33$ to $09 \mathrm{I}_{2}$, inclusive, resulted from pollinating different flowers of a single female, o8 I I4(4), with the pollen from ro different hermaphrodites taken consecutively in family o8II5, and the remaining is families were produced by pollinating the same female, o8I I4 (4), with pollen from I9 different hermaphrodites 
in family 08rig. This series of experiments, like those under case III, was calculated to discover any genetic differences which

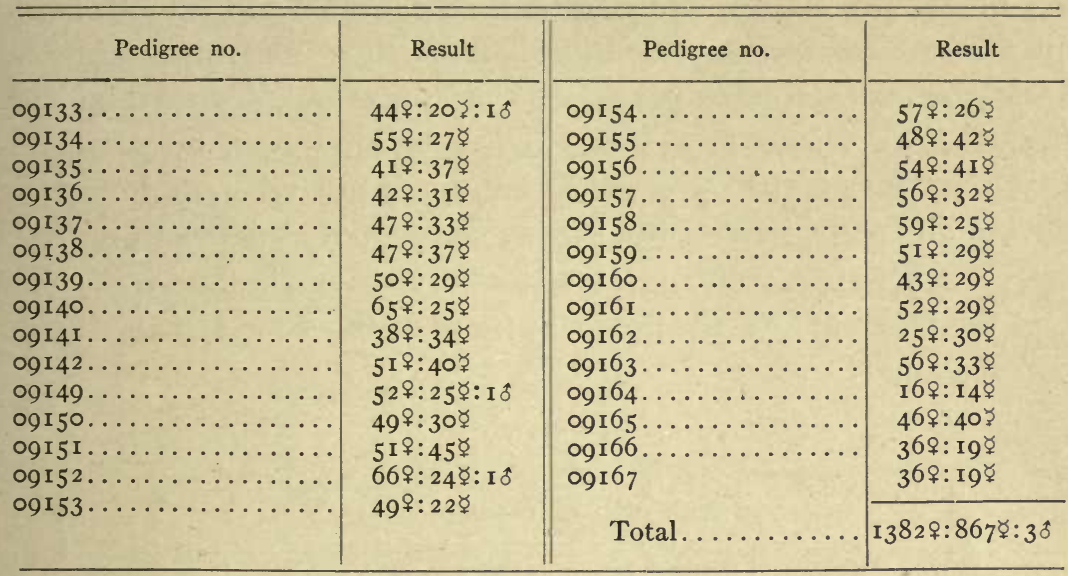

might exist among $F_{2}$ hermaphrodites, and the fact that these 29 different individuals when crossed with a single female produced

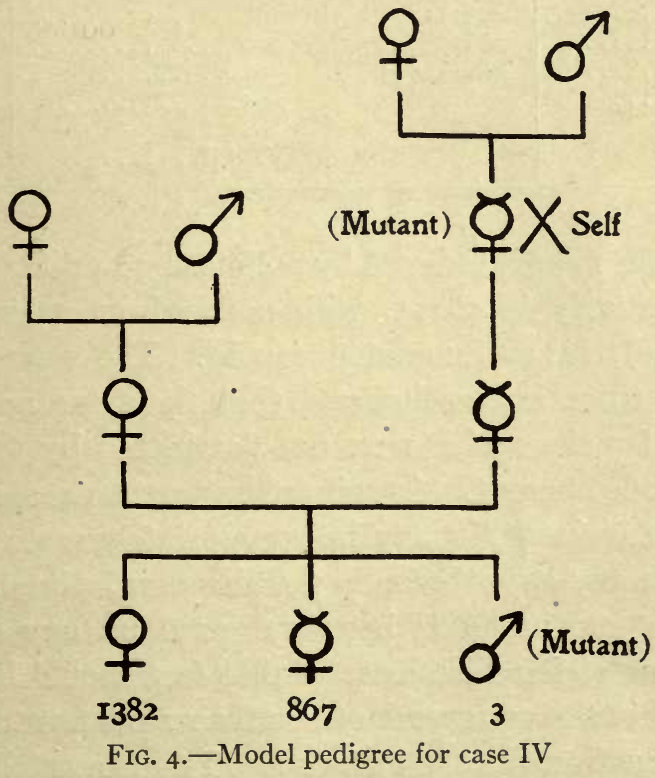

essentially identical results leads to the conclusion that no such genetic differences existed. This conclusion is apparently open to 
but one criticism; the characters of the female chosen to be the mother of all these families might dominate such different characters as were possessed by the hermaphrodites, in which case all families would show identical composition regardless of the variations in the pollen parents. This suggested dominating influence of the female is rendered untenable, however, by the fact that the same female was pollinated by 7 other hermaphrodites having different histories from those considered under the present case, and also by I I different males of diverse origin, and in every case the males among the progenies were of the same type as their pollen parent.

\section{CASE V}

WhEN FEMALE OFFSPRING OF SELF-FERTILIZED HERMAPHRODITES ARE CROSSED WITH AN UNRELATED MALE

\begin{tabular}{|c|c|c|c|}
\hline Pedigree no. & Result & Pedigree no. & Result \\
\hline 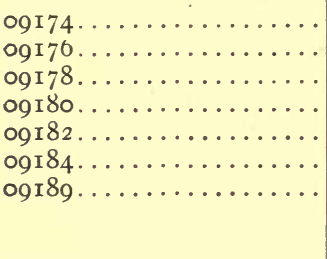 & 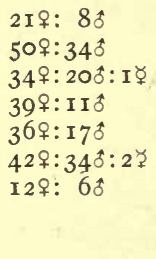 & 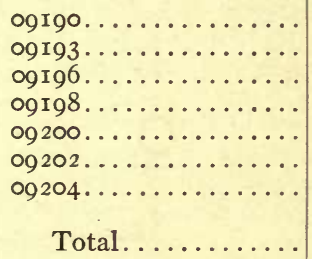 & 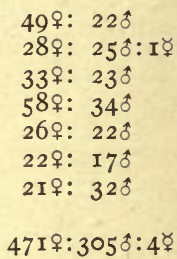 \\
\hline
\end{tabular}

These families were produced by pollinating $\cdot \mathrm{I}_{4}$ different females, taken consecutively in 08 II $_{5}$, with pollen from a single normal male, $0855(36)$, in an unrelated family. The essentially equal results of all these crosses indicate that there are no differences among these females which were not dominated by the sex character of the pollen parent. As this pollen parent was a male from a normal male parentage, it may be appropriately assumed to have been free from any hypothetically possible hermaphrodite modifier $H$. If such a modifier had been possessed by any of these I4 females, a more striking evidence of that fact should be presented than is found in the occurrence of less than I per cent of hermaphrodite individuals among the offspring. This is a smaller percentage of hermaphrodites than has been found in one or two cases among the offspring of a female pollinated by a normal male, neither 
parent having had any hermaphrodite connections. It appears fair, therefore, to consider these four hermaphrodites simply as mutants, and not as genetic derivatives from their maternal grandfather. The few hermaphrodites occurring in the families included under the present case may be related to the fact, however, that the females belong to a hermaphrodite family, for the same male $0855(36)$ was crossed with seven other females and with one hermaphrodite, and among the 443 offspring produced there were no other hermaphrodites.

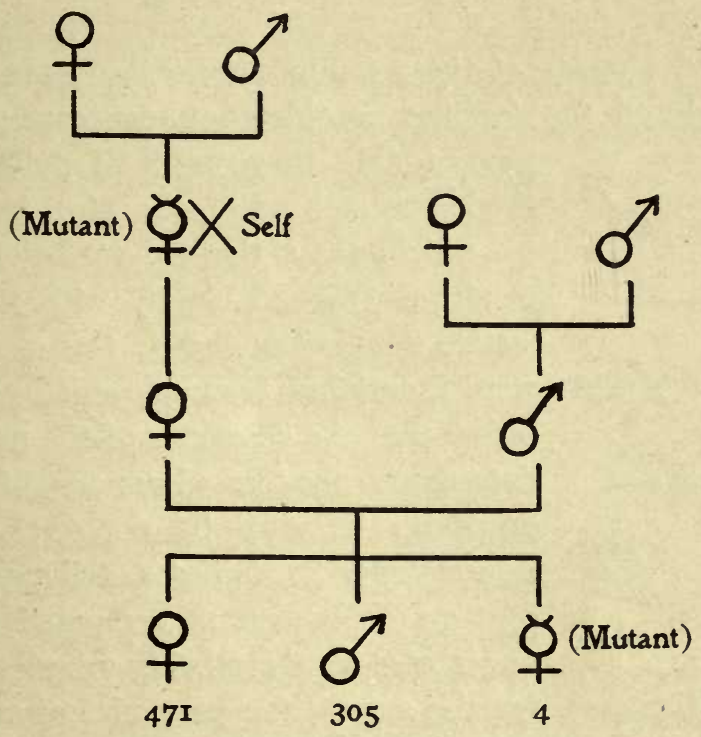

FIG. 5.-Model pedigree for case $\mathrm{V}$

Allowing for the same frequency of occurrence of hermaphrodites as shown in the table above, there should have appeared among these 443 individuals derived from the same male crossed with other females at least two hermaphrodite mutants. This number is so small that they may possibly have been omitted through the errors of random sampling, but the suggestion may be made that while a female cannot transmit hermaphroditism to its offspring, it may perhaps supply an intracellular environment favorable to the mutation of the male genes into hermaphrodite genes. 
CASE VI

WHEN THE DAUGHTERS OF A SELF-FERTILIZED HERMAPHRODITE ARE CROSSED WITH ONE OF THEIR HERMAPHRODITE SIBS

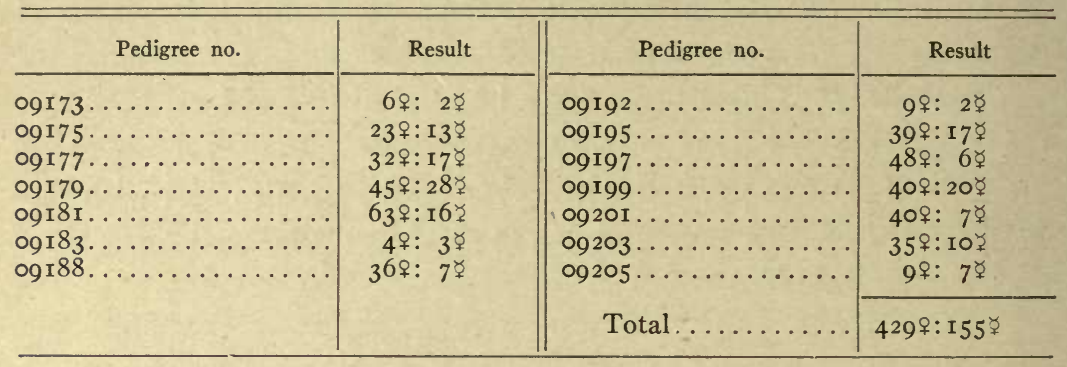

The seed parents of these families were the same I4 females which produced the families considered under case $V$. In the

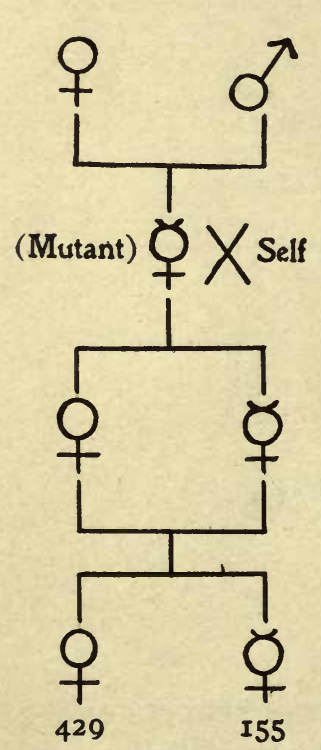

FIG. 6.-Model pedigree for case VI. present case they were all pollinated by a single hermaphrodite, o8II5(9), in the family to which they themselves belonged. The results correspond closely with those of the last section, except that in this case the males were invariably hermaphrodites, showing as before that the character of the pollen parent determines the sex character of the male offspring. It may be noted that most of these families contained a strikingly high percentage of females, as compared with those under case $\mathrm{V}$, there being 73.46 per cent of females among the progenies of case VI, and only 60.7 per cent among those of case $\mathrm{V}$. The meaning of such differences in the sex ratios is quite unknown at the present time, and no discussion of the series of experiments which are in progress for the purpose of finding an interpretation of such variable ratios will be undertaken here. It is believed, however, that the question of the sex-ratios constitutes an altogether different problem, and has no direct bearing upon matters relative to the genetic interrelationships of the different sexual types, which are alone under consideration in this paper. 
CASE VII

WHEN HERMAPHRODITE OFFSPRING OF AN OUT-CROSSED HERMAPHRODITE MUTANT ARE CROSSED WITH UNRELATED FEMALES

\begin{tabular}{|c|c|c|c|}
\hline Pedigree no. & Result & Pedigree no. & Result \\
\hline \multirow[t]{2}{*}{ 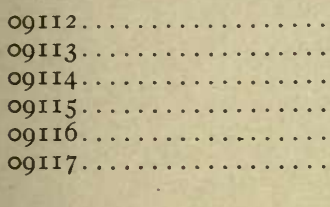 } & \multirow{2}{*}{ 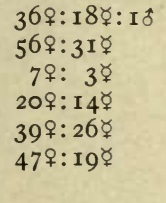 } & $\begin{array}{l}\text { og } 118 \ldots \ldots \ldots \ldots \ldots \\
\text { ogr } 45 \ldots \ldots \ldots \ldots \ldots \ldots \\
\text { ogr } 46 \ldots \ldots \ldots \ldots \ldots \ldots \\
\text { ogr } 48 \ldots \ldots \ldots \ldots \ldots \ldots \\
\text { og } 260 \ldots \ldots \ldots \ldots \ldots \ldots\end{array}$ & 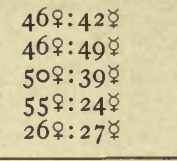 \\
\hline & & Total........ & 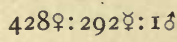 \\
\hline
\end{tabular}

These families are essentially similar in nature to those considered under case IV, except that in the present case the mutant was crossed with an unrelated female instead of being self-fertilized. The first 7 of these families were produced by crossing 7 different

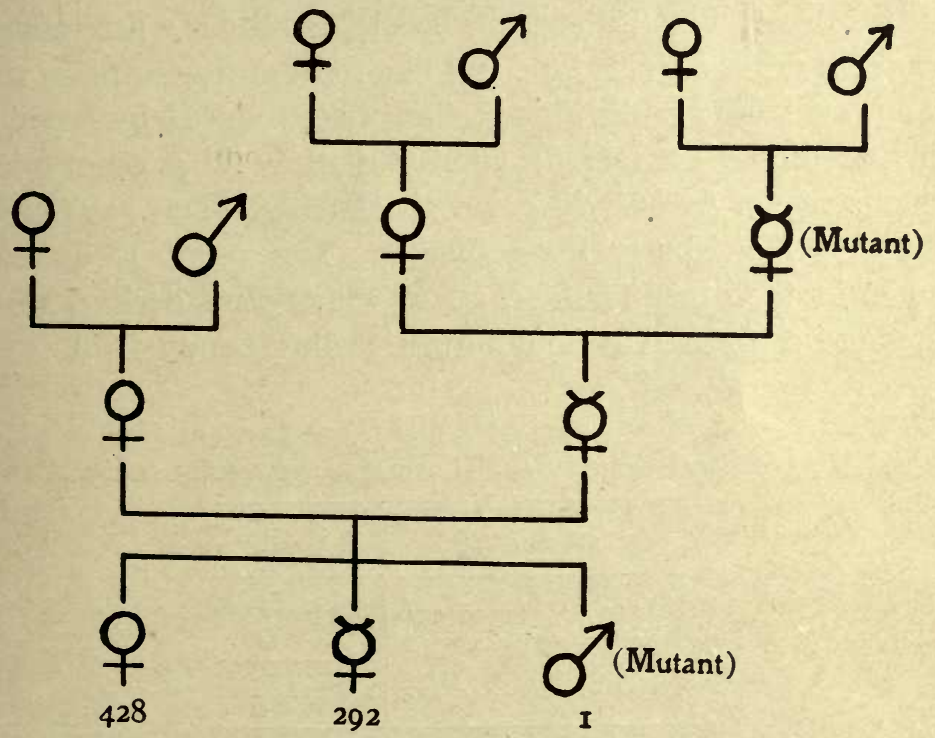

Fig. 7.-Model pedigree for case VII

hermaphrodites in $08 \mathrm{II} 8$, upon a single female, 08rog(r); the next three (09145-09148) were the result of using three of the same hermaphrodite individuals in the pollination of the female, $08 \mathrm{II}_{4}(4)$, which was used as the seed parent of all the families included under 
case IV. The genetic equivalence of the different hermaphrodites again stands out clearly in these results, and when the ratios of the two series are compared, it is found that the percentage of hermaphrodites produced by the hermaphrodite offspring of a self-fertilized hermaphrodite is slightly lower than that produced by the offspring of these cross-bred hermaphrodites, the former producing only 38.2 per cent of hermaphrodites and the latter 42.6 per cent. The difference is too small to be of significance, particularly in view of the fact that much wider differences than this are found in families produced from different seed capsules on a single plant when pollinated by a single male. It might have been expected, perhaps, that a self-fertilized hermaphrodite would have produced a larger percentage of hermaphrodites than would be produced by the same hermaphrodite crossed upon a female of a normal family. The fact that such a result does not appear is further proof that, although the hermaphrodite is a heterozygote, its egg cells are of a single type and like those of the normal females.

The last family under this section was produced by crossing a hermaphrodite of 08I28(I6) upon a female in a genotypically distinct strain of Lychnis dioica, received several years ago from the vicinity of Harrisburg, Pennsylvania. The result is quite the same as in the other families, all of which were derived from a common stock secured at Cold Spring Harbor, Long Island.

CASE VIII

WHEN HERMAPHRODITES ARE POLLINATED BY NORMAL MALES

\begin{tabular}{|c|c|}
\hline Pedigree no. & Result \\
\hline $\begin{array}{l}\circ 8 \mathrm{II} 6 \ldots \\
09215 \ldots\end{array}$ & 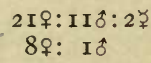 \\
\hline Total. & $29 \uparrow: 12 \widehat{\delta}: 2 ఫ$ \\
\hline
\end{tabular}

I have already remarked the difficulties encountered in the use of hermaphrodites as self-fertilized seed parents. The difficulties are still greater when the problem requires the crossing of the hermaphrodites with other males, for nearly all the numerous castrations which have been made have resulted in the dropping 
of the flowers without further development. Only one family (08II6) was produced in 1909 from a cross of this kind. It was

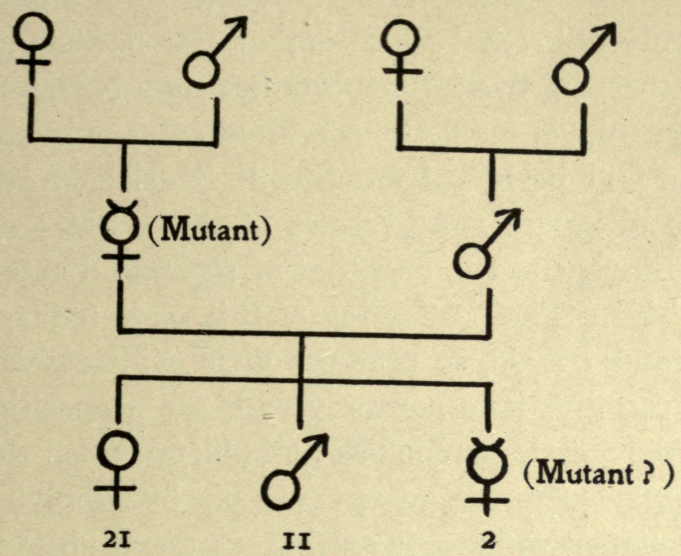

FIG. 8.-First model pedigree for case VIII .

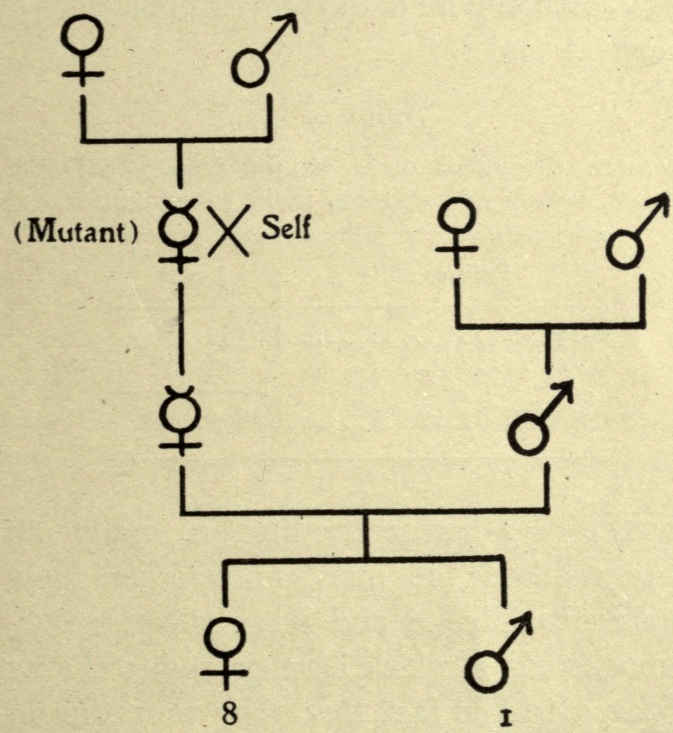

FIG. 9.-Second model pedigree for case VIII

reported upon in my preliminary paper, and is repeated here. The occurrence of two hermaphrodites in this small family suggested that the hermaphrodite character might be inherited from the 
mother as well as the father. On this account the cross between hermaphrodites and males must be considered the most important of all combinations in interpreting the relations of the sexes. The difficulty involved in the castration of the flowers permits the question whether the two hermaphrodites may not have been due to a faulty technique, for males produced from unintentional selfpollinations would be hermaphrodites. ${ }^{3}$ Special efforts were put forth in 1909 to secure more crosses of this character, but these resulted in a single success, and that of so limited extent as to be wholly indecisive. The 9 offspring of this cross (092 I 5) consisted of 8 females and I male, so that the little evidence which such a small family can give is in harmony with the proposition that the character of the female parent has no influence upon the sex characters of the male offspring, except possibly by supplying an intracellular environment which is favorable or unfavorable to the occurrence of sex mutation, suggested under case V. Continued efforts are being made to secure more data from combinations of hermaphrodites with normal males.

CASE IX

WHEN HERMAPHRODITE OFFSPRING OF A HERMAPHRODITE MOTHER AND NORMAL MALE FATHER ARE CROSSED UPON AN UNRELATED FEMALE

\begin{tabular}{|c|c|}
\hline Pedigree no. & Result \\
\hline 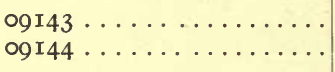 & $\begin{array}{l}38 \%: 38 \% \\
519: 33 \text { 우 }\end{array}$ \\
\hline 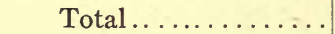 & $89 \%: 71 \not$ \\
\hline
\end{tabular}

The appearance of 2 hermaphrodites in family $081 x 6$ of case VIII immediately raised the question whether they were true genetic hermaphrodites like $A$ and $B$, or whether they might not be somatic hermaphrodites whose hermaphrodite character was not in any way related to the fact that they were the offspring of a hermaphrodite seed parent. If they should prove to be somatic

3 In a family grown in I9I I from a cross between a white-flowered hermaphrodite and a homozygous blue-flowered male, all the offspring were blue-flowered and several (less than 6 per cent) were hermaphrodite, thus showing that such hermaphrodites are not in this instance due to any unintentional self-fertilization. 
hermaphrodites, they would be in reality of the same genotype as their pollen parent, thus offering no exception to the general rule that the male parent determines the sexual type of its male offispring.

Both of these hermaphrodites were crossed upon female o8 I I 4 (4), already mentioned in cases IV and VII. No influence of the male grandparent appears, as all of the male offspring in these two

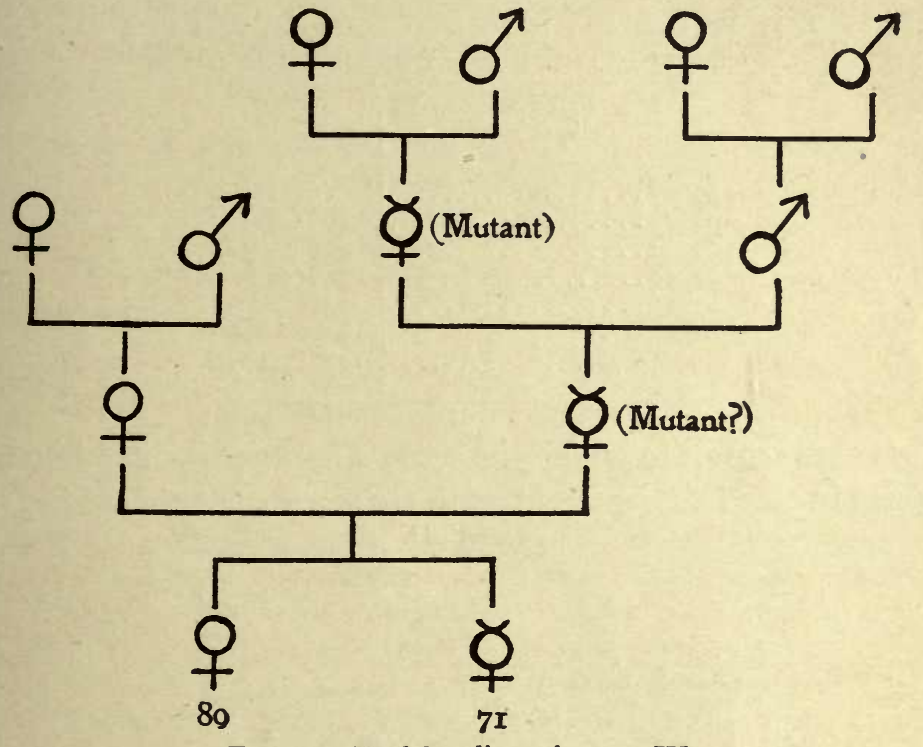

Fig. I0.-Model pedigree for case IX

families were hermaphrodites. This result proves that the 2 hermaphrodites of o8rir6 were genetic hermaphrodites. One of these hermaphrodites was also self-fertilized and gave a progeny of a single hermaphrodite, constituting family number o92 го. It would be rash to draw a conclusion from a family consisting of a single individual, and nothing could have been derived from it if by chance that individual had been a female. The fact that it was hermaphrodite instead of normal male, however, confirms the conclusion that the hermaphrodite parent was a genetic hermaphrodite like its own seed parent.

Whether these two hermaphrodites owed their hermaphrodite character directly to their hermaphrodite mother, or whether it 
resulted from a mutation of the male genes received from their father, cannot be definitely decided, but further experiments are in progress to test the possibility that the eggs of hermaphrodites can carry hermaphroditism and may therefore sometimes transmit it to their offspring. The evidence thus far is against their doing so to any considerable extent.

\section{CASE X}

Crosses between females and their hermaphrodite Sibs in a Family proDUCED BY CROSSING HERMAPHRODITE AND MALE

\begin{tabular}{|c|c|}
\hline Pedigree no. & Result \\
\hline 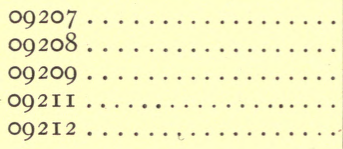 & 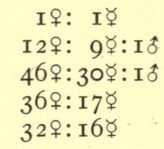 \\
\hline Total.. & I $27+9: 73 \not{q}: 2 \hat{0}$ \\
\hline
\end{tabular}

These are crosses in which the same 2 hermaphrodites of 08 II6, discussed in case IX, were used as the pollen parents in crosses with

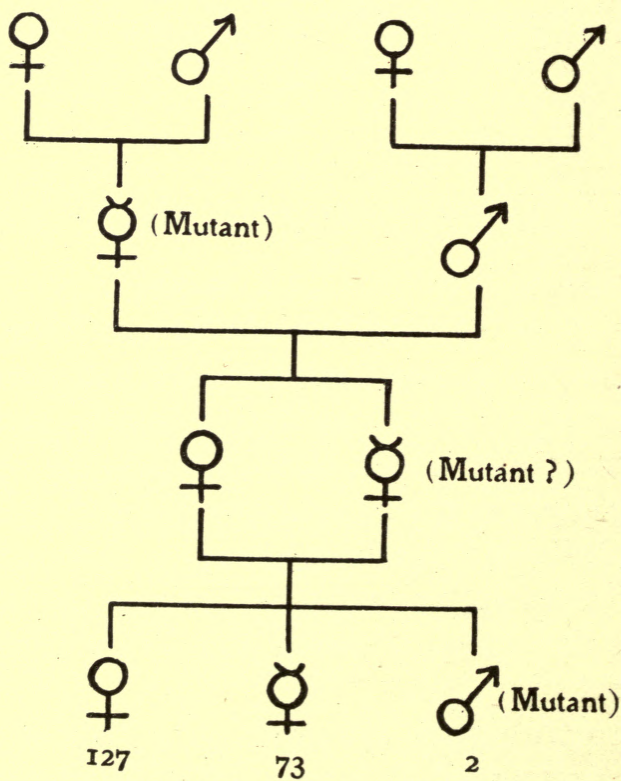

FIG. II.-Model pedigree for case $\mathrm{X}$ 
three different females in the same family. The results may be compared with those under case VI, where sib crosses were also dealt with. The comparison shows that the results were identical, though in one case the parents were the progeny of a self-fertilized hermaphrodite, while in the other the parents resulted from the cross of a hermaphrodite fertilized by a male. Thus is given still further evidence that these hermaphrodites in $081 \mathrm{I} 6$ were genetic hermaphrodites and that such hermaphrodites are of like hereditary capacity, whatever their origin.

\section{CASE XI}

WHEN DAUGHTERS OF A HERMAPHRODITE MOTHER AND MALE FATHER ARE CROSSED WITH AN UNRELATED MALE

\begin{tabular}{|c|c|}
\hline Pedigree no. & Result \\
\hline 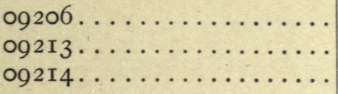 & 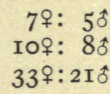 \\
\hline Total... & $50 \%: 34^{\wedge}$ \\
\hline
\end{tabular}

The first of these families (09206) had the same seed parent as the first two families (09207 and 09208) under case $\mathrm{X}$, and the

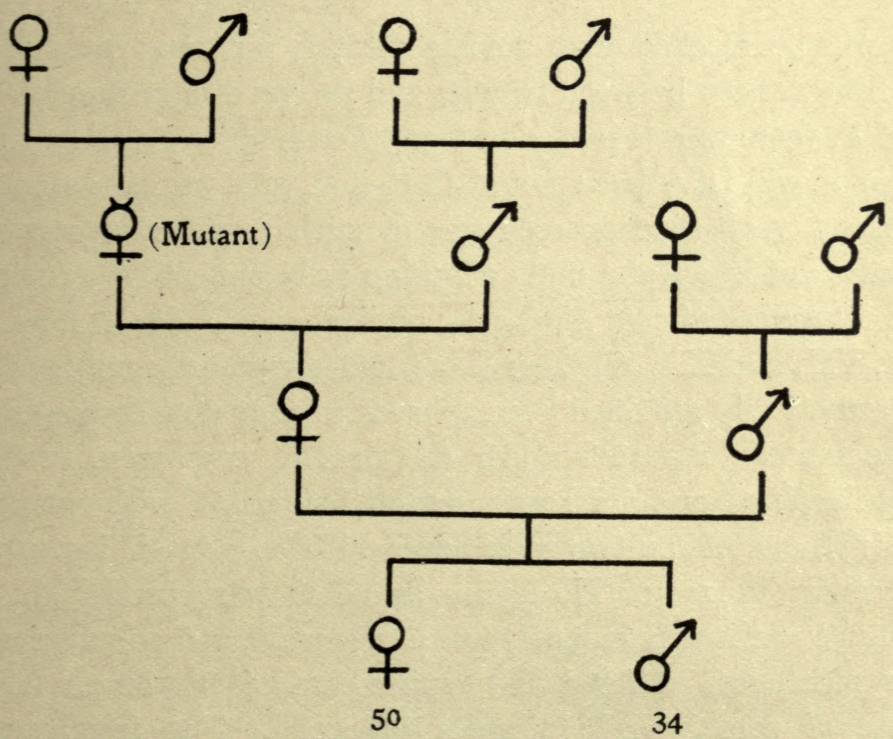

Fig. 12.-Model pedigree for case XI 
second $\left(092 \mathrm{I}_{3}\right)$ had the same seed parent as the last two families (092 I I and 092 I2) under that case. The pollen parent in all three families of the present case was the same normal male, $0855(36)$, that was used for all the crosses in case V. It is consequently fair to assume that the differences in the result under case $\mathrm{X}$ and case $\mathrm{XI}$ are wholly referable to the male parent, and that such differences as appear between case $\mathrm{X}$ and case $\mathrm{V}$ are referable to the seed parents. There is no difference in the latter instance, while the fundamental difference in the former is that in case $\mathrm{X}$ the males were hermaphrodite, while in the present case they were normal males, thus showing again the correspondence between the male offspring and their pollen parent.

\section{CASE XII}

When Male MUtants aRe CRossed With UNRElated females

\begin{tabular}{|c|c|}
\hline Pedigree no. & Result \\
\hline 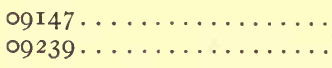 & 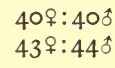 \\
\hline Total.... & $83^{\circ}: 84^{\dagger}$ \\
\hline
\end{tabular}

It will be recalled that among the 705 offspring produced in r 909 from crosses between females and the genetic hermaphrodites, $A$ and $B$, there were 2 males and 305 hermaphrodites. In similar manner it will have been noted that in a number of the cultures of rgro a very small percentage of such males have appeared in families of which the male offspring were generally hermaphrodite. Instances of this kind are noted above, under cases I, III, IV, V, VII, and X. Whether these males were true males or possibly somatically modified hermaphrodites may now be considered.

The families reported under the present case were produced by pollinating two different unrelated females with pollen of o81 I8(13), one of the two males derived from genetic hermaphrodite fathers in 1909. No hermaphrodites were produced, thus showing that the pollen parent was a true male, and not a hermaphrodite which had suffered the suppression of the female organs because of some purely somatic influence. The frequency of occurrence of such male mutants may be inferred from the fact 
that II of them appeared among progenies comprising a total of $333 \mathrm{I}$ females and $2 \mathrm{I} 26$ hermaphrodites. In other words, they constitute about 0.2 per cent of the total progeny of the genetic hermaphrodites when the latter are used as pollen parents. In no single family did more than one such male mutant occur. While these numbers are too small to allow an accurate estimate of the

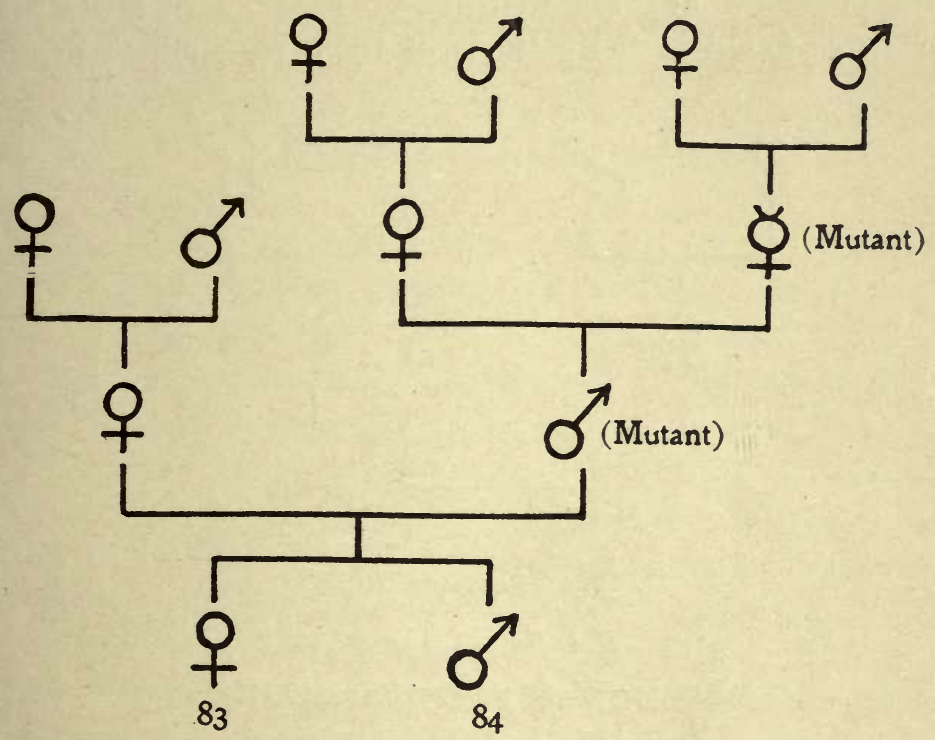

FIG. I3.-Model pedigree for case XII

relative frequency of hermaphrodite and male mutants, the evidence seems to indicate that there is no striking difference between the capacity of males to give rise to hermaphrodite mutants, and that of hermaphrodites to give rise to male mutants, though male mutants have appeared with slightly greater frequency than hermaphrodite mutants.

CASE XIII

WHEN SOMATIC HERMAPHRODITES ARE CROSSED WITH UNRELATED FEMALES

\begin{tabular}{|c|c|}
\hline Pedigree no. & Result \\
\hline 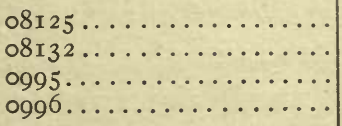 & 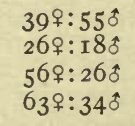 \\
\hline Total. & I $84^{\circ}: 133^{\hat{1}}$ \\
\hline
\end{tabular}


The pedigrees 08I25 and 08I32 are those of hermaphrodites $C$ and $D$ among the cultures of 1909 , which were reported upon last year. If the "model pedigree" illustrated in the diagram (fig. I4) be compared with that under case I (fig. I), the two will be seen to correspond perfectly. In fact, the hermaphrodites $A$ and $B$ included under case I were full sibs of hermaphrodites $C$ and $D$ whose progenies are repeated here. These 4 hermaphrodites which were found in the cultures of 1908 were indistinguishable

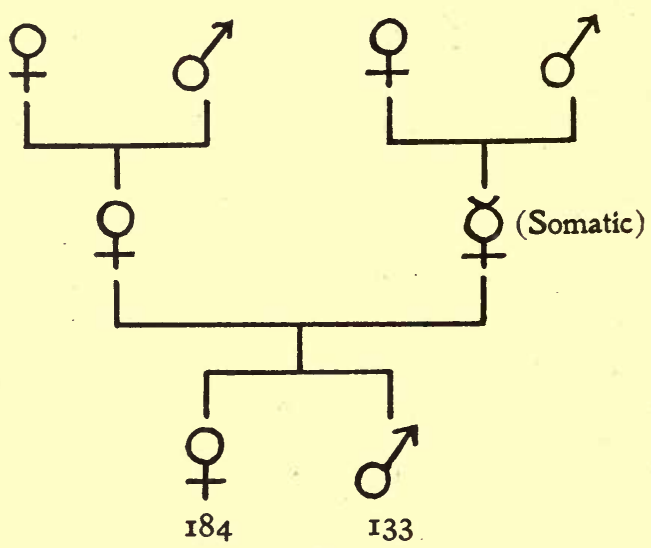

FIG. I4.-Model pedigree for case XIII

from one another in their external characters, and the fact that they belonged in two different categories was only demonstrated by the breeding tests.

No additional instances have been found in which a hermaphrodite indistinguishable from the usual type of "genetic hermaphrodites" has proved to be simply a somatic variation of the male. However, 2 peculiar variant individuals found in one family of the I909 cultures exhibited an analogous behavior, and consequently their progenies have been added to those of $C$ and $D$ under this case. The 2 individuals used as pollen parents of the families 0995 and 0996 had several lobes of the calyx prolonged and modified to the form and structure of stigmas, and one of the flowers had in the center a small unicarpellary ovary with an apparently functional stigma. Both of these plants had func- 
tional stamens, and both approached more nearly to the type of normal males as the season advanced. On account of the anomalous position of the stigmas in these plants, they are not to be included in the same class with the other hermaphrodites which have been considered, but it may not be unfair to accept the appearance of stigmatic calyx teeth in these male plants as additional evidence that the male is heterozygous in regard to sex, but normally has the presence of the female character completely hidden by the dominance of the male character. A somatic derangement may be assumed as the proximate cause of the appearance of the misplaced stigmas.

These 2 abnormal plants were crossed upon a female sib, 08Io9(I), and produced together II9 females and 60 normal males, not one of which showed any development of stigmatic calyx lobes or other female characteristics. The female 08Iog(I) was the one used in case VII for a number of crosses with genetic hermaphrodites, and it was also used as the seed parent in 20 crosses with males of various origin. In all of the other crosses upon this female, the males among the progenies were of the same type as the male parent used in the particular cross from which they sprang, thus showing that this female exerted no modifying influence upon the sex character of her male offspring. This makes it reasonable to conclude that the stigmatic calyx lobes were a purely somatic variation.

CASE XIV

The SECOND GENERATION FROM A SOMATIC MALE

\begin{tabular}{|c|c|}
\hline Pedigree no. & Result \\
\hline 09119. & $509: 27{ }^{\wedge}$ \\
\hline ogr $20 \ldots \ldots \ldots \ldots \ldots \ldots$ & $37 q: 16 \hat{\jmath}$ \\
\hline ogr $21 \ldots \ldots \ldots \ldots \ldots$ & $33 f: 26 \hat{s}$ \\
\hline $09122 \ldots$ & $619: 14 \hat{b}$ \\
\hline ogr $68 \ldots \ldots \ldots \ldots$ & $49 \Re: 42 \hat{b}$ \\
\hline$\circ q_{169} \ldots \ldots \ldots \ldots \ldots \ldots$ & 45 : $10 \hat{~}$ \\
\hline $09170 \ldots \ldots \ldots \ldots \ldots$ & $589: 33^{6}$ \\
\hline Total.. & $333^{\circ}: 168$ \\
\hline
\end{tabular}

In order to make sure that the conclusions drawn from the first generation regarding the character of the hermaphrodites $C$ and $D$, 
as discussed in case XII, were sound, and that there was not simply the temporary disappearance of the hermaphrodite character through some thinkable vagary of dominance in the $F_{I}, 5$ males in 08I25 were tested in crosses with 2 different females. The resultant progenies consisted of 333 females and I68 males. Not a single hermaphrodite appeared, thus convincingly supporting

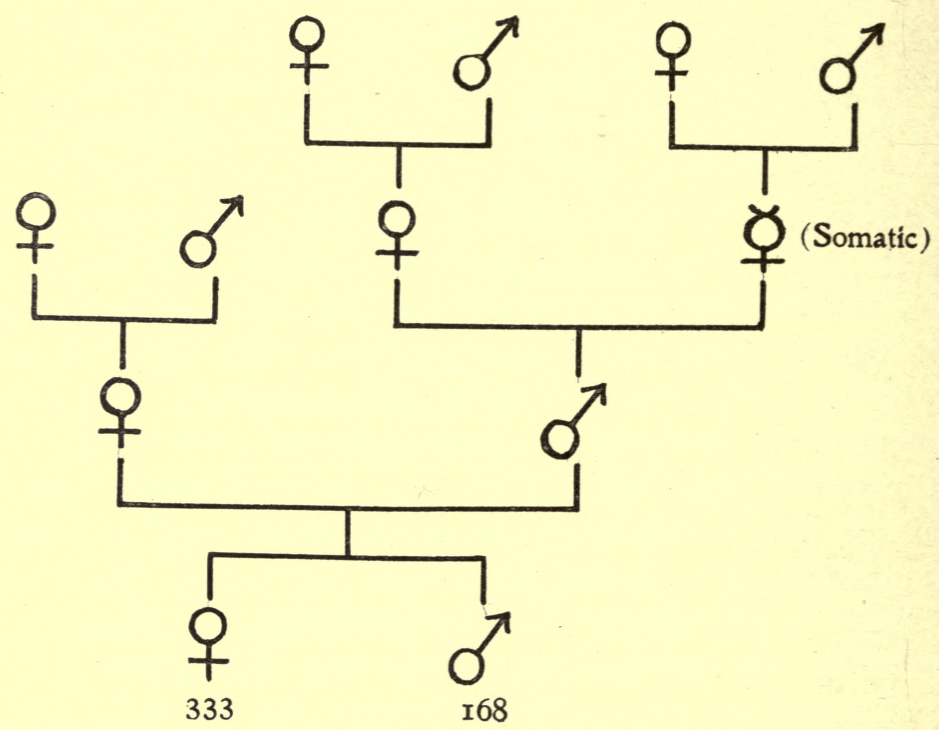

FIG. I5.-Model pedigree for case XIV

the view that the appearance of hermaphroditism in $C$ and $D$ was illusive, and that they were therefore only superficially like the genetic hermaphrodites $A$ and $B$. These results fully justify my conclusion that the hermaphrodites of Lychnis dioica belong to two genotypes, one of which is the same as the normal male, the other different from it.

\section{Discussion and conclusions}

Although these data from the breeding of hermaphrodites of Lychnis dioica are presented in fourteen sections, each representing a somewhat different direction of attack upon the genetic problems involved, the results under the various sections are remarkably consistent. The hermaphrodites are clearly of two kinds. Those 
included under cases I-XII produced male offspring like themselves when they were used as male parents (but not when used as female parents). These have been called "genetic hermaphrodites," to distinguish them from occasional genetic males which possess female organs as a purely somatic modification, and which I have therefore called "somatic hermaphrodites." These "somatic hermaphrodites" will be omitted from the discussion for the present.

Under cases II and III it is shown that genetic hermaphrodites, of whatever origin, when self-fertilized, yield dimorphic progenies consisting of females and hermaphrodites, thus confirming the conclusions derived from the $F_{\mathrm{r}}$. This fact, together with the apparent relative ease with which males are made to exhibit the organs of both sexes, has been accepted as conclusive evidence that the hermaphrodites (and therefore also the males) are heterozygous with respect to sex, and the females homozygous (SHULL 26). In this regard Lychnis dioica L. agrees with Bryonia dioica (CoRRENS 6); with many species of Coleoptera, Orthoptera, Hemiptera, Diptera, Odonata, and perhaps also with Myriapoda and Arachnida (McClung 19, Wilson 38-42, Morgan 20, 2 I, Stevens 31-34, etc.); and with the nematode worms, Heterakis (BOVERI 4) and Ascaris megalocephala (BORING 3). In man, GUYER (I6) has demonstrated that there are two types of sperms, and while the relation of one or other of these types to the type of the egg is unknown, there can hardly be a doubt that here also the female is homozygous and the male heterozygous. ${ }^{4}$

Although these widely divergent groups of plants and animals agree in having homozygous females and heterozygous males, there may still be fundamental differences in the different groups, since there may be three different kinds of homozygotes, and correspondingly different kinds of heterozygotes. This question

${ }^{4}$ Heterozygous females have now been recognized in Abraxas (DONCASTER and Raynor Io, and Doncaster 8, 9), sea urchins (BALTZER I), canaries (DurhaM and Marryat I I), and in domestic fowl (Bateson 2, Spillman 28, 29, Goodale I 2, I3, Hagedoorn 17, Pearl and Surface 24, 25, Sturtevant 37). Guyer (I4, I 5 ) reports two types of sperms in both the guinea fowl and the common fowl, but these observations are out of harmony with all the genetic studies in which sex-limited characters of the Gallinaceae have been involved. The considerable difficulties encountered in the cytological studies on these species suggest the advisability of a repetition of this work. 
will be discussed later in connection with the nature of the hermaphrodites.

CoRrens $(6$, p. I7), with undoubted justification, maintained that the germ cells of monoecious, hermaphrodite, and dioecious species possess the tendency to develop into individuals having the distribution of sex organs characteristic of the particular genotype to which they belong; but when he likens the association of organs of both sexes in the same individual to the mosaic of red and white colors in striped flowers, and of pigmented and white spots in the coats of spotted animals, his justification becomes less obvious. Both striped flowers and spotted pelages are known from many investigations to be due to the presence or absence of a definite Mendelian gene, a so-called "spotting factor" or "pattern factor."

One of the chief aims in the arrangement of my cultures for I9ro was to test the possible existence of such a mosaic or "pattern factor," $H$, as a proximate cause of hermaphroditism in Lychnis, and the most striking result secured is the decisive manner in which such a possibility is denied. The hermaphrodite character is not only incapable of reaching expression in the female ${ }^{5}$ (as might be expected, since the female is homozygous), but it is also as a rule not transmitted through the egg cell to the male offspring. The males in the progeny of any cross agree in their sexual type with the male parent of that cross, regardless of its antedecent history. All the assumptions and implications involved in the first section of table $\mathrm{I}$, in which an independent gene $H$ was postulated, may therefore be rejected.

5 I refer here only to the normal functional hermaphroditism with which this paper deals, and not the pseudo-hermaphroditism which results when females of Lychnis dioica are attacked by the smut, Ustilago violacea, as reported by STRASBURGER (35). I was inclined (SHULL 26) to consider StRASBURGER's interpretation of the effect of Ustilago a mistake, as it appeared to me more probable that the infected plants were males whose heterozygous nature easily permitted the development of the female characters. Strasburger $(36)$ has shown conclusively, however, by two series of facts, that his original observations and interpretation were correct. These facts are $(a)$ that diseased plants are not always completely infected, in which case the uninfected branches of the pseudo-hermaphrodites have normal female flowers; and $(b)$ that male plants may be infected also, but such infection does not in this case result in the development of the female organs. 
In the second section of table I the hypothetical gene $H$ for hermaphroditism is given limitations which make it fit all the empirical results of both the first and subsequent generations; but when the significance of the limitations is taken into account, it becomes evident that there is small advantage gained by the postulation of such a gene. Indeed the only advantage lies in the fact that in case the female is a positive homozygote, it keeps open the question whether or not there is a synaptic mate of $F$ in the normal male; for a newly arisen hermaphrodite gene $(H)$ might conceivably become a synaptic mate of $F$, even though the latter had had no synaptic mate in the normal male.

If the female is a neutral homozygote, that is, if the female gene $F$ has a male gene $M$ as its synaptic mate in the male, the hermaphrodite gene (if it exist at all) must be absolutely coupled with this male gene. In like manner, if the female is a negative homozygote $F F m m$, the $H$ (if present) must be coupled with the male gene $M$. It is simpler, however, to assume that the hermaphrodite determiner is a modified form of the sex gene itself, than to suppose that it is a separate gene invariably coupled with the sex gene. This conception that hermaphroditism results from a mutative change in the sex gene, or in its homologue (?), the "Yelement," is made the basis of the last section of table I, but can apply only to those cases in which a male gene is present, or if not a male gene, then its homologue, a sexually indifferent gene which takes the place of $M$ in the male; for if the hermaphrodite character is assumed to be due to a change in the female gene $(F)$, as it must be if the latter has no "synaptic mate," the scheme will not work.

It appears to me impossible at the present time to determine whether the females of Lychnis are positive, neutral, or negative homozygotes. The facts seem to be equally well met by any of these assumptions; but the definite limitations of the hermaphrodite character to the males makes inapplicable the extreme form of the "presence and absence" hypothesis (that is, the hypothesis of unpaired genes) unless the female is a negative homozygote with reference to a male sex gene $(M)$. While the possibility must be kept open that this is the relationship of the sexes in Lychnis, 
it seems to me more probable that the female is a neutral homozygote $(F F)$, the male having the formula $F M$, and the hermaphrodite the formula $F M_{H}$. The gradually increasing number of known instances of "spurious allelomorphism" proves that the pairing of unlike or unequal genes in the heterozygote is, if not the general condition, at least a not uncommon one.

The question whether the sex genes are paired or unpaired in the heterozygote, and if unpaired, whether the female is a positive or a negative homozygote, might be settled by simple observation, if it could be known that the chromosomes are the sex determiners, as a number of recent cytological studies clearly suggest. It is not at all certain, however, whether the unequal chromosome groups in the male-producing and female-producing germ cells are active determiners or simply passive indicators of other more fundamental differences. The latter possibility is strongly emphasized by Morgan (20), who shows that the pole to which the accessory chromosome in Phylloxera is to proceed, is already determined before that chromosome has given any indication, by its own motion, to which pole it will go. This suggests that the poles of the dividing spermacyte may be sexually differentiated in advance by some other factor. If the chromosomes are not the sexdeterminers, but only passive indicators, the fact that they are paired or unpaired, equal or unequal, has no decisive bearing upon the question whether the female is a positive, neutral, or negative homozygote, or whether the genes are paired or unpaired in the heterozygote, for it is quite as easy to assume that the movement of the accessory chromosome or "X-element" to the female pole takes place in response to a tension caused by the absence of a positive male sex-determiner at that pole, as that it is attracted by the presence of a positive female determiner. If the "X-element" should move into the vacancy caused by the absence of the sexdeterminer, the presence of the added chromosome or group of chromosomes would become the evidence of the absence of the sex gene; in other words, the female possessing the added chromosome would be a negative homozygote. All this is highly speculative, and as there appears to be no way as yet to put the matter to experimental test, it seems futile to discuss further the question 
whether the female of Lychnis dioica is a positive, neutral, or negative homozygote, or whether the synaptic mate of the female gene is qualitatively male or not. The matter has been considered at such length only because it is important that no unwarranted conclusions should be drawn from the configuration of the chromosomes in any given case.

There appears to be no very strong evidence at present that the chromosomes are the representatives or producers of particular Mendelian unit characters, though attempts have been made a number of times during the past decade to identify them as such. On the other hand, there is still no positive and complete demonstration that the chromosomes are not the determiners of the Mendelian characters, and until this demonstration is provided, the relation of the chromosomes to the unit characters must be kept open. Whether the chromosomes are responsible directly for sex may well remain likewise an open question for the present, especially in view of the fact that in many animals, and in the few plants which have been thus far investigated, no chromosome differences have been found to differentiate the sexes.

There can be no doubt of course that the sex characters are associated with chromosome differences in the considerable number of animals which have been found to present such differences, but, as we have just seen, the nature of this association is not clear. Where two types of sperms are found in the male, the one type corresponding in its chromosome complex with the single type presented by the eggs, the inference is fully justified that such males are heterozygous and the females homozygous in respect to sex, whether one or more chromosomes be the sex-determiner, or whether these chromosomes are merely symptomatic of other fundamental differences which are the true sex-determiners; and vice versa, when two types of eggs having different chromosome groups are found in the female, one of which agrees with the only type found in the sperms, the inference is fair that the female is heterozygous and the male homozygous in respect to sex. So consistent have been the results in those species in which both male and female germ cells have been investigated, that it has not seemed improper to assume that in any given species the one sex will have 
uniform germ cells, and is to be considered homozygous, if the other sex is demonstrated to have two types of germ cells. ${ }^{6}$

No chromosome differences have been found in Lychnis dioica L. by Strasburger (36), who has studied a form of this species known in German taxonomic works as Melandrium rubrum Garcke. His careful investigation of germ cells and root tips showed 24 chromosomes to be the somatic number, one pair of these chromosomes being notably larger than the rest, thus resembling the accessory chromosomes or supposed sex chromosomes of the insects. However, in Lychnis, the two members of this pair are indistinguishable from each other in both the male and the female. The same results have been independently secured by Miss LuTz during the past year, but have not yet been published. Lychnis appears to agree, therefore, with Nezara, Oncopeltus, etc. (WILson 39, 40), among the Hemiptera, as in these the two types of sperms, which doubtless exist, are not visibly differentiated. Strasburger (36) reports also that an investigation of Bryonia dioica has not revealed the two types of sperms that might $a$ priori have been expected.

The hypothesis of unpaired determiners implies that a new Mendelian character originates by the formation of a new gene or the loss of an old one. My interpretation of hermaphroditism in Lychnis dioica as due to an alteration in a sex gene already in existence, which alteration does not in any way change the homology of the gene in question, calls for a fundamentally different method of origin of new characters from that involved in this extreme form of the "presence and absence" hypothesis. The new genotype which arises by mutation from the old one has in this case neither more nor fewer genes than had the genotype from which it originated.

The occurrence of male mutants among the offspring of my genetic hermaphrodites appears to me to have a bearing upon this question, as to the mode of origin of new characters. Among the offspring of genetic hermaphrodites tabulated in this paper, II male mutants appeared, and under case XII it is shown conclusively that these are true males, and do not again give hermaphrodite offspring, except probably in the extremely small proportion given

${ }^{6}$ As already noted, GuYER's (14, I 5) studies on spermatogenesis in the domestic fowl and in the guinea fowl appear at present to be exceptions. 
by males not derived from a hermaphrodite family. These II males appeared in hermaphrodite families comprising a total of 5467 individuals, thus possibly indicating a somewhat greater coefficient of mutability than that reported for the production of hermaphrodites from normal males. It seems therefore that the modification of the gene $M$ (or $f$ ) into a hermaphrodite gene $H$, and the reversal of this modification so that a normal male gene is again produced from a hermaphrodite gene, occurs with somewhat unequal facility, but the difference is not great enough to warrant the belief that mutation in the one direction is caused by the appearance of a new, independent organ, while its reversal is due to the disappearance of that organ. It seems to me more probable that these reversible mutations are due to reversible modifications of an element or organ continuously in existence, and not to the production of a new element or the dropping out of an old one.

The change from a male to a hermaphrodite condition and the reverse are processes both striking and sudden. Perhaps they are as fundamental mutations as those observed among the oenotheras. The interpretation given here of the process of mutation in the sex character of Lychnis seems to be available for other mutations as well. The sudden acquirement of new functions by a gene already in existence is different from the conception presented by DE VRIEs in Die Mutationstheorie, to account for the origin of the Oenothera mutants, and is in accord with Spillman's "teleone hypothesis." Sprllman (30) is inclined to attribute the remarkable mutations in Oenothera to irregularities of mitosis, but in these sex mutants of Lychnis, abrupt genotypic modifications have taken place which can hardly be assigned to such irregular mitoses.

One puzzling feature of the inheritance of sex in Lychnis is the fact that self-fertilized hermaphrodites produce similar ratios of females and hermaphrodites as are produced when unrelated females are fertilized by sperms from hermaphrodites. Since it is obvious that the two types of offspring are due to the heterozygous character of the male, we are led to the conclusion that even though the hermaphrodite individual is heterozygous in respect to sex, its egg cells ${ }^{7}$ are of a single type like those of the normal female

7 Perhaps I should say "its successful egg cells." 
and carry only the female tendency, while its sperm cells are of two types, one of which has the same sex character as the egg cells, the other bearing the hermaphrodite condition. In my preliminary paper, it was suggested that those eggs may fail to develop which lack the female gene $F$, or which possess the male gene $M$; or that in case the female is a negative homozygote, there might be an extrusion of the male gene during oogenesis. As there are no visible cytological differences between the females and the hermaphrodites, it may not be possible to decide these questions. The relatively small number of seeds in the hermaphrodites, as compared with the females, appears to be favorable to a selective elimination of male-bearing eggs. Another explanation seems possible. A segregation of the female and male genes may conceivably take place earlier than the time at which the germ cells are formed, though it must be admitted that there is little evidence at present that such early segregations regularly ${ }^{8}$ take place in any plant or animal. Such a suggestion has been made by BATESon (2, p. I59), however, in the effort to account for certain interesting instances of coupling. If a segregation of female and hermaphrodite genes could be assumed to take place as early as the formation of a certain primordial cell from which the entire reproductive tissue of the ovary develops, so that the ovules are supplied only with the female genes, the observed uniformity of the egg cells would result. If segregation may take place thus before the spermacytes are developed, this might also offer an explanation of the exceedingly variable sex ratios which occur in Lychnis, for an unequally rapid development of tissues derived from female-bearing cells and male-beäring cells, from the moment of segregation until the spermacytes are produced, would give an unequal number of female-bearing and male-bearing sperms, and variability in this process would produce irregular ratios. I place no stress upon this hypothesis, however, and am inclined to look for an explanation of the observed phenomena in some sort of selective elimination.

There remains to be considered the relation of the somatic hermaphrodites to the problems of sex determination. The results under cases XIII and XIV show that the hermaphrodite

${ }^{8}$ They are known to take place occasionally in the production of bud sports. 
character of these plants was purely superficial and did not affect the germ cells in any recognizable manner. The only bearing these plants have upon the question of sex determination, I think, is in the evidence they give that genetically normal males may be induced in some unknown way to exhibit female characters. When the male is interpreted as a Mendelian heterozygote in respect to the sex-producing gene, the occurrence of such a somatic modification has the appearance of a simple case of imperfect dominance, such as has been noted not infrequently in other Mendelian heterozygotes. However, the development of male organs (non-functional) in the supposedly homozygous female, when the latter is attacked by the smut (Ustilago violacea), gives support to the view held by STRASBURGer (36), that not only the heterozygous sex but both sexes contain in some degree the elements of the opposite sex or the capacity to react in the sexually opposite manner. This fact may perhaps indicate that sex is a more fundamental condition than might be inferred from the frequency with which it behaves as a Mendelian unit character. MoRGan (23) suggests a way in which the appearance of the organs or characters of one sex in individuals of the opposite sex may be explained in harmony with the Mendelian interpretation of sex determination. He assumes that there may be present, underlying the female sex gene, a male element with respect to which all individuals of both sexes are homozygous. This he indicates by introducing $m$ into all of his sex formulae. In keeping with common usage among geneticists, he should have used $M$, since he intends to denote the presence of maleness.

While recognizing the aptness of this suggestion in removing some of the difficulties in the way of a general application of the Mendelian explanation of sex, I am inclined to the view that the Mendelian nature of sex is of secondary rather than of primary consequence. May not maleness and femaleness be thought of as alternative states, which can be crudely analogized with the acidity and alkalinity of chemical solutions? Just as solutions may be made acid or alkaline in different ways, either by qualitative or by quantitative additions, subtractions, or substitutions, or by a combination of qualitative and quantitative changes, it 
is conceivable that the alternative sexual types may be determined in different cases by very different methods, some qualitative, some quantitative, and others both qualitative and quantitative. In some species the sexes appear to represent a much more strongly polarized (?) condition than in other species, and a transition from the characters of the one sex to those of the other is attained only with the greatest rarity, if at all; while in other species the sex conditions may be so nearly balanced or neutral that individuals are not so absolutely determined in their sex relations by their genotypic nature, thus resulting in ever-sporting varieties in respect to sex, such as Correns (7) has found in Plantago lanceolata.

With such a conception of sex, it also appears probable that sex may be influenced sometimes by external factors as well as by internal ones, and in this case the preponderance of one sex over the other, which has been observed in many animals and plants, need not be attributed alone to a selective disorganization of germ cells, a selective fertilization, or a selective death rate, but might conceivably be controlled to a certain extent by environmental conditions acting at some particular "sensitive period" in the ontogeny of the organism in question. However this may be, there is little or no evidence at present that such environmental influences on sex can be more than relatively slight in the case of dioecious plants and animals. In such organisms recent genetic and cytological studies prove conclusively that sex is generally determined by the genotypic nature of the individual.

\section{Summary}

The hermaphrodites of Lychnis dioica are modified males. They are of two kinds, which are here distinguished as "genetic" and "somatic" hermaphrodites.

When the genetic hermaphrodites are used as pollen parents, either when self-fertilized or in crosses with females, their progenies consist of females and hermaphrodites. When they are used as pistil parents, and fertilized by normal males, they produce females and normal males.

Somatic hermaphrodites may be externally indistinguishable 
from the genetic hermaphrodites, but when used as pollen parents they produce no hermaphrodite offspring, but only females and normal males.

The fact that males can be modified so as to produce functional organs of both sexes, indicates that they are sex heterozygotes, and the production of both females and hermaphrodites by selffertilized hermaphrodites strongly supports the same interpretation.

The hermaphrodite character can neither find expression in the females, nor can it be transmitted by their eggs to the male offspring. Consequently it is not determined by an independent gene, $H$, but by a modification of the male sex gene, $M$, or of the "synaptic mate" of the female gene, $F$.

If the males and hermaphrodites are heterozygous, it follows that the females are homozygous; but this does not offer an ultimate solution to the relationship between females and males, since there may be several different kinds of homozygotes and heterozygotes. As applied to the relation of the sexes, these may be indicated by the following formulae: (a) The female may be a "positive" homozygote; then $F F=q, F f=\hat{\phi}, " F f_{H}$ or $F H=\varnothing$. (b) If the female is a "negative" homozygote, $F F m m=q, F F M m=\hat{\delta}$, $F F M_{H} m=\varnothing$. (c) When the female is a "neutral" homozygote, $F F=q, F M=\hat{\text { o }}, F M_{H}=\varnothing$. In each of these formulae the subscript $H$ is intended to represent a modification of the gene to whose symbol it is appended, such that the male is changed to a hermaphrodite. Which of these formulae correctly represents the condition in Lychnis can not be determined, but the modified gene which results in hermaphroditism is allelomorphic to $F$ unless the female is a negative homozygote.

Among the offspring of genetic hermaphrodites were a small number of male mutants (I in 5467), which on breeding proved to be normal males. The occurrence of these male mutants indicates that the modification to the hermaphrodite condition, and back again to the male condition, occurs with but slightly unequal facility, and this circumstance is believed to favor the view that mutation in this case depends upon reversible modifications of some permanent element or organ, rather than upon the origination of a new unit, and its disappearance. This interpretation 
bears both upon the nature of mutation and upon the real significance of the "presence and absence" hypothesis.

STRASBURGER has shown that females of Lychnis dioica attacked by Ustilago violacea become pseudo-hermaphrodites through the production of stamens, which however are non-functional, owing to the fact that the smut produces its spores in the anthers. This seems to justify his conclusion that each sex possesses some of the potentialities of the opposite sex.

The view is expressed that the sexes represent alternative states which in different species may be attained in various ways, through either quantitative or qualitative changes, additions, subtractions, substitutions, or transformations, and that in some instances the action of environment may prove effective in determining which of these states shall find expression. Nearly all the recent investigations indicate, however, that sex is at least predominantly dependent upon the genotypic nature of the individual.

Carnegie Station for Experimental Evolution

Cold Spring Harbor, N.Y.

\section{LITERATURE CITED}

I. Baltzer, F., Ueber die Grösse und Form der Chromosomen bei Seeigeleiern. Verhand. Deutsch. Zool. Gesells. I908.

2. Bateson, W., Mendel's principles of heredity. pp. xiv+396. Cambridge: University Press. I909.

3. Boring, Alice M., A small chromosome in Ascaris megalocephala. Arch. f. Zellf. $4^{\mathrm{x}}$ : I 20-I3I. pl. I. I909.

4. Boveri, T., Ueber "Geschlechtschromosomen" bei Nematoden. Arch. f. Zellf. $4^{\mathrm{I}}$ :132-I4I. figs. 2. I909.

5. Castle, W. E., A Mendelian view of sex heredity. Science N.S. 29:395400. I909.

6. Correns, C., Die Bestimmung und Vererbung des Geschlechtes, nach neuen Versuchen mit höheren Pflanzen. pp. vii+8r. figs. 9. Berlin: Gebr. Borntraeger. 1907.

7. — Die Rolle der männlichen Keimzellen bei der Geschlechtsbestimmung der gynodioecischen Pflanzen. Ber. Deutsch. Bot. Gesells. 26a: 686-701. 1908.

8. Doncaster, L., Sex inheritance in the moth Abraxas grossulariata and its var. lacticolor. Report Evol. Comm. IV. pp. 53-57. 1908. 
9. Doncaster, L., Recent work on the determination of sex. Sci. Prog. no. I3. pp. 90-104. I909.

I0. — and RAynoR, G. H., Breeding experiments with Lepidoptera. Proc. Zool. Soc. London I : I 25. Igo6.

I I. Durham, F. M., and Marryat, D. C. E., Inheritance of sex in canaries. Report Evol. Comm. IV. pp. 57-60. I908.

12. Goodale, H. D., Sex and its relation to the barring factor in poultry. Science N.S. 29: 1004, 1005. I909.

13. - Breeding experiments in poultry. Proc. Soc. Exp. Biol. Med. 7:I78-I79. I9IO.

14. GuYER; M. F., The spermatogenesis of the domestic guinea (Numida meleagris Dom.). Anat. Anz. 34:502-513. pls. 2. 1909.

15. - The spermatogenesis of the domestic chicken (Gallus gallus Dom.). Anat. Anz. 34:573-580. pls. 2. r 909.

16. - Accessory chromosomes in man. Biol. Bull. 19:219-234. pl. I. I9IO.

I7. Hagedoorn, A. L., Mendelian inheritance of sex. Arch. f. Entwick. Mech. 28: I-34. I909.

18. Hurst, C. C., Mendelian characters in plants and animals. Report 3d Internat. Conf. on Genetics. Jour. Roy. Hort. Soc. pp. II4-I 28. 1906.

I9. McClung, C. E., The accessory chromosome-sex determinant? Biol. Bull. 3:43-84. 1902 .

20. Morgan, T. H., A biological and cytological study of sex determination in phylloxerans and aphids. . Jour. Exp. Zool. 7:239-352. pl. I. I909.

21. - Sex limited inheritance in Drosophila. Science N.S. 32:I20-I 22. I9IO.

22. - Chromosomes and heredity. Amer. Nat. 44:449-496. Igro.

23. - The application of the conception of pure lines to sex-limited inheritance and to sexual dimorphism. Amer. Nat. 45:65-78. IgII.

24. Pearl R., and Surface, F. M., On the inheritance of the barred color pattern in poultry. Arch. Entwick. Mech. 30:45-6I. pls. 2. fig. I. I9IO.

25. — Further data regarding the sex-limited inheritance of the barred color pattern in poultry. Science N.S. 32:870-874. I9I0.

26. Shuld, G. H., Inheritance of sex in Lychnis. Bot. Gaz. 49:IIO-I25. figs. 2. I9I0.

27. —- The "presence and absence" hypothesis. Amer. Nat. 43:4104I9. I909.

28. SpIllman, W. J., Barring in barred Plymouth Rocks. Poultry $5: 7,8$. I909.

29. - , Spurious allelomorphism: results of recent investigations. Amer. Nat. 42:610-615. I909.

30. - Mendelian phenomena without De Vriesian theory. Amer. Nat. $44: 214-228$. I9IO. 
31. Stevens, N. M., Studies in spermatogenesis with special reference to the "accessory chromosome." Pub. 36, Carnegie Institution of Washington. 1905.

32. — Studies in spermatogenesis. II. A comparative study of the heterochromosomes in certain species of Coleoptera, Hemiptera, and Lepidoptera, with special reference to sex determination. Pub. 36, Carnegie Institution of Washington. Igo6.

33. — A study of the germ cells of certain Diptera with reference to the heterochromosomes and the phenomena of synapsis. Jour. Exp. Zool. 5:359-374. pls. 4. 1908 .

34. - The chromosomes in Diabrotica vittata, Diabrotica soror, and Diabrotica I2-punctata. Jour. Exp. Zool. 5:453-470. pls. 3. 1908.

35. Strasburger, E., Versuche mit dioïcischen Pflanzen in Rücksicht auf Geschlechtsverteilung. Biol. Centralbl. 20:657-665, 689-698, 72I-73I, 753-785. figs. 5. I 900 .

36. — Ueber geschlechtbestimmende Ursachen. Jahrb. Wiss. Bot. 48:427-520. pls. 2. I910.

37. Sturtevant, A. H., Another sex-limited character in fowls. Science N.S. 33:337-338. I 9 II.

38. Wilson, E. B., Studies on chromosomes. II. The paired microchromosomes, idiochromosomes, and heterotropic chromosomes in Hemiptera. Jour. Exp. Zool. 2:507-545. figs. 4. I905.

39. - Studies on chromosomes. III. The sexual differences of the chromosome groups in Hemiptera, with some considerations on the determination and inheritance of sex. Jour. Exp. Zool. 3:I-40. figs. 6. 1906.

40. - Studies on chromosomes. IV. The "accessory" chromosome in Syromastes and Pyrrochoris, with a comparative review of the types of sexual differences of the chromosome groups. Jour. Exp. Zool. 6:69-99. pls. 2. fig. I. 1909 .

4I. — Recent researches on the determination and heredity of sex. Science N.S. 29:53-70. 1909.

42. - The chromosomes in relation to the determination of sex. Science Prog. no. I6, pp. 570-592. figs. 3. IgIo. 
Reprinted from the Botanical Gazette, 52: $480-485$, No. 6, December, I9II

\section{NOTES FOR STUDENTS}

Experiments with maize.-Several years ago BLARINGHEM ${ }^{3}$ published a monograph on his now well known experiments in the production of anomalies in various plants as the result of mutilation. The mutilations forced into development buds which ordinarily remain latent, and the branches produced from these buds frequently possessed characters not recognized as normal features of the plants operated on. In a small percentage of cases the abnormalities thus brought to light were found to be inherited to a greater or less degree, and the conclusion was reached that mutilation is a very general and easy means of provoking mutability and an important factor in the evolution of vegetable forms. Most of his experiments were made with maize, though some apparently corroboratory evidence was derived from barley $(H$. distichum and $H$. tetrastichum) and mustard (Sinapis alba). All of the new characters, abnormal or otherwise, which came to light in his experiments with maize

3 Blaringhem, L., Mutation et traumatismes. Étude sur l'évolution des formes végétales. pp. 248. pls. 8. Paris: Felix Alcan. 1908. 
were discovered in the descendants of one original plant mutilated by the author in I902. After that time the pedigrees were kept carefully controlled, either by hand-pollinations or by cultivation in isolated plots.

The reviewer, ${ }^{4}$ at about the time this monograph appeared, demonstrated the occurrence of numerous biotypes in hybrid combination in what appeared to be a fairly uniform population of maize, and believes this to be the general situation in this species. JoHANNSEN 5 has pointed out that the reviewer's results favor a different interpretation of BLARINGHEM's experiences, since the new types which proved to be hereditary may have appeared as the result of segregation of biotypes which were already present in the original plant chosen for mutilation, this segregation being due, not to the mutilation, but to the subsequent method of breeding. GRIFFON ${ }^{6}$ has given further support to this interpretation of BLARINGHEM'S results, by showing that similar abnormalities appear when no mutilations have been practiced, and the reviewer has had the same experience. GRIFFON shows that the abnormalities which characterized BLARINGHEM's forms are strongly dependent upon seasonal conditions for their development, being much more abundant in all cultures in some seasons and less abundant in all in other seasons. He does not agree with BLARINGHEM that with respect to these abnormalities these maize families constitute ever-sporting varieties. It does not follow, however, that abnormalities are not hereditary because they are strongly affected by the environment. Hus and MURDOCK ${ }^{7}$ have shown the inheritance of fasciation in a strain of popcorn, the offspring of two fasciated ears giving progenies over 50 per cent of which produced fasciated ears, while an unfasciated ear from the same strain gave only 3 per cent fasciated ears. It will be understood, of course, that the strain from which these ears were selected was complexly hybrid, and that pure-bred derivatives might have shown either approximately roo per cent fasciated or approximately no fasciation, under favorable conditions. There is evidence that the fasciation is strongly fluctuating, the two ears on a single stem being not infrequently one fasciated and the other normal. The significance of the percentage inheritance is doubtful in complex material of this kind.

The reviewer ${ }^{8}$ has presented additional evidence of the hybrid nature of ordinary vigorous maize plants, and their dependence for their vigor upon

${ }^{4}$ Shull, G. H., The composition of a field of maize. Report Am. Breeders' Association 4:296-30r. r9o8.

${ }^{5}$ Johannsen, W., Elemente der exacten Erblichkeitslehre. pp. vi+5i6. figs. 3 I. Jena: Gustav Fischer. 1909. See p. 451.

${ }^{6}$ GrIfron, E., Observations et recherches expérimentales sur la variation chez le maïs. Bull. Soc. Bot. France 57:604-6r5. rgro.

7 Hus, H., and Murdock, A. W., Inheritance of fasciation in Zea Mays. Plant World I4:88-96. I9II.

${ }^{8}$ Shull, G. H., Hybridization methods in corn breeding. Amer. Breeders' Mag. I :98-ro7. I910. 
this hybridity. Previous conclusions that $F_{I}$ hybrids between self-fertilized strains are on the average equal in yielding capacity, and in certain combinations much superior, to strains cross-bred in the normal manner, have been confirmed; also that reciprocal crosses are essentially equal. In addition it is shown that the yield and quality of the crop are functions of the particular hybrid combination, the results being the same whenever the cross is repeated. The $F_{x}$ was found no more variable than the pure self-fertilized parental strains, but the $\mathrm{F}_{2}$ was considerably more variable, the coefficients of variability in number of rows on the ears in pure biotypes and in their $F_{r}$ hybrids being respectively $9.08 \mathrm{I}$ per cent and 9.063 per cent, while that of the $F_{2}$ was 12.63 per cent. This greater variability in the $F_{2}$, also noted by EMERSON9 in respect to size of seeds and height of stalks, though the latter gives no coefficients, clearly demonstrates the segregation of different grades of such purely quantitative characters. EAST ${ }^{\text {Io }}$ has also presented similar evidence of the segregation of a quantitative character (height of stalk) in the $F_{2}$, but he makes no reference to the reviewer's corresponding results published a year earlier. He gives no coefficient of variability for pure strains, but his coefficient for the $F_{x}$ was 8.68 per cent, while in the several $\mathrm{F}_{2}$ families it ranged from $\mathrm{I} 2.02$ per cent to 15.75 per cent.

The theory that the increased vigor of cross-bred maize plants is due to a stimulation accompanying heterozygosis requires that crossing within the same biotype or within the same $F_{I}$ shall give no advantage over self-fertilization in the same biotype or in the same $F_{1}$. The reviewer ${ }^{r I}$ has reported on a number of such sib-crosses in comparison with corresponding self-fertilizations, the advantage in favor of the crosses being so slight that they can be fairly accounted for by the lack of complete genotypic purity in some of the self-fertilized families. Crosses between sibs in ten self-fertilized families had an average height of $20 \mathrm{dm}$. and gave an average yield per acre of 30.17 bushels as compared with a height of $19.28 \mathrm{dm}$. and a yield of 29.04 bushels in the offspring of self-fertilized parents. In the $F_{2}$ those families which sprang from sib-crosses in the $F_{I}$ had an average height of $23.30 \mathrm{dm}$. as compared with $23.55 \mathrm{dm}$. in families produced from self-fertilized parents, and the corresponding yields per acre were 47.46 and 4 I.77 bushels respectively. These results show that cross-fertilization is of no (or little) advantage except when it brings together unlike hereditary elements. The relations of $F_{I}$ and $F_{2}$ in regard to height of plants and yield per acre strikingly emphasize the economic importance of using hybridized seed corn. Ten $F_{I}$ families had an average height of $25 \mathrm{dm}$. and produced an average yield of 68.07 bushels,

9 Emerson, R. A., The inheritance of sizes and shapes in plants. Amer. Nat. 44:739-746. I9ro.

xo EAst, E. M., The genotype hypothesis and hybridization. Amer. Nat. 45: 160-174. figs. 6. 1911.

II Shull, G. H., The genotypes of maize. Amer. Nat. 45:234-252. fig. I. I9r1. 
while twenty corresponding $F_{2}$ families had an average height of $23.42 \mathrm{dm}$. and gave a yield of only 44.62 bushels per acre.

HAYES and EAST ${ }^{\text {I2 }}$ have also shown a similar relation between first and second generation crosses, one such cross giving 105.5 bushels per acre in $F_{x}$ and only ${ }_{5}$ I. 5 bushels in $F_{2}$, another cross giving respectively II 7.5 bushels per acre and 98.4 bushels per acre. These authors give a good discussion of the economic bearings of these results and methods of putting them to practical use.

CoLLINS $^{13}$ has also shown the practical value of hybridization methods in corn growing, reporting on the results of sixteen rather wide crosses, all but two of which gave higher yields than the average of the parents, and all but four exceeding the better parent in yielding capacity. PEARL and Surface, ${ }^{14}$ while subscribing to the correctness of the genotype idea as applied to maize, are of the opinion that the ordinary ear-to-the-row selection method "in a much cruder and less precise way, really makes use of the same principle" as the reviewer's "pure-line method," and they advocate simply the relaxation of the selection after a few years, by which time the "more strikingly undesirable genotypes will have been automatically eliminated." This view fails to take account of the relatively greater vigor in the $F_{I}$ hybrids. EAST, ${ }^{15}$ HAYES and $\mathrm{EAST}^{16}{ }^{16}$ and Collins, ${ }^{17}$ on the other hand, urge the use of the method of MORROW and GARDNER, ${ }^{18}$ as the most practical means of utilizing the greater vigor produced by heterozygosis, and the reviewer believes that the attitude of these authors is justifiable. The method of MORRow and GARDNER is identical with the "pure-line" method, except that highly developed commercial varieties are used in the place of pure self-fertilized strains. The two chosen parental types are grown in alternate rows in an isolated plot, and one variety is detasseled. The seed for the general crop is harvested from the detasseled row, and the process is repeated year after year, using the same parental varieties.

EAST and HAYES ${ }^{\text {I9 }}$ have made a most important contribution to knowledge

${ }^{12}$ Hayes, H. K., and East, E. M., Improvement in corn. Bull. Conn. Agr. Exp. Sta. pp. 2 I. pls. 4. I9I I.

${ }^{13}$ Collins, G. N., The value of first-generation hybrids in corn. Bull. r9r, B.P.I., U.S. Dept. Agr. pp. 45. I9IO.

${ }^{14}$ Pearl, R., and Surface, F. M., Experiments in breeding sweet corn. Ann. Rep. Maine Agr. Exp. Sta. I910. pp. 249-307. Bull. 183. figs. 220-233.

${ }_{15}$ EAST, E. M., The distinction between development and heredity in inbreeding. Amer. Nat. 43: I73-I8I. Iø̧o9.

${ }^{16} \mathrm{Op}$. cit.

${ }_{77}$ Op. cit.

${ }^{8}$ Morrow, G. E., and Gardner, F. D., Field experiments with corn 1892. Bull. 25, Ill. Agr. Exp. Sta. pp. 173-203. I893.

19 East, E. M., and Hayes, H. K., Inheritance in maize. Bull. Conn. Agr. Exp. Sta. pp. I37. pls. 25. I9II. 
of the inheritance of unit characters in maize, and have succeeded in clearing up most of the difficulties met by CORRENS and LOCK, and simply by the expedient of applying a strictly individual analysis, instead of permitting pollinations from a number of individuals possessing the same characteristics. Only a few of the more striking results can be mentioned. There are two independent genes for yellow endosperm color, giving $F_{2}$ ratios $3: 1$ and ${ }_{5}$ : I. These are so related as respects dominance that the intensity of the yellow color agrees approximately with the actual number of $\mathrm{Y}$ genes present, i.e., the color is most intense in seeds having both genes homozygous, less intense when one gene is homozygous and the other heterozygous, still less intense when both are heterozygous or when either is absent and the other homozygous, etc. This situation results in a distribution of individuals in the form of the probable error curve, and is therefore superficially like that of fluctuating variations, from which it differs however in that the different grades are inherited. In another paper the senior author ${ }^{20}$ makes use of these facts in extending Mendelian theory to include variation that is apparently continuous. In the purple aleurone color, EAST and HAYES find no less than four independent genes involved in different varieties, a full purple color requiring the simultaneous presence of both $\mathrm{P}$ and $\mathrm{C}$, a full red color the presence of $\mathrm{R}$ and $\mathrm{C}$. In the absence of $\mathrm{C}$, both $\mathrm{P}$ and $\mathrm{R}$ are capable of producing some pigment, giving "particolored" seeds. In addition to these three genes, there was found in a cross between Tom Thumb pop corn and Black Mexican sweet corn, a factor which partially or wholly inhibited the production of the purple aleurone color. This inhibitor or "dominant white" is strictly normal in its hereditary behavior, and its presence in some of Lock's strains undoubtedly accounts for that investigator's aberrant results. In pericarp colors the authors recognize five independent red-producing genes, $R_{\mathrm{I}}$ the ordinary red of "red" maize, $R_{2}$ the striped red of "calico corn," $R_{3}$ a dirty red most abundant at the base of the grains and apparently completely coupled with red silks, $R_{4}$ and $R_{5}$ a rose red which reaches full development only on exposure to the sunlight. The red cob-color is a simple Mendelian dominant, independent of pericarp color. While starchiness is an endosperm character and shows xenia, the quality of the starch, whether flinty or floury, is a "plant character," and affects all the grains of an ear. Crosses between flint and dent varieties show undoubted segregation with the flint character recessive, but there are probably several genes involved, and the results are obscured by physiological correlation. Further evidence is given that size characters, such as height of stalk, length of ear, and size of grains, segregate normally in the $\mathbf{F}_{2}$. Several abnormalities are mentioned, dwarfness, striped leaves, split and furrowed cobs, branched ears, and hermaphrodite flowers. With exception of the last, these characters are thought to be inherited in Mendelian fashion, though the

${ }^{20}$ EAST, E. M., A Mendelian interpretation of variation that is apparently continuous. Amer. Nat. 44:65-82. I9ro. 
possibility is suggested that fasciation of the ears may be a purely physiological effect of disturbed nutrition.

EMERSON $^{21}$ reports the discovery of red aleurone color as a latent character in a cross between Queen's Golden pop corn and Black Mexican sweet corn, though other crosses between these two varieties gave only purple aleurone. Crosses between a tested homozygous red-aleurone strain and White Rice pop corn and Evergreen sweet corn produced $F_{\mathrm{I}}$ 's with only purple aleurone cells, thus demonstrating the presence of $P$ as a latent character in both of these white varieties. Dark and light yellow endosperm colors were also seen to be latent as a result of a cross between the orange-colored Queen's Golden and the Black Mexican with colorless endosperm.

While not experimental, two papers by ILTrS $^{22}$ on abnormalities are worthy of mention. Both of these abnormalities are assumed to have been induced by the traumatic action of Ustilago Maydis. In the first the glumes of the female flowers were somewhat enlarged, and in place of the carpel arose a tubular structure $10-20 \mathrm{~cm}$. long, terminated by a long pistil-like thread $20 \mathrm{~cm}$. long. The occurrence of a ligule on this structure served to identify it as a phyllode, and leads the author to the conclusion that the ovary, which afterward forms the seed coat, is homologous with the leaf sheath, and the style with the leaf blade. Within this tube, as a prolongation of the axis, grew an abnormal leafy branch. In the second paper ${ }^{23}$ the author describes abnormal inflorescences in which the flowers are paired, each pair consisting of a sessile female or hermaphrodite flower and a stalked male flower. This is an arrangement characteristic of the Andropogoneae, and the author looks upon its appearance in maize as a reversion. On this basis he would rank the Zeae as a subtribe of the Andropogoneae, in support of HACKEL and STAPF, who had adopted this arrangement on other grounds.-GEO. H. SHULL.

${ }^{2 x}$ Emerson, R. A., Latent colors in corn. Ann. Rept. Amer. Breeders' Ass. 6: 233-237. I910.

${ }^{22}$ ILTIS, H., Ueber eine durch Maisbrand verursachte intracarpellare Prolifikation bei Zea Mays L. Sitzungsber. Akad. Wiss. Wien. Math.-naturw. Klasse I I $9^{x}$. pp. 15. pls. 2. 1910.

${ }^{23}$ Iltis, H., Ueber einige bei Zea Mays L. beobachtete Atavismen, ihre Verursachung durch den Maisbrand, Ustilago Maydis (DC.) Corda über die Stellung der Gattung Zea im System. Zeitschr. Abst. Vererb. 5:38-57. pls. 2. I9Ir. 
Sonderabdruck aus der

\section{Zeitschrift für induktive Abstammungs-}

und Vererbungslehre

1912 Bd. VI Heft 5.

Verlag von Gebrüder Borntraeger in Berlin W 35

\section{SHULL, GEORGE HARRISON:}

Inheritance of the heptandra-form of Digitalis purpurea L. 



\section{Inheritance of the heptandra-form of Digitalis purpurea L.}

(Mit Tafel XV-XVI.)

By George Harrison Shull.

(Station for Experimental Evolution, Cold Spring Harbor, Long Island.)

There is an extraordinary form of the common foxglove (Digitalis ourpurea), which differs from the normal type of that species by having the ventral portion of the corolla, representing three of its lobes, commonly replaced by three additional stamens. Instead of the long and shapely tubular flower including four gracefully curved stamens, a strap-shaped or more or less laciniated petaloid structure usually represents the dorsal portion of the corolla and seven (often nine) widely divergent stamens give the flower a grotesque and unattractive appearance. (See PlateXVI.)

In the Figwort family (Scrophulariaceae) to which the foxglove belongs, the flower is constructed on the pentamerous plan, but the number of stamens is most commonly reduced to four as they are in the normal foxglove, and the carpels are always normally two. In Verbascum there are five functional stamens and in many other genera of this family the fifth stamen is represented by a more or less strongly developed staminode. In Ilysanthes and some species of Gratiola there are two functional stamens and two staminodes, and in Veronica and its relatives and in some Gratiola species the stamens are reduced to two without a trace of the other three which would be required to complete the pentamerous plan. Since five stamens is the maximum number normally present in any member of this very large family, and there exists in the family an obviously strong tendency to a reduction of this number, the occurrence of a sevenstamened form is remarkable, transgressing as it does such a strongly marked family character. 
DE CHAMISSO ${ }^{1}$ ) in 1826 first described this peculiar form of Digitalis and it has been found and noted a number of times since. A specimen sent by Mr. EARLEY to the GARDENERS CHRONICLE was figured and briefly described ${ }^{2}$ ) in 1874 . This was said to be a seedling of one similarly deformed. In I882 HENSLOW ${ }^{3}$ ) described specimens received by him from Tilburstow Hill, Surrey, England. The same form appeared about I903 in the Daisy Hill Nursery, Newry, England. In I905 Mr. T. SMITH, the owner of this nursery, sent flowers from the offspring of the original plant to the GARDENERS CHRONICLE with the statement that all the offspring of that plant possessed the same characters as their parent4). In Igro Professor DENDY showed before the Linnean Society a specimen which he had received from Dr. N. C. MACNAMARA ${ }^{5}$ ). This plant was also known to be the offspring of a similar plant found in I907. In 1906 the attention of Miss SAUNDERS was called to a specimen of the heptandraform which had appeared unexpectedly in the garden of a friend in Cambridge, England, and from unguarded seeds of this plant she has grown two generations which flowered in I908 and I9Io. Both in self-fertilizations and in crosses of $F_{1}$ plants with the heptandra-form, Miss SAUNDERS has demonstrated ${ }^{6}$ ) that the latter is a typical Mendelian recessive to the normal type. In I903 Dr. TINE TAMmES found a single individual of heptandra growing in the Botanic Garden of the Rijks-Universiteit at Groningen, Holland, and she, too, has conducted crossing experiments which have led her to the same conclusions regarding the Mendelian behavior of the abnormal form in crosses with normal Digitalis purpurea, but she had not yet published her results when Miss SAUNDERS's paper appeared, and will not now publish.

Until the appearance of Miss SAUNDERS'S account of her experiments, the published evidence as to the inheritance of the heptandra-character had been somewhat conflicting. DE CHAMISSO had

1) Chamisso, A. DE, De Digitali purpurea heptandra. Linnaea 1: 571-575, Pl. VI, I 826.

2) Gardeners Chronicle 2: 78 . I 874 .

3) Hensiow, G., Note on staminiferous corollas of Digitalis purpurea and Solanum tuberosum. Linn. Soc. Journ. Bot. 19: 216-218, Pl. 33. I882.

4) Gardeners Chronicle 36 (3 $3^{\text {rd }}$ Ser.): 208. Fig. 1904.

5) Proceedings of Linnean Society for 1910, p. 106.

6) SAUnders, E. R., On inheritance of a mutation in the common foxglove (Digitalis purpurea). The New Phytologist 10:47-63, I Pl. I2 Figs. IgI. 
reported $^{1}$ ) that several seedling offspring of the type-specimen showed no indications of the peculiar character of their parent; but as already noted, the specimens both of Mr. EARLEY and of Mr. SMITH were derived from parent plants of the same type. These apparently conflicting observations as well as the fact that in each case the original plant of the unexpected form was presumably derived from a seed of a normal Digitalis purpurea, are readily explainable on the supposition that the heptandra-form is a Mendelian recessive to the normal type, as Miss SAUNDERS has shown it be, for in this case DE CHAMISSO's experience can be accounted for by assuming that his seedlings were the result of cross-pollinations from the dominant normal type. However, such explanation could have no security until the hereditary relationship of these two forms had been tested by properly controlled genetic experiments.

I became interested in this question in 1905 on reading in the GARDENERS CHRONICLE the account of the Daisy Hill Nursery specimens, and I immediately wrote to the proprietor of the nursery for seeds. These were received in September 1905 through the generosity of Mr. SMITH, to whom I here render grateful acknowledgment. I have now grown from these seeds, three generations of plants which bloomed in 1907, I909, and I9II.

The culture of Digitalis at the Station for Experimental Evolution has been attended with great difficulty, owing chiefly to the depredations of a species of thrips (probably Anaphothrips striatus ${ }^{2}$ ) which flourishes amazingly on Digitalis during the hot, dry summer season. The destruction of the epidermis by these insects, leaves the plants so illy protected against the severe summer climatic conditions that all the green parts become rusty brown and the ovaries usually split open, exposing the young ovules. For this reason $I$ have failed in many controlled crosses to secure any viable seeds ${ }^{3}$ ). In fact, in one year (1908) I did not obtain a single plant from guarded seed, and was obliged to begin again with unguarded material. As I am abandoning work with this species because of these cultural difficulties, it seems desirable to record the results of my studies, although they can add little of essential consequence to the excellent account given by Miss SAUNDERS.

1) Linnaea 4:77. I829.

2) According to Dr. A. F. Shull.

3) Miss Tammes writes that she also had trouble with thrips on Digitalis but was able to control them by spraying with Bordeaux Mixture, a remedy not tried by me. 
My observations fully confirm most of the statements made by her, both as to the variability and the heredity of the heptandra-form. I, too, have noted that "offspring of heptandra parents all show dialysis and staminody of the corolla, but the extent to which these malformations are exhibited varies not only in different individuals, but in different flowers on the same individual". The least-developed heptandra-plants in my cultures have had tubular corollas of the same general form as those of the normal foxglove, but with the three lobes of the lower lip inrolled at the margin and forming obvious rudimentary anthers. (See Plate $\boldsymbol{X}$, fig. 2.) The corollas modified in this way are often more or less split between two or more of the lobes on the ventral side of the corolla. At the opposite extreme of the fluctuating series exhibited in my cultures was a form with nine stamens, each of the five lobes of the corolla being represented by a functional stamen of normal form. None was found with ten stamens as reported by Miss SAUNDERS, nor was any observed in which there was complete absence of petaloid structures, for even in the most marked cases, containing nine stamens, there appeared two linear-subulate petal-like phyllodes subtending the lower pair of normal stamens, as shown (i, i) in most of the figures in Plate $\mathbf{y}$. Not infrequently still larger lobes ( $f, f$, in figs. 6,8 , and 9 ) of similar character subtend the upper pair of normal stamens, and the dorsal lip which is commonly undivided and truncate (figs. 3 and 4), becomes in the plus-fluctuations laciniate, then divided into two filaments, each with a half-anther ( $p, p$, in the figures), and finally sometimes with complete anthers, and between these two filaments a median dorsal petaloid process (e).

It is to be noted that these remnants of the corolla (i, i, f, f, e) four of which subtend the four normal stamens and the fifth (e) standing opposite the position which would be occupied by the fifth stamen, were it present, constitute the five lobes of an "accessory corolla" in which the symmetry is exactly the reverse of that seen in the normal corolla, two lobes of the accessory corolla being ventral and three dorsal and each occupying the position of one of the sinuses of the normal corolla. The ventral components (i, i) of the accessory corolla are much more frequently in evidence than the three dorsal lobes. They appear in a number of the published figures of the heptandra-form, while the dorsal components are much more rarely seen, partly, no doubt, because the figures most commonly represent flowers in which the dorsal lobe of the corolla is not suffi- 
ciently reduced to show them, but partly also because the dorsal segments of the accessory corolla are not as generally present even in flowers showing the highest development of the heptandra-character as are the ventral lobes. DE CHAMISSO'S figures 2 and $3^{1}$ ) (reproduced in my Plate $\mathbf{X}$ as figs. A and B) show the complete accessory corolla, to the parts of which DE CHAMISSO gave distinctive names as follows: The median dorsal lobe = "(e) lacinula postica impari"; the lateral dorsal lobes $=$ "(f) alae laterales"; the ventral lobes = "(i) lacinulae corollinae stamina primaria stipantes". The median dorsal component of the accessory corolla is also shown in two of Miss SAUNDERS's figures (her figs. 4 and 5) though in both the flowers represented by these figures it was very small.

The morphological significance of these five petaloid structures forming the accessory corolla, seemingly occupying the sinuses of the normal corolla and becoming visible only when the lobes of the normal corolla have been completely modified to stamens, is not clear. Perhaps they are in some way related to the filaments of the stamens which they subtend. The filaments are normally adherent to the tube of the corolla at base, and this may indicate such close approximation of the fundaments of petals and stamens that the morphogenic activities operating to produce these two sets of organs may become slightly confused, the activities belonging normally to the one cycle being transferred in some degree to the adjoining cycle. In other words, it may be conceived that the "growth-wave" which in the staminal cycle gives rise to a stamen, affects to a slight degree the adjacent corollar cycle and there produces a petaloid out-growth lying in the same radial plane as the stamen.

The nature of the mutation by which the heptandra variety was produced from the normal Digitalis purpurea may be thought of in similar terms as a still more extensive confusion of morphogenic activities which are normally limited to two separate, closely adjacent cycles of development, namely, the staminal and the corollar. This confusion is still further evidenced by the frequency with which union of normal stamens with the accessory stamens appears in the flowers of the heptandra-form. DE CHAMIsso's fig. 3 (my fig. B, PlateXy shows a striking example of this phenomenon, three of the four normal stamens being each united with an adjoining accessory stamen. Three of my figures (figs. 7,8 , and 9) show similar con-

1) Linnaea 1: $57 \mathrm{I}-575$, Plate VI. I 826. 
ditions, but in each of these the ventral pair of normal stamens $(k, k)$ are united with the median ventral accessory stamen $(\mathrm{g})$ to form a triple structure, and in figs. 8 and 9 there is a partial or complete union of the dorsal normal stamens $(1,1)$ with the lateral accessory stamens $(h, h)$.

The periodicity in the degree to which the heptandra-character is developed at different levels on the raceme, observed by Miss SAUNDERS, was also noted in my cultures; but in no case was a completely normal corolla found on any heptandra plant grown at the Station for Experimental Evolution, such as Miss SAUNDERS states appeared rarely in the higher parts of the racemes on certain plants in her cultures which had the lowest grade of development of the heptandra-character. My failure to find such normal flowers may very well be due to the comparatively early death of the flower-stems owing to injury from thrips. In my cultures as in those of Miss SAUNDERS, the distinction between the heptandra plants and the normal was absolute.

Miss SAUNDERS has demonstrated that the heptandra-form is a simple Mendelian recessive to the normal type, and the following account of my experiences completely supports this conclusion.

In' January, Igo6, the seeds received the previous autumn from Mr. SMITH, proprietor of the Daisy Hill Nursery, Newry, England, were sown in sterilized soil in the propagating-house, and there resulted I6I plants which, on May $2 \mathrm{I}$, were set into the garden at intervals of one meter. Despite this early start, none bloomed in 1906, but all formed large rosettes, a few of which were destroyed by rot in the latter part of summer. Many more were lost during winter and spring, so that not over one hundred plants bloomed in I907. All of these were of the heptandra-form. The seeds had been collected, according to Mr. SMITH, from a plant which had "lost its corolla entirely, but not much its fecundity". It was therefore of the extreme heptandra-type. The offspring showed a great preponderance of the more highly developed heptandra-form, but varying to the least developed extreme.

A number of attempts were made to secure seeds by crossing reciprocally both the least developed and the most highly developed specimens in this family, with normal Digitalis purpurea, both whiteflowered and purple-flowered, which was growing in a neighboring garden at a distance of about roo meters from my cultures. The attacks of thrips were so disastrous that almost no seeds were secured 
from these hand-pollinations, and to insure against the loss of the strain, seeds were also collected from specimens whose flowers had been unguarded against promiscuous cross-pollination. In 1908 sowings were made from all of the attempted crosses and also from these unguarded seeds, but only the latter resulted in germinations. Of the plants so produced (family 0745) I84 came to bloom in I9o9, I54 having white flowers of the heptandra-type in various grades of development, thus agreeing with their seed-parent, and 30 with flowers of the normal type, I8 with purple flowers and I2 with white flowers.

Of the heptandra plants in this family ro produced no open flowers because of damage from thrips; 3 had corollas of nearly normal form but with the lower lip margined with half-anthers, or partially split into three segments, each bearing an anther with divergent lobes; 95 had a well developed upper lip, truncate or but little divided, and seven stamens; 40 had the upper lip more or less strongly cleft or laciniate, with two of its segments frequently bearing half-anthers, or rarely with complete anthers; 4 had the upper lip almost entirely absent in most of the flowers; one had the corollas split into four segments, the lateral and lower segments each being terminated with rudimentary anthers, thus standing intermediate between the class with the least development of the heptandra-characters and the, modal condition in which the lower lip is replaced by three perfect stamens, while the upper lip remains but little modified. These categories are not quite the equivalent of Miss SAUNDERS's classes, for the plants here recorded as having the upper lip laciniate, doubtless represent the transitional stages from the simple 7 -stamened to the 9-stamened classes in her grouping, and while many of them would probably have been placed in her "heptandra-form proper (i. e. with a large number of flowers having a petaloid upper lip", some of the more extreme cases of laciniated upper lips might have been classified without much violence with her "extreme heptandra-form". It must be borne in mind that all of these classes are artificial, and are connected by intermediate forms; but the records of the fluctuations among my plants correspond so closely with those of Miss SAUNDERS, that it may be fairly inferred that the range of fluctuation in the two cases has been essentially the same.

No doubt was entertained that the normal plants which appeared in this family were produced as a result of pollination by insects coming from normal plants in the adjoining garden. Such normal 
plants in Family 0745, coming as they did from seeds of the heptandra-form, should therefore prove to be heterozygous. This expectation was fully realized, as will be noted in the following table which gives the results of crosses among these normal-flowered individuals, and between them and their abnormal sibs.

$\begin{array}{cc}\text { Family } & \text { Cross } \\ 0930 & \mathrm{NW} \times \mathrm{AW} 1 \text { ) } \\ 0932 & \mathrm{AW} \times \mathrm{NW} \\ 0931 & \mathrm{NW} \times \mathrm{NP} \\ 0936 & \mathrm{NP} \times \mathrm{NW} \\ 0934 & \mathrm{AW} \times \mathrm{NP} \\ 0937 & \mathrm{NP} \times \mathrm{AW} \\ 0935 & \mathrm{NP} \times \mathrm{Self} \\ 0933 & \mathrm{AW} \times \mathrm{Self}\end{array}$

\begin{tabular}{|c|c|c|c|c|}
\hline & \multicolumn{4}{|c|}{ Results } \\
\hline & NP & NW & : $\mathrm{AP}$ & $\mathrm{AW}$ \\
\hline & & $\begin{array}{l}31 \\
31\end{array}$ & & $\begin{array}{l}24 \\
3 \mathrm{I}\end{array}$ \\
\hline $\begin{array}{l}\text { Total } \\
\text { Expected }\end{array}$ & & $\begin{array}{l}62 \\
58.5\end{array}$ & & $\begin{array}{l}55 \\
58.5\end{array}$ \\
\hline & $\begin{array}{l}17 \\
23\end{array}$ & $\begin{array}{l}23 \\
26\end{array}$ & $\begin{array}{r}3 \\
10\end{array}$ & $\begin{array}{l}5 \\
7\end{array}$ \\
\hline $\begin{array}{l}\text { Total } \\
\text { Expected }\end{array}$ & $\begin{array}{l}40 \\
42.75\end{array}$ & $\begin{array}{l}49 \\
42.75\end{array}$ & $\begin{array}{l}33 \\
14.25\end{array}$ & $\begin{array}{l}12 \\
14.25\end{array}$ \\
\hline & $\begin{array}{l}30 \\
23 \\
\end{array}$ & $\begin{array}{l}21 \\
14\end{array}$ & $\begin{array}{l}16 \\
14\end{array}$ & $\begin{array}{l}2 \mathrm{I} \\
12\end{array}$ \\
\hline $\begin{array}{l}\text { Total } \\
\text { Expected }\end{array}$ & $\begin{array}{c}53 \\
37.75\end{array}$ & $\begin{array}{l}35 \\
37.75\end{array}$ & $\begin{array}{l}30 \\
37.75\end{array}$ & $\overline{33}$ \\
\hline Expected & $\begin{array}{l}41 \\
41.06\end{array}$ & $\begin{array}{l}15 \\
13.69\end{array}$ & $\stackrel{9}{13.69}$ & $\begin{array}{l}8 \\
4.56\end{array}$ \\
\hline Expecte & & & & $\begin{array}{l}82 \\
82\end{array}$ \\
\hline
\end{tabular}

Only in crosses between abnormal white and normal purple (Families 0934 and 0937) is there a departure from expectation sufficiently great to suggest that the discrepancies are possibly not solely due to errors of random sampling. In the offspring of these reciprocal crosses the number of normal purple individuals is nearly 40 per cent in excess of expectation. Considering the small numbers employed, this departure should not be given undue weight, however, especially as the deviation comes within three times the-probable error, which is usually taken as the limit beyond which a variation may be pretty safely assumed to be due to some other than a chance distribution. Accepting this view of the exceptionally large proportion of normal purple-flowered individuals in this one pair of reciprocal crosses, it is seen that the constitution of all these families is in accord with the view that the heptandra-form differs from the normal Digitalis purpurea in a single Mendelian unit-character, and that it is recessive to the normal form.

While the origin of such new forms by gradual changes in the genotype is not absolutely unthinkable, the recent accumulations of

1) $\mathrm{N}=$ Normal; $\mathrm{A}=$ Abnormal; $\mathrm{P}=$ Purple; $\mathrm{W}=$ White. 
genetic science render it very probable that this remarkable deviation from the ordinary form of Digitalis originated by a sudden mutation involving the loss of one of the genes necessary to the production of the normal type. This conception leads one to contemplate the normal type as the resultant of an aggregate of genes of quite unsurmizable nature and number. How many "abnormal" strains are potentially present in such a species, each derivable from it by the loss of one or more genes, cannot be guessed, but the number may be large, and there may be many other genes quite as capable of independent loss, but whose loss would prove fatal to the derivative genotypes. Mutations resulting in the loss of such essential genes can not usually be demonstrated, of course, and even in those cases in which genes are lost which are necessary to sexual reproduction, but which are not essential to successful vegetation, giving rise to sterile forms which may be vegetatively reproduced, it may be impossible to determine whether the new form is due to a genotypic change or only to a somatic derangement of some sort. Due restraint should be exercised, therefore, in calling such sterile individuals of any species, "mutants", since their genotypic character in relation to the parent form can not be demonstrated.

As we have seen, the heptundra-form of Digitalis purpurea has been discovered independently a number of times. As it is wholly worthless as a garden variety and would never be grown except as a curiosity or for scientific study, it is fairly certain that at each recorded discovery it has been derived from the normal form. Whether its first recorded appearance in DE CHAMISSO'S garden or any of its later appearances among seedlings of the normal form, was the result of an immediately antecedent mutation, can not be known, for its status as a Mendelian recessive would make it possible to account for all of its occurrences, - widely separated as they have been both in space and time, - as the results of the normal progress of Mendelian inheritance following a single original mutation of unknown antiquity. If the several recorded origins of the heptandra variety have not been due to repeated mutations, this case affords a pretty illustration, albeit a somewhat artificial one, of an important principle which I pointed out several years ago ${ }^{1}$ ), namely, that recessiveness, instead of being a handicap as some have supposed, is in reality a great advantage to a new form which is in any way less well adapted to its environment than its parent. This advantage lies in

1) Science N. S. 25: 590-591, 12 Ap 1907. 
the fact that the heterozygote has the successful parental type and can preserve the less successful new form for an indefinite number of generations until a situation may arise in which it can become a success. Thus, although the gardeners have regularly sown seeds only of the normal type of Digitalis purpurea, the heptandra-form has probably been kept potentially in existence for more than threequarters of a century until it found, for a few years at least, an environment adapted to its successful existence, - in the gardens of the geneticists.

\section{Legends for figures.}

All flowers figured in Plate $\mathrm{XV}$ are viewed from the ventral side and drawn to the same scale. With exception of Figs. A and B, all were drawn from nature by MR. KAKO MORITA. The lettering follows that of DE CHAMISSO'S figures, two of which are reproduced here as Figs. A and B. As none of DE CHAMISSO'S figures show the posterior pair of accessory stamens, these are labeled here $p, p-k, 1,1=$ normal stamens; $p, p, h, h, g=$ accessory stamens; $e, f, f, i, i=$ segments of "accessory corolla".

Fig. A. Drawn from a tracing of DE CHAmisso's fig. 2, showing seven stamens and the five lobes of the accessory corolla, the "lacinulae corollinae", all distinct and equal.

Fig. B. Drawn from a tracing of DE. CHAmisso's fig. 3. The three accessory stamens, $\mathrm{h}, \mathrm{h}$, an $\mathrm{g}$, are severally united with adjacent normal stamens, $1, \mathrm{l}$, and $\mathrm{k}$. Posterior lateral lobes, $f, f$, of the accessory corolla relatively long.

Fig. I. A normal corolla of Digitalis purpurea L. Ventral lip longer than the dorsal. Treated as a transparency to show the normal position of the stamens.

Fig. 2. A low-grade heptandra flower; the ventral lip considerably shortened, the three accessory stamens, $\mathrm{h}, \mathrm{h}$, and $\mathrm{g}$, appearing as imperfectly developed anthers on the margin of the corolla. The lobes of the accessory corolla are also fore-shadowed.

Fig. 3. A very common heptandra-type with seven free stamens and only the ventral lobes, $\mathbf{i}, \mathbf{i}$, of the accessory corolla apparent, the dorsal lip of the corolla remaining almost entire.

Fig. 4. A similar form in which the dialysis of the corolla is less complete, the ventral lobes of the accessory corolla not apparent because they remain united with the ventral accessory stamen, $g$. The lateral accessory stamens, $h, h$, are also partially united with the dorsal lip of the corolla.

Fig. 5. A similar fluwer in which the ventral lobes, i, i, of the accessory Corolla are partially free from the accessory stamen, $g$, and in which the configuration of the dorsal lip of the corolla foreshadows the two dorsal accessory stamens, p, p, and the "lateral wings", $f, f$, of the accessory corolla.

Fig. 6. All parts of both accessory androecium and accessory corolla are clearly present, but the posterior accessory stamens, p, p, are club-shaped and yellowish, showing no other indication of antherformation. 
Fig. 7. All parts are clearly present, but the stamens are united into three groups. Note the prominence of the posterior median lobe, e, of the accessory corolla.

Fig. 8. All accessory organs are clearly present, the posterior accessory stamens being represented by sessile, non-functional halfanthers. The ventral pair of normal stamens, k, k, are united with the ventral accessory stamen, g.

Fig. 9. One ventral lobe, $i$, of the accessory corolla is missing, presumably remaining attached to the the ventral triple stamen formed by the union of the ventral pair of normal stamens with the ventral accessory stamen. The dorsal pair of normal stamens, 1,1 , are alsơ united with the lateral accessory stamens, $h, h$. The dorsal accessory stamens, p, p, are more completely developed, but still non-functional.

\section{Plate XVI.}

Fig. Io. A raceme of normal Digitalis purpurea L.

Fig. II. A raceme of the more common form of Digitalis purpurea heptandra De Chamisso.

Fig. 12. Extreme heptandra-form shown by a plant grown in the Botanic Garden of the Rijks-Universiteit, Groningen, Holland, in 1908. Photograph kindly supplied by Dr. Tine Tammes. 

Zeitschrift f. ind. Abstammungs- u. Vererbungslehre. Bd. 6 Taf. XV

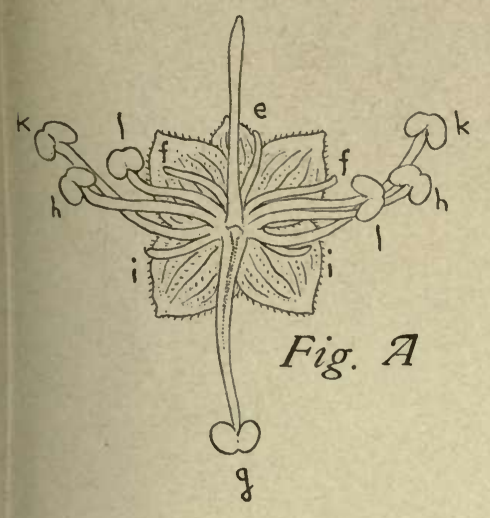

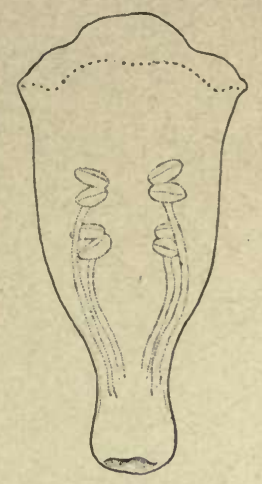

Fig. I

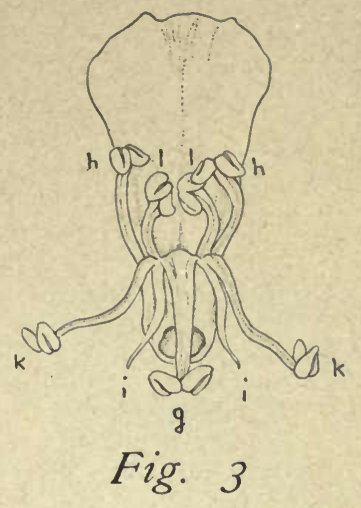

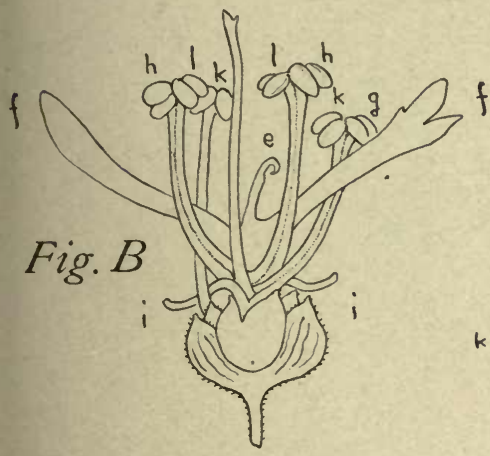
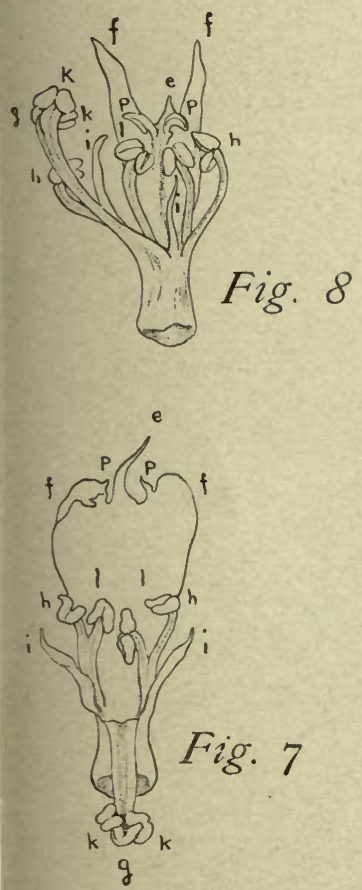

SHULL: Digitalis.
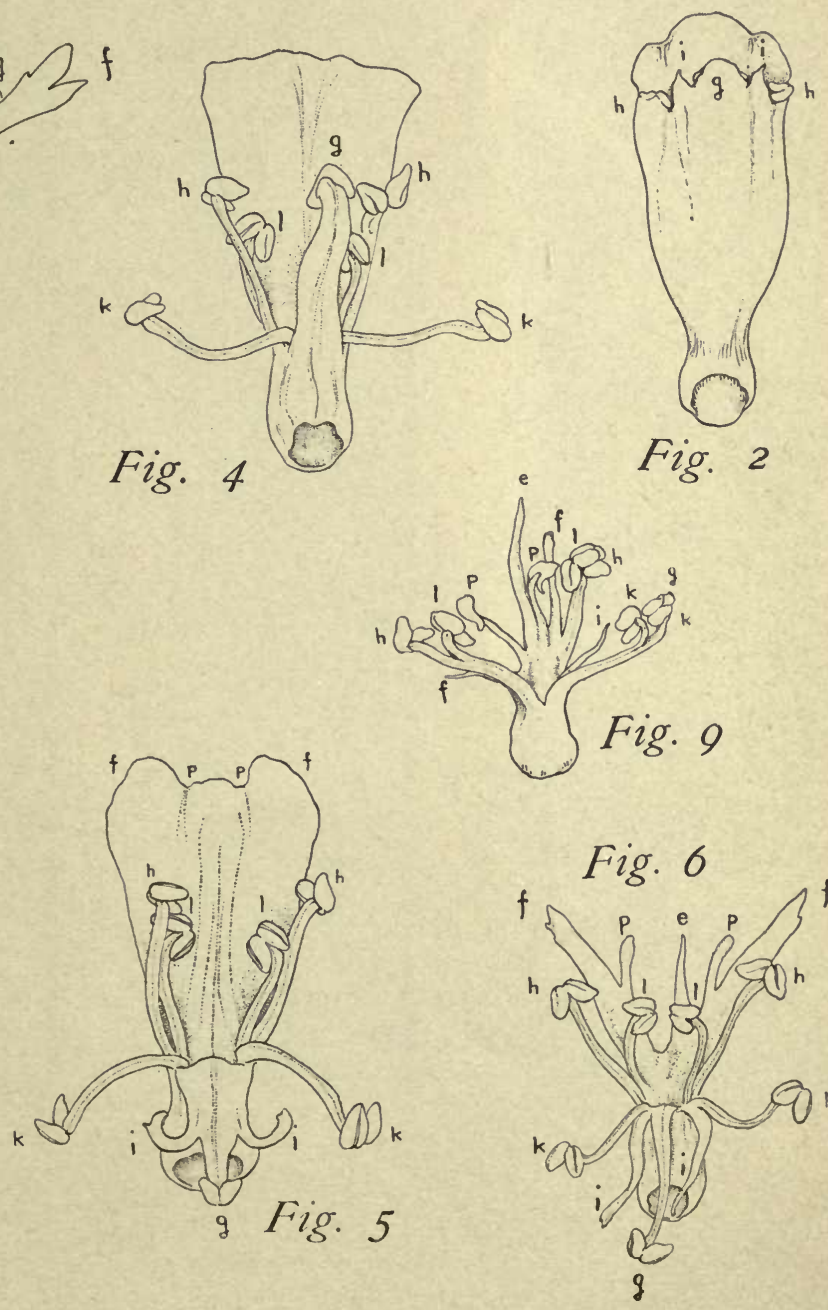

Fig. 2

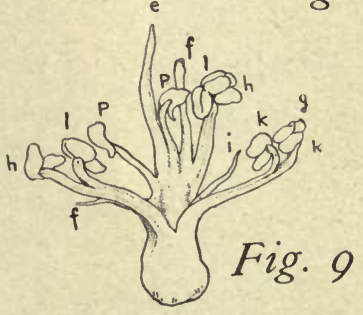

Fig. 6

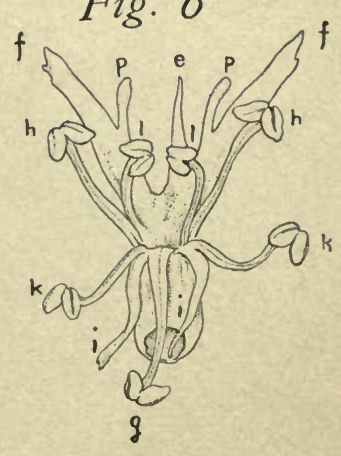




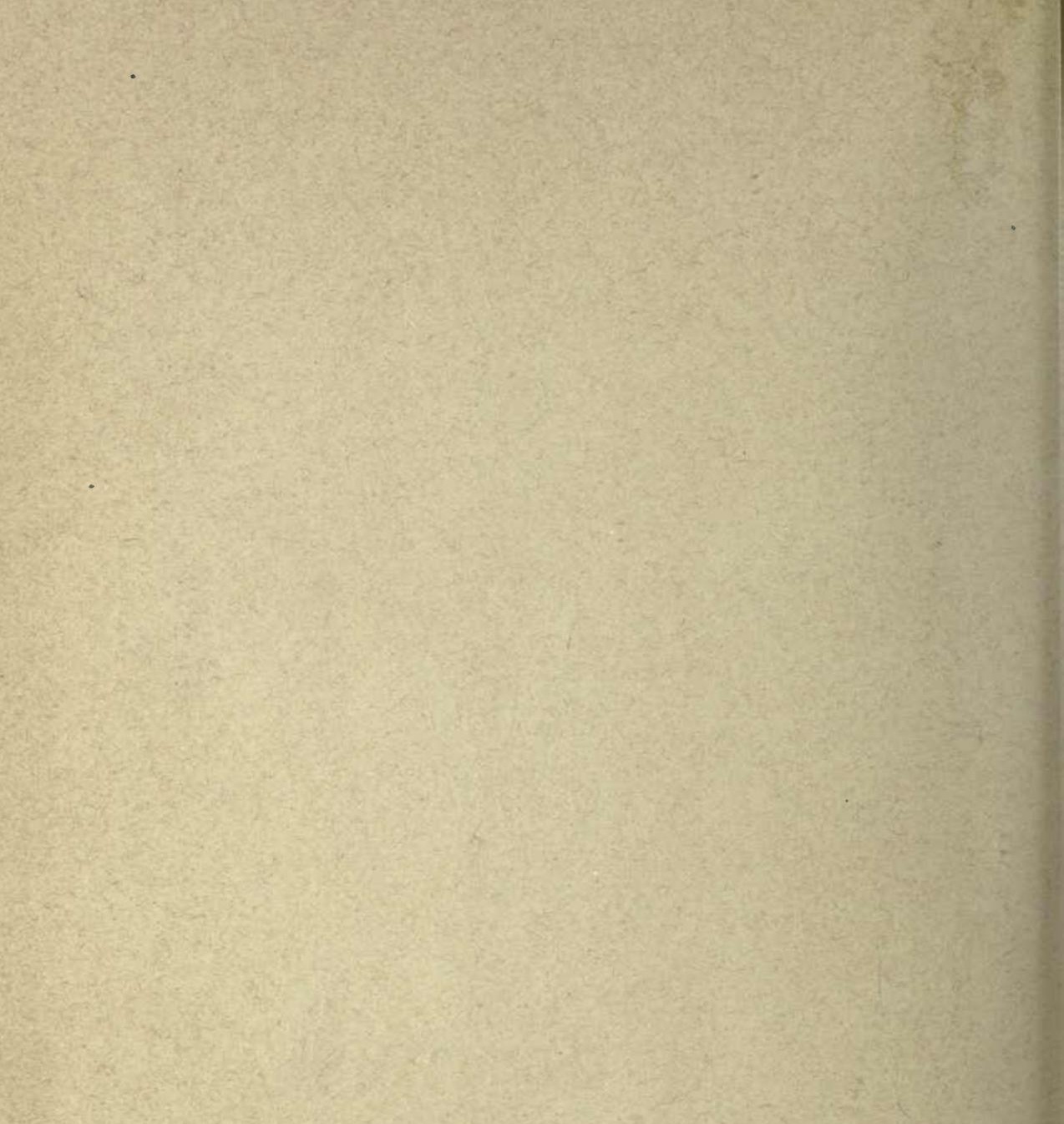




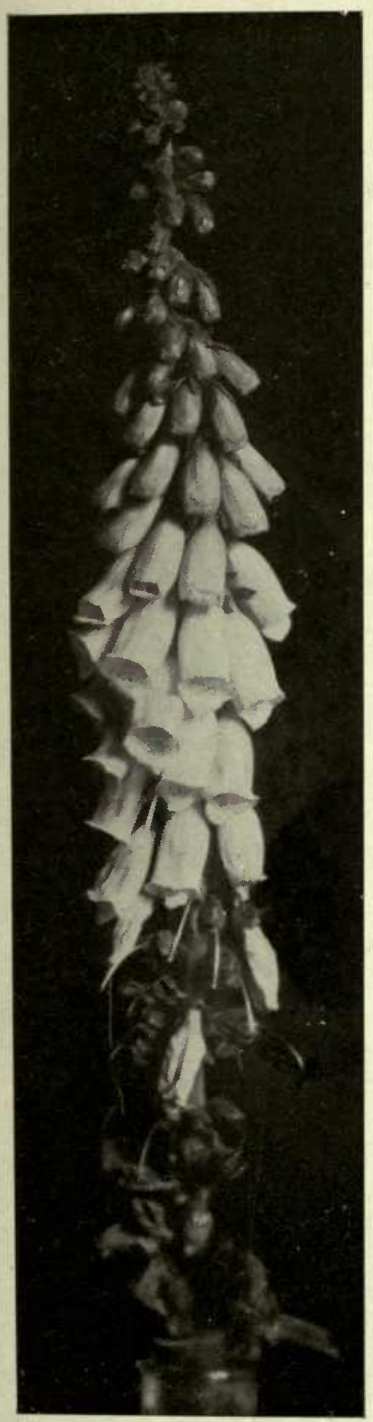

Fig. 10

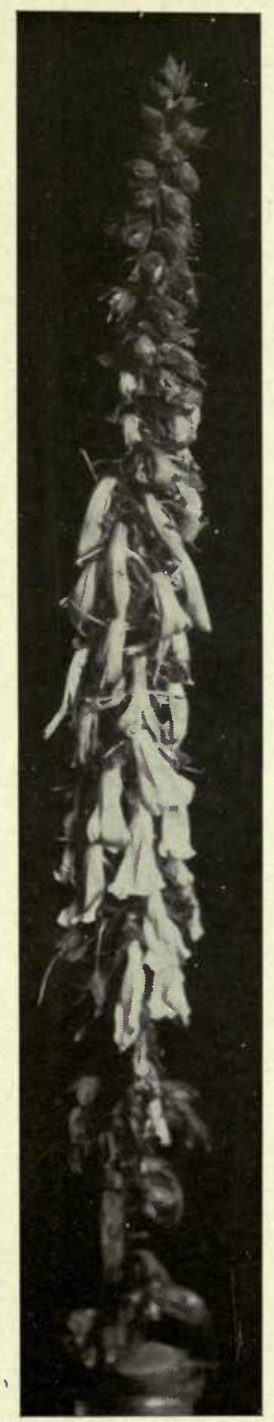

Fíg. 11

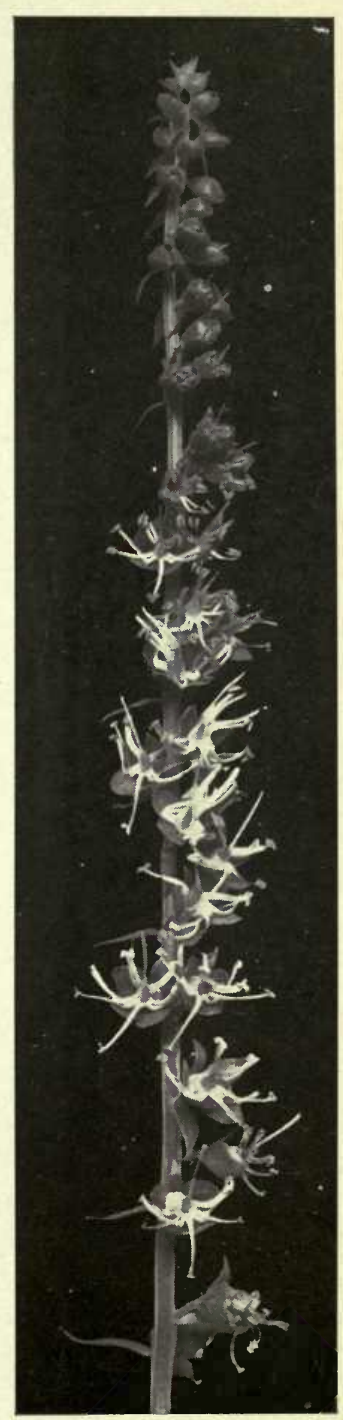

Fig. 12 

s.m.

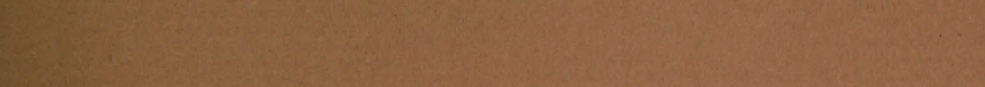

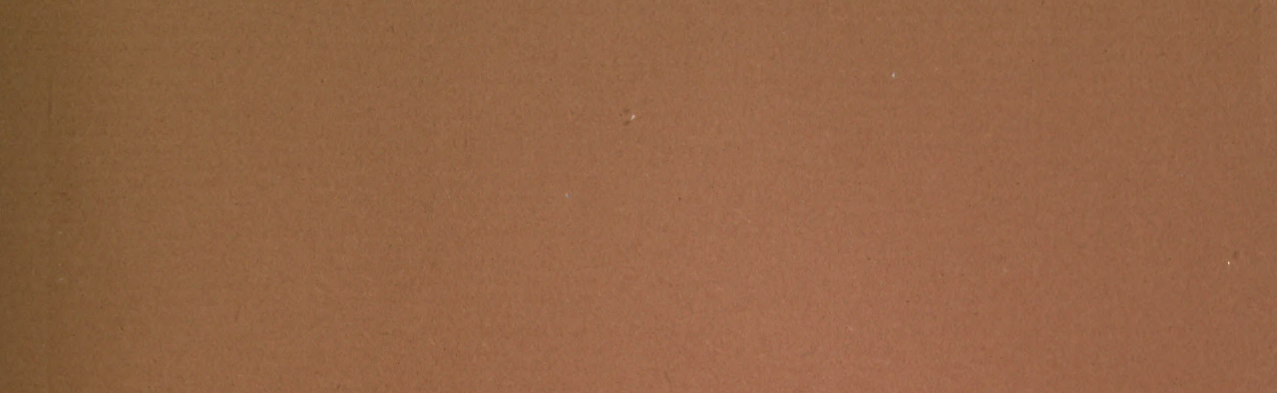

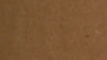

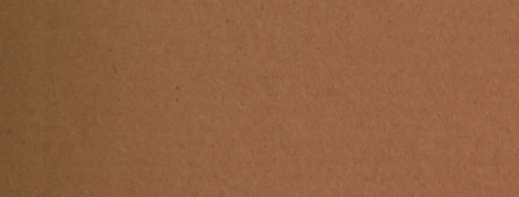

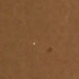

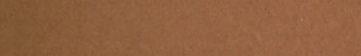

(2).

$\sin 2$

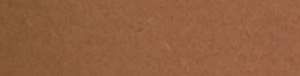

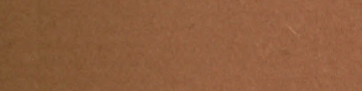

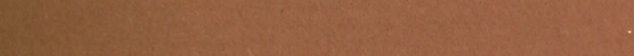

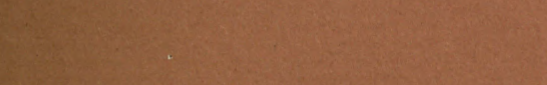

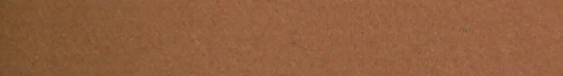

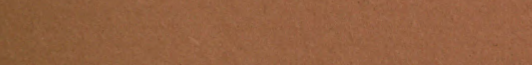

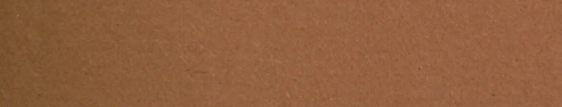

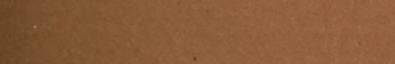

(3)

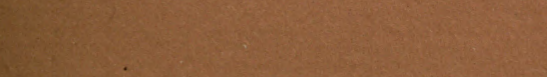

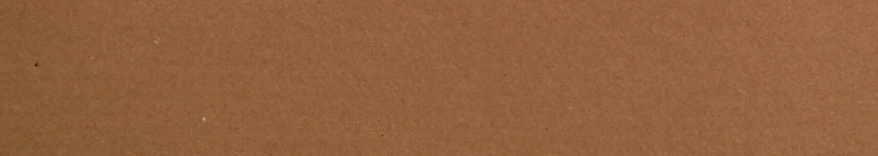

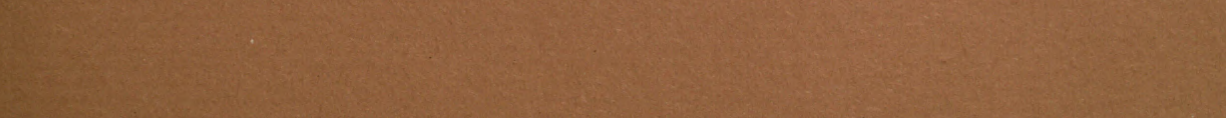


its utterance, by Dr. Johannsen, and his word "biotype" " was immediately adopted in my paper" on "The Composition of a Field of Maize" "and made a part of the title of my work on "Bursa bursa-pastoris and Bursa Heegeri: Biotypes and Hybrids." " In view of these facts there was no excuse for my use of the word "genotype" in a taxonomic sense.

Dr. Jennings also calls attention to an important misuse of the expression "pure line," and here I must again admit a certain amount of guilt, as I was probably the first to include under this term groups of individuals related through the process of budding or any other method of vegetative reproduction. In 1904 I wrote: :

By the "pure line" Johannsen means a series of individuals related only through the process of self-fertilization. On a priori grounds it seems proper to apply the term to every series of individuals that do not combine elements of two or more aneestral lines through the equivalent of a sexual process. Thus, so far as hereditary qualities are concerned, there should be no reason to expect in a self-fertilizing population conditions different from those in a population related through budding or other method of vegetative reproduction, provided, of course, that the selffertilizing population has not been so recently modified by a cross as to allow the analysis and

This word was first proposed in 1905 in "Arvelighedslærens Elementer," the Danish forerunner of "Elemente der exakten Erblichkeitslehre," and was first used in English at the Third International Conference on Genetics in 1906. (See Report Third International Conference on Genetics, p. 98, 1906.)

'Report American Breeders' Association, IV., 296-301, 1908.

- Carnegie Institution of Washington Publication No. 112, 1909.

- Torreya, V., 22, February, 1905. 
recombination of eharacters derived from different ancestral lines.

For this early departure from "the narrow path" I have in part atoned in my recent paper on the "Genotypes of Maize," " by referring to the vegetatively reproduced potato and paramecium as "clonal varieties," in contradistinction to the self-fertilizing "pure lines" of beans, barley, etc. I might equally well have called them simply "clones," as "clonal varieties" and "clones" should have identical meaning. The word "clone" (Greek $\kappa \lambda \omega \nu$, a twig, spray, or slip) was proposed by Webber" for "groups of plants that are propagated by the use of any form of vegetative parts, such as bulbs, tubers, cuttings, grafts, buds, etc., and which are simply parts of the same individual seedling." I believe that no violence will be done by extending this term to include animals which are similarly propagated by any asexual method, and I suggest the general adoption of the word "clone" for all groups of individuals having identical genotypic character," and arising by asexual reproduction of any sort, including apogamy (i. e., so-called "parthenogenesis" unaccompanied by a reduction division).

For the purposes of my discussion in 1904 the distinction between "pure lines" and "clones" was of no consequence, because the particular hereditary principle then under consideration was common to both. The same thing is no doubt true of many of the recent investigations of others, but it is well to re-

'Amer. Nat., XLV., 234-252, April, 1911.

${ }^{8}$ SCIENCE, XVIII., 501-503, October 16, 1903. For a discussion of the spelling of the word "clone" see SCIEnCE, XXII., 89, July 21, 1905.

- This restriction is necessary in order to avoid confusion through the appearance of bud-mutations. Such a mutation if propagated vegetatively represents the origin of a new clone. 
member that there are certain fundamental differences between "pure lines" and "clones," which render it very important to maintain the distinction between them. I will mention but two of these differences by way of example: (1) In the "clone" it is possible to retain as a permanent feature of the group any purely heterozygous character, as for instance the vigorous constitution dependent upon the stimulation of heterozygosis; such a phenomenon is impossible in the "pure line." (2) When clonal individuals reproduce sexually, either by self-fertilization or by crossing with other individuals, they need not, and usually do not, produce genotypically equal offspring, because the individuals of the "clone" are not necessarily homozygous, as the individuals of the "pure line" generally are. The "clones" of horticultural plants are notorious for the heterogeneity of their seedling offspring. The investigator of intermittently parthenogenetic organisms like aphids, rotifers and Hieracium, and of intermittently vegetatively produced organisms like paramecium and many plants, can not properly assume that their races are genotypically pure in the sense that they are homozygous, while the worker with "pure lines" can make such assumption with small probability of error, in case his self-fertilizations have been controlled with adequate care during a sufficiently large number of generations.

There is another prevalent misconception regarding "pure lines," to which attention needs to be called. The word "pure" in this connection does not refer to the genotypic equality of the individuals, but only to the exclusion of all crossing as a source of genotypic differentiation.

In Dr. Harris's criticism ${ }^{10}$ of Roemer's work with peas, he points out with very evi-

${ }^{10}$ Amer. Nat., XLV., 686-700, November, 1911. 
dent satisfaction that two of Roemer's populations are historically traceable to individual selections and that they are therefore really "pure lines" (i. e., providing, of course, that those originally selected individuals were strictly homozygous, and that no chance crossing has taken place since). If no genotypic changes can take place within the "pure line" all evolution is impossible in the large number of forms which naturally maintain "pure lines" by obligate self-fertilization. The only point to be made in regard to this feature of Roemer's results, is that, if his populations were really "pure lines," the numerous distinct biotypes he discovered by the "pure line" method in those populations, were the result of mutations which have taken place since the original selections were made. The occurrence of such mutations does not affect in the least the value of the genotype theory, nor the importance of the "pure line" method for the study of heredity.

Definitions:

Genotype, the fundamental hereditary constitution or combination of genes of an organism.

Biotype, a group of individuals possessing the same genotype.

Pure line, a group of individuals traceable through solely self-fertilized lines to a single homozygous ancestor.

Clone, a group of individuals like trpiconstion through asexual reproductions to a single ancestral zygote, or else perpetually asexual.

Geo. H. Shull

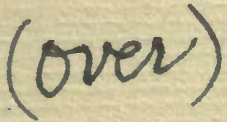



[Reprinted from Sciknce, N. S., Vol. $X X X V$., No. 892, Pages 182-183, February 2, 1912]

$$
\text { " PHENOTYPE" AND " CLONE" }
$$

Is calling attention to the frequent misuse of the words "genotype" and "pure line," Jennings says" that the word "phenotype" "designates a group of organisms which in outward appearance seem to belong to one type, although in hereditary constitution they may actually differ greatly. Genotype, in Johannsen's usage, is not directly contrasted with phenotype," etc.

As I have also used "phenotype" with the meaning indicated by Jennings, I did not recognize the fundamental misconception involved in the quotation given above, when I wrote $m y$ note $^{2}$ in response to the article from which this quotation is taken. My attention has been called to this point by Dr. Johannsen, and it seems best to set the matter straight at once, in connection with the attempt made by Jennings and seconded by myself, to restrict to their original meanings, the other terms introduced by Johannsen. "Phenotype" and "genotype," when both are rightly used, are contrasted terms, both being abstractions referring to the type to which an individual or group of individuals belongs, and not to the group of individuals belonging to that type. To illustrate the use of "phenotype" in its correct sense, reference may be made to the $F_{3}$ of a Mendelian hybrid. When an $F_{1}$ hybrid whose genotypic constitution may be represented by the formula, $X X A a B b$, is self-fertilized or crossed with

${ }^{2}$ Science, December 15, 1911.

${ }^{2}$ ScIEnce, January 5, 1912. 
another individual having the same formula, there will be possessed by different individuals among the offspring nine different genotypes, but only four different phenotypes. The nine genotypes may be represented by the formula: $X X A A B B, X X A A B b, X X A a B B, X X A A b b$, $X X a a B B, X X A a B b, X X A a b b, X X a a B b$ and $X X a a b b$. The four phenotypes may in similar manner be indicated by the formulæ: $X A B$, $X A b, X a B$ and $X a b$. As the "phenotype" is the "type of the phenomenon"-the type of that which actually appears-there must always be as many distinguishable groups of individuals as there are phenotypes; hence, the readiness with which the word "phenotype" has been misinterpreted and applied to the group of apparently equal individuals instead of the constitution or assemblage of characteristics with respect to which such a group of individuals is apparently homogeneous.

There is at present no satisfactory word universally applicable to all groups of individuals possessing the same phenotype-the concept for which the word "phenotype" itself has been misused. The words "spocies" and "sub-species" used by taxonomists are applicable, at least in some cases, to groups of such phenotypically equal individuals, but no one would think at present of applying either of these words to all the numerous slightly differentiated groups which the geneticist is now able to distinguish and with which he is obliged to work. A short and appropriate word for all such groups of individuals, of whatever degree of differentiation, is greatly needed.

In my discussion of "clone" as a suitable name for any group of plants or animals which has been formed from a single original individual by purely vegetative methods of reproduction, I suggested the restriction of the term to groups of genotypically identical 
individuals. Further consideration convinces me that this restriction is highly undesirable because it is impracticable. It would be quite impossible to know for a certainty that two twigs used as cuttings or cions from the same tree had the same genotypic constitution, and consequently there could be no security in the assumption that they were members of the same clone, if the definition given in my previous note should be maintained. I wish, therefore, to offer an amendment to that definition by striking out such restriction. The definition may then read: "Clone, a group of individuals traceable through asexual reproductions (including parthenogenesis when unaccompanied by genotypic segregation) to a single ancestral zygote, or else perpetually asexual." This definition puts the word "clone" on exactly the same footing as the expression "pure line," making it a purely genealogical term and involving no implication whatever as to the genotypic equality of the individuals included in the single clone.

Geo. H. ShULL 



\section{CURRENT LITERATURE}

\section{BOOK REVIEWS}

\section{Heredity}

Several courses of public lectures on heredity have been made the basis of a very readable book by CASTLE, ${ }^{\mathrm{r}}$ in which the principles of Mendelian heredity and other related topics are discussed with special reference to their bearing upon evolution and animal breeding. The rapidly increasing number of expositions of Mendelism are warranted both by the growth of scientific knowledge, and by the popular demand for information, and it is gratifying to have the subject presented in easily comprehensible language by one who is among the foremost investigators of the phenomena with which the book deals. The manner of origin of this book makes it natural that the author should illustrate the various principles of heredity by examples from his own extensive experiments, whenever such examples are available, and this method gives the book a unique value.

The brief introductory chapter on "Genetics a new science" recognizes the profound influence exercised by the theory of evolution in many fields of human activity, and shows how the evolutionary idea has forced man to consider his own probable future and to seek to control that future. As the "existence of civilized man rests ultimately on his ability to produce from the earth in sufficient abundance cultivated plants and domesticated animals," "civilization may be advanced in a very direct and practical way" by an increased knowledge of plant and animal breeding. To the solution of the problem "how to create new and improved breeds better adapted to the conditions of present-day agriculture," and especially to an exposition of the "operations" of Mendel's law of heredity, the author specifically addresses himself.

Chap. i on "the duality of inheritance" defines heredity as "organic resemblance based on descent," and discusses fertilization, pointing out that either eggs or sperms can under certain experimental conditions produce a complete organism without union with another gamete, and that such a result is realized regularly in nature in the case of male bees and wasps. The $x$ and $2 x$ generations of Lotsy are then considered under the designation $N$ and $2 N$ generations, a change of terminology which has nothing to commend it. Chap. ii distinguishes between "germ-plasm" and the body or "soma," and cites experiments in the transplantation of eggs to an alien soma as proof of

${ }^{x}$ Castle, W. E., Heredity in relation to evolution and animal breeding. 8vo, pp. xii+r84. figs. 53. New York: D. Appleton \& Co. I9r I. 
the correctness of WEISMANN's contention that "body and germ-cells are physiologically distinct," and that "body (or somatic) influences are not inherited." The next six chapters deal with "the greatest single discovery ever made in the field of heredity, Mendel's law," with illustrations drawn chiefly from the author's experiments with guinea-pigs, rabbits, rats, and mice. After a careful statement of the general principles with concrete examples, attention is given to the determination of dominance, heterozygous characters and their "fixation," atavism or reversion, evolution by loss or gain of characters, evolution of new races by variations in the potency of characters, modification of unitcharacters by selection, and "blending" inheritance. This enumeration of the subjects treated suffices to indicate that the author's discussion is no merely formal presentation of the ramifications of the Mendelian system. Instead, it deals lucidly and entertainingly with a number of moot questions. It is pleasing to note that the author does not follow some other recent writers (BATESON, Punnetr, DAvenPoRT) in the view that dominance is always due to the presence of a gene which is absent from the recessive form. He mentions several cases for which this explanation is not available, and leaves the problem as to the cause of dominance unsolved. $\mathrm{He}$ is convinced that unit-characters may be modified by selection. He says (p. I 20): "In several cases I have observed characters at first feebly manifested gradually improve under selection until they became established racial traits." While this must be accepted as an observed fact, the statement that it is due to selection is apt to be misleading. The difficulty remains that selection can only pick out individuals already possessing the observed degree of development of any characteristic under consideration, and does not in any manner modify the qualities which will be possessed by the offspring of the selected individuals. It only permits such individuals to reproduce to the exclusion of other individuals which do not have the character in question. Under "blending inheritance" the now wellknown case of skull-size and ear-length in rabbits is discussed, and the view is expressed that in the light of experimental results of Nilsson-Ehle, EAST, and others, such cases of apparent blending may really prove to be segregating inheritance in which a considerable number of units are involved.

In chap. ix the effects of in-breeding are considered, and the reviewer's conception is indorsed, that the apparent deterioration is generally due to the formation of homozygous strains, whereby the stimulus is lost which comes from the "bringing together of differentiated gametes, which, reacting on each other, produce greater metabolic activity." In this connection the statement is made ( $p$. 150) that "under self-pollination for one generation following a cross, half the offspring become homozygous; after two generations threefourths of the offspring are homozygous"; and so on. This statement is misleading as it stands, and is literally true only in the case of monohybrids. A second cause recognized for deterioration following in-breeding is the appearance of recessive defects, such as albinism, etc., a cause which has been specifically pointed out by DAVENPORT. 
The tenth and last chapter deals with heredity and sex. The hypothesis offered by the author several years ago that the female regularly possesses a chromatic element, or something else in addition to the possessions of the male, is made the key to the entire discussion of this subject, and a series of facts is presented which give the hypothesis considerable apparent plausibility, although the philosophical basis for it seems to the reviewer to be a little strained. This basis is found in the statement that the female as compared with the male has an additional function, namely the supplying of nourishment to the young zygote. On the other hand, it may be pointed out that the male differs from the female in many functions, and is in many respects morphologically and physiologically the superior sex, and it is possible to interpret these differences as additions to the female. If the egg has the added function of nourishing the young zygote, the sperm has the added function of motility, and there seems no better a priori ground for expecting an additional chromatin element to represent one of these additions than the other. The reviewer believes that there is no sufficient ground at present for the assumption that sex is always determined in the same manner. It cannot be determined as yet whether the basic differences between the sexes are quantitative or qualitative, and in either case the same results might be attained by any one of several different methods. The attempt to bring the sex-phenomena of all organisms under a single viewpoint is premature.

Each of the chapters is followed by a "bibliography," but the meagerness of the literature lists may be judged from the fact that they include only 46 titles from 26 authors, including I 4 of CAstLE's own papers. This may be compared with the bibliography appended to another recent book on heredity which includes 426 titles from I70 authors. However, this is not intended as an adverse criticism of CASTLE's excellent book, but is mentioned to show the limitations of its author's aims. Extensive literature lists are indispensable to students, but would defeat their own purpose in a book intended primarily for popular reading.

The press work is excellent and typographical errors are few, though "reversion" is rendered "revision" in the heading of chap. iv.-GEORGE H. SHull. 



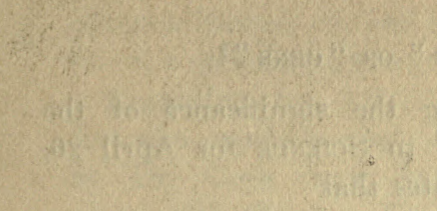

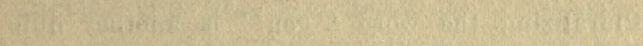

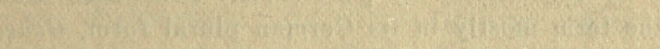

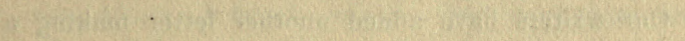

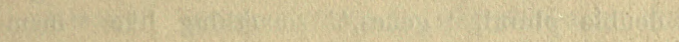

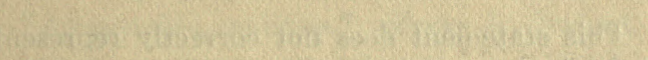

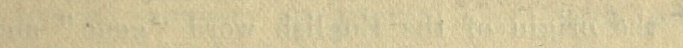

(3.

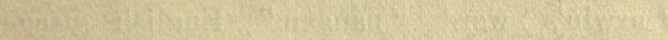

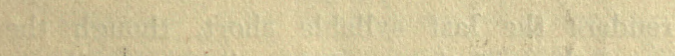

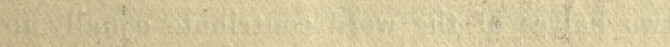

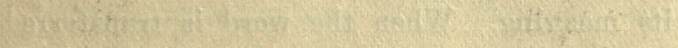

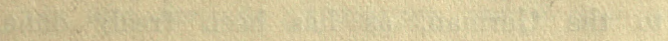

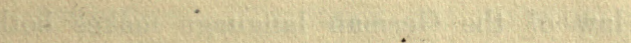

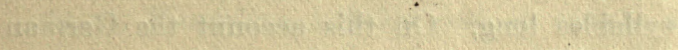

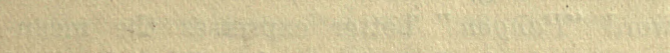

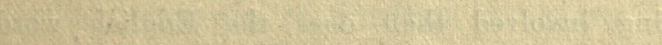

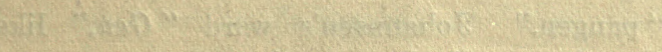

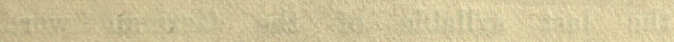

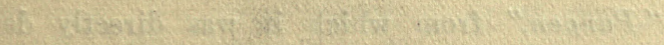

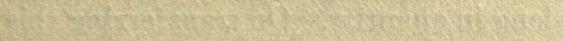

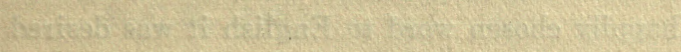

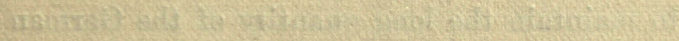

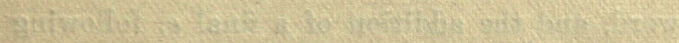

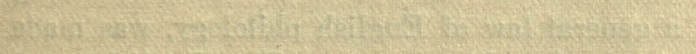

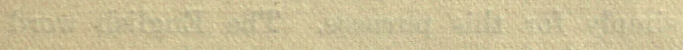

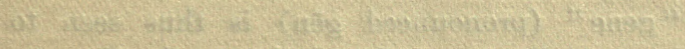

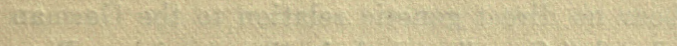

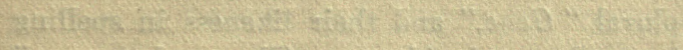

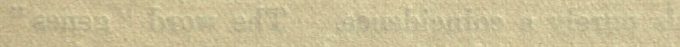

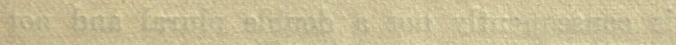

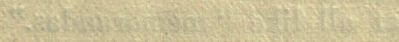

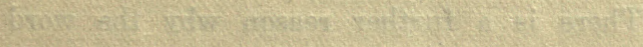




\section{DIVISION OF GENFTICS}

[Reprinted from ScIENCE, N. S., Vol. XXXV., No. 908, Page 819, Mag 24, 1912]

\section{"GENES" OR " GENS"?}

AFTER discussing the significance of the word "phenotype" in Scrence for April 26, Dr. O. F. Cook states that

Pluralizing the word "gen" is another difficulty encountered by geneticists. Johannsen used the term mostly in its German plural form, Gene. Our writers have added another letter making a double plural, "genes," something like "'memorandas."

This statement does not correctly represent the origin of the English word "gene" and its plural "genes," now generally used by writers of English papers on genetics. In Darwin's word "pangen" English usage renders the last syllable short, though the two halves of the word contribute equally to its meaning. When the word is transferred to the German, as has been freely done, a law of the German language makes both syllables long. On this account the German word "Pangen" better expresses the meaning involved than does the English word "pangen." Johannsen's word "Gen," like the last syllable of the German word "Pangen," from which it was directly derived, is long in quantity. On transferring this happily chosen word to English it was desired to maintain the long quantity of the German word, and the addition of a final $e$, following a general law of English philology, was made simply for this purpose. The English word "gene" (pronounced gēn) is thus seen to bear no direct genetic relation to the German plural "Gene," and their likeness in spelling is purely a coincidence. The word "genes" is consequently not a double plural and not at all like "memorandas."

There is a further reason why the word 
"gene" should be preferred. This word must be used commonly in the plural form, but there is already a word "gens" in rather common literary use and having, at least sometimes, a genetic meaning.

Regarding the definition of "phenotype," few who carefully read the passage translated by Dr. Cook from Johannsen's book will agree with the translator that "phenotype" as used by its author was ever anything but an abstraction. "Centers among series of variations around which the variants are grouped" must always be abstractions, and yet they are, as Johannsen rightly says, " measurable realities." Every individual organism possesses an external appearance and a fundamental constitution, and is therefore a representative of some phenotype and of some genotype. The words "phenotype" and "genotype" were never intended to be limited to statistically investigated organisms. Statistical investigation may discover, measure and describe phenotypes, but it does not create them. Phenotypes and genotypes exist among Mendelian hybrids just as among all other organisms, and my use of the Mendelian categories to illustrate the proper use of these two words involves no "new version of phenotype."

COLD SPRING HARBoR, L. I.,

G. H. SHULL

April 29, 1912 

Reprinted from 'The Antiochian Vol. 2, No. 8, June 1912

\section{A PILGRIMAGE TO BRÜNN}

\section{By Dr. George H. Shull (B. S., Antioch, 1901) Station for Experimental Evolution, of the Carnegie Institution of Washington, Cold Spring Harbor, L. I.}

Before me on the wall of my study hangs a little clock of oldfashioned design, its three-inch enameled dial set in a small square of pressed brass borne on a single ebonized stave, the pendulum and cylindrical brass weights attached to long, thin cords hanging free below. A visitor's attention is at once arrested by this clock because its design is unlike that of any modern American timepiece, and because its very simplicity and unpretentiousness suggests that it must have a history.

This rather obvious' inference is correct, for this little clock once hung on the chamber wall of a man to whose memory the scientific men of all nations joined in 1910 in erecting a beautiful white marble statue in Brünn, Austria, the capital city of the province of Moravia. This man was Johann Gregor Mendel, an Augustinian monk, Abbot of the old Monastery or "Königskloster" of Brünn, who, by a carefil study of garden peas during eight years, discovered the key to the age-long riddle of heredity; and this little clock is a memento of my visit to Brïnn, given to me by the present Abbot, Mendel's successor, Father Salesius Barcina (pronounced Bärsena). 
elapsed before dinner was announced and we descended to the first foor or basement, to the dining room. This is a long room with high ceiling, well lighted by a row of windows on one side. Walls and ceiling are absolutely devoid of all decoration. In the center of the room and extending nearly its whole length was a long $\mathrm{U}$-shaped table with chairs arranged along the outside of the $\mathrm{U}$, the inside providing a free passage-way for the waiters who served the meals. In the dining room, when we entered it, were over twenty monks assembled, all dressed in long black gowns, their hair cut short and crowns shaven. Father Barcina took his place at the center of the $\mathrm{U}$, placing me on his right and Doctor Iltis on his left, the monks then arranging themselves along the sides. After a short grace spoken by Father Barcina a simple but bounteous meal was served. Eatables and drinkables of varions kinds were there in abundance, but being a teetotaler, I was permitted to devote my attention chiefly to the former. After the meal was finished, ail stood while a lengthy responsive thanksgiving service was chanted, one long, thin, hollow-eyed monk taking the solo part, the rest responding in unison, making the great hall resound with their sonorous voices. This was all in Latin, of course, and as $I$ had been forgetting in eleven years what I had learned of Latin at Antioch in as many weeks, few of the words were intelligible to me, but the fervent expressions and the music belonged to that universal language which is neither learned nor forgotten; they were understood.

After dinner, in company with the Abbot and several other monks, we made a tour of the buildings and grounds. Several of the reception rooms have been purposely kept essentially in the condition in which they were when occupied by Mendel, even to having his chess-board resting open on a small table and arranged ready for his favorite game. A second favorite recreation was bowling, and the monks pointed out on the walls of the bowling-alley penciled scores which they declared had been placed there by the former prelate. On the third flcor, which is occupied by an extensive library containing many very old books, Mendel's tëlescope still stood on the ledge of one of the small dormer windows, for it nust be remembered that Mendel's scientific interests were not narrowly limited to the problems of heredity, but he studied sunspots, and made important contributions to meteorology as well, faithfully collecting for many years the first climatological data for that section of the world. The splendid results of his investigations in heredity tend to obscure the importance of his work in other lines. Just beside the Monastery is the little garden spot in which the classic pea-experiments were performed, still enclosed by a picket fence, but not now used as a garden. On a high terrace back of 
the chapel is a row of bee-houses, long since unoccupied and fallen into decay, in which Mendel was also attempting to carry on experiments in heredity, but of which no detailed account lias yet been found. Near by is the shelter in which were kept the weatherrecording instruments so faithfully used almost up to the day of Mendel's death, and so completely neglected during the quarter century which has elapsed since. Below the terrace, but still in the rear of the chapel, several pear trees were pointed out which were selected seedlings of much merit, the selection having been made by Mendel.

It was with much interest that I examined Mendel's microscope, a small compound microscope without rack-and-pinion adjustment, but with a sliding tube to be simply operated by hand. A number of microscopic slides made by Mendel were also carefully examined to see whether possibly their maker might not have known something more of modern microscopic methods than could ordinarily be expected, for in rccent years the development of microscopic technique has enabled cytologists to discover bodies in the cells of plants and animals, whose behavior in the germ-cells accords exactly with the observed behavior of unit-characters in Mendelian inheritance. It is now generally believed that these bodies-the chromosomes-are the producers of hereditary qualitics. Not the slightest evidence was found, however, that Mendel conld have anticipated the more recent discoveries regarding the structure of the living cell, though his inferences from the breeding of peas give an accurate picture of what has since been fully. demonstrated by observation. His microscopic slides contained only the simplest objects, - such as the epidermis stripped from the stems of plants, petals, trichomes, the feet and wings of a fly, etc. One could not fail to be impressed while examining these simple objects, and the modest instruments which Mendel had at his command, that greatness lies within the human spirit and not in elaborate equipment.

In the parlor of the Abbey hangs a splendid life-sized oil painting of Mendel in his official robes, with miter and crosier beside him. This painting is of such excellence that I securcd the privilege of taking a photographic copy of it. Mendel is shown here, as in all the photographs of him which are extant, as a man of sturdy physique, with strong, handsome, square-set face, firm but sensitive mouth, a straight and shapely, rather pugnacious nose, strong but not too large, a prominent forehead and brow, and very clear dark eyes that seem capable of penetrating to the very center of whatever they look at. His was obviously a harmonious organization, characterized by great strength loth of intellect and will, with keen powers of analysis, indomitable courage, and unswerving devotion 
to whatever task he undertook. These were the qualities that led him to analyze the problems of heredity on the basis of single characteristics, and which led him (quite unlike all previous students of the phenomena of hybridization) to continue his studies on the simplest possible material until the problems were solved, though it required seven years of close application and painstaking labor. The same characteristics probably cost him the satisfaction of seeing before he died, the triumph which, by fortunate acciclent, has come to his work two decades after his death; for about two years after the publication of his great work he was elected to the Prelacy of the Monastery and his time and attention were thenceforth absorbed with heavy administrative duties and his investigations in the field of heredity came to an end. The intensity of his devotion to the problems of his institution thus prevented his following up his first report with others which would have sooner or later attracted the attention of other students.

As we proceeded on our tour of inspection, Father Barcina told me of this little clock which still hung on the chamber wall where it had been placed by Mendel, and said that when I departed he would give it to me as an "Andenken" of my visit. Such care had been obviously taken to preserve the relics of Mendel's existence and work at the Monastery that I feared I might be misunderstanding the Father's rather swift-moving German, but when he had repeated the statement and scnt. one of his brethren to pack it up for me, I knew that I had not mistaken his intention. Thus it is that I have on my study wall this little alarm-clock which may many times have wakened Mendel to an early-morning competition with the bees in hybridization experiments on the peas in his garden. I have permitted it to awaken me in the same manner, and it has also assisted in keeping awake the enthusiasm which must sustain the long-continued effort necessary to the solution of evolutionary problems.

Before taking my cleparture we also spent a few moments in the chapel which is attached to the Monastery by a short covered passage. This is said to be the most beautiful small chapel in central Europe. It is built of gray stone in Gothic style in the form of a cross, is splendidly harmonious in its proportions, and is not spoiled by over-decoration. The groups of statuary overlooking the apse are of real artistic merit as well as of allegorical significance, and so also are the large stained-glass windows. There is none of that cheap and tawdry over-display which spoils so many European chapels.

With many expressions of mutual pleasure in my visit, I took leave of Father Barcina, and bearing my precious little "Andenken," 


\section{A PIIGRIMAGE TO BRUNN}

I went in company with Doctor Iltis to the rooms of the Natural History Society, where in $186 \pi$ Mendel rcad the account of hi, experiments with peas. The room in which the scientific meetings are held is a plain second-floor room with simple seats and desks, resembling somewhat an ordinary school-room. Doctor Iltis endeavored to find a copy of the report of that momentous meeting, to present to me, but the very limited supply had doubtless been exhausted. He then accompanied me to the station, where I took train about 4:00 P. M. for Vienna, feeling that my day in Briinn had been well worth all my effort.

In recognition of his great service to science, and regretful that this service should have been so long unknown and unappreciated, the biologists of the whole world united in 1910 in erecting in the public square beside the Königskloster a marble statue to the memory of Gregor Mendel. This statue is of heroic size and represents Menclel in his priestly robes standing before a wall against which pea vines are growing. On the peclestal are the kneeling forms of a man and woman clasping hands to symbolize marriage and human heredity, and the words "To the Nature-Student P. Gregor Nendel, 1822-1884. Erected by friends of science." At the same time a memorial volume was issued containing Mendel's original memoirs and fourteen papers by investigators who are at the present time engaged in following out the lines of work suggested by those memoirs. The contributors to this volume belong to seven nations and have been written in three different languages, thus bearing witness to the present wide-spread and eager interest in the work so well begun by a keen-minded Moravian monk more than fifty years ago. The name of Mendel will soon be as familiar a household word as that of Darwin, and as knowledge of the laws of heredity become generally diffused his name will be revered as a benediction to the human race. 



\section{THE PRIMARY COLOR-FACTORS OF LYCHNIS} AND COLOR-INHIBITORS OF PAPAVER GE RHOEAS

GEORGE HARRISON SHULL 


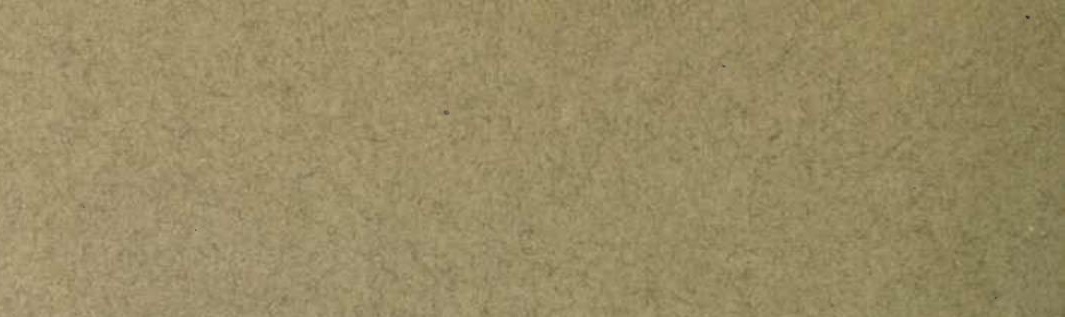
(6) (5)

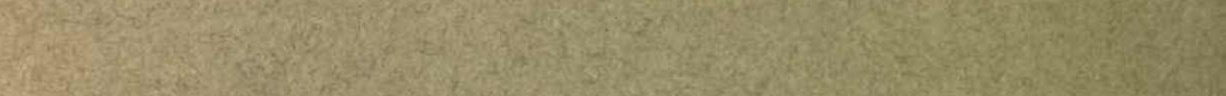

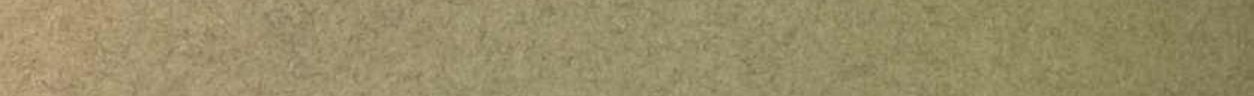
W.

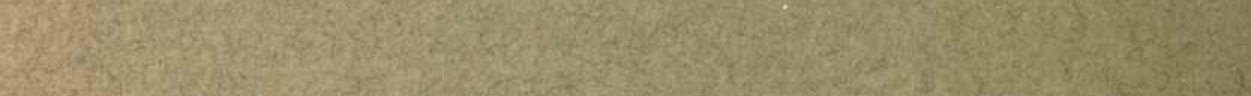
W.

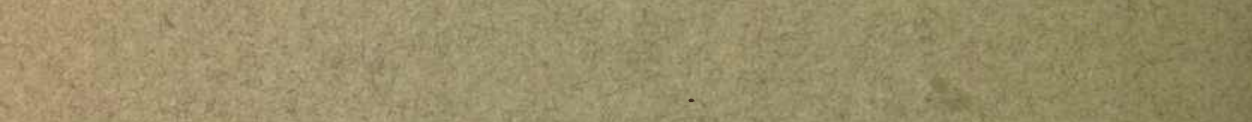

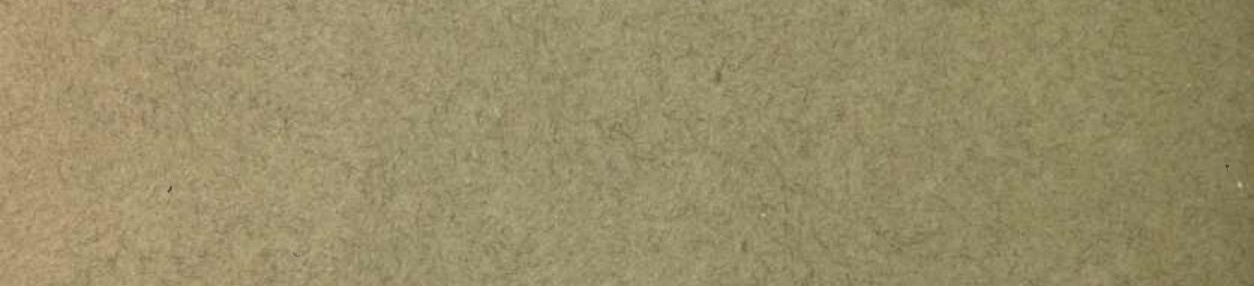

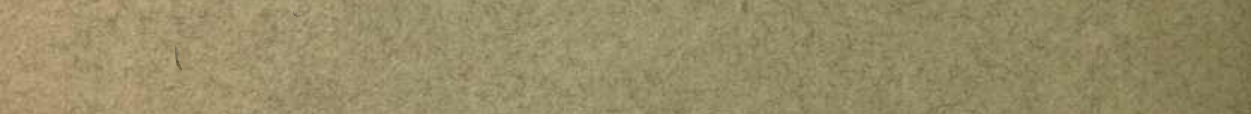

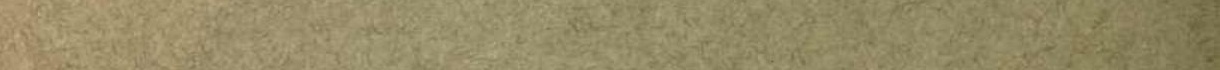

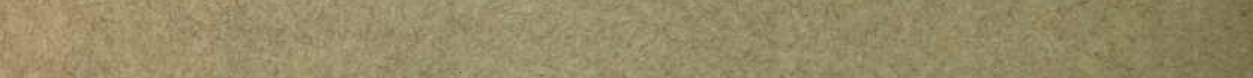
9.

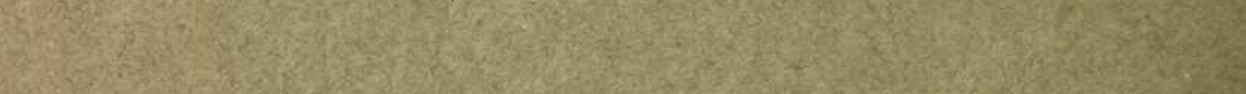
17. Q78.

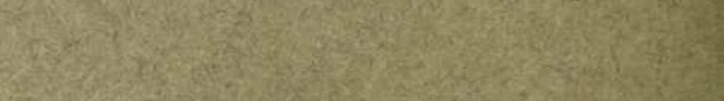

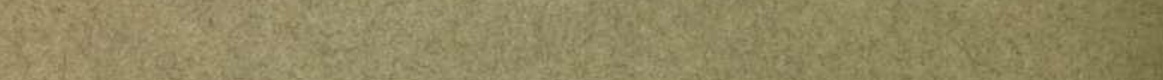
W.

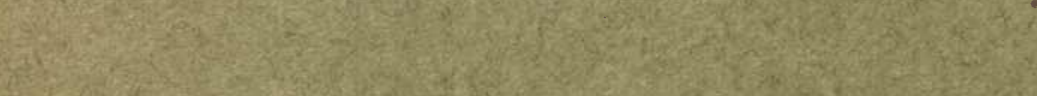




\section{THE PRIMARY COLOR-FACTORS OF LYCHNIS AND COLOR-INHIBITORS OF PAPAVER RHOEAS ${ }^{x}$}

GEORGE HARRISON SHULL

The frequency with which the presence of hereditary characters is dominant over their absence naturally suggests that inhibiting factors may be operating when the reverse relation appears to exist, as when the hornless character of polled cattle dominates over horns, and the "smooth" character over the "bearded" in wheat, oats, etc. Some writers (DAvenport, Bateson, PunNetr) have even taken the extreme view that dominance is in all cases a criterion of "presence." That this position is untenable I have shown several years ago (ShUll I I, I2), and CASTle (2) also opposes such an idea, calling attention to WooD's well known sheep hybrids (WOOD 15), in which the horned condition is dominant in the male and hornlessness in the female offspring from the same cross, as a proof that no such sweeping generalization is permissible. It may be granted, however, that presence is usually dominant, and that the dominance of the apparent absence of a character is probably in most cases, but not in all, the dominance of an inhibiting factor over its own absence. It is only necessary to keep the mental reservation that in any single instance of a putative inhibitor another hypothesis is always available, namely, that the gene for the character that is supposed to be inhibited, when existing singly as in the heterozygote, may be nearly or quite incapable of reaching the threshold of visible expression.

Both of the characters mentioned above by way of examplethe polled condition in cattle and the lack of long awns in wheatare structural characters. When a color-character is inhibited, the

"Under the title "Inhibiting factors in Lychnis and Papaver," this paper was read before the Botanical Society of America, Washington, D.C., December 28, rgri. The change of title and slight changes in the text have been rendered necessary by the discovery that the purple-flowered male parent of family 10201 discussed below was probably heterozygous in both the primary factors for color. This discovery in no wise affects the general considerations presented in the paper as read, but it withdraws Lychnis dioica for the present as an example of dominant $w$ te. 
result is a "dominant white" if the inhibition of all pigmentation is practically complete, or there may result parti-colored forms exhibiting various color-patterns, or the dominance of what appears to be a lower grade of pigmentation over a higher grade when the inhibition is localized or otherwise incomplete.

One of the earliest known and most familiar examples of dominant white is found in the plumage of domestic fowl, in most breeds of which white is epistatic to all colors, but not always quite perfectly so. It was soon found, however, that not all of the plumages of white fowl are of the same nature, for the "Silkie" fowl's white plumage is recessive to colors. Dominant and recessive whites have been discovered in a number of other cases, both in plants and in animals. BAteson (I, p. I05) and GREgory (7) found that white-flowered primulas with red stems are dominant whites, while those with green stems are recessive whites; KeEble, Pellew, and Jones (9), and Miss Saunders (10) have demonstrated dominant and recessive whites in Digitalis purpurea; and EAST (4,pp. 8I f.) has shown that an inhibitor for blue aleurone-color exists in some maize plants though absent in others.

In many cases, perhaps generally, the inhibition is not quite complete, and dominant whites are often distinguishable by the possession of patches or washings of color not found in recessive whites. Similar incompleteness of action of inhibitors is seen i1, the occasional appearance of rudimentary horns or "scurs" in pure-bred polled cattle, in the development of a few feathers on the legs of pure clean-legged fowls, the production of short awns or "beards" on "smooth" wheat, oats, etc.

Not only are there dominant and recessive whites, but there are also different kinds of these, dependent upon the fact, now well known, that the same visible effect may be attained in various ways. It has been demonstrated that pigmentation is generally due to the interaction of at least two independent factors. When only two such factors are required, e.g., $C$ and $R$, there may be three kinds of recessive whites, one lacking $C$, one lacking $R$, and

${ }^{2}$ While this is the general rule, Keeble and Pellew (8) have found exceptions in the variety "Pearl," which has dominant white flowers and green stems, and in "Snow King," in which both dominant and recessive whites were found associated with dark red stems. 
one lacking both $C$ and $R$; and each of these whites will behave differently in certain crosses, though all are recessive to colors and may be quite indistinguishable from one another when purebred. Individuals lacking either $C$ or $R$, when crossed with other individuals having the same genotypic constitution, or when crossed with individuals of the third type, which lack both $C$ and $R$, will produce only white offspring; but when recessive whites of the first two types are crossed together, the complementary factors, $C$ and $R$, necessary for the production of color, are brought together and a colored $F_{I}$ results, as exemplified by the classic case of "Emily Henderson" sweet peas, in which two white-flowered plants, differing externally only in the form of the pollen-grains, produced "reversionary" purple offspring when crossed together. Many similar "reversions" have been discovered by experimental breeders in a considerable number both of plants and of animals, and the old riddle of "reversion on crossing," exemplified by these phenomena, has been given a satisfactory solution in the "factor hypothesis." In so many organisms have different kinds of recessive whites been found, that their discovery in additional species no longer occasions surprise.

Less is known of the chemistry of dominant whites, but it is conceivable that these may also be of several kinds. It is plain that any pigment which is readily converted into an allied colorless compound would give a basis for a dominant white in which the pigment nucleus coexists with a factor which changes it to its colorless derivative. A suggestive illustration in vitro of such a reaction is the ready reduction of indigo blue $\left(\mathrm{C}_{\mathrm{I} 6} \mathrm{H}_{\mathrm{I0}} \mathrm{N}_{2} \mathrm{O}_{2}\right)$ in alkaline solutions to indigo white $\left(\mathrm{C}_{\mathrm{I} 6} \mathrm{H}_{\mathrm{I} 2} \mathrm{~N}_{2} \mathrm{O}_{2}\right)$. SPIEGLER (I4) believed that he had succeeded in isolating a "white melanin" from white wool and white horsehair, and while Gortner $(5,6)$ has been unable to confirm SPIEGLER's conclusions in this regard, the general type of reaction suggested by SPIEGLER may be retained as possibly explaining some cases of dominant white. GORTNER (5) has proposed a very different hypothesis, namely, that as melanin is the product of an oxidase acting on a chromogen (tyrosin), dominant whites may be the result of anti-enzymes which inhibit the action of the oxidase. The same hypothesis is applicable to 
the widely distributed plant-pigment anthocyanin, whose method of origin appears to be in essential agreement with that of melanin. More recently GoRTNer (6) has shown that anti-enzymes are not necessary for the inhibition in question, as the oxidizing action of tyrosinase is prevented by the presence of small quantities of such relatively simple $m$-dihydroxyl phenolic compounds as orcin, resorcin, and phloroglucin. GORTNER shows that on the basis of his investigations a satisfactory explanation can be given of those rare cases in which a white is dominant in some crosses and recessive in others, as apparently exemplified by the Shirley poppies described below.

I have been making numerous crosses among strains of Lychnis dioica L., and during the past six years have grown about 660 pedigreed families of this species. Nearly 300 of these families have resulted from matings between white-flowered individuals, many of the matings having been arranged for the specific purpose of finding different kinds of whites possessing complementary color-factors. Until the past summer (I9II) all of these crosses between white-flowered parents have given uniformly whiteflowered progenies, ${ }^{3}$ and a similar number of crosses between white and colored individuals have invariably shown the whites to be recessive to colors, though they differed genotypically in that some of the whites carried a factor for reddish-purple and others a factor for bluish-purple, the red being epistatic to blue.

With the bringing in of two new strains of Lychnis dioica from their native habitats in Germany (for seeds of which I am indebted to Dr. BAUR), I have realized the complementary factors for color for which I had been looking thus far in vain. ${ }^{4}$

3 The several purple-flowered individuals from white-flowered parents, mentioned in an earlier paper (SHULL I3), appear now to have been plus-fluctuants of a "tinged white" which had not been recognized as such at the time that paper was written. They have no bearing on the problem of complementary color-factors here under consideration.

${ }_{4}$ That the several kinds of recessive whites exist among my Cold Spring Harbor strains, though I have not yet made a mating among them between two whites which resulted in a purple-flowered $F_{x}$, is sufficiently demonstrated by the facts presented in my earlier paper. My failure thus far to secure a purple-flowered $F_{x}$ from two whites among these strains must be due to the mere chance that $\mathbf{I}$ have not selected whites from the proper families. 
These two forms of Lychnis from Germany are with apparent good reason classified by German taxonomists as distinct species, the white-flowered form being called Melandrium album Garcke, and the purple-flowered form $M$. rubrum Garcke. Melandrium album, as it appears in my cultures, has relatively narrow, spatulate, moderately ascending rosette-leaves of a rather dull dark green; the corollas are white, slender, and long-exserted from the calyxtube; the styles are long and slender, with inconspicuous stigmatic papillae. The plants are easily grown as annuals by early sowing. Melandrium rubrum Garcke, grown under the same conditions, has the rosette-leaves broader, with more rounded apices; the leaves are nearly horizontal, a little darker green, and more shining. The corollas are reddish-purple, shorter, scarcely extending beyond the mouth of the calyx; the styles shorter and relatively heavy, with prominent stigmatic papillae. A very small percentage of the plants are forced to bloom as annuals, even when seeds are sown early in February. In so far as visible characters are concerned, these two forms have shown but slight fluctuations, except that in $M$. album the calyx varies from plain green through green striped with purple to a rather deep dull crimson. They have kept quite distinct from each other in regard to the characteristics enumerated, but because they breed together with undiminished fertility and because I have many other strains showing similar differences and various degrees of intermediacy, I must continue for the sake of convenience the use of the Linnean name (Lychnis dioica) for the entire aggregation. To what extent the other forms in my cultures may have been derived from hybridizations between $M$. album and $M$. rubrum cannot be surmised, but all strains which I have thus far found in America have presented one or more characteristics which are not directly traceable to either of the German forms, nor obviously derivable from them by recombinations of their characters. For instance, my original material of this species, collected at Cold Spring Harbor, has considerably lighter green foliage than either $M$. album or $M$. rubrum, and from the vicinity of Harrisburg, Pa., I have secured a "chlorina" (CORRENS 3) variety having light yellow-green foliage.

Three crosses were made in I9ro between the German Melan- 
drium album and my original white-flowered strain from Cold Spring Harbor. Two of these families (I0200 and 10202) were the result of crossing two different German white-flowered females with pollen from a single Cold Spring Harbor white-flowered male. Both of these matings produced only white-flowered offspring, totaling 182 individuals. The young seedlings were indistinguishable from Cold Spring Harbor seedlings of the same age, but later they became darker green and were intermediate between the parents. A third family (I068) was essentially reciprocal to the two just described, being produced by crossing a female sib of the male used in 10200 and 10202 with pollen from a German white-flowered male. The 77 offspring were vegetatively indistinguishable from the reciprocal families, but the flowers were all reddish-purple. These different results in supposedly reciprocal crosses probably indicate that there was an unsuspected heterogeneity in the German strain. That the difference was due to heterogeneity in the Cold Spring Harbor parental family is rendered improbable by the fact that a mating (I060) between the female used as the mother of 1068 and the male used as the father of I0200 and 10202 resulted in a progeny of 73 white-flowered plants. It is unfortunate that a similar check was not applied to the German plants entering into these families, by also crossing them together. The only cross (10203) made between two specimens of $M$. album resulted in 84 offspring, all white-flowered. The mother of this family was also the mother of 10202 , but the father was not the same as the father of Io68.

Several crosses were also made between the purple-flowered German Melandrium rubrum and my Cold Spring Harbor strains, both white-flowered and purple-flowered. Families I092 and ro93 were produced by crossing a single white-flowered female of the Cold Spring Harbor strain with two males of $M$. rubrum, one derived from seeds collected at Furtwangen in the Schwarzwald, and the other from Oefingen in Baden. A female sib of the lastmentioned plant (i.e., from Baden) was crossed (I0204) with pollen from a white-flowered sib of the mother of families rog2 and 1093. It represented a cross, therefore, as nearly reciprocal to ro93 as is possible in dioecious material. Two other families 
(I0206 and 10207) were produced by crossing two females grown from the Baden seeds with pollen of a single pure-bred purpleflowered male from Cold Spring Harbor. As Melandrium rubrum has reddish-purple flowers and as this color has been shown to be epistatic to bluish-purple (which may have been carried as a latent character by the white-flowered plants), there was no reason to expect that the $F_{x}$ progeny of any of these five crosses would present any noticeable difference in flower-color from that of their $M$. rubrum parent. This expectation was realized, the 262 offspring from these crosses all having reddish-purple flowers. The young plants in these families were generally indistinguishable from pure-bred $M$. rubrum, but later they differed by being notably more vigorous, having enormous rosettes of broad, shining, darkgreen leaves. They were also much more easily grown as annuals by early sowing, being in this regard intermediate between the parents. Almost all of the hybrids were blooming by the middle of July, before the first flowers of any pure $M$. rubrum had opened.

Compared with these crosses between Melandrium rubrum and the Cold Spring Harbor plants, a cross of M. rubrum with $M$. album gave a totally different and unexpected result. A mating between a female of the white-flowered album and a purple-flowered male rubrum produced an $\mathrm{F}_{\mathrm{I}}$ (IO2OI) consisting of 23 white-flowered individuals and 3 (probably 4) purple-flowered ones. The whiteflowered plants were unlike either parent in vegetative characters, having relatively short, sharp-pointed, grayish-green leaves which were strongly ascending in the fully developed rosette, while both parents have long, spreading, dark-green leaves. The flowers were not only white like those of their white-flowered mother, but they were also nearly identical with them in form. It was noted that rarely some of the flowers became faintly and unevenly streaked and washed with purple just as they were fading, a feature never observed in the flowers of any of my other whiteflowered plants. These white-flowered hybrids were a little later in blooming than their white-flowered parent, but were still easily induced by early sowing to behave as annuals. The purpleflowered offspring of this cross were of an altogether different character, and were not readily distinguishable in rosette and 
floral characters from their purple-flowered male parent. They were also like pure $M$. rubrum in not blooming until late in the season. One plant having a rosette identical with those of the three purple-flowered specimens remained a rosette, but will doubtless have purple flowers if it survives the winter. ${ }^{5}$

Why there should be this segregation of types in the $F_{1}$, and why one of these types should so completely resemble the male parent, while the other type was goneoclinic to the female parent, though abundantly distinct from it in the rosettes, are mysteries. Perhaps this unexpected segregation of characters in a putative $F_{I}$ is further evidence of the heterogeneity of the $M$. album material. If the white-flowered mother were heterozygous in a dominant white factor, the expected result of a cross with $\boldsymbol{M}$. rubrum would be white-flowered to I purple-flowered, or in this particular family 18 white-flowered to $\$$ purple-flowered, to which expectation the observed result is in sufficiently close agreement considering the small number of individuals.

9 the rubrum parent were heterozygous in respect to both the primary factors for color, $C$ and $R$, it being assumed that the album parent lacked both these factors dence of heterogeneity in $\boldsymbol{M}$. rubrum has yet appeared in my cultures. It should be remarked that neither of these German strains had been pedigreed in controlled cultures, but were simply collected in separate regions in nature, so that questions as to their genotypic purity are legitimate.

In the derivatives of the corn-poppy (Papaser Rhocas L.), among which are the dainty and beautiful "Shirley" poppies of our gardens, color-inhibitors are found. According to his own statement, Rev. W. Wilks was first induced to pursue the course of selection, which resulted in the strain known as the "Shirley poppies," by discovering a bud-variation on a wild corn-poppy growing in a corner of his garden. Several flowers on this plant differed from the rest in having petals with a narrow white margin. Such a white margin is now a frequent feature of garden poppies, and when appropriate crosses are made, it is found that the presence

s Note added June 5, 1912. This plant is now blooming and has purple Howers as predicted. 
of a margin is dominant over its absence. It is probable, therefore, that the white margin is due to the presence of an inhibitor whose action is localized in the margins of the petals.

In IgIr, among 73 pedigreed families of Paparer Rhoeas grown at the Station for Experimental Evolution, 45 resulted from crosses between plants of which the presence or absence of a margin had been recorded, and of which a goodly proportion of the offspring were capable of being similarly recorded. The rest either had one white-flowered parent whose possession (or lack) of a margin could not be determined by inspection, or for some other reason one or both parents or the offspring could not be safely characterized with respect to margins. Of the 45 families having the margins of parents and offspring recorded, 3 represented crosses between plants both of which had margined petals, i7 were from crosses between one margined and one unmargined parent, and 25 resulted from matings between plants none of which had margined petals. The three families from matings between margined parents consisted of 236 individuals, including in each family a mixture of plants with margined and with unmargined petals. Records of the margins were often impossible, owing to the interference of other factors not yet fully investigated, so that the numbers of each type of offspring have no special significance in the present connection and they will be reserved for discussion at another time.

Of the I7 families produced by mating plants with margined and with unmargined petals, 12 were composed of a mixture of plants, some with margins and some without, 3 contained only plants with unmargined petals, and in 2 families practically all of the individuals had margins. With margins dominant over their absence, only two kinds of families were to be expected from this type of mating, namely, all margined if the margined parent chanced to be homozygous, and mixtures of plants with margined and unmargined petals if the margined parent was heterozygous. The three families (10272, 10273,10274 ) in which no margins appeared, though one of the parents had a margin, are exceptions. One margined individual was the mother of all three of these exceptional families. The records show that this plant differed 
from the usual type, the margin being in this case red-violet ${ }^{6}$ instead of nearly white. Whether this red-violet margin was a purely somatic modification of the dark-red body-color, or whether it was germinal, it was clearly of a different nature from the white margins involved in the other families.

In the two families (10287, IO289) whose margined parents were evidently homozygous, a small number of plants were recorded without margins. These exceptional plants occurred among those set into the garden, while larger numbers of plants from the same families, which were grown to maturity in pots in the propagatinghouse, were all margined. In family $\mathrm{IO}_{2} 87$ there were 6 plants with unmargined petals among 40 grown to maturity in the garden, and none among I33 which flowered in pots, and in family I0289 one was noted as unmargined among 47 plants in the garden and none among 83 which developed in pots. However these seven unmargined specimens are to be accounted for, it is clear that each of these families is the offspring of a homozygous margined parent.

In the 25 matings between plants, neither of which possessed margined petals, there appeared only ${ }_{5} 5$ plants with margins among a total of 1402 offspring, and in a number of those recorded as margined the margin was merely a trace of lighter color of more or less doubtful character. Only in one family (IO29I) were the margins unmistakable, and in this family the margined plants occurred only among those which were retained in the greenhouse. Of 2I which matured in the garden none had margins, while among 99 which flowered in pots in the greenhouse there were ro with margins, several having only a trace, while others had well marked white margins $2 \mathrm{~mm}$. wide -in one plant $3 \mathrm{~mm}$. wide. No attempt need be made at present to account for these few margined plants, for their number is too small to vitiate the conclusion that the unmargined condition is recessive, and that typically all the offspring of two unmargined parents are unmargined.

The most interesting matings in which margins were involved

${ }^{6}$ The color-nomenclature adopted in this paper is based on the spectrum colors, as arranged in the Milton Bradley system. Exact shades and tints have been recorded, but for the sake of simplicity these have not been reproduced here. 
were those in which the wild poppy was crossed with its garden derivatives, for as already noted the margin is a new character which does not normally occur in the wild poppy. In the two families representing such matings, the wild poppy was used as the mother in 10298 and as the father in I0310. Both families consisted of mixtures of margined and unmargined plants, showing conclusively here also that margined petals is a dominant character, since the wild plants are certainly homozygous in the lack of such margins. The dominance of the margined condition of the garden poppies over the unmargined condition of their wild prototype is in marked contrast to all the other color-characters of Papaver Rhoeas yet investigated, for the dark red-orange body-color ${ }^{7}$ of the wild poppy is epistatic to all the body-colors presented by the numerous garden forms. If dominance were a secure criterion of the presence of a gene which is absent in the recessive type, these results would indicate that while the various body-colors of the garden forms originated as retrogressive mutations, i.e., by losses of characters, the white margins of the petals represent a progressive mutation through the addition of a gene which inhibits the development of color in that region. Doubleness also proves to be dominant over the single type of the wild poppy, and, on the basis of the same assumption, would have to be classed as a progressive mutation. I cannot forbear, however, to repeat the caution that dominance does not necessarily demonstrate the progressiveness of a mutation, since the alternative hypothesis, mentioned above in the first paragraph, allows for the dominance of a character which has originated by a retrogressive mutation.

There is still one other color-inhibitor (possibly several) in the derivatives of Papaver Rhoeas, which is in some respects more noteworthy than that which produces the white margins. This affects the body-color of the petals, producing what is essentially a dominant white, though in this case the inhibition is not usually complete and the flowers often show some irregular striation of dull violet, reddish, or bluish color on the petals, especially in the presence of purple stamens.

"By the expression "body-color" it is intended to indicate the color of the general intermediate region of the petals as distinct from "center" (proximal) and "margin" (distal). 
A single white-flowered plant with yellow stamens was crossed in I909 with three red-flowered plants (yielding families I0275, IO28I, IO282) and with two plants having dull striations on the petals (families $10280, \mathrm{IO}_{28}$ ), and the offspring of these five matings were generally white or whitish-flowered. Of 559 plants in these families only 25 were neither pure white nor white with traces of reddish color, and of these 25 , all that had a full red (i.e., not striated) parent were lighter in color than that parent. These fully pigmented offspring may simply represent minus-fluctuations in the action of the inhibitor derived from the white-flowered parent. If this is the correct interpretation of these few plants with colored flowers, it should be possible to secure from them progenies displaying the presence of the inhibitor though it be invisible in both parents. While I have as yet grown no offspring from the colored plants of these families, I have two other families (I0270, I0308) in which the same whitish offspring have appeared, though both parents in each case were fully pigmented. Family I0270 was produced by mating two dark-red parents which were sibs in a family consisting of red, red-orange, pink (light violetred), and white. The progeny of these two dark-red plants consisted of 68 white or whitish and 70 pigmented, the latter often striated and generally much less intensely pigmented than either parent. Only two of the offspring showed as deep shade as that of their parents. The parents of family 10308 were also redflowered sibs in a family containing red, red-orange, pink, and white. They were considerably lighter red than the parents of I0270, but were fully and evenly pigmented. Their offspring consisted of 80 white- and whitish-flowered plants and 13 with pigmented flowers, none of which were as deeply pigmented as either parent, and several of which showed the peculiar striation which seems to be one of the manifestations of the inhibitor believed to be operating in these crosses. Similar results were obtained in seven families (10266, I0273, I0274, 10297, I0303, 10305, I0311) produced from mating together two plants with striated petals, or a striated with a plain red, and only in one family, containing three individuals (I0268), did the "dominant white" fail to manifest itself in progenies from matings of this character. In the latter family a 
cross between a light-red and a striated individual produced three offspring, all with flowers slightly darker red than those of their redflowered parent. Considering the complexity of some of these families, this number of individuals is entirely inadequate for the deduction that family 10268 was really exceptional.

While I have laid no emphasis thus far on the fact, it may have been noted that all of these poppy-families in which a "dominant white" has made its appearance have been derived from red or striated parents, never from red-orange or pink (light violet-red). It seems that the factor under discussion is not a general inhibitor of color but only of pure spectrum-red. The following facts seem to prove this: The same white-flowered plant with yellow stamens which we have seen producing white-flowered progenies when mated with red (families 10275 and I028I) was also mated with two homozygous pink-flowered plants (families 10277 and 10278) and a homozygous red-orange plant (10279) and in all of these three crosses the white-flowered parent proved to be a recessive white. Families 10277 and 10278 consisted of 43 pink-flowered and 25 red-flowered plants, and 10279 contained. 226 red-orangeflowered plants and I red-flowered. Not a single individual in any of these three families had white or whitish flowers. In keeping with these results are families in which striated plants were mated with pink (10295) and red-orange (I030r), for in neither of these families appeared a white-flowered offspring or one with striations, I0295 yielding 37 pink-flowered and 33 redflowered and I030I giving 22 which were red-orange and 5 intermediate between this and red.

The occurrence of many red-flowered plants in these families, when one of the parents supposedly contained an inhibitor for red, is not satisfactorily explainable on the assumption made above, that there is a single inhibitor for red whose effectiveness fluctuates to such an extent that its presence may not be detected in its extreme minus-fluctuations. An alternative hypothesis may be suggested, which must await further experimentation for its confirmation or rejection. If there be two factors, $A$ and $B$, which are ineffective when existing apart from each other, but which become an inhibitor when acting together, the observed 
results could be explained by assuming that in those matings which produced whitish-flowered offspring, the one parent possessed $A$, the other $B$, while in those matings in which a fully pigmented progeny was produced, the two parents had the same factor-either both $A$ or both $B$-or else one of them lacked both $A$ and $B$ and the other parent lacked one of them. The occurrence of fully pigmented individuals in association with "dominant whites" need not then be minus-fluctuations of a single inhibitor, but might be the result of segregation of inhibiting factors, one or more of which were heterozygous in one or both parents.

\section{Summary}

Dominant and recessive whites have been discovered in a number of different plants and animals. Both the dominant whites and the recessive whites may be of different kinds, though externally indistinguishable.

Dominance does not necessarily indicate presence of an added gene, but when the absence of a character appears to be dominant over its presence, the action of an inhibiting factor may usually be inferred. An alternative hypothesis is always available, however, which should prevent a too dogmatic assertion that dominance is synonymous with presence.

A white-flowered form (Melandrium album) of Lychnis dioica L. from Germany, when crossed with the purple-flowered form ( $M$. rubrum) from the same country, produced 23 white-flowered and 4 purple-flowered offspring, but in certain crosses with a whiteflowered strain derived from plants growing at Cold Spring Harbor, the German white-flowered plants produced purple-flowered offspring in the $F_{I}$, in other crosses only white-flowered offspring were produced.

In the "Shirley" poppies (Papaver Rhoeas L.), the presence of a white margin of the petals is a dominant character and is probably due to an inhibitor limited in its effective action to the margins of the petals.

These white margins and doubleness of the flowers are the only characters in the garden poppies which were found dominant over the corresponding characters of the wild type from which they 
were derived. They may represent the results of progressive mutations, but here again caution is necessary because of the alternative hypothesis.

There is also an inhibitor which affects the body of the petals in the "Shirley" poppies, producing what is essentially a dominant white, though the inhibition is often very imperfect, in which case the flowers are more or less washed and striated with color, though generally whitish.

This supposed inhibitor was evident only in crosses involving at least one red-flowered or striated parent. The same whiteflowered plant which was a dominant white in crosses with redflowered and striated plants was a recessive white in crosses with pink-flowered and red-orange-flowered plants.

In several cases red-flowered plants crossed together produced a whitish progeny and a similar result was produced when two striated plants were mated or when striated was crossed with red.

Two hypotheses to account for these facts are considered: (a) that there is one inhibitor affecting only the pure spectrum-red and having no effect on pink and red-orange; the minus-fluctuations of this inhibitor pass the limit of visibility; $(b)$ that there are two factors, $A$ and $B$, which have no visible effect when existing alone, but which act as an inhibitor when brought together. These two hypotheses must be tested by further breeding.

I take pleasure in acknowledging here the faithful work of Mr. E. E. BARKER, who assisted me in making the records upon which this paper is based.

Station for Experimental Evolution

COLd Spring Harbor, L.I.

\section{LITERATURE CITED}

I. Bateson, W., Mendel's principles of heredity. Cambridge: University Press. I909.

2. Castle, W. E., Heredity in relation to evolution and animal breeding. New York: D. Appleton \& Co. Igrr.

3. Correns, C., Vererbungsversuche mit blass(gelb)grünen und buntblättrigen Sippen bei Mirabilis Jalapa, Urtica pilulifera, und Lunaria annua. Zeitschr. Ind. Abst. Vererb. I:291-329. figs. 2. I909. 
4. East, E. M., Inheritance in maize. Bull. I67, Conn. Agr. Exp. Sta. pp. I42. pls. 25. I9II.

5. Gortner, R. A., Spiegler's "white melanin" as related to dominant and recessive whites. Amer. Nat. 44:497. I910.

6. - Studies on melanin: III. The inhibitory action of certain phenolic substances upon tyrosinase. Jour. Biol. Chem. 10:II3-I22. I911.

7. Gregory, R. P., Experiments with Primula sinensis. Jour. Genetics I:73-I32. pls. 3. figs. 2. I9II.

8. Keeble, F., and Pellew, Miss C., White-flowered varieties of Primula sinensis. Jour. Genetics $\mathrm{I}: \mathrm{I}-5$. I9I I.

9. Keeble, F., Pellew, Miss C., and Jones, W. N., The inheritance of peloria and flower-color in foxgloves (Digitalis purpurea). New Phytol. 9:68-77. fig. I. I910.

Io. SAUnders, Miss E. R., On inheritance of a mutation in the common foxglove (Digitalis purpurea). New Phytol. 10:47-63. pl. I. figs. I4. I9II.

I I. Shull, G. H., The "presence and absence" hypothesis. Amer. Nat. 43:410-4I9. I909.

I2. - - A simple chemical device to illustrate Mendelian inheritance. Plant World I2: I45-I53. pl. I. fig. I. I909.

13. —— Color inheritance in Lychnis dioica L. Amer. Nat. 44:83-9I. I9I0.

I4. Spiegler, E., Ueber das Haarpigment. Beitr. Chem. Physiol. Path. 4:40. 1904 .

15. Woon, T. B., Note on the inheritance of horns and face-color in sheep. Jour. Agr. Sci. I: 364 . 1906. 
10.

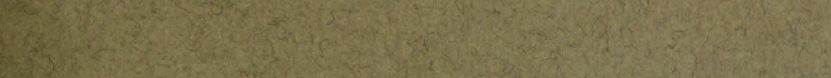

(3)

W.

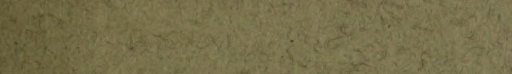

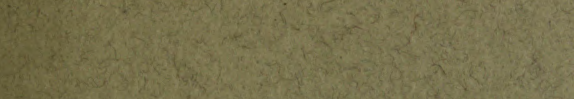

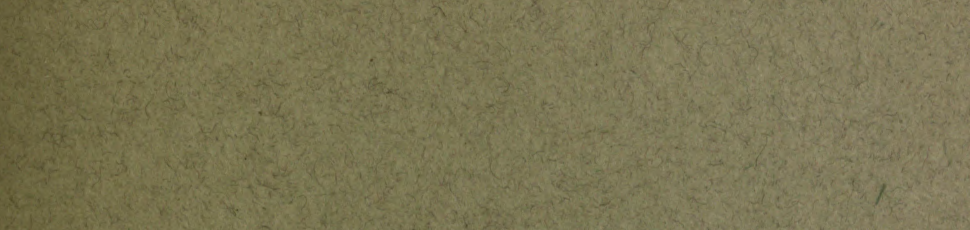

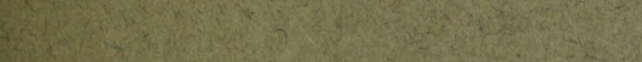

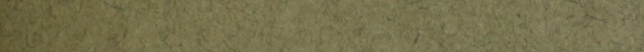

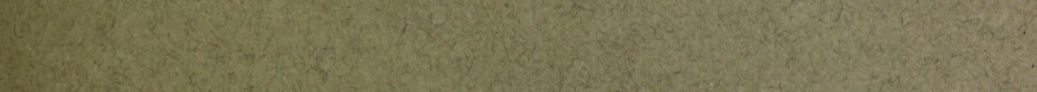

2.

E.

2.

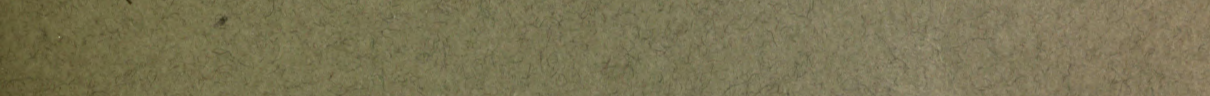

A19.

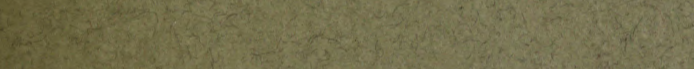

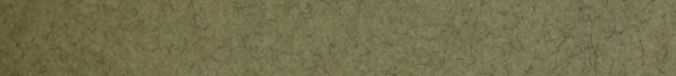

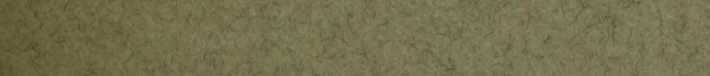

3.95

Q3

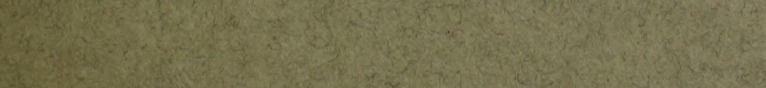

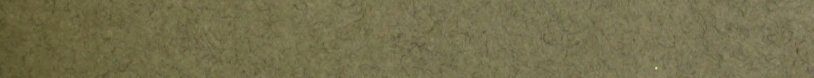

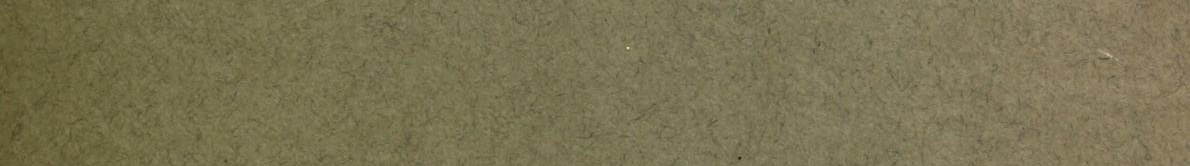

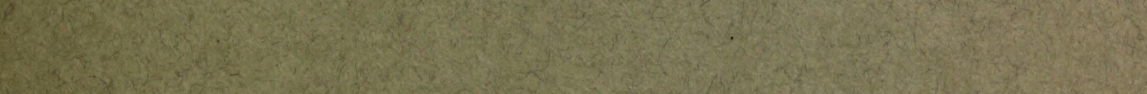

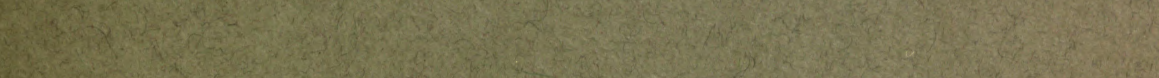

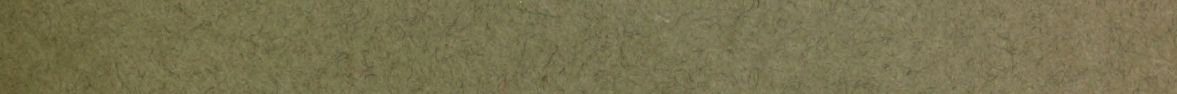

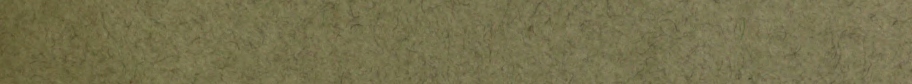

8.

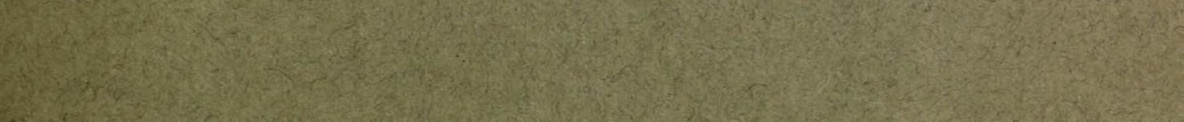

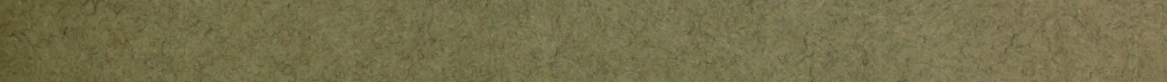

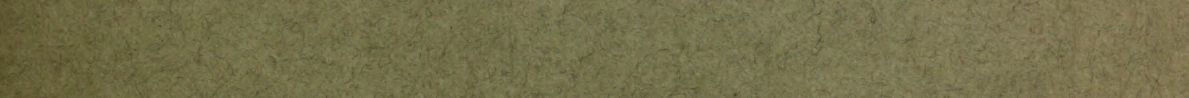

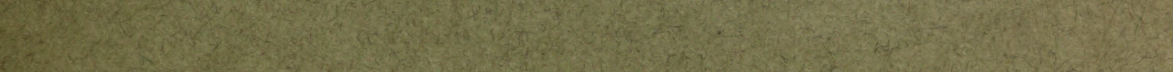

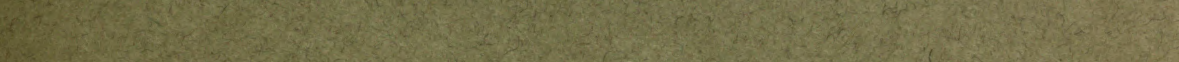

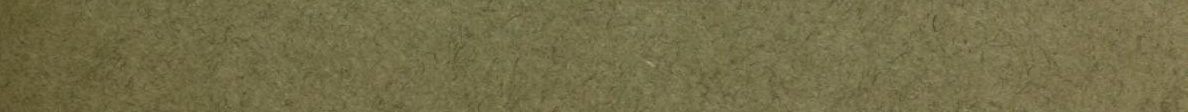
W(1)

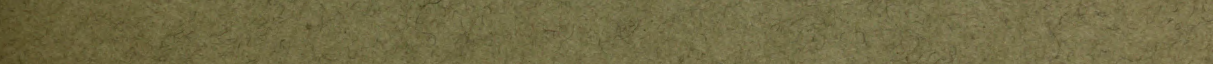


The 
[Reprinted from ScizNce, N. S., Vol. XXXVI., No. 928, Pages 482-483, October 11, 1912]

HERMAPHRODITE FEMALES IN LYCHNIS DIOICA

Some years ago Strasburger ${ }^{1}$ reported that female specimens of Melandrium rubrum Garcke (a form of Lychnis dioica L.) growing in his experimental garden at Bonn, were changed to apparent hermaphrodites as a result of infection with the anther-smut, Ustilago violacea. The infected plants had fully developed stamens, but the sporogenous tissue of the anthers was completely replaced by the spores of the smut. Strasburger suggested that all the cases of hermaphroditism which had been occasionally reported in this species were probably due to infection by Ustilago.

When I discovered functional hermaphrodite mutants in Lychnis dioica and demonstrated by numerous genetic experiments ${ }^{2}$ that these functional hermaphrodites are modified males, I believed that Strasburger had misinterpreted his material and that his hermaphrodites which resulted from infection by Ustilago were produced by the development of female organs in the male, and not as he supposed by the development of male organs in the female. Strasburger was correct, however, as to the nature of his apparent hermaphrodites, as demonstrated by two facts which he has recently pointed out, ${ }^{3}$ namely, $(a)$ that the females are not always completely infected, in which case the uninfected branches bear normal female flowers, and (b) that infected

${ }^{1}$ Biologisches Centralblatt, XX,, 657 et seq., 1900.

-Botanical Gazette, XLIX., 110, 1910.

- Jahrbuch für wissenschaftlichen Botanik, XLVIII., 427, 1910. 
males show no development of the female organs.

Professor Doncaster, of the University of Cambridge, England, has tested the influence of Ustilago violacea upon Lychnis dioica by artificial infections, and his results completely corroborate the conclusions of Strasburger. He sends for publication in Science the following brief account of his experiments:

It is well known that Lychnis vespertina is diœcious, but that all plants infected with the fungus Ustilago have well-developed stamens. Some of these plants have the typical male form, without trace of ovary; others have a vestigial ovary and styles in addition to the stamens and anthers filled with Ustilago spores. This suggests that when a female plant is affected by the parasite, the stamens are caused to develop and the ovary is reduced, while the form of the flower of a male which is infected is not altered. In order to test this suggestion, I planted some ustilaginized plants in my garden in the late summer of 1910, and put with them some uninfected plants which I attempted to infect by sprinkling them with spores and by rubbing spores into parts of the stem from which I had scraped away the epidermis. The results were as follows: Of seven females which I attempted to inoculate in August, 1910, one became infected, and had the typical "'hermaphrodite" form of flower in October, but in June, 1911, was again quite free from Ustilago, and had typical female flowers. A second female plant showed infection in June, 1911, but only on part of the plant; one branch was quite clean and had typical female flowers, the rest of the plant was infected and had "hermaphrodite" flowers.

Of eight male plants which were inoculated in August, three showed some infected flowers before the end of September; the anthers contained Ustilago spores, but there was no change from the male type of flower. Three of these eight plants were dead in June, 1911; one of the remaining five was infected.

Of five ustilaginized plants transferred to the garden, four had the hermaphrodite type of flower and one the male. One of the hermaphrodites so 
far recovered in September, 1910, as to set some seed; in 1911 all were still infected. One of them had some branehes with hermaphrodite flowers containing large ovary, short styles and stamens with little or no pollen, but without Ustilago spores, while the rest of the plant had flowers with anthers full of spores, and the ovary and styles more reduced.

These observations seem to prove that infection with Ustilago can turn the female flower into the apparent hermaphrodite, but that no production of female organs takes place in a male flower when it becomes infected.

\section{Doncastrar}

Cambridge, England

The occurrence of uninfected hermaphrodite flowers on one of Doncaster's original infected plants may possibly indicate that this plant was not a female previous to its infection, but a hermaphrodite. If it were possible to secure pollen from a ustilaginized female, certain genetic problems of very great interest might be solved. It is of great theoretic importance to know whether infection by Ustilago affects the genotypic nature of the host. If the effect is purely somatic, as seems to me the more probable, the offspring of a self-fertilized hermaphroditic female, or of a normal female fertilized by sperms from a hermaphroditic female, should consist only of females (if uninfected), and not of females and hermaphrodites, as I have shown to be the case when a female is fertilized by a hermaphroditic male. If infection by Ustilago produces a genotypic modification, it would be interesting to know whether such induced hermaphrodites are homozygous like the females by whose modification they are produced; they should in that case yield only hermaphrodite offspring. Hermaphroditic males produce both female and hermaphroditic male offspring, because the males are sex-heterozygotes.

As I have been fortunate enough thus far 
not to have a single infection from Ustilago among the many thousands of individuals of Lychnis dioica which have been involved in my cultures during the past seven years, I do not care to take up at present the here suggested line of investigation upon ustilaginized females. I do not wish to jeopardize by importing infected material, the solution of many other genetic problems now under investigation, but I hope that Professor Doncaster or some one else who is in a position to do so, will give attention to breeding from hermaphroditic females if this proves to be technically possible.

Geo. H. Shull 


\section{RETURN TO DIVISION OF GENETICS HILGARL HALL}

\section{Über die Verepbung der}

\section{Blattfarbe bei Melandrium.}

(Mit 2 Abbildungen im Text und Doppeltafel XXIII.)

\section{Von}

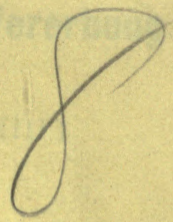

\section{George Harrison Shull,}

Station für experimentelle Evolution der Carnegie Institntion of Washington, Cold Spring Harbor, Long Island,

Sonderabdruck aus den Berichten der Deutschen Botanischen Gesellschaft, Jahrgang 1913, Band XXXI, Generalversammlungs-Heft.

\section{BERLIN,}

GEBRÜDER BORNTRÆGER, 


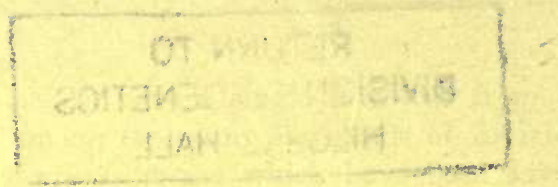

906 griud $990 \mathrm{~V}$ eib qodl

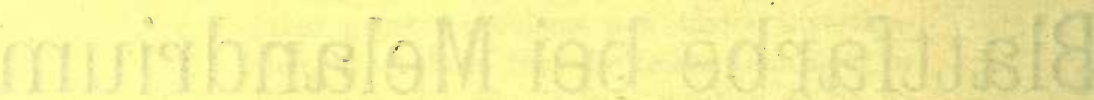

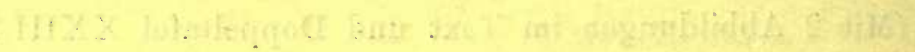

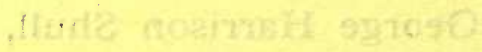

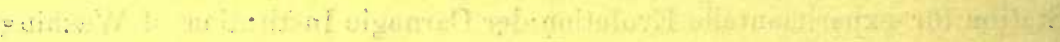

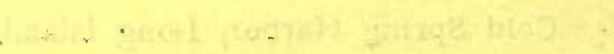

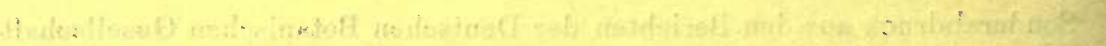

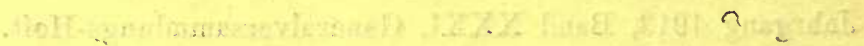

WTIBTHE:

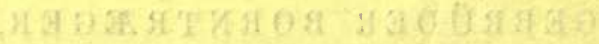


Sonderabdruck aus den Berichten der Deutschen Botanischen Gesellschaft, Jalirgang 1913, Band XXXI, Generalversammlungs-Heft.

\section{George Harris on Shull: Über die Vererbung der Blattfarbe bei Melandrium ${ }^{1}$ ).}

(Mit 2 Abbildungen im Text urd Doppeltafel XXIII.)

(Eingegangen am 15. Januar 1914.)

Einleitung.

Gelegentlich haben diejenigen, die dem Mendelismus seine große Bedeutung absprechen, betont, daß nur solche Merkmale mendeln, die wenig oder nichts mit den wesentlichen Lebensprozessen zu tun haben. Sie kamen zu diesem Trugschluß, da anfänglich die große Mehrzahl genetischer Untersuchungen scheinbar weniger wichtigen Eigenschaften der Pflanzen und Tiere galt, z. B. den Pigmenten. Bei nicht grünen Pigmenten mag es sich um unwesentliche Merkmale handeln; keinesfalls gilt dies aber für die grüne Färbung der Pflanzen, denn wir wissen, daß fast alle Algen, Lebermoose, Laubmoose, Farnkräuter, Coniferen und Angiospermen einer Reihe grüner Pigmente zum dauernden Leben bedürfen; sekundär hängt somit auch die Existenz aller hochorganisierten Tiere von dem Chlorophyll der Pflanzen ab.

Unter dem Namen Chlorophyll faßt man die verschiedenen grïnen Pflanzenfarbstoffe zusammen. STOKES ${ }^{2}$ ) und SORBI ${ }^{3}$ ) unter-

1) Zum Schluß des Vortrages wurden 24 Lumière-Lichtbilder gezeigt.

2) StoKes, G. G., On the supposed identity of biliverdin with chlorophyll, with remarks on the constitution of chlorophyll. Proc. Roy. Soc. London, 13: S. 144, 1864.

3) SoRBY, H. C., On comparative vegetable chromatology. Proc. Roy. Soc. London, 21: S. 442-483, 1873. 
scheiden drei verschiedene Chlorophylle, von denen zwei in jeder diesbezüglich untersuchten Landpflanze gefunden worden sind. Diese beiden sind das Allochlorophyll (MARCHLEWSKI) ${ }^{1}$ ) und das häufigere Neochlorophy!l (JACOI3SON und MARCHLEWSKI) ${ }^{2}$ ). Das Verhältnis, in dem diese Chlorophylle vorkommen, ist durchaus kein konstantes; es wechselt sogar in ein und derselben Pflanze ${ }^{2}$ ).

Außer den Chlorophyllen kennen wir bei den Pflanzen noch eine ganze Reihe von gelben, roten und blauen Pigmenten. Die zwei letztgenannten heißen Anthocyane; auf diese will ich nicht näher eingehen, da sie in keinem direkten Zusammenhang mit dieser Abhandlung stehen. Wir kennen schon eine ganze Anzahl von gelben Earbstoffen, von denen ich aber nur das Karotin und Xanthophyll erwähnen will. Diese beiden haben wir, genau wie das Chlorophyll, nur als Sammelbegriffe aufzufassen.

Fast alles, was wir über die Vererbung des Chlorophylls wissen, danken wir den wichtigen Untersuchungen von CORRENS ${ }^{3}$ ) und $\mathrm{BAUR}^{3}$ ) sowie den erst vor kurzem erschienenen Beiträgen von EMERSON $^{4}$ ) und NILSSON-EHLE ${ }^{5}$ ). Da ich im folgenden über einige eigene, derartige Versuche berichten werde, brauche ich hier auf die Ergebnisse der früheren Forscher nicht einzugehen; zumal die meinen mit den ihrigen fast durchweg übereinstimmen.

Die gelben Pigmente wurden genetisch bisher relativ wenig untersucht. Dies mag auf zweierlei Gründe zurückzuführen sein: Erstens ist unser Auge für Gelb lange nicht so empfindlich wie z. B. für Rot oder Grün, und zweitens sind die gelben Pigmente durch das Chlorophyll oder Anthocyan sehr oft mehroder weniger vollständig verdeckt und entziehen sich so meistens der genaueren Beobachtung. Ich beabsichtige, im folgenden einiges über die Be-

1) Marchlewski, L., Die Chemie der Chlorophylle und ihre Beziehung zur Chemie des Blutfarbstoffs. S. 187, 1909, Braunschweig, FRIEDRICH VIEWEG \& SOHN.

2) JAcoBson, C. A., und Marchlewski, L., On the duality of chlorophyll and the variable ratio of the two constituents. Bull. de l'Acad. Sci. de Cracovie, Serie A, S. 28-40. 1912.

3) Siehe das Literaturverzeichnis am Ende der Abhandlung.

4) Emerson, R. A., The inheritance of certain forms of chlorophyll reduction in corn leaves. Ann. Rep. Nebraska Agr. Exp. Sta. 25: S. 89 bis $105, \cdot 1912$.

b) Nilsson-EHLE, H., Einige Beobachtungen über erbliche Variationen der Chlorophylleigenschaft bei den Getreidearten. Zeitschr. f. ind. Abstamm. u. Vererb. 9: S. 289-300, 1913. 
schaffenheit und Vererbung der grünen und gelben Blattpigmente mitzuteilen.

\section{Untersuchungsmaterial.}

Seit acht Jahren habe ich jährlich eine oder mehrere Rassen von Melandrium (Lychnis dioica L.) auf den Versuchsfeldern der Station for. Experimental Evolution bei Cold Spring Harbor, New York, großgezogen und ihre Merkmale und Verschiedenheiten sorgfältig beobachtet. Ich operierte mit folgenden Sippen:

1. „Cold Spring Harbor" oder abgekürzt „CSH“ sind die Nachkommen einer Kreuzung zwischen zwei wildwachsenden Individuen, die ich 1905 in der Nähe der Station for Experimental Evolution vor dem Hörsaal des Marine Biologischen Laboratoriums des Brooklyn Instituts gefunden habe.

2. "Harrisburg“ - stammt von Samen, der mir im Jahre 1909 von dem Samenlaboratorium des U. S. Department of Agriculture zugeschickt worden war. Der Same war in der Nähe von Harrisburg, Pennsylvania, gesammelt worden.

3. "Melandrium album Garcke"; den Samen verdanke ic̀ meinem Freund, Herrn Prof. Dr. BAUR, der ihn mir 1911 in liebenswürdiger Weise geschickt hat.

4. "Melandrium rubrum Garcke" habe ich gleichzeitig mit Melandrium album von Herrn Prof. Dr. BAUR erhalten. Die Samen von 3. und 4. wurden in Deutschland und Norwegen gesammelt.

Diese vier Sippen haben normal dunkelgrüne Blätter. Die Unterschiede lassen sich nur schlecht beschreiben; sie sind aber deutlich erkennbar, wenn die verschiedenen Rassen in größeren Beständen nebeneinander wachsen. Ich habe die Blattfarbe dieser vier Formen des öfteren mit den MAXWELLschen Farbenscheiben gemessen. Ich habe den Farbenkreisel, wie ihn die Milton Bradley Company, Springfield, Massachusetts, herstellt, benutzt. "Harrisburg" und "Melandrium album" zeigten beinahe die gleiche Intensität der Blattfarbe. Sio lassen sich auf dem Farbenkreisel mit 13,5 pCt. grün, 4,5 pCt. orangegelb und 82,0 pCt. schwarz wiedergeben. Die „CSH"-Sippe ist merklich heller mit 12,0 pCt. grün, 6,5 pCt. gelb und 81,5 pCt. schwarz. Melandrium rubrum ist etwas dunkler mit 10,5 pCt. grün, 3,0 pCt. orangegelb und 86,5 pCt. schwarz.

$\mathrm{Zu}$ diesen vier sattgrünen Sippen stehen zwei andere in auffallendem Gegensatz. Sie haben nur 57,0--62,0 pCt. schwarz; diese sind: 
5. "Chlorina", die 1911 in einem "Harrisburg"-Bestand auftrat, und

6. „Pallida“, die ich 1911 aus Samen zog, den ich vom Samenlaboratorium des U. S. Department of Agriculture bekommen hatte. Weiter unten Näheres über die zwei hellgrünen Rassen.

\section{Chloraỉbinismus ${ }^{\mathbf{1}}$ ).}

Vor drei Jahren hat $\mathrm{BAUR}^{2}$ ) bei Melandrium album, Antirrhinum latifolium und A. rupestre einen Faktor $\mathrm{Z}$ erkannt, der grundlegend für Chlorophyllbildung überhaupt ist. In Abwesenheit dieser Erbeinheit ist die Pflanze gänzlich chlorophyllfrei und nicht lebensfähig. Dasselbe Gen haben EMERSON ${ }^{3}$ ) und GERNERT ${ }^{4}$ ) bei Mais und NILSSON-EHLE ${ }^{5}$ bei Roggen und Gerste nachgewiesen.

Ich bin nun imstande, BAURs Beobachtungen über die Anwesenheit dieses Faktors bei Melandrium zu bestätigen. Im Frühjahr 1911 habe ich von BAUR Samen erhalten, der von zwei Kreuzungen seines ursprünglichen weißrandigen Exemplares $\boldsymbol{M}$. album Nr. „M 1“ mit einer schmalblättrigen Mutante derselben Rasse stammte. 200 Samen gaben mir 1911 (Saat Nr. 10244 und 10245)

\section{Berichtigung: Die Lutterpflanzon der Aussaat 10244 und 10245 waron Baurs L.2 und H. 4: sie stammten aus samon von dom poriklinal woissen Ast von H.I; also waren die pflanzen aleser z Aussaaten Bnkelkinder und nicht Kinder ron li. 1.}

heterozygotisch in bezug auf das Gen $\mathrm{Z}$ war. Die Krouzung dieser zwei Pflanzen hatte also die Formel $\mathrm{ZZ} \times \mathrm{Z} \mathrm{z}$ oder umgekehrt $\mathrm{Zz} \times \mathrm{ZZ}$. Die aus dieser Kreuzung erhaltenen Samen gaben mir 1912 (Saat Nr. 11335) 50 grüne und 0 weiße Keimlinge. Alle 50 waren gleichmäßig dunkelgrün, da der Faktor Z in diesem Falle vollkommen dominierte. Wenn die genotypische Formulie-

1) Ich benutze das Wort „Chloralbinismus“ und die ảavon abgeleiteten Worte „Chloralbinisten" und ,chloralbinotisch", um das Fehlen der grünen Blattpigmente auszudrücken. Das Wort „Albinismus" halte ich für ungeeignet, da solche Blätter häufig nicht weiß sondern gelb sind.

2) Zeitschr. f. ind. Abstamm. u. Vererb. 4: S. 81, 1910.

3) Ann. Rep. Nebraska Agr. Exp. Sta. 25: S. 89, 1912.

4) GeRnerT, W. B., The analysis of characters in corn and their behavior in transmission. S.58, 1912. Herausgegeben von dem Verfasser, Champaign, Illinois.

5) Zeitschr. f. ind. Abstamm. u. Vererb. 9: S. 289, 1913. 
rung der Eltern stimmt, dann müssen 50 pCt. der Nachkommen homozygotisch $\mathrm{ZZ}$ und $50 \mathrm{pCt}$. heterozygotisch $\mathrm{Z}_{\mathrm{z}}$ sein. Kreuzt man die Geschwisterpflanzen miteinander, so sollte man theoretisch zweierlei Nachkommenschaft erwarten: Erstens eine mit nur grünen Pflanzen, wenn der eine ev. beide Eltern homozygotisch waren und zweitens eine mit $75 \mathrm{pCt}$. grünen und $25 \mathrm{pCt}$. chloralbinotischen Pflanzen, wenn beide Eltern für diesen Faktor heterozygotisch waren. 1912 habe ich viele solche Geschwisterkreuzungen unter den Individuen von Saat Nr. 11335 ausgeführt und habe dieses Jahr 60 auf diese Art erzeugte Nachkommenschaften großgezogen. Wie sich herausstellte, hatte ich häufig Homozygoten miteinander oder mit Heterozygoten gekreuzt, denn ich erhielt viele reingrüne Nachkommenschaften. Da ja, wie schon erwähnt, die Heterozygoten von den Homozygoten nicht zu unterscheiden sind, war es mir nicht möglich, alle Pflanzen genetisch daraufhin zu prüfen. Deswegen kann ich nur von 27 Individuen der Aussaat Nr. 11335 mit Bestimmtheit sagen, ob sie hetero- oder homozygotisch für $\mathrm{Z}$ waren. Von diesen waren 13 heterozygotisch und 14 homozygotisch. Aus Tabelle I sind die Resultate der von mir ausgeführten Kreuzungen zwischen je zwei heterozygotischen Pflanzen zu ersehen.

Tabelle I.

Erbformel: XXZzYYNN $\times$ XXZzYYNN.

\begin{tabular}{c|c|c|c|c}
\hline \multirow{2}{*}{$\begin{array}{c}\text { Eltern } \\
11335\end{array}$} & Saat Nr. & \multicolumn{2}{|c|}{ Keimlinge } & pCt. grün \\
\cline { 2 - 5 } & & grün & weiß & \\
\hline$(20) \times(4)$ & 12235 & 60 & 27 & 68,97 \\
$(24) \times(3)$ & 12238 & 69 & 22 & 75,82 \\
$(26) \times(4)$ & 12239 & 69 & 21 & 76,67 \\
$(29) \times(4)$ & 12241 & 40 & 12 & 76,92 \\
$(30) \times(4)$ & 12242 & 60 & 24 & 71,43 \\
$(32) \times(3)$ & 12244 & 65 & 15 & 81,25 \\
$(34) \times(5)$ & 12245 & 72 & 28 & 72,00 \\
$(47) \times(4)$ & 12255 & 69 & 27 & 71,87 \\
$(48) \times(4)$ & 12256 & 64 & 23 & 73,56 \\
$(50) \times(4)$ & 12259 & 77 & 21 & 78,67 \\
\hline
\end{tabular}

Aus dieser Tabelle geht hervor, daß dis 10 Mutterpflanzen und 3 Vaterpflanzen Heterozygoten waren, und daß man eine Aufspaltung erhält, die das theoretisch zu erwartende Verhältnis $3: 1$ nahezu genau aufweist. Schon die einzelnen Familien kommen diesem Verhältnis sehr nahe. Zwei dieser 3 Vaterpflanzen wurden außerdem mit 13 anderen Schwesterpflanzen gekreuzt (Tảbelle II). 
Tabelle II.

Erbformel: XXZZYYNN $\times$ XXZzYYNN.

\begin{tabular}{c|r|r|c}
\hline \hline \multirow{2}{*}{\begin{tabular}{c|c|c} 
Eltern \\
11335
\end{tabular}} & Saat Nr. & grün & Weimlinge \\
\cline { 2 - 4 } & & 101 & - \\
$(8) \times(4)$ & 12200 & 97 & - \\
$(19) \times(3)$ & 12225 & 57 & - \\
$(22) \times(4)$ & 12237 & 87 & - \\
$(28) \times(4)$ & 12240 & 97 & - \\
$(31) \times(4)$ & 12243 & 73 & - \\
$(35) \times(4)$ & 12246 & 93 & - \\
$(36) \times(4)$ & 12247 & 59 & - \\
$(38) \times(3)$ & 12249 & 87 & - \\
$(40) \times(4)$ & 12251 & 95 & - \\
$(42) \times(3)$ & 12252 & 96 & - \\
$(43) \times(3)$ & 12253 & 55 & - \\
$(45) \times(3)$ & 12254 & 95 & - \\
$(49) \times(4)$ & 12257 & Sa. 1092 & \\
\hline
\end{tabular}

Da die Vaterpflanzen geprüfte Heterozygoten waren, müssen alle Mutterpflanzen Homozygoten gewesen sein. Außerdem wurden 9 weitere Schwesterpflanzen mit einer Bruderpflanze gekreuzt, die ganz zweifellos homozygotisch war. Aus diesen Kreuzungen stammen 839 grüne Sämlinge und nur 1 weißer. Die Resultate sind in Tabelle III zusammengestellt.

Tabelle III.

Erbformeln: XXZZYYNN $\times$ XXZZYYNN und $X X Z_{z Y Y N N} \times X X Z Z Y Y N N$.

\begin{tabular}{c|c|r|c}
\hline \multirow{2}{*}{$\begin{array}{c}\text { Eltern } \\
11335\end{array}$} & Saat Nr. & grün & weiß \\
\cline { 2 - 4 } & & 97 & - \\
$(9) \times(1)$ & 12201 & 91 & - \\
$(11) \times(1)$ & 12202 & 87 & - \\
$(12) \times(1)$ & 12203 & 101 & - \\
$(13) \times(1)$ & 12204 & 95 & - \\
$(14) \times(1)$ & 12214 & 87 & - \\
$(21) \times(1)$ & 12224 & 92 & - \\
$(37) \times(1)$ & 12236 & 99 & 1 \\
$(39) \times(1)$ & 12248 & 90 & -
\end{tabular}

Der einzige weiße Keimling in Saat $\mathrm{Nr}$. 12224 war sehr wahrscheinlich eine Mutante; denn wäre 11335(1) heterozygotisch gewesen, so hätte ungefähr die Hälfte der in Tabelle III angeführten Kreuzungen 25 pCt. weiße Keimlinge liefern müssen. Leider habe ich die Mutterpflanze 11335(17) in keiner anderen Kreuzung verwendet, und deswegen kann ich nicht unbedingt sicher sagen, ob diese Pflanze heterozygotisch oder homozygotisch war. 


\section{Di e „chlorina"-Sippen.}

Im Jahre 1902 erwähnte CORRENS ${ }^{1}$ ) kurz eine samenbeständige, chlorophyllarme, gelbgrüne Sippe von Mirabilis Jalapa, die, wenn mit den dunkelgrünen typica-Sippen gekreuzt, nur einheitlich dunkelgrüne $\mathrm{F}_{1}$-Pflanzen gibt; die $\mathrm{F}_{1}$-Pflanzen waren aber merklich heller als ihre typica-Eltern. In einem späteren Bericht ${ }^{2}$ ) geht er ausführlicher auf die unvollkommene Dominanz des typica-Merkmals ein. Er gibt die Verhältniszahlen der $\mathrm{F}_{2}$-Generation nicht an, doch glaubt er, daß es sich bei der gelbgrünen Färbung um eine mendelnde, rezessive Eigenschaft handelt. Diese Sippe war früher als aurea bezeichnet worden, vor kurzem aber hat CORRENS ${ }^{3}$ ) vorgeschlagen, alle die Sorten chlorina zu nennen, bei denen „die Quantität des Chlorophylls und des Xanthophylls sowie der Carotine abgenommen hat, ohne sehr auffällige Verschiebung im Verhältnis der grünen und gelben Bestandteile zueinander", und den aureaNamen auf jene Sorten zu beschränken, „bei denen der alkoholische Blattauszug gegenüber dem der typischen Sorten relativ viel mehr Xanthophyll und Carotin enthält". CORRENS glaubt, daß sich auf diesem Wege wenigstens eine künstliche Grenze zwischen den zwei Rassen ziehen lasse, doch er hofft, daß auf Grund genetischer Untersuchungen eine schärfere und zugleich natürlichere Trennung zu erzielen sei, da, wie er meint, BAURs aurea dominiert, während seine eigene chlorina rezessiv ist. Hierin kann ich nicht mit CORRENS übereinstimmen, da es sich bei BAURs aurea-Sippe von Antirrinum gar nicht um einen echten Fall von Dominanz handelt; denn bei vollkommener Dominanz eines positiven aurea-Merkmals wären nux die rezessiven, grünen Pflanzen lebensfähig, während die heterozygotischen aurea-Individuen genau wio dio homozygotischen schon als ganz junge Sämlinge absterben müßten ${ }^{4}$ ). Außerdem dominiert die typisch grüne Form von Mirabilis Jalapa auch nicht vollkommen über die chlorina-Form, wie ja aus CORRENS' Versuchen zu ersehen ist. Meiner Meinung nach gibt es überhaupt keinen prinzipiellen Unterschied zwischen aurea- und chlorina-Sippen. Ich glaube, daß die gelben Pigmente in den meisten Fällen vollkommen unabhängig von den grünen vererbt

1) Correns, C., Über Bastardierungsversuche mit Mirabilis-Sippen. Ber. d. Deutsch. Bot. Gesell., 20: S. 594-608, 1902.

2) Correns, C., Über die dominierenden Merkmale der Bastarde. Ber d. Deutsch. Bot. Gesell., 21: S. 133-147, 1903.

3) Zeitschr. f. ind. Abstamm. u. Vererb., 1: S. 291, 1909.

4) BAUR, E., Einführung in die experimentelle Vererbungslehre. S. 293, 1911, Berlin, Gebr. Borntraeger. Siehe S. 119. 
werden, und daß auf diese Weise die verschiedene Färbung der Chloralbinisten und der chloralbinotischen Flecken zu erklären ist. Bei einem Blatt, das viel Chlorophyll führt, können wir nichts über die gelbe Unterfärbung aussagen; sie ist vollkommen verdeckt. Ist nun aber das Chlorophyll aus irgendeinem Grunde weniger oder gar nicht ausgebildet, dann kommen die vorher verdeckten, gelben Pigmente zum Vorschein. Als Beispiel hierfür sollen $M$. album und $M$. rubrum dienen, die in ihren ausgewachsenen Blättern fast die gleiche Menge Chlorophyll haben. Dennoch ist in der Blattfarbe ein Unterschied zwischen den beiden. Die Blätter von $M$. rubrum sind etwas dunkler grün als die von M. album. Dagegen ist der Unterschied in den jungen Blättern, in denen noch relativ wenig Chlorophyll gebildet ist, viel deutlicher; bei $M$. rubrum sind sie entschieden gelber als bei $M$. album. Verschwindet nun das Chlorophyll gänzlich, wie bei den chloralbinotischen Sämlingen oder den mosaikartig zusammengesetzten, grïn-weißen Chimären, so erhält man von $M$. album gelblichweiße Sämlinge bzw. Blattteile; für $M$. rubrum nehme ich an, daß die chloralbinotischen Sämlinge oder Blattteile gelb sind; leider habe ich aber noch keine ${ }^{1}$ ).

Dasselbe gilt auch wohl für NILSSON-EHLEs ${ }^{2}$ ) chloralbinotische Roggensämlinge, die des öfteren weiflich und seltener gelb waren. NILSSON-EHLE glaubt daraus schließen zu müssen, daß Chlorophyllbildung überhaupt von zwei Faktoren abhängt. Ich glaube nicht, daß diese Folgerung nötig ist, da die gelbe Farbe ebenso gut durch Chlorophyll überdeckt sein kann wie die weißliche.

Den ersten sicheren Beweis, daß blaßgrüne Sippen in $\mathrm{F}_{2}$ regelrecht aufmendeln, haben PRICE und DRINKARD ${ }^{3}$ ) mit Tomatenkreuzungen erbracht. CORRENS ${ }^{4}$ ) und $\mathrm{BAUR}^{5}$ ) haben dies für die chlorina-Sippen von Mirabilis, Urtica, Antirrhinum und Aquilegia bestätigt.

Ich kann nun dasselbe für Melandrium mitteilen. 1911 traten bei mir ganz unerwartet in drei verschiedenen Aussaaten von Melandrium in jeder etwa $25 \mathrm{pCt}$. chlorina-Pflanzen auf. In Tabelle IV sind die genauen Zahlen angeführt.

1) Das Aussehen von grün-weißen Chimären der Bastarde, (M. album $\times$ rubrum und reziprok) bestätigt diese Annahme. Siehe S. 62 und 65.

2) Zeitsch. f. ind. Abstamm. u. Vererb. 9: S. 298, 1918.

3) Price, H. L., und DRINKaRd, A. W, Jr., Inheritance in tomato hybrids. Virginia Agr. Exp. Sta. Bull. Nr. 177. S. 17-53, 1908.

4) Zeitschr, f. ind. Abstamm. u. Vererb. 1: S. 291, 1909.

5) Zeitschr. f. ind. Abstamm. u. Vererb. 4: S. 81, 1910. 
Tabelle IV.

Erbformel: XXZZYYNn X XXZZYYNn.

\begin{tabular}{c|c|c|c}
\hline \hline \multirow{2}{*}{ Eltern } & \multirow{2}{*}{ Saat Nr. } & \multicolumn{2}{|c}{ Nachkommen } \\
\cline { 2 - 4 } & & grün & chlorina \\
\hline $09255 \times 09255$ & 10191 & 75 & 20 \\
$09256 \times 09256$ & 10195 & 60 & 26 \\
$09260 \times 09260$ & 10199 & 71 & 24 \\
\hline & Gefunden: & Sa. 206 & 70 \\
& Theoretisch: & $\mathbf{2 0 7}$ & $\mathbf{6 9}$
\end{tabular}

Ich war erstaunt über das dreimalige, unerwartete Auftreten der chlorina-Pflanzen. Um das Rätsel aufzuklären, verfolgte ich den Stammbaum der drei Aussaaten und fand, daß alle drei Sippen die Enkelkinder einer weiblichen Pflanze aus der ersten Aussaat meiner HARRISBURGschen Sippe waren (siehe Fig. 1). Um die Aufspaltung zu verstehen, braucht man nur anzunehmen, daß die Pflanze 08248(2) heterozygotisch grün $(\times$ chlorina $)$ war und ursprünglich nur von homozygotisch grünen Pflanzen befruchtet worden war. Die Nachkommen müssen dann zu etwa 50 pCt. Heterozygoten bzw. Homozygoten sein, die allerdings̀ nicht zu unterscheiden sind. Verbastardiert man diese Geschwisterpflanzen miteinander, so wird dem Zufallsgesetz entsprechend jede vierte Kreuzung eine Heterozygotenkreuzung sein, deren Deszendenzen zu 25 pCt. rein rezessiv chlorina sind. Aussaaten 10191, 10195 und 10199 sind die Nachkommenschaften solcher Heterozygotenkreuzungen.

Die chlorina-Pflanzen sind als Sämlinge in bezug auf die Blattfarbe ziemlich einheitlich und sind in diesem Entwickelungsstadium sehr leicht von ihren dunkelgrünen Geschwisterpflanzen zu unterscheiden. Bei den ausgewachsenen chlorina-Pflanzen ist die gelbgrüne Blattfarbe keineswegs einheitlich, sie wechselt sogar an ein und derselben Pflanze. Allen chlorina-Blättern gemeinsam - den dunkelsten wie den hellsten - ist die Eigenschaft, im hellen Sonnenlicht zu bleichen. Ganz besonders stark bleichen die Blattmitten, was wohl mit der stärkeren Bestrahlung zusammenhängt. Diese Eigenschaft habe ich bei den typisch grünen Sippen nie wahrgenommen. Im Jahre 1912 habe ich die Farbenzusammensetzung der hellen und der dunklen chlorina-Blätter mit dem Farbenkreisel bestimmt. Die dunkelste Blattfarbe konnte mit 14 pCt. grün, 24 pCt. gelb, 62 pCt. schwarz, die hellste mit 45 pCt. grün, $20 \mathrm{pCt}$. orangegelb, $12 \mathrm{pCt}$. weiß, 23 pCt. schwarz anpähernd wiedergegeben werden. Vergleicht man diese Resultato 
mit den oben für die dunkelgrünen Sippen mitgeteilten, so fällt dort der bedeutend höhere Prozentsatz an schwarz $(81,5$ bis 86,5 pCt.) auf.

Es ist keineswegs überraschend, daß die chlorina-Pflanzen viel weniger Chlorophyll als die typisch dunkelgrünen haben." Vergleicht man alkoholische Blattauszüge kolorimetrisch, so findet man, daß die dunkelsten Blattteile von chlorina nicht halb so viel Chlorophyll enthalten wie die Blätter von $\boldsymbol{M}$. rubrum $^{1}$ ).

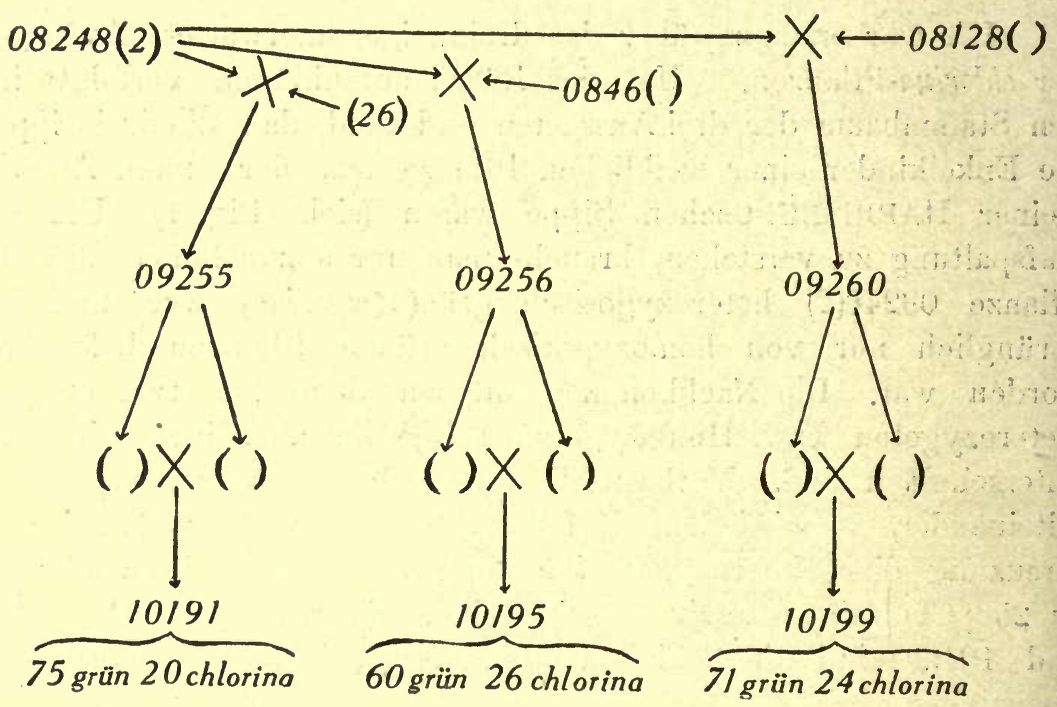

Fig. 1. Die Klammern bedeuten je ein Individuum aus der Saat, mit dcren Nummer sie in direkter Verbindung stehen.

Die gemeinsame Großmutter meiner ersten chlorina-Familien stammte von einer wildwachsenden Pflanze ab. Ob chlorinaFormen von Melandrium wild vorkommen, weiß ich nicht. Es ist möglich, daß eine derartige Sippe im Kampf ums Dasein unterliegt, und dann könnte das chlorina-Merkmal nur mit Hilfe eines

1) Für die kolorimetrischen Messungen wurden gleiche Gewichtsteile frischer Blätter jeweils mit einer gleichen Menge Alkohol extrahiert. Die Extrakte wurden in einander gleiche Probiergläser gegossen und zwar immer so viel, bis sie bei gedämpftem Licht von oben gesehen alle die gleichie. Farbendichte hatten. Die Menge der Flüssigkeit ist dann umgekehrt proportional dem Chlorophyllgehalt dieser Flüssigkeit. 
heterozygotischen Ergänzungsfaktors, den ich N nennen will, potentiell existieren. Im Garten und unter anderen günstigen Bedingungen kann die chlorina-Form fortkommen.

Ich habe die ursprünglichen chlorina-Pflanzen miteinander gekreuzt und 9 Nachkommenschaften mit insgesamt 815 Individuen erhalten. Als Sämlinge waren sie alle gelbgrün; als ausgewachsene Pflanzen, wie schon oben erwähnt, von sehr verschiedener Intensität der Blattfarbe. Zwei der 815 Pflanzen waren in diesem Entwicklungsstadium von den dunkelgrünen Sippen nicht zu untersckeiden. Möglicherweise gibt es noch eine ganze Reihe bisher unbekannter Chlorophyllfaktoren, die diese Abstufungen hervorrufen. Ich habe Versuche eingeleitet, die hoffentlich zu einer befriedigenden Entscheidung dieser Frage führen werden.

Kreuzt man chlorina mit typica, so erhält man zwei verschiedene Nachikommenschaften, je nachdem, ob die typische Form homozygotisch oder heterozygotisch ist. Im ersten Falle sind alle unmittelbaren Nachkommen dunkelgrün (aus 14 solchen Kreuzungen erhielt ich insgesamt 874 dunkelgrüne Pflanzen); während im zweiten Falle annährend 50 pCt. dunkelgrün und 50 pCt. chlorina-farben sind. Die Resultate von fünf derartigen Kreuzungen sind in Tabelle $V$ zusammengestellt. Mit Ausnahme von Saat Nr. 12294 stimmen die gefundenen Zahlenverhältnisse mit den theoretisch $\mathrm{zu}$ erwartenden recht gut überein.

Tabelle $\mathrm{V}$.

Erbformel: XXZZYYnn $\times$ XXZZYYNn.

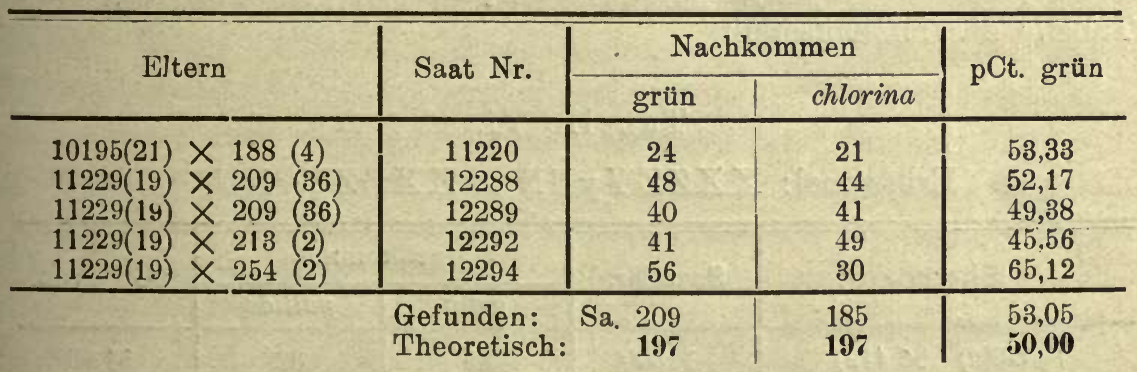

Im Jahre 1911 entdeckte ich noch eine zweite blaßgrüne Form in meinem Versuchsgarten. Es handelt sich um zwei Pflanzen, die aus Samen gezogen worden waren, der mir vom Samenlaboratorium des U. S. Department of Agriculture, leider ohne nähere Angaben, zugeschickt worden war. Ich vermute, 
daß der Same von wildwachsenden Pflanzen stammt. Alle übrigen Individuen der gleichen Aussaat (Nr. 10208) waren dunkelgrün und glichen $M$. album. Die blaßgrünen Pflanzen hatten auffallend große Blüten, die Kelche waren gebauscht und die großen Samenkapseln lieferten sehr viel Samen. Glücklicherweise war eine Pflanze männlich und die andere weiblich. Die Nachkommenschaft war in bezug auf die blaßgrüne Blattfarbe völlig einheitlich, und gut von allen anderen Sippen zu unterscheiden. Nach der Definition von CORRENS gehören sie zu dem chlorina-Typus; um Verwirrung zu vermeiden, habe ich diese neue Sippe „pallida“ genannt. Sie unterscheidet sich von meinen chlorina-Formen in der Intensität und besonders in der Verteilung der grünen Farbe. Pallida ist etwas dunkler grün als chlorina durchschnittlich ist. Die Farbe ist gleichmäBig über die Blattspreite verteilt, also nicht wie bei chlorina, deren Blattmitte heller ist als Basis und Spitze; das Bleichen im Sonnenlicht habe ich bei pallida nie bemerkt. Die Blattfarbe kann auf dem Farbenkreisel mit 30,5 pCt. grün, 12,0 pCt. orangegelb, 57,5 pCt. schwarz wiedergegeben werden. Um den Unterschied dieses Resultates mit den oben gefundenen zu erkennen, vergleicht man am besten nur den Prozentsatz an schwarz. Für die dunkelste chlorina-Farbe war 62 pCt. schwarz gefunden worden, für typica $81,5-86,5 \mathrm{pCt}$.

Pallida liefert, mit homozygotisch dunkelgrünen Pflanzen gekreuzt, ebenso wie chlorina, nur dunkelgrüne Nachkommen. Ich führte vier derartige Kreuzungen aus und erhielt 345 dunkelgrüne Pflanzen $\left(\mathrm{F}_{1}\right)$. Ich habe nur zwei Geschwisterkreuzungen unter den $\mathrm{F}_{1}$-Individuen ausgeführt. Die $\mathrm{F}_{2}$-Resultate sind in folgender Tabelle zusammengestellt.

Tabelle VI.

Erbformel: XXZZYyNN $\times$ XXZZYyNN.

\begin{tabular}{c|c|c|c|c}
\hline \multirow{2}{*}{ Eltern } & \multirow{2}{*}{ Saat Nr. } & \multicolumn{2}{|c|}{ Nachkommen } & \multirow{2}{*}{ pCt. grün } \\
\cline { 3 - 5 } & & grün & pallida & \\
\hline $11320(2) \times(12)$ & 12300 & 39 & 32 & 54,93 \\
$11351(53) \times(30)$ & 12329 & 63 & 27 & 70,00 \\
\hline \multicolumn{3}{c}{ Gefunden: Sa. 102} & 59 & 63,35 \\
& Theoretisch: & $\mathbf{1 2 1}$ & $\mathbf{4 0}$ & $\mathbf{7 5 , 0 0}$
\end{tabular}

Die gefundenen Zahlen weichen hier ziemlich beträchtlich von den theoretisch erwarteten ab. Man darf aber darauf wegen der geringen Anzahl von Pflanzen keinen zu großen Wert legen. 
Allerdings habe ich öfters einen deutlichen Überschuß von blaßgrünen Pflanzen erhalten, wio aus einigen der nachfolgenden Tabellon ersichtlich ist. Diese Tatsache kann verschiedene Ursachon haben. So scheint mir als wahrscheinlich, daß Pflanzen, die allo Erbeinheiten, die für dunkelgrüne Blattfarbe wesentlich sind, enthalten, trotzdem gelbgrün oder blaßgrün sein können. Die Gründe hierfür kennen wir heute noch nicht. CORRENS') hat bei Mirabilis Jalapa solche phaenotypisch blasse Pflanzen gefunden. Mit Rücksicht auf die Blattfarbe variieren die verschiedenen homozygotischen Melandrium-Sippen mit Ausnahme der chlorina-Sippe sehr wenig. Möglicherweise steht die phaenotypische Blässe der Bastarde auch mit unvollkommener Dominanz in Zusammenhang.

Bei Rückkreuzungen von typica $(\times$ pallida) Heterozygoten mit pallida werden die erwarteten 50 pCt. grün und 50 pCt. pallida annähernd erreicht (Tabelle VII).

Tabelle VII.

Erbformeln: XXZZY y NN $\times$ XXZZyyNN und umgekehrt.

\begin{tabular}{|c|c|c|c|c|}
\hline \multirow{2}{*}{ Eltern } & \multirow{2}{*}{ Saat $\mathrm{Nr}$. } & \multicolumn{2}{|c|}{ Nachkommen } & \multirow{2}{*}{ pCt. grün } \\
\hline & & grün & pallida & \\
\hline $\begin{array}{c}11320(2) \times 334(34) \\
11324(7) \times 351(49) \\
11351(53) \times 324(34)\end{array}$ & $\begin{array}{l}12301 \\
12325 \\
12332\end{array}$ & $\begin{array}{l}48 \\
38 \\
42\end{array}$ & $\begin{array}{l}51 \\
59 \\
40\end{array}$ & $\begin{array}{l}48,48 \\
39,18 \\
51,22\end{array}$ \\
\hline & Theor & $\begin{array}{l}128 \\
139\end{array}$ & $\begin{array}{l}150 \\
139\end{array}$ & $\begin{array}{l}46,04 \\
50,00\end{array}$ \\
\hline
\end{tabular}

Wir sehen also, daß sich chlorina und pallida gegen typica in gleicher Weise verhalten. Beide sind mendelndo Rezessiven und stellen deshalb aller Wahrscheinlichkeit nach beide Verlustmutationen dar. Da sie ganz zweifellos nicht identisch sind, ist es billig anzunehmen, daß jede Sippe ursprünglich einen verschiedenen Faktor verloren hat. Wenn diese Vermutung zutrifft, so müßte eine Kreuzung zwischen pallida und chlorina eine einheitlich dunkelgrüne $\mathrm{F}_{1}$-Generation ergeben. Ich habe fünf solche Kreuzungen ausgeführt, und alle gaben das gleiche Resultat. Ich erhielt insgesamt $432 \mathrm{~F}_{1}$-Pflanzen, die alle ebenso dunkelgrün waren wie normale typica.Pflanzen. Von drei dieser fünf $\mathrm{F}_{1}-$ Familien wurde die Blattfarbe je einer typischen Pflanze mit dem Farbenkreisel gemessen. In Tabelle VIII sind diese Messungen, sowie zum Vergleich einige schon erwähnte, zusammengestellt. 
Tabelle VIII.

\begin{tabular}{c|c|c|c}
\hline \hline Saat Nr. & & & \\
\hline 11223 & 11,0 pCt grün & 2,6 pCt. orangegelb & 86,4 pCt. schwarz \\
11321 & 12,0 pCt. grün & 4,5 pCt. orangegelb & 83,5 pCt. schwarz \\
11322 & 14,0 pCt. grün & 5,0 pCt. orangegelb & 81,0 pCt. schwarz \\
\hline
\end{tabular}

Zum Vergleich:

\begin{tabular}{l|l|l|l|l}
\hline dunkel chlorina: & 14,0 pCt. grün & 24,0 pCt. gelb & 62,0 pCt. schwarz \\
pallida: & 30,5 pCt. grün & 12,0 pCt. orangegelb & 57,5 pCt. schwarz \\
hellste typica: & 12,0 pCt. grün & 6,5 pCt. gelb & 81,5 C Ct. schwarz \\
dunkelste typica: & 10,5 pCt. grün & 3,0 pCt. orangegelb & 86,5 pCt. schwarz \\
\hline
\end{tabular}

Die kolorimetrische Bestimmung der alkoholischen Blattauszüge aus chlorina- und pallida-Pflanzen sowie aus deren Bastarden gab ein entsprechendes Ergebnis. Setzt man die für die $\mathrm{F}_{\mathbf{1}^{-}}$ Pflanzen gefundene Dichte des Blattauszuges gleich 100, so ergibt sich im Durchschnitt für chlorina 42,9, für pallida 64,7. Die Löslichkeit der Blattfarbstoffe ist auffallend verschieden. Pallida hat schon nach einstündigem Kochen in Alkohol alles Chlorophyll verloren, während man in chlorina und der Bastardform selbst nach vierstündigem Kochen noch Spuren von Chlorophyll in den Blättern finden kann.

Es fragt sich nun, wie sich diese dunkelgrünen $\mathrm{F}_{\mathbf{1}}$-Pflanzen verhalten, erstens wenn man sie untereinander kreuzt, und zweitens wenn man sie mit pallida und chlorina rückkreuzt. Die Voraussetzung, daß es sich um zwei unabhängig voneinander mendelnde Faktoren, und um vollkommene Dominanz der dunkelgrünen Farbe handelt, läßt uns die dihybriden Spaltungszahlen, 9 grün: 3 chlorina : 3 pallida: 1 ?? erwarten. Das Schachbrettschema (Fig. 2) macht dies ohne weiteres verständlich. Wie die Pflanzen, die für die Faktoren $\mathrm{Y}$ und $\mathrm{N}$ rezessiv sind, aussehen, läßt sich ohne weiteres nicht sagen, da ihr Aussehen von den Genen $\mathrm{X}$ und $\mathrm{Z}$ abhängt. Z ist der Grundfaktor für Chlorophyllbildung überhaupt und $\mathrm{X}$ ist die Bezeichnung ${ }^{1}$ ) für den noch nicht analysierten Rest des Genotypus. BAUR ${ }^{2}$ ) behauptet für Melandrium und Antirrhinum, daß alle $\mathrm{XXzz}$-Pflanzen "rein weiß" sind und daB. Z, chne die Gegenwart eines der anderen Blattfarbenfaktoren, mit $\mathrm{X}$ gelbe Farbe bewirkt. In beiden Fällen sind die

1) Johannsen, W., Elemente der exakten Erblichkeitslehre, I. Auflage, 1909, S. 304; II. Auflage, 1913, S. 387.

2) Zeitschr. f. ind. Abstamm. u. Vererb. 4: S. 89, 1910. 
Pflanzen nicht selbständig lebensfähig. Wenn dies auch für meine chlorina- und pallida-Sippen richtig wäre, dann müßte ihre $\mathrm{F}_{2}$-Generation Chloralbinisten liefern ( $j e$ eine in 16). Dies ist aber nicht der Fall, denn unter mehr als $1000 \mathrm{~F}_{2}$-Pflanzen trat kein einziger chloralbinotischer Sämling auf. Nach moiner Ansicht muß man aus diesem Resultat schließen, daß entweder bei alleiniger Gegenwart von $X$ und $Z$ ïberhaupt keine Keimlinge gebildet werden,

\begin{tabular}{|c|c|c|c|c|}
\hline$\tilde{\delta} \rightarrow$ & $\mathrm{XZYN}$ & $\mathrm{XZYn}$ & $\mathrm{XZyN}$ & XZyn \\
\hline$\stackrel{+}{1}$ & XZYN & XZYn & & XZyn \\
\hline $\mathrm{XZYN}$ & $\mathrm{XZYN}$ & $\mathrm{XZYN}$ & $\mathrm{XZYN}$ & $\mathrm{XZYN}$ \\
\hline & grün & grün & grün & grün \\
\hline & XZYN & XZYn & $\mathrm{XZyN}$ & XZyn \\
\hline $\mathrm{ZYn}$ & $\mathrm{XZYn}$ & XZYn & XZYn & XZYn \\
\hline & grün & chlorina & grün & chlorina \\
\hline & XZYN & XZYn & $\mathrm{XZ}_{\mathrm{y}} \mathrm{N}$ & XZyn \\
\hline $\mathrm{XZyN}$ & XZy y & $\mathrm{XZyN}$ & XZyN & $\mathrm{XZyN}$ \\
\hline & grün & grün & pallida & pallida \\
\hline & $\mathrm{XZYN}$ & XZYn & XZy N & XZyn \\
\hline$y n$ & $\mathrm{XZyn}$ & $\mathrm{XZyn}$ & $\mathrm{XZyn}$ & XZyn \\
\hline & grün & chlorina & pallida & ??? \\
\hline
\end{tabular}

Fig. 2.

oder aber, daß diese genügend Chlorophyll haben, um lebensfähig zu sein. Dann wäre dies eine neue chlorina-Form (XXZZy ynn), die ich fernerhin als subchlorina bezeichnen werde. Subchlorina ist äußerlich wohl nur sehr schwer von pallida und chlorina zu unterscheiden; bei Verbastardierung müßte sich diese Form aber ganz anders als diese verhalten. Mit pallida und chlorina gekreuzt müßten alle Nachkommen blaßgrün sein; mit typica wäre eine einheitliche, dunkelgrüne $F_{1}$-Generation zu erwarten, während $F_{2}$ in 9 
dunkelgrün $\mathrm{zu}$ i hellgrün aufspalten müßte. Die hellgrünen Pflanzen wären zum Teil pallida (je 3), zum Teil chlorina (je 3) und zum Teil subchlorina (je 1). Ich habe viele Kreuzungen ausgeführt, um eine subchlorina-Pflanze zu finden; doch man braucht wenigstens noch die $\mathrm{F}_{3}$-Generation, und diese habe ich noch nicht gezogen. Ich konnte leicht feststellen, daß die zweite Generation wenigstens aus drei verschiedenen Typen zusammengesetzt war, grün, chlorina und pallida. $\mathrm{Da}$ es aber nicht immer möglich war chlorina und pallida absolut sicher zu unterscheiden, habo ich die $\mathrm{F}_{2}$-Generation nur in dunkelgrüne und blaßgrüne Pflanzen eingeteilt. Je nachdem nun subchlorina.Individuen existieren oder nicht, muB man die Spaltungszahlen 9 dunkelgrün zu 7 blaßgrün (3 chlorina + 3 pallida +1 subchlorina) oder 9 dunkelgrün zu 6 blaßgrün ( 3 chlorina + 3 pallida) erwarten. Der schon oben erwähnte häufige Überschuß von blaßgrünen Pflanzen erschwert die Entscheidung, um welche dieser beiden Spaltungsverhältnisse es sich handelt. Der folgenden Tabelle habe ich das Verhältnis $9: 7$ als theoretisch zugrunde gelegt.

Tabelle IX.

Erbformel: XXZZYyNn×XXZZYyNn.

\begin{tabular}{|c|c|c|c|c|}
\hline \multirow{2}{*}{ Eltern } & \multirow{2}{*}{ Saat $\mathrm{Nr}$. } & \multicolumn{2}{|c|}{ Nachkommen } & \multirow{2}{*}{$\underset{\text { grün }}{\text { pCt. dunkel- }}$} \\
\hline & & dunkelgrün & blaßgrün & \\
\hline $\begin{array}{l}11223(5) \times(7) \\
11321(3) \times(4) \\
11321(3) \times \times 223(7) \\
11321(3) \times \times 322(31) \\
\left.11322(8) \times(8)^{*}\right) \\
\left.11322(15) \times(15)^{*}\right) \\
\left.11322(40) \times(40)^{*}\right) \\
11322(51) \times(33) \\
11322(51) \times 223(7) \\
11322(51) \times 320(5) \\
11322(51) \times 321(2)\end{array}$ & $\begin{array}{l}12285 \\
12302 \\
12304 \\
12305 \\
12307 \\
12308 \\
12309 \\
12310 \\
12311 \\
12312 \\
12313\end{array}$ & $\begin{array}{l}45 \\
39 \\
41 \\
40 \\
59 \\
53 \\
51 \\
54 \\
44 \\
66 \\
48\end{array}$ & $\begin{array}{l}35 \\
47 \\
55 \\
43 \\
43 \\
39 \\
43 \\
48 \\
59 \\
25 \\
50\end{array}$ & $\begin{array}{l}56,25 \\
45,35 \\
42,71 \\
48,19 \\
57,84 \\
57,61 \\
54,26 \\
52,94 \\
42,72 \\
72,53 \\
48,98\end{array}$ \\
\hline & $\begin{array}{l}\text { Gefunder } \\
\text { Theoreti }\end{array}$ & จ. $\begin{array}{r}540 \\
578\end{array}$ & $\begin{array}{l}487 \\
\mathbf{4 4 9}\end{array}$ & $\begin{array}{l}52,58 \\
\mathbf{5 6 , 2 5}\end{array}$ \\
\hline
\end{tabular}

*) Selbstbefruchtete Hermaphroditen.

Bei Rückkreuzung der dunkelgrünen $\mathrm{F}_{\mathbf{1}}$-Individuen mit den blaßgrünen Individuen ist es einerlei, ob man eine pallida- oder chlorina-Pflanze benutzt, solange es sich nur um eine Unterscheidung zwischen dunkelgrün und blaßgrün handelt. In dem einen Fall sind eben die 50 pCt. blaßgrünen Nachkommen pallida-Pflanzen, im anderen Falle chlorina-Pflanzen. Zu den zwei ersten Kreuzungen der Tabelle $\mathrm{X}$ wurde eine chlorina-Pflanze benutzt, zu den übrigen drei verschiedene pallida-Pflanzen. 
Tabelle X.

Erbformeln: XXZZYyNn XXXZZYYnn, XXZZYyNn $\times$

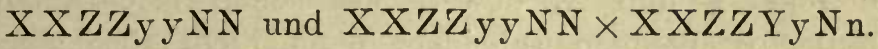

\begin{tabular}{|c|c|c|c|c|c|}
\hline \multirow{2}{*}{\multicolumn{2}{|c|}{ Eltern }} & \multirow{3}{*}{$\begin{array}{c}\text { Saut Nr. } \\
12286 \\
12303\end{array}$} & \multicolumn{2}{|c|}{ Nachkommen } & \multirow{3}{*}{$\begin{array}{c}\begin{array}{c}\text { pCt. dunkel- } \\
\text { grün }\end{array} \\
\begin{array}{c}47,19 \\
48,28\end{array}\end{array}$} \\
\hline & & & \multirow{2}{*}{$\begin{array}{c}\text { dunkelgrün } \\
42 \\
42\end{array}$} & \multirow{2}{*}{ 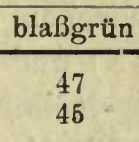 } & \\
\hline $\begin{array}{l}11223(5) \\
11321(3)\end{array}$ & $\begin{array}{r}211(81) \\
\times 211(81)\end{array}$ & & & & \\
\hline $\begin{array}{l}11223(5) \\
11321(3) \\
11322(51) \\
11323(19) \\
11324(7) \\
11324(7) \\
11324(7)\end{array}$ & $\begin{array}{l}\times 324(34) \\
\times 324(34) \\
\times 324(31) \\
\times 324(34) \\
\times 223(7) \\
\times 321(4) \\
\times 322(40)\end{array}$ & $\begin{array}{l}12287 \\
12306 \\
12314 \\
12316 \\
12321 \\
12322 \\
12323\end{array}$ & $\begin{array}{l}42 \\
42 \\
34 \\
44 \\
46 \\
38 \\
43\end{array}$ & $\begin{array}{l}36 \\
46 \\
48 \\
33 \\
42 \\
58 \\
52\end{array}$ & $\begin{array}{l}53,85 \\
47,73 \\
41,46 \\
59,26 \\
52,27 \\
39,58 \\
45,26\end{array}$ \\
\hline $11324(7)$ & & Theoret & a. $\begin{array}{l}377 \\
392\end{array}$ & $\begin{array}{l}407 \\
392\end{array}$ & $\begin{array}{l}48,09 \\
50,00\end{array}$ \\
\hline
\end{tabular}

Aus dieser 'Tabelle ist abermals ein kleiner Überschuß von blaßgrünen Pflanzen zu ersehen. Doch ist in dieser sowie in der vorhergehenden Tabelle die Übereinstimmung der gefundenen mit den theoretischen Zahlen wohl gut genug, um die Richtigkeit meiner Annahme zu bestätigen. Besonders möchte ich auf die Saat Nrn. 12286, 12287 und 12303, 12306 hinweisen; denn aus ihnen ist klar zu ersehen, daß die $\mathrm{F}_{1}$-Pflanzen für beide Genen $\left(\mathrm{Y}\right.$ und $\mathrm{N}$ ) heterozygotisch waren, da in diesen Fällen die $\mathrm{F}_{\mathbf{1}^{-}}$ Pflanzen sowohl mit pallida als auch mit chlorina gekreuzt worden waren.

Das Verhalten der chlorina- und pallida-Pflanzen bei Vererbung erinnert uns an zwei andere wohlbekannte Merkmalskategorien; als Vertreter der ersten Kategorie seien die rote Kornfarbe des Weizens (NILSSON-EHLE) ${ }^{1}$ ) und die gelbe Endospermfarbe des Mais $\left.(\mathrm{EAST})^{2}\right)$ angeführt. Die Blütenaṇthocyane mögen die zweite Kategorie repräsentieren. Wir haben schon gehört, daß die typicaPflanzen von Melandrium fast genau so viel Chlorophyll enthalten wie chlorina und pallida zusammen, und deswegen könnte man vermuten, daß es sich um einen Fall der ersten Kategorio handelt. Es wären also die Gene $\mathrm{Y}$ und $\mathrm{N}$ gleichsinnig, d. h. jedes Gen für sich erzeugt schon Chlorophyll, und es tritt lediglich eine Ver-

1) Nilsson-Ehle, H, Kreuzungsuntersuchungen an Hafer und Weizen. Lunds Universitets Årsskrift N. F. Afd. 2, Bd. 5, Nr. 2, S. 122, 1909.

2) EAsT, E.M., A Mendelian interpretation of variation that is apparently continuous. Amer. Nat. 44: S. 65-82, 1910. 
mehrung des Chlorophylls ein, wenn die beiden Gene gemeinsam vorhanden sind. Bei genauer Betrachtung wird man aber finden, daB die Gene $\mathrm{Y}$ und $\mathrm{N}$, obwohl sie schon beide allein etwas Chlorophyll zu erzeugen scheinen, doch nicht gleichsinnig sein können, denn dann müßten die Bastarde (XXZZY yNn) intermediär ${ }^{1}$ ) sein zwischen rein chlorina (XXZZYYnn) und rein pallida (XXZZ y y N ) und nicht dunkelgrün wie sie es tatsächlich sind. Nach meiner Meinung handelt es sich bei der Erzeugung von dunkelgrüner Blattfarbe um einen Fall, der mit der zweiten Kategorie viel Ähnlichkeit hat, allerdings mit dem Unterschied, daß bei Anthocyanbildung der Ausfall von $\mathrm{C}$ bzw. $\mathrm{R}$ gänzliche Farb. losigkeit bedingt, während in unserem Falle die Abwesenheit von $\mathrm{Y}$ bzw. $\mathrm{N}$ nur eine Verminderung des Chlorophyllgehalts von etwa 50 pCt. hervorruft. Möglicherweise ist dies aber gar kein prinzipieller Unterschied, da das Chlorophyll der pallida- und chlorinaSippen vielleicht gar nicht von $\mathrm{Y}$ und $\mathrm{N}$, sondern von andern bisher unbekannten Faktoren gebildet wird. Vielleicht sind also $\mathrm{Y}$ und $\mathrm{N}$ lediglich Gene, die eine gemeinsame Unterlage geringfügig modifizieren. Der volle Chlorophyllgehalt der dunkelgrünen Rassen entstände dann nur, wenn $\mathrm{Y}$ und $\mathrm{N}$ auf der gemeinsamen Unterlage zusammenträfen. Das wahrscheinliche Vorkommen der subchlorina-Sippe (XXZZyynn), die ja nícht von chlorina und pallida zu unterscheiden ist, stützt diese Erklärung wesentlich. Mit einem ganz ähnlichen Fall haben wir es vielleicht auch in der Vererbung von bläulicher Aleuronfarbe bei $\mathrm{Mais}^{2}$ ) zu tun, wo das Zusammenwirken von zwei Genen C und $\mathrm{P}$ blaue Farbe bewirkt, während jeder Faktor allein ganz hellblaue Farbe hervorruft.

BAUR (1911) hat bei Antirrhinum die Bezeichnungen Z, Y und $\mathrm{N}$ für die Chlorophyllfaktoren benutzt. Ich habe diese Bezeichnungen für Melandrium übernommen, da gewisse Übereinstimmungen offenbar bestehen; ich will aber damit keineswegs sagen, daß $\mathrm{Y}$ und $\mathrm{N}$ bei Melandrium mit $\mathrm{Y}$ und $\mathrm{N}$ bei Antirrhinum identisch sind. Es wäre keineswegs ausgeschlossen, daß mein $\mathrm{Y}$ BAURs $\mathrm{N}$ entspricht und vice versa. Genetisch läßt sich die

1) y y $\mathrm{NN}=$ pallida $=60 \mathrm{pCt}$. Ohlorophyll (d. i. $\mathrm{N}=30 \mathrm{pCt}$.)

Y Y n $\mathrm{n}=$ chlorina $=40$ pCt. Ohlorophyll (d. i. $\mathrm{Y}=20 \mathrm{pCt}$.)

Y yNn $=$ Bastard $=100$ pCt. Chlorophyll (gefunden); wenn aber $\mathrm{N}$ und $\mathrm{Y}$ gleichsinnige Faktoren wären, dann dürfte der Bastard nur 50 pCt. Chlorophyll haben (d. i. $\mathrm{N}+\mathrm{Y}=30+20=50 \mathrm{pCt}$.).

2) EAST, E. M., und HAYES, H. K., Inheritance in maize. Connecticut Agr. Exp. Sta. Bull. 167: S. 142, 1911. Siehe S. (68). 
Identität ja nicht beweisen, da eine Bastardierung der zwei in Frage stehenden Spezies unmöglich ist. Nur auf chemischem Wege könnte diese Frage ev. entschieden werden. Wir wollen zunächst ruhig annehmen, daß Z, Y und $\mathrm{N}$ bei Antirrhinum entsprechend sind mit $\mathrm{Z}, \mathrm{Y}$ und $\mathrm{N}$ bei Melandrium and die Verschiedenheiten und Übereinstimmungen kurz betrachten.

In beiden Spezies ist Z der Grundfaktor für Chlorophyllbildung. BAUR ist der Ansicht, daß ZZ oder $\mathrm{Z} z$ ohne Y Gelbfärbung bedingen; dagegen glaube ich, daß Z lediglich ein Chlorophyllfaktor ist und keinen Einfluß auf die gelben Blattpigmente ausübt; diese werden ganz unabhängig von $\mathrm{Z}$ vererbt.

Antirrhinum und Melandrium sind chlorina-farben, wenn die Pflanze bei Anwesenheit von $\mathrm{Z}$ homozygotisch für $\mathrm{Y}$ ist; in beiden Fällen ist chlorina rezessiv gegen normaldunnkelgrün. Nach BAURs Annahme ist seine aurea-Rasse von Antirrhinum bei Gegenwart von $\mathrm{Z}$ und $\mathrm{N}$ heterozygotisch für $\mathrm{Y}$; bei der aurea-Rasse handelt es sich demnach um unvollständige Dominanz der Erbeinheit Y. Bei Melandrium dagegen haben wir es mit vollkommener Dominanz dieses Faktors zu tun; die Heterozygoten ( $\mathrm{YyNN}$ ) sind von den dunkelgrünen Homozygoten nicht $\mathrm{zu}$ unterscheiden.

Der Faktor $\mathrm{N}$ bedingt sowohl bei Antirrhinum als auch bei Melandrium mit ZZ und Y Y normal dunkelgrüne Blattfarbe. Hier aber hört die Übereinstimmung auf; denn während bei Antirrhinum XXZZY y NN die genotypische Formel der aurea-Sippe ist, sind Melandrium-Pflanzen dieser Kombination dunkelgrün. Ferner erhalten wir bei Abwesenheit von Y die pallida-Rasse (XXZZ y y NN) von Melandrium, während Antirrhinum in diesem Falle anscheinend chlorophyllfrei und deswegen nicht selbständig lebensfähig ist.

Es gibt verschiedene Möglichkeiten, diese Verschiedenheiten zwischen Antirrhimum und Melandrium zu erklären. Wir können z. B. annehmen, daß in beiden Spezies $\mathrm{Y}$ und N Ergänzungsfaktoren sind, die, wenn sie zusammentreffen, normal dunkelgrüne Blätter geben, ohne aber gezwungen zu sein, für $\mathrm{Y}$ und $\mathrm{N}$ immer die gleichen Werte vorauszusetzen. Wir wollen dies an Zahlen illustrieren; so wollen wir für sattgrün den Wert 10 annehmen. Die zwei Ergänzungsfaktoren sollen wechselnde Werte haben, die sich aber jeweils zu 10 ergänzen; also: $\mathrm{YY}=1, \mathrm{NN}=9 ; \mathrm{YY}=2$, $\mathrm{NN}=8 ; \mathrm{YY}=3, \mathrm{NN}=7 ; \ldots . \mathrm{YY}=9, \mathrm{NN}=1$. Wenn nun bei Antirrhinum $\mathrm{YY}=9, \mathrm{NN}=1$ wären, dann wäre es durchaus möglich, daß bei Abwesenheit von $Y$ ein so geringer Wert $N$ nicht genügend Chlorophyll bilden könnte, damit die Pflanze lebensfähig wäre. Wenn dagegen bei Melandrium $\mathrm{YY}=4, \mathrm{NN}=6$ 
wären, dann wäre es nicht unmöglich, daß bei Anwesenheit von $\mathrm{Z}$ schon jeder Faktor allein genug Chlorophyll zum selbständigen Leben hervorbringen könnte. Wir erhielten so die zwei lebens. fähigen Melandrium-Sippen chlorina und pallida. Mit der Annahme verschiedener Werte für $\mathrm{Y}$ und $\mathrm{N}$ bei Antirrhinum und Melandrium ist es möglich, die Verschiedenheiten zu erklären. Die aurea-Sippe (XXZZ Y y N ) von Antirrhinum hätte nur den Wert 5,5 statt 10 (sattgrün), der grüne $(\times$ chlorina) Heterozygot $(\mathrm{XXZZY} \mathrm{y} \mathrm{N} \mathrm{N)} \mathrm{von}$ Melandrium aber den Wert 8 statt 10; die nicht lebensfähige Form von Antirrhinum (XXZZy yNN) bätte den Wert 1 statt 10; die pallida-Sippe von Melandrium (XXZZy y N ) aber 6 statt 10.

Eine andere Erklärung, die vielleicht den Vorteil größerer Einfachheit hat, ergibt sich aus BAURs ursprünglicher Deutung des ,aurea"-Merkmals seiner Antirrhinum-Sippe. Er nimmt einen teilweise dominierenden Hemmungsfaktor $\mathrm{H}$ an, der, wenn er homozygotisch vorhanden ist, jede Chlorophyllbildung verhindert; ist $\mathrm{H}$ heterozygotisch, so entsteht der ,aurea"-Typus (XXZZYYNNHh); der Widerspruch in der Vererbung der Blattfarbe bei Melantrium und Antirrhinum ließe sich so allein auf $\mathrm{H}$ zurückführen; $\mathrm{Z}, \mathrm{Y}$ und $\mathrm{N}$ würden sich dann in beiden Spezies entsprechen.

Es bedarf noch weiterer Versuche mit Antirrhinum und Melandrium, um die Frage über die grundlegende Übereinstimmung und Verschiedenheit der Chlorophyllfaktoren endgültig zu entscheiden.

\section{Marmorierte Sippen.}

Das Vorkommen verschiedener Unregelmäßigkeiten in der Verteilung des Chlorophylls auf den Pflanzen, wio Flecken, Streifen, weiße. Blattränder usw., sowie das Vorkommen ganz weißer ev. gelber Blätter oder Äste, ist eine der verbreitetsten teratologischen Erscheinungen bei den grünen Pflanzen. Wir kennen verhältnismäßig wenig verschiedene Arten von Panaschierung; dies mag uns auf den ersten Blick merkwürdig erscheinen, da wir Panaschierung ja bei beinahe allen Pflanzen finden, weshalb von Vererbung natürlich kaum die Rede sein kann. Panaschierung muß also häufig ganz spontan gebildet werden und zwar immer nur in einer der wenigen Formen. Dies wird uns sofort verständlich, wenn wir berücksichtigen, daß Panaschierung lediglich eine Zerstörung des bei allen höheren Pflanzen im Prinzip gleichen Chlorophyllapparates ist.

In der Literatur finden sich viele Stellen über die Vererbung der Marmorierung oder Panaschierung der Blätter, doch nur die Arbeiten von CORRENS und BAUR geben eine genügend klare 
Übersicht dieser Erscheinungen in dem Lichte der modernen Vererbungslehre. Dank der Versuche dieser zwei Forscher steht es heute fest, daß die Marmorierung sogar bei ein und derselben Pflanze verschiedener Natur sein kann.

Von der infektiösen Chlorose der Malvaceen usw., die gar keine erbliche Eigenschaft ist, abgesehen, unterscheidet BAUR') auf Grund des Verhaltens bei Vererbung viererlei verschiedene, marmorierte Sippen, von denen zwei mendeln und zwei nicht mendeln. Die mendelnden Sippen sind: a) variegata-Sippen von Mirabilis Jalapa (Correns) und Aquilegia vulgaris (Baur); b) eine albomarginata-Sippe von Lunaria biennis (Correns). Die nicht mendelnden sind: c) eine albomaculata-Sippe von Mirabilis Jalapa, in der die Buntblättrigkeit nur durch die Mutter vererbt wird, und zwar ist die Anzahl der buntblättrigen Nachkommen von dem Grad der Buntheit des Fruchtknotens der Mutterpflanze abhängig (CORRENS); d) eine albomarginata-Sippe von Pelargonium zonale, bei welcher die weißen oder weißrandigen Teile bei Selbstbefruchtung weiRe, die grünen Teile grüne Deszendenz liefern. Wenn aber diese weißen oder weißrandigen Äste mit rein grünen Pflanzen oder Pflanzenteilen gekreuzt werden, so ist die Nachkommenschaft zum weitaus größten Teil rein grün (etwa nur 5,8 pCt. sind marmoriert). Rein weiße Pflanzen traten, wenn auch in sehr kleiner Zahl (0,2 pCt.), nur dann auf, wenn der Vater weiß oder weißrandig war (BAUR).

In meinen Versuchen traten gelegentlich marmorierte Pflanzen auf und natürlich habe ich ihre Vererbung verfolgt. Einige derartige Versuche blieben erfolglos und einige sind noch nicht abgeschlossen. Im folgenden kann ich deswegen nur einen vorläufigen Bericht über meine noch lückenhaften Beobachtungen geben. Ich kann mit Sicherheit heute schon drei prinzipiell verschiedene Typen von Marmorierung bei Melandrium unterscheiden. Alle "drei gehören zu den nicht mendelnden Sippen und zwar stimmen zwei von ihnen wenigstens in einigen Punkten mit den oben beschriebenen Typen (c) und (d) überein. In anderen Punkten sind sie aber sicher verschieden. Bei der dritten handelt es sich um einen ganz eigenartigen, neuen Typus.

1. Grün-weiße Chimären. Chlorophyllfreie Gewebe von verschiedenster Ausdehnung sind bei Blättern von Melandrium

1) Zeitschr. f. ind. Abstamm. u. Vererb. 4: S. 97, 1910. 
sehr häufig. Es ist fast immer leicht zu sehen, daß das entfärbte Gewebe einheitlich weiß ist ${ }^{1}$ ); es mag nun ein ganzer Sektor der Pflanze sein oder nur ein schmaler Streifen auf der Blattspreite. Die Grenze zwischen den grünen und weißen Teilen ist immer scharf gezogen, und es ist wohl richtig, mit BAUR anzunehmen, daß der ganze weiße Gewebekomplex seinen Ursprung aus einer einzigen Zelle genommen hat, die ihre grünen Plastiden ganz oder fast ganz verloren hat, ohne aber ihrer anderen Eigenschaften, hauptsächlich des Zellteilungsvermögens, verlustig gegangen $\mathrm{zu}$ sein. Eine solche Pflanze ist demnach eine Chimäre, deren weißer Teil einer einzigen mehr oder minder verzweigten Zelllinie entspricht. Mit Rücksicht auf die Farbe der chlorophyllfreien Teile kann man verschiedene Arten von Chimären unterscheiden. Die Verschiedenheiten sind nach meiner Ansicht lediglich durch die Beschaffenheit und Quantität der gelben Farbstoffe, die ja sonst von den dunkelgrünen Farben mehr oder weniger verdeckt sind, bedingt.

Von den zahlreichen grün-weißen Chimären, die in meinem Garten aufgetreten sind, habe ich nur folgende vier auf ihre Vererbung genauer untersucht:

i) Die merkwürdigste, grün-weiße Pflanze war Nr. 1069(5), welche 1911 in meinen Kulturen auftrat. Als junger Sämling war diese Pflanze reingrün, aber schon das dritte Laubblatt zeigte zwei keilförmige, gelbweiße Felder, die etwa ein Drittel der ganzen Blattspreite einnahmen. Später wurde die eine Hälfte dieser Pflanze vollkommen gelbweiß, während die andere Hälfte teils einheitlich grün, teils gestreift war. Einige weiße Blätter hatten in der Mitte je einen blaßgrünen Fleck, der auf den ersten Blick wie eine grüne Insel aussah; erst mit der Lupe konnte man sehen, daß ein grüner Zellstrang diese grüne Insel mit anderen grünen Teilen der Pflanze verband; die Pflanze war also aus zwei Teilen zusammengesetzt, von denen nur der eine Chloroplasten ausgebildet hatte. Ein Querschnitt durch solche Blätter zeigte, daß die grüne Zellschicht, die nur eine oder höchstens zwei Zellen dick war, zwischen zwei weißen Schichten, die beide vier, stellenweise aber auch nur drei Zellen dick waren (inkl. Epidermis), eingebettet lag. Ich hatte also hier ein gutes Beispiel einer Periklinalchimäre. In

1) Das chlorophyllfreie Gewebe ist nie reinweiß, sondern immer mehr oder weniger gelblich bis intensiv gelb. Aus Bequemlichkeit werde ich das Wort „weiß“" synonymisch für „,chlorophyllfrei“" gebrauchen. 
dem grünen Teil der Pflanze fanden sich ebenso schön ausgebildete Sektorialchimären. Wie sich erwarten ließ, war die Pflanze bedeutend schwächer als ihre dunkelgrünen Geschwister. Blütenknospen wurden nur spärlich im Spätsommer gebildet, und die auf den rein weißen Sprossen kamen nicht zur Blüte. Die Pflanze war weiblich, und deswegen hatte ich gehofft, ihre Vererbung verhältnismäßig leicht studieren zu können. Leider wurde aber nur ein Fruchtknoten reif, welcher drei scheinbar gut ausgebildete Samen enthielt. Dieser Fruchtknoten war eine Sektorialchimäre (zu $110^{\circ}$ grün, $250^{\circ}$ gelbweiß). Es ist aber nicht ausguschlossen, daß er gleichzeitig auch eine Periklinalchimäre war. Alle drei Samen waren gelbweiß und hatten einen Stich ins Rosa. Leider waren alle drei nicht keimfähig, und deswegen kann ich nicht sagen, ob sie gelbweiße, chloralbinotische Keimlinge geliefert hätten, was ich eigentlich sicher annehme.

ii) Die zweite Chimäre (Nr. 11311(68)), die ich etwas näher untersucht habe, trat in einem $\mathrm{F}_{1}$-Bustand einer Kreuzung $\mathbf{M e}$ landrium album $\times$ rubrum auf. Prinzipiell war sie mit i) gleich, aber sie wich in vielen Einzelheiten von dieser ab. Sie zeigte nur einen kleinen chlorophyllfreien Sektor (Tafel XXIII, A), der zum Unterschied von i) nicht gelbweiß sondern gelb war. Mit dem Farbenkreisel kann man die Farbe des Sektors mit 64 pCt. gelb und 35 pCt. weiß wiedergeben. Die Pflanze war ebenfalls weiblich; ich habe sie mit zwei verschiedenen männlichen gekreuzt. Die Blüten der reingrünen $\mathrm{Z}$ weige bestäubte ich erstens mit Pollen einer chlorina-Pflanze (11211(81)) und zweitens mit Pollen, der von einem chlorophylllosen Ast des weiter unten unter iii) beschricbenen Individuums (11209(36)) stammte. In beiden Fällen war die Deszendenz durchweg dunkelgrün. Von der ersten Kreuzung zog ich 93, von der zweiten $96 \mathrm{~F}_{1}$-Pflanzen. Auf dem marmorierten Zweig konnte ich nur eine Blüte benutzen, die ich mit der schon erwähnten chlorina.Pflanze (11211(81)) kreuzte. Ich erhielt zahlreiche, reingelbe Keimlinge (Saat Nr. 12299), die nur einige Tage am Leben blieben; nur ein Individuum dieser Aussaat, dessen Kotyledonen schon gelb und grün waren, bildete Blätter aus, die gelb und grün gestreift waren. Leider gedieh diese Pflanze schlecht, und als ich sie Ende Mai ins freie Land auspflanzte, ging sie bald zu Grunde. Die Blüte der Mutterpflanze dieser Kreuzung hatte einen fast reingelben Kelch, der Fruchtknoten war gelb und von feinen, grünen Streifen durchzogen. Natürlich konnte ich die Verteilung der gelben und grünen Gewebekomplexe am Fruchtknoten nicht genau feststellen; aber ich zweifle nicht, daß es sich auch hier 
um eine. Verquickung von Periklinal- und Sektorialchimäre handelte, mit chlorophyllfreien Zellschichten nach innen und außen. Wenn diese Vermutung richtig ist, dann müssen die Eizellen ihren Ursprung im gelben Gewebe haben.

iii) Individuum 11209(36) war eine männliche $\mathrm{F}_{1}$-Pflanze (chlorina $\times$ typica). Die junge Pflanze war vollkommen dunkelgrün, und die Marmorierung trat erst auf, als ich sie schon ins Freiland ausgepflanzt hatte. Ende Juli 1912 waren einige Blätter der großen Rosette zur Hälfte blaßgelb, zur Hälfte grün, und ein Blatt war ganz chlorophyllfrei. Die ausgewachsene Pflanze hatte

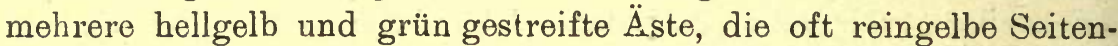
äste trugen. Um mit dem Farbenkreisel die Farbe der gelben Teile dieser Pflanze genau wiederzugeben, stelle man auf $59 \mathrm{pCt}$. gelb und $41 \mathrm{pCt}$. weiß ein. Den Pollen von Blüten auf reingelben Ästen benutzte ich zu Kreuzungen mit den in Tabelle XI angeführten Pflanzen. Die Resultate sind aus der letzten Spalte dieser Tabelle zu ersehen.

Tabelle XI.

\begin{tabular}{|c|c|c|c|}
\hline $\begin{array}{l}\text { Mutterpflanzen, die } \\
\text { mit,,gelben“ Blüten } \\
\text { von } 11209 \text { (36) be- } \\
\text { fruchtet wurden }\end{array}$ & $\begin{array}{l}\text { Saat } \\
\text { Nr. }\end{array}$ & $\begin{array}{l}\text { Beschaffenheit } \\
\text { der Mutter }\end{array}$ & $\begin{array}{l}\text { Beschaffenheit der } \\
\text { Nachkommenschaft }\end{array}$ \\
\hline $11209(1)$ & 12279 & $\begin{array}{l}\text { Grün (X chlorina) } \\
\text { Schwesterpflanze }\end{array}$ & 66 grün, 23 chlorina \\
\hline $11229(19)$ & 12289 & Rein chlorina & 40 grün, 41 chlorina \\
\hline $11324(7)$ & 12318 & Rein pallida & 97 dunkelgrün \\
\hline $11311(68)$ & 12297 & $\begin{array}{c}\text { Grüner Teil der grün- } \\
\text { weißen Chimäre oben als } \\
\text { ii) beschrieben }\end{array}$ & 96 sämtlich grün \\
\hline $11112(74)$ & 12272 & $\left|\begin{array}{c}\text { Rein gelbgrüner Teil der } \\
\text { ursprünglichen chlorino. } \\
\text { maculata-Pflanze unten } \\
\text { unter } 2 \text {. beschriebon }\end{array}\right|$ & $\begin{array}{l}\text { Zahlreiche Keimlinge } \\
\text { sämtlich hell grüngelb, } \\
\text { die alle bald abstarben. }\end{array}$ \\
\hline $11112(74)$ & 12274 & $\begin{array}{c}\text { Eine Blüte von 2. deren } \\
\text { Kelch zur Hälfte gelb- } \\
\text { grün, zur Hälfte grün } \\
\text { und gelbgrün gestreift } \\
\text { war }\end{array}$ & $\begin{array}{l}12 \text { grün, } 14 \text { chlorino- } \\
\text { maculata; dazu viele } \\
\text { chlorophyllfreie Säm- } \\
\text { linge }\end{array}$ \\
\hline $11112(74)$ & 12273 & $\begin{array}{c}\text { Eine Blüte von 2. deren } \\
\text { Kelch jedenfalls rein } \\
\text { grün war }\end{array}$ & 78 grün, 1 ?*) $^{*}$ \\
\hline
\end{tabular}

*) Als Sämling hatte diese Pflanze unregelmäBig̀e, gelbgrüne Flecken; ausgewachsen war sie aber rein dunkelgrün. 
Die Resultate wären dieselben, wenn eine normal grüne Bruderpflanze zu diesen Kreuzungen verwendet worden wäre. Drei dieser weiblichen Pflanzen (Nrn. 11209(1), 11229(19) und $11112(74))$ waren mit Pollen, der von rein grünen Ästen des Individuums 11209(36) stammte, belegt worden, und ich erhielt genau dieselben Resultate wio von den entsprechenden Kreuzungen der Tabelle.

iv) Eine weitere, merkwürdige Chimäre fand ich in einem Bestand von Lychnis coeli-rosa Desr. Der obere Teil eines Blütenstengels war zur Hälfte grün bzw. gelbweiß. Ein ganzes Nebenblatt war chlorophyllfrei und der oberständige Kelch und Frucht. knoten am Ende dieses Stengels waren scharf in eine grüne und eine gelbe Hälfte geteilt. Als ich diese auffallende, "halbgelbweiße" Blüte bemerkte, war sie leider aufgeblüht, so daß es keinen Zweck mehr hatte, sie künstlich zu bestäuben. Sie kann aber beinahe nur ausschließlich von normal grünen Pflanzen befruchtet worden sein, erstens, weil keine andere Lychnis coeli.rosa-Chimäre in meinem Garten war, und zweitens, weil der Pollen einige Tage eher reif ist als die Narbe und deswegen wohl schon von den Insekten entfernt war, ehe die Blüte befruchtet werden konnte. Der Samen aus diesem Fruchtknoten wurde unter Nr. 11348 ausgesät und lieferte ungefähr 60 rein gelbweiße Sämlinge und nur einen einzigen grünen. Es ist sehr auffallend, daß ein nur zur Hälfte gelbweißer Fruchtknoten fast lauter chlorophyllfreie Nachkommen hat. Es ist also möglich, daß die grüne Hälfte des Fruchtknotens nur äußerlich grün war, und daß er innen mit einer weißen Schicht, aus der sich die Eizellen entwickelt haben, fast gänzlich ausgekleidet war.

Trotz dieser sehr lückenhaften Versuche scheint mir die Folgerung, daß das Fehlen von Chlorophyll in Melandrium (auch in Lychnis coeli-rosa Desr.) nur durch die Mutter übertragbar sei, gerechtfertigt, daß also die Chlorophyllbeschaffenheit eines beliebigen Sämlings dieselbe sein muß wie die der Eizelle, aus der er entstanden ist. Diese Chimären sind also in ihrem anatomischen Bau mit BAURs Pelargonium zonale albomarginatum identisch, in der Vererbungsweise stimmen sie dagegen vielmehr mit CORRENS' Mirabilis Jalapa albomarginata überein. Nur die einzige grün-weiß malmorierte Pflanze der Saat Nr. 12299 (oben unter ii) beschrieben) hatte vielleicht einen Ursprung, welcher dem von BAURs marmorierten Pelargonium-Sämlingen entsprechen kann. Es ist aber nicht ausgeschlossen, daß gerade die Eizelle, der diese marmorierte Pflanze entsprungen ist, etwas Chlorophyll führte, da 
die Wand des mütterlichen Fruchtknotens teilweise grün gefärbt war.

An dieser Stelle möchte ich nun noch zwei weitere grün. weiße Chimären erwähnen, weil sie für mich Beweise zu sein scheinen, daß die gelbe Grundfarbe unabhängig von dem Faktor Z vererbt wird $\left.{ }^{1}\right)$. Wir haben oben unter ii) gesehen, daß die chlorophyllfreien Teile einer $\mathrm{F}_{1}$-Pflanze $(M$. album $\times$ rubrum $)$ gelb waren und daß sie entsprechend gelbe Nachkommenschaft lieferten. Im vergangenen Sommer habe ich in der reziproken Kreuzung ebenfalls eine Sektorialchimäre (12268(1)) mit derselben eigentümlichen, gelben Farbe beobachtet. Eine Pflanze $(12218(71)$ ) aus einem Bestand von reinen $M$.-album-Individuen hatte gelblichweiße ("rein weiße", BAUR²)) Blätter und Blattteile, und wie wir schon gehört haben, sind die chlorophyllfreien Keimlinge dieser Sippe ebenfalls „rein weiß“. Leider habe ich von $M$. rubrum noch nie grün-weiße Chimären oder chloralbinotische Sämlinge gesehen, aber die jungen Rosettenblätter dieser Rasse haben eine merklich gelbe Nüance, die meiner Meinung nach auf eine gelbe Grundfarbe schließen läßt. Sollte diese Vermutung richtig sein, dann könnte die gelbe Grundfarbe der zwei eben erwähnten, reziproken $\mathrm{F}_{1}$-Pflanzen durch M. rubrum bedingt sein und zwar durch Eizelle und Spermazelle. Die gelbe Grundfarbe wäre dann ein gewöhnliches, dominierendes Merkmal, über dessen Vererbung ich noch nichts weiteres aussagen kann.

2. Die chlorinomaculata-Sippe. Mit diesem Namen bezeichne ich eine Rasse, die von einer Pflanze (11112(74)) abstammt, die 1912 in einem sonst reingrünen Bestand von mehrfach miteinander gekreuzten CSH und HARRISBURGschen Pflanzen auftrat. Am 5. Juni 1912, als diese chlorinomaculata-Pflanze zum ersten Male als Neuheit notiert wurde, waren mehrere, sonst dunkelgrüne Blätter der Rosette gelbgrün gefleckt und gestreift. Manchmal war die Hälfte eines Blattes gelbgrün, meistens aber waren die Flecken nur klein und undeutlich. Die ausgewachsene Pflanze war ungefähr zur einen Hälfte normal dunkelgrün, die andere Hälfte zeigte durchweg eine grün-chlorina Marmorierung; die chlorina-Farbe trat in langgezogenen Flecken, Linien und Streifen auf (Tafel XXIII, B). Einige Äste waren überwiegend grün, andere überwiegend chlorina-

1) BAUR nimmt an, daß $\mathrm{Z}$ ohne die anderen Chlorophyllfaktoren gelbe Blattfarbe bedingt; dann müßten aber den verschiedenen gelben Grundfärbun. gen Veränderungen von $\mathrm{Z}$ entsprechen. Dies mag ja. vielleicht der Fall sein, ich aber halte es für sehr unwahrscheinlich.

2) Zeitschr. f. ind. Abstamm. u. Vererb. 4: S. 81, 1910. 
farben und an den letztgenannten war manchmal ein ganzes Nebenblatt mit dem axelständigen Blütenstengel und Blumenkelch von reiner chlorina-Färbung. Die Form und Anordnung der einzelnen Farbfelder zeigten deutlich, daß sie nicht aus einer einzigen ursprünglich modifizierten Zelle durch Folgeteilung entstanden sein konnten. Die Verlängerung der Flecken dagegen mag auf Folgeteilung zurückzuführen sein. Es besteht weder zwischen den beiden grünen und chlorinomaculata-Pflanzenhälften noch zwischen den grünen und chlorina-gefärbten Feldern der chlorinomaculata. Hälfte eine scharfe Grenze. Danach ist diese Pflanze keine grünchlorina Chimäre, sondern etwas prinzipiell Verschiedenes. Die Pflanze war weiblich; ich habe mehrere Blüten befruchtet. Die Kreuzungen und Resultate sind in Tabelle XII zusammengestellt.

Tabelle XII.

\begin{tabular}{|c|c|c|c|c|}
\hline $\begin{array}{c}\text { Pflanzen, die zur } \\
\text { Befruchtung } \\
\text { von } 11112(74) \\
\text { verwendet } \\
\text { wurden }\end{array}$ & $\begin{array}{l}\text { Saat } \\
\text { Nr. }\end{array}$ & $\begin{array}{l}\text { Farbe des } \\
\text { mütterlicheo } \\
\text { Kelches }\end{array}$ & $\begin{array}{c}\text { Farbe des } \\
\text { väterlichen } \\
\text { Kelches }\end{array}$ & $\begin{array}{c}\text { Beschaffenheit } \\
\text { der } \\
\text { Nachlzommenschaft }\end{array}$ \\
\hline $\begin{array}{c}11112(17) \\
\text { (Geschwister- } \\
\text { pflanze) }\end{array}$ & 12270 & $\begin{array}{l}40 \text { pCt. chlorina } \\
\text { und grün gestreift, } \\
60 \text { pCt. rein grün }\end{array}$ & Rein grün & $\begin{array}{l}13 \text { rein grün, } \\
20 \text { chlorinomaculata, } \\
\text { ?? grüngelbe Keim- } \\
\text { linge, die starben } \\
\text { ohne Laubblätter } \\
\text { zu bilden }\end{array}$ \\
\hline $\begin{array}{c}11106(13) \\
\text { (CSH-Sippe) }\end{array}$ & 12271 & $\begin{array}{c}\text { Rein grün bis auf } \\
\text { eine Stelle, die von } \\
\text { schmalen chlorina- } \\
\text { Streifen durch- } \\
\text { zogen war }\end{array}$ & Rein grün & $\begin{array}{l}44 \text { grün, } \\
25 \text { chlorinomaculata, } \\
7 \text { (?) gelbgrün, } \\
\text { starben ohne Laub- } \\
\text { blätter zu bilden }\end{array}$ \\
\hline $11209(36)$ & 12272 & $\begin{array}{c}\text { Gänzlich chlorina- } \\
\text { gefärbt }\end{array}$ & Chlorophyllfrei & $\begin{array}{l}\text { Alle grüngelb; } \\
\text { starben ohne Laub- } \\
\text { blätter zu bilden }\end{array}$ \\
\hline $11209(36)$ & 12273 & Gänzlich grün & $\begin{array}{l}90 \text { pCt. grün } \\
10 \text { pCt. chloro- } \\
\text { phyllfrei }\end{array}$ & $\begin{array}{l}79 \text { rein grün, } \\
1 ? * j\end{array}$ \\
\hline $11209(36)$ & 12274 & $\begin{array}{l}50 \text { pCt. chlorina, } \\
50 \text { pCt. grün und } \\
\text { chlorina, feiu ge- } \\
\text { streift }\end{array}$ & Chlorophyllfrei & $\begin{array}{l}12 \text { rein grün, } \\
14 \text { chlorinomaculata, } \\
\text { und viele grüngelbe } \\
\text { Keimlinge, die alle } \\
\text { abstarben }\end{array}$ \\
\hline $11211(81)$ & 12275 & $\begin{array}{c}\text { Gänzlich chlorina- } \\
\text { gefärbt }\end{array}$ & Rein chlorina & $\begin{array}{l}\text { Alle grüngelb, } \\
\text { starben als Keim- } \\
\text { linge }\end{array}$ \\
\hline $11211(81)$ & 12276 & Gänzlich grün & Rein chlorina & 91 rein grün \\
\hline
\end{tabular}

*) Als Keimling hatte diese Pflanze undeutliche, gelbgrüne Flecken; die ausgewachsene Pflanze war sicher rein grün. 
Aus den Resultaten dieser Versuchsreihe geht hervor, da $B$ die Deszendenz mit Rücksicht auf die chlorinomaculata-Eigenschaft von dem Grade der chlorina-Fürbung des mütterlichen Kelches (Fruchtknotens) abhängig ist; und daß die Beschaffenheit des väterlichen Kelches (Pollen) ohne Einfluß bleibt. Soweit stimmt die Vererbung des chlorinomaculata-Merkmals mit dem überein, was CORRENS $^{1}$ ) für das albomaculata-Merkmal bei Mirabilis Jalapa gefunden hat. $\mathrm{Ob}$ die chlorinomaculata-Eigenschaft durch den Vater vererbt wird, konnte ich noch nicht prüfen, weil ich leider nur eine weibliche Pflanze dieser Sippe verwenden konnte; da aber von den 59 chlorinomaculata-Nachkommen dieser Pflanze viele männlich sind, hoffe ich, späterhin auf diese Frage zurückkommen zu können.

Man kann aus den Resultaten dieser Kreuzungen ferner orkennen, daß die Farbe der helleren Teile der chlorinomaculata.Pflanzen, obwohl der chlorina-Farbe sehr ähnlich, doch nicht mit dieser identisch ist, da die Keimlinge nicht lebensfähig sind, wenn die betreffenden Fruchtknoten der Mutterpflanzen rein chlorina-farben waren. Ein weiterer Beweis für die Nichtidentität dieser zwei Färbungen ist die Tatsache, daß infolge andauernder Insolation die hellgrünen Teile der chlorinomaculata.Pflanzen vollkommen gebleicht werden, während die chlorina.Sippen entschieden lichtbeständiger sind.

3. Die „aurea"-Sippen. Ich komme nun zu einer Reihe von buntblättrigen Pflanzen, die eine merkwürdige Herkunft und Vererbungsweise haben. Im Jahre 1911 fiel mir eine Pflanze (10111(54)) einer meiner CSH-Familien auf; die Blätter dieser Pflanze zeigten gelbe Flecken, die meistens klein und rundlich waren; nur vereinzelt war ein halbes Blatt verfärbt. Einige Blütenstengel waren ebenfalls verfärbt und die Blütenkelche an diesen waren, von den Spitzen der Kelchblätter abgesehen, blaßgelb und rosa anstatt grün und dunkelrot. Die Blüten an normalen Stengeln waren purpurrot, ebenso die Blüten an den verfärbten Stengeln; nur eine solche Blüte hatte weißrot gestreifte Blumenblätter. $\mathrm{Da}$ ich bisher nur eine einzige, derartige Blüte an „aurea"-Pflanzen beobachtet habe, darf man die Streifung wohl nicht mit der "aurea"-Eigenschaft in Zusammenhang bringen.

Die gelben Flecken der Blätter waren augenscheinlich von normalem, grünen Gewebe umgeben, und die Kelche an den gelben

1) Zeitschr. f. ind. Abstamm. u. Vererb. 1: S. 313, 1909 
Stengeln waren ebenfalls rein gelb bis auf die Spitzen der Kelchblätter, die oft normal dunkelgrün waren. Es waren grüne Gewebe von gelben Geweben ebenso gut wie gelbe von grünen umgeben. Auf dem Querschnitt durch derartige bunte Blätter konnte man keine scharfe Grenze zwischen den grünen und gelben Blattteilen erkennen; die grünen Chloroplasten werden gegen den Mittelpunkt des Fleckens zu immer bräunlicher oder gelber und verschwinden bald ganz. Das Übergangsgewebe ist nur wenige Zellen dick. Ich kenne die chemische Beschaffenheit dieser bräunlichgelben Pigmente nicht, aber ich vermute, daß sie größtenteils Zersetzungsprodukte des Chlorophylls sind und nicht unabhängige, unterliegende Färbungen, wie ich das bei den grün-weißen Chimären annehme. Da diese gelbgefleckte Pflanze (Nr. 10111(54)) männlich war, bezweifelte ich den Erfolg einer genetischen Untersuchung, zumal das neue Merkmal so auffällig nur ein lokaler, pathologischer Zustand der cytoplasmischen Organe zu sein schien. Dennoch führte ich einige Kreuzungen mit dieser Pflanze aus. Ich bestäubte je eine chlorina- und pallida-Pflanze mit Pollen, der von einer dieser "gelben" Blüten stammte, und außerdem noch zwei chlorina. Pflanzen, mit Pollen von normalen Blüten dieser gescheckten Pflanze. (Vergleiche Tabelle XIII.)

Tabelle XIII.

\begin{tabular}{|c|c|c|c|c|}
\hline \multirow{2}{*}{$\begin{array}{l}\text { OPflanzen, die von } \\
10111(54) \\
\text { befruchtet wurden }\end{array}$} & \multirow{2}{*}{ Saat Nr. } & \multicolumn{2}{|c|}{$\begin{array}{l}\text { Beschaffenheit der } \\
\text { Eltern }\end{array}$} & \multirow{2}{*}{ Nachkommen } \\
\hline & & Mutter & $\begin{array}{l}\text { Kelch des } \\
\text { Vaters }\end{array}$ & \\
\hline $\begin{array}{l}10192(2) \\
10195(21) \\
10195(21) \\
10208(1)\end{array}$ & $\begin{array}{l}11210 \\
11217 \\
11218 \\
11351\end{array}$ & $\begin{array}{l}\text { Chlorina } \\
\text { Chlorina } \\
\text { Chlorina } \\
\text { Pallida }\end{array}$ & $\begin{array}{l}\text { Grün } \\
\text { Grün } \\
\text { Gelb } \\
\text { Gelb }\end{array}$ & $\begin{array}{l}\text { Keine } \\
48 \text { dunkelgrün } \\
59 \text { grün, } 5 \text { „aurea } \\
81 \text { grün, } 6 \text {,aurea }{ }^{4}, 2 \text { ?*) }\end{array}$ \\
\hline
\end{tabular}

*) Diese zwei Pflanzen waren anfangs etwas blasser als ihre normalen Geschwister; später aber waren sie nicht mehr zu unterscheiden.

Ich gebrauche in dieser Tabelle und weiterhin den Namen "aurea" einheitlich für alle Pflanzen, die von meiner Stammpflanze 10111(54) abstammen und wie diese anormal gefärbte Blätter oder Äste haben, obschon die Nachkommen in bezug auf diese Eigenschaft sehr verschieden waren und fast nie ihrem Vater auch nur ähnelten. Die fünf "aurea"-Pflanzen von Saat Nr. 11218 sahen wie folgt aus:

Pflanze 11218(1). Die Kotyledonen waren rein gelb, und da keine Laubblätter gebildet wurden, verhungerte sie als Keimling. 
Pflanze 11218(2). Die ersten zwei Laubblätter hatten rein gelbe Adern und die dazwischenliegenden Blattflächen waren grünlichgelb. Alle Blätter der jungen Rosette waren dunkel grüngelb. Die ausgewachsene Pflanze war einheitlich gelbgrün ${ }^{1}$ ) (Farbenkreisel: 22,0 pCt. grün, 9,5 pCt. orangegelb, 68,5 pCt. schwarz).

Pflanze 11218(3). Die ersten Blätter waren gelbgrün, die späteren bis auf einzelne, die gelbgrün waren, sattgrün. Zur Zeit der Blüte waren die gelben Blätter ganz verschwunden, und es war dinn unmöglich, diese Pflanze von ihren normalen Geschwistern $\mathrm{zu}$ unterscheiden (Farbenkreisel: 12,5 pCt. grün, 3,0 pCt. orangegelb, 84,5 pCt. schwarz).

Pflanze 11218(4). Die ersten zwei Blätter waren beide halb sattgrün, halb gelbgrün; die Mittelrippe bildete die Grenzlinie. Die zwei Monate alte Pflanze sah wie eine sattgrün-gelbgrüne Sektorialchimäre aus, in der die hellere Farbe etwa ein Fünftel einnahm. Die ausgewachsene Pflanze war eigentlich vollständig sattgrün.

Pflanze 11218(5). Das eine der ersten beiden Blätter war genau so wie bei der vorhergehenden Pflanze, halb sattgrün, halb gelbgrün; das andere war dunkelgrün bis auf ein schmales, grüngelbes Dreieck. Die ausgewachsene Pflanze war gelbgrün.

Mit Ausnahme dieser fünf. "aurea"-Pflanzen war die ganze Aussaat dunkelgrün, wie das ja auch bei einer Kreuzung von chlorina $\times$ homozygotisch typica zu erwarten war.

Noch auffallender als bei dieser Kreuzung war der Einfluß der „aurea"-Eigenschaft der väterlichen Pflanze (10111(54)) auf die Deszendenz (Saat Nr.11351), die sie mit einem pallida-Individuum gekreuzt ergab. Von 89 Pflanzen waren 6 sicher ,aurea" (in Saat Nr. 11218 waren von 64 Pflanzen 5 ,aurea"). Da ich fürchtete, daß die fünf auffallendsten ,aurea"-Keimlinge (A), (B), (C), (D), (E), unter den gewöhnlichen Freilandkulturbedingungen zu Grunde gehen möchten, pflanzte ich sie in die Nähe des Laboratoriums aus, da dort der Boden etwas besser ist; auch konnte ich sie so besser beobachten. Alle fünf hatten grüne Kotyledonen und die ersten Laubblätter waren beinah chlorophyllfrei. Später wurde auch noch Individuum 11351(8) derselben Aussaat zu den ,aurea“-

1) Ich habe mich des MiLTON-BRAdLEYschen Farbensystems bedient, in dem das Sonnenspektrum in 18 Farbengrade eingeteilt ist. Es fügt zwischen je zwei Hauptfarben zwei weitere Abtönungen ein, z. B zwischen rot und orangegelb, orangegelbrot und rotorangegelb; zwischen orangegelb und gelb, gelborangegelb und orangegelbgelb; zwischen gelb und grün, grüngelb und gelbgrün usw. 
Pflanzen gezählt, wạhhrend 11351(1) und (2), obwohl als Sämlinge etwas gelblichgrün, unter die normal dunkelgrünen Pflanzen gerechnet wurden, da sie später von diesen nicht mehr sicher unterschieden werden konnten. Im folgenden will ich nun die sechs „aurea"-Pflanzen näher beschreiben.

(A) und (B) waren eigentlich rein weiß mit Ausnahme der blaßgrünen Kotyledonen und einiger der ersten Blätter, die etwas Chlorophyll in den Basen führten; sie lebten etwa zwei Monate anscheinend gesund und bildeten 4-6 kleine, weißliche Blätter, wonach sie eingingen. Die jungen Rosetten von (C), (D) und (E) waren grüngelb; (D) war etwas dunkler grün als (C) und (E); (E) war am hellsten und ihre grüngelben Blätter hatten etwa die Tönung des grüngelben Streifens des Sonnenspektrums; (E) war nicht einheitlich gefärbt, sondern wies undeutlich umgrenzte, dunkler grüne Schattierungen auf. (D) war vollkommen einheitlich in der Farbe, während (C) einige Blätter hatte, die auf der einen Seite der Mittelrippe etwas grüner waren als auf der anderen. Obwohl (C), (D) und (E) ganz deutlich weniger Chlorophyll besaßen als normale Pflanzen, bildeten sie dennoch sehr üppige Rosetten aus; im. Spätsommer trieben (C) und (E) einige Blütenstengel. Wider mein Erwarten überwinterten alle drei sogar ohne irgendwelchen Schutz sehr gut und bildeten im Frühling dieses Jahres (1913) ungewöhnlich große ;,aurea"-farbene Rosetten.

Die blühenden Pflanzen (C) und (E) waren einander sehr ähnlich; (C) war männlich und (E) weiblich. Sie waren schön marmoriert und sahen auf den ersten Blick dunkelorangegelb bis dunkelgelb aus; bei genauerer Untersuchung waren die Stengel und untersten Blätter entschieden grüner als die übrigen Teile. Manchmal war die grüne Marmorierung willkürlich über die ganze Blattspreite verteilt; doch häufig war die grüne Farbe in einem länglichen, unregelmäßigen Flecken in der Blattmitte ange: sammelt. Verschiedene Blätter hatten einzelne reingelbe Streifen; das Gelb war etwas dunkler als das des Sonnenspektrums. Da (C) und (E) so weitgehend in ihrer Färbung übereinstimmten, gelten die für (E) gefundenen, kolorimetrischen und FarbenkreiselResultate ebenșo gut für (C), abgesehen von den unteren schattierten Blättern, die mir bei (E) etwas grüner zu sein schienen als bei (C). Für die untersten Blätter der Pflanze (E) fand ich mit dem Farbenkreisel 45,5 pCt. grün, 19,5 pCt. orangegelb und 35,0 pCt. schwarz. Nach den obersten Blättern zu nimmt das Chlorophyll immer mehr und mehr ab, bis es schließlich scheinbar gänzlich verschwindet, so daß ich für die obersten Blätter 64,0 pCt. gelb, 
28,5 pCt. orangegelb und 7,5 pCt. weiß fand. Ich halte es für zweckmäßig, diese Messungen mit den schon angeführten der pallida-Mutterpflanzen zu vergleichen $(30,5 \mathrm{pCt}$. grün, $12,0 \mathrm{pCt}$. orangegelb, $57,5 \mathrm{pCt}$. schwarz).

Alkoholische Chlorophyllauszüge aus pallida-Blättern haben, mit Auszügen aus einem gleichen Gewicht von den grünsten, untersten Blättern von (E) verglichen, genau um die Hälfte mehr Chlorophyll. Da nun ferner $(\mathrm{E})$ in den obersten Blättern überbaupt kein Chlorophyll hat, während pallida solches durchweg führt, ist es klar, dab der Gesamtbetrag von Chlorophyll in pallidaPflanzen vielmal größer ist als in (E) und (C). Trotzdem waren (E) und (C) üppiger als pallida und selbst als tiefgrüne Sippen es gewöhnlich sind ${ }^{1}$ ). Soll man daraus schließen, daß normale, grüne Pflanzen stets ein Übermaß von Chlorophyll haben? Oder soll man annehmen, daB die gelben Farbstoffe dieser „aurea"-Pflanzen $\mathrm{CO}_{2} \mathrm{zu}$ assimilieren vermögen? Die zweite Vermutung gewinnt an Wahrscheinlichkeit, wenn man mit mir der Ansicht ist, daß die gelben Pigmente in diesem Falle Zersetzungsprodukte des Chloro. phylls sind.

Obschon (D) als junge Pflanze grüner war als (C) und (E), wurde sie in diesem Frühjahr merklich gelber als diese, und Anfang Juni war sie bis auf einige untere Blätter, die grüngelb waren, scheinbar chlorophyllfrei (eines der untersten Blätter war zur Hälfte dunkelgrün). Die Stengel, die bei (C) und (E) blaBgrün waren, waren bei (D) schwach gelb. Bis Mitte Juni wuchs (D) fast so schnell wie (C) und (E), aber dann hörte sie auf weiter zu wachsen, und ein Blatt nach dem anderen begann schwarz zu werden und einzuschrumpfen, wohl infolge der stärkeren Insolation. Einige Wochen später war die ganze Pflanze abgestorben, ohne geblüht zu haben.

Die Pflanze 11351(8) schien anfangs vollkommen normal; dies änderte sich erst, nachdem sie ausgepflanzt worden war. Anfang August war die auffallend große Rosette bedeutend heller als die der normalen Geschwisterpflanzen (Farbenkreisel: 17 pCt. grün, 6 pCt. orangegelb, 77 pCt. schwarz); einige Rosettenblätter waren gelb $(56,5$ pCt. gelb, 19,0 pCt. orangegelb, 24,5 pCt. weiß). Diese Pflanze trieb im ersten Sommer keine Stengel. Sie wurde nicht überwintert.

1) Der bessere Boden, in dem diese Pflanzen wuchsen, mag etwas mit dem üppigen Wachstum zu tun haben; allerdings habe ich auch andere normale Melandrium-Sippen in diesem Boden gezogen, und auch mit diesen verglichen, waren die "aurea"-Pflanzen üppiger. 
Ich habe diese ,aurca"-Pflanzen so ausführlich beschrieben, um die Ungleichheit mit ihrem gelbgefleckten Vater und die Verschiedenheit der ,aurea"-Eigenschaft zu betonen.

Wie schon erwähnt, kamen die wenigen ,aurea"-Pflanzen im Sommer 1912 gar nicht oder nur spät zur Blüte, weshalb ich natürlich nur wenige Kreuzungon ausführen konnte (Tabelle XIV).

Tabelle XIV.

\begin{tabular}{|c|c|c|c|}
\hline Eltern & $\begin{array}{c}\text { Saat } \\
\text { Nr. }\end{array}$ & $\begin{array}{l}\text { Beschaffenheit } \\
\text { der Eltern }\end{array}$ & $\begin{array}{l}\text { Beschaffenheit der } \\
\text { Nachkommen }\end{array}$ \\
\hline $11218(3) \times(2)$ & 12282 & 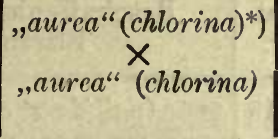 & $\begin{array}{l}23 \text { dunkelgrün, } 46 \text {,aurea } \\
\text { von den letzten waren } 12 \\
\text { hellgelb und anscheinend } \\
\text { chlorophyllfrei }\end{array}$ \\
\hline $11218(3) \times 212(75)$ & $1: 283$ & $\begin{array}{c}\text {,aurea" } \\
\text { chlorina }\end{array}$ & $\begin{array}{c}44 \text { dunkelgrün, } 49 \text {,aurea“" } \\
\text { bis chlorina, meistens sicher } \\
\text {,aurea“" }\end{array}$ \\
\hline $11229(19) \times 218(2)$ & $12 \cdot 293$ & $\stackrel{\text { chlorina }}{\times}{ }_{\text {,aurea }}^{\text {"chlorina })}$ & $\begin{array}{c}24 \text { dunkelgrün, } 46 \text {,aurea“ } \\
\text { bis chlorina, meistens } \\
\text { „aurea“ }\end{array}$ \\
\hline $11218(3) \times 324(34)$ & 12284 & $\begin{array}{c}\text { "aurea" } \\
\times \\
\text { pallida }\end{array}$ & $\begin{array}{l}62 \text { dunkelgrün, } 6 \text { als Keim- } \\
\text { linge undeutlich gescheckt, } \\
\text { später dunkelgrün }\end{array}$ \\
\hline $11324(7) \times 218(2)$ & 12320 & 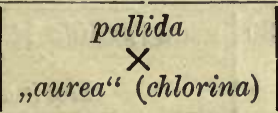 & 56 dunkelgrün, 44 „,aurea“ \\
\hline $11229(19) \times 351(C)$ & 12296 & $\stackrel{\text { chlorina }}{\times}$ & 68 dunkelgrün, 18 „aurea“ \\
\hline $11324(7) \times 351(C)$ & 12324 & $\underset{\substack{\times \\
\text {,aurea“ }}}{\text { pallidallida })}$ & $\begin{array}{l}41 \text { dunkelgrün, } 47 \text {,aurea" } \\
\text { bis pallida, von diesen waren } \\
38 \text { sicher, aurea" }\end{array}$ \\
\hline $11351(\mathrm{E}) \times(\mathrm{C})$ & 12327 & $\begin{array}{c}\text { "aurea" }(\text { pallida }) \\
\times \text { "aurea" (pallida })\end{array}$ & $\begin{array}{l}1 \text { dunkelgrün, } 24 \text { gleichge- } \\
\text { färbt griun, fast so hell wie } \\
\text { pallida, 33 ,, aurea } \\
\text { dieson waren } 10 \text { rein orange- } \\
\text { gelb, die aber mebrere Laub- } \\
\text { blätter erzeugten }\end{array}$ \\
\hline $11351(63) \times(C)$ & 12328 & $\begin{array}{l}\text { grün }(\text { pallida }) \\
\text { "aurea }^{\text {" }}(\text { pallida })\end{array}$ & $\begin{array}{l}40 \text { dunkelgrün, } 21 \text { gelblich } \\
\text { grün (,,aurea"? und pallida), } \\
\text { und } 35 \text { sicher ,aurea" }\end{array}$ \\
\hline
\end{tabular}

*) Die großväterliche Pflanze ist meine ursprüngliche „aurea“-Pflanze“ (10111(54)); die Beschaffenheit der großmütterlichen Pflanze ist jeweils in Klammern hinter ,aurea" zugefügt.

In Tabelle XIV sind nur die Nachkommenschaften zusammengestellt, deren einer Elter bezw. beide „aurea“-Eigenschaft hatten. 
In der folgenden Tabelle sind noch weitere 6 Familien angeführt. Die Eltern hatten keine „aurea“-Färbung, aber als Großvater diente Pollen der "gelben“ Blüten von der ursprünglichen „aurea“-Pflanze (10111(54)). Wenn das „aurea"-Merkmal latent oder rezessiv unter der grünen Farbe des Heterozygoten verborgen wäre, dann müßten diese Kreuzungen ebenfalls „aurea"-Pflanzen liefern; aber wie aus Tabelle XV zu ersehen ist, waren alle 523 Nachkommen rein grün oder pallida, was sich aus anderen Gründen erklärt.

Tabelle XV.

\begin{tabular}{|c|c|c|c|}
\hline Eltern & $\begin{array}{l}\text { Saat } \\
\mathrm{Nr} \text {. }\end{array}$ & $\begin{array}{l}\text { Beschaffenheit der } \\
\text { Eltern }\end{array}$ & $\begin{array}{c}\text { Beschaffenheit der } \\
\text { Nachkommen }\end{array}$ \\
\hline $11322(51) \times 351(30)$ & 12315 & $\begin{array}{c}\left.\text { grün }(\text { pallida } \times \text { chlorina })^{*}\right) \\
\text { grün }(\text { pallida } \times \text {,aurea“ })\end{array}$ & $\begin{array}{l}50 \text { tiefgrün } \\
17 \text { pallida }\end{array}$ \\
\hline $11324(7) \times 351(49)$ & 12325 & $\begin{array}{c}\text { pallida } \\
\times \\
\text { grün }(\text { pallida } \times \text {,aurea“ })\end{array}$ & $\begin{array}{l}38 \text { dunkelgrün } \\
12 \text { ein wenig heller } \\
\text { (unvollkommene } \\
\text { Dominanz?) } \\
47 \text { pallida }\end{array}$ \\
\hline $11351(53) \times(30)$ & 12329 & $\begin{array}{c}\text { grün (pallida } \times \text {,aurea") } \\
\times \text { grün (pallida } \times \text {,aurea") }\end{array}$ & $\begin{array}{l}63 \text { dunkelgrün } \\
4 \text { intermediär } \\
23 \text { pallida }\end{array}$ \\
\hline $11351(53) \times 211(81)$ & 12330 & grün $($ pallida $\times$, ,aurea" $)$ & 97 dunkelgrün \\
\hline $11351(53) \times 321(4)$ & 12331 & $\begin{array}{r}\text { grün (pallida } \times \text {, ,aurea") } \\
\times \text { grün (pallida } \times \text { chlorina })\end{array}$ & $\begin{array}{l}55 \text { dunkelgrün } \\
5 \text { intermediär } \\
30 \text { pallida }\end{array}$ \\
\hline $11351(53) \times 324(34)$ & 12332 & grün (pallida $\underset{\text { pallida }}{\times} \times$,aurea & $\begin{array}{l}42 \text { dunkelgrïn } \\
11 \text { intermediär } \\
29 \text { pallida }\end{array}$ \\
\hline
\end{tabular}

*) Wenn die Beschaffenheit der großelterlichen Pflanzen nicht mit der der Eltern übereinstimmt, so ist sie in Klammern beigefügt.

Aus den zwei letzten Tabellen ersehen wir, daß die „aurea“Eigenschaft scheinbar ,dominiert", und daß sie nur auftritt, wenn wenigstens der eine Elter eine „aurea"-Pflanze war. Die in Tabelle XV angeführten Resultate entsprechen der Erwartung vollkommen, wenn man von der Voraussetzung ausgeht, daß die große Mehrzahl der Spermazellen der chlorophyllfreien Blüten der ursprünglichen „aurea“-Pflanze (10111(54)) mit allen Genen (XZYN) ausgestattet waren, die zur Hervorbringung von tiefgrüner Farbe notwendig sind, daß sie aber nichts, was das "dominierende" "aurea"-Merkmal erzeugt, führten. 
Was nun das Aussehen der mehr als $250 \mathrm{~F}_{2^{-}}$, aurea"-Pflanzen betrifft (Tabelle XIV), so sei nur kurz gesagt, daß diese in bezug auf die „aurea“-Eigenschaft sehr stark variierten, wie dies ja aus der oben geschilderten Verschiedenheit der vorhergehenden Generation nicht anders zu erwarten war. Nur zwei Individuen (12296(45)) und (12328(32)) hatten ungefähr die gleiche, eigentümliche Scheckung ihres Großvaters, - also isolierte gelbe Flecken auf mehr oder weniger tiefgrünem Grunde. Im folgenden will ich einige der auffallendsten „aurea"-Variationen kurz beschreiben. Eine Klassifizierung der Pflanzen in verschiedene „aurea"-Typen war vollkommen ausgeschlossen.

Über die grünlichgelben Keimlinge, die ohne Blätter zu bilden abstarben, ist nichts weiter zu sagen. Außer diesen waren die meisten „aurea"-Sämlinge gelbgrün bis grüngelb, häufig einfarbig; aber wohl noch häufiger gescheckt (grüne Flecken und Streifen auf gelbem oder orangegelbem Grund) (Tafel XXIII, C). Zum Teil waren die ausgewachsenen Pflanzen einheitlich blaßgrün, gelbgrün oder orangegelb, zum Teil waren sie gescheckt. Im letzteren Falle waren tiefgrüne Flecken und unregelmäßige Streifen auf rein orangegelbem, gelblichem oder hellgrünem Grunde unregelmäßig zerstreut; während bei den meisten Pflanzen die Flecken anscheinend direkt unter der Epidermis lagen und infolgedessen intensiv grün waren, zeigte eine einzige Pflanze verschwommene, grüne Flecken. Ich nehme an, daß in diesem Falle das grüne Gewebe zwischen gelbweißen Zellschichten eingebettet lag. Die Marmorierung der Blätter war ebenfalls sehr verschieden. Ich will nur einige anführen: Längliche, grüne Flecken mitten auf der Spreite; verschieden intensiv gelbgefärbte Blatthälften; verschieden stark gelb geränderte, sattgrüne Blätter usw. Des öfteren zeigte eine Pflanze auf verschiedenen Zweigen verschiedene Marmorierung. (Tafel XXIII, D). Zuweilen sah dann wohl eine derartige Pflanze wie eine Sektorialchimäre aus, aber die Grenzen waren niemals scharf gezogen.

Was ist nun diese „aurea"-Eigenschaft? Diese Frage kann ich noch nicht mit Sicherheit beantworten, aber ich halte es für nicht unwahrscheinlich, daß wir es hier mit einem Fall von infelktiöser Chlorose zu tun haben. Wenn sich meine Vermutung als richtig erweisen sollte, dann wäre diese Chlorose von den uns bekannten im Prinzip insofern verschieden als in diesem Falle, die Buntblättrigkeit sowohl durch die Eizellen als auch durch die Spermazellen auf einen Teil der Deszendenz übertragbar ist. Die Nachkommen von chlorotischen Individuen von Abutilon und 
anderen Malvaceen, sowie von Ligustrum, sind immer rein grün $\left.(\mathrm{BAUR})^{1}\right)$. Wenn man auf eine für Chlorose empfindliche, grüne Unterlage einen chlorotischen Ast pfropft, so wird die ganze Pflanze infiziert. Dies gilt natürlich auch für die umgekehrte Pfropfung.

Leider habe ich mit der ,aurea"-Sippe von Melandrium noch keine Pfropfungsversuche ausgeführt. Mehrere Versuche, die wachsenden Teile grüner Individuen mit dem ausgepreßten Saft einer „aurea"-Pflanze zu infizieren, blieben erfolglos. Die Blätter, die nach der Einspritzung gebildet worden waren, waren rein grün. Ich habe folgende Gründe, um trotzdem an eine Infektionserscheinung zu glauben:

1. Die runden Flecken der ursprünglichen „aurea"-Pflanze sind in ihrer Mitte rein gelb, und nach dem Rande zu werden sie immer grüner, so daß ein allmählicher Übergang von dem gelben zu dem normalgrünen Gewebe stattfindet. Dies spricht für ein "Virus" oder dergleichen, das sich von einer Zelle aus exzentrisch ausbreitet. Vielleicht können nur junge Zellen vergiftet werden, da sich die Flecken in einer ausgewachsenen Pflanze nicht vergrößern.

2. Die vielen verschiedenen Formen, in denen wir der „aurea"-Farbe nicht nur bei verschiedenen Pflanzen, sondern häufig auch bei ein und derselben Pflanze begegnen, ist ebenfalls mit der Annahme einer Infektion leichter zu erklären.

3. Die für die ,aurea"-Sippe charakteristischen gelben und braunen Farbstoffe sind aller Wahrscheinlichkeit nach Zersetzungsprodukte des Chlorophylls und keine selbständigen Pigmente.

Wenn aber die „aurea"-Eigenschaft keine Infektionserscheinung sein sollte, sondern richtig vererbt wird, dann würde die Vererbungsweise noch am ersten an BAURs weißrandiges Pelargonium zonale erinnern. In beiden Fällen geben Kreuzungen $\mathrm{zwischen}$ normalen und anormalen Blüten einen Teil rein grüne und einen Teil marmorierte Nachkommen. Von Pelargonium waren alle Nachkommen „rein weiß“, wenn beide elterlichen Keimzellen aus rein weißem Gewebe entstanden waren; bei Melandrium dagegen war die Deszendenz nur zum Teil chlorophyllfrei, wenn beide Keimzellen

1) Zur Ätiologie der infektiösen Panaschierung. Ber. d. Deutsch. Bot. Gesell., 22: S. 453-460, 1904.

Über die infektiöse Chlorose der Malvaceen. Sitz.-Ber. Kgl. Preuß. Akad. d. Wiss., 1: S. 11-29, 1906.

Über infektiöse Chlorosen bei Ligustrum, Laburnum, Fraxinus, Sorbus und Ptelea. Ber. d. Deutsch. Bot. Gesell., 25: S. 410-413, 1907. 
von „aurea“-farbenen Blüten stammten; sie waren niemals chloralbinotisch, wenn nur der eine Elter gescheckt war.

Ich glaube, daß wir es bei den grün-weißen Chimären und bei den Marmorierungen von chlorinomaculata- und "aurea"-Sippen nur mit pathologischen Erscheinungen zu tun haben, und daß es sich in diesen Fällen um gar keine wirkliche Vererbung handelt; allerdings erschwert diese Annahme die Definition des Begriffs "Vererbung“. Vorerst wissen wir auch noch nicht genau was ,pathologisch“ und was „,normal“" ist.

\section{Zus ammenfassung.}

Die Anwesenheit der zuerst von BAUR nachgewiesenen Erbeinheit Z, die für "Chlorophyllbildung überhaupt" notwendig ist wird hier völlig bestätigt. Ohne diesen Faktor ist die Pflanze gïnzlich chlorophyllfrei und muß als Keimling verhungern.

Die chloralbinotischen Formen von $M$. album sind fast rein weiß, während die von anderen Sippen mehr oder weniger intensiv gelb sind. Ferner sind die chlorophyllfreien Teile der Sektorialund Periklinalchimären von $M$. album weißlich, während die entsprechenden Teile der $\mathrm{F}_{1}$ Bastarde $(\boldsymbol{M}$. album $\times \boldsymbol{M}$. rubrum und rezipruk) gelb sind. Diese Tatsichen sprechen gegen BAURs Vermutung, daß die gelben Blattpigmente von dem Gen Z hervorgebracht werden. Vielmehr werden die gelben Farbstoffe unabhängig von $\mathrm{Z}$ gebildet. In nurmalen Blättern sind sie durch das Chlorophyll verdeckt, bei dessen Vurminderung sie erst zum Vorschein kommen.

Zwei verschiedene, blaßgrüne Sippen von Melandrium "chiorina" und "pallida“ - sind beide rezessiv gegen die dunkelgrünen „typica“-Sippen. Kreuzungen zwischen chlorina und pallida geben eine einheitlich dunkelgrüne $\mathrm{F}_{1}$-Generation. In $\mathrm{F}_{2}$ erhält man eine Aufspaltung von ungefähr 9 dunkelgrün zu 7 hellgrün. Unter den hellgrünen Pflanzen kann man deutlich pallida- und chlorina-Individuen erkennen. Daraus folgt, daß die normale Chlorophyllmenge außer von Z von noch mindestens zwei weiteren Faktoren abhängt, von denen die chlorina-Sippe den einen und die pallida Sippe den anderen führt. Für chlorina haben wir dann die Erbformel XXZZYYnn, für pallida XXZZy yNN und für typica XXZZYYNN anzunehmen.

In dieser Arbeit werden ferner drei verschiedene, nichtmendelnde Fälle von Buntblättrigkeit beschrieben, und es wird ein vorläufiger Bericht über ihre Vererbung gegeben. Es handelt sich um folgende drei Sippen: 
1. Grün-wuiße Chimären. Die Chimären sind aus einem dunkelgrünen und einem chlorophyllfreien Teil zusammengesetzt, nur die Mutterpflanze hat einen Einfluß auf die Nachkommen, der merkwürdig ist. Die Samen von chlorophyllfreien Ästen geben chlorophyllfreie Sämlinge; die Beschaffenheit des Vaters bleibt ohne Einflulb. Von Blüten an grünen Ästen erhält man nur grüne Sämlinge.

2. Chlorinomaculata. Die Pflanzen sind grün und chlorina. farben marmoriert, die Grenze zwischen den grünen und chlorinaTeilen ist nicht scharf gezogen; sie sind also keine Chimären. Ich habe bisher nur die Nachkommenschaft von einer weiblichen chlorinomaculata-Pflanze ziehen können und erhielt folgende Resultate: Die Deszendenz von Blüten an marmorierten Stengeln war zusammengesetzt aus grünen, marmorierten und chlorophyllfreien Pflanzen. Die Blüten an grünen Zweigen gaben nur grüne, die an chlorina-farbenen Zweigen nur chlorophyllfreie Sämlinge, die nicht lebensfähig waren. Ob diese Eigenschaft durch den Vater vererbt wird, konnte noch nicht geprüft werden.

3. Aurea. Die Pflanzen waren auffallend kräftig, obwohl sie nur gelbgrün, gelb oder orangegelb waren. Sie wiesen häufig eine sehr ejgenartige Marmorierung auf. Sie stammten alle von einer dunkelgrünen, männlichen Pflanze ab, die mehrere kleine, rundliche, gelbe Flecken auf den Blättern hatte. Ich vermute, daß es sich hier um einen neuen allerdings in bezug auf Vererbung prinzipiell verschiedenen Fall von infektiöser Chlorose handelt. Während die Chlorose von Abutilon und anderen Malvaceen usw. weder durch die Eizellen noch durch die Spermazellen vererbt wird, wird diese "aurea"-Eigenschaft durch beide Keimzellen auf einen Teil der Nachkommenschaft übertragen.

Für die Liebenswürdigkeit, mit der Herr RICHARD FREUDENBERG sich um die Verbesserung des Manuskripts und die Korrekturen bemüht hat, spreche ich ihm meinen herzlichsten Dank aus.

Station for:Experimental Evolution, of the Carnegie Institution, Cold Spring Harbor, Long Island, N. Y.

\section{Anhang.}

Herr Prof. Dr. MARCHLEWSKI hatte sich in freundlichster Weise erboten, Blätter meiner verschiedenen Melandrium-Sippen auf ihren Gehalt an Neo- und Allochlorophyll zu prüfen. Ich schickte ihm Blätter, die sorgfältig im Vakuum über $\mathrm{KOH}$ getrocknet 
worden waren. Vor einigen Tagen teilte or mir die Resultate mit, die ich weiter unten vollständig wiedergebe. Die Resultate überraschten mich sehr; ich hatte es näınlich für möglich gehalten, daß z. B. das Chlorophyll der chlorina-Sippe chemisch verschieden von dem Chlorophyll der pallida-Sippe oder dem der normal grünen Sippen sei; oder aber, daß wenigstens in den verschiedenen Rassen ein Unterschied im Verhältnis von Allo- zu Neochlorophyll bestände. MARCHLEWSKIs Resultate sprechen aber ganz deutlich gegen eine solche Vermutung; denn sie zeigen, daß das Verhältnis von Allo- zu Neochlorophyll in allen untersuchten MelandriumBlättern ziemlich konstant ist. Wir haben also wohl anzunehmen, daß die Verschiedenheit der Blattfarbe lediglich durch die Quan. tität und nicht durch die Qualität des Chlorophylls verursacht wird. Herr Prof. Dr. MARCHLEWSKI hat die Methode von JACOBSON und MARCHLEWSKI benutzt; er gibt den Versuchsfehler auf etwa \pm 2 pCt. an.

\begin{tabular}{|c|c|c|c|}
\hline \multirow{2}{*}{$\begin{array}{l}\text { Stammbuch- } \\
\text { Nummern }\end{array}$} & \multirow{2}{*}{ Beschaffenheit der Pflanze } & \multicolumn{2}{|c|}{ Prozent-Gehalt von } \\
\hline & & $\begin{array}{c}\text { Neo- } \\
\text { chlorophyll }\end{array}$ & $\begin{array}{l}\text { Allo- } \\
\text { chlorophyll }\end{array}$ \\
\hline $12249(35)$ & M. album & 72 & 28 \\
\hline $12263(43)$ & M. album $\times$ rubrum $\mathrm{F}_{1}$ & 72 & 28 \\
\hline $12337(6)$ & CSH-Sippe & 70 & 30 \\
\hline $12290(31)$ & chlorina & 74 & 26 \\
\hline $12317(2)$ & pallida & 74 & 26 \\
\hline $12319(7)$ & pallida $\times$ chlorina $\mathrm{F}_{1}$ & 68 & 32 \\
\hline $12285(41)$ & pallida $\times$ chlorina $\mathrm{F}_{2}$ (grün) & 68 & 32 \\
\hline $12285(42)$ & pallida $\times$ chlorina $\mathrm{F}_{2}($ chlorina $)$ & 68 & 32 \\
\hline $12285(77)$ & pallida $\times$ chlorina $\mathrm{F}_{\mathbf{2}}($ pallida $)$ & 74 & 26 \\
\hline $12320(58)$ & „aurea" & 70 & 30 \\
\hline
\end{tabular}

\section{Literaturverzeichnis.}

BAUR, E., Zur Aetiologie der infektiösen Panachierung. Ber. d. Deutsch. Bot. Gesell., 22: S. $453-460,1904$.

- Über die infektiöse Chlorose der Malvaceen. Sitz.-Ber. d. Kgl. Preuß. Akad. d. Wiss., 1: S. 11-29, 1906.

- Üher infektiöse Chlorosen bei Ligustrum, Laburnum, Fraxinus, Sorbus und Ptelea. Ber. Deutsch. Bot. Gesell., 25: S. 410-413, 1907.

- Untersuchungen über die Erblichkeitsverhältnisse einer nur in Bastardform lebensfähigen Sippe von Antirrhinum majus. Ber. d. Deutsch. Bot. Gesell., 25: S. 44 4 -454. 1907.

- Das Wesen und die Erblichkeitsverhältnisse der „Varietates albomarginatae Hort." von Pelargonium zonale. Zeitschr. f. ind. Abstamm. u. Vererb. 1: $330-351,1909$.

- Vererbungs- und Bastardierungsversuche mit Antirrhinum. Zeitschr. f. ind Abstamm. u. Vererb. 3: S. 34-98, 1910. 
BAUR, E., Untersuchungen über die Vererbung von Chromatophorenmerkmalen bei Melandrium, Antirrhinum und Aquilegia. Zeitschr. f. ind. Abstamm. u. Vererb. 4: S. 81-102, 1910.

- Einführung in die experimentelle Vererbungslehre. S. 293, 1911. Berlin: Gebr. BORNTRAEGER.

- Vererbungs- und Bastardierungsversuche mit Antirrhinum. II. Faktorenkoppelung. Zeitschr. f. ind. Abstamm. u. Vererb. 6: S. 201-216, 1912. Correns, C., Über Bastardierungsversuche mit Mirabilis-Sippen. Ber. d. Deutsch. Bot. Gesell., 20: S. $594-608,1902$.

- Über die dominierenden Merkmale der Bastarde. Ber, d. Deutsch. Bot Gesell., 21: 133-147, 1903.

- Vererbungsversuche mit blaß(gelb)grünen und buntblättrigen Sippen bei Mirabilis Jalapa, Urtica pilulifera und Lunaria annua. Zeitschr. f. ind. Abstamm. u. Vererb. 1: S. 291-329, 1909.

- Zur Kenntnis der Rolle von Kern und Plasma bei der Vererbung. Zeitschr. f. ind. Abstamm. u. Vererb. 2: S. 331-340, 1909.

- Der Übergang aus dem homozygotischen in einen heterozygotischen Zustand im selben Individuum bei buntblättrigen und gestreiftblühenden Mirabilis-Sippen. Ber. d. Deutsch. Bot. Gesell., 28: S. 418-434, 1910.

EAST, E. M., A Mendelian interpretation of variation that is apparently continuous. Amer. Nat. 44: S. 65-82, 1910.

EAST, E. M., und HAYeS, H. K., Inheritance in maize. Conn. Agr. Exp. Sta. Bull. 167. S. 142, 1911.

EMERSON, R. A., The inheritance of certain forms of chlorophyll reduction in corn leaves. Ann. rep. Nebraska Agr. Exp. Sta. 25: S. 89-105, 1912.

GERNERT, W. B., The analysis of characters in corn and their behavior in transmission. S. 58, 1912. Herausgegeben von dem Verfasser, Champaign, Illinois.

JaCOBSON, C. A., und MARChlEWSKI, L., On the duality of chlorophyll and the variable ratio of the two constituents. Bull. de l'Acad. Sci. de Cracovie, Serie A, S. 28-40. 1912.

JoHANNSEN, W., Elemente der exakten Erblichkeitslehre. I. Auflage pp. VI u. 516, 1909. II. Aufl. pp. XI u. 724, 1913. Jena: GUSTAV FisCHER.

MaRCHLEWSKI, L., Die Chemie der Chlorophylle und ihre Beziehung zur Chemie des Blutfarbstoffs. S. 187, 1909, Braunschweig, FRIEDRICH VIEWEG \& SOHN.

NILSSON-EHLE, H., Kreuzungsuntersuchungen an Hafer und Weizen. LUNDs Universitets Årsskrift, N. F., Afd. 2, Bd. 5, Nr. 2, S. 122, 1909.

- Einige Beobachtungen über erbliche Variationen der Chlorophylleigenschaft bei den Getreidearten. Zeitschr. f. ind. Abstamm. u. Vererb. 9: S. $289-300,1913$.

PRICE, H. L., und DRINKARD, A. W., Jr., Inheritance in tomato hybrids. Virginia Agr. Exp. Sta. Bull. Nr. 177, S. 17-53, 1908.

SORBY, H. C., On comparative vegetable chromatology. Proc. Roy. Soc. London, 21: S. 442-483, 1873.

STOKES, G. G., On the supposed identity of biliverdin with chlorophyll, with remarks on the constitution of chlorophyll. Proc. Roy. Soc. London, 13: S. 144,1864 . 


\section{Erklärung der Doppeltafel XXIII.}

A. Grün-weiße Chimäre (Nr. 11311(68)) von Melandrium album $\times$ rubrum $\mathrm{F}_{1}$. Das chlorophyllfreie Gewebe ist gelb.

B. Chlorinomaculata-Pflanze Nr. 12270(9). Die hellen Streifen und Flecken sind chlorinafarben.

C. Grün und "aurea" Nachkommen einer pallida-Mutter und eines ,aurea"-Vaters (Saat Nr. 12320).

D. „Aurea-Pflanze mit verschieden marmorierten Blättern. 


Berichte d. Deutsch. Bot. Gesellsch. Bd. XXXI.

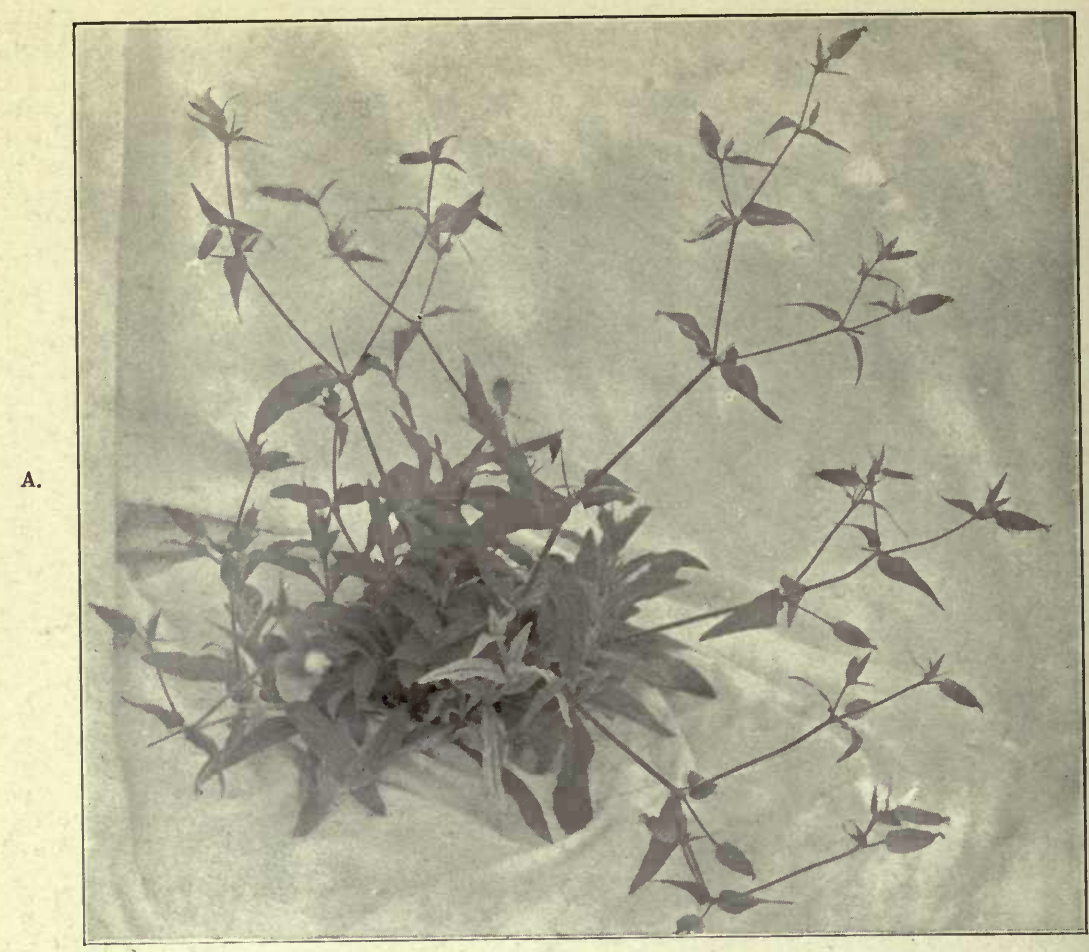

c.

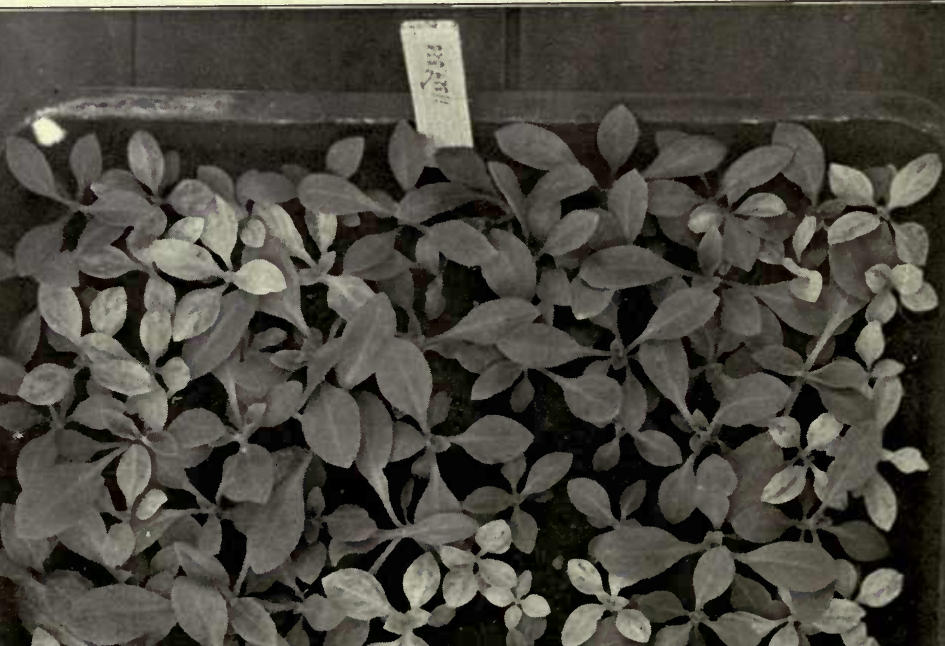

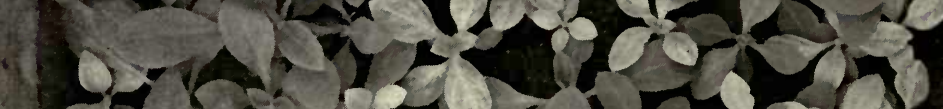

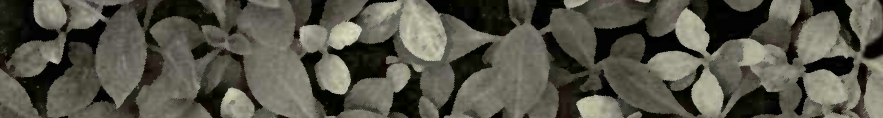
(1) 


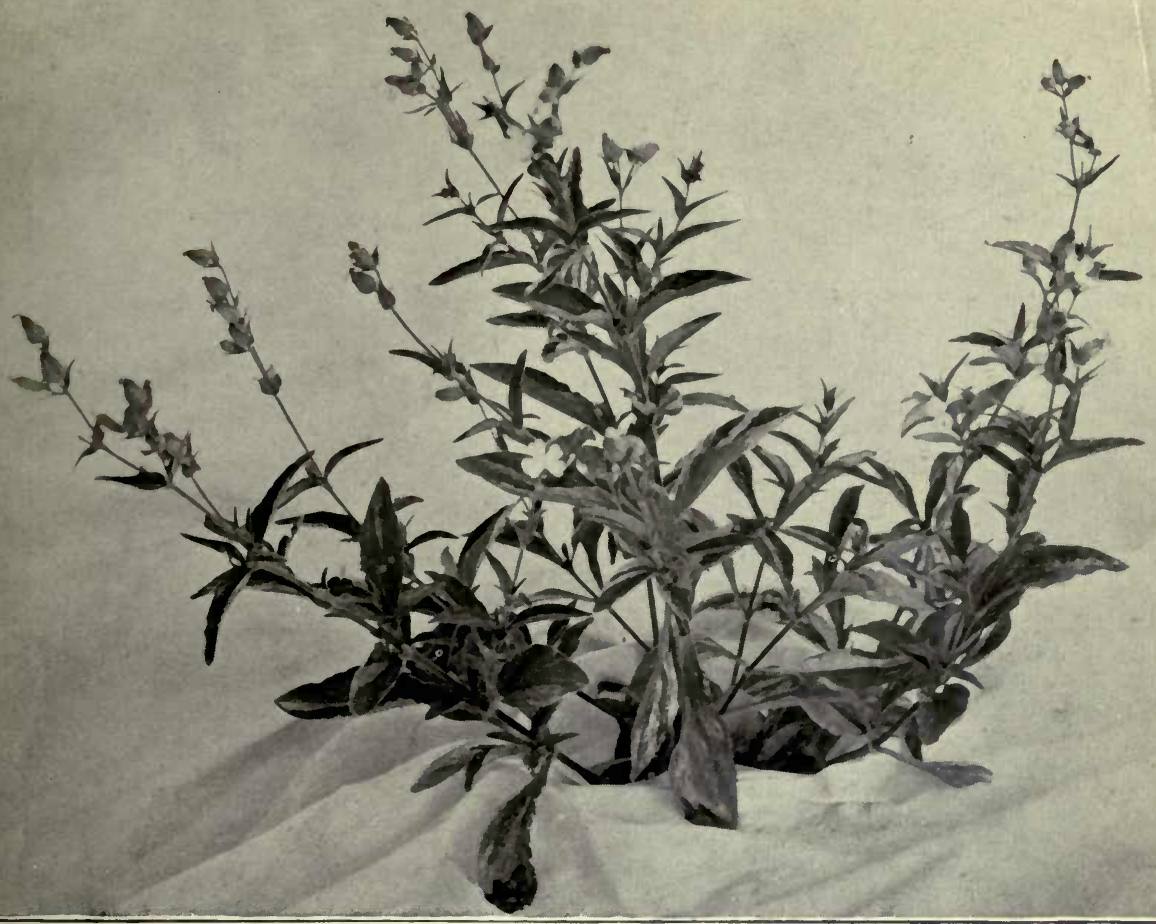

D.

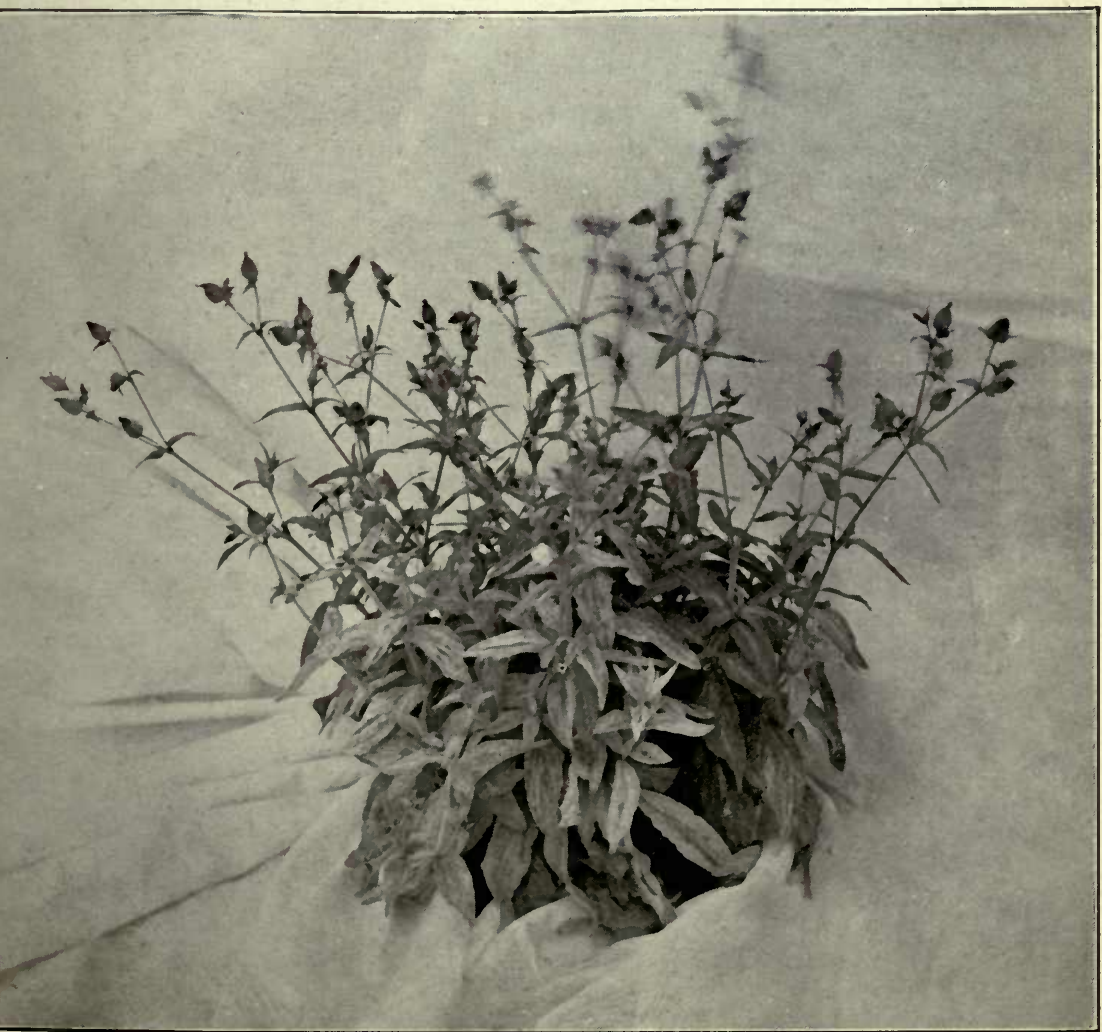

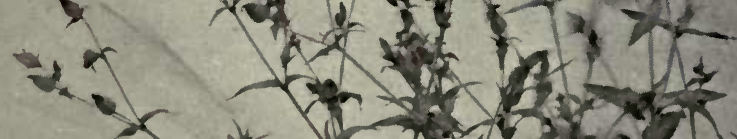

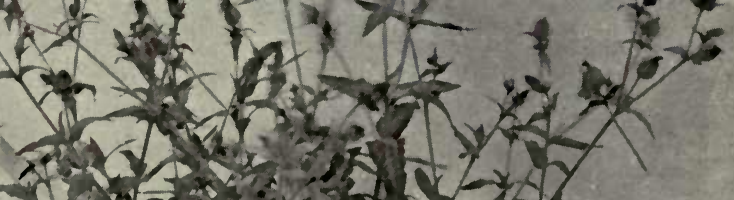
$1+2 x<y$ 



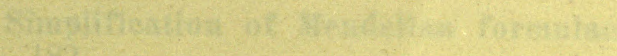

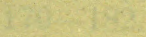





\section{"Zeitschrift für induktive Abstammungs- und Vererbungslehre" Band XII Heft 21914}

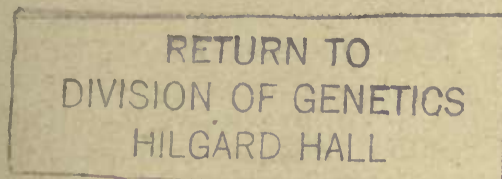

\section{Referate.}
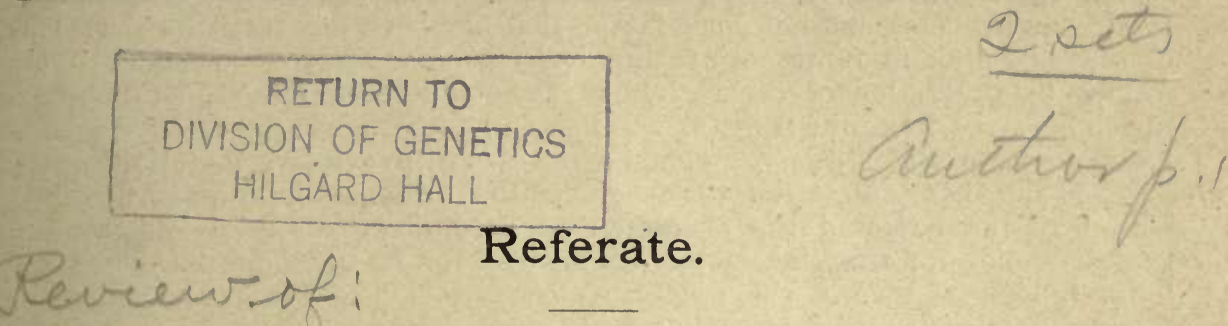

Morgan, T. H. Factors and unit characters in Mendelian heredity. Amer. Nat. 47 1913. S. 5-16.

Castle, W. E. Simplification of Mendelian formulae. Amer. Nat. 47 1913. S. $170-182$.

Morgan, T. I. Simplicity versus adequacy in Mendelian formulae. Amer. Nat. 47 1913. S. $372-374$.

Emerson, R. A. Simplified Mendelian formulae. Amer. Nat. 471913. S. $307-311$.

These papers contain suggestions and criticisms regarding changes in the present system of recording Mendelian formulae. Morgan points out that although genetists have repeatedly repudiated any intention of regarding a unit character as the product of a single unit factor, confusion between the two terms still persists. In Mendel's original work no such trouble existed because Mendel did not meet with a complex case of inheritance. $\mathrm{He}$ designated the gametic representatives of segregating characters by capitals and small letters, but his a was as much a reality as his A. When Bates on found that rose comb and pea comb were both dominant to single comb in fowls, however, the system broke down and caused the elaboration of the presence and absence hypothesis. The letter $\mathbf{p}$ henceforth meant simply the absence of a germinal unit $\mathbf{P}$, and the letter $\mathbf{r}$ the absence of a germinal unit R. The fact that $\mathbf{P}$ and $\mathbf{R}$ together brought about the production of the walnut comb was not in the least disturbing.

Morgan finds, however, that even the system of Bateson is confusing when dealing with complexities such as he has found in the eye color of Drosophila ampelophila. For example, certain eye colors have been represented as follows:

$$
\begin{array}{llll}
\text { P V } & \text { O } & \text { red } \\
p & \text { V } & \text { v } & \text { vermilion } \\
\text { P } & \text { O } & \text { pink } \\
\text { p } & \text { v } & \text { O } & \text { orange }
\end{array}
$$

Now the presence and absence hypothesis, he says, implies that something is lost from the original germplasm PVo when the vermilion $p$ VO arises. "The vermilion color is supposed to be the product of what is left when this something (called P) is lost. It is not supposed on this hypothesis that the vermilion factor alone is responsible for the vermilion color, for it is hypothetically only a part of what is left when something (P) is lost. Yet it is the identification of the vermilion factor with the vermilion eyecolor that the opponents of Mendelism seem anxious to impute to the Mendelians." 
In this series the pink eye was assumed to be the result of all that is left when $\mathbf{V}$ is lost, and not the result of the activity of the factor $\mathbf{P}$. On the other hand, the letter $\mathbf{O}$ was on a different basis from the letters $\mathbf{P}$ and $\mathbf{V}$. It stood for a color and not for a factor that had been lost from the germcells of the wild fly. In representing thus by $\mathbf{0}$ a color which of course is really formed by the action of other residual factors besides $\mathbf{0}$, considerable confusion occurred when the $\mathbf{0}$ factor was lost and an eosin eye appeared. Furthermore the case was still more complicated when progressive mutations arose.

Morgan's suggestion to overcome the difficulty follows. An abbreviation of the name of the character, as heretofore, stands as its symbol; thus $\mathbf{P}$ stands for the pink factor and small $\mathbf{p}$ for its allelomorph. The large letter represents merely the dominant character. The eye color series for Drosophila then becomes:

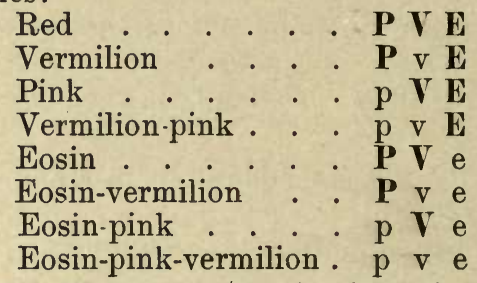

Castle agrees with Morgan that simplification of Mendelian formulae is needed, but he says that Morgan's suggestion to allow the absence of a factor to stand for a character,-i.e. the factor $\mathbf{P}$ being present only in animals that are not pink eyed,-- "is confusion worse confounded". Further Castle states that investigators using the presence and absence notation have made the system ridiculous. They start out, he says, as if small a stands for nothing, then they make it stand for something. They couple $\mathbf{a}$ und b. He asks, "How can nothing be inseparably bound up with nothing"? All of which shows that Castle uses his factors as realities and not as a notation. Castle's suggestion is to abandon the dual terminology. A small letter is used to designate a variation which is recessive in crosses with the normal, and a capital letter for a variation that is dominant in crosses with the normal.

This would give the following formulae for the Drosophila eye colors.

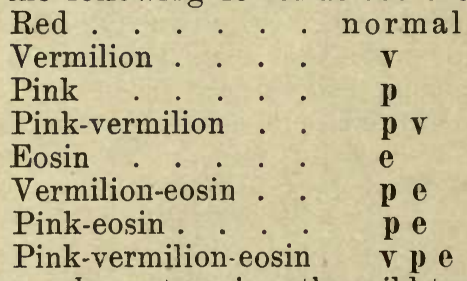

Applying the same scheme to mice, the wild type and the seven mutations in hair color that have occurred would be designated as follows:

$$
\begin{array}{ll}
\text { 1. } & \text { Wild }=\text { gray } \\
\text { 2. } & \mathbf{a}=\text { albino } \\
\text { 3. } & \mathbf{b}=\text { black } \\
\text { 4. } & \mathbf{c}=\text { cinnamon } \\
\text { 5. } & \mathbf{d}=\text { dilute } \\
6 . & \mathbf{p}=\text { pink-eyed } \\
\text { 7. } & \mathbf{s}=\text { spotted } \\
\text { 8. } & \mathbf{y}=\text { yellow }
\end{array}
$$


In Morgan's second paper he admits the simplicity of Castle's suggestions, but says they are not adequate. His criticism is that without the dual system it is difficult to represent heterozygous forms and practically impossible to show gametic coupling.

Emerson, in a very fair criticism of these three papers, shows that the reforms suggested will not fit other Mendelian work. For example, he says, "Who can point out the normal color of a bean?" Emerson believes, with justice, that no simplified terminology should be adopted unless it will express all of the current Mendelian formulae. Further, he thinks that there is no great difficultiy in understandig the present terminology or in teaching it to students. He also emphasizes the fact that in cases where "whites" exist with a number of different gametic formulae, as in the seeds of maize, neither Morgan's nor Castle's scheme is simple or adequate.

The trouble seems to be, as Emerson shows, that though both Morgan and $\mathrm{C}$ astle seem to see the difficulty, they both attempt to force a letter to represent a character, rather than a factor in a system of notation that is concerned with other factors in the development of a character.

E. M. East.

Morgan, T. H. Heredity and sex. pp. 282, figs. 121, 1913. New York: Columbia University Press.

The Jesup lectures of the Columbia University, for 1912-13, give a comprehensive view of the results of modern work on the inheritance of sex. The eight lectures are published essentially as delivered, though only a few of the splendid lantern-slides with which they were illustrated could be included, and some of these have suffered considerably in the reproduction. The lectures were addressed to a general biological audience and are intended to give the general student of biology a fair notion of the work which is being done in this special field, rather than to provide a handbook for the specialist. For simplicity and clarity of statement, the work is a model.

The treatment is almost wholly zoological and references to the sexproblems in plants are made only when they have obvious bearing on those of animals. The more or less incidental sex-relations among animals are discussed at some length. The chief disadvantages of this strong zoological orientation are seen in the implication that certain phenomena are fundamental which are, in fact, only incidental; thus in the second chapter, on the mechanism of sex-determination, as in much of the modern zoological literature, undue emphasis is laid upon the significance of the polar bodies. Under ordinary circumstances, the peculiarities of the polar bodies have nothing whatever to do with the mechanism of sex-determination. The naive statement that "the egg deliberately, as it were, twice throws away its valuable heritage" places the emphasis on a wholly irrelevant fact. The reduction of chromosomes, which is the one important feature in connection with the formation of the polar bodies, takes place in the formation of the sperms, where no chromosomes degenerate, quite as well as in the maturation of eggs; and in the eggs of plants the cells which correspond to the polar bodies of animals, apparently differ in no other way from the successful egg-cells, except that they usually occupy a relatively more superficial position. In plants also, they quickly degenerate and disappear, leaving to the single remaining egg the capacity for further development and the production of a new individual, but this process probably relates, in plants as in animals, 
only to the advantages of giving to a single offspring the nutritional and spatial relations that would otherwise be divided among several. Another irrelevant feature which is discussed at some length, is the early isolation of the germ-cells. In plants no such early isolation occurs although it is always theoretically possible of course, to trace a direct cell-lineage from one generation of germ-cells to the next.

The third lecture develops the Mendelian principles of heredity in the briefest possible manner and then proceeds to the application of these principles to the determination of sex as illustrated by sex-limited characters. The author attempts to substitute the term "sex-linked" for the older and more generally used term "sex-limited", and applies the latter term to secondary sexual characters, but to the reviewer this does not seem a desirable change. As nearly all cases to which the term "sex-limited" have been applied are of the kind which Morgan calls "sex-linked" it would be a more desirable reform to simply drop the usage of "sex-limited" in referring to secondary sexual characters.

The fourth, fifth and sixth lectures deal mainly with secondary sexual relations; these include secondary sexual characters, sexual selection, the effects of castration and the transplantation of ovaries, gynandromorphism, hermaphroditism and parthenogenesis. About two pages of these chapters are devoted to hermaphroditism in plants. Here the terms "hermaphroditic" and "sporophytic" are used as if they were synonymous, and at another place "sexual" and "hermaphroditic" are used as antonyms, but hermaphrodites are sexual, too, and sporophytes may be either hermaphroditic or dioeciou: The classic case of Bryonia, in which crosses between a hermaphroditic and a dioecious species led Correns to the first clear statement of the Mendelian interpretation of sex-inheritance which is now generally accepted, was worthy of more than a single short paragraph closed with the statement that it is difficult to bring this case into harmony with other theories of sex-cletermination. The author's difficulty here seems to be that he limits himself to a single genotypic formulation for the sexes in those cases in which the female is homozygous; he assumes that the female is FF, and the heterozygous male Ff. The homozygous female could be FF or $\mathbf{~ m m}$ and the heterozygous male Ff, FM or $\mathbf{M m}$. With the recognition of these available formulae the results in Bryonia may be easily harmonized with other cases of sex-inheritance. The mixture of chromosome-terminology with genotypic formulae tends to confusion. Morgan ${ }^{1}$ ) has shown plausibly that the sexdeterminer is not the X-chromosome but is only a part of it, or associated with it. It is not proper, therefore, to indicate the sex-determiner by $\mathbf{X}$, as he has done in these lectures. It would be much better to substitute $\mathbf{F}$ for this $\mathbf{X}$.

Morgan assumes that all Mendelian determiners lie in the chromosomes, 1 but that they need not remain in the same chromosome. As a working hypothesis his adoption of "chiasmatype" to explain the coupling and repulsion of characters and the breaking of correlations between coupled characters, is proving fruitful, in that it is leading to interesting discoveries regarding the relative frequency of such "cross overs", or broken correlations, in different sets of coupled characters. It seems that two sex-limiterl chapacters carried by the unpaired chromosome of the male are incapable of

1) T. H. Morgan, An attempt to analyze the constitution of the chromosomes on the basis of sex-limited inheritance in Drosophilo. Jour. Expar. Zool. 11, 365 bis $411,1911$. 
exhibiting the phenomenon of "cross-over", presumably for the reason that there is no homologous chromosome into which the character in question may be transferred, while in the corresponding female in which the sexchromosomes are paired, such "cross-overs" or separations of the coupled characters do occasionally occur.

Various problems and hypotheses relating to fertility and sterility are discussed in chapter VII, and the last chapter takes up certain special cases of sex-inheritance, considering in particular, various modifications of the sex-ratios. The author holds that disturbances in the sex-ratios give no proper basis for the formulation of far-reaching conclusions in regard to sex-determination itself. In the final section sex-inheritance in man is considered and it is shown that both cytological and genetic evidence point to the probability that in man the female is homozygous and the male heterozygous.

In public lectures it is impossible to give a large number of references to the literature, but the author has made up this necessary defect by presenting a bibliography including 475 titles which will be valuable to those who. wish to go more deeply into any particular phase of the subject. When bibliographies become so extensive it is not fair to expect that they shall be complete. One important omission noted in the present case is the work of von Winiwarter ${ }^{1}$ ) whose studies on human cytology are prominently presented in the text, but not included in the bibliography.

G. H. Shull.

1) H. von Winiwarter, Etudes sur la spermatogénèse humaine. Arch. de Biol. 27, 1912. 


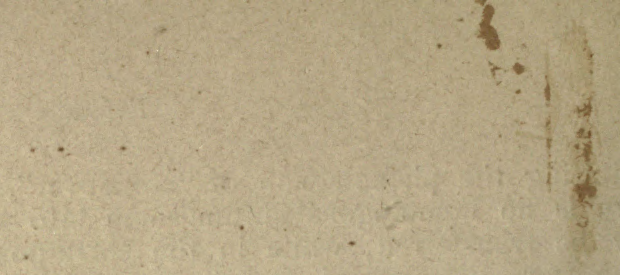

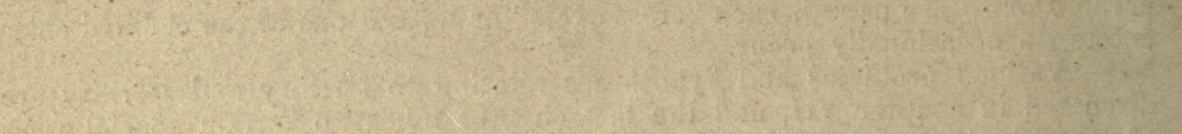

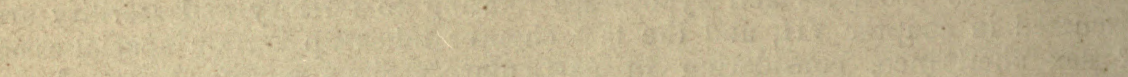

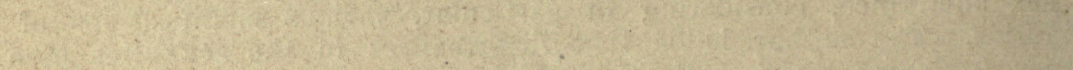

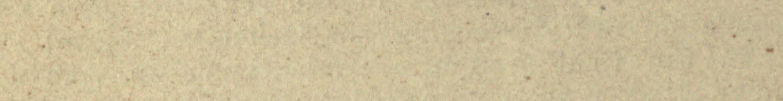




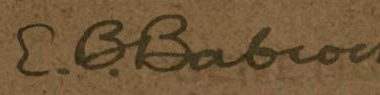

Überreicht von dem Verfass

Sonderabdruck aus der

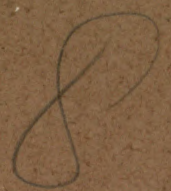

\section{Zeitschrift für induktive Abstammungs-}

\section{und Vererbungslehre}

\section{Bd. XII Heft 5}

Verlag von Gebrüder Borntraeger in Berlin W 35

GEORGE HARRISON SHULL:

Sex-limited inheritance in Lychnis dioica L. 
W. 


\title{
Sex-limited inheritance in Lychnis dioica $\mathrm{L}$.
}

\author{
By George Harrison Shull, \\ Station for Experimental Evolution, Cold Spring Harbor, Long Island.
}

(Eingegangen am 12. März 1914.)

The hereditary distribution of sex-limited characters has been by far the most fruitful source of experimental evidence concerning the inheritance of sex, though crosses between hermaphroditic and dioecious organisms have also given important data supporting the same conclusions. It was in fact a cross of the latter sort between Bryonia alba and $B$. dioica which led CORRENS (1907) to the clear formulation of the genetic relationship between the sexes which has now been generally adopted, the one sex being recognized as homozygous, the other heterozygous, with respect to a sex-determiner which is inherited as a Mendelian gene. The first statement of this hypothesis seems to have been made by GEOFFREY SMITH (1906) as a deduction from the effects of parasitic castration of the crab, Inachus, together with the discoveries of the cytologists that the sexes are in many cases characterized by chromosome differences which lead necessarily to the inference that, in these cases at least, the one sex is homogametic, the other heterogametic. SMrTH laid no special stress upon this hypothesis as a generally applicable Mendelian interpretation of the sexes, and as his statement was not based upon genetic experiments, it has been generally overlooked by geneticists.

The greater value of sex-limited characters for the genetic study of the sexes, is due solely to the fact that such characters are relatively common, while the occurrence of hermaphrodites in sufficiently near relationship to dioecious species that crosses between them yield fertile offspring, is very rare. The fact that the $\mathrm{F}_{1}$ hybrids between Bryonia dioica and $B$. alba are sterile, makes it impossible to go beyond the simple demonstration that when the cross is executed in the one direction a uniform progeny results, while a cross in the opposite direction 
produces a dimorphic progeny. As it is the male of the dioecious species that produces in each case a dimorphic progeny, there can be no reasonable doubt of the correctness of CORRENS's inference that the male is, in Bryonia dioica, the heterozygous sex.

The discovery of hermaphrodite mutants in Lychnis dioica L. which is usually strictly dioecious, has enabled me to verify in this species the hypothesis that the sexes are related to each other as Mendelian homozygotes to heterozygotes. I have shown (SHULL 1910, 1911) that these hermaphrodite mutants result from a modification of the male sex-determiner or its homolog and that this modification can be reversed, so that the male type again appears with great rarity among the offspring of the hermaphrodites. In crosses of either males or "genetic" hermaphrodites upon females, the type of the non-female offspring is determined by the type of the pollen-parent, regardless of the ancestry of the latter; in other words, crosses between females and males produce females and males, while crosses between females and hermaphrodites produce females and hermaphrodites. From the fact that the male can become modified into a functional hermaphrodite, I inferred that the male of this species is heterozygous with respect to the sex-determiner. The crucial tests of this hypothesis were found in self-fertilizations of the hermaphrodites, and in crosses between hermaphrodites and males, for both of these combinations ought to yield mixed progenies if the males and hermaphrodites are heterozygous, and uniform progenies if both are homozygous. The actual results in every case were in accord with my hypothesis, in so far that in each a mixed progeny was produced, but the proportions in which the different sexual types appeared, presented some difficulties which could not be cleared up at that time, and which are still only partially understood. I have some further data bearing on these difficulties and an continuing the search for a complete solution. The new results will be presented in detail in another connection and it need only be stated here, that it has become certain that my earlier inference that the egg-cells of the hermaphrodites are unable to produce hermaphroditic offspring unless fertilized by a hermaphroditebearing sperm, was erroneous, though it appears that the hermaphroditebearing eggs are usually rare. Since such eggs are now known to be produced, even though usually in relatively small numbers, it should be possible, unless selective fertilization or non-viability contravene, to produce homozygous hermaphrodites and so to reconstruct, out of the dioecious race, a pure-breeding hermaphroditic race. Although this goal has not yet been attained, the irregularities in the genetic behavior of 
the eggs in the hermaphrodites constitutes no basis for doubting the heterozygous nature of this bisexual form and of its homologous form, the male.

By the discovery of a unit-character in Lychnis dioica, whose relationship to the sex-characters is the same as that of many sexlimited characters which have been found in animals, it is now possible to completely confirm by a totally new line of evidence, the conclusion that the female in this species is a sex-homozygote, while both the males and the hermaphrodites are sex-heterozygotes, as will be obvious from the data presented below.

This first sex-limited character of the ordinary sort, to be demonstrated in any plant, is the normal width of the leaves. As in the case of any other unit-character, the existence of the gene $B$, which, in conjunction with the rest of the Lychnis dioica genotype, $X X$, produces the broad leaves characteristic of the ordinary strains of this species, could be discovered only when an individual had been found from which this determiner $B$ had disappeared. This important individual was found by BAUR in 1910 in a culture of Melandrium album Garcke (Lychnis dioica L. in part), the parents of which were derived from nature in the vicinity of Sadowa near Berlin, Germany. It is briefly mentioned by BAUR (1911 p. 189) with a photographic illustration in his "Einführung in die experimentelle Vererbungslehre". This plant, which was a male, was crossed by BAUR, with broad-leafed females, and the offspring were all broadleafed, some males and some females. Without knowing that the narrowleafed character of the mutant had any special significance for the sexproblems, Dr. BAuR generously shared with me his hybridized seeds, so that the $\mathrm{F}_{1}$ was grown simultaneously in 1911 in his garden and in mine, and likewise the $\mathrm{F}_{2}$ in 1912. This circumstance gave me the pleasure of an independent discovery of the sex-limited nature of the broad-leaf gene, $B$. BAUR (1912) has published a brief note on his experiences with this form, the further study of which he has, with characteristic generosity, resigned to me.

\section{Description of Lychnis dioica angustifolia.}

From the day on which a seed of this new form germinates it is distinguishable from the broad-leafed type by the longer, narrower cotyledons (fig. 1). The earlier leaves are linear and nearly grass-like, though the surface, color and texture are not markedly different from those of the typical races of Lychnis dioica. In the adult rosette the leaves of 
the narrow-leafed and broad-leafed forms differ little in length, but the rosette-leaves of angustifolia are usually less than half the width of those of the ordinary "typica"-strains. The apexes in angustifolia are longacuminate, and often more or less curled into delicate hooks or loose croziers, the normal form being only sharply acute, as seen in Plate I. The adult leaves of angustifolia are generally of slightly firmer texture than those of the "typica"-races, but a section of a leaf shows no very

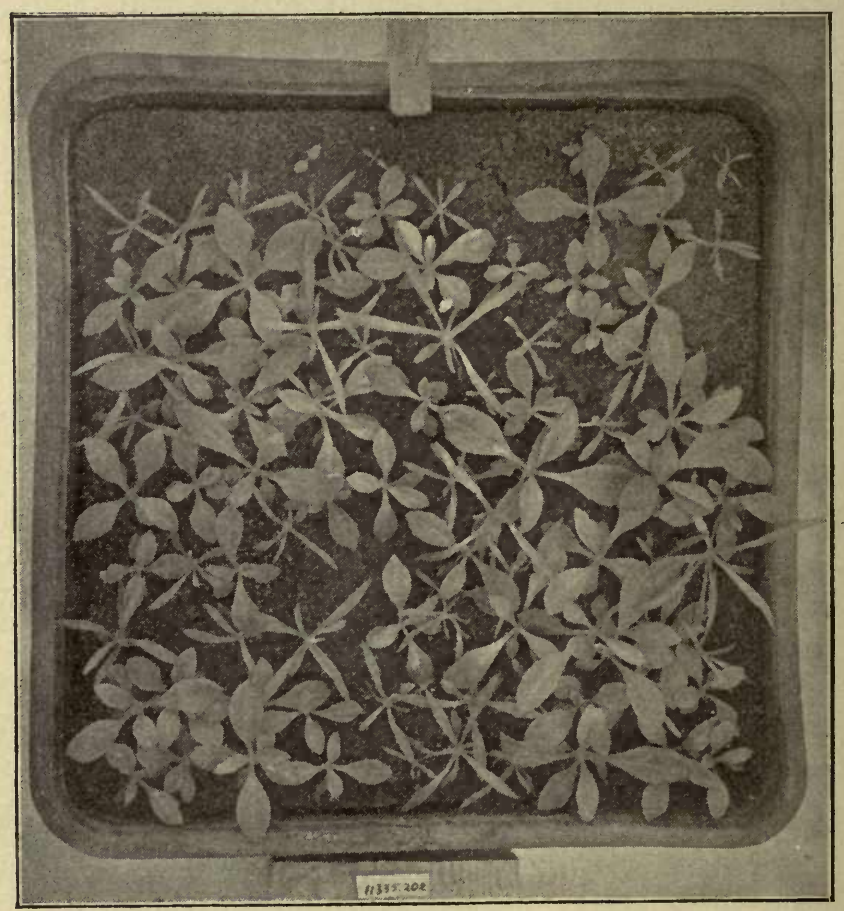

Fig. 1. Seedlings of Lychnis dioica from a cross between a heterozygous female and a narrow-leafed male sib (Ped. No. 12202). Photo Mar. 22, 1913. Oldest plants are 29 days old. The seed-pan is $30 \mathrm{~cm}$ broad.

obvious difference in structure, except that the intercellular spaces among the cells of the mesophyll are smaller in angustifolia, while the cells themselves are not notably different in size, form or arrangement; con sequently the difference in width of the leaves is mainly dependent or a difference in the number of cell-divisions which take place in the transverse direction, while the number of cells in the longitudinal direc tion is not much different from the number in the normal form. 
In the normal or "typica"-forms of Lychnis dioica, the only obvious difference between the rosette-leaves and the stem-leaves, is the rapid disappearance of the petioles of the latter, but in angustifolia the very first stem-leaf is noticeably wider than the rosette-leaves, and this widening is more marked in the successive leaves, until they are of about the same width as the corresponding leaves in the broad-leafed forms, but these broad stem-leaves of angustifolia are still usually distinguishable from the corresponding leaves of the normal plants, because of their long-acuminate apexes (see Plate II).

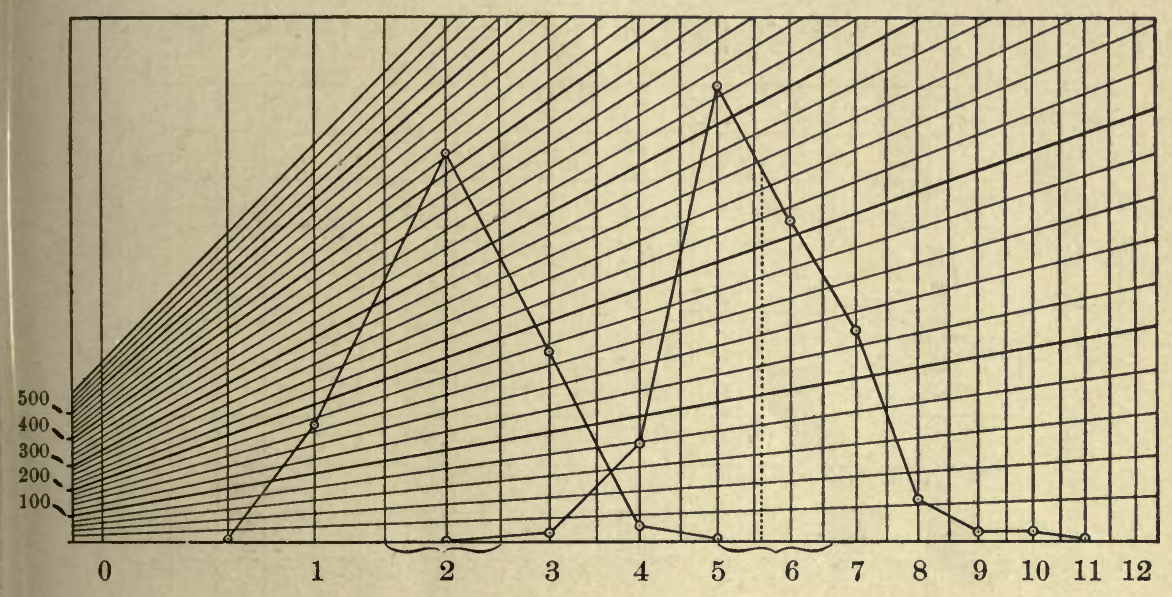

Fig. 2. Curves showing the variation in length of rudimentary ovaries in the males of typical Lychnis dioica (at left) and of $L$. dioica angustifolia belonging to the same families. The curves are so drawn as to represent the relative variability of the two groups of variates. Coefficients of variation: "typica", $30 \cdot 97 \pm 0.57$; angustifolia, 19.08 \pm 0.34 . The ranges of average lengths in the several families are indicated by the braces below the two curves.

The floral characters of angustifolia are variable, as in the "typica"races, so that they can not be taken as definitely diagnostic, but they show certain characteristic tendencies which deserve mention. It will be remembered that the ordinary Lychnis petal has a median cleft of greater or less depth; this cleft is very often absent from the petals of angustifolia. The petals are as a rule smaller and narrower, and in place of the cleft there is frequently a greenish line of virescent tissue, which, in the most marked cases, extends beyond the white part of the petal, forming a spine-like apiculation. The extent to which these virescent lines are developed is characteristic of particular individuals, but whether the differences between different individuals in this regard may be made 
the characteristics of distinct hereditary lines, is not yet known. Over 150 specimens with the virescent lines on the petals were noted in 27 families which together included 664 narrow-leafed individuals, i.e., more than 23 iper cent of the narrow-leafed plants in these families were modified in this way. No attempt was made to be exhaustive in the records of this characteristic, and there is no doubt that a few other individuals in these families showed traces of the same abnormality, but the number recorded will give a fairly accurate idea of the prevalence

Length of bristle in angustifolia males.

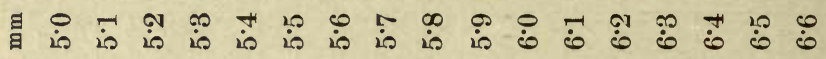

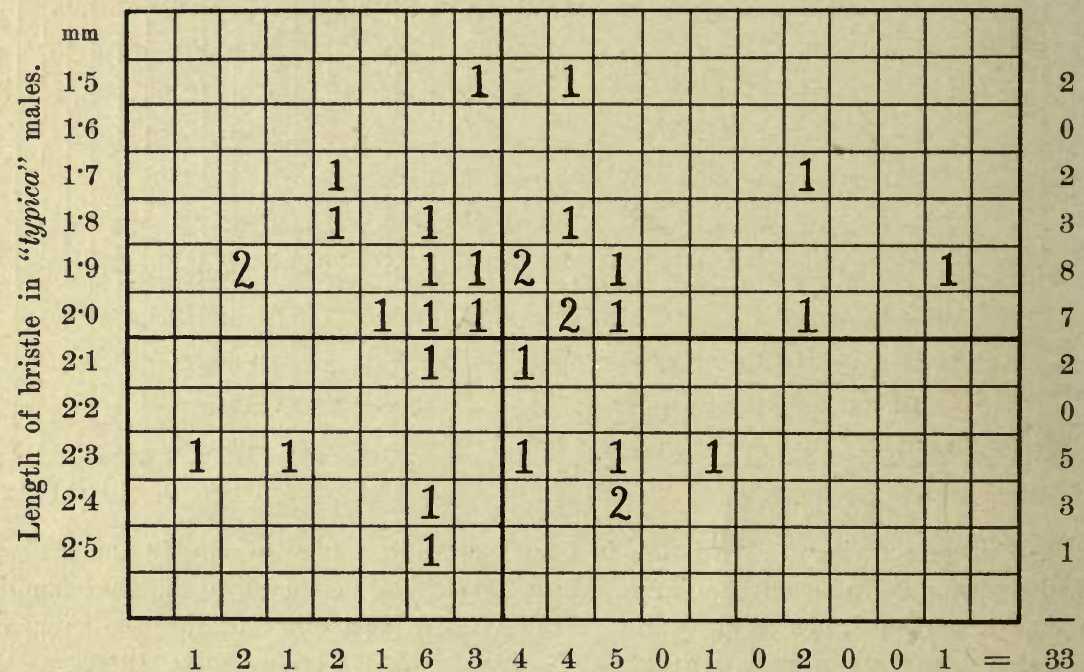

Fig. 3. Correlation-table showing the relations of the average lengths of the rudimentary ovaries in the males of Lychnis dioica angustifolia, to their average lengths in the males of typical Lychnis dioica belonging to the same families. Coefficient of correlation $-0 \cdot 024 \pm 0 \cdot 117$.

of this type of variation in the narrow-leafed plants. I have not yet noticed a similar abnormality in any of my many thousand broadleafed plants.

With regard to the sex-organs there is little that can be said at present, as only males of angustifolia have been available for study. These show no very striking departures from the males of the broadleafed plants, except in the length of the delicate bristle-like rudimentary ovary, which occurs almost without exception, in the center of every male flower of Lychnis dioica. The length of this "bristle" has 
been recorded for all the males, both broad-leafed and narrow-leafed. In Figure 2 the length of bristles in all of the 788 narrow-leafed males that bloomed, and those in a like number of broad-leafed males, taken without selection, from the same families, are presented in the form of variation-curves for comparison, the curves being drawn in such manner as to correct for the changing basis of comparison, so that the difference in variability as well as in the actual and average lengths of the "bristle" appear to the eye with little distortion ${ }^{1}$ ). It is seen that the curves overlap slightly, because a few of the narrow-leafed plants have bristles shorter than the longest bristles which occur in the broad-leafed plants. When the lengths of the bristles in the narrow-leafed plants of each family are averaged and compared with the average lengths of those in the broad-leafed plants of the same family, the difference between the two groups is always striking. The ranges of these averages for the 33 families in which narrow-leafed males appeared, are indicated by the braces at the base of the two curves in Figure 2. Numerically these averages for the narrow-leafed plants are $5 \cdot 0-6 \cdot 6 \mathrm{~mm}$ and for the broad-leafed plants only $1 \cdot 5-2 \cdot 5 \mathrm{~mm}$. It is clear, therefore, that the factor $B$, which increases the width of the leaf, also definitely decreases the length of the rudimentary ovary of the male. Perhaps this increase in the size of the rudimentary ovary in the angustifolia male represents a variation in the direction of hermaphroditism, but there was no other evidence to support such an inference. Of the two hermaphrodite mutants found in these families, one was included among about 800 narrowleafed males, while the other occurred among a little more than 1,000 broad-leafed males.

1) The reader may be interested in the method of construction of this diagram. The theoretical values of maximum frequency, $Y_{0}=\frac{n}{\sigma \sqrt{2 \pi}}$, were calculated for both curves referred to the mean value of the right-hand curve. Vertical lines representing these two maximum frequencies were erected on the base-line at a convenient distance apart, and their summits connected by a line extended to the left until it intersected the base-line. Through the point of intersection radiating lines were drawn, dividing the vertical lines into equal segments. The remaining vertical lines were then erected, dividing the sectors thus formed into equal areas of such magnitude that the distance between the originally erected vertical lines included the number of classes required by the actual calculated difference between the means of the two curves. Had these two curves been drawn in the usual manner on ordinary coordinate paper, the left-hand curve would have been tall and narrow, apparently indicating relatively slight variability, but actual calculation shows the left-hand curve to have a coefficient of variability more than 50 per cent greater than the right-hand curve. I gratefully acknowledge the assistance of my brother, S. P. SHULL, in solving the mathematical problems involved in the method of plotting here used. 
There appears to be no correlation between the average length of "bristles" in the narrow-leafed group of any family and the broad-leafed group of the same family, as shown by Fig. 3, the coefficient of correlation being $-0.024 \pm 0.117$. This indicates that there was probably no other genetic factor affecting differentially the length of the rudimentary ovary in these families.

\section{Genetic studies with Lychnis dioica angustifolia.}

As already indicated, the original narrow-leafed plant appeared singly among more than 100 broad-leafed offspring of a controlled cross between two specimens of Melandrium album secured from nature at Sadowa near Berlin. This plant was a male, and the $\mathrm{F}_{1}$ produced by crossing it with broad-leafed females, consisted entirely of broad-leafed males and broad-leafed females. Dr. BAUR did not make an exact determination of the sex-ratio in this family, but noted that there was a marked excess of males, as he had found generally in his cultures of Melandrium album (BAUR 1912). In my garden the $\mathrm{F}_{1}\left(\right.$ Ped. No. 10244) ${ }^{1}$ ) consisted of 72 males and 8 females. In the $\mathrm{F}_{2}$ grown in 1912, BAUR found among 151 plants, 112 broad-leafed and 39 narrow-leafed, a ratio of $2 \cdot 87: 1$, a sufficiently close agreement with the monohybrid ratio. His total cultures of the $\mathrm{F}_{2}$ contained about 300 plants, a part of which were not exactly counted. Of these, 52 narrow-leafed individuals and 3 broadleafed were set into the garden to complete their development. All of the former were males, and by a peculiar coincidence, since males were normally present in excessive proportion in this strain, all of the three broad-leafed plants were females. In my cultures of the $\mathrm{F}_{2}$ grown at the same time, no selection had been made. Only 50 seeds were sown, and every seed germinated, producing 50 plants, 44 of which were broad-leafed and 6 narrow-leafed. One of the broad-leafed plants failed to bloom, and the rest consisted of 32 females and 11 males. All of the narrow-leafed plants were males. BAUR then made a second sowing from the same lot of seeds and secured 55 broad-leafed and 21 narrow-leafed plants. Owing to the lateness of the season when these were sown, none of the narrow-leafed plants bloomed, but 20 broad-

1) By an oversight in a recent paper (Ber. d. Deutsch. bot. Gesell. 31: Generalversammlungs-Heft, pp. $40-80,1914)$ I have represented this family as the offspring of BAUR's white-margined plant, M. 1. The mother of my 10244 was BAUR's M. 2, which was itself produced from a seed of the white-margined part of M. 1. The father of 10244 , as there correctly indicated, was the narrow-leafed mutant M. 3. 
leafed plants which bloomed consisted of $12 \%$ and $8 \sigma^{7}$, thus showing that in his cultures, as in mine, the $\mathrm{F}_{2}$ progeny consisted of three phenotypes, broad-leafed females, broad-leafed males and narrow-leafed males, and in my cultures, in which there had been no selection, the ratios of these types, $32: 11: 6$, gave as near approach to the expected ratios, $25: 12: 12$, as the smallness of the numbers requires.

To one familiar with the characteristic ratios in sex-limited inheritance, this result in the $\mathrm{F}_{2}$ is at once a sufficient demonstration that the females are sexually homozygous and the males heterozygous, as I had already decided to be the case from my investigations of the genetic relations of the hermaphrodites (SHULL 1910, 1911). The situation may be illustrated schematically by the conventional method, assuming that the female is a positive homozygote, $X X F F^{1}$ ), and the male a heterozygote, $X X F f$, and that the determiner of the typical broad leaves, $B$, is coupled $^{2}$ ) with the sex-gene, $F$. We have then the somatic formula of the normal female $X \widehat{B F} \cdot X \widehat{B F}$ and that of the normal male either $X \widehat{B F} \cdot X \widehat{B f}$ or $X \widehat{B F} . X \widehat{b f}$. The mutation by which the narrow-leafed form was produced, consisted in the disappearance of the gene $B$ from its association with the sex-gene $F$, (and from $f$, the homolog of $F$, in case the normal male has the first of the two mentioned formulae, $X \widehat{B F} \cdot X \widehat{B f})$. As it is easier to comprehend a mutation which results in the loss of a single gene than the simultaneous loss of two genes, we will, for the sake of the present schematic representation, adopt the second formula for the normal male, $X \widehat{B F} . X \widehat{b f}$. Then the narrow-leafed male which had lost the $B$ from its $\widehat{B F}$ gene, will have the formula $X b \widehat{F} . X b \widehat{f}$ and will produce sperms of two kinds, $X \widehat{b F}$ and $X \widehat{b f}$, while the broad-leafed female produces only one sort of egg, $X \widehat{B F}$. The $\mathrm{F}_{1}$ produced by the union of broad-leafed females and narrow-leafed males will consist, therefore, of heterozygous broad-leafed females, $X \widehat{B F}$. $X \widehat{b F}$, and heterozygous broadleafed males, $X \widehat{B F} \cdot X \widehat{b f}$.

1) The " $X X$ " in my formulae represent the unanalyzed rest of the genotype. They can be omitted without in any way affecting the manipulation of the formulae, but it must not be forgotten that they are a part of every Mendelian formula, whether expressed or omitted in the written symbols.

${ }^{2}$ ) It must be understood that this is a figurative expression, as all the known facts will be met quite as well by assuming that the gene $F$ itself determines not only the sex, but also the broad leaves. On the latter assumption, the narrow-leafed condition must result from a modification of the gene $F$, as a result of which it no longer produces such great breadth of leaves as before, though continuing to have the same relation to the determination of sex. 
The $F_{1}$ females being heterozygous for the broad leaf will produce two kinds of eggs, $X \widehat{B F}$ and $X \widehat{b F}$, and the $\mathrm{F}_{1}$ males will produce two kinds of sperms, $X \overparen{B F}$ and $X \widehat{b f}$, thus permitting four combinations when crosses are made among the $\mathrm{F}_{1}$ plants. The $\mathrm{F}_{2}$ zygotes produced from these combinations will be as follows:

$$
\begin{aligned}
& X \widehat{B F} \cdot X \widehat{B F}=\text { homozygous broad-leafed females, } \\
& X \widehat{b F} \cdot X \widehat{B F}=\text { heterozygous broad-leafed females, } \\
& X \widehat{B F} \cdot X \widehat{b f}=\text { heterozygous broad-leafed males, } \\
& X \overrightarrow{b F} \cdot X \overrightarrow{b f}=\text { homozygous narrow-leafed males. }
\end{aligned}
$$

These hypotheses regarding the genetic constitutions of the $\mathrm{F}_{2}$ plants, formed a working basis for genetic experiments involving these plants. The following points are to be emphasized: namely, (a) that although there were only three phenotypes present in the $F_{2}$, there were four genotypes; (b) that half of the $\mathrm{F}_{2}$ females must be heterozygous and half homozygous with respect to the gene $B$; and (c) that all the broad-leafed males must be heterozygous for the gene $B$. To test these inferences a genetic analysis was made of all the broad-leafed plants of my $\mathrm{F}_{2}$ generation. For the sake of clarity the results of these analyses will be presented in four sections, based on the nature of the germcells which were brought together in each particular mating. The series of crosses which form the basis of these four sections were as follows: (1) Every female was fertilized by one of its narrow-leafed sibs. As the females were genotypically of two kinds the results fall into two groups, (a) and (b), according as the females were heterozygous or homozygous for the factor $B$; (2) All the broad-leafed males were crossed with one or more of their female sibs, and as some of the females chosen for the crosses were homozygous, others heterozygous, this series of crosses also falls into two groups, (c) and (d). A remarkable and wholly unexpected situation in regard to the sex-ratios will be noticed in these tables. They present an independent problem which must not be permitted to confuse the otherwise perfectly consistent results of all these matings.

\section{(a) Crosses between heterozygous females and their narrow-leafed sibs.}

On the supposition that the heterozygous females of the $\mathrm{F}_{2}$ have the formula $X \widehat{B F} \cdot X b \hat{F}$, and the narrow-leafed males $X b \hat{F} \cdot X \overrightarrow{b f}$, there should be again two kinds of eggs, $X \widehat{B F}$ and $X \widehat{b F}$ and two kinds of sperms, $X \widehat{b F}$ and $X \widehat{b f}$, giving the four following combinations in equal numbers: 
$X \widehat{B F} \cdot X \widehat{b F}=$ heterozygous broad-leafed females, $X \widehat{b F} \cdot X \widehat{b F}=$ homozygous narrow-leafed females, $X \widehat{B F} \cdot X \widehat{b f}=$ heterozygous broad-leafed males, $X \widehat{b F} \cdot X \widehat{b f}=$ homozygous narrow-leafed males.

It will be noted that this expectation differs from that for the $\mathrm{F}_{2}$ in only one point, namely, in the appearance of homozygous narrowleafed females instead of homozygous broad-leafed females. I confidently predicted the fulfilment of these expectations and laid plans for the extensive use of narrow-leafed females in subsequent breeding; imagine my astonishment at the results presented in Table I.

Table I.

Genetic formula: $X \widehat{B F} . X \widehat{b F} \times X \widehat{b F} . X \widehat{b f}$.

\begin{tabular}{|c|c|c|c|c|c|c|c|c|}
\hline \multirow{2}{*}{$\begin{array}{c}\text { Ped. } \\
\text { No. } 11335\end{array}$} & \multirow{2}{*}{$\begin{array}{c}\text { Culture } \\
\text { No. }\end{array}$} & \multicolumn{2}{|c|}{ Rosettes } & \multicolumn{2}{|c|}{ Broad-leafed } & \multicolumn{2}{|c|}{ Narrow-leafed } & \multirow{2}{*}{$\begin{array}{l}\text { Percentage } \\
\text { Broad-leafed }\end{array}$} \\
\hline & & Broad & Narrow & Female & Male & Female & Male & \\
\hline$(11) \times(1)$ & 12202 & 53 & 39 & - & 53 & - & 37 & $57 \cdot 61$ \\
\hline$(12) \times(1)$ & 12203 & 55 & 32 & - & 55 & - & 32 & $63 \cdot 22$ \\
\hline$(13) \times(1)$ & 12204 & 55 & 48 & 1 & 54 & - & 43 & $53 \cdot 40$ \\
\hline (14) $\times(1)$ & 12214 & 52 & 44 & - & 51 & - & 39 & $54 \cdot 17$ \\
\hline (17) $\times(1)$ & 12224 & 46 & 41 & 1 & 40 & - & 35 & $52 \cdot 87$ \\
\hline$(20) \times(4)$ & 12235 & 36 & 25 & - & $34(+1$ 하 & - & 19 & $59 \cdot 02$ \\
\hline$(26) \times(4)$ & 12239 & 41 & 28 & - & 36 & - & 20 & $59 \cdot 42$ \\
\hline$(28) \times(4)$ & 12240 & 48 & 39 & - & 46 & - & $35(+1$ ్ $)$ & $55 \cdot 17$ \\
\hline (29) $\times(4)$ & 12241 & 31 & 12 & - & 28 & - & 11 & $72 \cdot 09$ \\
\hline$(30) \times(4)$ & 12242 & 31 & 29 & - & 29 & - & 27 & $51 \cdot 67$ \\
\hline$(34) \times(5)$ & 12245 & 37 & 37 & - & 37 & - & 34 & $50 \cdot 00$ \\
\hline$(39) \times(1)$ & 12250 & 48 & 41 & - & 47 & - & 35 & $53 \cdot 93$ \\
\hline$(40) \times(4)$ & 12251 & 46 & 41 & - & 42 & - & 32 & $52 \cdot 87$ \\
\hline$(42) \times(3)$ & 12252 & 50 & 46 & - & 50 & - & 45 & $52 \cdot 08$ \\
\hline$(48) \times(4)$ & 12256 & 37 & 27 & - & 28 & - & 19 & $57 \cdot 81$ \\
\hline \multicolumn{2}{|c|}{ Total } & 666 & 529 & 2 & $630(+1$ 우 & - & $\mid 463(+1$ ఫ) & $55 \cdot 73$ \\
\hline \multicolumn{2}{|c|}{ Expected } & 597 & 597 & 274 & 274 & 274 & 274 & $50 \cdot 00$ \\
\hline
\end{tabular}

It is seen that the two expected rosette-types, broad and narrow, occurred in each family, in ratios not more divergent from the expected $1: 1$, than might well be accounted for by the obviously less vigorous constitution of the angustifolia-form, but in the entire series of families, aggregating 1097 individuals, there were only 2 females! No adequate explanation can be offered at the present time for this remarkable result. It must be made the subject of further experimental inquiry. That there 
must have been some fundamentally important genotypic feature of the narrow-leafed males used in these crosses is obvious from several considerations. The consistency of the results in every mating, regardless of the fact that four different males were used, shows that this absence of females must be due to something more fundamental than a mere individual idiosyncracy, and the same conclusion is further impressed by the results of crosses between these same narrow-leafed males and homozygous broad-leafed females, as given in Table $\amalg$.

(b) Crosses between homozygous broad-leafed females and the $F_{2}$ narrow-leafed males.

As the homozygous broad-leafed female is here assumed to have the formula $X \widehat{B F} \cdot X \widehat{B F}$ and the homozygous angustifolia-male $X \widehat{b F} . X \widehat{b f}$ there are only two classes of offspring expected; namely,

$X \widehat{B F} \cdot X \widehat{b F}=$ heterozygous broad-leafed females,

$X \widehat{B F} \cdot X \widehat{b f}=$ heterozygous broad-leafed males.

The results are given below in Table II. This table is divided into two sections; the first section contains 17 sib-crosses and the second presents the results of five "out-crosses" in which the same narrowleafed males were used.

Table II.

Formula: $X \widehat{B F} \cdot X \widehat{B F} \times X b \widehat{F} \cdot X \widehat{b f}$.

\begin{tabular}{|c|c|c|c|c|}
\hline \multirow{2}{*}{ Ped. No. 11335} & \multirow{2}{*}{ Culture No. } & \multirow{2}{*}{$\begin{array}{c}\text { Rosettes } \\
\text { Broad }\end{array}$} & \multicolumn{2}{|c|}{ Adult plants } \\
\hline & & & Female & Male \\
\hline$(8) \times(4)$ & 12200 & 101 & 1 & 95 \\
\hline$(9) \times(1)$ & 12201 & 97 & - & 96 \\
\hline$(19) \times(3)$ & 12225 & 97 & - & 97 \\
\hline$(21) \times(1)$ & 12236 & 92 & - & 86 \\
\hline$(22) \times(4)$ & 12237 & 57 & - & 54 \\
\hline$(24) \times(3)$ & 12238 & 65 & - & 65 \\
\hline$(31) \times(4)$ & 12243 & 97 & - & 97 \\
\hline$(32) \times(3)$ & 12244 & 64 & - & 64 \\
\hline$(35) \times(4)$ & 12246 & 73 & - & 69 \\
\hline$(36) \times(4)$ & 12247 & 93 & - & 70 \\
\hline$(37) \times(1)$ & 12248 & 99 & - & 97 \\
\hline$(38) \times(3)$ & 12249 & 59 & 2 & 56 \\
\hline$(43) \times(3)$ & 12253 & 96 & - & 96 \\
\hline$(45) \times(3)$ & 12254 & 55 & - & 52 \\
\hline$(47) \times(4)$ & 12255 & 68 & - & 68 \\
\hline$(49) \times(4)$ & 12257 & 95 & - & 68 \\
\hline$(50) \times(4)$ & 12259 & 76 & - & 68 \\
\hline
\end{tabular}


Table II (continued).

\begin{tabular}{c|c|c|c|c}
\hline \hline \multirow{2}{*}{ Pedigree numbers } & Culture No. & $\begin{array}{c}\text { Rosettes } \\
\text { Broad }\end{array}$ & \multicolumn{2}{|c}{ Adult plants } \\
\cline { 4 - 5 } & & Female & Male \\
\hline \hline $1193(74) \times 11335(1)$ & 12264 & 97 & - & 92 \\
$1193(74) \times 11335(3)$ & 12265 & 95 & 1 & 88 \\
$11315(42) \times 11335(1)$ & 12266 & 93 & 8 & 58 \\
$11324(7) \times 11335(4)$ & 12267 & 102 & - & 89 \\
$11337(16) \times 11335(1)$ & 12268 & 28 & - & 19 \\
\hline & Total & 1799 & 12 & 1644 \\
& Expected & $\mathbf{1 7 9 9}$ & $\mathbf{8 2 8}$ & $\mathbf{8 2 8}$
\end{tabular}

In this table again is seen the nearly total absence of females, there being only 12 to offset 1644 males, and of these few females, 8 occurred in a single rather small family, leaving to the remaining 21 families only 4 females among 1590 individuals. We might almost conclude that the narrow-leafed $\mathrm{F}_{2}$ males were negative homozygotes with respect to the sex-gene $F$ as well as its usual companion, the broad-leaf-gene, $B$. That narrow-leafed males need not be so constituted as to produce only male offspring, however, we have already seen, for the original narrow-leafed mutant gave 10 per cent females in my cultures and 42 per cent females in those of Dr. BAUR.

Leaving aside this peculiar absence of females, we are now prepared to show from Tables I and II that the first deduction from the formulation proposed for the genotypic constitution of the broad-leafed and narrow-leafed types, has been realized; namely, it has been shown that the 32 broad-leafed females in the $\mathrm{F}_{2}$ family (No. 11335) were genotypically of two kinds, though phenotypically all were alike. Table I shows that 15 were heterozygous, Table II that 17 were homozygous, almost exactly the equal numbers of these two types, which were to be expected.

\section{(c) Crosses between the broad-leafed $F_{2}$ males and their heterozygous female sibs.}

According to our hypothesis, all of the broad-leafed males in the $\mathrm{F}_{2}$ family should have been heterozygous for the gene $B$, and therefore of the same genotype as the $\mathbf{F}_{1}$ males. When crossed with their heterozygous sibs, if this hypothesis holds, they should give in the $F_{3}$ a repetition of the phenomena seen in $\mathrm{F}_{2}$; i.e., there should appear again the three phenotypes, broad-leafed females, broad-leafed males and narrow-leafed males, in approximately the series $2: 1: 1$. The essential attainment of this expected result is shown in Table III. 
Table III.

Formula: $X \widehat{B F} \cdot X \widehat{b F} \times X \widehat{B F} \cdot X \widehat{b f}$.

\begin{tabular}{|c|c|c|c|c|c|c|c|c|}
\hline \multirow{2}{*}{$\begin{array}{c}\text { Ped. } \\
\text { No. } 11335\end{array}$} & \multirow{2}{*}{$\begin{array}{c}\text { Culture } \\
\text { No. }\end{array}$} & \multicolumn{2}{|c|}{ Rosettes } & \multicolumn{2}{|c|}{ Broad-leafed } & \multicolumn{2}{|c|}{ Narrow-leafed } & \multirow{2}{*}{$\begin{array}{c}\text { Percentage } \\
\text { Broad }\end{array}$} \\
\hline & & Broad & Narrow & Females & Males & Females & Males & \\
\hline$(13) \times(7)$ & 12205 & 76 & 25 & 48 & 27 & - & 25 & $75 \cdot 25$ \\
\hline$(13) \times(10)$ & 12206 & 78 & 20 & 50 & 24 & - & 18 & $79 \cdot 59$ \\
\hline$(13) \times(15)$ & 12207 & 81 & 19 & 56 & 24 & - & 18 & $81 \cdot 00$ \\
\hline$(13) \times(16)$ & 12208 & 78 & 24 & 56 & 20 & - & 22 & $76 \cdot 47$ \\
\hline$(13) \times(18)$ & 12209 & 70 & 24 & 48 & 16 & - & 21 & $74 \cdot 47$ \\
\hline$(13) \times(23)$ & 12210 & 76 & 19 & 47 & 26 & 1 & 16 & $80 \cdot 00$ \\
\hline$(13) \times(27)$ & 12211 & 76 & 17 & 45 & 28 & - & 16 & $81 \cdot 72$ \\
\hline$(13) \times(33)$ & 12212 & 68 & 23 & 47 & 21 & - & 23 & $74 \cdot 73$ \\
\hline$(13) \times(41)$ & 12213 & 70 & 18 & 45 & 18 & - & 17 & $79 \cdot 55$ \\
\hline$(14) \times(7)$ & 12215 & 64 & 19 & 42 & 19 & 一 & 19 & $77 \cdot 11$ \\
\hline$(14) \times(10)$ & 12216 & 64 & 21 & 30 & 24 & - & 17 & $75 \cdot 29$ \\
\hline$(14) \times(16)$ & 12217 & 74 & 21 & 54 & 18 & - & 20 & $77 \cdot 89$ \\
\hline (14) $\times(23)$ & 12218 & 80 & 16 & 36 & 26 & - & 12 & $83 \cdot 33$ \\
\hline$(14) \times(27)$ & 12219 & 76 & 21 & 41 & 25 & - & 19 & $78 \cdot 35$ \\
\hline$(14) \times(33)$ & 12220 & 71 & 19 & 44 & 23 & - & 19 & $78 \cdot 89$ \\
\hline$(14) \times(41)$ & 12221 & 68 & 20 & 28 & 21 & - & 15 & $77 \cdot 27$ \\
\hline$(14) \times(44)$ & 12222 & 67 & 24 & 50 & 15 & - & 24 & $73 \cdot 63$ \\
\hline$(14) \times(46)$ & 12223 & 75 & 19 & 52 & 20 & - & 18 & $79 \cdot 79$ \\
\hline $\begin{array}{l}\mathrm{Tc} \\
\mathrm{E}\end{array}$ & , & $\begin{array}{l}1312 \\
1261\end{array}$ & $\begin{array}{l}369 \\
420\end{array}$ & $\begin{array}{l}819 \\
778\end{array}$ & $\begin{array}{l}395 \\
388\end{array}$ & $\begin{array}{l}1 \\
0\end{array}$ & $\begin{array}{l}339 \\
388\end{array}$ & $\begin{array}{l}77 \cdot 96 \\
75 \cdot 00\end{array}$ \\
\hline
\end{tabular}

As all of the 11 broad-leafed males which occurred in the $\mathrm{F}_{2}$ family were used in these crosses, we are now able to classify that family definitely on the basis of genotypic constitution, and to show that the $49 \mathrm{~F}_{2}$ individuals consisted of

17 homozygous broad-leafed females,

15 heterozygous broad-leafed females,

11 heterozygous broad-leafed males, and

6 homozygous narrow-leafed males.

It will be noticed in Table III that the proportions of the sexes are almost completely normal, although the mother-plants for these crosses were included in Table I among those which produced almost no females when crossed with narrow-leafed males. The only striking departure from the expected result, as shown in this table, is the occurrence of a narrow-leafed female. It is certainly a peeuliar circumstance 
that in the families in which hundreds of narrow-leafed females were expected, not one appeared, while the only narrow-leafed female which did appear was included in a family (No. 12210) in which no narrow-leafed females were to be expected. This narrow-leafed female was remarkable in several respects; it was the smallest adult Lychnis plant which I have yet seen, attaining a height of no more than $16 \mathrm{~cm}$ as compared with $33-114 \mathrm{~cm}$ among my other Lychnis cultures. Although the young rosette was of the usual angustifolia-type, it remained smaller than the

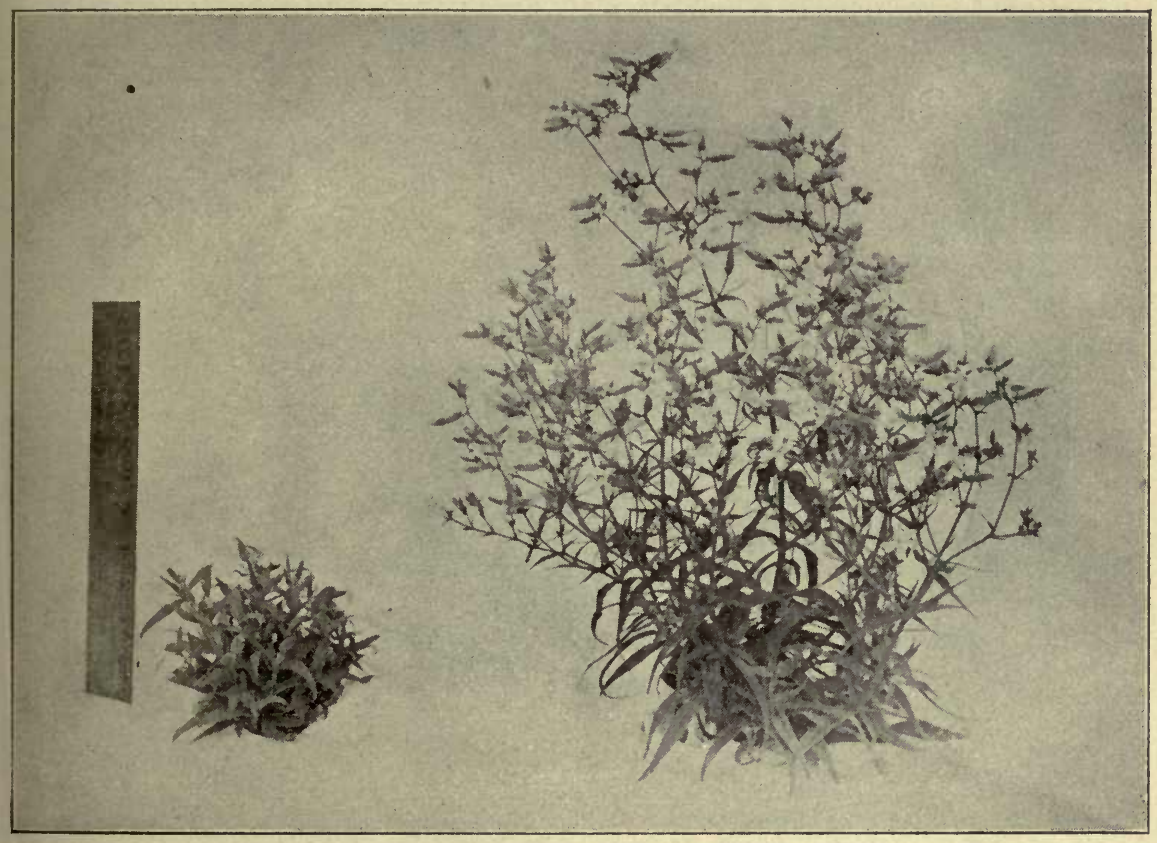

Fig. 4. A narrow-leafed female "mutant" (at left) (Ped. No. $12210(2)$ ) compared with one of its narrow-leafed male sibs (5). A $30 \mathrm{~cm}$ rule included for comparison.

rosettes of its narrow-leafed sibs. The stem-leaves were broad, as in other narrow-leafed plants, but not so strikingly acuminate. In this regard they were intermediate between the stem-leaves of the angustifolia males and those of "typica" females. The calyx was smooth and nearly cylindric, the ribs being much less prominent than is usual in this species, and without a trace of red pigmentation. The flowers were about $1.8 \mathrm{~cm}$ in diameter, scarcely exceeding the calyx-tube, while the females of Melandrium album Garcke, of which this plant was a pure derivative, usually have the corolla rather strongly exserted 
from the calyx-tube. The petals were of the usual type, broad, with an apical cleft about one-third their length; stigmas long and very slender, exserted from the corolla about $5 \mathrm{~mm}$. (In typical $M$. album they are usually included or nearly so, while in M. rubrum they are exserted). Capsules narrowly conical, slender. Another remarkable feature was the apparent absence of the absciss-layer, which, in most other strains of Lychnis dioica, and especially in Melandrium album, causes the buds and flowers to drop off or to be broken off readily. In Figure 4 this

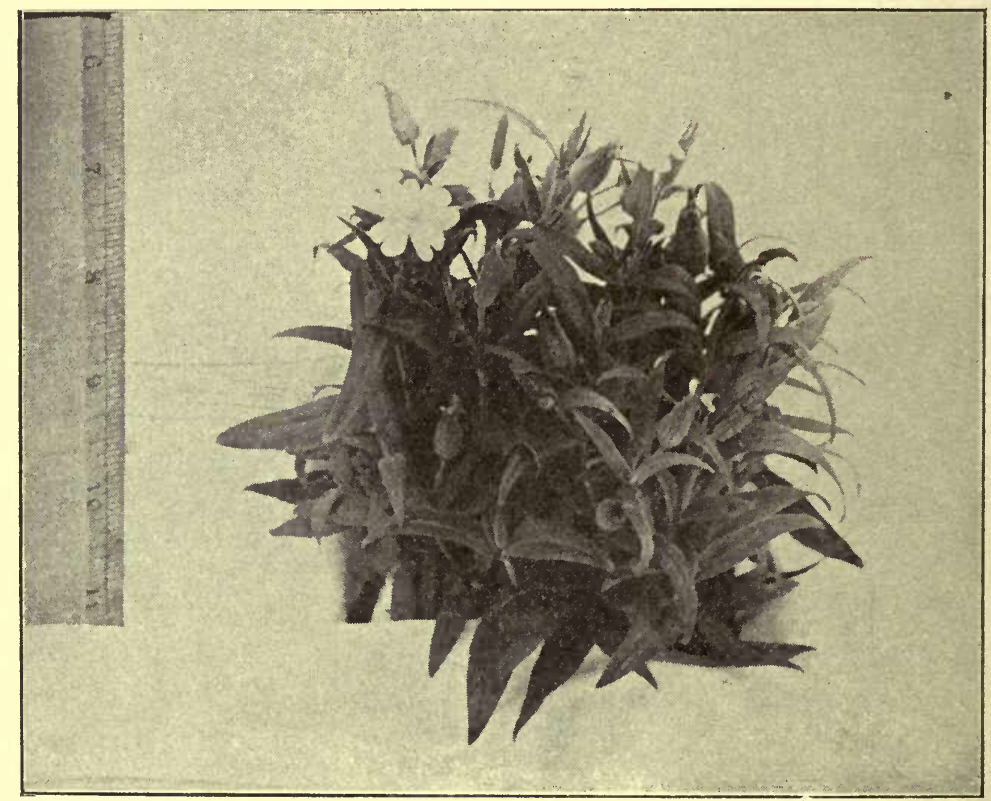

Fig. 5. Narrow-leafed female No. $12210(2)$. Numbered divisions on the rule are inches $(1 \mathrm{inch}=2.5 \mathrm{~cm})$.

plant is shown in comparison with one of its narrow-leafed male sibs. See also Fig. 5.

That this peculiar plant represents a mutation of some sort, there can be little doubt, but its genotypic relationship with its sibs can not be surmised. It may have been a sex-mutant from a narrow-leafed male or a leaf-mutant from a heterozygous broad-leafed female, or something independent of both. Whether its characters are those which will be found in narrow-leafed females, when the latter are produced by normal segregation and recombination, - as I have no doubt they will be in some future mating between heterozygous broad $(X$ narrow)-leafed females 
and narrow-leafed males, - is also an important question for whose answer there is at present no basis for a guess.

(d) Crosses between heterozygous broad-leafed males and homozygous broad-leafed females.

A cross between a heterozygote and a homozygote, when dominance is complete, as it is in the case of the particular characters here involved, is expected to give no result of special genetic interest. The data from such matings in Table IV serve to complete the records of the crosses made in the $\mathrm{F}_{2}$ family under discussion, and emphasize further the difference between the genetic behavior of the narrow-leafed males and that of their broad-leafed male sibs, with respect to the distribution of the sexes. In Table II it may be noted that the two females (19) and (49) which were the mothers of all the families included in Table IV, produced no female offspring when crossed with narrow-leafed males, while Table IV shows that, when crossed with broadleafed sibs of those same narrow-leafed males, they produced practically as many females as males.

Table IV.

Formula: $X \widehat{B F} \cdot X \widehat{B F} \times X \widehat{B F} \cdot X \widehat{b f}$.

\begin{tabular}{|c|c|c|c|c|}
\hline \multirow{2}{*}{$\begin{array}{c}\text { Ped. No. } \\
11335\end{array}$} & \multirow{2}{*}{$\begin{array}{c}\text { Culture } \\
\text { No. }\end{array}$} & \multirow{2}{*}{$\begin{array}{c}\text { Rosettes } \\
\text { Broad-leafed }\end{array}$} & \multicolumn{2}{|c|}{$\begin{array}{c}\text { Adults } \\
\text { Broad-leafed }\end{array}$} \\
\hline & & & Female & Male \\
\hline$(19) \times(15)$ & 12226 & 85 & 38 & 42 \\
\hline$(19) \times(16)$ & 12227 & 89 & 42 & 41 \\
\hline$(19) \times(18)$ & 12228 & 92 & 42 & 31 \\
\hline$(19) \times(23)$ & 12229 & 88 & 37 & 37 \\
\hline$(19) \times(27)$ & 12230 & 82 & 37 & 42 \\
\hline$(19) \times(33)$ & 12231 & 96 & 43 & 53 \\
\hline (19) $\times(41)$ & 12232 & 74 & 28 & 32 \\
\hline$(19) \times(44)$ & 12233 & 76 & 42 & 33 \\
\hline$(19) \times(46)$ & 12234 & 94 & 43 & 47 \\
\hline \multirow[t]{3}{*}{$(49) \times(44)$} & 12258 & $\left.93+1(?)^{1}\right)$ & 47 & $\left.43+1(?)^{1}\right)$ \\
\hline & Total & $897+1(?)$ & 399 & $401+1(?)$ \\
\hline & Expected & 898 & 400 & 400 \\
\hline
\end{tabular}

1) This plant had leaves almost as narrow as those of angustifolia, but the bristlelike rudiment of the ovary was only $1 \mathrm{~mm}$ long. Its genotypic character is not known. 


\section{Other crosses bearing upon the relation of the gene $B$ to the gene $F$.}

It is now a familiar idea, first suggested by DE VRIES $(1903$, p. 504) and frequently mentioned by recent writers, that a mutation which takes place in the preparation of any gamete may be expected to produce a heterozygous mutant, because of the practically prohibitive chances against the meeting of two gametes which have independently undergone the same mutation. Those supposed mutants which breed true from the first, and especially those which possess a new dominant character, would seem to require some other interpretation, unless, as appears to be true to a large extent in the Oenotheras, a hereditary mechanism is involved by which heterozygotes may breed true.

If the mutant is a Mendelian recessive, as in the case of $L$. dioica angustifolia, it may be supposed to make its appearance only when a union takes place between a sperm and an egg, both of which lack the determiner for the dominant character of the parent type. The question presents itself, therefore, as to the condition of the broad-leaf determiner, $B$, in the strain which gave rise to the original angustifolia mutant. From the results of the crosses recorded above in Tables II and IV, it is clear that the females of normal broad-leafed strains are homozygous for both the female gene $F$ and its coupled broad-leaf gene $B$. The broad-leafed males in the $\mathrm{F}_{2}$ families, as we have also seen in Table III, were heterozygous for both of the genes $B$ and $F$, but what is the condition of $B$ in the males of normal broad-leafed strains? It might be either homozygous, $X \widehat{B F} . X \widehat{B f}$, or heterozygous, $X \widehat{B F} . X \widehat{b}$. If homozygous, the occurrence of a narrow-leafed male mutant must have required a double mutation, affecting both an egg and a sperm. If on the other hand, the males of the normal strains, generally, are simultaneously heterozygous for the genes $B$ and $F$, a single mutation which removed the $B$ from its combination with the $F$ might give rise to a narrow-leafed male, for in every cross half of the available sperms $(X \widehat{b f})$ would already lack the gene $B$, and would thus be ready to unite with any $X \widehat{b F}$ egg which might be presented. Prior to the presentation of such a mutated egg, the existence of $X \widehat{b f}$ sperms could not be discovered because they must always fertilize eggs of the type $X \widehat{B F}$.

Now that heterozygous females are available, half of whose eggs are of the type $X \widehat{b F}$, it will be easy to test the males and hermaphrodites of all the various normal strains with regard to the homozygous 
or heterozygous condition of their broad-leaf determiners. I have three such crosses to report at present, and have made the requisite pollinations to test the condition of the males in all of the other independent strains in my hands. I will greatly appreciate the consideration of any one who will send me seeds of other strains of Lychnis dioica from as many localities as possible, in order that similar tests may be made upon them to ascertain whether the males of this species have the same genotypic configuration, generally, with respect to the $F$ and $B$ genes.

The three $\mathrm{F}_{1}$ families produced by crosses of males or hermaphrodites of independent families, upon heterozygous broad $(\times$ narrow)-leafed females, may be given separately as follows:

(1) Pedigree Numbers: $10244(9) \times 1090(64)=11334$. The mother of this cross was a female in my $F_{1}$ broad $(\times$ narrow)-leafed family reported above, for which the seeds were sent me by Dr. BAUR in 1911. The father was a white-flowered male in a family representing a combination of two strains coming originally from Cold Spring Harbor, Long Island, and from Harrisburg, Pennsylvania, respectively. The seeds were sown early in March 1912 and produced 20 plants, all of which bloomed. There were 14 broad-leafed females, 5 broad-leafed males and 1 narrow-leafed male. The numbers are too small to be of much value, but the parallel with the family No. 11335 , which was the offspring of the same mother, is striking. The single narrow-leafed male was in all respects typical of the angustifolia-form. It had a bristle $5.5 \mathrm{~mm}$ long as compared with $2 \cdot 0-3.5 \mathrm{~mm}$ in its five male sibs. Its occurrence in this family indicates that the father was probably heterozygous for the gene $B$, although certainly separated by a very long series of intervening generations from any individual which could have been in the ancestral line of the Sadowa plants which gave rise to the narrow-leafed mutant. Since only one narrow-leafed plant appeared in this pedigree it is not absolutely impossible that it may have been itself a mutant. Five narrowleafed individuals were theoretically due to appear if both parents had been heterozygous, but as we have already seen, angustifolia is a weaker form than "typica" and is consequently usually deficient in numbers.

(2) Pedigree numbers: $11335(13) \times 11127(11)=12260$. The mother plant (11335 (13)) is shown above in Tables I and III to have been heterozygous for the gene $B$. The father was a hermaphrodite containing elements derived from the two original strains mentioned in the last paragraph, but tracing back in one line to my original hermaphrodite mutants discovered in 1908 in pure cultures of the Cold Spring Harbor strain. The seeds were sown in February 1913, and yielded 
86 broad-leafed rosettes and 9 narrow-leafed. The 91 plants which bloomed consisted of 67 broad-leafed females, 15 broad-leafed hermaphrodites and 9 narrow-leafed hermaphrodites. The narrow-leafed plants were indistinguishable from the angustifolia males of other pedigrees in any respect other than the development of well-marked ovaries, some of which were functional. Not only were the leaves identical at all stages with those of angustifolia males, but the petals also were narrow and frequently exhibited the virescent lines mentioned above in the description of the angustifolia form.

This demonstration that my "genetic" hermaphrodites have the same constitution with respect to the broad-leaf character as the males, being like them, heterozygous for the gene $B$, is of much interest because of the strong support it gives to my conclusion, arrived at on other grounds, that the hermaphrodites are modified males; at the same time it strengthens the indication given by the preceding family, that the males of Lychnis dioica may be generally heterozygous with respect to the gene $B$ as well as the gene $F$. Morgan (1913, p. 173) assumes that my hermaphrodite mutants were derived from the female, by a modification of one of the female sex-genes, $F$. He gives the female the formula $F F$ and the hermaphrodite $F H$, just as I gave them in one of my formulae (SHULL 1911, pp. 357 and 365), though he states that the symbols he uses are not those used by me. The only difference consists, not in the symbols, but in MorGaN's assumption that the $H$ is a modified $F$, while I supposed that it might have an origin independent of $F$ and was inclined to the view that it resulted from the modification of a male gene $M$, or perhaps a sexually indifferent "synaptic mate" of $F$. If the genetic hermaphrodites were modified females, would it not be strange that in the process of modification the sex should be only half way converted to the male condition, while the accompanying broad-leaf character is completely reduced to the condition seen in the angustifolia males? While such an interpretation is possible, it appears to me simpler to conclude that the mutation which gave rise to the hermaphrodite took place in that element of the male which already lacked the gene $B$ and which may possibly be a male gene, $M$. A further convincing evidence that hermaphrodites are derivable from the males is the occurrence of a narrow-leafed hermaphrodite mutant in family No. 12240 (see Table I), for in that family no narrow-leafed females were due to appear.

(3) Pedigree Numbers: $11335(14) \times 11266(51)=12261$. The mother plant has been shown to be heterozygous with respect to $B$ 
(Tables I and III). The father was a well developed hermaphroditic plant in a family of pure Melandrium album, being the only hermaphrodite I have yet seen in this strain. The original material of this line had been sent to me in September 1909 by Dr. BAUR, and had come from nature in the vicinity of Friedrichshagen near Berlin, Germany. It may have been rather closely related, therefore, to the line which produced, in that vicinity, the angustifolia mutant. The rarity of hermaphrodites in this $M$. album strain may be inferred from the fact that in the same year (1912) in which this hermaphroditic plant appeared, I had 57 families of pure first cousins of this plant, which contained a total of 1861 females and 1911 males and no hermaphrodites. The sibs of the hermaphrodite plant consisted of 34 females and 36 males.

The seeds from this cross, sown February 15, 1913, produced 96 broad-leafed and 0 narrow-leafed rosettes. Of these plants 95 bloomed and all were female! In many hundreds of families of Lychnis dioica which I have grown during the past eight years, I have had no sizable family before 1913 in which no males (or hermaphrodites) were included, though there have been wide differences in the ratios. The result is the more remarkable when it is recalled that both in BAUR's cultures (BAUR 1912) and in mine there has generally been an excess of males in the pure $M$. album families, though in other strains of Lychnis dioica there has been, on the whole, a marked excess of females.

With some hesitancy I offer a hypothesis for this unexpected result, since it may not be possible to determine experimentally whether the hypothesis is correct or not. In my earlier papers (SHULL 1910, 1911) I have shown that certain hermaphrodites gave the same results, on breeding, as if they had been normal males. These I have called "somatic hermaphrodites" because their genotypic condition was apparently the same as that of the normal males. On the other hand, STRASBURGER (1900, 1910) and DoNCASTER (1912) have shown that females affected by the anther-smut (Ustilago violacea) are changed to apparent (i.e. somatic) hermaphrodites, and DONCASTER (loc. cit.) has found in one such case pollen-mother-cells formed. I am now inclined to the view that the hermaphrodite plant $(11266(51))$ used in the cross described in this section, was merely a somatic hermaphrodite, and that instead of being derived from the male as in my former somatic hermaphrodites, this one was produced by a somatic modification of the female, whose genotypic condition remained unchanged, so that all of its sperms were of a single sort, all carrying both of the determiners $B$ and $F$ in their normal condition. 
In full harmony with this hypothesis are two crosses in which the same hermaphroditic plant was crossed upon other females. In the first of these (Ped. Nos. $11335(19) \times 11266(51)=12262)$ the mother was a homozygous sib of the female plant which was used as mother of the preceding family. The offspring were all broad-leafed as expected, and the 96 plants which bloomed were all female! In the second (Ped. Nos. $11286(2) \times 11266(51)=12339)$ the mother was likewise a pure Melandrium album, a pure first cousin of the hermaphroditic specimen under consideration. All of the 85 plants bloomed and all were female! Unfortunately these were the only crosses made with this hermaphrodite, and repeated attempts to get self-fertilized seeds of it failed because of the ease with which the buds, flowers, and immature capsules were caused to drop off, - a feature more strongly developed in $M$. album than in any of my other strains of Lychnis dioica, and also generally more prominent in the hermaphrodites of any family than in the females of the same family. This greater tendency to cut off the buds and flowers by an absciss-layer in the hermaphrodites as compared with the females, is not strange, as the formation of such absciss-layers is universal in the males.

The failure of this remarkable hermaphrodite to produce any hermaphroditic offspring renders it impossible to derive from it any information concerning the state of normal males and genetic hermaphrodites with respect to the gene $B$. Doncaster $(1913$ a) has reported a somewhat similar experience with Abraxas grossulariata in which certain females produced only female offspring, and he was able to show that these females were characterized by an abnormal chromosome-number. No cytological studies were made on my female-producing hermaphrodite Lychnis, but I do not think it probable that such an examination, if made, would have discovered a cytological basis for its peculiar behavior ${ }^{1}$ ).

\section{Concerning genotypic formulae of the sexes.}

Various attitudes may be taken with regard to genetic formulae. Some writers appear to hold (though appearances may be misleading in this case) that such formulae present a picture of the actual constitution

1) These wholly female progenies may suggest to some the possibility of parthenogenesis, but against the plausibility of such a suggestion may be cited the fact that in hundreds of the crosses which I have made among various strains of Lychnis dioica, the occurrence of an individual having only the characters of the mother would have strikingly differentiated such individual from its sibs, but I have never seen a single case of this kind, and do not believe that parthenogenesis occurs in this species. 
of the "hereditary mechanism"; others go to the opposite extreme and maintain that they are simply a shorthand description of the results of genetic experiments. It appears to me that an intermediate position is the proper one, and I believe that this is the attitude taken, consciously or unconsciously, by most of those who use genetic formulae. A formulation must represent with approximate correctness the experimental results, - otherwise it can have no value; but a formula is both something less and something more than such a description. It constitutes not only an abbreviated statement of results already secured, but at the same time it involves a prophecy as to future results. Both of these functions of genetic formulae are imperfectly performed; instead of saying that they describe the results of genetic experiments, it would be more correct to say that they approximate those results so closely that they may be advantageously used as standards of comparison, and that, although they may not correctly represent in any case the actual genotypic apparatus, they do symbolize an internal mechanism which could give rise to approximately the observed genetic results.

Predictions as to the outcome of genetic experiments, based upon this hypothetical internal mechanism, fail with a frequency sufficient to add zest to the work of every geneticist. To those who are following genetic investigations on a fairly extensive scale, these constant surprises are a sufficient safeguard against giving the genetic formula too important a significance as a picture of the actual constitution of the genotype. The limitations of genetic formulae in this respect become even more convincing when it is seen that each genetic situation, including both empirical results and prophetic inferences, can be equally well expressed by several different sets of formulae. On several occasions I have pointed out such alternative formulations for given sets of genetic facts (SHULL 1909, $1910 \mathrm{a}, \mathrm{b}, 1911$ ), and the literature abounds in examples of the application of different formulae by different writers, to the same or essentially the same situation.

As the literature increases in volume it becomes more important that genetic terminology be simplified as far as this may be done consistently with the necessities of a correct statement of the ideas intended to be conveyed. Several suggestions have already been made toward simplification (LANG 1910, MORGaN 1913 a, b, CASTle 1913, EMERSON 1913), but the more radical of these schemes, even if generally adopted, would lead to confusion rather than to simplification. No scheme which would overturn or change beyond recognition, the formulae which are now in use, and which would consequently disconnect future genetic 
literature from that which is already published, can produce the desired simplification; but there are certain principles of conduct in the choice of symbols which writers might elect to follow in the future, which would avoid unnecessary addition to the confusion.

(1) The most important of these principles is that of conformity to those elements of past and present usage which have become so general as to be fairly considered "standard". There appear to me to be two such standard elements: namely, (a) The use of capital letters for the assumed presence of determiners, and the corresponding small letters for the assumed absence of those determiners; and (b) such a choice of symbols that the specific reactions for which the designated genes are believed to be responsible, shall be definitely suggested by the symbols, thus assisting the memory and rendering less necessary the constant reference to keys. The most feasible method of accomplishing this object, and that which is so generally used as to be most nearly "standard", is the adoption of the initial letter, or of some other prominent letter in the name of the reaction which the given gene produces, the name of the character being in that one of the three scientifically international languages, English, French, or German, which is being used at the time by the writer who proposes the symbol. LANG's suggestion that the initial of the Greek name of the given character should be used, does not appeal to me except in certain special cases, because the Greek (or Latin) names are not as familiar to most geneticists as the corresponding words in the three languages, English, German and French, in which nearly all of the genetic literature is being published.

(2) A second principle whose general adoption would avoid much confusion is the principle of priority. This should not be applied in the rigid manner of the taxonomist, but whenever a writer is dealing with genes which have been investigated and which already have published symbols, he should use those symbols in preference to new ones, unless, as will sometimes happen, a situation arises which makes the use of certain prior symbols impossible in which case these unavailable symbols, and these only, should be replaced by new ones. If a number of formulations already occur in the literature, strict priority need not be insisted upon; but preference should be given to those symbols which were used in the paper which has made the most fundamental contribution to the knowledge of the particular genotypic complex under consideration, always, when other things are equal, giving the preference to symbols which conform most nearly to the spirit of the preceding section. If this rule were generally adopted, regardless of the manner in which the 
prior symbols were themselves chosen, it being required only that they conform to the conventional method stated above under (1) (a), most of the symbols would soon become as familiar to the geneticist as are the characteristics they represent.

(3) The use of subscripts, exponents, accents and different styles of type should be abandoned, except as temporary didactic expedients. The positive homozygote is by some writers represented by a subscript-two, as $\mathrm{A}_{2}$, instead of $A A$, but if such a scheme were generally adopted the homozygous purple-flowered individual would be $\mathrm{P}_{2}$ and a homozygous female $F_{2}$, but these symbols already have general genetic usage with other meanings. Duplicate determiners might be represented by successive alphabetic symbols, the first being the initial of the character which is represented in duplicate; thus instead of $R_{1}, R_{2}, R_{3}$ or $R^{\prime}, R^{\prime \prime}, R^{\prime \prime \prime}$ for NILSSON-EHLE's red pericarp-colors of wheat, we would have $R, S, T$; for the ligula in oats, $L, M, N, O$; and for EAST's two determiners for yellow endosperm in maize, $Y$ and $Z$.

(4) It sometimes happens that a character is not easily described or suggested by any single word. In such a case any unoccupied alphabetical symbol may be used, and except for good and sufficient reasons such a symbol once adopted should be accepted by other writers who deal with the same character. Several writers have intentionally abandoned the use of suggestive symbols, for the laudable purpose of emphasizing the fact that no unit-character is the product of the single gene represented by the symbol. It appears to me that the true relation between the symbol and the unit-character can be made sufficiently clear without sacrificing the didactic advantages of the suggestive symbol.

While none of these suggestions, except perhaps the first, should be made a hard and fast rule, a general adherence to them would greatly aid in making genetic literature more lucid. At present the number of synonymous symbols and formulae involving any given character are roughly proportional to the number of investigators who have studied that character. This is well illustrated by the genetic formulae of the sexes, for the sex-phenomena are more universally interesting than any other single unit-character-complex with which geneticists deal and the number of different formulations has become surprisingly large.

In this case the confusion has been more than doubled by the fact that cytology and experimental breeding have each developed a formulation of the sexes, and that the formulae derived from these two fields of investigation have not only had considerable influence upon each other, but that they have often been more or less intermingled. 
Cytological studies have shown in a considerable number of animals belonging to several different natural groups, that the female contains a pair of special chromosomes, the "X-chromosomes", while the male has only one similar chromosome, either unpaired or paired with a chromosome which is often smaller than the $\mathrm{X}$-chromosome, and which is known as the "Y-chromosome". Sometimes instead of the Y-chromosome there is a group of chromosomes which remain associated together as a "Y-element", this group being included, as a whole, in 50 per cent of the sperms, the X-chromosome being present in the rest.

These results have led to the rather frequent adoption of the letters $X$ and $Y$ in the genotypic formulations of the sexes, but there appear to me to be certain more or less serious disadvantages of such use. It was entirely proper that CAstue (1909) should use the formulae, $X X=$ q, $X$ or $X Y=\sigma^{\pi}$, in assisting WILson (1909) to clear up his difficulties in aligning the cytological observations on the " $X$ "- and "Y"-chromosomes, with current interpretations of Mendelian inheritance; but now that these difficulties have entirely disappeared, there seems to be no good reason why the earlier method which represents the determiner for femaleness by $F$ and its absence by $f$, and the determiner for maleness by $M$ and its absence by $m$, should not be universally followed in constructing the genetic formulae of the sexes. These symbols are in entire accord with the principles of standardization suggested above.

Besides the X-and Y-chromosomes we now have references in the literature to the "Second" and "Third" chromosomes. There has been established in this way a rather definite chromosome terminology whose advantages are manifest and which should be made permanent by consistent usage. The relations between the Mendelian genes and the chromosomes are still a matter for speculation and discussion, and just because these relations must doubtless long remain problematic, it seems inadvisable to continually imply by the symbols, that chromosomes and Mendelian genes are synonymous terms. The inadvisability of the use of $X$ for the sex-gene is rendered more obvious by the apparent demonstration, through the brilliant work of MorGan (1911c) with Drosophila, that the sex-determiner is probably not the $\mathrm{X}$-chromosome, as a whole, but only a part,-perhaps a very small part,- - of it. We should be able to say without tautology that the sex-determiner ( $F$ rather than $X$ ) is probably carried by the X-chromosome.

The difficulties due to the introduction of chromosome-terminology into genotypic formulae are accentuated when the symbol $Y$, representing a gene for yellow pigmentation, is introduced into the same formula in 
which $X$ represents the gene for sex (Morgan $1912 \mathrm{~b}$, Morgan and LYNCH 1912, LITTLE 1912), or when $O$ for orange pigmentation appears together with $X$ in the genetic formulae used by a writer who has frequently used $O$ to represent the absence of $X$ (MorGaN 1911c).

Aside from formulae whose manipulations involve assumptions of selective fertilization (CASTLE 1903, DONCASTER and RAYNOR 1906, Goodale 1910, DoNCASTER 1911, SCHLEIP 1912), reversible dominance and changing potencies of determiners (SMITH 1910, de MEIJERE 1911, Goldschmidt 1912, Strong $1912 \mathrm{a}, \mathrm{b})$, - processes having no adequate experimental support and too elusive to be of use in working out genetic problems, six different types of formulae may be used to represent the genotypic relations between the sexes, - three for those cases in which the female is a sex-homozygote and three for those in which she is a sex-heterozygote. Several of these formulae have appeared in many variations, while others have been little used and consequently have few synonyms. The classification of the various formulations given below is intended to be fairly comprehensive, but not exhaustive. Since chromosome-terminology has been blended with the genetic, both are included. For this reason it has not always been easy to decide where a particular formula should be placed, since some of those who have discussed the cytological phenomena have carefully avoided committing themselves regarding the functions of the Y-chromosome. For the sake of the classification I have assumed that the Y-chromosome is sexually indifferent, as that seems to be the view generally held by those who have discussed the cytological basis of sex-determination. The division into those cases (A) in which the female is homozygous, and (B) in which she is heterozygous, now rests upon such a large body of harmonious evidence, that it must be accepted as a natural division; but the three sets of formulae available for each of these natural divisions, are largely hypothetical and may be used interchangeably for nearly all of the cases thus far investigated. These three hypothetical formulations differ from each other according as they assume that a member of the homozygous sex is a positive, a negative, or a neutral, homozygote.

\section{A. The female is homozygous, the male heterozygous.}

Examples: Bryonia, Lychnis, Nematoda, Crustacea, Myriapoda, Arachnida, Odonata, Orthoptera, Hemiptera, Diptera, Mammalia.

1. The female is assumed to be a positive homozygote.

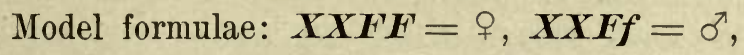
or simply, $\boldsymbol{F} \boldsymbol{F}=q, \boldsymbol{F f}=\sigma^{7}$. 
These formulae have been used by Morgan (1911a), SHuLl (1911), Sturtevant (1912a), and Goldschmidt (1913).

Synonymous formulae:

(1) FFmm = +, Ffmm = $\sigma^{\top}$ (Morgan 1911a, Stevens 1911a).

(2) $M M F F=$, $M M F f=\sigma^{7}$ (Goldschmidt 1911, 1913, Lang 1912, Correns and Goldschmidt 1913).

(3) $M M F F=$ ㅇ, $M M F=\sigma^{\nearrow}$ (Morgan 1911 c).

(4) $F F=$ ㅇ, $F O=\sigma^{7}$ (Morgan 1911a).

(5) $F F=$ ㅇ $\left.F Y^{1}\right)=\sigma^{\nearrow}$ (Spillman 1911).

(6) $X X=$ ㅇ, $X=\sigma^{7}$ (Wilson 1909, 1910, Castle 1909, Morgan 1910a, b, 1911c, 1912a, b, 1913 c, Pinney 1911, Morgan and Lynch 1912, Dexter 1912, Morgan and Cattell 1912, 1913, Sturtevant 1913).

(7) $M M X X=$ ㅇ, $M M X=\sigma^{7}$ (Morgan 1911c).

(8) $X X=$ ㅇ, $X-=\sigma^{7}$ (Castle 1912, Little 1912, Morgan 1911b, 1912c, Bridges $1913 b)$.

(9) $X X=$ ㅇ, $X O=\sigma^{7}$ (Morgan 1911a, 1913c).

(10) $x x=$ o, $x 0=\sigma^{7}$ (Hertwig 1912).

(11) $X X=q, X x=\sigma^{\pi}$ (Arkell 1912, Arkell and Davenport 1912, Doncaster $1913 \mathrm{~b})$.

(12) $X X=$ ㅇ, $\left.X Y^{1}\right)=\sigma^{\nearrow}$ (Wilson 1909, 1910, Stevens 1911b, Edwards 1911).

(13) $\left.x x=q, x y^{1}\right)=\sigma^{\pi}$ (Hertwig 1912, Schleip 1912).

(14) 우우 = 우 우 $=\sigma^{\top}$ (Shull 1910).

2. The female is assumed to be a negative homozygote.

Model formulae: $\boldsymbol{X X \boldsymbol { X } m}=$ ㅇ, $\boldsymbol{X X \boldsymbol { X } \boldsymbol { M }}=\sigma^{7}$, or simply, $\boldsymbol{m m}=$ 우, $\boldsymbol{M} \boldsymbol{m}=\sigma^{7}$.

These formulae have been used by GoLDSCHMIDT (1913) and JoHANNSEN (1913).

1) Formulae (5)( $(2)$ and $(\boldsymbol{\beta})$ have the same construction as those included below under the case in which the female is assumed to be a neutral homozygote. They are inclnded in the present case because the $\mathrm{Y}$ or $\mathrm{y}$ has been generally represented as a sexually indifferent element. The authors who have used these formulae have not always expressly stated this point, however, so there may be some doubt in such a case whether they should be included here or below under 3 . 
Synonymous formulae:
(1) $F F m m=$ क FFMm $=\sigma^{\top}$ (Shull 1911).
(2) $\left.0 O=9, f O^{1}\right)=\sigma^{\top}$ (Morgan 1911a).
(3) $O O=$ =,$X O=\sigma$ (Pinney 1911).
(4) $Y Y=q, X Y=\sigma^{\top}$ (Wilson 1910, Stevens 1911b).
(5) $a a=$ = $A a=\sigma^{\top}$ (Baur 1911).
(6) 우우 $=$ 우, 우웅 $=\sigma^{\top}$ (Shull 1910).

3. The female is assumed to be a neutral homozygote.

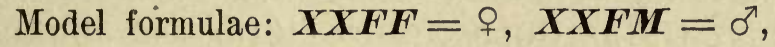
or simply, $\boldsymbol{F} \boldsymbol{F}=$ ㅇ, $\boldsymbol{F} \boldsymbol{M}=\sigma^{7}$.

This formula has been used by SHULL (1911).

Synonymous formulae:
(1) $\mathrm{ff}=$ ㅇ, (f) $m=\sigma^{7}$ (Schleip 1912).
(2) $w w=q, w m=\sigma^{7}$ (Correns 1907).
(3) 우우 $=$ 우, 웅 $\sigma^{\pi}$ (Morgan 1911a, Smith 1906, 1910).
(4) 우 $=$ ㅇ, $\left(\right.$ ㅇ) $\sigma^{\top}=\sigma^{\top}$ (Wilson 1909).

B. The female is heterozygous, the male homozygous.

Examples: Echinoidea, Lepidoptera, Aves.

4. The male is assumed to be a positive homozygote.

Model formulae: $\boldsymbol{X X} \boldsymbol{M} \boldsymbol{m}=$, $\boldsymbol{X X} \boldsymbol{M} \boldsymbol{M}=\sigma^{7}$, or simply, $\boldsymbol{M} \boldsymbol{m}=$ \%, $\boldsymbol{M} \boldsymbol{M}=\sigma^{\top}$.

These formulae have been used by STURTEVANT $(1912 \mathrm{a}, \mathrm{b})$ and BRIDGES (1913a).

Synonymous formulae:

(1) $F F M m=$ ㅇ $F F M M=\sigma^{7}$ (Goldschmidt 1912, 1913, Sturtevant $1912 \mathrm{a}$, Correns and Goldschmidt1913, Goldschmidt and Poppelbaum 1914, Gerschler 1914).

(2) $X O=q, X X=\sigma^{\pi}$ (Gerould 1911).

(3) $x_{0}=$ क,$x x=\sigma^{\pi}$ (Gerould 1911).

(4) $x 0=$ क,$x x=\sigma^{\pi}$ (Hertwig 1912).

(5) $Y O=$ ㅇ, $Y Y=\sigma^{\top}$ (Gulick 1911).

1) This formula hecomes comprehensible when it is known that in the paper in which the formula appears, MorgaN does not follow the usual method of letting the lower-case letter denote the absence of a determiner whose presence is indicated by the corresponding capital. 
(6) $y 0=$, yy = $\sigma^{\top}$ (Gulick 1911, Hertwig 1912).

(7) $z 0=9, z z=\sigma^{7}$ (Hertwig 1912).

5. The male is assumed to be a negative homozygote.

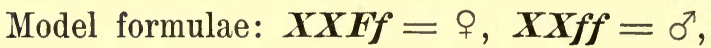
or simply, $\boldsymbol{F f}=$ i, $\boldsymbol{f f}=\sigma^{7}$.

These formulae have been used by SpILlman (1908), Goodale (1909), Hurst (1909), Pearl and Surface (1910a, b), Morgan (1911a), Bateson and Punnett (1911), Cole (1912), Morgan and Goodale (1912), Hadley (1913), Goldschmidt (1913), and Johannsen (1913).

Synonymous formulae:

(1) Ffmm $=$ f, ffmm $=\sigma^{\top}$ (Morgan 1911a).

(2) $F O=$ ㅇ, $O O=\sigma^{\top}$ (Morgan 1911a, Morgan and Goodale 1912).

(3) $F G=$ =,$G G=\sigma^{\nearrow}$ (Wilson 1910, Pinney 1911).

(4) $X=+$, no-X $=\sigma^{7}$ (Castle 1909).

(5) $X-=$, $--=\sigma^{7}$ (Castle 1912).

(6) $X O=+, O O=\sigma^{\nearrow}$ (Morgan 1911a).

(7) $x O=q, 00=\sigma^{7}$ (Hertwig 1912).

(8) $X O=q, O=\sigma^{\pi}$ (Pinney 1911).

(9) $X Y=$, $Y Y=\sigma^{7}$ (Wilson 1910, Morgan and Goodale 1912).

(10) $x y=$ ㅇ, $y y=\sigma^{\top}$ (Schleip 1912).

6. The male is assumed to be a neutral homozygote.

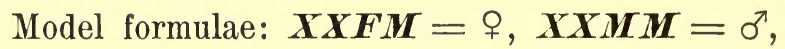
or simply, $\boldsymbol{F M}=$ q, $\boldsymbol{M M}=\sigma^{7}$.

These formulae have been used by FEDERLEY (1911).

Synonymous formulae:

(1) $f(m)=q, m m=\sigma^{7}$ (Schleip 1912).

(2) $+\sigma^{\top}=$ ․ $\sigma^{\top} \sigma^{\top}=\sigma^{\top}$ (Punnett and Bateson 1908, Doncaster 1908, Bates on 1909, Hurst 1909, Goodale 1910, Smith 1910, 1911, 1913, Morgan 1911a).

(3) $q\left(\sigma^{\pi}\right)=q, \sigma^{\pi}=\sigma^{\pi}($ Wilson 1909).

The 50 sets of formulae here listed are not all that have been used to represent these 6 fundamentally distinct ideas regarding the genotypic interrelations of the sexes, but they are sufficient, I think, to indicate the degree of simplification which would result from a general 
adoption of the "standard" formulae. I have introduced into all the formulae of the sexes used in the present paper, the symbols $X X$, to call attention to the fact that, whether symbolized or not, the rest of the genotype with which the special factors for sex are associated, is important. This is the $X X$ of JOHANNsEN which represents all those elements or characteristics of the genotype which are not otherwise specifically mentioned in the given formula. They pertain logically to every genetic formula, and when not expressed they must be, nevertheless, always understood as taken for granted. Several writers have introduced $M M$ or $F F$ into the formulae for both sexes; but in such cases there can be no experimental basis for the assumption of the specific determiners implied by these symbols. All we can say is that in one case $X X$ is essentially male and becomes female only when brought into relation with one or more added $F$ determiners; and in the other case that $X X$ is essentially female and becomes male only in reactions with added $M$ factors. Whether the essential maleness or essential femaleness of the unanalyzable part of the genotype, is due to the presence of a single pair of $F$ or of $M$ factors, or of forty such pairs, or of none at all, there is in these cases no basis for a judgment.

As already stated, there are three sets of formulae which may be used in describing the facts of sex-inheritance in any organism, and the choice of a particular formulation must be made arbitrarily, though a decision in favor of one particular set of formulae may be suggested by some feature of the evidence. Thus in those animals in which there is no $\mathrm{Y}$-chromosome in the male, it is natural, though not necessary, to prefer the formulae $F F=q$, and $F f=\sigma^{\top}$. As pointed out by several writers, it is possible to think of the chromosomes as sexually indifferent, - simply as passive indicators of sex, rather than sexdeterminers, - and if this be their status no limitation is placed even in these cases, upon the use of the other two sets of formulae. When a $\mathrm{Y}$-chromosome is present, there is no longer such strong reasons for considering the female a positive homozygote, for if the sex-determiner should be related to only a small part of a chromosome, as MoRGAN (1911c) has made plausible, the difference in size of the X-and Y-chromosomes is no barrier to the inclusion of a male sex-determiner in the $\mathrm{Y}$-chromosome, which is absent from the X-chromosomes, the difference in the size of the $\mathrm{X}$ - and $\mathrm{Y}$-chromosomes then perhaps having relation to other factors concerned with the greater anabolic activity required of the female, or with other secondary sexual relations. This would make the female a negative sex-homozygote, despite the pair of large 
chromosomes. On the other hand the X-chromosomes may contain one or more factors for femaleness and the Y-chromosomes one or more factors for maleness, the latter being epistatic. All of the empirical phenomena would be fully met by any of these hypotheses.

In the present paper I have used the formulae $X X F F=$ 우 and $X X F f=\sigma^{7}$, not because I consider these formulae better adapted than the other two sets of available formulae, to express the observed results in Lychnis, but because these formulae have been used more frequently than either of the other two sets for cases in which the female is a sexhomozygote. The preponderant use of the $F F-F f$ scheme is due, I think, solely to cytological phenomena observed in certain classes of animals, but which are absent in others and which have not yet been observed in plants. I have shown, in fact, (SHULL 1911) that the origin and genetic behavior of the hermaphrodite mutants favor one of the other formulations, because it seems more in harmony with other known cases of mutation to assume that the hermaphrodites result from a retrogressive variation in a dominant male determiner than from a progressive variation in a cryptomeric female determiner.

It can be readily shown that the discovery of sex-limited characters does not help us to reach a decision as to whether the female is positive, negative or neutral. STURTEVANT (1912), writing of Bryonia and Lychnis, remarks that "in the absence of cytological evidence or sexlinkage phenomena a chromosome interpretation would perhaps be out of place." The cytology of Lychnis dioica was worked out by STRASBURGER (1910), and a sex-limited character is now available, but there is still no way in which we can decide with any security just what the sex-determiners in Lychnis are. There are 24 chromosomes in the somatic cells of both males and females, and one pair of these are larger than the rest, but no consistent difference has been detected between the members of this pair in either sex. The sex-limited character can be related to the other available genetic formulae just as well as to the set of formulae used in the present paper. Thus, if we assume that the female is a negative homozygote, $X X m m$, and the male $X X M m$, the broadleaf factor, $B$, must be described simply as allelomorphic to $M$ instead of coupled with $F$. The formulae would then be as follows:-

$X \widehat{B m} . X \widehat{B m}$ homozygous broad-leafed female, $X \widehat{B m} . X \widehat{b m}$ heterozygous broad-leafed female, $X \widehat{B m} . X \widehat{b M}$ broad-leafed male, $X \widehat{b m} . X \widehat{b M}$ narrow-leafed male. 
If the female is a neutral homozygote, the $B$ factor must be coupled with $F$, just as if she were a positive homozygote, the only difference in the formulae being that an $M$ replaces the $f$ in the formulae of the males. The formulation on this basis appears as follows:-

$X \overparen{B F} . X \widehat{B F}$ homozygous broad-leafed female, $X \widehat{B F} . X \hat{b F}$ heterozygous broad-leafed female, $X \widehat{B F} . X \widehat{b M}$ broad-leafed male, $X \widehat{b F} . X b \widehat{M}$ narrow-leafed male.

All three formulations fit the genetic facts and all can be with equal propriety related to a chromosome explanation of sex. Something more is needed before it can be decided that one of these formulae is more likely to be a correct symbolization of the genotypic mechanism of sex in Lychnis, than the others, and what is true of Lychnis in this regard, is likewise true of sex-inheritance in most other species.

A number of cases are now known in which genetic factors which affect the same character, act in opposite directions, some producing or intensifying a given character which others inhibit, diminish, or modify in other ways. The same end-result may be produced by a retrogressive variation from a more advanced condition, or by a progressive variation from a less advanced condition. This being the case we are scarcely warranted in assuming that such universally distributed differential characters as femaleness and maleness are produced in all different organisms by the same method, even though we can successfully apply to all of them the same set of formulae. The fact seems to be that the genotypic nucleus which is common to both the males and females of any species, contains in itself nearly all of the elements necessary to the production of both the male and the female of that species, and it is therefore to a large degree essentially hermaphroditic. This is evidenced by the general occurrence in each sex, of vestigial organs which function only in the other sex. Under rare circumstances which are largely or entirely unknown at present, some or all of these vestigial organs may becone more highly developed or even functional in an individual. The statement often made that the male contains femaleness and the female contains maleness is based npon such facts as these, but the expression is not particularly appropriate, since it rests, not upon what the two sexes possess, which is distinctive to each, but upon that which they possess in common. There seems no good reason for calling those things which both sexes possess in common, either maleness or femaleness. It must be remembered only that what they 
both possess in common is nearly all of that which they each possess, and that the additional element or elements which are required for the actual realization of the one or the other sex, are conceivably of relatively minor value.

\section{Summary.}

The discovery by BAUR of a narrow-leafed mutant in Lychnis dioica has led to the demonstration that the relatively broad leaves of the normal or "typica" form is a sex-limited character.

This narrow-leafed form is here described and illustrated under the name Lyclinis divica angustifolia. It differs from the normal type not only in its narrow leaves, but also in having narrower petals and longer rudimentary ovaries.

In crosses with the normal type angustifolia is a recessive which reappears in the $\mathrm{F}_{2}$ only in half of the males. A complete analysis of the $\mathrm{F}_{2}$ family showed that all of the broad-leafed males were heterozygous for the broad-leaf factor $B$, and that half of the females were homozygous, and half heterozygous, for the same factor. This confirms the conclusion gained from the breeding of hermaphrodites, that the female of Lychnis dioica is a sex-homozygote and the male a sex-heterozygote.

There was a remarkable absence of females in families produced by matings between.the $\mathrm{F}_{2}$ narrow-leafed males and numerous females; the total progenies from such matings included 2741 males and only 14 females, and 8 of the latter occurred in one small family of 66 individuals. The broad-leafed males in the same $\mathrm{F}_{2}$ family produced males and females in approximately equal numbers.

The $\mathrm{F}_{2}$ families resulting from crosses between heterozygous females and narrow-leafed males were expected to produce about 25 per cent narrow-leafed females. Only 2 females appeared in such families among a total progeny of 1097 and these 2 happened to be broad-leafed.

One narrow-leafed female did appear, but in a family in which it was not expected, having been produced by a cross between a heterozygous female and a broad-leafed male. This female was a very small dwarf; its genotypic relationship to the angustifolia form is unknown.

Evidence is presented indicating that the males of normal strains not directly related to the original angustifolia mutant, are heterozygous for the broad-leaf gene $B$, but further investigation will be necessary to determine whether this is generally the case.

The hermaphroditic plants in families derived from my original hermaphroditic mutants are also heterozygous for the gene $B$, which 
strongly supports my conclusion that the hermaphrodite mutants resulted from a modification in the male genotype. A further proof of this relation between the hermaphrodites and males is given by the occurrence of a narrow-leafed hermaphrodite mutant in a family which included narrowleafed males, but in which no narrow-leafed females were due to appear.

One well developed hermaphroditic specimen, when used as the pollen-parent in three different crosses, produced a progeny of 276 females and no males nor hermaphrodites. It is suggested that this plant may have been a "somatic" hermaphrodite whose genotypic constitution was that of a normal female.

Suggestions are made for the standardization of genetic formulae and a list of 50 formulae which have been used to represent the genotypic interrelations of the sexes, are arranged under the six standard formulae which represent the various conceptions which may be logically related to current Mendelian methods of interpretation.

There are two natural divisions of the sex-formulae, in one of which the female is a sex-homozygote, in the other a sex-heterozygote. Under each of these there are three available sets of formulae which differ from each other according as an individual of the homozygous sex is represented as a positive, a negative, or a neutral, homozygote. In most cases there is no basis for other than a purely arbitrary choice among these formulae, since they all fit equally well the genetic facts and can be related in most cases with equal ease to the cytological observations.

Males do not possess femaleness, nor do females exhibit maleness, but what they both possess in common is nearly all of what they each are. For this reason it is not difficult to believe that the actual realization of the one or the other sex may rest upon relatively minor circumstances which may be totally different in different cases.

\section{Literature cited.}

ARKELL, T. R., 1912: Further report on inheritance of horn and wool covering in sheep. Ann. Rep. Amer. Breeders Ass. 8: $561-568$.

ArkelL, T. R., and DAvenporT, C. B., 1912: Horns in sheep as a typical sex-limited character. Science N. S. 35: $375-377$.

Bateson, W., 1909: Mendel's principles of heredity. pp. 396. Cambridge: The University Press.

Bateson, W., and Punnett, R. C., 1911: The inheritance of the peculiar pigmentation of the silky fowl. Jour. Genet. 1: 185-203.

BAUR, E., 1911: Einführung in die experimentelle Vererbungslehre. pp. 293. Berlin: Gebrüder Borntraeger. 
BAUR, E., 1912: Ein Fall von geschlechtsbegrenzter Vererbung bei Melandrium album.

Zeilschr. f. indukt. Abstamm. u. Vererb. 8: 335-336.

Bridges, C. B., 1913a: Partial sex-linkage in the pigeon. Science N. S. 37: 112-113.

- 1913 b: Non-disjunction of the sex chromosomes of Drosophila. Jour. Exper. Zool.

15: $587-606$.

Castle, W. E., 1903: The heredity of sex. Bull. Mus. Comp. Zool. Harvard College

40: $187-218$.

- 1909: A Mendelian view of sex-heredity. Science N. S. 29: 395-400.

- 1912: Heredity and sex. Chapter IV in "Heredity and eugenics" by Castle, Coulter,

Davenport, East and Tower, pp. 62-79. Chicago: University of Chicago Press.

- 1913: Simplification of Mendelian formulae. Amer. Nat. 47: 170-182.

CoLE, L. J., 1912: A case of sex-linked inheritance in the domestic pigeon. Science N. S. 36: $190-192$.

Correns, C., 1907: Die Bestimmung und Vererbung des Geschlechtes nach neuen Versuchen mit höheren Pflauzen. pp. 81. Berlin: Gebrüder Borntraeger.

Correns, C., and Golnschmidt, R., 1913: Die Vererbung und Bestimmung des Geschlechtes. pp. 149 . Berlin: Gebrüder Borntraeger.

Dexter, J. S., 1912: On coupling of certain sex-linked characters in Drosophila. Biol. Bull. 23: $183-194$.

Doncaster, L., 1908: Sex inheritance in the moth Abraxas grossulariata and its var. lacticolor. Report Evol. Comm. IV, pp. 53-57.

- 1911: Note on the inheritance of characters in which dominance appears to be influenced by sex. Jour. Genet. 1: $377-379$.

- 1912: Hermaphrodite females in Lychnis dioica. Science N. S. 36: 282-283.

- 1913a: On an inherited tendency to produce purely female families in Abraxas grossulariata, and its relation to an abnormal chromosome number. Jour. Genet. 3: 1-10.

- 1913b: On sex-limited inheritance in cats, and its bearing on the sex-limited transmission of certain human abnormalities. Jour. Genet. 3: 11-23.

Doncaster, L, and RAynor, G. H., 1906: Breeding experiments with Lepidoptera.

Proc. Zool. Soc. London 1: 125.

Edwards, C. L., 1911: The sex-chromosomes in Ascaris felis. Archiv f. Zellf. 7: 309-313. Emerson, R. A., 1913: Simplified Mendelian formulae. Amer. Nat. 47: 307-311.

Federley, H., 1911: Vererbungsstudien an der Lepidopteren-Gattung Pygaera. Archiv

f. Rassen- u. Gesell.-Biol. 3. Heft, 1-60.

Gerould, J. H., 1911: The inheritance of polymorphism and sex in Colias philodice. Amer. Nat. 45: 257-283.

Gerschler, M. W., 1914: Über alternative Vererbung bei Kreuzung von Cyprinodontiden-Gattungen. Zeitschr. f. indukt. Abstamm. u. Vererb. 12: 73-96.

Golnschмірт, R., 1911: Einführung in die Vererbungswissenschaft. pp. 546. Leipzig u. Berlin: Wilh. Engelmann.

- 1912: Erblichkeitsstudien an Schmetterlingen 1. 1. Untersuchuugen über die Vererbung der sekundären Geschlechtscharaktere und des Geschlechts. Zeitschr. f. indukt. Abstamm. u. Vererb. 7: 1-62.

- 1913: Einführung in die Vererbungswissenschaft. 2. Aufl. pp. 546. Leipzig u. Berlin: Wilh. Engelmann.

Goldschmidt, R., and Poppelbaum, H., 1914: Erblichkeitsstudien an Schmetterlingen I1. 2. Weitere Untersuchungen über die Vererbung der sekundären Geschlechtscharaktere und des Geschlechts. Zeitschr. f. indukt. Abstamm. u. Vererb. 11: 280-316. 
Goodale, H. D., 1909: Sex and its relation to the barring factor in poultry. Science N. S. 29: $1004-1005$.

- 1910: Some results of castration in ducks. Biol. Bull. 20: $35-66$.

- 1911: Sex-limited inheritance and sexual dimorphism in poultry. Sci. N. S. 33: 939-940.

- 1913: Castration in relation to the secondary sexual characters of Brown Leghorns. Amer. Nat. 47: 159-169.

Gulıck, A., 1911: Über die Geschlechtschromosomen bei einigen Nematoden nebst Bemerkungen über die Bedeutung dieser Chromosomen. Arcliv f. Zellf. 6: 339-382.

HADLEY, P. B., 1913: The presence of the barred plumage pattern in the White Leghorn breed of fowls. Amer. Nat. 47: 418-428.

Hertwig, R., 1912: Über den derzeitigen Stand des Sexualitätsproblems nebst eigenen Untersuchungen. Biol. Centralbl. 32: 1-45, 65-111, 129-146.

Hurst, C. C., 1909: Mendelism and sex. pp. 34. London: Taylor, Garnett, Evans \& Co. Johannsen, W., 1913: Elemente der exakten Erblichkeitslehre. 2. Aufl. pp. XI + 724. Jena: Gustar Fischer.

LANG, A., 1910: Referat über: W. E. Castle, H. E. Walter, R. C. Mullenix and S. Cobb. Studies of inheritance in rabbits. Zeitschr. f. indukt. Abstamm. u. Vererb. 4: 29-41. See pp. 40-41.

- 1912: Vererbungswissenschaftliche Miszellen. Zeitschr. f. indukt. Abstamm. u. Vererb. 8: $233-283$.

LitTle, C. C., 1912: Preliminary note on the occurrence of a sex-limited character in cats. Science N. S. 35: 784-785.

MFiJERE, J. C. H. DE, 1910: Über Jacobsons Züchtungsversuche bezüglich des Polymorphismus von Papilio Memnon L. $q$ und über die Vererbung sekundärer Geschlechtsmerkmale. Zeitschr. f. indukt. Abstamm. u. Vererb. 3: 161-181.

- 1911: Über getrennte Vererbung der Geschlechter. Archiv f. Rassen- u. Gesell.-Biol. 5. Heft. pp. 553-752.

Morgan, T. H., 1910a: Sex-limited inheritance in Drosophila. Science N. S. 32: 120-122.

- $1910 \mathrm{~b}$ : The method of inheritance of two sex-limited characters in the same animal. Proc. Soc. Exper. Biol. Med. 8: 17-19.

- 1911a: The application of the conception of pure lines to sex-limited inheritance and to sexual dimorphism. Amer. Nat. 45: 65-78.

- 1911 b: A dominant sex-limited character. Proc. Soc. Exper. Biol. Med. 9: 14-15.

- 1911c: An attempt to analyze the constitution of the chromosomes on the basis of sex-limited inheritance in Drosophila. Jour. Exper. Zool. 11: 365-412.

- 1912a: A modification of the sex ratio, and of other ratios, in Drosophila through linkage. Zeitschr. f. indukt. Abstamm. u. Vererb. 7: 323-345.

- 1912 b: Heredity of body color in Drosophila. Jour. Exper. Zool. 13: 27-44.

- 1912c: The explanation of a new sex ratio in Drosophila. Science N. S. 36: 718-720.

- 1913a: Factors and unit characters in Mendelian heredity. Amer. Nat. 47: 5-16.

- 1913 b: Simplicity versus adequacy in Mendelian formulae. Amer. Nat. 47: 372 - 374.

- 1913c: Heredity and sex. pp. 282. New York: Columbia University Press.

Morgan, T. H., and Cattell, E., 1912: Data for the study of sex-linked inheritance in Drosophila. Jour. Exper. Zool. 13: 79-101.

- 1913: Additional data for the study of sex-linked inheritance in Drosophila. Jour. Exper. Zool. 14: 33-42.

Morgan, T. H., and Goodale, H. D., 1912: Sex-linked inheritance in poultry. Annals N. Y. Acad. Sci. 22: 113-133. 
Morgan, T. H., and LYNCH, C. J., 1912: The linkage of two factors in Drosophila that are not sex-linked. Biol. Bull. 23: 174-182.

PEARI, R., 1912: The Mendelian inheritance of fecundity in the domestic fowl. Amer. Nat. 45: $697-711$.

PEARL, R., and SuRface, F. M., 1910a: On the inheritance of the barred color pattern in poultry. Archiv f. Entwicklungsmechanik der Organismen 30: 45-61.

- $1910 \mathrm{~b}$ : Further data regarding the sex-limited inheritance of the barred color pattern in poultry. Science N. S. 32: 870-874.

Pinney, E., 1911: A study of the chromosomes of Hipponoe esculenta and Moira alropos. Biol. Bull. 21: 168-186.

Punnett, R. C., and Bateson, W., 1908: The heredity of sex. Science N.S. 27: $785-787$. Schleip, W., 1912: Geschlechtsbestimmende Ursachen im Tierreich. Ergebn. u. Fortschr. d. Zool. 3: $165-328$.

Shull, G. H., 1909: The "presence and absence" hypothesis. Amer. Nat 43: $410-419$. - 1910a: Color inheritance in Lychnis dioica L. Amer. Nat. 44: 83-91.

- 1910 b: Inheritance of sex in Lychnis. Bot. Gaz. 49: 110-125.

- 1911: Reversible sex-mutants in Lychnis dioica. Bot. Gaz. 52: 329-368.

- 1912: Hermaphrodite females in Lychnis dioica. Science N. S. 36: 282-283.

Sмrтн, G., 1906: Rhizocephala. Fauna und Flora des Golfes von Neapel und der angrenzenden Meeres-Abschnitte. Monograph 29. pp. 123, pls. 8. See p. 89.

- 1910: Studies in the experimental analysis of sex. Quarterly Jour. Microscop. Sci.

N. S. 54: $577-604$.

Spillman, W. J., 1908: Spurious allelomorphism: Results of some recent investigations.

Amer. Nat. 42: $610-615$.

- 1911: Notes on heredity. Amer. Nat. 45: 507-512.

Stevens, N. M., 1911a: Further studies on heterochromosomes in mosquitoes. Biol. Bull. 20: $109-120$.

- $1911 \mathrm{~b}$ : Heterochromosomes in the guinea-pig. Biol. Bull. 21: 155-167.

Strasburger, E., 1900: Versuche mit diöcischen Pflanzen in Rücksicht auf Geschlechtsverteilung. Biol. Centralbl. 20: $657-665,689-698,721-731,753-785$.

- 1910: Über geschlechtsbestimmende Ursachen. Jahrb. f. wiss. Bot. 48: 427-520.

Strong, R. M., 1912 a: Another view of sex-limited inheritance. Science N. S. 36: $443-445$.

- $1912 \mathrm{~b}$ : Results of hybridizing ring-doves, including sex-linked inheritance. Biol. Bull. 23: 293-320.

Sturtevant, A. H., 1912 a: An experiment dealing with sex-linkage in fowls. Jour. Exper. Zool. 12: $499-518$.

1912 b: Federley's breeding experiments with the moth Pygaera. Amer. Nat. 46: $565-568$.

- 1913: The linear arrangement of six sex-linked factors in Drosophila, as shown by their mode of association. Jour. Exper. Zool. 14: 43-59.

VRIES, H. DE, 1903: Die Mutationstheorie II. Elementare Bastardlehre. pp. XIV + 752. Leipzig: Veit \& Co.

Wilson, E. B., 1909: Recent researches on the determination and heredity of sex.

Science N. S. 29: $53-70$.

- 1910: The chromosomes in relation to the determination of sex. Sci. Prog. pp. 570-592. 

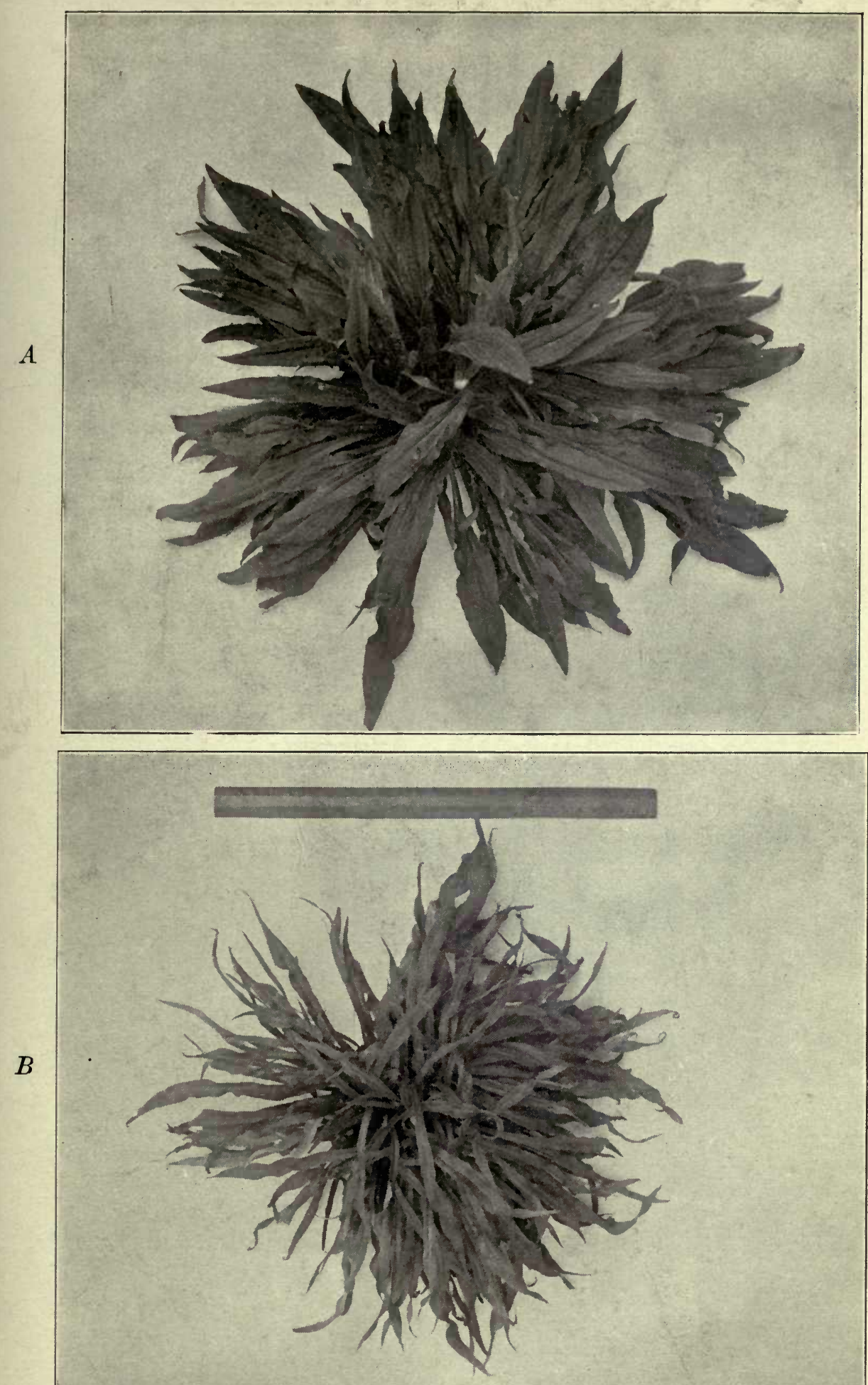

Plate I. Adult rosettes of Lychnis dioica (A) and L. dioica angustifolia (B). $30 \mathrm{~cm}$ rule included for comparison. Photographed by C. W. Palmer. 



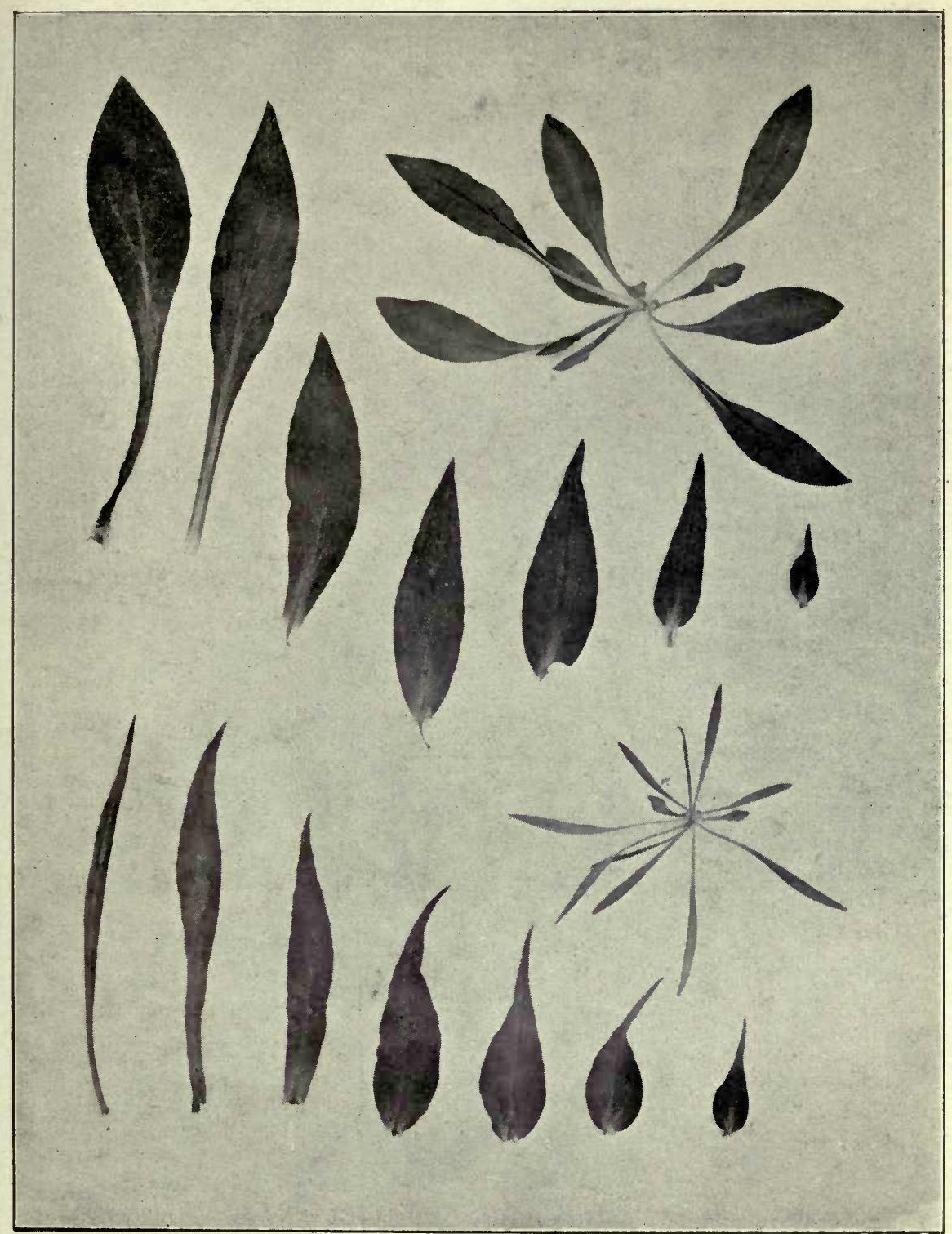

Plate II. Succession of leaves in typical Lychnis dioica (above) and in L. dioica angustifolia. The leaves of the two series are severally homologous, the first leaf at the left being a "climax"-leaf (i.e. the longest leaf) of the rosette; the second leaf is from the base of the stem, and the remaining leaves are from the successive nodes of one of the principal axes. 

2.

(3)

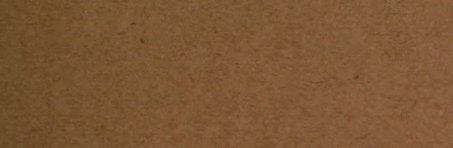

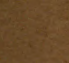

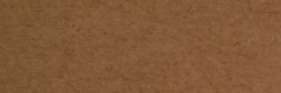

S.t. -

28

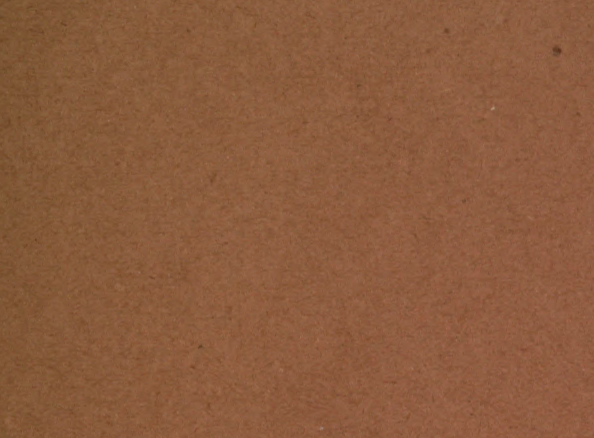

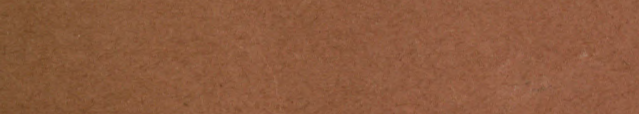




\section{Zeitschrift für induktive Abstammungs- und Vererbungslehre}

\section{Inhaltsverzeichnis von Bd. XII Heft 5}

Abhandlungen

Castle, W. E., Variation and Selection; a Reply

Shull, G. H., Sex-limited inheritance in Lychnis dioica L. (mit Tafel 1 und 2) . . . . . . . . . . . . 2 265-302

Belling, J, The Mode of Inheritance of Semi-Sterility in the Offspring of Certain Hybrid Plants . . . . . . . . . . . . . 303-342

\section{Kleinere Mitteilungen}

Internationaler Botanischer Kongreß London 1915 , . . . . . . 343

Internationaler Kongreß für Vererbungs- und Züchtungsforsehung $\quad 343$

Titel und Inhalt von Band XII 
[From JOURNAL OF GenetiCS, Vot. iv. No. 1, June, 1914.] [All Rights reserved] 



\title{
A PECULIAR NEGATIVE CORRELATION IN OENOTHERA HYBRIDS.
}

\author{
By GEORGE HARRISON SHULL.
}

Station for Experimental Evolution, of the Carnegie Institution of Washington, Cold Spring Harbor, Long Island.

WITH all the careful attention which has been given to the Oenotheras by students of genetic phenomena, there is no other group of organisms in which these phenomena seem so far from a satisfactory interpretation. Fundamental difficulties are encountered whenever attempts are made to apply to the Oenotheras rules of genetic behavior which are readily demonstrated in other groups of organisms. Equal confusion has arisen by the application of genetic experiences with the Oenotheras to species in which typical Mendelian phenomena appear. A hereditary mechanism must exist in Oenothera fundamentally different from that which distributes the Mendelian unit-characters. It becomes increasingly clear that the data on record are inadequate for the discovery of the essential features of this mechanism. It is conceivable that different mechanisms for the distribution of the hereditary characters may exist in different sections of the genus and that this may partly account for the slowness with which a comprehensive view of the genetic phenomena in this field has been made possible: Under these circumstances there is demanded a large amount of purely inductive work by the strictest pedigree methods and individual analysis. Before we can hope to explain the phenomena we must know what phenomena are presented for explanation. As a modest contribution to our knowledge of the genetic facts in Oenothera which need interpretation the following data are presented.

Early in June 1912 I received from Dr. A. F. Blakeslee, Storrs, Connecticut, three rosettes grown from unguarded seeds of Oenothera rubricalyx Gates, the seeds having been received by him from Dr. R. R. Gates, the discoverer of this particularly beautiful form. 


\section{Negative Correlation in Oenothera Hybrids}

The rosettes had the general morphological features of my 0 . rubrinervis forms, but the leaves were darker green and of more shining surface. The buds were more slender and more nearly terete, but the capsules were long and decorated, when young, with the 2-4 deep-red longitudinal bands usually seen on immature rubrinervis-capsules. The buds were brilliantly red-pigmented throughout, except on the ovary and the distal portion of the free tips, which were green. The stems were also brilliantly red-pigmented, as described by Gates. All three of the plants were of the same type, and it seems probable that they were true 0. rubricalyx Gates, despite the fact that they came from unguarded seeds under circumstances doubtless favorable to crossing with many other Oenotheras'. As my results in breeding these plants differ strikingly from those reported by Gates, the possibility of the hybrid nature of my original plants of this form must be kept in mind ; but it must also be kept in mind that even if these three plants were hybrids, they must each have been produced by the union of only two germ-cells, and as I have used in the self-fertilization and in all the crosses described below only one of the three original plants, any complication from their possibly hybrid nature is reduced to the lowest possible minimum. Such a possible hybrid origin will not detract in the least from the interesting correlations with which this paper deals. It should be added that in making reciprocal crosses generally, I have used in both crosses the same pair of individuals, so that no unsuspected complications can have been introduced by genotypic differences in individuals wrongly assumed to be identical because they were phenotypically alike. This is a detail of technique of the utmost importance; it will be found that the genetic problems in Oenothera are sufficiently complex when the elements which enter into the related crosses are reduced to the lowest possible terms.

Oenothera rubricalyx appeared in 1907 in a mixed progeny consisting of 112 offspring of four self-fertilized rubrinervis-plants grown at Woods Hole the preceding year, from unguarded ${ }^{2}$ seeds received from de Vries

1 Several features of my plants suggested a relationship to o. grandifora, particularly the rather lax rosettes, strong red spotting of the young rosette-leaves, and the development of buds more slender and rounder than in my $O$. rubrinervis cultures, but as none of the characters presented by the offspring showed accentuated resemblance to 0 . grandiflora, I doubt the reality of this suggested relationship.

2 Gates does not state that the seeds received from de Vries were from open pollinations, but the experience of Oenothera-students with rubrinervis-cultures, makes probable no other interpretation of the occurrence of 20 . Lamarckiana and 10 .oblonga among the 45 plants which included the rubrinervis-parent of the original 0 . rubricalyx. 
(Gates, $1913 \mathrm{~b}$ ). The original rubricalyx-plant, self-fertilized, yielded 9 O. rubricalyx, 1 rubrinervis, 2 undetermined ${ }^{1}$. Three self-fertilizations of these rubricalyx-specimens produced in the next generation 57 rubricalyx and 22 rubrinervis, showing in one family of 44 plants a ratio $3: 1$, and another family grown from seeds of an open-pollinated rubricalyx-plant in the same generation, yielded 71 rubricalyx, 38 rubrinervis and 4 doubtful ${ }^{2}$. Owing to various misfortunes the progenies from several other self-fertilizations could not be classified, but both rubricalyx- and rubrinervis-plants were apparently present. From these results Gates concluded that rubricalyx originated as a heterozygote which differed from the parent in a single, dominant, purely quantitative, Mendelian character, and strangely enough at the same time concluded that it would always split into rubricalyx and rubrinervis (Gates, 1911).

The small number of plants which reached maturity in Gates's pedigrees aroused the desire to test more thoroughly the apparently unique behavior of the rubricalyx-character. Since my experiments with this form were undertaken, Gates (1912) has announced the discovery of a "homozygous" individual of $O$. rubricalyx, which breeds true to this character, thus proving the incorrectness of his conclusion regarding the continued splitting of the rubricalyx-progenies, but on the other hand strengthening his assumption that the rubricalyxcharacter is Mendelian in inheritance. His oft repeated emphasis of the view that 0 . rubricalyx differs from 0 . rubrinervis in a purely quantitative character which acts as a monohybrid Mendelian dominant over the rubrinervis-type of pigmentation will have a special interest in relation to the results described below. In a paper published since this was written Gates (1914) wavers between the treatment of the rubricaly $x$ type of pigmentation as a Mendelian and as a non-Mendelian character. His most positive declarations on the subject are that it is non-Mendelian; but if he sincerely holds to this conviction it is strange that he should continue to treat the genetic behavior of this character as if it threw valuable sidelights on Mendelian phenomena.

During the past season (1913) I have had five pedigrees derived from one of the three individuals of 0 . rubricalyx grown by me in 1912 . These were 0 . rubricaly $x$ self-fertilized, 0 . rubricaly $x \times$ rubrinervis,

1 These two undetermined plants are listed in one place as 0 . rubricalyx. See Gates, 1911, Table II, p. 365.

${ }^{2}$ In the same place (Gates 1911, Table II) this family is erroneously indicated as the product of a self-fertilization and the four doubtful plants are included under $O$.rubrinervis. 
O. rubrinervis $\times$ rubricaly $x, 0$. rubricaly $x \times$ Lamarckiana and $O$. Lamarckiana $\times$ rubricalyx. All of these were sown on the same day, grown under the same conditions, potted, described and photographed at approximately the same ages, and set into the field in adjacent rows on the same day. Each of these families will be considered under a separate heading.

\section{Oenothera rubricalyx Gates.}

\section{Pedigree No. $11410(1) \times$ Self $=1231$.}

Seeds sown January 30th, 1913, germinated in 16-20 days and yielded 119 plants, which at 10 weeks of age were clearly divisible into two groups in respect to morphological characters, and into two independent groups in regard to the pigmentation of the leaves. These four groups may be briefly characterized as $(a)$ the rubrinervis-like group, (b) the nanella-like group, (c) the spotted group, and $(d)$ the unspotted group. Two plants died unclassified. The remaining 117 were distributed as follows: (ac) 59, (ad) 48, (bc) 8, (bd) 2. These categories may be briefly characterized as follows:

Plants of the (a) group containing 107 individuals, or 91.45 per cent. of the entire family, had rather lax, ascending rosettes of relatively narrow leaves, resembling those of 0 .rubrinervis, but in general a little darker green and with more reddening of petioles and midribs in the older leaves, the spotted sub-group (c) usually being a little more strongly crinkled and a little more prominently dentate toward the base of the blades than the unspotted sub-group $(d)$, as seen in the upper series of plants in Plate V. These may be compared with purebred O. rubrinervis-rosettes of similar age, which are shown in the lower series on the same Plate. The adult plants all had the tall, wide-branching form usually seen in vigorous strains of 0 . rubrinervis, with the characteristic long capsules of that species, but the buds were as in the parent, relatively a little more slender than in my long-controlled strains of $O$. rubrinervis.

In the following table, bud-measurements of these rubricalyx-plants both red-stemmed $(a c)$, and pale-stemmed $(a d)$, may be compared with corresponding measurements from four pure 0 . rubrinervis-pedigrees taken at about the same time (Aug. 1) and under similar conditions. Each measurement entered in the table is an average from five buds taken at random from five different plants in the indicated pedigree. All 
measurements are in centimeters and were made with a micrometercaliper by my scientific assistant, W. F. Friedman, to whose faithful work and painstaking care it is a pleasure to give this grateful recognition.

\begin{tabular}{|c|c|c|c|c|c|c|c|c|}
\hline \multirow[b]{2}{*}{ Character } & \multirow[b]{2}{*}{ Ped. No. } & \multicolumn{5}{|c|}{ o. rubrinervis } & \multicolumn{2}{|c|}{ o. rubricalyx } \\
\hline & & 1213 & 1214 & 1215 & 1216 & Av. & $1231(a c)$ & $1231(\mathrm{ad})$ \\
\hline Length of ovary ... & $\ldots$ & $1 \cdot 14$ & $1 \cdot 13$ & 1.07 & $1 \cdot 17$ & $1 \cdot 13$ & $1 \cdot 06$ & 1.00 \\
\hline Thickness of ovary ${ }^{1}$ & ... & $0 \cdot 33$ & 0.32 & 0.32 & $0 \cdot 31$ & 0.32 & 0.30 & $0 \cdot 28$ \\
\hline Length of hypanthium & n ... & $3 \cdot 63$ & $3 \cdot 93$ & $3 \cdot 55$ & 3.03 & $3 \cdot 53$ & $4 \cdot 13$ & $3 \cdot 77$ \\
\hline Thickness of hypanthiv & $u^{2}$ & $0 \cdot 31$ & 0.28 & 0.25 & 0.28 & $0 \cdot 26$ & 0.24 & $0 \cdot 24$ \\
\hline Length of cone ... & $\ldots$ & $4 \cdot 21$ & $4 \cdot 43$ & $3 \cdot 95$ & $4 \cdot 06$ & $4 \cdot 16$ & $4 \cdot 61$ & $4 \cdot 07$ \\
\hline Thickness of cone ${ }^{3}$ & $\cdots$ & 0.81 & 0.84 & 0.75 & 0.74 & 0.78 & 0.74 & 0.72 \\
\hline Length of free tip & ... & 0.59 & 0.63 & 0.66 & 0.66 & 0.63 & 0.74 & $0 \cdot 60$ \\
\hline Length of anther & $\ldots$ & 1.54 & 1.58 & - & $1 \cdot 66$ & 1.59 & $1 \cdot 49$ & $1 \cdot 45$ \\
\hline
\end{tabular}

Plants of the $(b)$ group were distinguishable by their closer rosettes, caused by the relatively short, ascending petioles and nearly horizontal blades. They were recognized at once as nanella-like rosettes, though their longer, narrower, less crinkled leaves distinguished them strikingly from the nanella-form derived from 0 . Lamarckiana. The adult plants proved to be of little more than nanella-stature $(25-40 \mathrm{~cm}$.) as shown in fig. 1, but of very unique aspect, due to the broad-lanceolate, acuminate, dark green, nearly uncrinkled leaves. The buds were long, slender and the cones nearly terete. Gates (1914) reports the occurrence of dwarfs in his cultures of both 0 . rubricalyx and 0. grandiflora, and their recurrence in some of the $F_{2}$ families from crosses between these two species.

The group $(c)$ differed from the $(d)$ group in having conspicuous red spots on the dorsal surface of the leaf-blades, as shown in the accompanying Plates. As Gates had found self-fertilized rubricalyx yielding progenies containing both rubricalyx and rubrinervis, I at once inferred that I was getting the same result, and that the spotted rosettes belonged to the rubricalyx- and the unspotted rosettes to the rubrinervis-type. This did not prove to be true, however, as the entire (a) group, both spotted and unspotted, had the intensely pigmented hypanthia and budcones characteristic of 0 . rubricalyx. In the adult stage the spotted and unspotted groups, $(c)$ and $(d)$, were definitely differentiated from one another in only one feature, namely, in the pigmentation of the stems. The group grown from spotted rosettes, $(a c)$, had intensely red-pigmented stems, the pigment being particularly conspicuous about the base of the

\footnotetext{
1 Greatest diameter.

2 Least diameter.

3 Greatest diameter parallel with sides.
} 
central spike and on the upper lateral branches. They were in this respect like their parent. The unspotted group, on the other hand, had the stems only slightly reddened, with the upper part of the main-stem and upper laterals not conspicuously pigmented with anthocyan. In the dwarf group, $(b)$, only six plants bloomed, all belonging to the sub-group with spotted rosettes. Five of them had no anthocyan in the buds, and one had the pigmentation of 0 . rubricalyx. All had strongly reddened stems.

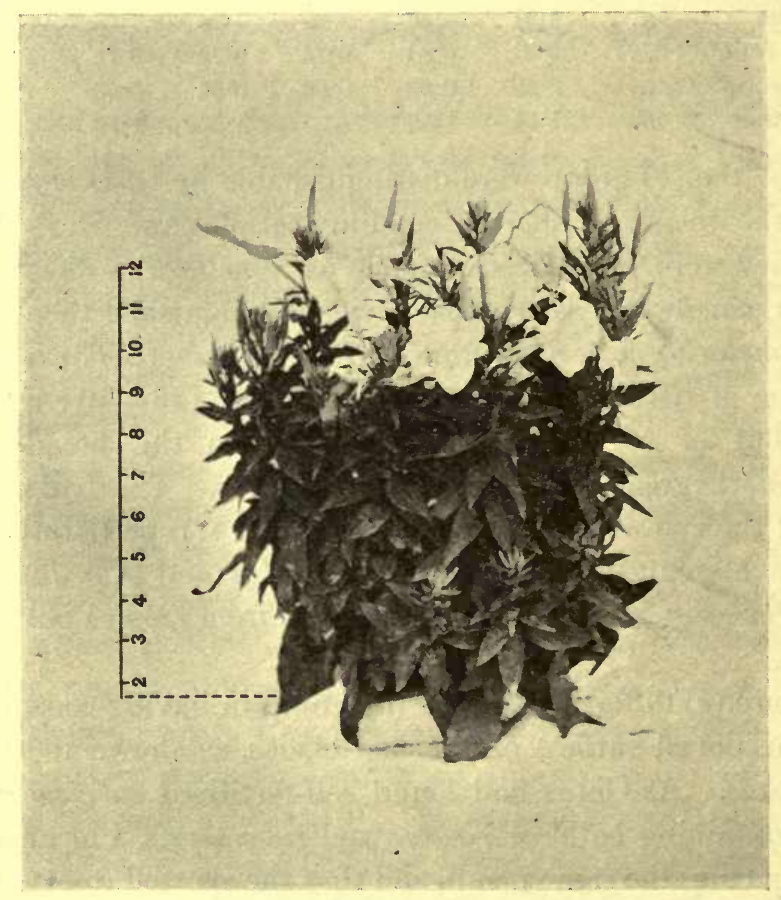

Fig. 1. A dwarf plant with dark red stems and green buds from selffertilized O. rubricalyx. (The included scale of inches was drawn from a rule photographed on the same negative; 1 inch $=2.5 \mathrm{~cm}$.)

Gates has not mentioned red-spotting of the young rosette-leaves as a characteristic of 0 . rubricalyx, but on the other hand he now includes this character among the features which differentiate 0 . grandiflora from 0. rubricalyx (Gates, 1914). The fact that half of my rubricalyxplants had strongly spotted leaves may perhaps mean, therefore, that my original rubricalyx-plants were hybrids. Another possibility may be suggested. Gates (1914) characterizes the rosette of $\theta$. grandiflora 
as having "pale red blotches"; in my cultures that species had deep red blotches. There can be no doubt that anthocyan production is strongly influenced by sunlight and temperature, and possibly other environmental factors, which differ in different localities. The deeper red spots on $O$. grandiflora in my cultures might be due to genotypic differences between Gates's material of that species and mine, but it seems rather more likely that they indicate a condition in my cultures favorable to the intensification of the anthocyan colors. It is conceivable that a form which does not possess red spots at all under one condition may be strongly spotted under some other condition. This is directly illustrated by the spotted plants themselves, for the spotting is an evanescent character, which completely disappears as the rosettes grow older. The occurrence of spotting in the young rosette-leaves in my cultures of 0 . rubricalyx, does not necessarily prove, therefore, that my plants are hybrid derivatives of 0 . rubricalyx instead of that species itself, although on certain grounds the assumption that they are hybrids may seem to be the more tenable hypothesis.

As the rubricalyx-plants in this pedigree were of two types with respect to the pigmentation of the stems, it was thought possible that such splitting might also occur in the homozygous strain discovered by Gates, but a letter from A. W. Sutton, Esq., Reading, England, informs me that all of the 0 . rubricalyx-plants from Gates's pure-breeding stock have the brilliant red stems?

\section{Oenothera rubricaly $x \times$ rubrinervis $F_{1}$.}

$$
\text { Pedigree Nos. } 11410(1) \times 1123(8)=1232 \text {. }
$$

The male parent was from a pure cross-bred strain of Oenothera rubrinervis which had been fully controlled in my cultures in seven consecutive generations, each of these ancestral generations having resulted from a cross between two typical rubrinervis-specimens of as widely separate relationship as my several cultures provided. All the preceding generations had been uniformly typical $O$. rubrinervis with the exception of an extremely small percentage of aberrant individuals which have appeared from time to time as characteristic mutants(?).

Seeds from this cross yielded 205 individuals of which 8 died unclassified. These were, like the progeny from the selfed 0 . rubricalyx

1 Seeds of this form are now being offered for sale by Sutton and Sons under the name "Oenothera Afterglow.' 
(No. 1231), divisible into a spotted and an unspotted group, in the ratio 103:94. No nanella-plants were seen, and all were similar in morphological features to 0 . rubrinervis, as shown in the second row of rosettes from the top of Plate $\mathrm{V}$, though in the 10 -weeks-old rosettes about two-thirds of the unspotted group had somewhat broader, darker green leaves than the remaining one-third. Later, however, this distinction could no longer be seen; the broader- and narrower-leafed groups were kept separate throughout their development, but no distinction was visible between them in the mature plants. With the exception of three plants which were slightly divergent in characters of foliage and branching, all were of the same vegetative form, and indistinguishable in this respect from the (a) group of selfed 0 . rubricalyx. In pigmentation, however, a striking situation was presented. The unspotted rosettes developed into plants of the same type as the corresponding group in the selfed $O$. rubricalyx family, having greenish stems and brilliantly pigmented buds of the rubricalyx-type. The spotted rosettes, on the other hand, produced brilliantly red-pigmented stems, with buds of the Lamarckiana-type of pigmentation, the cones being merely pink in longitudinal bands of greater or lesser width, and the hypanthia green. No plant of the latter group had buds as strongly pigmented as in 0 . rubrinervis, the male parent, and the minus variations ranged to cones nearly, though not quite, completely free from anthocyan.

\section{Oenothera rubrinervis $\times$ rubricalyx $F_{1}$.}

\section{Pedigree Nos. $1123(8) \times 11410(1)=1233$.}

Seeds from this cross produced 152 plants which were likewise clearly separable into a spotted and an unspotted group, but with no other apparent distinction. Two-months-old rosettes are shown in the second row from the bottom of Plate V. The two groups consisted of 62 spotted and 89 unspotted rosettes, 1 having died unclassified. Plants of both groups had red on the under side of petioles and leaf-blades, especially when the latter were going into decline. Several plants which had one or two obscure red spots or fine red specks, but proved on their subsequent development to belong to the unspotted group, are included with that group in the ratio given above. One plant which was marked on May 2 as spotted, was found two weeks later without spots, and was transferred to the unspotted group, where its identity was lost. Among the adult plants one individual from the unspotted 
group had the bud- and stem-characters of the spotted plants, from which I infer that the original determination of the character of this plant was the correct one, and it is therefore included with the spotted plants in the above reckoning. Only 25 of each group were set into the field to grow to maturity. All the adult plants had the branching habit and long capsules characteristic of $O$. rubriculyx and O. rubrinervis. Eight in the spotted group had slightly broader, darker green, more strongly crinkled leaves than their sibs, in these particulars more closely approaching the characters of 0 . Lamarckiana, but their stems and buds did not differ in pigmentation from those of the other plants in the spotted group. One plant in the spotted group had the buds colored as in 0 . rubrinervis, but was so heavy in all its parts as to suggest the likelihood that it was a triploid form. Its capsules were notably thicker and shorter than those of its sibs. I have no special record as to the color of the stem of this plant, but as it stood in the red-stemmed group, it would probably have been noticed if different in this respect from the rest of the group. All the rest of the spotted group had brilliant red stems and Lamarckiana-like buds with pink cones and green hypanthia. The unspotted group, with the one exception already mentioned, had greenish stems and the typical rubricalyx-coloration of the buds, i.e., with hypanthia and cones uniformly and intensely red-pigmented, except on the distal portion of the free tips of the sepals, which were free from anthocyan.

\section{Oenothera rubricalyx $\times$ Lamarckiana $F_{1}$.}

Pedigree Nos. $11410(1) \times 118(10)=1234$.

Of this pedigree I secured 123 plants which were again divisible into spotted and unspotted groups, but the spotting was not so pronounced as in the rubricalyx-rubrinervis-hybrids, so that it was not quite certain whether the unspotted group was a natural group or simply the minus-end of a single fluctuating series. The grouping of the young rosettes gave 97 spotted and 26 unspotted plants. None of these rosettes closely resembled 0 . Lamarckiana, being more lax, the leaves having longer petioles and more tapering bases; and they were also more ascending than in the rosettes of 0 . Lamarckiana. They may be compared with $O$. Lamarckiana in Plate VI, in which O. rubricalyx-rosettes are at the top, O. Lamarckiana at the bottom, and the present series of hybrids in the second row from the top. The foliage 
in the spotted group was perceptibly darker green and the leaves slightly broader and more crinkled than in the unspotted group. As the rosettes grew older, a small number were noted, which were differentiated from the rest in being rather coarsely crinkled, with the obvate to oblong-obvate blades abruptly contracted, with a slight undulation, to a triangular-winged petiole. The leaves of these had more numerous and more conspicuous red spots than their spotted sibs. That this group grades into the more common type of spotted plants, is shown by the fact that I separated only 6 out of the 9 plants which subsequent development proved to belong to the same natural group. Fifty plants were set in the field, including 30 spotted and 20 unspotted plants, the members of each of these groups being taken at random, with the exception of one of the crinkled-leafed, strongly spotted plants just described, which was discovered before the reservations were made for the field cultures. Aside from this one individual, therefore, the distribution of the different types in the field should accurately represent, within the limits of the probable error, the composition of the entire progeny. The adult plants were all of one type with respect to general habit of branching and the long capsules $(2.5-2 \cdot 7 \mathrm{~cm}$.), being in these regards like my selfed 0 . rubricalyx. Among them were three classes of individuals sharply distinct from one another, namely,

(a) $9[27]^{1}$ with very dark, dull-red stems, and buds wholly free from red pigment;

(b) 5 [17] with brilliant red stems, and Lamarckiana-like buds, i.e., with pink cones and green hypanthia; and

(c) 36 [79] with greenish stems and rubricalyx-buds, i.e., brilliant red hypanthia and cones.

It would have been desirable to make an actual determination of the relative amounts of anthocyan in the stems of groups $(a)$ and $(b)$, but for lack of time this determination was not made. It was my impression, however, that the dull blackish-red stems of the former contained more anthocyan than the brilliant stems of the latter. If this impression is correct, the three grades of pigmentation of the buds are completely associated with three grades of pigmentation in the stems, but the bud-series and the stem-series run in opposite directions,

1 In brackets are given the number of each type which should have been present if the entire progeny had been grown to maturity. These are the significant numbers, as the proportionality among the observed numbers is distorted by the fact that a relatively larger number of unspotted rosettes were grown to maturity, than of spotted ones. 
thus: green with dark red, pink with bright red, red with green. The fine red specks often seen on the ovaries show a partially independent series, being absent in $(a)$, numerous in $(b)$ and scarce in $(c)$.

The distribution of the adult plants shows that the classification of the rosettes into spotted and unspotted categories was not in this case a natural grouping, as it was in the reciprocal rubricalyx-rubrinerviscrosses, and this difference is doubtless due to the fact that my $O$. Lamarckiana has moderately spotted rosettes while 0 . rubrinervis is unspotted, as shown by Plates V and VI (bottom series in each). All plants of groups $(a)$ and $(b)$ had spotted rosettes, and two-thirds of the former were noticeably more strongly spotted than the rest of the spotted plants. All the unspotted rosettes and most of the moderately spotted ones developed into plants of type $(c)$. It is thus seen that although the pigmentation of the rosette is not in this hybrid combination, as sharply diagnostic of the natural groups, the nature of the association between the pigmentation of the rosette and that of the adult organs is the same, in direction, in the rubricalyx-Lamarckianacross as in the rubricalyx-rubrinervis-cross. Stated more generally, there is a positive correlation between the red-pigmentation of the rosette-leaves and that of the adult stems, and a corresponding negative correlation between the red-pigmentation of the rosette and the redpigmentation of the buds.

\section{Oenothera Lamarckiana $\times$ rubricalyx $F_{1}$.}

$$
\text { Pedigree Nos. } 118(10) \times 11410(1)=1235 \text {. }
$$

Seeds of this cross produced 117 plants which were at one time grouped provisionally into 36 Lamarckiana-like, 18 rubricalyx-like and 37 rubrinervis-like, besides a considerable number of individual aberrants. As these names proved later to be inappropriate it is desirable to translate them for our present use into terms of their pigment-characters. The "Lamarckiana-like" group and the "rubricalyx-like" group had red-spotted rosettes, and the "rubrinervis-like" group had unspotted rosettes. Unfortunately, several of the divergent rosettes which received individual description, were not noted with reference to the presence or absence of red spots, and only 113 are now classifiable on the basis of the red spotting of the rosette-leaves; of these, 64 were spotted and 49 unspotted. Most of the slightly aberrant rosettes developed into adult plants not perceptibly divergent from other plants which had not been noticeably aberrant in the rosette- 
stage. Two had heavier buds than the rest, nearly like those of pure rubrinervis in size and form, but each of these had greenish stems and rubricalyx-colored buds; they are included among the number referred to group $(c)$ below. Aside from these two plants and several specimens which failed to bloom, the 78 plants which had been set into the field were referable to three phenotypes, indistinguishable from those presented by the reciprocal family. The several types of pigmentation were distributed in the following proportions:

(a) $7[9]^{1}$ with dull, dark-red stems and buds devoid of anthocyan.

(b) 17 [22] with brilliant red stems, pink cones and green hypanthia.

(c) 51 [82] with greenish stems and intensely red hypanthia and cones.

The relation of these groups to the rosette-characters of the same family are the same as in the reciprocal family; both $(a)$ and $(b)$ were completely included among the spotted rosettes, while $(c)$ included all the unspotted rosettes and 26 (i.e. essentially 50 per cent) of the spotted ones.

\section{Similar phenomena in other Oenothera-crosses.}

The negative correlation between the redness of the stems and that of the buds, which has been so strikingly manifested in these rubricalyxcrosses, is by no means limited to the combinations of 0 . rubricalyx. In an extensive series of crosses between O. Lamarckiana and several biotypes of 0 . cruciata, glimpses of the same inverse relation in the distribution of the red pigmentation, have been frequently seen. The results of the latter crosses will be published in detail in another place, and only several examples of many that might be presented will be introduced here to illustrate the criss-cross distribution of the red pigment on the stems and buds. The forms of 0 . cruciata have green buds and usually more or less strongly reddened stems. O. Lamarckiana has pink cones and green hypanthia associated with only moderate reddening of the stems, the stems having a degree of red-pigmentation similar to that of the above-described "greenish"-stemmed plants among the rubricalyx-hybrids. A cross between a certain elementary form of 0 . cruciata $q$ and 0 . Lamarckiana $\delta$ produced in the $F_{1}$ (Ped. No. 1140) four types characterized as follows with respect to pigmentation :

(a) $2[2]^{1}$ with red stems and buds entirely green.

1 See footnote on p. 92. 
(b) 10 [18] with bright red stems, very slightly reddened bud-cones and green hypanthia.

(c) 2 [3] with pink stems, and with cones and hypanthia red throughout.

(d) $5[5]$ with stems nearly green and buds entirely green.

A plant of the type $(b)$, which type made up the bulk of the $F_{1}$ family, pollinated by a plant of the parental biotype of $O$. cruciata (i.e., a sesqui-reciprocal hybridization of the form $(A \times B) \times A)$, produced 43 [97] offspring with red hypanthia and red cones on pink stems, and 5 [6] with faint reddening on the cones, green hypanthia and intensely red stems (Ped. No. 12107). The parental form of 0 . cruciata had only moderate reddening of the stems, so that the red stems of the hybrid types $(a)$ and $(b)$ represent a marked intensification of the pigmentation as compared with both parents, just as the red hypanthia and strongly red-pigmented bud-cones of hybrid type (c) show a remarkable advance over the pigmentation of the buds in O. Lamarckiana, the only one of the parents which had any red coloration of the buds.

In crosses between 0. Lamarckiana and two other biotypes of O. cruciata, the $F_{1}$ hybrids, when $O$. Lamarckiana is the mother, are in each cross of uniform type, having the bud-cones reddened to about the same extent as in 0 . rubrinervis (i.e., much more strongly reddened than in O. Lamarckiana) while all the vegetative parts are pale green and absolutely devoid of red-pigmentation. The reciprocal crosses produced in the one case two types, in the other case four types, some of which had pink cones and green hypanthia, others entirely green buds, but all had strongly reddened stems, the green-budded plants having a stronger pigmentation of the stems than the pink-coned plants. In the latter pedigrees the green-budded forms can be partially sorted out from the pink-coned forms in the rosette-stage, because of the more prominent red spots on the dorsal surface of the young rosette-leaves in the former.

The pigmentation of 0 . rubrinervis as compared with its parent O. Laniarckiana, may be related to the same phenomenon, for the budcones of the mutant-form are much more strongly pigmented than those of 0 . Lamarckiana, while the rosettes are entirely free from red spots and the stems are nearly green, while 0 . Lamarckiana, on the other hand, has the rosettes sparsely spotted and the stems moderately reddened. Thus, 0 . rubrinervis shows a progressive variation in the amount of red pigment in the buds, while the leaves and stems present a retrogressive variation in respect to red pigmentation. 


\section{Discussion.}

The behavior of the red pigments in these 0 . rubricalyx-hybrids, is in striking contrast with that reported by Gates (1909 to $1913 \mathrm{~b}$ ), whose reciprocal crosses between 0 . rubricalyx and 0 . Lamarckiana appeared to consist predominantly of rubricalyx and Lamarckiana. To what extent the differences between his results and mine are due to the very slender basis for the conclusions regarding his crosses of these species, cannot be determined, and consequently it is impossible to say how much of these differences is to be referred to putative genotypic differences between the particular individuals which entered into his crosses and those which were used in mine. Whatever may be the fact in regard to the lack of genotypic identity between his parent plants and mine, the results described in this paper have an important bearing on the several propositions set forth by Gates in regard to the origin and genetic behavior of the rubricalyx-character, namely $(a)$ that the difference between 0 . rubricalyx and O. rubrinervis is a purely quantitative one; $(b)$ that the rubricalyx-character is a typical monohybrid Mendelian character; and (c) that the method of inheritance of a character is determined by the nature of that character itself.

Many Mendelian color-patterns have been discovered in various plants and animals, which are inherited quite independently of the actual quantities of pigment present in the organism as a whole, the same pattern being associated sometimes with weak pigmentation at other times with intense pigmentation; dissimilar and independently inheritable patterns may also affect different parts of the same individual, and in every such case it is demonstrable that a "qualitative" and not alone a "quantitative" difference is present. Such experiences incline the geneticist to the interpretation of any striking change in a color-pattern as something more than a quantitative change in the amount of pigmentation present. Still, in a case as simple as that which Gates's material seemed to him at first to present, in which the new color-pattern completely includes the original one, the interpretation of the change as a purely quantitative one is possible, though perhaps not in any case particularly probable. Gates (1914) has now shown that in the case of the rubricalyx-character, also, the pattern is measurably independent of the actual quantity of pigment, since he secured pale red buds with the characteristic rubricalyx-distribution of the pigment. The results from my crosses show even more strikingly 


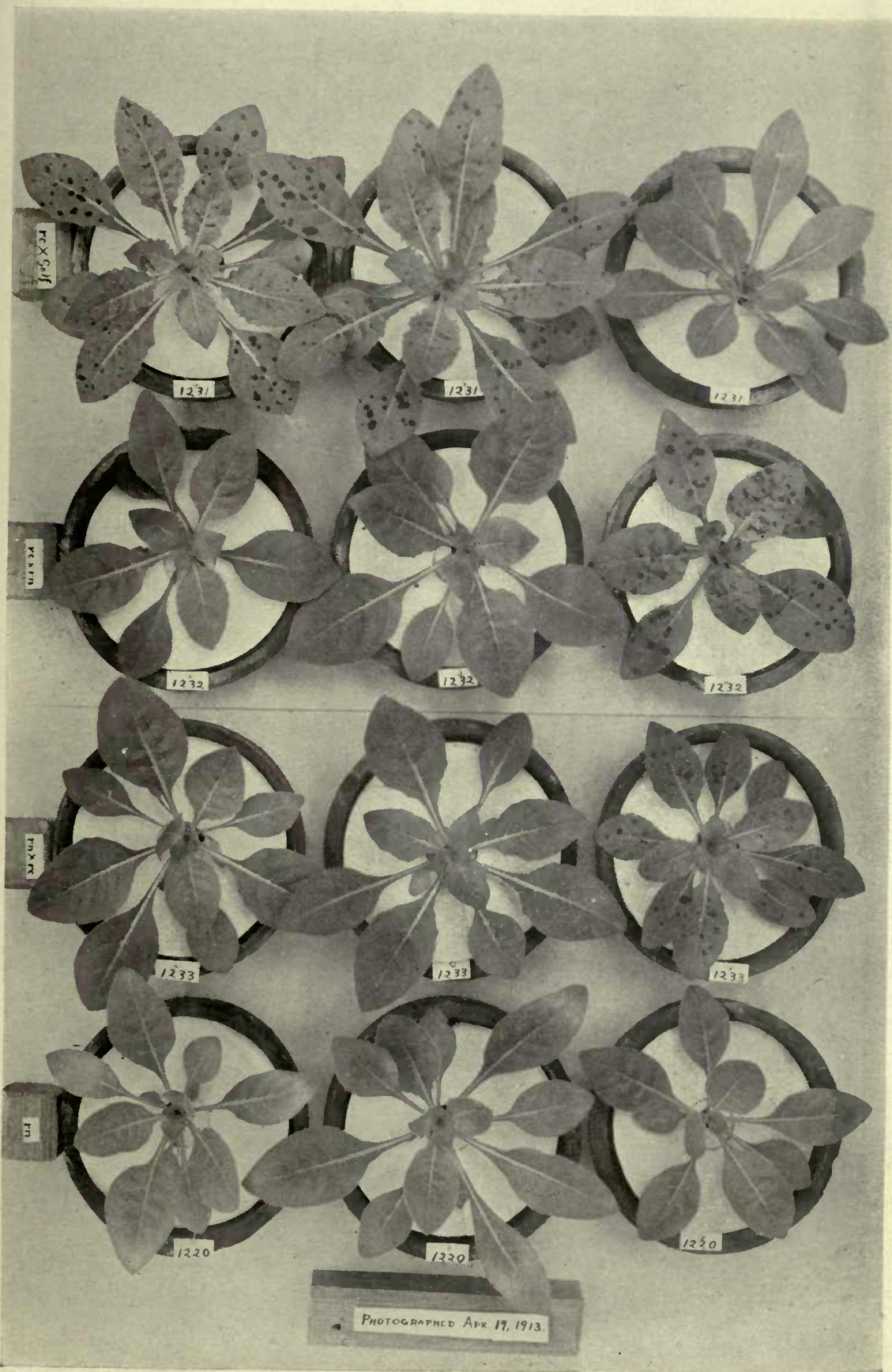





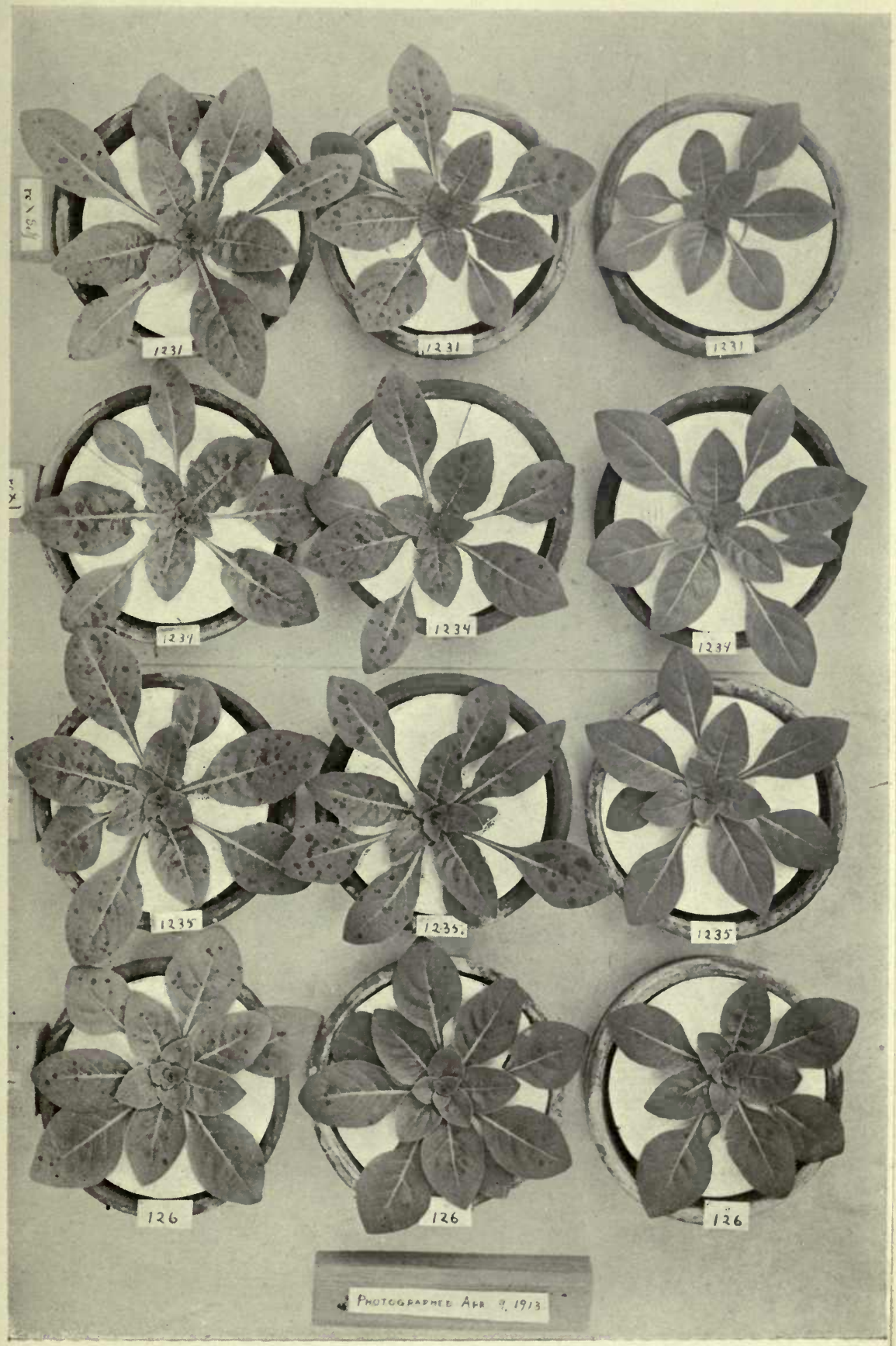


that the mere quantity of anthocyan which a plant produces does not make the difference between the color-pattern of 0 . rubricalyx and that of 0 . rubrinervis, for certainly those plants with red-spotted leaves and dark-red or brilliant-red stems, produced many times as much anthocyan as the greenish-stemmed plants, though only the latter had the rubricalyx-type of buds.

It is interesting to see that even Gates (1914) occasionally catches a glimpse of the fact that the difference between 0 . rubrinervis and 0 . rubricalyx is something more than a mere quantitative difference in the amount of red pigment produced by each. He says (1914, p. 246) "It is then perfectly clear that, although extent of pigment on the buds behaves, with very few exceptions, as a definite unit-character, showing the phenomena of dominance and absence; yet the amount of pigment is very probably reduced to a half in the $F_{1}$ hybrids, and it is certainly diluted very much (probably one-half) again on crossing back with grandiflora....The cause of the definiteness of distribution of the pigment in the buds is a problem in morphogenesis." After this statement one may be surprised to read (p. 270) that "the most careful observation and study shows that the red pigmentation-character $R$ is in the last analysis, inherited in quantitative fashion."

The Mendelian behavior of the rubricalyx-character was inferred by Gates from very insufficient evidence. When an attempt is made to interpret my results on a Mendelian basis, difficulties are encountered which seem at present nearly insurmountable. If we suppose that my original rubricalyx-plant (No. $11410(1))$ was a heterozygote in respect to two independent determiners for the rubricalyx-type of buds, the progeny secured by self-fertilizing this plant was in as close agreement with expectation, as the small number of plants would require, the ratio being $10 \cdot 7: 1$ instead of $15: 1$; but if there were no further complications, such a constitution for the rubricalyx parent would lead to the expectation of rubricalyx and non-rubricalyx in the ratio $3: 1$ in every cross between this plant and other plants which lacked both of the supposed determiners for the rubricalyx-character. Instead of this, the ratios in the four hybrid families here reported, were $0.91: 1,1 \cdot 44: 1$, $1 \cdot 80: 1$ and $2 \cdot 65: 1$, or if we combine the two rubricalyx-rubrinervis families, the result is $165: 183$ or nearly $1: 1$, while the two Lamarckiana crosses combined give $161: 75$, or about $2: 1$. Both of these ratios actually occur in typical Mendelian inheritance, and could be accounted for here by such additional assumptions as these; namely, $(a)$ that my rubrinervis plant, No. 1123(8), not only lacked the two independent 
rubricalyx-determiners, but that it was also heterozygous for a third gene which inhibits the action of one of the genes for the rubricalyxtype of pigmentation, but not the other ; and (b) that my Lumarckianaplant, No. 118(10), contained a factor whose union with one of the rubricalyx-determiners produces, in the absence of the other, a non-viable zygote. While such assumptions are perfectly proper as working hypotheses, they have no other value. Assumptions of a still more radical kind would have to be made to account for the peculiar alternative relation between the pigmentation of leaves and stems on the one hand and that of the buds on the other hand.

In the enormous mass of genetic data already recorded for the Oenotheras, there is but here and there a situation which bears more than a remote resemblance to a Mendelian behavior, and in these cases the observed phenomena usually present only a more or less misshapen caricature of the beautiful regularity of procedure which has such far-reaching applicability among many other groups of organisms. It appears to me undesirable therefore to speak of the rubricalyxcharacter as a Mendelian unit-character because it happened to constitute 75 per cent of one family of 44 plants. I believe that the only other character in Oenotheras which has been accepted as Mendelian in inheritance, namely, the brevistylis-character, may well be put to the test of a fuller genetic analysis.

In view of these facts, one can only view with astonishment the performance of Heribert-Nilsson (1912) in maintaining that the remarkable series of genetic puzzles presented by the Oenotheras can find an explanation through the recombinations of plural Mendelian determiners. His entire thesis falls to the ground the instant we begin to figure out some of the very simplest and most obvious consequences of such an explanation. His abandon in the application of this hypothesis was made possible only by his belief that students of the Oenotheras have generally failed to use strictly individual analysis in their investigations. Having myself never mixed the seeds from two different mothers or from two different crosses, I am unwilling to believe that HeribertNilsson has not greatly overestimated this source of difficulty in interpreting the genetic phenomena in Oenothera, although it is a valuable service to have pointed out so strongly as he has done the importance of the strictest possible adherence to the "isolation principle." The importance of this emphasis may be seen, when so careful a worker as Dr. Gates grows a culture from the mixed seed of four different selffertilized parents, belonging to a group in which there was obvious 
genotypic impurity, on the ground that "this made no difference in the experiments [he] then had in view" (Gates, $1913 \mathrm{~b}, \mathrm{p} .143$ ). It is a little difficult at the present time to imagine the nature of those genetic experiments which would not be injuriously affected by such an origin of the foundation stock. It can only be a matter for regret that Gates (1914) has also used for his so-called "reciprocal" crosses between 0 . rubricalyx and 0. grandiftora, a strain of the latter species from Alabama, for the one cross, and one from Birkenhead, England, for the "reciprocal" cross, especially in view of the fact that he has found much evidence of complex hybridizations in the latter locality (Gates, 1913 a). Finally, his "back-crosses" of the $F_{1}$ plants from this second hybrid family have been made not upon plants of the parental strain, but upon those of the Alabama strain. It is hardly to be hoped that the elements of genetic behavior in Oenothera will be discovered by these methods.

The conclusion reached by Gates (1910) that the method of inheritance of a character is determined by the nature of the character itself, is also brought sharply into question by the peculiar situation in my 0 . rubricalyx-crosses, for I am clearly dealing with the same character which Gates has repeatedly called a monohybrid Mendelian dominant; but in my cultures it is either not Mendelian at all, or, if Mendelian, is affected in a complex way by several different determiners. The remarkable diversity in the nature of the characters which have been proved to be typically Mendelian in inheritance in various plants and animals, should have made Gates's conclusion impossible. It is not the externally visible, physical or chemical nature of a character which determines the method of its inheritance, but the nature of the inheriting-"mechanism" to which it is related, and the manner in which its determiner or determiners are related to that mechanism. Baur (1910) has discovered a case which clearly illustrates this point, and I also have been able to confirm his results (Shull, 1914). We have shown that "chloralbinism" in plants may result from several different causes. In some cases the absence of chlorophyll is due to the absence of a definite Mendelian gene, inherited equally well through both the male and female gametes; in other cases it appears to be a purely cytoplasmic defect, inherited in characteristically non-Mendelian ways, sometimes only through the mother, sometimes through the father and the mother, but with such irregularity that its inheritance cannot be related to Mendelian phenomena. Whether Mendelian or non-Mendelian, the character itself appears to be the same, namely, the 
absence of the chloroplasts. Numerous investigators have shown that the anthocyan pigments of many plants are determined in quantity, quality and distribution by normal Mendelian genes, but the studies described in this paper, as well as those of Gates, strongly indicate that in Oenothera the inheritance of the red-pigmentation is determined by some other hereditary system, and the.same inference may be drawn from most of the other genetic phenomena thus far recorded for Oenothera. Further experimentation must discover a mechanism adequate for the interpretation of these genetic phenomena. Until that mechanism is found it will be impossible to decide what constitutes a unit-character in Oenothera, or to decide whether any particular genetic differentiation represents a case of segregation, "fractionation," or some other method of distribution of characters.

\section{SUMmary.}

An investigation of the genetic phenomena presented by Oenothera rubricalyx Gates and its hybrids has shown that the bright red hypanthia and cones of that species are separable in inheritance from the brilliant red stems with which, according to Gates's description, it was always associated in his cultures.

In the $F_{1}$ hybrids from reciprocal crosses between this species and O. rubrinervis and O. Lamarckiana a remarkable series of negative correlations appear in the distribution of the red pigment, the brilliantly pigmented buds characteristic of 0 . rubricaly $x$ being invariably associated with a low degree of red-pigmentation in the stems and rosettes; pinkconed buds with green hypanthia, characteristic of 0 . Lamarckiana, being on the other hand, invariably associated with brilliant red stems, while buds entirely free from anthocyan are associated with dull darkred stems.

A self-fertilized plant of 0 . rubricalyx produced offspring having rubricalyx-buds and green buds in the ratio $10 \cdot 7: 1$, all the greenbudded plants having nanella-stature and characteristic dark-red stems. One plant having rubricalyx-pigmentation was likewise of the dwarf type.

The ratio of rubricalyx-budded plants to non-rubricalyx in the crosses with rubrinervis was approximately $1: 1$, and in crosses with Lamarckiana $2: 1$.

By complicated auxiliary hypotheses these ratios could be explained in accord with Mendelian inheritance, but the fact that Oenothera apparently has a unique mechanism for the distribution of hereditary 
characters makes such subsidiary hypotheses of no value except as a basis for further investigations.

Other Oenothera crosses are cited, which indicate that the inverse relation between the red-pigmentation of the buds and that of the stems is not limited to erosses of 0 . rubricalyx. Oenothera rubrinervis represents a progressive variation in the pigmentation of the bud-cones and a retrogressive variation in the pigmentation of the stems.

It is held that three conclusions arrived at by Gates regarding the origin and genetic nature of the rubricalyx-character are erroneous, namely, (a) that the character represents a purely quantitative difference from 0 . rubrinervis, $(b)$ that it differs from the latter species in a single monohybrid Mendelian unit, and $(c)$ that the nature of a character itself, instead of the nature of the inheriting-mechanism to which it is related, determines the manner of inheritance of that character.

\section{LEGENDS FOR PLATES.}

PLATE V.

Rosettes of $O$. rubricalyx (upper series), O. rubrinervis (lower series) and of their reciprocal $F_{1}$ hybrids. Each set of 3 hybrid rosettes stands next to those of the seed-parent type. Inside diameter of pots about $7.5 \mathrm{~cm}$. Photographed April 19, 1913, about 11 weeks after the seeds were sown.

\section{PLATE VI.}

Rosettes of O. rubricalyx (upper series), O. Lamarckiana (lower series) and of their reciprocal $F_{1}$ hybrids. Arrangement the same as in Plate V. Photographed April 9, 1913 , about 10 weeks after the seeds were sown.

\section{LITERATURE CITED.}

BAUR, E. 1910. "Untersuchungen über die Vererbung von Chromatophorenmerkmalen bei Melandrium, Antirrhinum und Aquilegia. Zeitschr. f. ind. Abstamm. u. Vererb. Bd. Iv. pp. 81-102.

Heribert-Nilsson, N. 1912. "Die Variabilität der Oenothera Lamarckiana und das Problem der Mutation." Zeitschr. f. ind. Abstamm: u. Vererb. Bd. viII. pp. $89-231$.

Gates, R. R. 1909. " An Analytical Key to some of the Segregates of Oenothera." Ann. Rep. Missouri Bot. Gard. Vol. xx. pp. 123-137. See p. 133.

- 1910. "The Material Basis of Mendelian Phenomena." Amer. Nat. Vol. XLIV. pp. $203-213$. 
Gates, R. R. 1911. "Studies on the Variability and Heritability of Pigmentation in Oenothera." Zeitschr. f. ind. Abstamm. u. Vererb. Bd. Iv. pp. 337-372. 2 figs. of 0 . rubricalyx and 1 colored plate showing contrast between buds of 0 . rubricalyx and 0 . rubrinervis.

—_. 1912. "Mutations in Plants." Bot. Jour. Vol. II. pp. $84-87.1$ fig. of 0. rubricalyx.

—. 1913a. "A Contribution to a Knowledge of the Mutating Oenotheras." Trans. Linnean Soc. London, Botany, Vol. viII. pp. 1-67. 6 pls., including 6 figs. of 0 . rubricalyx.

1913b. "Tetraploid Mutants and Chromosome Mechanisms." Biol. Centralbl. Bd. xxxiIr. pp. $92-150$.

-. 1914. "Breeding Experiments which show that Hybridization and Mutation are Independent Phenomena." Zeitschr. f. ind. Abstamm. u. Vererb. Bd. XI. pp. 209-279. 3 figs. of 0 . rubricalyx.

Shull, G. H. 1914. "Über die Vererbung der Blattfarbe bei Melandrium." Ber. d. Deutsch. Bot. Gesell. Bd. xxxi. Generalversammlungs-Heft, pp. 40-80. 




\section{DIVISION OF GENETICS \\ HILGARD HALL}

[Reprinted from The American Naturalist, Vol. XLIX.. Jan., 1915]

\section{GENETIC DEFINITIONS IN THE NEW STANDARD DICTIONARY}

THE widely advertised aim of the Funk \& Wagnalls Company to include in their "New Standard Dictionary of the English Language " all of the new additions to scientific terminology naturally invites the specialist in each branch of science to examine the definitions of the new words in his own field. Professor Miller ${ }^{1}$ has called attention to the fact that the mathematical definitions are not reliable. The same criticism must be made regarding the definitions of many terms now familiar in the literature of genetics. For some of the errors in these definitions the editorial staff can not be blamed, because the errors were passing current among genetic writers themselves, at a time when further changes in the dictionary probably became impossible; other errors are less easily explained. While such a monumental work as the Standard Dictionary tends to fix the usage of language, the shorteomings of the genetic definitions may not be expected to seriously affect the terminology actually used by the specialists in this fietd; but for those who are engaged in other scientific. fields, who have only a casual interest in geneties, and who must, therefore, depend upon the dictionary for the meaning of any genetic terms they may happen to meet, the erroneous definitions are unfortunate. While very few of the genetic definitions are free from defects, either of omission or of commission, only those which seem most obviously defective will be considered here. In the following list of words the definition of the New Standard Dictionary is stated first, and then follows, in italic type, a definition which I believe will meet with the approval of most geneticists.

Acquired. Transmitted by inheritance to subsequent generations; as, acquired characters.

Acquired character. A modification of bodily structure or habit which is impressed on the organism in the course of individual life.

Both of these definitions occur in the New Standard Dictionary, the first under "acquired," the second under "character." Although "impressed on" may not be the best figure of speech to use in this connection, the second definition represents fairly well the correct usage of this phrase. It is difficult to understand why

1 Science, N. S., 38: 772, November 28, 1913. 
essentially the same definition should not have been given at both places.

Allelomorph. "In Mendelian inheritance a pair of contrasted characters which become segregated in the formation of reproductive cells."

Allelomorph. One of a pair of contrasted characters which are alternative to each other in Mendelian inheritance. Often used with doubtful propriety as a synonym for gene, factor or determiner.

The defects in the dictionary definition in this case are two: (a) The definition is plural, while "allelomorph" is singular; the "allelomorph" is not a pair of characters, but a single character. (b) No segregation of allelomorphs takes place in the formation of asexual reproductive cells.

Allelomorphism. "The presence of allelomorphic pairs of characters."

Allelomorphism. A relation between two characters, such that the de. terminers of both do not enter the same gamete, but are separated into sister gametes.

Alternative inheritance. "The transmission to alternating generations of descendants of the characteristies of either parent, as that of the father to the odd, and of the mother to the even generations."

Alternative inheritance. A distribution of contrasting parental or ancestral characters among offspring or descendants, such that the individuals exhibit one or other of the characters in question, combinations or blends of these characters being absent or exceptional.

Biotype. "In Mendelian inheritance a race or strain that breeds true or almost true; a term introduced by Johannsen."

Biotype. A group of individuals all of which have the same genotype.

The word "biotype" was introduced into English by Dr. Johannsen $^{2}$ in 1906 with the definition "one single 'sort' of organisms." It is a term of general applicability and not limited to Mendelian races, as stated in the New Standard Dictionary. Although homozygous biotypes generally do breed true, this is not an essential feature and therefore should not be included in the definition. Ever-sporting varieties are now well known which do not breed true, but which, so far as present evidence goes, do constitute single homozygous biotypes. Heterozygous biotypes generally do not breed true.

Clon. "A plant-group the members of which have been grown from an original stock, but which do not come true from seed."

Clone. A group of individuals produced from a single original individual by some process of asexual reproduction, such as division, budding, stipping, grafting, parthenogenesis (when unaccompanied by a reduction of the chromosomes), etc.

There are several defects in the dictionary definition of this word, even if restricted to a plant-group in accord with the original meaning given to it by Webber, who introduced the word.

2 Report of the third International Conference of Genetics, p. 98. 
The defects consist, first, in the ambiguity of the word "stock," because we may grow plants "from an original stock" of seeds, quite as well as from cuttings, while a clone is derived from a single individual; second, the statement that clones do not come true from seed is incorrect, for a clone formed by cuttings, etc., from a homozygous individual does "breed true," $i$. e., it produces seedling offspring of its own type. The word is now being generally applied to animals as well as to plants.

Coupling. ("Genetic coupling" is not defined in the dictionary.) Such a relation between the genes of two unit-characters that they have a more or less marked tendency to be included in the same gamete when the individual is heterozygous for both of the genes in question.

Cross-over. (Not given a genetic definition in the dictionary.) A separation into different gametes, of determiners that are usually coupled, and the association of determiners in the same gamete, which are generally allelomorphic.

Cryptomere. "A plant character which may exist in the germ-cells without making its presence visible."

Cryptomere: A factor or gene whose presence can not be inferred from an inspection of the individual, but whose existence can be demonstrated by means of suitable crosses.

The chief defect in the dictionary definition is the restriction of this term to plant characters. "Cryptomere" is a general genetic term which may be applied as well to animals as to plants.

Determiner. "The same as determinant 3."

Determiner. An element or condition in a germ-cell which is essential to the development of a particular feature, quality or manner of reaction of the organism which arises from that germ-cell; a gene or factor.

The word "determiner," as used in recent years, is not the equivalent of "determinant 3," which latter is correctly defined in the dictionary in terms of Weismann's complicated hypothesis. "Determiner," "factor" and "gene" are now quite generally used interchangeably without implication as to their fundamental nature, simply in the generic sense, as "that which determines."

Dominance. "In the cross-bred offspring of parents with marked mutually antagonistic characteristics, the exhibition by such offspring or its descendants of one of these characteristics to the exclusion of the other."

Dominance. In Mendelian hybrids the capacity of a character which is derived from only one of the two generating gametes to develop to an extent nearly or quite equal to that exhibited by an individual which has derived the same character from both of the generating gametes. In the absence of dominance the given character of the hybrid usually presents a "blend" or intermediate conldition between the two parents, but may present new features not found in either parent.

There are several defects in the dictionary definition. In the first place, the parents used in a given cross may not themselves 
be homozygous, in which case some of their offspring will resemble one parent and some the other; in such a case, according to the dictionary, both of the contrasted characters would exhibit dominance. The phrase "or its descendants" would make it possible, in any case, to include both recessives and dominants, since among the descendants of such cross-bred individuals there will also be recessive individuals which "exhibit one of the characteristics to the exclusion of the other."

Dominant. "(1) A marked parental character exhibited by a cross-bred organism and its descendants. (2) The parent, cross-bred organism, or descendant exhibiting such character. Parental characters latent in a crossbred organism, but actively evidenced by its descendants, are called recessives, as are the descendants which exhibit them."

Dominant. (1) A character which exhibits dominance, i. e., that one of two contrasted parental characters which appears in the individuals of the first hybrid generation to the exclusion of the alternative, "recessive," character. (2) An individual possessing a dominant character, in contrast to those individuals which lack that character, which are called "recessives."

An "extracted dominant," as defined in the dictionary, is not distinguishable from the pure homozygous dominant used in the cross from which the dominant in question was "extracted," as no mention is made of the essential historical fact that it is of hybrid origin and that its parent or other known ancestor did not breed true to the same dominant character.

Factors. "Latent physiological units which upon crossing give rise to the new characters found in the hybrid."

Factor. An independently inheritable element of the genotype whose presence makes possible any specific reaction or the development of any particular unit-character of the organism which possesses that genotype; a gene or determiner.

The limitation of the term "factor" to those cases in which new characters appear in hybrids, is not in accord with present usage. All the various characters of organisms are to an important degree dependent upon the existence of genotypic factors, regardless of the behavior of these organisms in crosses.

Gen. "A minute hypothetical particle supposed to be the bearer of hereditary qualities."

Gene. An element of the genotype; a genetic factor; a determiner.

The treatment of this word in the dictionary is particularly mischievous. When I introduced the word "gene" to Englishreading students, I said:" "This word is proposed by Dr. Johannsen ... to denote an internal something or condition upon whose presence an elementary morphological or-physiological characteristic depends. The word 'gene' has the advan-

3 Am. NaT., 43, p. 414, 1909. 
tage that it does not assume by its form or derivation any hypothesis as to the ultimate character, origin or behavior of the determining factor." In adopting the word "Gen" in the German, Johannsen said:4 "Das Wort Gen ist völlig frei von jeder Hypothese; es drückt nur die sichergestellte Tatsache aus, dass jedenfalls viele Eigenschaften des Organismus durch in den Gameten vorkommende besondere, trennbare und somit selbständige 'Zustände,' 'Grundlagen,' 'Anlagen'-kurz, was wir eben Gene nennen wollen-bedingt sind. ... die Gene sehr vieler Eigenschaften glatt trennbar sind, während andere nicht oder nicht glatt sich trennen. Dies alles erinnert an das Verhalten chemischer Körper. Damit ist aber noch gar nicht gesagt, dass die Gene selbst chemische Gebilde oder Zustände seien-darüber wissen wir vorläufig noch gar nichts." How different is all this from "a minute hypothetical particle"! It is obviously improper, therefore, to define a gene as a "minute particle." Neither is it correct to say that it is "supposed to be the bearer of hereditary qualities." It is only the something of unascertained nature, which must lie at the foundation of any elementary hereditary quality. The spelling "gene" is not even mentioned in the dictionary as a variant, yet this was the original spelling and is now in practically universal use among geneticists, while no one uses "gen."

Genotype. "A race of organisms different from another in its hereditary qualities; contrasted with phenotype."

Genotype. The fundamental hereditary constitution or sum of all the genes of an organism.

The unfortunate definition of "genotype" given in the dictionary was current in America at the time when the dictionary forms were probably closed, so that the editors are not in any way to blame for the totally erroneous definition. The definition given by the dictionary for "genotype" fits fairly well the word "biotype."

Heredity. "The tendency manifested by an organism to develop in the likeness of a progenitor."

Heredity. The distribution of genotypic elements of ancestors among the descendants; the resemblance of an organism to its parents and other ancestors with respect to genotypic constitution.

The results of modern experimental work on heredity show that the definition given by the dictionary is entirely too restricted. Heredity must be so defined that it may apply to characters that were never exhibited by any ancestor.

Heterozygosity. "In Mendelian inheritance, the state or condition due to an organism having developed from a heterozygote."

4 “Elemente der exakten Erblichkeitslehre," 1. Auf., 1909, pp. 124-125. 
Heterozygosity. The condition of an organism due to the fact that it is a heterozygote; the state of being heterozygous; the extent to which an individual is heterozygous.

Heterozygote. "A Mendelian hybrid resulting from the fusion of two gametes that bear different allelomorphs of the same character and which in consequence does not breed true; contrasted with homozygote."

Heterozygote. A zygotic individual in which any given genetic factor has been derived from only one of the two generating gametes. Both eggs and sperms produced by such an individual are typically of two kinds, half of them containing the gene in question, the rest lacking this gene; consequently the off spring of heterozygotes usually consist of a mixture of individuals, some of which possess the corresponding character while others lack it.

Homozygosis. "Development from a zygote originating from a union of two gametes of the same kind."

Homozygosis. The state of being homozygous; the extent to which an individual is homozygous.

Homozygote. "A zygote formed by the conjugation of two gametes of the same stock; any animal or plant that receives and retains the dominant or recessive characters of both its parents, and is therefore said to be true to type, and breeds true to type."

Homozygote. An individual in which any given genetic factor is doubly present, due usually to the fact that the two gametes which gave rise to this individual were alike with respect to the determiner, in question. Such an individual, having been formed by the union of like gametes, in turn generally produces gametes of only one kind with respect to the given character, thus giving rise to offspring which are, in this regard, like the parents; in other words, homozygotes usually "breed true." A "positive" homozygote with respect to any character contains a pair of determiners for that character, while a "negative" homozygote lacks this pair of determiners.

"Two gametes of the same stock" is ambiguous because of the indefiniteness of the word "stock." Many homozygotes receive some dominant and some recessive characteristics of the two parents; and what can be intended by the statement that a plant or animal which receives certain characteristics also "retains" them? How could it do otherwise?

Hypostasis. (Not given a genetic definition in the dictionary.) That relation of a gene in which its usual reaction fails to appear because of the masking or inhibitory effect of another gene; contrasted with "epistasis."

The corresponding adjective "hypostatic" is also not given a genetic definition in the dictionary.

Mendelize. "To cause to follow Mendel's law of inheritance."

Mendelize. To follow Mendel's law of inheritance.

The word is rightly indicated in the dictionary, as an intransitive verb; it is manifestly incorrect to define it by the use of a transitive verb. 
Mutant. "That which admits of or undergoes mutation or change; specifically, an individual or a species which shows significant changes in form or character in a single generation."

Mutant. An individual possessing a genotypic character differing from that of its parent or those of its parents, and not derived from them by a normal process of segregation.

The expression "significant changes" is ambiguous, since every change is significant of something.

Mutate. "To 'sport.',"

Mutate. To undergo a change in genotypic character independently of normal segregation.

The word "sport" which is used in the dictionary definition of "mutate" is defined thus: "To vary suddenly or spontaneously from the normal type; said of an animal or plant or of one of its parts." It is well known that many such sudden and spontaneous variations from the normal type are not due to mutations. The word "mutation" is defined in the dictionary as "a permanent transmissible yariation in organisms, as distinct from fuctuation." This definition is good as far as it goes, but should expressly exclude transmissible variations which are due to normal segregation and recombination of determiners.

Phenotype. "A type or strain of organisms distinguishable from others by some character or characters, whether their observable differences from other organisms be due to their inherent hereditary differences or to the direct action of the environment upon them: contrasted with genotype."

Phenotype. The apparent type of an individual or group of individuals, i. e., the sum of the externally obvious characteristics which an individual possesses, or which a group of individuals possesses in common; contrasted with genotype.

"Phenotype" and "genotype" are both abstractions; the qualities which distinguish the phenotype are always capable of direct observation, while those of the genotype can only be inferred from the results of genetic experiments.

Presence and absence hypothesis, "in the Mendelian doctrine of inheritance, the theory that an allelomorphic pair of characters in every zygote has two contrasted factors or determinants, one representing the positive character of the generated organism and the other denoting its absence."

Presence and absence hypothesis. The hypothesis that any simple Mendelian difference between two individuals, results solely from the presence of a factor in the genotype of the one individual, which is absent from that of the other. Presence and absence of unit-differences as a convenient method of describing the results of genetic experiments should be carefully distinguished from the presence and absence hypothesis. The method is purely objective and entirely free from hypothetical implications.

It will be noted that the dictionary definition of this phrase is directly opposite in significance to the one here set forth. 
Pure line (Not included in the dictionary.) A group of individuals derived solely by one or more self-fertilizations from a common homozygous ancestor. Sometimes erroneously applied to groups of individuals believed to be genotypically homogeneous (a homozygous biotype or a clone) without regard to their method of reproduction.

Repulsion. (Not given a genetic definition in the dictionary.) Such a relation between two genetic factors that both are not, as a rule, included in the same gamete, referring especially to cases in which the factors in question give rise to obviously different characteristics; also called "spurious allelomorphism."

Sex-limited inheritance. (Not defined in the dictionary.) The association of the determiner for any unit-character, with a sex-determiner, in such a manner that the two determiners are either generally included in the same gamete, or that they are generally included in different gametes. This method of inheritance is also called "sex-linked" inheritance by Professor T. H. Morgan and his students.

Segregate. "To become separated from the rest; specif., of Mendelian hybrids, to separate, by a numerical law, into dominants, hybrids and recessives."

Segregate. With reference to Mendelian unit-characters, to become separated through the independent distribution of the genetic factors before or at the time of the formation of the gametes.

The dictionary definition goes too far; the formation of dominants, hybrids and recessives depends not alone upon the fact that the factors segregate, but that the segregated factors recombine. The word "segregation" receives a fairly satisfactory definition.

Unit-character. (Not included in the dictionary.) In Mendelian inheritance a character or alternative difference of any kind, which is either present or absent, as a whole, in each individual, and which is capable of: becoming associated in new combinations with other unit-characters.

I have made no systematic study of the definitions of technical terms in other related fields, but have noted incidentally that there is no recognition in the New Standard Dictionary of the generally familiar usage of the words "meristic" and "substantive" as applied to types of variation.

G. H. SHULL 





\section{RETURN TO the circulation desk of any University of California Library}

NORTHERN REGIONAL Or the

BIdg. 400, Rich ONAL LIBRARY FACILITY

Univer, Richmond Field Station

Richersity of California

Richmond, CA 94804-4698

ALL BOOKS MAY BE RECALLED AFTER 7 DAYS

- 2-month loans may be renewed by calling

books to NRLF

- Renewals

days prior a recharges may be made 4 days prior to due date.

DUE AS STAMPED BELOW

SEP 2 21998 


\section{U.C. BERKELEY LIBRARIES}

QH 308

S 53

1905-15 
